Sven Wydra

Produktions- und

Beschäftigungseffekte neuer Technologien 
Sven Wydra

\section{Produktions- und Beschäftigungseffekte neuer Technologien}

Die Biotechnologie wird häufig als eine sehr bedeutende Technologie des 21 . Jahrhunderts angesehen. Allerdings sind die empirischen Erkenntnisse über ihre wirtschaftlichen Effekte bislang gering. Daran setzt diese Arbeit an. Auf der Basis von theoretischen Wirkungszusammenhängen untersucht der Autor mögliche zukünftige Produktions- und Beschäftigungseffekte der Biotechnologie. Dabei werden in verschiedenen Szenarien für das Jahr 2020 technologische Entwicklungen für verschiedene Anwendungsfelder projiziert und mit einem Input-Output-Modell gekoppelt. Die Ergebnisse ermöglichen Aussagen über die Nettoveränderung der Produktion und Beschäftigung sowie Veränderungen im sektoralen Strukturwandel durch die Diffusion der Biotechnologie.

Sven Wydra studierte Wirtschaftswissenschaften an der Universität Hohenheim. Seit 2005 ist er Wissenschaftlicher Mitarbeiter und seit 2009 Projektleiter am Fraunhofer Institut für System- und Innovationsforschung im Competence Center „Neue Technologien“ in Karlsruhe. 
Produktions- und Beschäftigungseffekte neuer Technologien 


\section{Hohenheimer Volkswirtschaftliche Schriften}

Herausgegeben von

Prof. Dr. Michael Ahlheim, Prof. Dr. Thomas Beißinger, Prof. Dr. Ansgar Belke, Prof. Dr. Rolf Caesar, Prof. Dr. Gabriel Felbermayr, Prof. Dr. Harald Hagemann, Prof. Dr. Klaus Herdzina,Prof. Dr. Walter Piesch, Prof. Dr. Andreas Pyka,

Prof. Dr. Ingo Schmidt, Prof. Dr. Ulrich Schwalbe, Prof. Dr. Peter Spahn, Prof. Dr. Jochen Streb, Prof. Dr. Gerhard Wagenhals,

\section{Band 62}

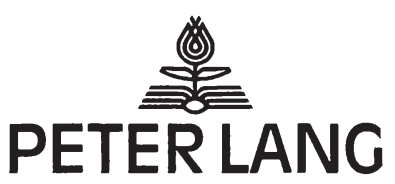

Frankfurt am Main · Berlin · Bern · Bruxelles · New York · Oxford · Wien 


\section{Sven Wydra}

\section{Produktions- und Beschäftigungseffekte neuer Technologien Am Beispiel der Biotechnologie}

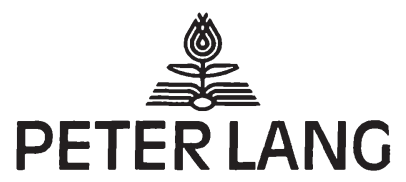

Frankfurt am Main · Berlin · Bern · Bruxelles - New York · Oxford · Wien 


\section{Bibliografische Information der Deutschen Nationalbibliothek}

Die Deutsche Nationalbibliothek verzeichnet diese Publikation in der Deutschen Nationalbibliografie; detaillierte bibliografische Daten sind im Internet über http://dnb.d-nb.de abrufbar.

Open Access: The online version of this publication is published on www.peterlang.com and www.econstor.eu under the international Creative Commons License CC-BY 4.0. Learn more on how you can use and share this work: http://creativecommons.org/licenses/ by/4.0.

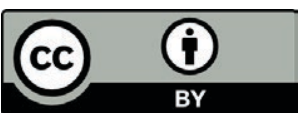

This book is available Open Access thanks to the kind support of ZBW - Leibniz-Informationszentrum Wirtschaft.

Zugl.: Hohenheim, Univ., Diss., 2009

Gedruckt auf alterungsbeständigem, säurefreiem Papier.

$$
\text { D } 100
$$

ISSN 0721-3085

ISBN 978-3-631-59602-9

ISBN 978-3-631-75392-7 (eBook)

(C) Peter Lang GmbH Internationaler Verlag der Wissenschaften

Frankfurt am Main 2010

Alle Rechte vorbehalten.

Das Werk einschließlich aller seiner Teile ist urheberrechtlich geschützt. Jede Verwertung außerhalb der engen Grenzen des

Urheberrechtsgesetzes ist ohne Zustimmung des Verlages unzulässig und strafbar. Das gilt insbesondere für Vervielfältigungen, Übersetzungen, Mikroverfilmungen und die Einspeicherung und Verarbeitung in elektronischen Systemen. 


\section{Vorwort}

Die vorliegende Arbeit ist im Rahmen meiner Tätigkeit als wissenschaftlicher Mitarbeiter am Fraunhofer Institut für System- und Innovationsforschung (ISI) in Karlsruhe entstanden. Die Arbeit wurde als Dissertation an der Universität Hohenheim angenommen. Für die Betreuung der Arbeit möchte ich mich bei Herrn Prof. Dr. Harald Hagemann sehr bedanken. Ebenso danke ich Herrn Prof. Dr. Gerhard Wagenhals für die Übernahme des Zweitgutachtens.

Große Unterstützung und Freiraum für diese Arbeit fand ich bei meinen Kolleginnen und Kollegen in meinem Competence Center „Neue Technologien“ und anderen Kollegen am Institut. Dabei möchte ich mich herzlich bei meinem internen Betreuer Prof. Dr. Michael Nusser für die Hilfe und die anregenden Diskussionen bedanken. Daneben haben mir in zahlreichen Gesprächen PD Dr. Rainer Walz, Dr. Sibylle Gaisser, Dr. Bärbel Hüsing, Dr. Ralf Lindner, Dr. Frank Marscheider-Weidemann und Dr. Thomas Reiss sehr weitergeholfen.

Mein ganz besonderer Dank gilt Gaby Somaggio für ihr großes Verständnis und ihre tolle Unterstützung.

Sven Wydra

Karlsruhe, August 2009 
Sven Wydra - 978-3-631-75392-7

Downloaded from PubFactory at 01/11/2019 05:32:25AM

via free access 


\section{Inhaltsverzeichnis}

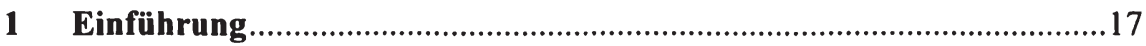

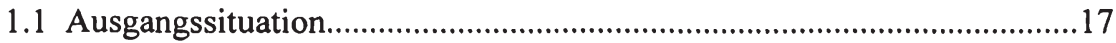

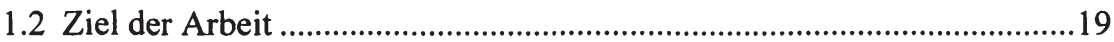

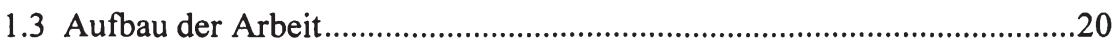

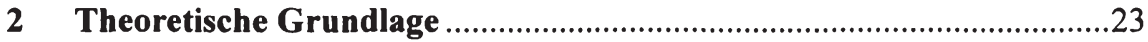

2.1 Konzeptionelle Erläuterungen .............................................................23

2.1.1 Charakterisierung von Technologien und technischer Fortschritt ...23

2.1.2 Definition und Charakterisierung von Innovationen........................24

2.1.3 Analyseebenen der wirtschaftlichen Effekte von Technologien

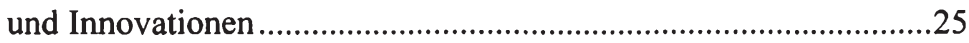

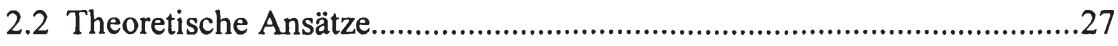

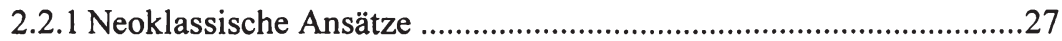

2.2.2 Post-Keynesianische Ansätze ..........................................................30

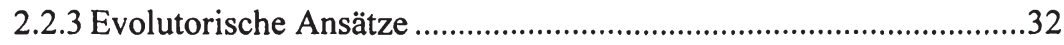

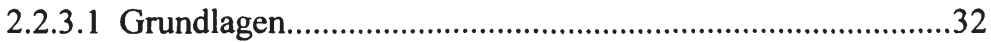

2.2.3.2 Konzept der General Purpose Technologies ........................40

2.2.4 Kritische Würdigung ....................................................................50

2.3 Auswirkungen neuer Technologien auf Beschäftigung und Produktion ..52

2.3.1 Produktivitätswirkung von Prozessinnovationen .............................54

2.3.2 Produktinnovationen ......................................................................57

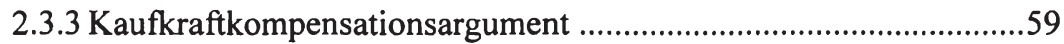

2.3.4 Maschinenherstellungsargument und sektoraler Strukturwandel ....62

2.3.5 Internationale Wettbewerbsfähigkeit und Außenhandel ..................64

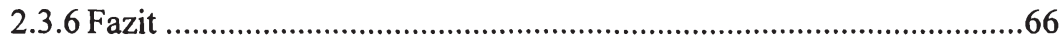

3 Charakterisierung und Entwicklung der Biotechnologie .....................69

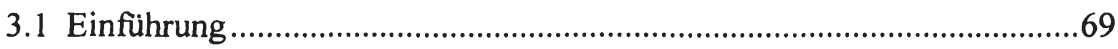

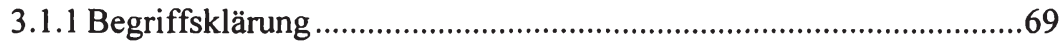

3.1.2 Historische Entwicklung................................................................70

3.1.3 Wissenschafts- und Technikvielfalt der Biotechnologie...................71 
3.1.4 Bedeutung der Biotechnologie in F\&E und Anwendung.................72

3.1.5 Anwendungsfelder der Biotechnologie ...........................................74

3.1.5.1 Pharmazeutika und Gesundheit .........................................75

3.1.5.2 Industrielle Anwendungen ..................................................77

3.1.5.3 Landwirtschaft und Lebensmittel (Agro-Food)....................79

3.1.5.4 Umweltbiotechnologie..........................................................80

3.2 Biotechnologie als General Purpose Technology ....................................81

3.2.1 Erfüllung der Charakteristika einer GPT? .......................................81

3.2.2 Diffusion und Entwicklung der Biotechnologie...............................85

3.2.2.1 Bisherige kommerzielle Entwicklung ................................85

3.2.2.2 Hohe Erwartungshaltung und schwierige Messbarkeit von

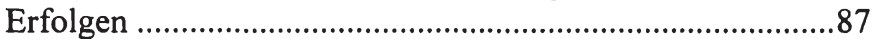

3.2.2.3 Technologische Charakteristika und Entwicklungs-

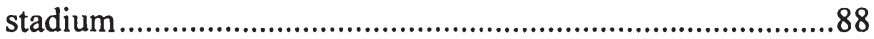

3.2.2.4 Kosten- und zeitintensive Anpassungsprozesse ...................90

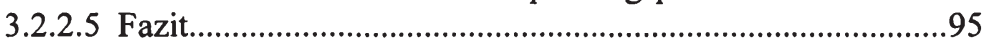

3.3 Wirkungsmechanismen für Produktions- und Beschäftigungswirkungen

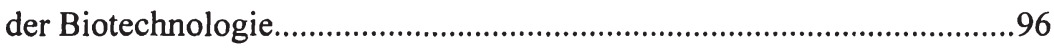

4 Messung von wirtschaftlichen Effekten der Biotechnologie.................101

4.1 Überblick zur Messung wirtschaftlicher Effekte von Technologien ......101

4.2 Indikatoren für die wirtschaftliche Bedeutung der Biotechnologie ........102 4.2.1 Relevanz der Technologiecharakteristika für die Indikatoren-

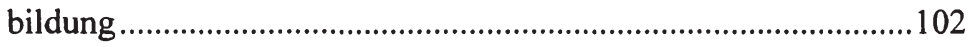

4.2.2 Messung des "Biotechnologiesektors" ...........................................105

4.2.2.1 Erfassung der Biotechnologieunternehmen........................105

4.2.2.2 Erfassung der Biotechnologie in der Produktionsstatistik.. 107

4.2.3 Technologischer Fortschritt und Diffusion der Biotechnologie.....108

4.2.3.1 Indikatoren für den technologischen Wandel.....................108

4.2.3.2 Indikatoren für die Diffusion in Anwendersektoren ..........110

4.2.4 Wirtschaftliche Effekte der Biotechnologie ...................................112

4.3 Input-Output-Analyse für die wirtschaftlichen Effekte neuer

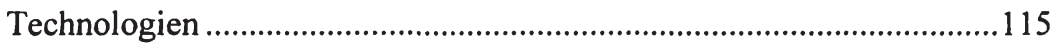

4.3.1 Darstellung der Input-Output-Analyse .......................................115

4.3.1.1 Input-Output-Tabelle ......................................................115 
4.3.1.2 Grundformen des Input-Output-Modells...........................117

4.3.1.3 Erweiterungen des Input-Output-Modells..........................119

4.3.2 Bestimmungsgründe für die Änderung von Koeffizienten .............121

4.3.3 Zukünftige Technologieentwicklung im Input-Output Modell......123

4.3.3.1 Übersicht zu Methoden der Projektion................................123

4.3.3.2 Vorgehensweise bei pragmatischen Ansätzen.....................124

4.3.4 Stärken und Schwächen der Input-Output-Analyse .......................132

4.3.4.1 Vergleich zu anderen gesamtwirtschaftlichen Modellen ...133

4.3.4.2 Allgemeine Einschränkungen bei Modellösungen..............135

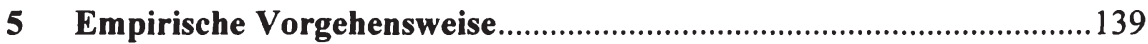

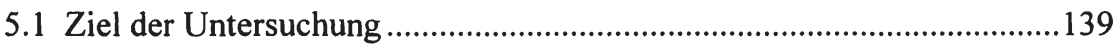

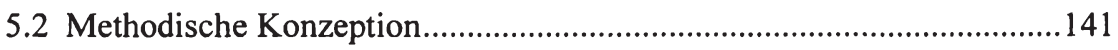

5.2.1 Auswahl der betrachteten Anwendungsfelder.................................141

5.2.2 Erfassung und Modellierung der Wirkungszusammenhänge..........143

5.2.3 Bildung von Szenarien............................................................151

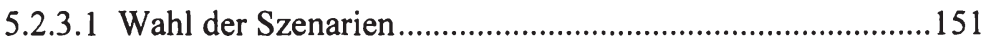

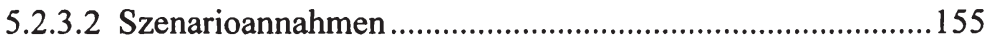

5.2.4 Bestimmung der ökonomischen Impulse ........................................ 164

5.2.5 Input-Output-Modellrechnungen .................................................167

6 Auswirkungen der Biotechnologie in ausgewählten

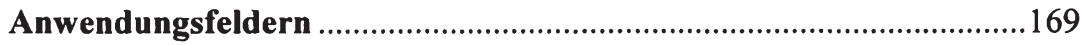

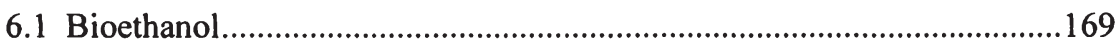

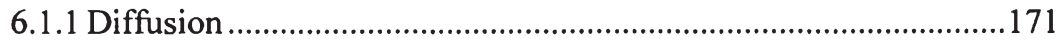

6.1.2 Internationaler Wettbewerb und Außenhandel..............................174

6.1.3 Herstellungskosten für Bioethanol ...............................................175

6.1.4 Zusammenfassende Darstellung der Szenariowerte........................181

6.1.5 Produktions- und Beschäftigungseffekte.....................................185

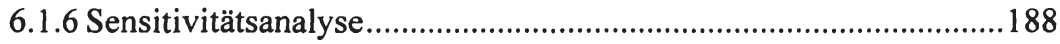

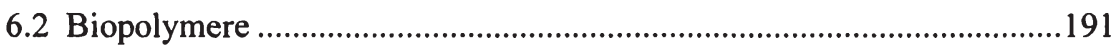

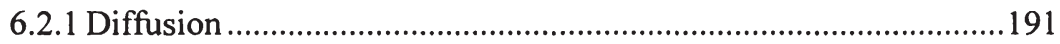

6.2.2 Internationaler Wettbewerb und Außenhandel................................194

6.2.3 Herstellungskosten für Biopolymere..........................................195 
6.2.4 Zusammenfassende Darstellung der Szenariowerte ........................200

6.2.5 Produktions- und Beschäftigungseffekte.......................................205

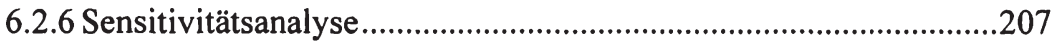

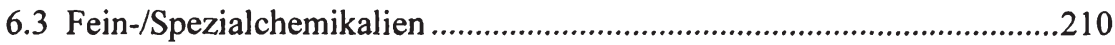

6.3.1 Diffusion und internationaler Wettbewerb...................................211

6.3.2 Bedeutung der Biotechnologie für Produktinnovationen...............213

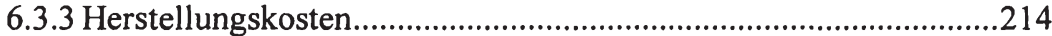

6.3.4 Zusammenfassende Darstellung der Szenariowerte.......................218

6.3.5 Produktions- und Beschäftigungseffekte........................................221

6.3.6 Sensitivitätsanalyse ........................................................................2223

6.4 Biopharmazeutika..............................................................................226

6.4.1 Diffusion und internationaler Wettbewerb..................................227

6.4.2 Bedeutung der Biotechnologie für Produktinnovationen................232

6.4.3 Effizienz in Forschung und Herstellung........................................235

6.4.4 Zusammenfassende Darstellung der Szenariowerte ........................241

6.4.5 Produktions- und Beschäftigungseffekte.......................................243

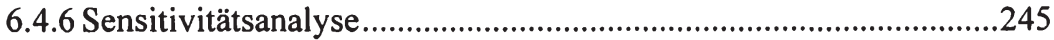

6.5 Zusammenfassende Ergebnisse und Diskussion der Modell-

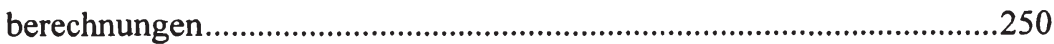

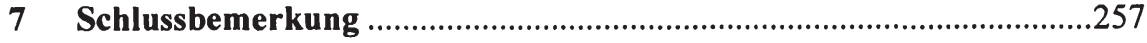

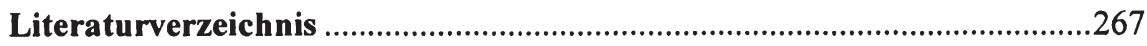

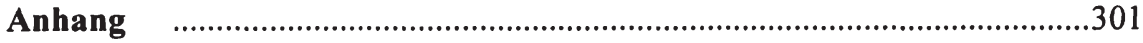




\section{Abbildungsverzeichnis}

Abbildung 1: Analyseebenen wirtschaftlicher Auswirkungen neuer Technologien

Abbildung 2: Reichweite und Innovationskomplementaritäten einer GPT.

Abbildung 3: Produktionsprozess in der ,structuralist evolutionary“ Dekomposition

Abbildung 4: Entwicklung der Biotechnologie-Patente (Index $1980=100)$

Abbildung 5: Anwendersektoren der Biotechnologie .75

Abbildung 6: Wertschöpfungskette von (Bio-)Pharmazeutika .76

Abbildung 7: Einsatz der industriellen Biotechnologie in verschiedenen Wertschöpfungsketten

Abbildung 8: Biotechnologie in der Wertschöpfungskette im AgroFood Bereich .80

Abbildung 9: Rahmen zur Erfassung der Akteure und Indikatoren für die Biotechnologie.

Abbildung 10: Input-Output-Tabelle

Abbildung 11: Vorgehensweise zur Ermittlung gesamtwirtschaftlicher Effekte der Biotechnologie.

Abbildung 12: Modellierung der Auswirkungen von Produkt/Prozessinnovationen auf die Nachfrage

Abbildung 13: Vorgehen der Szenariobildung

Abbildung 14: Flächen- und Verwendungskonkurrenzen von pflanzlichen Erzeugnissen

Abbildung 15: Nettoproduktionswirkungen durch zusätzliche Diffusion von Bioethanol im Vergleich zum Basisszenario (in Mio. €). 
Abbildung 16: Nettobeschäftigungswirkungen durch zusätzliche Diffusion von Bioethanol im Vergleich zum Basisszenario (in Erwerbstätige).

Abbildung 17: Nettoproduktionswirkungen für Bioethanol in Sensitivitätsrechnungen im Vergleich zum Basisszenario (in Mio. €) 190

Abbildung 18: Nettobeschäftigungswirkungen für Bioethanol in Sensitivitätsrechnungen im Vergleich zum Basisszenario (in Erwerbstätige) 190

Abbildung 19: Nettoproduktionswirkungen durch zusätzliche Diffusion von Biopolymeren im Vergleich zum Basisszenario (in Mio. €) 206

Abbildung 20: Nettobeschäftigungswirkungen durch zusätzliche Diffusion von Biopolymeren im Vergleich zum Basisszenario (in Erwerbstätige) 207

Abbildung 21: Nettoproduktionswirkungen für Biopolymere in Sensitivitätsrechnungen im Vergleich zum Basisszenario (in Mio. €). 209

Abbildung 22: Nettobeschäftigungswirkungen für Biopolymere in Sensitivitätsrechnungen im Vergleich zum Basisszenario (in Erwerbstätige) .209

Abbildung 23: Nettoproduktionswirkungen durch zusätzliche Diffusion der Biotechnologie bei Fein-/Spezialchemikalien im Vergleich zum Basisszenario (in Mio. $€$ ).

Abbildung 24: Nettobeschäftigungswirkungen durch zusätzliche Diffusion der Biotechnologie bei Fein-/Spezial-chemikalien im Vergleich zum Basisszenario (in Erwerbstätige)

Abbildung 25: Nettoproduktionswirkungen für biotechnologische Produktion von Fein-/Spezialchemikalien in Sensitivitätsrechnungen .225

Abbildung 26: Nettobeschäftigungswirkungen für biotechnologische Produktion von Fein-/Spezialchemikalien in Sensitivitätsrechnungen .225 
Abbildung 27: Anzahl der jährlich weltweit eingeführten „New Molecular Entities".

Abbildung 28: Nettoproduktionswirkungen durch zusätzliche Diffusion von Biopharmazeutika im Vergleich zum Basisszenario (in Mio. $€$ )

Abbildung 29: Nettobeschäftigungswirkungen durch zusätzliche Diffusion von Biopharmazeutika im Vergleich zum Basisszenario (in Erwerbstätige)

Abbildung 30: Nettoproduktionswirkungen für Biopharmazeutika in Sensitivitätsrechnungen (in Mio. €)

Abbildung 31: Nettobeschäftigungswirkungen für Biopharmazeutika in Sensitivitätsrechnungen (in Erwerbstätige). 


\section{Tabellenverzeichnis}

Tabelle 1: Übersicht über verschiedene Biotechniken ......................................71

Tabelle 2: Anteile der Biotechnologie an den gesamten Patenten und Publikationen im Zeitraum 1995 bis 2004 .......................................73

Tabelle 3: „Impact“-Indikatoren für die Biotechnologie....................................114

Tabelle 4: Ausgewählte Anwendungsfelder der Biotechnologie ...................... 142

Tabelle 5: Berücksichtigung der Wirkungsmechanismen in der empirischen Analyse ...................................................................... 144

Tabelle 6: Auszug aus dem Fragebogenleitfaden für Biopolymere ..................166

Tabelle 7: Weltweite Produktion von Bioethanol im Jahr 2007 .....................171

Tabelle 8: Diffusion von Bioethanol in den Szenarien ...................................173

Tabelle 9: Anteil der Ethanolproduktion auf konkurrierenden Flächen........... 178

Tabelle 10: Kosten für Bioethanol im Referenz-Szenario $(\ell$ je $t) \ldots \ldots \ldots \ldots \ldots \ldots . . . . . . .183$

Tabelle 11: Kosten für Bioethanol im HD-Szenario $(€$ je $t)$.............................. 183

Tabelle 12: Szenarienannahmen für die Produktion von Bioethanol im Jahr 2020 (in Mio. $€$ )................................................................... 185

Tabelle 13: Sensitivitätsannahmen für Bioethanol...........................................188

Tabelle 14: Diffusion von Biopolymeren in den Szenarien .................................194

Tabelle 15: Anteil der Produktion von Biopolymeren auf konkurrieren-

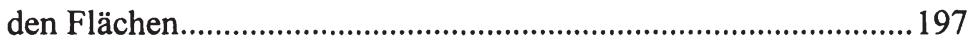

Tabelle 16: Kosten für Biopolymere im Referenz-Szenario (in $€$ je $t$ ) ............. 202

Tabelle 17: Kosten für Biopolymere im HD-Szenario (in $€$ je $t$ ).......................203

Tabelle 18: Szenarienannahmen für die Produktion von Biopolymeren im Jahr 2020 (in Mio. €)..............................................................205

Tabelle 19: Sensitivitätsannahmen für Biopolymere .......................................208

Tabelle 20: Biotechnologische Produktion von Fein-/Spezialchemikalien in den Szenarien.....................................................213

Tabelle 21: I-O-Koeffizienten für Biotechnologie und Chemie in den Szenarien 
Tabelle 22: Szenarienannahmen für die biotechnologische Produktion von Fein-/Spezialchemikalien im Jahr 2020 (in Mio. €).

Tabelle 23: Sensitivitätsannahmen für die biotechnologische Produktion von Fein-/Spezialchemikalien ..................................................224

Tabelle 24: Diffusion von Biopharmazeutika in den Szenarien.

Tabelle 25: Szenarienannahmen für die Wirkung von Produktinnovationen bei Biopharmazeutika

Tabelle 26: Input-Output-Struktur für Biopharmazeutika (in \%)

Tabelle 27: Szenarienannahmen für Biopharmazeutika im Jahr 2020 (in Mio. €)

Tabelle 28: Nettoeffekte für Produktion und Beschäftigung bei alternativen Annahmen für Produktinnovationen und Exporte im Vergleich zum Basisszenario

Tabelle 29: Sensitivitätsannahmen für die Biopharmazeutika 248

Tabelle 30: Zusammenfassung der Ergebnissen und Wirkungsmechanismen in den Fallbeispielen.

Tabelle 31: Kennzahlen zur Biotechnologie - Branche in Deutschland 2005-2007 301

Tabelle 32: Verwendete Preis- und Einkommenselastizitäten für die Berechnung der Konsumvektoren 301

Tabelle 33: Befragte Akteure in den Experteninterviews 302

Tabelle 34: Zusätzlicher Flächenbedarf durch die Verwendung von Bioethanol 302

Tabelle 35: Zusätzlicher Flächenbedarf durch die Verwendung von Biopolymeren

Tabelle 36: Sektorale Produktionseffekte für Bioethanol im Referenzszenario (im Vergleich zum Basisszenario). 304

Tabelle 37: Sektorale Produktionseffekte für Bioethanol im HD-

Szenario (im Vergleich zum Basisszenario) 306

Tabelle 38: Sektorale Beschäftigungseffekte für Bioethanol im

Referenzszenario (im Vergleich zum Basisszenario) 308 
Tabelle 39: Sektorale Beschäftigungseffekte für Bioethanol im HD-

Szenario (im Vergleich zum Basisszenario)

Tabelle 40: Sektorale Produktionseffekte für Biopolymere im

Referenzszenario (im Vergleich zum Basisszenario)

Tabelle 41: Sektorale Produktionseffekte für Biopolymere im HD-

Szenario (im Vergleich zum Basisszenario)

Tabelle 42: Sektorale Beschäftigungseffekte für Biopolymere im

Referenzszenario (im Vergleich zum Basisszenario)

Tabelle 43: Sektorale Beschäftigungseffekte für Biopolymere im HD-

Szenario (im Vergleich zum Basisszenario)

Tabelle 44: Sektorale Produktionseffekte für biotechnologisch herge-

stellte Fein-/Spezialchemikalien im Referenzszenario (im

Vergleich zum Basisszenario)

Tabelle 45: Sektorale Produktionseffekte für biotechnologisch hergestellte Fein-/Spezialchemikalien im HD-Szenario (im Vergleich zum Basisszenario)

Tabelle 46: Sektorale Beschäftigungseffekte für biotechnologisch hergestellte Fein-/Spezialchemikalien im Referenzszenario (im Ver-gleich zum Basisszenario).

Tabelle 47: Sektorale Beschäftigungseffekte für biotechnologisch hergestellte Fein-/Spezialchemikalien im HD-Szenario (im Vergleich zum Basisszenario)

Tabelle 48: Sektorale Produktionseffekte für Biopharmazeutika im

Referenzszenario (im Vergleich zum Basisszenario)

Tabelle 49: Sektorale Produktionseffekte für Biopharmazeutika im HD-

Szenario (im Vergleich zum Basisszenario)

Tabelle 50: Sektorale Beschäftigungseffekte für Biopharmazeutika im

Referenzszenario (im Vergleich zum Basisszenario)

Tabelle 51: Sektorale Beschäftigungseffekte für Biopharmazeutika im HD-Szenario (im Vergleich zum Basisszenario). 


\section{Einführung}

\subsection{Ausgangssituation}

Bereits seit dem 18. Jahrhundert wird die Auswirkung neuer Technologien auf die Beschäftigung kontrovers diskutiert. Lange Zeit galt der technische Fortschritt als mögliche Ursache steigender (technologischer) Arbeitslosigkeit. Dabei wurde befürchtet, dass das induzierte Produktivitätswachstum höher ist als die Produktionszunahme und der Bedarf an Arbeitskräften sinkt. In jüngerer Vergangenheit lässt sich allerdings verstärkt ein Paradigmenwechsel in der öffentlichen und wirtschaftswissenschaftlichen Debatte beobachten (Welsch 2006, S.610). Neue Technologien und Innovationen gelten vielmehr als Schlüsselfaktor für eine hohe volkswirtschaftliche Dynamik und hohe Beschäftigung. Die Umsetzung der technologischen Möglichkeiten in neue Produkte und die Einsetzung neuer Verfahren sind für fortgeschrittene Industriestaaten demnach von essentieller Bedeutung zur Steigerung der Produktion und Beschäftigung. Qualifizierte Arbeitskräfte stellen dabei einen entscheidenden Faktor für die Generierung neuer Innovationen und Problemlösungen dar. Arbeitslosigkeit entsteht nach dieser Auffassung nur dann, wenn der Faktor Arbeit nicht ausreichend genutzt wird und die Innovationsleistung hinter anderen Ländern zurückbleibt. Wichtige Gründe für den zunehmenden Paradigmenwechsel liegen unter anderem im verstärkten globalen Standortwettbewerb. Die Entwicklung und Nutzung neuer Technologien sind in hoch entwickelten und rohstoffarmen Ländern (z.B. Deutschland) notwendig, um sich dauerhaft im internationalen Wettbewerb zu behaupten. Falls dies gelingt, können neue Arbeitsplätze im Inland geschaffen oder existierende Arbeitsplätze dauerhaft im Inland gehalten werden.

Daneben zeigen sich aber auch bei den Technologien, die hinter dem heutigen technischen Fortschritt stehen, deutliche Unterschiede in den Eigenschaften, Diffusion und Anwendungsbereichen gegenüber früheren Technologien. Diesen Charakteristika wird erhebliche Relevanz für deren wirtschaftliche Auswirkungen zugesprochen (Holwegler 2003). Ein Beispiel hierfür sind technologiespezifische Komplementaritäten zu anderen Produktionsfaktoren, z.B. der Bedarf an qualifizierten Arbeitskräften in einer wissensbasierten Ökonomie auf Basis neuer Technologien. Der zukünftige Zusammenhang zwischen neuen Technologien und Produktion bzw. Beschäftigung ist demnach nicht zuletzt von den konkreten Technologien selbst abhängig. Um Aussagen über diese Zusammenhänge treffen zu können, bedarf es einer expliziten Erfassung der technologischen Entwicklung, da Vergangenheitsanalysen nicht genug Aufschluss geben. Bei aktuellen Betrachtungen zukünftiger technologischer Veränderungen wird häufig einigen wenigen „Schlüsseltechnologien" ein besonders großer Einfluss auf Wirtschaft und Gesellschaft zugesprochen. Hierzu zählt insbesondere die Biotechnologie. Sie verfügt nach dieser Ansicht über ein hohes Potenzial für die Entwicklung neuer oder verbesserter Prozesse, Produkte und Dienstleistungen in sehr 
verschiedenen Sektoren wie z.B. Landwirtschaft, Lebensmittel, Pharmazie, Umwelttechnik, oder Chemie (McKelvey 2007, S.610). In einer Vielzahl von Ländern werden deshalb erhebliche öffentliche und private (Forschungs-) Investitionen in die Biotechnologie getätigt, in der Erwartung neben wissenschaftsund technologiepolitischen eine Reihe gesellschaftspolitischer Ziele zu erreichen (Feuerstein 2007, S.11). Neben signifikanten Verbesserungen in der Arzneimittelentwicklung und der gesamten Gesundheitsversorgung sowie der Umweltschonung werden durch die genannten Vorteile positive Effekte für die wirtschaftliche Entwicklung erhofft. Ob die wirtschaftlichen Erwartungen allerdings gerechtfertigt sind, kann aufgrund der vielschichtigen Zusammenhänge zwischen Technologien und Ökonomie nicht pauschal beurteilt werden. Hierfür bedarf es konkreter empirische Untersuchungen.

Die bisherigen wissenschaftlichen Erkenntnisse über die wirtschaftlichen Auswirkungen der Biotechnologie in ihrer Höhe aber auch in der Art und Weise (z.B. neue Produkte, Einsparung von Ressourcen zur Produktion etc.) sind allerdings gering. Viele bisherigen Studien und Untersuchungen konzentrieren sich auf Marktzahlen (z.B. Umsatz, Beschäftigung) der „Biotechnologieindustrie“. Dabei handelt es sich vorrangig um in der Pharmaindustrie zu findende spezialisierte Biotechnologieunternehmen. Diese Unternehmen weisen viele Besonderheiten wie die enge Verbindung zur Universitätswissenschaft, eine hohe Forschungsintensität und Abhängigkeit von Risikokapital auf. Somit werden Unternehmen eines besonderen Typs in einer besonderen Phase des Wachstums betrachtet (McKelvey 2007, S.610). Diese Analysen können kaum Hinweise für das Potenzial und die Bedeutung einer Technologie für die Volkswirtschaft geben. Die Bedeutung der Biotechnologie für Produkte und Prozesse in den oben genannten Anwenderbranchen wird mit diesen Analysen überhaupt nicht erfasst. McKelvey stellt deshalb fest: „[...] a broader perspective on modern biotechnology is necessary to capture all the relevant economic activities..." (McKelvey 2007, S.610). Die wenigen bisherigen Untersuchungen für Anwenderbranchen betrachten aber nur einzelne Aspekte möglicher gesamtwirtschaftlicher Auswirkungen. Meist werden Bruttogrößen (z.B. Produktionsanteil) untersucht (z.B. Menrad et al. 2003; Nusser et. al. 2007a) und keine Veränderungen, die durch eine Diffusion der Biotechnologie auftreten. Diese bisherigen Limitationen sind der schwierigen Datensituation für die Biotechnologie geschuldet. Erst in jüngerer Vergangenheit zeigt sich eine gewisse Zunahme bei Studien, die zumindest Teilgebiete und/oder Teile der Effekte der Biotechnologie aus technoökonomischer Sicht erfassen. Diese können eine wichtige Grundlage für aufbauende Analysen zu gesamtwirtschaftlichen Effekten geben. 


\subsection{Ziel der Arbeit}

Das Ziel dieser Arbeit ist die Analyse von möglichen zukünftigen Produktionsund Beschäftigungseffekten der Diffusion der Biotechnologie. Andere sozioökonomische und gesellschaftliche Dimensionen der Fortschritte in der Biotechnologie - z.B. für Gesundheit und Umwelt sowie deren Folgeeffekte - können im Rahmen dieser Arbeit nicht vertieft behandelt werden. Die Betrachtung ist dabei vor allem auf die Zukunft gerichtet, für die empirische Analyse wird das Jahr 2020 gewählt. Schließlich ist die Analyse der Effekte einer neuen Technologie für die Beurteilung ihrer Potenziale dann sinnvoll, wenn sie einen gewissen Diffusions- und Reifegrad erreicht hat. Während in einigen Anwendungsfeldern (z.B. Pharma) eine Diffusion der Biotechnologie zumindest teilweise bereits stattgefunden hat, ist dies für andere Felder (z.B. Kunststoffe) erst zu erwarten.

Um diese möglichen zukünftigen Auswirkungen der Biotechnologie zu analysieren, werden relevante Wirkungsmechanismen aus der Theorie abgeleitet, die Verfügbarkeit und Aussagekraft verschiedener Daten(-quellen) erörtert und eine quantitative empirische Untersuchung für ausgewählte Anwendungsfelder der Biotechnologie durchgeführt. Dabei werden folgende Teilfragen erörtert:

- Welche Bedeutung haben neue Technologien für die wirtschaftliche Entwicklung in verschiedenen Theorieansätzen? Welche Wirkungsmechanismen von Technologien auf Beschäftigung und Produktion sind von besonderer Relevanz und inwiefern sind diese von den Charakteristika einer Technologie abhängig?

- Welche technologischen Charakteristika lassen sich der Biotechnologie zuordnen und welche Implikationen für die wirtschaftlichen Effekte und deren Analyse ergeben sich daraus?

- Wie ist die Datenverfügbarkeit für die Biotechnologie einzustufen? Wie kann eine geeignete Modelllösung zur Erfassung gesamtwirtschaftlicher Effekte unter Berücksichtigung der Datenverfügbarkeit aussehen?

- Welche gesamtwirtschaftlichen Effekte können sich in projektiven Szenarien durch die Diffusion und Weiterentwicklung der Biotechnologie in verschiedenen Anwendungsfeldern ergeben? Welche Faktoren sind für diese Effekte von besonderer Bedeutung?

Für die Beantwortung der empirischen Teilfragen wird eine technoökonomische Vorgehensweise gewählt. So liegt der Schwerpunkt dieser Arbeit in einer Integration der biotechnologiespezifischen Impulse in ein volkswirtschaftliches Modell, da besonders hier umfangreiche Forschungsarbeiten fehlen. Dabei ergibt sich die Problematik, die vor allem bei umweltökonomischen Modellen bisher häufig diskutiert wird (z.B. Walz/Schleich 2009; Frohn et al. 1998), dass je nach Aggregationsebene eines Modells unterschiedliche Wirkungen neuer Technologien erfasst werden können. Während so genannte Top-Down-Modelle (z.B. 
Allgemeine Gleichgewichtsmodelle, Input-Output-Modelle) gesamtwirtschaftliche Zusammenhänge untersuchen, können Bottom-Up-Modelle genauer konkrete Techniken und Technikalternativen erfassen. Im Rahmen dieser Arbeit wird ein Zwischenweg gewählt, indem in einer Bottom-Up-Betrachtung technoökonomische Daten in ein gesamtwirtschaftliches Input-Output-Modell integriert werden. Der Grundgedanke dieses technoökonomischen Zugangs zu diesem Thema ist, dass das Wissen über technologische Prozesse nützliche Hinweise über die Art und Richtung wirtschaftlicher Effekte geben kann. Schließlich sind Charakteristika einzelner Technologien bereits häufig vor der breiten Diffusion zumindest in Grundzügen bekannt. Die Berücksichtigung indirekter wirtschaftlicher Effekte erfolgt in einem Input-Output-Modell. Dieses Modell zeichnet sich im Vergleich zu anderen Top-Down-Modellen in der Regel durch eine stärkere sektorale Disaggregation und eine relativ große Flexibilität aus, welche die Integration von technoökonomischen Informationen auf der Mesoebene ermöglicht. Dabei werden für vier Anwendungsfelder der Biotechnologie mehrere Szenarien gebildet. Diese sollen die aus heutiger Sicht für Experten plausibel erscheinenden Innovations- und Diffusionspfade der Biotechnologie abbilden. Für diese Szenarien werden die Art und Weise (z.B. neue Produkte, Produktivitätswachstum, Veränderung von Produktionsstrukturen) und die Größenordnungen möglicher Effekte der Biotechnologie analysiert und miteinander verglichen.

Diese Vorgehensweise mithilfe des Input-Output-Modells geht aber auch mit Einschränkungen bei der Modellierung gesamtwirtschaftlicher Rückkoppelungseffekte (z.B. Preiseffekte) und der Abbildung möglicher Innovations- und Diffusionsdynamiken einher. Die breite theoretische Diskussion und Erörterung der Implikationen für die Auswirkungen der Biotechnologie auf einer Makroebene, soll die interpretative Einordnung der empirischen Ergebnisse ermöglichen und ergänzen.

\subsection{Aufbau der Arbeit}

In Kapitel 2.1 erfolgt zunächst eine Klärung einiger relevanter Begriffe für diese Arbeit. Danach werden in Kapitel 2.2 Theorieansätze, die wichtige Erkenntnisse für die vorliegende Forschungsfrage liefern, in ihren Grundzügen dargestellt. Hierbei wird erörtert, wie der Einsatz neuer Technologien (bzw. technischer Fortschritt) spezifiziert wird, welche Bedeutung sie für die wirtschaftliche Entwicklung einnehmen und auf welche Art und Weise (z.B. Produktivität, strukturelle Wirkungen) sie sich auf die Gesamtwirtschaft auswirken. Dabei stehen Ansätze der Neoklassik, des Post-Keynesianismus und der Evolutorik im Fokus. In Kapitel 2.3 werden dann konkrete Wirkungsmechanismen für die Produktionsund Beschäftigungswirkungen von neuen Technologien diskutiert, welche die Grundlage für die empirische Modellierung bilden. In Kapitel 3 wird die Bio- 
technologie charakterisiert und mögliche Implikationen der theoretischen $\mathrm{Zu}$ sammenhänge für die wirtschaftlichen Auswirkungen der Biotechnologie erörtert. Dabei werden sowohl die beschriebenen Wirkungsmechanismen als auch eine evolutorische Interpretation des Konzepts der „General Purpose Technologies" für die Biotechnologie diskutiert. Die Charakteristika der Biotechnologie können mit diesem Grundgerüst spezifiziert, die bisherige Entwicklung der Biotechnologie nachgezeichnet und zumindest Ansatzpunkte über mögliche Entwicklungspfade gegeben werden. Gerade weil die Entwicklung der Biotechnologie bisher hinter vielen Erwartungen zurückgeblieben ist, bedarf es für eine prospektive Analyse einer Ursachenklärung. Sie unterstützt die Bildung möglichst plausibler und konsistenter Szenarien, indem sie Anhaltspunkte für die relevanten Rahmenbedingungen und Annahmen für die Modellparameter gibt. In Kapitel 4 stehen die Möglichkeiten der Messung wirtschaftlicher Auswirkungen der Biotechnologie im Fokus. Die Datenlage für die Biotechnologie wird bezüglich ihrer Verfügbarkeit, Aussagekraft und daraus folgenden Einschränkungen für gesamtwirtschaftliche Analysen erörtert. Im Anschluss werden die Grundzüge des Input-Output-Modells dargestellt und die Analyse der Wirkungen neuer Technologien mit diesem Modell diskutiert. Die empirische Vorgehensweise für die eigenen Modellsimulationen und die Auswahl der untersuchten Anwendungsfelder der Biotechnologie werden in Kapitel 5 erläutert. In Kapitel 6 werden für die gewählten Anwendungsfelder die Auswirkungen der Biotechnologie anhand verfügbarer Daten, Literatur und Experteninterviews diskutiert und Modellparameter für verschiedene Szenarien abgeleitet. Im Anschluss an die jeweilige Erörterung werden die Ergebnisse der Modellsimulationen dargestellt und interpretiert. Abschließend werden in Kapitel 7 die zentralen Ergebnisse zusammengefasst und Schlussfolgerungen gezogen. 
Sven Wydra - 978-3-631-75392-7

Downloaded from PubFactory at 01/11/2019 05:32:25AM

via free access 


\section{Theoretische Grundlage}

\subsection{Konzeptionelle Erläuterungen}

\subsubsection{Charakterisierung von Technologien und technischer Fortschritt}

Die Begriffe technologischer Fortschritt, technischer Fortschritt oder Auswirkungen neuer Technologien werden im Sprachgebrauch häufig synonym verwendet. Bei einer genaueren Begriffsklärung zeigen sich allerdings teilweise Unterschiede. Den Begriff der Technologie beschreibt bspw. Stoneman folgendermaßen: "Technology means the goods and services produced and the means by which they are produced in a firm, industry, or economy" (Stoneman 1983, S.5). Technologischer Fortschritt umschreibt Änderungen in der Technologie, bzw. die „Erweiterung des produktionstechnisch anwendbaren Wissens“ (Walter 1969, S.237). Dabei ist zu beachten, dass eine Veränderung nicht eine Verbesserung der Technologie in allen Belangen bedeutet. Technischer Fortschritt umfasst darüber hinaus die tatsächliche Anwendung dieses Wissens im Produktions- oder Wertschöpfungsprozess (Vogel 2000, S.44). Im Folgenden steht in der Regel technischer Fortschritt im Mittelpunkt, der Begriff Auswirkung neuer Technologien wird synonym verwendet.

Eine wichtige Unterscheidung zwischen einzelnen Technologien kann anhand des Ausmaßes der induzierten Änderungen erfolgen. Diese Änderungen können sowohl ökonomischer, organisatorischer, institutioneller oder sozialer Art sein. In der Regel wird zwischen zwei Arten von Technologien unterschieden. Allgemein wird zwischen Basistechnologien auf der einen Seite und gewöhnlichen, inkrementellen Verbesserungen auf der anderen Seite differenziert. Das Ziel dieser Unterscheidungen ist die Hervorhebung langfristiger Auswirkungen der Basistechnologien oder „General Purpose Technologies" (Meyer-Krahmer 1999). Diese neuen Technologien werden in einer Vielzahl von Sektoren eingesetzt und bewirken dort tiefgreifende Veränderungen (siehe Kapitel 2.2.3.2). Sie führen zur Schaffung neuer Produkte, verändern Industriestrukturen, indem neue Branchen entstehen und einige ältere an Bedeutung verlieren, beeinflussen Nachfragemuster der Konsumenten und verändern die Real- und Humankapitalbestände einer Volkswirtschaft. Allerdings entstehen auch Anpassungsfriktionen und Ungleichgewichte insbesondere im Zusammenspiel mit nicht-ökonomischen Faktoren. Inkrementelle Verbesserungen entstehen hingegen kontinuierlich in der gesamten Wirtschaft (OECD 1988, S.34). Sie verbessern bzw. erhöhen die Produktivität von existierenden Produkten, Dienstleistungen und Prozessen. Allerdings verursachen sie keine strukturellen Anpassungsprobleme, da sie in etablierten Industriestrukturen und bestehenden institutionellen Rahmenbedingungen stattfinden und keine völlig neuen Fähigkeiten oder Kapitalgüter benötigen (OECD 
1988). ${ }^{1}$ Als ein klassisches Beispiel gilt der Katalysator für Autos (Dolata 2007, S.26).

\subsubsection{Definition und Charakterisierung von Innovationen}

Wichtige Konzepte für die Analyse der Wirkung neuer Technologien beziehen sich nicht auf den Technologiebegriff, sondern auf Innovationen. Der Begriff Innovationen ist in der Regel weiter definiert und umfasst auch nichttechnologische Fortschritte. In weitem Sinne beziehen sich Innovationen auf eine realisierte Menge von Ideen (Grupp 1997). Häufig werden Innovationen als Teil eines Prozesses gesehen. Dieser wird in der Regel in drei Phasen untergliedert, der Invention, der Innovation und der Diffusion:

- Eine Invention ist eine neue Idee oder Entdeckung für neue Produkte, Prozesse oder Systeme. "Such inventions may often (not always) be patented but they do not necessarily lead to technical innovation" (Freeman 1982, S.6).

- Eine Innovation ist die erstmalige wirtschaftliche Verwertung einer Invention. $^{2}$

- Die Diffusion ist die Anwendung und Verbreitung von neuen Innovationen und deren Nachahmungen.

Dabei entspricht der Begriff der Invention dem technologischen Fortschritt, während Innovation und Diffusion zusammen den technischen Fortschritt darstellen (Holwegler 2003, S.10). Ähnlich wie Technologien werden auch Innovationen in verschiedenen Typologien unterschieden. Im Hinblick auf ihre ökonomischen Auswirkungen werden häufig Produkt- und Prozessinnovationen voneinander getrennt. Für diese Begriffe existieren wiederum verschiedene Definitionen, die zu großen Differenzen bei ihrer empirischen Messung führen können (Archibugi et al 1994; Tether 2003). Konsens besteht darin, dass es sich bei Produktinnovationen um neue oder merklich verbesserte Produkte handelt. ${ }^{3}$ Unter Prozessinnovationen werden neue oder verbesserte Verfahren verstanden. Allerdings ergeben sich Differenzen bei der Operationalisierung der Unterscheidung von Produkt- und Prozessinnovationen. Im Folgenden werden die für die

\footnotetext{
' Dabei stellt die OECD zu dem Erscheinen der Auswirkungen dieser Innovationen auf amtliche Statistiken fest: „They are reflected in the official economic statistics by changes in the coefficients of the existing input-output matrix and in technology statistics mainly through patents, but they do not necessarily involve organised R\&D" (OECD 1988, S.34).

${ }^{2}$ Hierbei ist die Betonung der "erstmaligen" Verwertung in Abgrenzung zum Begriff der Imitation notwendig. Darunter werden konkurrierende, identische Neuerscheinungen verstanden, die später am Markt eingeführt werden (Grupp 1997).

${ }^{3}$ Im OSLO Manual der OECD ist eine Produktinnovation folgendermaßen definiert: " $A$ technologically new product is a product whose technological characteristics or intended uses differ significantly from those of previously produced products" (OECD 1997, S.48).
} 
vorliegende Arbeit relevanten Abgrenzungen aus Sicht der Nachfrage und aus sektoraler Sicht erläutert.

Schumpeter (1934, S.100) unterscheidet die Innovationen anhand der Endnachfrage. Dabei handelt es sich bei Produktinnovationen um neue oder verbesserte Konsumgüter für die Haushaltsnachfrage. Neue Kapitalgüter (z.B. neue Maschinen) und Vorleistungsgüter für andere Unternehmen sind hingegen Prozessinnovationen. Somit wäre der PC gleichzeitig eine Produktinnovation in einem Privathaushalt, aber eine Prozessinnovation in einem Unternehmen.

Aus sektoraler Sicht sind zwei Unterscheidungen denkbar. Häufig werden dabei unter Produktinnovationen neue bzw. verbesserte Produkte, die außerhalb des Sektors verwendet werden, verstanden (z.B. Pavitt 1984). Prozessinnovationen werden hingegen in dem Sektor genutzt, in dem sie auch hergestellt werden. ${ }^{4}$ Eine leicht differierende, aber für die empirische Analyse dieser Arbeit bedeutende Unterscheidung, zielt auf den Nutzen aus Sicht eines einzelnen Sektors ab (Edquist et al. 2001). Produktinnovationen sind demnach neue bzw. verbesserte Produkte in einem Sektor, Prozessinnovation stellen ein neues Verfahren für einen Sektor dar. Bei diesen Unterscheidungen der Innovationen auf sektoraler Ebene muss allerdings die Interdependenz der Sektoren beachtet werden. Die Produktinnovation eines Sektors kann eine Prozessinnovation in einem anderen Sektor darstellen. Als Beispiel kann ein industrieller Roboter im Herstellungssektor eine Produktinnovation, für das Automobilunternehmen als Nutzer dieser Technologie aber eine Prozessinnovation sein.

Im Ergebnis lassen sich aufgrund der verschiedenen Perspektiven Innovationen meist nicht eindeutig einer Produkt- oder Prozessinnovation zuordnen. Archibugi et al. (1994) kommen anhand einer Datenbank über britische Innovationsdaten mit den unterschiedlichen Definitionen zu dem Ergebnis, dass nur $3,1 \%$ dieser Innovationen eindeutig einer der beiden Kategorien zugeordnet werden können. Trotz dieser empirischen Zuordnungsprobleme ist die Unterscheidung häufig explizit in der empirischen Analyse und implizit in den Theorien - meist erfolgt nur die Betrachtung einer von beiden Arten - von großer Bedeutung, da ihnen unterschiedliche Auswirkungen zugeschrieben werden (siehe Kapitel 2.3).

\subsubsection{Analyseebenen der wirtschaftlichen Effekte von Technologien und Innovationen}

Wie diese Unterscheidungsproblematik der Produkt- und Prozessinnovationen zeigt, sind die Ergebnisse der Analysen zu den Folgewirkungen von Technologien und Innovationen von der Aggregationsebene abhängig. Insgesamt können

\footnotetext{
${ }^{4}$ Dabei ist auch das Aggregationsniveau bei der Sektorenabgrenzung für die Unterscheidung bedeutend. Falls die Aggregation hoch ist, fallen die meisten Innovationen in die Kategorie der Prozessinnovationen.
} 
die wirtschaftlichen Auswirkungen neuer Technologien auf verschiedenen Aggregationsebenen theoretisch und empirisch untersucht werden. Je nach gewählter Aggregationsebene bestehen aber unterschiedliche Schwierigkeiten wirtschaftliche Entwicklungen kausal dem Einsatz von Technologien zuzuschreiben, Spezifika auf Unternehmens-, Sektor- oder Landesebene zu berücksichtigen und bestimmte Wirkungen des technologischen Fortschritts überhaupt zu erfassen. So sind viele kompensatorische und substitutive Wirkungen - z.B. Veränderungen in der Nachfrage aufgrund höherer Kaufkraft infolge von Preissenkungen - auf Unternehmensebene gar nicht erfassbar. Die Ergebnisse unterscheiden sich deshalb in der Regel je nach Analyseebene erheblich (Pianta 2005; Fröhlich et al. 1992; Edquist et al. 2001; RWI 2005). Während die Vor- und Nachteile einzelner Betrachtungsebenen in Kapitel 4.1 in Bezug auf die im Rahmen dieser Arbeit interessierenden Forschungsfragen erörtert werden, ist hier zunächst eine begriffliche Klärung von Interesse. In Anlehnung an Kaplinsky (1987) und Fröhlich et al. (1992) kann dabei zwischen den in Abbildung 1 dargestellten Analysenebenen unterschieden werden, die nach dem Grad der erfassten Wirkungszusammenhänge differieren.

Abbildung 1: Analyseebenen wirtschaftlicher Auswirkungen neuer Technologien

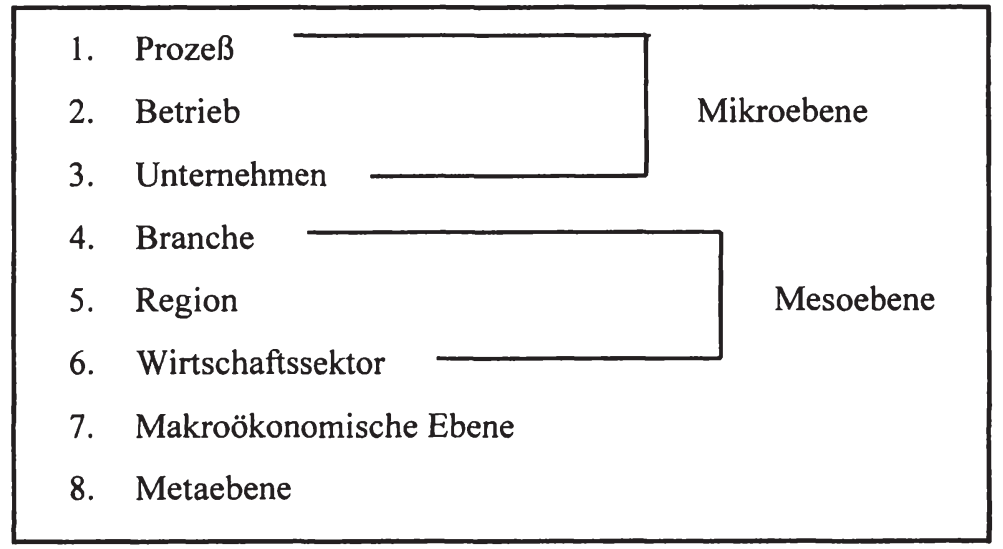

Quelle: Fröhlich et al. (1992); Kaplinsky (1987)

Im Folgenden werden die verschiedenen Analyseebenen vereinfacht in Mikroebene mit Bezug auf Unternehmen, Mesoekene mit Bezug auf Branchen bzw. „Anwendungsfelder einer Technologie" und Makroebene auf nationaler Ebene unterschieden.

Eine eng daran geknüpfte Frage zu dieser Unterscheidung von Analyseebenen besteht darin, was unter direkten und indirekten Wirkungen des technologischen 
Fortschritts zu verstehen ist. Schließlich wird gerade hier das Unterscheidungsmerkmal des Erkenntnisgewinns der einzelnen Analyseebenen gesehen. Über die genaue Definition besteht allerdings Unklarheit und es existiert ein unterschiedlicher Wortgebrauch (Mettelsiefen/Barens 1987). Im Rahmen dieser Arbeit werden unter direkten Wirkungen ökonomische Effekte verstanden, die sich dort einstellen und beobachtet werden können, wo eine Einführung neuer Technologien erfolgt. ${ }^{5}$ Ein Beispiel hierfür ist die Freisetzung von Arbeitskräften in einem Unternehmen, falls durch den dortigen Einsatz der neuen Technologie die Prozesse weniger arbeitsintensiv sind. Indirekte Effekte stellen sich an einem anderen Ort und meist zu einem anderen Zeitpunkt als dem des beobachtbaren Technologieeinsatzes ein. ${ }^{6}$ Ein Beispiel ist der Produktionsrückgang bei Vorleistungslieferanten, wenn die Anwender der neuen Technologie dieses Vorleistungsgut durch die neuen Prozesse einsparen können.

\subsection{Theoretische Ansätze}

Der Zusammenhang zwischen der technologischen und wirtschaftlichen Entwicklung lässt sich nur unzureichend durch eine Theorie beschreiben. Vielmehr unterscheiden sich einzelnen Theorieansätze in ihren Analyseschwerpunkten und Implikationen für die wirtschaftlichen Auswirkungen neuer Technologien. Im Folgenden wird kurz dargestellt, welche Bedeutung der technische Fortschritt für die wirtschaftliche Entwicklung in den Theorieansätzen einnimmt und durch welche Wirkungsmechanismen dies geschieht. Im Anschluss daran wird diskutiert, inwiefern sich die Theorien in ihren Kernaussagen unterscheiden und als Analysegerüst für diese Arbeit eignen.

\subsubsection{Neoklassische Ansätze}

Die Analyse des technischen Fortschritts in der neoklassischen Theorie erfolgt als Determinante des wirtschaftlichen Wachstums in einem Gleichgewichtsmodell. Grundlage hierfür bilden dabei die Arbeiten zum Wachstumsmodell von Solow (1956/1957). Ausgangspunkt ist dabei eine gesamtwirtschaftliche Produktionsfunktion, bei der das Produktionsvolumen einer Volkswirtschaft (Y) in

\footnotetext{
${ }^{5}$ Dabei besteht noch die Frage der exakten räumlichen Abgrenzung, die hier analog zur impliziten Behandlung in großen Teilen der Literatur auf Unternehmensebene definiert wird.

${ }^{6}$ Diese in der allgemeinen Literatur sehr geläufige Einteilung wird bei Analysen zur Biotechnologie bisher überraschend selten verwendet. Stattdessen werden Effekte in Anwenderbranchen der Biotechnologie häufig als indirekte Effekte deklariert, um sie analytisch von der Bereitstellung neuer biotechnologischer Verfahren, Produkte und Dienstleistungen zu unterscheiden (z.B. IPTS 2007).
} 
Abhängigkeit von den beiden Produktionsfaktoren Arbeit (L) und Kapital (K) gesetzt wird. ${ }^{7}$

$$
Y=f(K, L)
$$

Eine Änderung des Einsatzes der Faktoren Arbeit oder Kapital hat aufgrund der angenommenen abnehmenden Grenzerträge nur einen vorübergehenden Einfluss auf die Wachstumsrate. Langfristig wird die gleichgewichtige Wachstumsrate (Steady-State) allein vom technischen Fortschritt bestimmt, der als exogene Größe angenommen und im Modell selbst nicht erklärt wird. Technischer Fortschritt wird dabei als , a short hand expression for any kind of shift of the productions function" definiert (Solow 1957, S.312). ${ }^{8}$ Die Betrachtung wird dabei auf Prozessinnovationen eingeschränkt, Produktinnovationen sind im Grundmodell ausgeschlossen. In der Produktionsfunktion können diese Zusammenhänge über einen Index (A) dargestellt werden, der das technologische $\mathrm{Ni}$ veau beschreibt (Totale Faktorproduktivität, kurz: TFP).

$$
Y=A f(K, L)
$$

Die Wachstumsrate des Produktionsvolumens $\left(\frac{\dot{Y}}{Y}\right)$ ergibt sich aus den Veränderungen der Produktionsfaktoren und der totalen Faktorproduktivität.

$$
\frac{\dot{Y}}{Y}=\alpha \frac{\dot{K}}{K}+(1-\alpha) \frac{\dot{L}}{L}+\frac{\dot{A}}{A}
$$

Die totale Faktorproduktivität, welche den technischen Fortschritt widerspiegelt, ist dabei nicht direkt beobachtbar. Sie wird als diejenige Veränderung des Outputs, die nicht direkt auf Änderungen der Produktionsfaktoren Arbeit und Kapital zurückführbar ist, gemessen (Conte 2006). Deshalb wird sie häufig als Solow-Residuum bezeichnet.

$$
\frac{\dot{A}}{A}=\frac{\dot{Y}}{Y}-\alpha \frac{\dot{K}}{K}-(1-\alpha) \frac{\dot{L}}{L}
$$

\footnotetext{
${ }^{7}$ Grundlegend sind dabei folgende Annahmen: Die Skalenerträge der Produktionsfaktoren sind konstant, ihre Grenzerträge sind hingegen abnehmend. Arbeit und Kapital werden entsprechend den Grenzproduktivitäten entlohnt und sind dabei gegenseitig substituierbar. Es herrscht vollkommener Wettbewerb und alle Märkte sind im Gleichgewicht. Die Produktionsfaktoren sind in diesem Zustand voll ausgelastet, d.h. es existiert Vollbeschäftigung.

${ }^{8}$ Dabei wird nur unverkörperter technischer Fortschritt betrachtet. Er ist nicht an einer der Produktionsfaktoren gebunden und beeinflusst sowohl den zusätzlichen als auch den existierenden Kapital- und Arbeitsstock gleichermaßen. Diese Art von technischem Fortschritt wird auch als Hicks-neutral bezeichnet (siehe Kapitel 2.3.1).
} 
Dieses Modell bildet die Grundlage für zahlreiche theoretische und empirische Untersuchungen zu den Auswirkungen des technischen Fortschritts. Zum einen stehen dabei die Effekte auf die Höhe der langfristigen Wachstumsrate des Produktionsvolumens im Vordergrund. Zum anderen werden häufig die Auswirkungen auf die Produktionsfaktoren (z.B. Beschäftigungsentwicklung) und deren Entlohnung (quotale Einkommensverteilung) untersucht. Diese Wirkungen werden endogen im Modell bestimmt. Dabei sind verschiedene Weiterentwicklungen durch die Aufhebung einiger strikter Annahmen (z.B. vollkommener Wettbewerb) oder der Erweiterung um weitere Produktionsfaktoren (z.B. Humankapital) und Erklärung des technischen Fortschritts vorgenommen worden (Conte 2006, S.27; Petit 1995, S.376 ff). Bei den Weiterentwicklungen zu den Ursachen des technischen Fortschritts hat die größte Beachtung der investitionsinduzierte Ansatz von Arrow (1962) gefunden. ${ }^{9}$ In seinem „Learning-by-doing“Ansatz führt die Kapitalakkumulation zu technischem Fortschritt. Investitionen in neues Kapital beinhalten einerseits den neuesten Stand des technologischen Wissens, andererseits entstehen bei der Anwendung der neuen Maschinen Lerneffekte. Diese Lerneffekte fließen wiederum in die nächste Maschinengeneration ein. $\mathrm{Da}$ in diesem Ansatz weiterhin die Grenzerträge des Kapitals abnehmen, findet endogenes Wachstum nicht statt.

Häufig in der Diskussion steht die Anwendung des Gleichgewichtskonzepts der Neoklassik. Darin wird die (Wieder-)Anpassung eines Modells an einen Gleichgewichtszustand erklärt und der Endzustand der endogenen Variablen ermittelt. Bezogen auf die Analyse des technischen Fortschritts wird dessen exogene Behandlung kritisiert. Technologien werden dabei als öffentliches Gut behandelt, welches für die Akteure kostenfrei und unmittelbar verfügbar ist (Vivarelli 1995, S.13). Der Prozess des technischen Fortschritts selbst wird nicht genauer spezifiziert und erklärt (Cameron 1998). Rückschlüsse auf die Ursachen des technischen Fortschritts und damit auf die langfristigen Wachstumsdeterminanten sind nicht möglich. Daneben wird ein linearer Innovationsprozess angenommen, bei dem der Diffusionsprozess keine Rückwirkung auf Innovation selbst hat. Eine Weiterentwicklung einer bestehenden Innovation wird somit nicht betrachtet. Zudem wird im Grundmodell die Betrachtung des technischen Fortschritts auf Prozessinnovationen eingeschränkt. "To exclude product innovation from technical progress, especially when we considering long historical periods, is to play Hamlet without the prince" (Rosenberg 1982, S.4).

Diese mangelnden Erklärungsmöglichkeiten für den technischen Fortschritt bildeten einen wichtigen Ausgangspunkt für die Entwicklung von Modellen der so genannten Neuen Wachstumstheorie (Erber et al. 1998). Die wesentliche Zielsetzung der ab Mitte der 1980er Jahre entstandenen Ansätze der Neuen

9 Daneben existieren Ansätze zum Faktorpreis-induzierten Fortschritt. Siehe hierfür z.B. Hicks (1962), Ruttan (2001). 
Wachstumstheorie ist die Endogenisierung des technischen Fortschritts (Conte 2006, S.27). Der technische Fortschritt und die damit verbundenen Auswirkungen sind von Wachstumsdeterminanten abhängig, welche sowohl durch Wirtschaftssubjekte als auch durch die Politik beeinflusst werden können. Dabei wird besonders die Bedeutung von Forschung und Entwicklung (F\&E) sowie Humankapital als Determinanten des Wachstums untersucht. Bei den Modellen, welche die Bedeutung von Forschung und Entwicklung hervorheben (Grossman/Helpman 1991/1994; Aghion/Howitt 1992/1996), werden neben Prozessinnovationen auch Produktinnovationen modelliert. Ein besonderer Verdienst der Neuen Wachstumstheorie wird in der stärkeren Mikroökonomischen Fundierung und Verbindung mit der Makroebene gesehen (z.B. RWI 2005; Vosskamp/ Schmidt-Ehmke 2005). Dabei werden in einigen Modellen neoklassische Annahmen, wie vollkommener Wettbewerb oder perfekte Voraussicht der Akteure, aufgehoben. Kritiker der Neuen Wachstumstheorie, insbesondere Vertreter der Evolutorik, sehen die Probleme der neoklassischen Theorie allerdings als nur begrenzt gelöst an. Der Innovationsprozess wird demnach weiterhin relativ abstrakt dargestellt, und lässt eine Analyse der Interaktions- und Anpassungsprozesse bei der Entstehung von Innovationen nur eingeschränkt zu (Brusoni et al. 2006). Darüber hinaus werden grundlegende Prinzipien der Neoklassik wie rationale Akteure und geräumte Märkte beibehalten. Aus empirischer Sicht ist die Umsetzung dieser theoretischen Modelle aufgrund der sehr abstrakten Annahmen und der hohen Komplexität schwierig (Petit 1995; Vosskamp/SchmidtEhmke 2005). So werden in empirischen Studien (u.a. Barro 1991) häufig der Einfluss von Faktoren wie F\&E oder Bildung auf das Wirtschaftswachstum bestätigt, die beschriebenen Ursache-Wirkungszusammen-hänge in den Modellen lassen sich allerdings kaum überprüfen (Petit 1995, S.380). ${ }^{10}$

\subsubsection{Post-Keynesianische Ansätze}

Der angebotsorientierten neoklassischen Wachstumstheorie stehen nachfrageorientierte Post- oder Neo-Keynesianische Wachstumsmodelle gegenüber. Demnach spielt die Nachfrage eine wichtige Rolle sowohl für die Auswirkungen des technischen Fortschritts als auch für dessen Realisierung. Große Bedeutung haben dabei kaldorianische Wachstumsmodelle und Pasinettis Theorie des sektoralen Strukturwandels. Im Gegensatz zur Neoklassik wird nicht von vollkommenen, rationalen und informierten Akteuren ausgegangen. Folglich können Friktionen auf der Angebots- und Nachfrageseite auftreten.

Die Bedeutung der Nachfragedynamik für den technischen Fortschritt und den Wachstumsprozess steht im Mittelpunkt des kaldorianisches Konzepts zur kumulativen Verursachung des Wachstums (Seiter 1997). Das Produktivitäts-

${ }^{10}$ Für eine Übersicht zu empirischen Ergebnissen von Einflussgrößen (z.B. F\&E) auf das Wirtschaftswachstum siehe Cameron (1998) und Temple (1999). 
wachstum und die Nachfrageentwicklung beeinflussen sich dabei gegenseitig. Den Ausgangspunkt des Konzepts bildet die Nachfrage. Eine Expansion der Nachfrage führt zu einer Erhöhung der Produktivität. Die Gründe hierfür liegen vor allem in steigenden Skalenerträgen bei höherer Produktion, neuen Bruttoinvestitionen und Lerneffekten. Die Produktivitätsgewinne führen - unter bestimmten Voraussetzungen auf der Nachfrageseite (Petit 1995, S.387) - wiederum zu einer Erhöhung der Nachfrage und damit zu weiteren Expansionsmöglichkeiten. Im Ergebnis entsteht ein nachhaltiger, kumulativer Wachstumsprozess, der nicht zu einer gleichgewichtigen Wachstumsrate (Steady-State) wie im neoklassischen Modell führt. Der technische Fortschritt wird allerdings auch hier nicht genauer spezifiziert; bei Kaldor (1966/1972) wird er recht allgemein in der "technical progress function“ beschrieben. " Der Analysefokus liegt stattdessen bei den Komponenten der Nachfrage, die teilweise exogen als Ursache des technischen Fortschritts (z.B. öffentliche Ausgaben, private Investitionen) und teilweise endogen als Folge des technischen Fortschritts (z.B. Konsumausgaben, Auslandsnachfrage) auftreten (Pini 1995). Erweiterungen erfolgten durch die „French School of Regulation“ (u.a. Boyer 1988). Dabei werden institutionelle Faktoren stärker in die Analyse miteinbezogen. Diese haben einen entscheidenden Einfluss darauf, inwieweit Produktivitätsgewinne zu einer höheren Nachfrage führen (siehe Kapitel 2.3.3). Nur unter bestimmten institutionellen Entwicklungen wird ein erfolgreicher Wachstumsprozess erreicht. "If a good match between institutional forms and technological opportunities occurs, a mode of regulation is established which can assure the economic growth for a given historical period" (Vivarelli 1995, S.84).

Pasinetti $(1981,1993)$ untersucht in seinen bekannten Werken in einem mehrsektoralen Modell den Zusammenhang zwischen Strukturwandel und Wirtschaftswachstum (siehe Kapitel 2.3). Er unterscheidet dabei explizit Produktund Prozessinnovationen. Kernergebnis ist hier ebenfalls, dass hohe Nachfrageimpulse in Folge des technischen Fortschritts nur unter bestimmten Bedingungen (z.B. hohe Lerneffekte der Konsumenten) auftreten. Es existiert kein Automatismus bei dem sich technischer Fortschritt so in Nachfrageimpulse umsetzt, dass makroökonomische Ziele (z.B. Vollbeschäftigung) erreicht werden. Folglich kann technischer Fortschritt bei fehlendem staatlichen Eingriff möglicherweise zu einer wirtschaftlichen Destabilität mit Arbeitslosigkeit führen (Pasinetti 1981).

Diese in keynesianischer Tradition stehenden Theorien erlauben somit eine recht detaillierte Analyse der Bedeutung der Nachfrage. Sie ist zentral für die

"Bei der ,technical progress function“ stellen investitionsinduzierte Lerneffekte einen Zusammenhang zwischen der Kapitalakkumulation und der Entstehung des technischen Fortschritts her. Investitionen führen zu intertemporalen externen Effekten, da sie die Effizienz zukünftiger Kapitalgüter positiv beeinflussen. Die Veränderung des „technical dynamisn” wird bei Kaldor aber nicht genauer analysiert. 
Auswirkungen des technischen Fortschritts auf die Gesamtwirtschaft und sektorale Entwicklung. ${ }^{12}$ Daneben stellt sie selbst eine Ursache für den technischen Fortschritt dar. Allerdings bleibt die Analyse des technischen Fortschritts auch hier abstrakt. Zwar werden beim kaldorianischen Prozess der kumulativen Wachstumsverursachung wie bei der Neuen Wachstumstheorie Teile des technischen Fortschritts endogenisiert, der Innovations- und Diffusionsprozess wird allerdings ausgeblendet (Petit 1995, S.389).

\subsubsection{Evolutorische Ansätze}

Bei der Evolutorik handelt es sich um kein einheitliches Gedankengerüst, sondern es existiert eine Mehrzahl von Ansatzsträngen (Schwitalla 1993; Freeman 1994). Zunächst werden einige grundlegende Ideen der Evolutorik dargestellt und auf mikrofundierte Ansätze der Innovationsökonomik eingegangen. Im Anschluss wird eine evolutorische Interpretation des „General Purpose Technologies"-Konzept dargestellt, welches eine qualitative Diskussion der wirtschaftlichen Auswirkungen neuer Technologien auf der Makroebene ermöglicht.

\subsubsection{Grundlagen}

Die Evolutorik ist insbesondere als Kritik an der weit verbreiteten neoklassischen Theorie entstanden. Einigkeit der verschiedenen evolutorischen Ansätze besteht in der Ablehnung des neoklassischen Gleichgewichtsansatzes; stattdessen stehen Entstehungs- und Veränderungsprozesse im Zeitablauf im Fokus. Diese dynamische Betrachtungsweise hat ihre Wurzeln in den Arbeiten Schumpeters (1934) zur wirtschaftlichen Entwicklung. Die Entwicklung einer Wirtschaft strebt dabei nicht einem Gleichgewicht im Sinne eines „SteadyState" entgegen, stattdessen führen Innovationen zu spontanen und sprunghaften Veränderungen des Gleichgewichtszustandes. Diese Arbeiten Schumpeters sind in der Evolutorik modifiziert und erweitert worden. ${ }^{13}$ Es zeigen sich dabei einige grundsätzliche Charakterisierungsmerkmale der Evolutorik, wenngleich deren konkrete Zusammenstellung in verschiedenen Arbeiten etwas differiert (siehe z.B. Silverberg/Verspagen 2005; Lehmann-Waffenschmidt 2006). Im Folgenden werden in Anlehnung an Silverberg und Verspagen (2005) drei grundsätzliche Merkmale der Evolutorik dargestellt und im Anschluss auf die Analyse von Technologien konkretisiert.

Grundlegend ist die Annahme der Heterogenität der Akteure. Sie unterscheiden sich u.a. hinsichtlich ihrer Präferenzen, Ausstattung, Erwartungen und Kenntnisse (Nelson/Winter 1982; Dosi et al. 2003). Im Gegensatz zur Neoklas-

${ }^{12}$ Die sektorale Dimension wird dabei besonders im Modell von Pasinetti $(1981,1993)$ deutlich.

${ }^{13}$ Für eine Übersicht zu der Debatte, inwiefern die Evolutorik noch mit den Arbeiten Schumpeters vereinbar ist, siehe z.B. Fagerberg (2003). 
sik handeln sie dabei nicht allein gewinnmaximierend, sondern haben eine gebundene Rationalität. ${ }^{14}$ Als Folge der Heterogenität ergeben sich unterschiedliche Verhaltensweisen der Akteure. Zum Beispiel kann bei der Frage der Technologieadaption für einige Akteure das Risiko zu hoch sein, das Wissen für den Umgang mit der neuen Technologie nicht vorhanden sein oder nicht ins jeweilige Kalkül (Marktanteilsgewinn, Kostenreduktion) passen. ${ }^{15}$ Infolgedessen entsteht eine Vielfalt möglicher wirtschaftlicher Prozesse und eingesetzter Technologien (Silverberg/Verspagen 2005, S.516). Die Reduktion dieser Vielfalt erfolgt durch Selektionsmechanismen (Dosi 1988). ${ }^{16}$ Sie führen dazu, dass die Entwicklung auch durch Regelmäßigkeiten und Kontinuität gekennzeichnet ist. Im Gegensatz zur Neoklassik, bei der durch den Preismechanismus die entscheidende Koordinationsfunktion ausgeht, wirkt eine Vielzahl von Kriterien auf verschiedene Elemente. Es erfolgt dabei eine positive Auswahl von Elementen (z.B. Produkten, Technologien), seitens der einzelnen Wirtschaftsakteure (z.B. Unternehmer), durch die Konkurrenzmechanismen auf den Märkten oder durch vielfältige Institutionen (z.B. Staat) (Dosi et al. 2003; Knottenbauer 2000).

\section{Charakterisierung von Technologien}

Die Evolutorik unterscheidet sich in ihrer Konzeption von Technologien deutlich zur Neoklassik. Technologie wird nicht als Blaupause verstanden, deren Informationen allgemein verfügbar und anwendbar für alle Unternehmen sind. Stattdessen zeichnet sich Technologie besonders durch vier Charakteristika aus (Dosi 1988; Dosi et al. 2003; Metcalfe 1998, Dominguez-Lacasa 2005):

1. Teile einer Technologie sind unternehmensspezifisch. Anwender einer Technologie müssen sich häufig erst zusätzliches Wissen aneignen und Modifikationen in der Technologie vornehmen, um sie in ihren Produktionsprozess integrieren zu können. Dabei setzen die Unternehmen jeweils in eigenen Kombinationen technologisches Wissen, Kapital und Marktmacht ein, so dass im Ergebnis unternehmensspezifische technologische Lösungen entstehen.

\footnotetext{
14 Silverberg (1997, S.413) charakterisiert die gebundene Rationalität wie folgt: "bounded rationality of agents: behaviour may be goal directed (although often without an explicitly specified goal function) but agents have limited information, limited computational ability, and both are costly."

${ }^{15}$ Die Annahme des gewinnmaximierenden Verhaltens wird insbesondere bei der Entscheidung über technische Neuerungen als unrealistisch eingestuft. Diese ist mit hoher Unsicherheit verbunden, die Informationsgrundlage für eine Kalkulation der erwarteten Kosten und Erträge der Forschungs- und Entwicklungstätigkeiten ist nicht gegeben (Gerybadze 1982, S.44).

${ }^{16}$ Dabei werden (lose) Analogien des ökonomischen Selektionsprozesses zur Biologie gebildet.
} 
2. Eine Technologie ist lokal. Die Exploration und Entwicklung neuer Techniken von Unternehmen ist eng an ihre bisherigen Produktionstechniken und Organisation geknüpft. Technischer Fortschritt führt demnach nicht wie in der Neoklassik zu einer Verschiebung der gesamten Produktionsfunktion, die für alle Kombinationen der Produktionsfaktoren eine höhere Effizienz ermöglicht, sondern bezieht sich im Extremfall nur auf eine spezifische Faktorkombination (Stiglitz 1987).

3. Technologien bauen auf implizitem Wissen auf. Sie können nicht vollständig kodifiziert werden, sie können folglich nur bedingt über die Informationsinfrastruktur übertragen und verteilt werden. Insbesondere die spezifische Transformation von Wissen in technologische Lösungen baut auf implizitem Wissen der Individuen und Organisationen auf.

4. Eine Technologie ist kumulativ in ihrer Entwicklung. Die aktuelle technologische Entwicklung basiert auf dem im Zeitablauf individuell akkumulierten Wissen, das durch zielgerichtete Kombination und Ergänzung zur Entwicklung neuer Problemlösungen genutzt wird (Nelson/Winter 1982; Dosi et al. 2003).

Die von Unternehmen vorangetriebene technologische Entwicklung hängt demnach entscheidend von dem im Zeitablauf akkumulierten Wissen ab, das zu einem gewissen Anteil spezifischen, lokalen und impliziten Charakter aufweist. Insgesamt setzt sich das eingesetzte technologische Wissen in einer Volkswirtschaft zu einem Zeitpunkt aus der Imitation und Diffusion von „Best-PracticeTechnologien", aus der Suche nach neuen Technologien und aus der Marktselektion zwischen heterogenen Akteuren zusammen. Demnach gibt es keine „Best-Practice-Technologie“, die gleichzeitig von allen Akteuren eingesetzt wird. Ferner wird in der Evolutorik dem Kapital-ungebundenen technischen Fortschritt eine höhere Bedeutung beigemessen. Technologisches Wissen lässt sich nicht einfach über Investitionen in neues Sachkapital erwerben, sondern wird über vielfältige Mechanismen erlernt (Dosi et al. 2003; Freeman 1982; Dosi 1988). Dieser wissensbasierte Ansatz hat entscheidende Implikationen für die Bedeutung der Produktionsfaktoren im Wachstumsprozess. Der Faktor Arbeit verkörpert technischen Fortschritt und ist nicht der Produktionsfaktor, welcher bei technischem Fortschritt durch Kapital substituiert wird (Dopfer 2005, S.53; Welsch 2006, S.820). Daneben wird die Bedeutung der Produktinnovationen in evolutorischen Ansätzen hervorgehoben (z.B. Rosenberg 1982) und die unterschiedlichen Faktoren für die Entwicklung und die Diffusion im Vergleich zu Prozessinnovationen explizit untersucht (z.B. Lundvall 1988; Edquist et al. 2001).

\section{(Teil-)Endogenitität des technologischen Fortschritts}

Die dargestellte Spezifität von technologischem Wissen und ihre kumulative Erwerbung implizieren zunächst eine unkoordinierte technologische Entwick- 
lung (Pyka 1999). Wichtige Ansätze zur Erklärung der in der Realität dennoch über längere Zeit in mehr oder weniger geordneten Bahnen ablaufenden technologischen Entwicklung liefern die Ausführungen von Dosi $(1982,1988)$ zu Paradigmen und Trajektorien. Dosi definiert ein technologisches Paradigma ,as a 'model' and a 'pattern' of solution of selected technological problems, based on selected principles derived from natural science and on selected material technologies" (Dosi 1988, S.1334). Das Paradigma stellt somit eine Art Lösungsmuster für ausgewählte technoökonomische Probleme dar. Es umfasst dabei ein Cluster von verbundenen Technologien und organisatorischen Prinzipien. Eine bedeutende Rolle spielt dabei die Wissenschaft. Fortschritte im wissenschaftlichen Know-how erweitern den Pool an möglichen technologischen Paradigmen. Entscheidend dafür wie und welches Wissen weiterentwickelt und umgesetzt wird, sind die bereits erläuterten Selektionsprozesse. In Bezug auf die Technologie selbst sind hier besonders institutionelle Faktoren, „Trial-and-Error" Mechanismen bei der Exploration neuer Technologien und technoökonomische Voraussetzungen für „early user" zu nennen. "When a technological paradigm is established, it brings with it a reduction of uncertainty, in the sense that it focuses the directions of search and forms the grounds for formatting technological and market expectations more surely" (Dosi 1988, S.1334). Dies impliziert, dass andere alternative Technologiepfade und Lösungsmuster von den Akteuren kaum weiter verfolgt werden. Ein typisches Paradigma basiert dabei auf einigen konkreten Schlüsseltechnologien, Produkten, Rohstoffen, organisatorischen Aktivitäten und geographischen Kernpunkten. Beispiele für technologische Paradigmen sind der Verbrennungsmotor, die ölbasierte synthetische Chemie und Halbleiter (Dosi et al. 2003).

Innerhalb dieser Paradigmen vollzieht sich die technologische Entwicklung in technologischen Trajektorien (Bahnen). Diese definiert Dosi als "the pattern of normal problem solving activity (i.e. of. progress) on the ground of technological paradigm" (Dosi 1988, S.152). Technischer Fortschritt entlang einer Trajektorie erfolgt graduell und ist mit der Entwicklung von spezifischen Infrastrukturen, von komplementären Technologien, dem Aufbau von technischen Standards und Skaleneffekten im System verbunden. Der Prozess und das Ergebnis sind daher teilweise irreversibel. Somit ist die weitere Entwicklung von den historischen Entscheidungen und Gegebenheiten beeinflusst und pfadabhängig. Als Beispiele gelten aus dem Bereich der Mikroelektronik die ständige Miniaturisierung von Chips und die kontinuierliche Vergrößerung ihrer Speicherkapazität oder Polymertechniken in der organischen Chemie (Dosi et al. 2003). Allerdings ist der mögliche technische Fortschritt innerhalb einer Trajektorie nicht unerschöpflich und nimmt entlang einer Trajektorie im Zeitablauf ab, da die technologischen Möglichkeiten von naturwissenschaftlichen Gesetzen beschränkt werden. 
Dies bedeutet, dass neue technologische Paradigmen und Trajektorien notwendig sind, damit die technologische Entwicklung nicht langfristig stagniert. Dabei findet eine Teilendogenisierung des technischen Fortschritts statt. Innerhalb der Grenzen eines Paradigmas wird die Rate und Richtung des technischen Fortschritts stark endogen durch ökonomische und institutionelle Faktoren beeinflusst. ${ }^{17}$ Dosi et al. (2003) unterscheiden zwischen vier Kategorien von Anreizeffekten für die Richtung und Ausmaß von technologischen Aktivitäten der Akteure:

1. Veränderte Angebots- und Preisbedingungen haben entscheidenden Einfluss auf die Such- und Problemlösungsaktivitäten, die eine wichtige Voraussetzung für Innovationen bilden. Beispiele hierfür sind Angebotsschocks (z.B. Ölpreisschocks) oder technologische „Bottlenecks“ bei komplementären Prozessen und Produkten.

2. Anreize gehen auch vom Wachstum der Nachfrage und dessen Zusammensetzung aus. Investitionen in die F\&E und der Implementierung von Technologien sind abhängig von Profitanreizen. Die Profitanreize werden u.a. von der Marktgröße beeinflusst (Schmookler 1966).

3. Die Profitanreize von Akteuren hängen daneben davon $a b$, inwiefern Möglichkeiten bestehen, selbst von Innovationen profitieren zu können. Mögliche Mechanismen hierfür sind u.a. Patente, Geheimhaltung, Zeitvorsprünge, Kosten- und Zeitaufwand von Imitationen. Ihre Wirkungen unterscheiden sich dabei zwischen Technologien und Sektoren. Neben dem Anreizeffekt führen hohe Profitmöglichkeiten für die Innovatoren aber auch dazu, dass nur ein kleiner Teil des Nutzens einer Innovation im gesamten ökonomischen System Verbreitung durch Effizienzverbesserungen, Lernen durch Imitationen und/oder Preisveränderungen findet. Schließlich sind z.B. günstigere Imitationen nicht möglich, welche die Verbreitung und den Nutzen für Anwender und Konsumenten erhöhen könnten. ${ }^{18}$

4. Ein weiterer Faktor besteht in den Selektionsmechanismen. Die Selektionsdynamik von Technologien, Verhaltensweisen und Unternehmen wird entscheidend von ökonomischen Faktoren (z.B. Wettbewerb auf Gütermärkten, Auswahl durch Finanzinstitutionen) geprägt.

\footnotetext{
${ }^{17}$ Die in den 1960er und 1970er Jahren stark diskutierten Theorien des „technology push“ und des „demand pull“" werden jeweils als unzureichend angesehen, um ein theoretisches Gerüst für technologischen Fortschritt zu liefern. Diese Theorien fokussieren sich entweder auf Angebotsfaktoren als Ursache von technologischen Möglichkeiten oder auf Nachfragebedürfnisse als Haupttreiber für technologische Aktivitäten.

${ }^{18}$ Für eine Diskussion dieses zwiespältigen Anreizeffektes für die Biotechnologie siehe Kapitel 3.2.2.4.
} 
Die Hauptimplikation dieser Anerkennung von endogenen Mechanismen ist die Abkehr der Annahme des linearen Innovationsprozesses. Vielmehr wird eine koevolutionäre Sicht eingenommen, die permanente Rückkoppelungen zwischen Innovation, Diffusion und endogenen Mechanismen zur technologischen Weiterentwicklung zulässt (Dosi et al. 2003). Dies bedeutet auch, dass nicht eine homogene Technologie zu einem bestimmten Zeitpunkt allgemein verfügbar wird und diffundiert, sondern sich im Zeit- und Diffusionsablauf noch weiterentwickelt. „The fact is that most important innovations go through rather drastic changes over the lifetimes - changes that may, and often do, totally transform their economic significance" (Kline/Rosenberg 1986, S.283). Ein bekanntes Beispiel hierfür ist der Automobilbau, bei dem zahlreiche Verbesserungsinnovationen im Laufe der Zeit zu einem völlig veränderten Produkt geführt haben. Erst dadurch haben sich bedeutender technischer Fortschritt und wirtschaftliche Effekte für die Volkwirtschaft entfaltet (Freeman 1994, S.481). Diese Beobachtungen über den Innovationsprozess und der enge Zusammenhang mit sozialen, institutionellen und politischen Faktoren, führte zu einer stärkeren systemischen Betrachtung von Innovation und Diffusion von Technologien. Da diese Faktoren nach Ansicht vieler Autoren stark auf nationaler oder regionaler Ebene bestimmt werden, bekam die Analyse eine stärkere territoriale Dimension (Fagerberg 2003). ${ }^{19}$ Dies führte zu einer breiten Anzahl an Konzepten von nationalen und regionalen Innovationssystemen (u.a. Nelson 1988; Carlsson et al. 2002; Malerba 2002; Freeman 1987; Lundvall 1988) und zu einer großen Fülle empirischer Untersuchungen. ${ }^{20}$ Freeman (1987), der neben Lundvall den Begriff des Nationalen Innovationssystem prägte, umschreibt dieses als "the networks of institutions in the public and private sectors whose activities and interactions initiate, import, modify and diffuse new technologies" (Freeman 1987, S.4). Nach diesen Konzepten beeinflusst eine Vielzahl von Faktoren und Akteuren die Entwicklung, Diffusion oder Nutzung von Innovationen. Die Akteure agieren dabei nicht unabhängig voneinander, sondern erst das Zusammenspiel und die Vernetzung der Akteure ermöglichen die Schaffung und die Verbreitung neuer Lösungen (Lundvall 1992). Besonders für die Entwicklung und Diffusion neuer

\footnotetext{
${ }^{19}$ Nach Lundvall $(1988,1992)$ sprechen zwei Kernargumente für einen starken nationalen Charakter des Innovationssystems. Erstens führt die zeitliche Stabilität (nur langsame Veränderung) der Wirtschaftsstruktur eines Landes zu relativ stabilen Vernetzungsstrukturen zwischen den inländisch ansässigen Akteuren. Die Innovationsdynamik wird wiederum entscheidend von den Vernetzungen geprägt. Zweitens führen Kultur, Sprache und Institutionen zu einer Interaktion und Lernprozess von Unternehmen und ihrer Umwelt.

${ }^{20}$ Unterschiede zwischen den einzelnen Konzepten der Innovationssysteme bestehen dabei vor allem in der Abgrenzung des Systems. Sie unterscheiden sich dabei insbesondere bei der Breite der betrachteten Akteure, bezüglich des geographischen Bezugs, Bedeutung einzelner Teilelemente (z.B. Technologie, Nachfrage) und ihrem Fokus auf der Mikro-, Meso- oder Makroebene.
} 
Produktinnovationen sind gut funktionierende Innovationssysteme eine entscheidende Voraussetzung (Lundvall 1988).

\section{Betrachtung der wirtschaftlichen Effekte von Technologien}

Die Analyse der wirtschaftlichen Effekte von Innovationen und technischem Fortschritt ist aus dieser Perspektive schwierig, da sich Technologien im $\mathrm{Zu}$ sammenspiel mit vielen anderen Faktoren entwickeln und die Effekte hiervon abhängen (Carlsson/Eliasson 2003). „Die Funktions- und Leistungsfähigkeit eines nationalen Innovationssystems ist ausschlaggebend für das Hervorbringen von Innovationen und damit für die Sicherung und Erschließung von Beschäftigungspotenzialen" (Welsch 2006, S.819). Aussagen über falsifizierbare Ursache-Wirkungszusammenhänge und eine ex-ante-Betrachtung der Entwicklung sind bei der Fülle der Einflussfaktoren schwer möglich. Stattdessen konzentriert sich die Evolutorik auf die ex-post-Erklärung der Ursachen, die Analyse der wirtschaftlichen Effekte selbst nimmt eher eine untergeordnete Rolle ein (Verspagen 2001, S.6.).

Der Ausgangspunkt bei der Analyse der Effekte erfolgt in der Regel auf der Mikroebene, da die Heterogenität der Akteure und die Interaktionen von hoher Bedeutung sind (Carlsson/ Eliasson 2003; Brusoni et al. 2006). Einige Arbeiten zeigen dabei auf, dass bei der Integration der Mikroebene ein bestimmtes Ergebnis auf der Makroebene durch unterschiedliche Wirkungszusammenhänge entstehen kann (Ruttan 2001; Nelson/Winter 1982). Insgesamt ist eine Vielzahl an Erfolgsdimensionen und -indikatoren von Innovationen denkbar, es besteht aber kein Konsens, welche davon am relevantesten ist. Als Beispiele für Erfolgsindikatoren der Unternehmen sind die Profitabilität, das Umsatzwachstum, die Beschäftigung, Patentanteile, Marktanteile etc. zu nennen. Dabei besteht aber die Problematik - sowohl auf theoretischer als auch auf empirischer Ebene - mikroökomische Zusammenhänge und Ergebnisse auf die gesamtwirtschaftliche Ebene zu übertragen. Eine direkte Übertragung ist aufgrund möglicher kompensatorischer Effekte in der Regel nicht möglich (siehe Kapitel 4.1; Pianta 2005; Brusoni et al. 2006).

Eine ähnliche Problematik gilt für die Mesoebene. Diese wird dennoch häufig als sehr geeignete Analyseebene eingeschätzt (Pavitt 1984; Malerba 2002; Pianta 2005; Edquist et al. 2001). So zeigt sich, dass sich die betonte Heterogenität der Akteure stark in Unterschieden zwischen den Sektoren (z.B. verwendete Technologien, Wissensbasis, Profitmöglichkeiten, Nachfrage etc.) widerspiegelt, während Unternehmen innerhalb von Sektoren deutliche Ähnlichkeiten zueinander aufweisen (Pavitt 1984; Pianta 2005). Dabei können Technologien und technischer Fortschritt eine sehr unterschiedliche Bedeutung in einzelnen Sektoren haben. Dies hat insbesondere Implikationen für die Analyse von Querschnittstechnologien (z.B. der Biotechnologie), die für eine Mehrzahl von Sektoren relevant ist. Die Gründe für die unterschiedliche sektorale Bedeutung 
von Technologien, werden im Konzept von Malerba (2002) zu sektoralen Innovationssystemen deutlich. Die Sektoren haben einen unterschiedlichen Bezug zu wissenschaftlichen Zweigen und Technologiefeldern, zu Anwendungen und Nutzer auf der Nachfrageseite sowie verschiedene Rahmenbedingungen (z.B. Möglichkeiten zum Schutz des Wissens). Jeder Sektor zeichnet sich durch eine unterschiedliche Produkt-Technologie-Matrix aus. Folglich sind die sektoralen technologischen Möglichkeiten unterschiedlich und verändern sich im Zeitablauf. ${ }^{21}$ Hieraus wird deutlich, dass sich die sektoralen Effekte einer neuen Technologie deutlich unterscheiden können. Allerdings lassen diese Betrachtungen keine direkten Rückschlüsse auf wirtschaftliche Effekte in einem Sektor zu (RWI 2005), noch werden indirekte Effekte in anderen Sektoren ersichtlich.

Aufgrund der schwierigen Übertragung der Ergebnisse der Mikro- und Mesoebene auf die Makroebene fehlt folglich ein einheitliches oder limitiertes Set an Wachstumsmodellen wie in der neoklassischen oder der Neuen Wachstumstheorie. ${ }^{22},[\ldots]$ even when attention is limited to growth theory, it is impossible to define the evolutionary growth model" (Verspagen 2001, S.3). Es besteht lediglich Konsens über einige Aspekte. So wird auch in der Evolutorik dem technischen Fortschritt eine hohe Bedeutung für wirtschaftliches Wachstum beigemessen (Verspagen 2001, S.6; Conte 2006, S.34). Für die Realisierung der wirtschaftlichen Potenziale reicht das Aufkommen neuer technologischer Möglichkeiten allein aber nicht aus. Stattdessen findet technischer Fortschritt und wirtschaftliches Wachstum als ein von der historischen Zeit abhängiger Prozess statt und ist von ökonomischen und außerökonomischen (Kultur, Institutionen, Wissenschaft) Faktoren geprägt. Die wirtschaftliche Entwicklung bildet dabei keinen Konvergenzprozess zu einem Gleichgewichtspfad (Steady-State), sondern bildet einen Transformationsprozess mit ständigen strukturellen Veränderungen. ${ }^{23}$ In diesem Prozess kommt es nicht unbedingt zu einer Vollauslastung

${ }^{21}$ Dies liegt nach Malerba (2002) besonders an einem unterschiedlichen Wissenszugang und der jeweiligen Kumulierung des technologischen Wissens. Nach Dosi (1988) ist hierfür die technologische Nähe der Sektoren zum ,revolutionären Kern“, dem Ursprung eines neuen Paradigmas entscheidend, sowie der Entwicklungsstand der jeweiligen Trajektorien. Ebenso sind die technologischen Schwierigkeiten, die Produktion effizienter zu gestalten und die Leistung zu steigern, verschieden. Diese durch die jeweiligen Basistechnologien bestimmten Grenzen der technologischen Möglichkeiten sowie die Nachfrage können zu einer unterschiedlichen Bereitschaft zwischen Sektoren führen, in eine bestimmte Technologie zu investieren.

${ }^{22}$ Es existieren zwar einige Ansätze zur Modellierung evolutorischer Wachstumsprozesse. Allerdings müssen zur Komplexitätsreduktion deutliche Vereinfachungen und restriktivere Annahmen im Vergleich zu den mikroökonomischen Modellen getroffen werden (Silverberg/ Verspagen 2005).

${ }^{23}$ Eine zusätzliche Implikation, die aber im Folgenden nicht weiter betrachtet wird, ist die folglich schwierige Unterscheidungsmöglichkeit zwischen Trendwachstum und Konjunkturzyklen (Verspagen 2001, S.6). 
der Produktionsfaktoren (z.B. Vollbeschäftigung) oder einer Konvergenz der wirtschaftlichen Entwicklung zwischen Ländern. Stattdessen kann gerade die Entwicklung und Anwendung neuer Technologien zu Wachstumsunterschieden zwischen Ländern führen. Innovations- und Imitation/Diffusions-prozesse können zu einem ständigen Konvergenz- und Divergenzprozess führen (Fagerberg/ Verspagen 2002; Castaldi/Dosi 2008). Darüber hinaus können verschiedene Technologien unterschiedliche wirtschaftliche Folgen haben. Dies wird besonders im Konzept der „General Purpose Technologies“ deutlich.

\subsubsection{Konzept der General Purpose Technologies}

Die Theorien der „General Purpose Technologies" (GPT) oder der Basistechnologien analysieren die langfristigen makroökonomischen Auswirkungen besonders bedeutender Technologien. ${ }^{24}$ Diese Technologien führen zu tiefgreifenden Veränderungen in einer Volkswirtschaft. Sie haben einen großen Einfluss auf den wirtschaftlichen Wachstumsprozess und führen langfristig zu einem Anstieg der Produktion und Produktivität. Die Auswirkungen sind allerdings nicht nur ökonomischer Natur, sondern können auch organisatorischer, institutioneller oder sozialer Art sein. Gerade aufgrund der tiefgreifenden Veränderungen treten Anpassungsfriktionen und Ungleichgewichte auf, die zu Schwankungen (z.B. „productivity slowdowns“ ${ }^{25}$ ) in der wirtschaftlichen Entwicklung führen. Diese zeit- und kostenintensiven Anpassungsprozesse beim Auftreten neuer GPTs stehen im Mittelpunkt vieler dieser Konzepte und Modelle, für die es keine einheitliche Theorie gibt. ${ }^{26}$ Mittlerweile existieren eine Vielzahl an formalisierten Modellen sowie umfassende, nicht-formale Überlegungen. Die Konzepte unterscheiden sich teilweise in der Definition einer GPT, ihrer Sicht über die Entstehung $^{27}$, den Implikationen von GPTs und ihren Untersuchungsschwerpunkten (Helpman 1998, S.4). So stehen in formalen Modellen die zeitliche Entwicklung makroökonomischer Effekte (z.B. Helpman, Trajtenberg 1998a; Aghion/ Howitt 1998), die Rolle von Institutionen (Rosenberg 1998), der Diffusionsprozess, die Endogenität bzw. das zeitliche Auftreten der GPTs (Carlaw/Lipsey 2003; Helpman/ Trajtenberg 1998b) oder die Implikationen für die Faktormärkte

\footnotetext{
${ }^{24}$ Der Begriff und das Konzept der GPTs entstand Anfang der 1990er Jahre in einer Arbeit von Bresnahan/ Trajtenberg (1995).

${ }^{25}$ Zwischenzeitliche Rückgänge in der Wachstumsrate der Produktivität wurden vor allem für die beiden GTPs Elektrizität und IKT beobachtet (David 1990).

${ }^{26}$ Vor allem die Formalisierung der GPT grenzt diese Konzepte von früheren Ansätzen zu langfristigen Wachstumseffekten von Technologien ab. Dabei schlagen die Modelle eine Brücke zwischen der neuen Wachstumstheorie auf der einen Seite und evolutionsökonomischen Überlegungen, wie der kumulativen Verursachung oder Rückkoppelungseffekte, auf der anderen Seite.

27 Dabei bestehen insbesondere unterschiedliche Ansichten bezüglich der Exogenität vs. Endogenität sowie der zeitlichen Kontinuität von GPTs.
} 
(Aghion/Howitt 1998; Harris 1998) im Vordergrund. Aufgrund der Komplexität der formalen Modellierung gelingt es diesen Modellen nur einen Teil dieser Aspekte gleichzeitig explizit zu untersuchen. Im Folgenden wird deshalb ein nichtformalisiertes Konzept von Lipsey et al. (1998/2005) dargestellt, welches auf evolutorischen Ansätzen aufbaut. Es ist deutlich umfassender als andere Konzepte und zeigt differenzierter die Grundcharakteristika einer GPT sowie die Einflussfaktoren und Wirkungszusammenhänge für die Auswirkungen einer GPT auf. Es eignet sich daher gut für eine Diskussion über eine konkrete Technologie (Kapitel 3.2). Daneben stehen stärker die Voraussetzungen im Fokus, unter welchen eine Technologie zu einem langfristigen Wachstumsmotor wird (Palmberg/Nikulainen 2006).

\section{GPT-Kriterien im Konzept von Lipsey/Carlaw/Bekar}

Das Ziel des Konzepts von Lipsey, Carlaw und Bekar $(1998,2005)$ ist es, möglichst viele Aspekte zu erfassen, die bei der Analyse von GPT und ihrer Auswirkungen auf die Ökonomie, Politik und Gesellschaft von Bedeutung sein können (Hepperle 2004). Eine GPT ist dabei ,[...] a single generic technology, recognizable as such over its lifetime, that initially has much scope for improvement and eventually comes to be widely used, to have many uses, and to have many spillover effects" (Lipsey et al. 2005, S.98). Die Identifikation einer GPT und ihre Abgrenzung zu anderen Technologien gelten als schwierig. Abstrakt gesprochen wird unter einer GPT eine generische ${ }^{28}$ Technologie verstanden, die trotz ihrer stetigen Weiterentwicklung als solche erkennbar bleibt. Sie zeichnet sich durch mehrere Charakteristika aus, die gleichzeitig erfüllt sein müssen. ${ }^{29}$

Potenzial für Verbesserung und Leistungssteigerung (Scope for Improvement): Die Technologie hat im Laufe ihrer Entwicklung das Potenzial für deutliche Verbesserungen. Durch diese Verbesserungen verringern sich die Kosten bei bestehenden Anwendungen und es entstehen neue Anwendungsmöglichkeiten.

Große Anzahl verschiedener Anwendungsbereiche (range of use): Die Technologie hat eine große Reichweite bei den Anwendungen. Die Reichweite

\footnotetext{
${ }^{28}$ Mit dem Begriff ,generisch“ wird von einzelnen Ausprägungen von Technologien, z.B. verschiedene Varianten von Dampfmaschinen, abstrahiert. Die Erkennbarkeit einer generischen Technologie im Zeitablauf kann am Beispiel des Computers verdeutlicht werden. Der moderne Personalcomputer unterscheidet sich in vielerlei Weise von seinen Vorgängern, ist aber ebenso der generischen Technologie einer elektronischen Rechenmaschine zurechenbar.

${ }^{29}$ Während einige Autoren das Konzept der GPT enger eingrenzen und sich bei ihrer Analyse auf wenige, für sie zentral erscheinende GPTs beschränken, identifizieren Lipsey et al. $(1998,2005)$ eine breite Anzahl an GPTs. In ihrer historischen Analyse ermitteln sie 22 eigenständige GPTs, da sie auch Organisationsformen, wie z.B. Massenproduktion, als eigenständige GPTs mit einbeziehen. Im Unterschied hierzu stuft David (1990) viele dieser GPTs aus Sicht von Lipsey et al. (2005) nur als subsidiäre Technologien ein.
} 
bezieht sich auf den Anteil der Wirtschaft, in dem die Technologie genutzt wird. Eine GPT zeichnet sich dabei durch Anwendungen in vielen Sektoren und Produkten aus.

Große Vielfalt verschiedener Produkte und Prozesse (variety of use): Die Technologie hat eine große Vielfalt bei den Anwendungen. Die Vielfalt der Nutzung ergibt sich durch den Gebrauch der Technologie für verschiedene Zwecke. In Abgrenzung zum Begriff der Reichweite steht dabei nicht die Anzahl der Anwendersektoren, sondern die Verschiedenartigkeit der Nutzbarkeit der Technologie im Vordergrund. ${ }^{30}$

Starke Komplementaritäten zu anderen Technologien / Innovationen (complementarities): GPTs besitzen starke Komplementaritäten zu bestehenden oder neuen Technologien. Diese starke Einbettung der GPT in das technologische System ermöglicht das Auftreten zahlreicher Spillover-Effekte. Es profitieren auch viele Akteure außerhalb des Unternehmens oder des Sektors, in welchem die Technologie zunächst produziert wurde, vom technischen Fortschritt.

Viele Autoren heben dabei besonders die Bedeutung von komplementären Innovationen und Externalitäten hervor (z.B. Bresnahan/Trajtenberg 1995). Die GPT hat vor allem die Rolle einer „enabling technology“. Sie eröffnet in der Regel neue Möglichkeiten, anstatt direkt finale Lösungen in konkreten Anwendungen zu liefern. Um das vollständige Produktivitätspotenzial einer GPT zu realisieren, sind komplementäre Innovationen in den Anwendersektoren notwendig. Dabei wird besonders im Ansatz von Bresnahan und Trajtenberg (1995) die Bedeutung von vertikalen (GPT-Sektor - Anwendersektor) und horizontalen (zwischen Anwendersektoren) sektoralen Beziehungen für die Diffusion und Entfaltung der Potenziale deutlich. Zum einen erhöht sich die F\&E-Produktivität in Anwendersektoren einer GPT in Folge neuer Innovationen bei den GPTProduzenten in dieser Technologie. Zum anderen entstehen Spillover-Effekte zwischen Anwendersektoren durch die Übertragung von Lerneffekten. Diese Zusammenhänge sind in Abbildung 2 skizziert. Dabei werden die vertikalen (durchgezogene Linien) und horizontalen (gestrichelte Linien) Beziehungen zwischen dem GPT-Sektor (GPT) und den Anwendersektoren (AS) ersichtlich.

\section{Faktoren im Produktionsprozess}

Für diejenigen Technologien, die diese Kriterien erfüllen (z.B. IKT), werden die Zusammenhänge mit anderen Faktoren im gesamtwirtschaftlichen Produktionsprozess, die Diffusionsfaktoren und die Auswirkungen von Lipsey et al. (1998/2005) näher untersucht. Dabei wird der Produktionsprozess einer Volkswirtschaft im Gegensatz zur aggregierten Produktionsfunktion der Neoklassik in verschiedene Elemente zerlegt. In dieser „structuralist evolutionary“ Dekompo-

${ }^{30}$ Als Beispiel für eine hohe Reichweite, aber eingeschränkten Nutzungsmöglichkeiten, gilt die Glühbirne, welche nur für die Produktion von Licht eingesetzt wird (Lipsey et al. 2005). 
sition wird das technologische Wissen von allen strukturellen Faktoren (,Faciliating Structure“) und der Politik getrennt (Abbildung 3) ${ }^{31}$. Die „Faciliating Structure” (FS) beinhaltet alle Komponenten (physische Objekte, Leute und Strukturen), die in Verbindung mit dem technologischen Wissen für den physischen Produktionsprozess von Bedeutung sind. Darunter fallen eine Vielzahl von Elementen wie zum Beispiel das physische Kapital, Humankapital, Forschungseinrichtungen, Organisations- und Industriestrukturen, geographische Lage der Aktivitäten, usw..

\section{Abbildung 2: Reichweite und Innovationskomplementaritäten einer GPT}

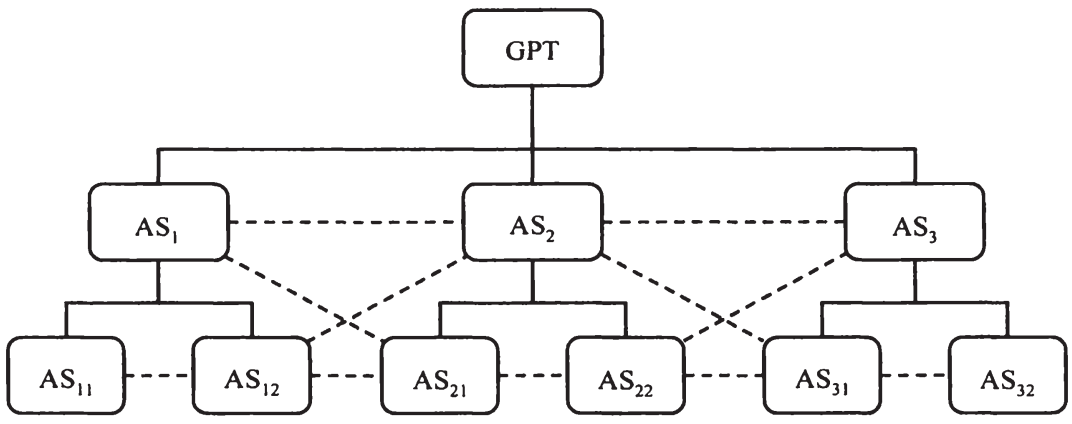

Quelle: Ott et al. 2008

Abbildung 3: Produktionsprozess in der "structuralist evolutionary“ Dekomposition

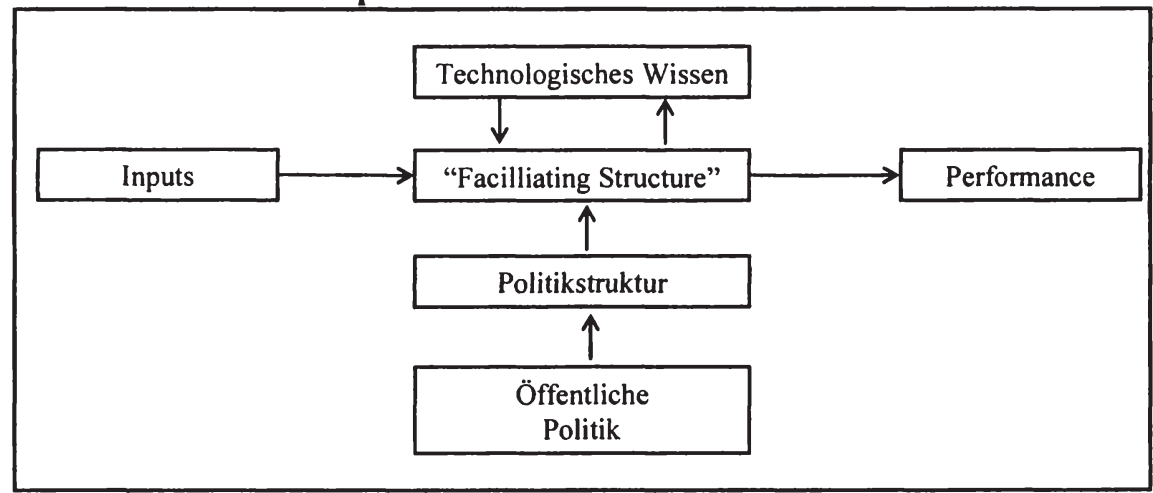

Quelle: Lipsey et al. (2005)

${ }^{31}$ Unter Technologien wird allein das Technologische Wissen verstanden, nicht die Verkörperung in Kapitalgüter. 
Durch das Auftreten neuer GPTs und ihrer Weiterentwicklung verändern sich der Produktionsprozess und dessen Ergebnisse in entscheidendem $\mathrm{Maße}$. $\mathrm{Zu}-$ nächst sollen hierbei die Rückwirkungen zwischen der neuen GPT und den bisher existierenden Technologien betrachtet werden: Eine neue GPT beeinflusst das bisherige technologische System einer Volkswirtschaft über zwei zentrale Wirkungskanäle: den Preis- und den Technologieeffekten. Durch die GPT lassen sich zum einen Kosten für Inputs senken, die in einer Vielzahl von Produktionsprozessen eingesetzt werden (z.B. Computer). Zum anderen führen technologische Zusammenhänge zu weit reichenden Effekten, die nicht als Folge von Preisveränderungen modelliert werden können. Beispielsweise ermöglichen der Computer und das Internet neue Produkte und Prozesse, deren Bereitstellung durch vorherige Technologien auch durch einen Preisverfall bei verschiedenen Inputfaktoren nicht möglich gewesen wären. Damit unterscheidet sich die Theorie von der neoklassischen Sichtweise, in der Preiseffekte im Vordergrund stehen. Lipsey et al. $(1998,2005)$ stellen eine Vielzahl von unterschiedlichen Möglichkeiten der Verhältnisse von Technologien zueinander heraus, welche zu verschiedenen Effekten führen. GPTs können den Wert oder die Anwendungsmöglichkeiten komplementärer Technologien erhöhen, die Entwicklung neuer Technologien ermöglichen, aber auch stark in Konkurrenz zu existierenden Technologien stehen. Dabei kann sich das Verhältnis im Zeitablauf ändern. Häufig kommen neue GPTs zunächst für bereits existierende Anwendungen in Betracht, für welche bisher bestehende Technologien genutzt werden. ${ }^{32}$ Dadurch entsteht eine starke Konkurrenz zwischen der neuen und der existierenden Technologie, insbesondere wenn sich die existierende Technologie selbst noch in der Weiterentwicklung befindet. Eine Konkurrenz kann aber auch erst dadurch auftreten, dass zusätzliche Anwendungen einer GPT in ihrer Entwicklungsphase entstehen und dort mit Technologien in Konkurrenz treten, die zunächst völlig losgelöst oder sogar komplementär zur GPT waren. ${ }^{33}$

Ähnlich vielfältig sind die Auswirkungen und Rückkoppelungen zwischen der GPT einerseits und der FS andererseits. Für eine breite Diffusion und besonders der vollständigen Realisierung des Potenzials einer GPT sind tiefgreifende Veränderungen in der FS notwendig. Die existierende Ausgestaltung der Produktionsfaktoren (Kapitalgüter, Humankapital etc.) aber auch organisatorische Formen und institutionelle Rahmenbedingungen sind nicht dafür geeignet, um

${ }^{32}$ Lipsey et al. $(1998,2005)$ sehen in der Geschichte nur wenige Fälle, bei denen eine GPT völlig neue Anwendungsnischen gefüllt hat. Eines der wenigen Beispiele ist demnach die lang andauernde Alleinstellung von dreimastrigen Segelschiffen für den transozeanischen Handel.

${ }^{33}$ Ein historisches Beispiel ist das Verhältnis zwischen Automobilen und Eisenbahn. Zunächst wurden Automobile für die Zulieferung von Materialien zu den Bahnhöfen für Züge eingesetzt, sie hatten demnach eine Komplementärfunktion. Durch die Verbesserung der Effizienz der Motoren und den verstärkten Aufbau von Straßen traten Automobile in Konkurrenz zur Eisenbahn für längere Transporte. 
die Technologie effizient einzusetzen und neue Möglichkeiten zu nutzen. Stattdessen ist u.a. die Entwicklung neuer Infrastrukturen (z.B. Telekommunikationsnetze und Internet zum Computer), neuer Kapitalgüter, komplementärer Technologien sowie neuer Produktions- und Organisationsformen notwendig (Welsch 2006, S.817; Holwegler 2003, S.356). Diese Veränderungen vollziehen sich in einem zeit- und kostenintensiven Lern- und Anpassungsprozess. Schließlich sind für die erfolgreiche Implementierung der GPT umfangreiche Investitionen in den F\&E-Bereich, in technologiespezifische Infrastrukturen, in neue Organisationsformen und in komplementäres Humankapital der Arbeitskräfte erforderlich. Dabei müssen aufgrund von technologischen und organisatorischen Komplementaritäten und intersektoralen Abhängigkeiten eine ganze Reihe von Anpassungs- und Koordinationsproblemen gelöst werden sowie Widerstände im sozialen und institutionellen Umfeld überwunden werden (Hagemann et al. 2001). Lipsey et al. $(1998,2005)$ betonen hierbei besonders die Bedeutung der Unsicherheit der Akteure. Zwar ist es für diese häufig offensichtlich, dass Strukturanpassungen notwendig sind, aber unklar wie diese Anpassungen auszusehen haben. Dies gilt für die politischen Akteure sowie Unternehmen, die ihre Strategien bezüglich Investitionen in Technologien, Standort und Größe der Aktivitäten sowie Organisation und Finanzierung überdenken müssen. Der Erfolg ihrer Reaktionen hängt aber entscheidend vom Handeln der anderen Akteure ab.

Im Ergebnis ändern sich durch die neue GPT wichtige Komponenten der FS. ${ }^{34}$ So ergeben sich große Veränderungen im realen Kapitalstock und in den Anforderungen an das Humankapital der Arbeitskräfte. Die Richtungswirkung ist dabei nicht eindeutig. Beispielsweise verringerten in der ersten industriellen Revolution Textilmaschinen die Anforderungen an die Arbeitskräfte im Vergleich zu den gebräuchlichen handbetriebenen Maschinen. Die Bedienung des Computers hingegen setzte zumindest in seinen Anfängen spezielle Fähigkeiten und Qualifikationen voraus. Daneben bedingt eine effiziente Nutzung neuer GPTs häufig neue Management- und Organisationsstrukturen. Als Beispiel gelten hier die Informations- und Kommunikationstechnologien (IKT), die eine Verflachung der Organisationshierarchien ermöglichten. Ganze Industriestrukturen können sich durch das Auftreten von Skaleneffekten bei den GPTs verändern. Während einige Technologien eher kleine Produktionseinheiten zur effizienten Nutzung benötigten (z.B. Wasserkraft), zeichnen sich andere Technologien durch steigende Skalenerträge aus (z.B. Dampfmaschine), die zu einer starken Konzentration in den Industrien führen können. Letztlich kann sich auch die Standortwahl der Produktionsprozesse verändern. Beispielsweise führte die moderne Kommunikationstechnologie in Verbindung mit günstigen Transportmög-

34 Dabei stellen Lipsey et al. (1998) Typologien bezüglich der Auswirkung der GPT auf. Während sich Informations- und Kommunikationstechnologien durch ihre Verkörperung in Kapitalgüter stärker auf diese selbst auswirken, führen neue Transporttechnologien zur Änderung von Standorten anderer Industrien. 
lichkeiten zu einer Dezentralisierung der Produktion. Daneben können GPTs auch neue Handlungsfelder für die Politik ermöglichen, z.B. die Kontrolle des Straßenverkehrs via GPS und Aufzeichnungsgeräte.

\section{Diffusion einer neuen GPT}

Die FS und ebenso auch die Politik werden allerdings nicht einseitig von Technologien determiniert, sondern haben ihrerseits Rückwirkungen auf die Entwicklung der GPT. Beispielsweise beeinflussen die Wahl der Organisation, die Industriestrukturen und das Humankapital die F\&E-Aktivitäten und damit die Generierung von technologischem Wissen. Die Politik kann z.B. direkt durch Fördermaßnahmen für die Technologie oder indirekt durch die Beseitigung von Monopolen in Industrien oder der Förderung von Humankapital Einfluss auf die Technologieentwicklung nehmen. Der Diffusionsverlauf einer GPT hängt entscheidend von den geschilderten mikro- und makroökonomischen sowie institutionellen Rahmenbedingungen ab. Lipsey et al. $(1998,2005)$ lassen dabei den Verlauf der Technologiediffusion recht offen. Es ist aber auf jeden Fall ein zeitund kostenintensiver Prozess, der von hohen Unsicherheiten geprägt ist. ${ }^{35}$ Die Unternehmen müssen häufig zusätzliche Forschung betreiben, um eine Technologie effizient nutzen zu können. ${ }^{36}$ Daneben tragen bereits getätigte (Fix-)Kosten stark zur Irreversibilität bei. Neue Technologien, für die neue Kapitalgüter benötigt werden, müssen häufig kostenmäßig mit bereits existierenden Technologien konkurrieren, die bereits Skaleneffekte generieren und deren Kapitalkosten schon getätigt wurden, also demnach nicht mehr entscheidungsrelevant sind. Die intensive Konkurrenz mit bestehenden Technologien kann sich zusätzlich verstärken, wenn diese ihrerseits noch weiteres Entwicklungspotenzial besitzen. Erst wenn sich im Laufe des Wettbewerbs die GPT gegenüber der bisherigen Technologie durchzusetzen scheint, werden die F\&E-Aktivitäten vollständig auf die neue GPT verlagert.

Interessante Ergänzungen zu den Diffusionsfaktoren arbeiten Bresnahan/ Trajtenberg (1995) und Helpman/Trajtenberg (1998b) heraus. Z.B. können Koordinationsprobleme zwischen den Unternehmen der technologieproduzierenden Sektoren und den Anwendersektoren entstehen (Bresnahan/ Trajtenberg 1995). Sofern die Erfinder oder Produzenten einer GPT nicht angemessen von ihrer Invention profitieren können - z.B. aufgrund institutioneller Rahmenbedingungen (siehe Kapitel 2.2.3.1) - wird das Angebot an die Anwendersektoren und weitere F\&E-Aktivitäten zur Weiterentwicklung zu gering ausfallen. Auf der Nachfrageseite verhindert eine fehlende Koordination zwischen den Anwen-

\footnotetext{
${ }^{35}$ Dabei beziehen sich Lipsey er al. (1998,2005) auf Rogers (1995), der zwischen drei Arten der Unsicherheiten unterscheidet: Der technologischen Unsicherheit, der finanziellen Unsicherheit und der sozialen Unsicherheit. Infolge dieser Unsicherheiten kann die Entscheidung selbst rationaler Akteure unterschiedlich ausfallen.

${ }^{36}$ Innovation und Diffusion lassen sich demnach nicht klar voneinander trennen.
} 
dersektoren eine abgestimmte, gleichzeitige Nachfrage nach der GPT, die ihre Profitabilität und Anreize zu Verbesserungen erhöhen würde. Ebenso bleibt die Übertragung von Lerneffekten in der Anwendung in diesem Falle aus. Daneben kann nach Helpman/Trajtenberg (1998) die Diffusion einer GPT zwischen Anwendersektoren differieren. ${ }^{37}$ Für die sektorale Diffusion ist der Produktivitätsvorteil der neuen GPT im Vergleich zur alten GPT im jeweiligen Sektor mit entscheidend. Daneben ist die Höhe der sektoralen F\&E-Kosten für die Herstellung komplementärer Güter sowie die Anzahl der komplementären Güter für die alte GPT von Bedeutung. Je größer die Anzahl ist, desto zeit- und ressourcenaufwändiger wird eine Adaption der neuen GPT. Zudem ist die Höhe der sektoralen Nachfrage entscheidend dafür, ob die Produktions- und Entwicklungskosten gedeckt werden können. Im formalen Modell wird sie bei Helpman/Trajtenberg (1998) durch die Homogenität und die Zahlungsbereitschaft der Konsumenten bestimmt.

\section{Wirtschaftliche Auswirkungen einer neuen GPT}

Die ökonomischen Auswirkungen von GPTs sind im Gegensatz zu anderen Modellen bei Lipsey et al. $(1998,2005)$ in vielerlei Hinsicht offen und können sich zwischen den einzelnen GPTs deutlich unterscheiden. ${ }^{38}$ Infolgedessen werden für viele konkrete volkswirtschaftliche Größen, wie Beschäftigung, sektorale Produktionsstruktur oder Einkommensverteilung, wenn überhaupt, nur Tendenzaussagen getroffen. Dabei wird hervorgehoben, dass eine von den existierenden Technologien und Strukturen losgelöste GPT-Betrachtung, wie es insbesondere in vielen formalen Modellen der Fall ist, zu irreführenden Folgerungen über die Effekte neuer GPTs führt. Für die Höhe der langfristigen Effekte einer GPT auf das Wachstum der Produktivität sind vier Faktoren entscheidend:

- Erstens ist die aktuelle und zukünftige Produktivität der GPT im Vergleich zu der existierenden Technologie, die vorrangig ersetzt wird, entscheidend.

\footnotetext{
${ }^{37}$ Helpman und Trajtenberg (1998) veranschaulichen dies anhand einer Fallstudie der Diffusion des Transistors. Auffällig bei dieser Technologie war die späte sektorale Diffusion in der Telekommunikations- und Automobilbranche, obwohl frühe Beobachtungen gerade für diese Sektoren eine große Anzahl von Anwendungsmöglichkeiten und einen großen -nutzen in diesen Branchen ausmachten. Die späte Diffusion der Telekommunikationsbranche führen die Autoren auf die Sektorgröße und des komplexen Zusammenspiels von Subsystemen und Komponenten zurück. Die Anzahl der benötigten komplementären Güter in diesem Sektor war demnach besonders hoch. In der Automobilbranche beeinträchtigten massive Investitionen in Vorgängertechnologien den unmittelbaren Produktivitätsvorteil des Transistors.

${ }^{38}$ Die unterschiedliche Ausgestaltung der FS in einzelnen Volkswirtschaften bildet hierbei eine wichtige Erklärung, weshalb manche Länder größere Probleme bei der Nutzung der Potenziale einer GPT haben.
} 
- Zweitens ist entscheidend, inwiefern die GPT ein Substitut oder ein Komplement zu bisherigen Technologien ist. Bei einer Komplementarität zu bisherigen Technologien sind die Effekte höher.

- Drittens beeinflusst das komplexe Zusammenspiel der Technologie mit ihrer FS und der Politik entscheidend die Marktergebnisse. Beispielsweise haben die Anzahl und Eigenschaften von neuen Kapitalgütern, die Unternehmensorganisation, die Standortwahl, die Finanzstruktur, die Infrastruktur und der Einsatz von Humankapital einen zentralen Einfluss auf die ökonomischen Ergebnisse. Dieses Zusammenspiel zwischen der Politik, der FS und den Technologien kann sich zwischen den einzelnen Volkswirtschaften unterscheiden und ist ein wichtiger Grund für die verschiedene Wachstumsperformance in den einzelnen Ländern.

- Viertens haben die Eigenschaften der GPT selbst Einfluss auf die Effekte. Diese Einflüsse werden von Lipsey et al. $(1998,2005)$ allerdings nicht genauer spezifiziert.

Die beschriebenen nötigen Anpassungsprozesse können zu einer zwischenzeitlichen Abschwächung der Produktivitätszuwächse (,productivity slowdown“) führen. Allerdings sehen Lipsey et al. $(1998,2005)$ im Gegensatz zu anderen GPT-Konzepten keinen zwingenden Grund dafür, sondern nur eine Möglichkeit: Die Unterschiede zwischen den GPTs, ihr diskontinuierliches Auftreten und die vielschichtigen Wirkungszusammenhänge mit ihrer FS lassen ihrer Ansicht nach keine zwingende Rückschlüsse auf einen solchen Rückgang des Produktivitätswachstums zu. Unterstützt sehen sie ihre These in der historischen Entwicklung, bei der nach ihren Angaben nur bei zwei von 22 GPTs, der Elektrizität und der IKT, ein zwischenzeitlicher relativer Produktivitätsrückgang zu beobachten war. Die Autoren relativieren allerdings auch die hohen Erwartungen der GPT auf das Produktivitätswachstum: Neue GPT erhöhen zwar das Niveau der Produktivität, nicht aber zwingend die durchschnittliche Wachstumsrate. Selbst bei GPT mit besonders großen Potenzialen ist das nicht der Fall, da sich durch die besonders große Reichweite an Anwendungsmöglichkeiten die Zeit der Diffusion verlängert. Die Zuwächse pro Jahr werden demnach kleiner. Folglich wird die wirtschaftliche Bedeutung der GPTs weniger in einer Beschleunigung als in einer Aufrechterhaltung des Wachstumsprozesses gesehen. Der Grundgedanke hierbei ist die im Laufe der Zeit abnehmenden Investitions- und Innovationsmöglichkeiten auf Basis bereits existierender GPTs. Diese Entwicklung würde langfristig zu einem Stillstand des Wachstums führen. Nur die Erfindung neuer GPTs bietet die Grundlage für die Entwicklung neuer Forschungsprogramme und technologischer Weiterentwicklung. Diese bieten neue Anwendungs- und lukrative Investitionsmöglichkeiten für die Akteure. „In summary, new GPTs rejuvenate the growth process, they do not necessarily accelerate it" (Lipsey et al. 2005, S.112). 


\section{Kritik und Erweiterungen des Konzepts von Lipsey/Carlaw/Bekar}

Das Konzept der GPT sowie vergleichbare Theorien zu Basisinnovationen und langen Wellen sind nicht frei von Kritik und werden nicht selten grundlegend in Frage gestellt. Häufig kritisiert wird, dass die abgeleiteten starken Auswirkungen von solchen Technologien nicht eindeutig belegbar sind (Smith 2001; Cordes 2007). Selbst einige Autoren dieser Ansätze betonen die Grenzen einer quantitativen Überprüfung, die nicht alle Wirkungen umfassen kann und häufig nicht statistisch von überlagernden Entwicklungen zu isolieren ist (z.B. Freeman/Louca 2001).

Die Bedeutung dieser Konzepte liegt besonders in den Erklärungsmustern für den möglichen zeitlichen Verlauf der wirtschaftlichen Effekte von Technologien. Das Konzept von Lipsey et al. $(1998,2005)$ zeichnet sich vor allem dadurch aus, dass es die Vielfalt und die unterschiedlichen Wirkungen einzelner Technologien verdeutlicht (Palmberg/Nikulainen 2006; Brusoni et al. 2006). Infolgedessen werden Faktoren für die Diffusion und die ökonomischen Folgen für eine Volkswirtschaft wenig konkretisiert. Interessante Ergänzungen, besonders aus sektoraler Sicht, zeigen sich bei den Konzepten von Freeman und Perez (Freeman/Perez 1988; Perez 1983/2003; Freeman/Louca 2001), die deutlich stärker auf stilisierten Fakten aufbauen.

Freeman/Perez (1988) thematisieren stärker einzelne Entwicklungsphasen der so genannten technoökonomischen Paradigmen sowie deren Folgen auf den Strukturwandel einer Volkswirtschaft. Sie geben auch Anhaltspunkte dafür, in welchem Entwicklungsstadium sich eine Technologie befinden könnte. Dabei zeichnet sich jedes Paradigma ${ }^{39}$ durch eigene Schlüsselfaktoren in einem Input (bzw. miteinander zusammenhängenden Inputs) aus, die sich durch sinkende Kosten, einem schnell zunehmenden Angebot und zunehmenden Anwendungen auszeichnen. Diese führen zu komparativen Vorteilen des neuen Weges und dessen Verbreitung. Die Auswirkungen der Paradigmen erfassen die gesamte Wirtschaft, allerdings werden die verschiedenen Sektoren sehr unterschiedlich beeinflusst. Die weit reichenden Neuerungen in Technologien und Organisationsformen beeinflussen dabei nicht nur viele existierende Industriebranchen, sondern führen auch zur Schaffung neuer Sektoren. Die Produzenten von Schlüsselfaktoren (z.B. Eisen, Kohle, Stahl, Öl, elektronische Chips) werden zu „motive branches“, die zu einer bedeutenden Branche heranwachsen. Durch neue Produkte und einigen komplementären Inputs entstehen in anderen Sektoren („carrier branches") Impulse (z.B. Automobile, elektronische Produkte,

39 Die Anforderungen an die Charakteristika dieser technoökonomischen Paradigmen sind dabei höher als im GPT-Konzept von Lipsey et al. $(1998,2005)$. So identifizieren Freeman und Perez (Freeman/Perez 1988; Freeman/Louca 2001; Perez 2003) in der historischen Analyse nur sechs Beispiele für technologische Paradigmen. Eine Anforderung, die im Konzept der GPT nicht explizit erwähnt wird, ist die soziale Akzeptanz einer Technologie, welche gerade für die Biotechnologie häufig in Frage gestellt wird. 
Computer), deren schnelles Wachstum und großes Marktpotenzial deutliche Impulse für die Gesamtwirtschaft gibt. Gerade durch diese wirtschaftliche Dynamik mit hohen Investitionstätigkeiten entstehen hohe gesamtwirtschaftliche Wachstumsimpulse. Dabei werden auch einige ,induced branches" als Vorleister oder Anbieter komplementärer Leistungen, wie z.B. Tankstellen und Werkstätten im Falle des Automobils, positiv beeinflusst. Eine Untersuchung des Einflusses der IKT auf diese Typen von Sektoren zeigt deutliche Steigerungen der Arbeitsproduktivität in den "motive" und „carrier" Sektoren, in ersteren konnte zudem eine deutliche Steigerung der Kapitalproduktivität beobachtet werden. (Freeman/Soete 1987)

Bei der Entwicklung einer Technologie werden explizit sechs Phasen des Technologiezyklus unterschieden (Freeman/Louca 2001; Perez 2003):

1. Labor-Erfindungsphase: Kennzeichnend hierfür ist das Entstehen erster Prototypen, „small-scale“ Demonstrationen und Patentierungen

2. Demonstration technologischer Machbarkeit und kommerzieller Nutzbarkeit mit vielen potenziellen Anwendungsbereichen

3. Explosives "take-off" und Wachstum in einer turbulenten Phase mit strukturellen Krisen und Problemen der politischen Koordinierung, da erst ein neues Regulationsregime geschaffen werden muss

4. Kontinuierliche Wachstumsphase mit breiter Akzeptanz, zunehmender Reichweite in Industrie und Dienstleistungen und einem dominanten technologischen Regime in den führenden Ländern

5. Verlangsamung des Wachstums und Erosion der Profitabilität, während das ausgereifte System von neuen Technologien angefochten wird

6. Reifephase mit einer möglichen Koexistenz zu neuen Technologien und eventuellem, langsamem Verschwinden

Diese Beschreibung der Phasen verdeutlicht, dass die Auswirkungen vom Entwicklungsstadium einer Technologie abhängig sind.

\subsubsection{Kritische Würdigung}

Alle dargestellten Theorieansätze schreiben dem technischen Fortschritt für den wirtschaftlichen Wachstumsprozess eine große Bedeutung zu. Es zeigen sich aber erhebliche Unterschiede in den verschiedenen Theorien bei den Eigenschaften von Technologien, den Ursachen und der Verbreitung des technischen Fortschritts, aber auch den wirtschaftlichen Auswirkungen. Dabei ist der Analysefokus der Theorien unterschiedlich. Die in neoklassischer und die in keynesianischer Tradition stehenden Theorien thematisieren, mit einem Fokus auf die Angebots- bzw. Nachfrageseite, die gesamtwirtschaftlichen Folgen von technischem Fortschritt. Sie zeigen dabei wichtige Wirkungszusammenhänge auf, wie sich der Einfluss des technischen Fortschritts in der Gesamtwirtschaft vollzieht. Allerdings wird der technische Fortschritt dabei kaum näher spezifi- 
ziert. Folglich ist der Erklärungsgehalt z.B. für zeitliche Verzögerungen von Effekten oder möglichen Unterschieden der Effekte zwischen Technologien eher gering.

Dagegen steht in evolutorischen Ansätzen die Analyse des Innovationsprozesses im Mittelpunkt. Sie zeigt dabei auch interessante Implikationen für mögliche Auswirkungen von Technologien auf. So unterscheiden sich einzelne Technologien deutlich voneinander in Art und Ausmaß der sektoralen und gesamtwirtschaftlichen Effekte. Diese ändern sich zudem erheblich im Zeitablauf der Diffusion. Schließlich diffundiert die Technologie nicht gleichmäßig im Zeitverlauf und entwickelt sich ständig weiter. Darüber hinaus können zeit- und kostenintensive Anpassungsprozesse zu einer Verzögerung der Effekte führen. Dieser größeren Realitätsnähe steht allerdings der Verlust an theoretischer Robustheit gegenüber (Conte 2006, S.29). Viele der evolutorischen Ansätze leiten keine empirisch falsifizierbaren Ursache-Wirkungszusammenhänge ab, sondern bilden eher eine verbale Beschreibung. Sie liefern bisher kein in sich geschlossenes Konzept, welches eine systematische gesamtwirtschaftliche Analyse der Effekte von Technologien zulässt.

Ein erheblicher Unterschied zwischen den Theorieansätzen besteht in der Analyse des Wachstumsprozesses. In der Neoklassik wird das Gleichgewichtskonzept angewendet, wonach die Wirtschaft nach den Impulsen des technischen Fortschritts wieder in ein Gleichgewicht zustrebt. Dies wird in der Evolutorik und in den post-keynesianischen Theorien abgelehnt. Stattdessen entstehen dynamische Entwicklungsprozesse. Dabei stehen die wirtschaftliche Entwicklung und der technische Fortschritt in enger Rückkoppelung zueinander. Die Diffusion und Weiterentwicklung von neuen Technologien und ihre damit verbundenen Auswirkungen hängen selbst von wirtschaftlichen Impulsen ab. ${ }^{40}$

Aus inhaltlichen Gesichtspunkten erscheint eine Verbindung der verschiedenen Kernaspekte dieser Theorien wünschenswert (Petit 1995). Solche Bemühungen der Synthese der verschiedenen Elemente zeigen sich auch bei verschiedenen theoretischen und empirischen Weiterentwicklungen. Beispiele hierfür sind formale Modelle der GPT, die neoklassische und evolutorische Elemente verbinden oder auch empirische Modelle zum sektoralen Strukturwandel, die post-keynesianische und evolutorische Argumente vereinen (z.B. Llerena/ Lorentz 2004; Verspagen 2002). In der folgenden vertiefenden Diskussion der Auswirkungen neuer Technologien sollen deshalb die z.T. komplementären Beiträge verschiedener Theorieansätze berücksichtigt werden.

\footnotetext{
${ }^{40}$ Diese Aspekte der Endogenisierung des technischen Fortschritts werden auch in der Neuen Wachstumstheorie berücksichtigt. Allerdings bauen diese Ansätze wie die Neoklassik auf dem Gleichgewichtsmodell auf.
} 


\subsection{Auswirkungen neuer Technologien auf Beschäftigung und Produktion}

Nach dieser allgemeinen Darstellung der Bedeutung des technischen Fortschritts in den einzelnen Theorien, werden im Folgenden konkrete Wirkungsmechanismen zu den meso- und makroökonomischen Folgen neuer Technologien erörtert. Sie dienen als Grundlage für eine empirische Analyse einer konkreten Technologie. Dabei stehen besonders die Beschäftigungswirkungen im Vordergrund. Die Beschäftigung wird in der Regel als sehr wichtiges Ziel einer Volkswirtschaft betrachtet, zugleich werden bei der Analyse der Beschäftigungswirkungen die vielfältigen Einflüsse neuer Technologien deutlich. Daneben stehen die Wirkungen auf Produktion und Wertschöpfung im Fokus, die eng mit den Beschäftigungsfolgen zusammenhängen können, aber nicht müssen.

Den Ausgangspunkt hierfür bildet die Debatte über die Freisetzung- und Kompensationseffekte zur Beschäftigungswirkung neuer Technologien. Hierbei wird die „Janusköpfigkeit“ des technischen Fortschritts im Bezug auf die Beschäftigung diskutiert (siehe z.B. Hagemann 1985; Klauder 1986; Mettelsiefen/ Barens 1987; Vivarelli 1995, 2007; Blattner 1996; Meyer-Krahmer 1999; Holwegler 2003). Die Freisetzungshypothese besagt, dass technischer Fortschritt bei konstantem Niveau und Struktur des Outputs die Arbeitsproduktivität erhöht und Arbeitskräfte freisetzt. Dieser Freisetzungseffekt ist allerdings eine hypothetische Größe, da sich Niveau und Struktur des Outputs ebenfalls verändern. Der technische Fortschritt löst verschiedene Kompensationsmechanismen (siehe unten) aus, die den Bedarf nach Arbeitskräften wiederum erhöhen und damit der Freisetzung entgegenwirken. Dabei unterscheiden sich die einzelnen Kompensationsmechanismen in ihrer zeitlichen Wirksamkeit. ${ }^{41}$ Einige Effekte sind kurzfristig mit dem Einsatz neuer Technologien verbunden, z.B. Investitionseffekte durch den Kauf neuer Maschinen. Andere Wirkungen, wie z.B. die Erhöhung der internationalen Wettbewerbsfähigkeit durch Einsatz neuer Technologien, sind eher mittel bis langfristig zu erwarten (Pfaffenberger et al. 1995, Zeddies 2006). Wenn zu einem Zeitpunkt der prozessinnovationsbedingte Anstieg der Arbeitsproduktivität allerdings weder durch wirtschaftliche, endogene Kompensationsmechanismen noch durch exogene Veränderungen (z.B. Reduktion von Arbeitszeit oder Erwerbsquote) ausgeglichen werden kann, entsteht die so genannte technologische Arbeitslosigkeit.

Die Diskussion über die Beschäftigungseffekte des technischen Fortschritts besitzt eine lange Historie. So wurden die Freisetzung- und Kompensationsthesen bereits in der Klassik ${ }^{42}$ ausgiebig diskutiert. Bedeutende Beiträge stammen insbesondere von Ricardo (1821), Say (1819) oder auch Marx (1872). Auch in

\footnotetext{
${ }^{41}$ Dieser zeitliche Unterschied des Auftretens wirtschaftlicher Effekte wird besonders bei der traversenanalytischen Betrachtung von Hicks (1973) deutlich.

${ }^{42}$ Bereits vor der Klassik wurden solche Wirkungen des technischen Fortschritts erwähnt (z.B. Steuart 1767), aber nicht systematisch untersucht (Fremann/Soete 1994).
} 
der Neoklassik und (Post-)Keynesianismus wurde diese Frage der Kompensation erörtert, wenngleich sie auch in der Neoklassik eher in den Hintergrund gedrängt wurde (Melzig-Thiel 2000, S.63). In jüngerer Vergangenheit wurden auch zunehmend evolutorische Aspekte ergänzt (z.B. Pianta 2000; Edquist et al. 2001; Vivarelli 1995; Freeman/Soete 1994). ${ }^{43}$ Die verschiedenen theoretischen Sichtweisen unterscheiden sich vor allem bei der Einschätzung der relativen Bedeutung einzelner Kompensationsmechanismen und in welcher Geschwindigkeit und Reibungslosigkeit sich diese Anpassungen vollziehen (Freeman/Soete 1994).

In den 1980er Jahren erlebte die Debatte eine Renaissance, dabei wurden besonders empirische Messversuche durchgeführt (siehe z.B. Wilson/Whitley 1982,1987; Howell 1985; Leontief/Duchin 1986; Kalmbach et al. 1989; Edler 1990), da befürchtet wurde, dass viele Anwendungen der IKT mit großer Arbeitsfreisetzung verbunden sind. Freeman und Soete (1994) stellen hierzu fest, dass dieses kontroverse Thema immer dann in den Vordergrund in der ökonomischen Forschung tritt, wenn das Problem der strukturellen Arbeitslosigkeit auf der politischen Agenda steht. In jüngerer Vergangenheit wird dieses Konzept in leicht abgewandelter Form zur Untersuchung der Wirkungen von Umwelttechnologien oder Maßnahmen zur Förderung alternativer Energieträger auf Produktion und Beschäftigung verwendet (Walz 2001; Zeddies 2006; Lehr et al. 2008). Der Schwerpunkt liegt dabei weniger auf der Konkretisierung der Produktions/Produktivitätsschere, d.h. ob sich Beschäftigungs- und Produktionsentwicklung voneinander unterscheiden, sondern ob und in welche Richtung sich Implikationen aus der Diffusion einer neuen Technologie auf die sektorale und gesamtwirtschaftliche Produktion sowie auf die Beschäftigung ergeben. Schließlich steht beim Einsatz dieser Technologien häufig nicht zwingend die Erhöhung der Arbeitsproduktivität im Vordergrund, sondern es sind auch andere Motive denkbar (z.B. Energieeffizienz, Umweltschonung). Die Freisetzungs- und Kompensationsthesen bilden aber für diese Analysen die theoretische Grundlage, indirekte Effekte zu berücksichtigen (z.B. Walz 2002). Schließlich spielt die Senkung von Produktionskosten auch bei diesen Gütern häufig eine wichtige Rolle, wodurch Vorleistungsgüter mitsamt der hierfür notwendigen Arbeitskraft eingespart werden und kompensatorische Kräfte wirksam werden. Zusätzlich treten

\footnotetext{
${ }^{43}$ Vereinzelt wird von evolutorischer Seite die Eignung der Freisetzungs- und Kompensationsthesen insgesamt als Konzept zur Untersuchung des Zusammenhangs zwischen neuen Technologien und Beschäftigung in Frage gestellt (z.B. Welsch 2006). Hauptkritikpunkt sind vereinfachenden Annahmen des technischen Fortschritts im Konzept, u.a. die exogene Behandlung, fehlende ,time-lags“ bei der Produktivität und die starke Affinität des Konzepts auf die industrielle Produktion (Petit 1995; Welsch 2006; Pianta 2005). Im empirischen Teil dieser Arbeit wird versucht wichtige Innovations-/Diffusionszusammenhänge und die Bedeutung der Produktinnovationen nach Möglichkeit zu berücksichtigen. Die Affinität zur industriellen Produktion stellt aufgrund des Analysegegenstands hier weniger ein Problem dar.
} 
bspw. Veränderungen von Wertschöpfungsketten sowie möglicher Budgeteffekte zur Förderung der Technologiediffusion (z.B. Subvention von erneuerbaren Energieträgern) in den Vordergrund. Auch beim Einsatz biotechnologischer Methoden steht nicht die Erhöhung der Arbeitsproduktivität durch neue Rationalisierungsmöglichkeiten im Vordergrund (Nusser et al 2007a). Deshalb werden im Rahmen dieser Arbeit diese Modifikationen bzw. Schwerpunkte bei einzelnen Argumenten berücksichtigt. Die Gliederung und Benennung der Argumente orientiert sich aber eng an der bekannten Freisetzung-/Kompensationsdebatte:

1. Produktivitätswirkung von Prozessinnovationen

2. Produktinnovationen

3. Kaufkraftkompensationsargument

4. Maschinenherstellungsargument und sektoraler Strukturwandel

5. Internationale Wettbewerbsfähigkeit und Außenhandel

Zunächst erfolgt demnach die allgemeine Betrachtung von Prozessinnovationen und die Frage, wann Freisetzungseffekte damit verbunden sind. Im Anschluss werden die Wirkungsmechanismen (Kompensationsmechanismen) erörtert, die eine Veränderung des Outputs bewirken können. ${ }^{44}$ Dabei werden die einzelnen Argumente nur aus inländischer Sicht diskutiert und sämtliche Effekte, die den Außenhandel einer Volkswirtschaft verändern, im vierten Argument erörtert. Neben der allgemeinen Darstellung und Beurteilung aus Sicht der einzelnen Theorien steht die Frage im Vordergrund, in welchem Ausmaß diese Effekte von den spezifischen Charakteristika einer Technologie abhängen.

\subsubsection{Produktivitätswirkung von Prozessinnovationen}

"No macroeconomic magnitude is more important for the future evolution of the economy than productivity growth..." (Gordon 2003, S.1).Produktivitätssteigerungen gelten als zentral für den Wohlstand und den langfristigen Wachstumsprozess einer Volkswirtschaft. Schließlich hängt das Produktionspotential einer Volkswirtschaft maßgeblich davon ab, ob Produktionsfaktoren (wie z.B. Arbeit und Kapital) effizient eingesetzt werden. Prozessinnovationen erhöhen die Produktivität, indem sie die Herstellung eines höheren Outputs bei gegeben Inputs oder eines gegebenen Outputs mit weniger Inputs (u.a. Arbeit, Kapital) ermöglichen. In letzterem Fall kann die Prozessinnovation aus Beschäftigungssicht aber negativ wirken, da sie (zumindest temporär) zur Freisetzung von Arbeitskräften führt. Der technische Fortschritt erhöht in diesem Fall vor allem das Rationalisierungspotential und treibt dadurch das Produktivitätstempo stärker voran als das Produktionswachstum, so dass möglicherweise mehr Arbeitskräfte

${ }^{44}$ Die neoklassische Sichtweise bezieht in die Freisetzungs-/Kompensationsdebatte zusätzlich die Faktorsubstitution als weiteren Kompensationsmechanismus ein. 
freigesetzt werden, als anderweitig wiederbeschäftigt werden können (Klauder 1986, S.2).

Die Wirkungsweise von Prozessinnovationen kann anhand des neoklassischen Modells verdeutlicht werden (RWI 2005). Ausgehend von einer Produktionsfunktion, die den Output in Abhängigkeit von den Faktoren Arbeit und Kapital erklärt, können die Wirkungen einer Prozessinnovation in drei verschiedene Effekte unterteilt werden:

1. Der Fortschrittseffekt verringert für eine gegebene Outputmenge sowohl den Arbeits- als auch den Kapitaleinsatz. In welchem Ausmaß dabei technischer Fortschritt die Produktivität erhöht und welcher Produktionsfaktor stärker eingespart wird, hängt maßgeblich von der Technologie selbst ab. Hicks (1932) unterscheidet für die Faktoreinsparung zwischen drei verschiedene Arten: Neben dem neutralen technischen Fortschritt - bei dem die Grenzproduktivitäten beider Faktoren im gleichen Verhältnis steigen sind arbeitssparender oder kapitalsparender technischer Fortschritt möglich. Beim arbeitssparenden technischen Fortschritt steigt die Grenzproduktivität des Kapitals stärker als die des Faktors Arbeit. Dadurch steigt die Kapitalintensität der Produktion, die relative Verminderung des Arbeitseinsatzes bei gegebenem Output ist höher. Umgekehrt wirkt der kapitalsparende technische Fortschritt.

2. Daneben tritt ein Substitutionseffekt zwischen beiden Produktionsfaktoren ein. Durch die Veränderung der Lohn-Zinsrelation bei nicht-neutralem technischem Fortschritt ändert sich das Einsatzverhältnis der beiden Produktionsfaktoren so lange, bis die Substitutionselastizität des Faktoreinsatzes wieder dem Lohn-Zins-Verhältnis entspricht.

3. Der Niveau- bzw. Kompensationseffekt führt zu einer Erhöhung der Produktionsmenge und folglich einer Ausweitung des Arbeits- und Kapitaleinsatzes. Ob diese Erhöhung ausreicht um die Faktoreinsparungen durch den Fortschrittseffekt zu kompensieren, ist zunächst offen. Neoklassischen Vorstellungen zufolge kommt es zur Vollauslastung der Produktionsfaktoren, da sich die Reallöhne entsprechend solange anpassen würden, bis der Arbeitsmarkt wieder ins Gleichgewicht kommt. ${ }^{45}$ Arbeitslosigkeit ist in den Grundmodellen nicht möglich. Ein Überwiegen der Freisetzungseffekte ist höchstens kurzfristig durch Unvollkommenheiten auf den Arbeitsmärkten denkbar (RWI 2005; Freeman/Soete 1994).

Eine Erweiterung dieser Grundbetrachtung zum Charakter des technischen Fortschritts bezüglich der Arbeitsfreisetzung bilden Konzepte über dessen

${ }^{45}$ Diesem Argument wird meist entgegengehalten, dass drastische Lohnsenkungen zwar die Einführung arbeitssparender Maschinen vorübergehend aufhalten können, aber spätestens langfristig hochentwickelte Maschinen die menschliche Arbeitskraft in den Kosten unterbieten wird (u.a. Leontief 1982; Walz 2002). 
Induzierung (Ruttan 2001, S.100 ff.). Nach Hicks bestimmen die relativen Faktorpreise die Richtung des technischen Fortschritts (Hicks 1932). Ist ein Faktor relativ teuer (z.B. hohe Arbeitskosten), so werden Innovationsbemühungen zur ökonomischeren Nutzung dieses Faktors ausgerichtet sein. Vertreter der Evolutorik betonen hingegen die Entwicklung von Trajektorien (Kapitel 2.2.3.1). Die Kumulierung und Irreversibilität des technischen Fortschritts führen demnach zu Pfadabhängigkeiten. Marktsignale (z.B. hohe Arbeitskosten) können zur Wahl einer Trajektorie mit arbeitssparendem technologischem Fortschritt führen (Vivarelli 1995, S. 14). Falls die Wahl einer bestimmten Trajektorie getroffen worden ist, bleibt sie auch dann bestehen, wenn der originäre Grund wegfällt (z.B. Arbeiterknappheit). Resultierende Marktsignale reichen folglich nicht aus, um die ursprüngliche Selektion von technologischen Lösungen rückgängig zu machen. Führende Vertreter der Evolutorik halten einen ,labor-saving bias" für ein häufiges Charakteristikum von Trajektorien, da die Einsparung von Arbeitskosten ein wichtiges Selektionskriterium darstellt (Nelson/Winter 1982; Dosi 1982; van Tunzelmann 1995). In Verbindung mit der sektorspezifischen Wirkung von Trajektorien spiegeln sich demnach auch Beschäftigungsunterschiede zwischen Ländern wider. „National growth patterns of low employment intensity can thus reflect the predominance of sectors in which the bias of technological change is labour saving" (Edquist et al. 2001, S.95).

Neben dieser Erweiterung bestehen einige Kritikpunkte an der neoklassischen Interpretation der Wirkung von Prozessinnovationen. Zum einen wird die Annahme der Substitutionalität zwischen Arbeit und Kapital bzw. Technologien zunehmend in Frage gestellt. Während dies für die industrielle Massenproduktion zutreffen mag, werden zumindest für andere Bereiche (z.B. Dienstleistungen) Komplementaritäten von qualifizierten Arbeitskräften und neuen Technologien postuliert (Welsch 2006; Pianta 2005). Neue Technologien wirken bei Arbeiten mit hohen kognitiven Anforderungen eher unterstützend als ersetzend und setzen gleichzeitig auch hohe kognitive Anforderungen an die qualifizierten Arbeitskräfte voraus (Nusser 2000). An diesen "Technology-skill"-Komplementaritäten setzt die Debatte des "skill-biased-technological change" an. Eine steigende Arbeitsnachfrage nach qualifizierten Arbeitskräften durch technologischen Fortschritt gilt weitestgehend als empirisch untermauert (Autor et al. 1998; Chennels/van Reenen 2002; Dunne et al. 2000).

Darüber hinaus stellen die in Kapitel 2.2.3.2 diskutierten Konzepte der GPTs die unmittelbare zeitliche Wirksamkeit der Produktivitätswirkung von Prozessinnovationen in Frage. Zwar ist das Ausmaß des Produktivitätsanstiegs an die Diffusion der Technologie gebunden, da die Reichweite und das Ausmaß des Innovationseinsatzes den durchschnittlichen Produktivitätsanstieg in einer Volkswirtschaft oder in einem Sektor bestimmen. Allerdings gilt dies nicht zwingend in gleich bleibender proportionaler Höhe über die Zeit, stattdessen können die Diffusion und ihre Effekte aufgrund der kostenintensiven Anpas- 
sungsprozesse zeitlich auseinanderfallen. Ähnliche Auswirkungen auf das zeitliche Auftreten der Produktivitätsgewinne können Lerneffekte haben (Arrow 1962; Freeman 1994). Während der komparative Vorteil der neuen Technologie zu Beginn der Diffusion möglicherweise nur gering ist, kann durch zunehmende Erfahrung die Herstellung und Nutzung der neuen Technologie weiter verbessert werden. Dies erhöht die Produktivitätsgewinne.

\subsubsection{Produktinnovationen}

Als einer der Kompensationsmechanismen gilt die Wirkung von Produktinnovationen, die häufig komplementär zu Prozessinnovationen auftreten (Kalmbach 1989, S.24). Produktinnovationen führen dazu, dass neue oder qualitativ verbesserte Produkte angeboten werden, die den Konsumenten zusätzlichen Nutzen stiften und helfen, Sättigungsgrenzen hinauszuschieben. Dadurch können neue Produktmärkte und Branchenzweige entstehen, die zusätzliche Beschäftigungsmöglichkeiten bieten. Entscheidend für die tatsächliche Beschäftigungswirksamkeit ist hierbei allerdings, ob es sich bei Produktinnovationen tatsächlich um ein neuartiges Produkt handelt (additiv) oder ob es bisherige Produkte ersetzt (substitutiv). Für die Beurteilung der Abgrenzung zwischen Additivität und Substitutionalität existieren allerdings verschiedene Perspektiven.

Im Folgenden wird aus Sicht der Endnachfrage und der sektoralen Sicht unterschieden. Zunächst werden additive Produktinnovationen aus Sicht der Endnachfrage betrachtet. Additive Produktinnovationen führen über die Stiftung eines zusätzlichen Nutzens zu einer Ausweitung der Endnachfrage und haben einen positiven Effekt auf Produktion und Beschäftigung. Als historische Beispiele gelten Automobile, Penicillin, Schwarz-Weiss-Fernseher, Faxgeräte (Edquist et al. S.97). Dabei wird insbesondere die (zunehmende) Bedeutung der Komplementarität von Gütern hervorgehoben (u.a. Stille/Bitzer 1998; Welsch 2006). Dies gilt im zunehmenden Maße für Dienstleistungsbereiche, die eine Komplementarität mit industriellen Produkten und Produktionsprozessen aufweisen. Im Rahmen der Industrieproduktion nehmen Tätigkeiten wie z.B. Organisation, Marketing, Kundenbetreuung einen immer größeren Raum ein. Industriell produzierte Waren werden zudem vielfach nicht mehr isoliert, sondern im System oder Paket mit begleitenden Dienstleistungen angeboten (Welsch 2006, S.817).

Inwiefern Produktinnovationen - oder deren Komplementärgüter - zu diesen additiven Impulsen führen, liegt zum einen am Grade der (technologischen) Verbesserung im Vergleich zu bisherigen Gütern. Die Nachfrage hängt aber auch vom verfügbaren Einkommen und der Einkommenselastizität nach diesen Gütern ab (RWI 2005; Holwegler 2003). Die Einkommenselastizität gibt an, um welchen Prozentsatz sich die Nachfrage nach einem Gut bei einer prozentualen Veränderung des Einkommens verändert. Zum Beispiel werden selbst deutliche Verbesserungen der chemischen Eigenschaften bei Textilien kaum wirtschaftli- 
che Folgewirkungen haben, da die Einkommenselastizität weiter gering bleiben wird (Kuznets 1972). ${ }^{46}$ Tendenziell haben neue Güter aufgrund der geringeren Sättigungsgrade in der Regel höhere Einkommenselastizitäten (Pasinetti 1981).

Insbesondere Vertreter der Evolutorik betonen die hohe Bedeutung von Produktinnovationen zur Vermeidung von Stagnation und Arbeitslosigkeit (z.B. Lundvall 1988). Nach dieser Ansicht kommt es häufig zur Entwicklung von völlig neuen Produkten oder zu einer deutlichen Differenzierung von reifen Produkten. (Vivarelli 2007, S.2; Castaldi/Dosi 2008, S.2). Die Produktinnovationen haben folglich einen additiven Charakter. Allerdings können sich einzelne Technologien in der Wahrscheinlichkeit neue Beschäftigungsmöglichkeiten durch Produktinnovationen zu schaffen, deutlich voneinander unterscheiden (Vivarelli 2007; IPTS 2002a). "As a matter of fact ..., the relative balance between the labour-saving effect of process innovation and the labour-intensive impact of product innovations can be considerably different" (Vivarelli/Pianta 2000, S.17). So erforderte die Verbreitung des Automobils einen höheren Arbeitseinsatz als die Diffusion von Computern (Vivarelli 2007).

Der Beschäftigungseffekt von substitutiven Produktinnovationen aus Endnachfragesicht ist hingegen offen. ${ }^{47}$ Die Endnachfrage bleibt identisch und der Beschäftigungseffekt der Produktinnovation ist abhängig von seiner Arbeitsintensität und der Arbeitsintensität der verdrängten Güter. Als häufiges Beispiel hierfür gilt der HDTV statt Farbfernseher (Edquist et al. 2001, S.97). Nur wenn die Innovation zu einer geänderten Nachfragestruktur führt, bei der Güter mit höherem Arbeitsbedarf produziert werden, ist der Effekt positiv (Stoneman 1983). ${ }^{48}$

Zusätzliche Implikationen für den Strukturwandel ergeben sich aus sektoraler Perspektive. Additive Produktinnovationen sind - in Anlehnung an die Definition von Edquist et al. (2001, siehe Kapitel 2.1.2) - solche Güter, die bisher nicht in dem betreffenden Sektor hergestellt wurden und kein Produkt im eigenen

\footnotetext{
${ }^{46}$ Kuznets stellt hierzu fest: „...the technological innovations that create new consumer goods differ substantially in magnitude, depending on the weight and magnitude of the consumption component and the elasticity of its response... In general, if a significantly large component of household consumption is comprised of low-cost, efficiently produced goods, with a low price elasticity of demand, even technically significant changes in it are not have much economic effect" (Kuznets 1972, S.436-437).

${ }^{47}$ Dabei sind auch temporäre Effekte denkbar. Auf makroökonomischer Ebene ist kurzfristig eine Steigerung der Nachfrage möglich, wenn z.B. die Sparquote gesenkt wird. Daneben kann sich das Verhältnis zwischen zwei Gütern im Zeitablauf ändern. Bspw. wird das Mobiltelefon bisher häufig zusätzlich zum Festnetztelefon eingesetzt, eine zunehmende Ersetzung ist aber denkbar, bzw. bereits teilweise beobachtbar (Holwegler 2003, S.267)

${ }^{48}$ Einen weiteren Fall, bei dem Produktinnovationen positive Beschäftigungseffekte haben, zeigt Katsoulacos (1986) auf. Ein höherer Nutzen für die Konsumenten aus neuen Produkten führt zu einem steigenden Arbeitsangebot, um sich diese Güter leisten zu können. Dieses ist gleichzeitig notwendig, um diese zusätzlichen Güter zu produzieren (Katsoulacos 1986).
} 
Sektor ersetzen. ${ }^{49}$ Somit werden gegenüber dem obigen Fall nicht nur Konsumgüter, sondern auch Investitions- und Vorleistungsgüter in der Betrachtung mit erfasst. Dies kann dazu führen, dass aus dieser Sichtweise eine Innovation in einem Sektor eine Produktinnovation, in einem anderen aber eine Prozessinnovation ist. Während die Herstellung einer neuartigen Maschine für den Maschinenbau eine Produktinnovation darstellt, dient sie in einem anderen Sektor (z.B. zur Herstellung von Papier) als Prozessinnovation. Für die sektorale Struktur können diese sektoralen Produktinnovationen von erheblicher Bedeutung sein (Edquist et al. 2001). Nur in bestimmten Sektoren wird ein hoher Anteil an neuen Produkten generiert. Nach Edquist et al. (2001) haben vor allem Sektoren mit einer hohen F\&E-Intensität eine Neigung zur Einführung neuer Produktinnovationen. ${ }^{50}$ Daneben unterscheiden sich die Einkommenselastizitäten für diese Produkte der einzelnen Sektoren voneinander. Folglich wird eine neue Technologie durch ihre Produktinnovationen in einigen Sektoren tendenziell einen deutlich expansiveren Einfluss als in anderen Sektoren haben. So zeigen Analysen, dass ein positiver Zusammenhang zwischen technologischem Fortschritt und Produktionswachstum vor allem für Hochtechnologiesektoren besteht (Brusoni et al. 2006; Edquist et al. 2001; RWI 2005).

\subsubsection{Kaufkraftkompensationsargument}

Ein weiterer Kompensationsmechanismus der arbeitsfreisetzenden Wirkung von Prozessinnovationen besteht in der Ausweitung der Nachfrage. ${ }^{51}$ Prozessinnovationen führen häufig zu einer Senkung der Kosten pro Outputeinheit. Diese Kostensenkungen können entweder zu Preissenkungen und/oder zusätzlichen Verteilungsspielräumen für Gewinne und Löhne bzw. Einkommen führen. Beides kann zu einer höheren (Real-)Nachfrage von Gütern führen und damit zu einer zusätzlichen Produktion und Beschäftigung.

In welchem Ausmaß und in welcher Struktur diese zusätzliche Nachfrage stattfindet, hängt von einer Reihe von Anpassungsreaktionen ab. Im Folgenden wird zunächst die Wirkung über Preissenkungen analysiert. Unter der Voraussetzung von wettbewerblichen Marktstrukturen und flexiblen Preisen führen Produktivitätsgewinne zu einer Senkung der Preise. Dabei sinkt sowohl der relative Preis der mit neuen Technologien produzierten Güter als auch das absolute

${ }^{49} \mathrm{Ob}$ sich die Endnachfrage dadurch erhöht und/oder ein anderes Produkt aus einem anderen Produktionssektor hierfür substituiert wird, ist hierbei unerheblich.

${ }^{50}$ Eine Ausnahme bildet der im Rahmen dieser Arbeit wichtige Sektor Chemie, der trotz überdurchschnittlicher F\&E-Ausgaben eine hohe Neigung $\mathrm{zu}$ Prozessinnovationen hat (Edquist et al. 2001).

${ }^{51}$ Die Argumentation dieses Wirkungsmechanismus geht zurück auf das Say'sche Gesetz (Say 1819). Demnach ist eine allgemeine Überproduktion nicht möglich, sondern eine Volkswirtschaft tendiert bei jedem Produktionsniveau zu einem Ausgleich zwischen Angebot und Nachfrage. 
Preisniveau. Während die Absenkung der relativen Preise zu einer größeren Nachfrage nach diesen Gütern führen kann, erhöht die steigende Kaufkraft durch den absoluten Fall der Preise die Nachfrage auch nach anderen Gütern. $\mathrm{Ob}$ die Nachfragesteigerung im Sektor mit der Produktivitätssteigerung stattfindet, hängt entscheidend von der Preiselastizität des Gutes ab. Die Preiselastizität gibt an, zu welchen prozentualen Nachfrageänderungen es kommt, wenn sich die Preise bestimmter Güter um 1\% verändern. Bei einer Preiselastizität gleich eins kommt es zu einer proportionalen Nachfragesteigerung in dem entsprechenden Sektor, bei Elastizitäten kleiner (größer) eins ist die Nachfragesteigerung unterproportional (überproportional). Die empirischen Erfahrungen zeigen bei vielen Gütern eine Preiselastizität von kleiner als eins. Demnach findet die Kompensation in anderen Sektoren statt, als dort wo die Prozessinnovation mit dem arbeitssparenden Fortschritt aufgetreten ist (OECD 1994; Edquist et al. 2001).

Die Kompensationswirkungen über steigende Einkommen und Profite in Folge von Produktivitätssteigerungen können vor allem aus keynesianischer Sicht zum Tragen kommen. Steigende Profite können entweder durch zusätzliche Investitionen die Nachfrage erhöhen ${ }^{52}$ oder über mögliche Dividendenzahlungen ebenfalls zu steigenden Einkommen der Konsumenten führen. Ein steigendes Einkommen erhöht die gesamtwirtschaftliche Nachfrage analog zur Senkung der absoluten Preise. Die Auswirkungen auf die sektorale Struktur ergeben sich aus den Einkommenselastizitäten (Hagemann 1985, S.301). In Sektoren mit Einkommenselastizitäten der privaten Haushalte von größer eins kommt es zu einer überproportionalen Nachfragesteigerung, Sektoren mit einer Einkommenselastizität gleich eins haben einen proportionalen Nachfrageanstieg, während Sektoren mit Werten kleiner eins langfristig in einem Wachstumsprozess an Bedeutung verlieren.

Welche dieser drei Kaufkraftmechanismen - Preise, Löhne, Profit - durch Produktivitätswachstum zum Tragen kommt, wird von institutionellen Rahmenbedingungen bestimmt (Pini 1996, S. 131; Villeval 2002, S.135). Bei vollkommenem Wettbewerb werden Kostensenkungen vollständig durch niedrigere Preise an den Markt weitergegeben. Wenn kein vollkommener Wettbewerb herrscht, wird höchstens ein Teil der Produktivitätssteigerungen in Preissenkungen umgesetzt (Sylos Labini 1969, S.160). Bei der daraus folgenden Profit- und Lohnverteilung wird - nach der in kaldorianischer Tradition stehenden "French School of Regulation" - zwischen verschiedenen Nachfrageregimen unterschieden (z.B. Boyer 1988): ${ }^{53}$ Beim keynesianischen Regime führt der institutionelle

${ }^{52}$ Dabei ist allerdings im Hinblick auf die Wirkung die Investitionsstruktur zu beachten. Wenn neue Investitionen vor allem zu zusätzlichen Rationalisierungen dienen sollen, kann es zu weiteren Freisetzungen von Arbeitern kommen.

${ }^{53}$ Diese institutionellen Regime können sich im Zeitablauf deutlich verändern. Während in den 1950er und 1960er Jahren die Arbeiter einen hohen Anteil an den Produktivitätsgewinnen 
Lohnfindungsprozess zu einem Anstieg der Löhne und infolgedessen zu einer Steigerung der Konsumnachfrage. Beim klassischen Regime führt ein hoher Wettbewerb auf dem Arbeitsmarkt zu einer Steigerung der Profite bei Produktivitätsgewinnen. Diese werden annahmegemäß investiert. ${ }^{54}$ Dabei hat es für den Wachstumsprozess und die Beschäftigung unterschiedliche Folgen, je nachdem, welche dieser Nachfragekomponenten die Produktivitätsgewinne erhält. Die unterschiedlichen Komponenten der Nachfrage können zu verschiedenen Wachstumsdynamiken führen, ihre jeweilige Wirksamkeit ist dabei von unterschiedlichen institutionellen Faktoren abhängig (Pini 1995). „These mechanisms trigger substantially different employment compensation effects, and different economic and institutional factors mediate their functioning and effectiveness" (Pini, 1996, S.130). Dabei wird nicht spezifiziert, wie die institutionellen Faktoren ausgeprägt sein müssen. Wichtig für den Wachstumsprozess ist die Passfähigkeit von institutionellen Formen und technologischem Fortschritt (Vivarelli 1995, S.84).

Insgesamt herrscht aus keynesianischer Sicht Skepsis, ob sich die Gesamtnachfrage in ausreichendem Maße erhöht und zu einer vollen Kompensation der Freisetzungseffekte führt (Hagemann 1985, S.302). Mill (1848) bekräftigt in seiner berühmten Kritik, dass die Nachfrage nach Gütern nicht der Nachfrage nach Arbeit entspricht. Demnach findet zunächst nur eine Umverteilung statt, bei welcher der Nachfrageausfall von freigesetzten Arbeitern durch Nachfragesteigerungen an anderer Stelle kompensiert wird. Hingegen ist eine Erhöhung der Gesamtnachfrage an eine Reihe von Bedingungen geknüpft. Lowe (1976) hebt bspw. die Notwendigkeit zusätzlicher Kapitalakkumulation hervor. Pasinetti $(1981,1993)$ gibt in seinen Modellen zum Wachstum und Strukturwandel zu bedenken, dass eine Mehrnachfrage infolge eines steigenden Realeinkommens keineswegs automatisch erfolgt. Aufgrund von Sättigungstendenzen im Konsum wird eine Nachfrageerhöhung in Sektoren mit Produktivitätssteigerungen die Preissenkungen in der Regel nicht ausgleichen. Ein Ausgleich der Freisetzung im gleichen Sektor ist demnach unwahrscheinlich. Ob gesamtwirtschaftlich eine Vollbeschäftigung erreicht werden kann, ist dagegen von der Entstehung neuer Sektoren durch additive Produktinnovation abhängig. ${ }^{55}$ Hier-

\footnotetext{
zugesprochen bekamen - und so Anreize für zusätzlichen arbeitssparenden technologischen Fortschritt entstanden - wurde für Ende des 20. Jahrhunderts eine deutliche Abschwächung der Bedeutung der Steigerung von (Real-)Einkommen als Kompensationsmechanismus beobachtet (Vivarelli 2007, S.7).

${ }^{54}$ Daneben sind weitere Regime denkbar, insbesondere für ungleichgewichtige Wachstumsprozesse (Pini 1995).

${ }^{55}$ Dabei wird in diesem Modell der enge Zusammenhang zwischen Prozess- und Produktinnovationen deutlich. Während erstere vor allem über die Steigerung der Produktivität eine Erhöhung der Kaufkraft ermöglichen, führen Produktinnovationen zu einer Verschiebung der Sättigungsgrenze.
} 
für ist allerdings eine entsprechende Nachfrage erforderlich. Die Konsumenten müssen dabei die Vorteile und Nutzung der neuen Produkte erst erlernen. Wenn die Lerneffekte nicht ausreichen, wird nur ein Teil des zusätzlichen Einkommens konsumiert und die Nachfrage reicht nicht aus, um die Arbeitsfreisetzung zu kompensieren (Vivarelli 1995, S.63). Darüber hinaus können Ungleichgewichte aufgrund verschiedener Arbeitskoeffizienten entstehen. Ist die Arbeitsintensität im Sektor mit Prozessinnovationen höher als in neuen Sektoren mit Produktinnovationen, nimmt die gesamtwirtschaftliche Beschäftigung ab. Pasinetti kommt deshalb insgesamt zum Schluss: "For the time being, we may draw the important conclusion that the structural dynamics of the economic system inevitably tend to generate what has rightly been called technological unemployment. At the same time, the very same structural dynamics produce counterbalancing movements which are capable of bringing macro-economic condition towards fulfilment, but not automatically" (Pasinetti 1981, S.90).

Zudem ist $\mathrm{zu}$ beachten, dass sich die Wirkungsrichtung von diesem Kaufkraftmechanismus auch umgekehrt vollziehen kann. Unter bestimmten Umständen kann die Technologiediffusion auch zu einer Erhöhung der Kosten führen. ${ }^{56}$ So ist die Diffusion von Technologien in bestimmten Anwendungsfeldern (z.B. Energie, Gesundheit, Umwelt) stark von politischen Maßnahmen abhängig, da sie von allein noch nicht wettbewerbsfähig sind. So sind sie häufig teurer. Für die Unterstützung der Diffusion sind deshalb häufig finanzielle Mittel nötig, die folglich nicht für andere Zwecke verwendet werden können. Ein Beispiel hierfür sind Steuerbefreiungen, die Mindereinnahmen zu Folge haben. Eine ähnliche Wirkung haben Festsetzungen von Preisen (z.B. Einspeisungsgesetz erneuerbarer Energieträger) oder Quoten (z.B. Mindestquote von Biokraftstoffen). Sofern die Anbieter diese in höhere Preise umwälzen können, wird der Konsument höher belastet. Diese Mehrbelastungen für Staat und Verbraucher führen in der Regel zu geringeren Ausgaben für andere Güter und Dienstleistungen.

\subsubsection{Maschinenherstellungsargument und sektoraler Strukturwandel}

Das Maschinenherstellungsargument nimmt unter den Kompensationsthesen eine besondere Stellung ein, da dessen Formulierung bereits zu Beginn des 19. Jahrhunderts erfolgte (Holwegler 2003). ${ }^{57}$ Ausgangspunkt ist die Hypothese, dass der technische Fortschritt durch die Prozessinnovationen in Maschinen oder andere Kapitalgüter inkorporiert ist. Die Einführung von Maschinen bedarf zunächst deren Herstellung, so dass positive Beschäftigungseffekte bei den Herstellern und ihren Vorlieferanten entstehen können.

\footnotetext{
${ }^{56}$ Dies gilt streng genommen auch für die finanzielle Forschungsförderung, deren Höhe und Folgewirkung für die Technologieentwicklung aber kaum abschätzbar sind.

${ }^{57}$ Zentrale Arbeiten hierfür stammen von Ricardo (1821) und Say (1819).
} 
Auch wenn dieser Wirkungsmechanismus in der Freisetzungs-/ Kompensationsdebatte im Kern nicht bestritten wird, so bestehen Einwände gegenüber der quantitativen Bedeutung dieser möglichen Kompensation und deren Fristigkeit (Hagemann 1995, S.39). Marx (1872) verwies darauf, dass eine vollständige Kompensation durch die Maschinenherstellung nicht erfolgen kann, da sonst die Einführung der Technologie nicht profitabel wäre. ${ }^{58}$ Von entscheidender Bedeutung für die Stärke dieses Arguments ist aber der Nettoeffekt der Maschinenherstellung. Hierbei stellt sich die Frage, ob die Investitionen der Anwender additiv oder substitutiv als Ersatz für die Herstellung der alten Kapitalgüter stattfinden. Im Fall der substitutiven Investitionen - welcher als häufiger Fall gilt ${ }^{59}$ - findet höchstens eine geringe Kompensation statt, die von den Charakteristika der neuen gegenüber denen der alten Technologie abhängt. $\mathrm{Ob}$ additive Investitionen stattfinden, hängt in der Theorie vor allem von der Annahme hinsichtlich der Investitionsfunktion $a b$. Vorrausetzung für die Investition in zusätzlichen Maschinen ist in einigen formalen Modellen, wie in der Traversenanalyse von Hicks (1973), die Erzielung von höheren Profiten. Wird hingegen eine stärkere Abhängigkeit der Investition von Erwartungen angenommen, werden in expansiven Phasen zusätzliche Investitionen stattfinden, in Zeiten der Stagnation hingegen ausbleiben.

Die Fristigkeit der Effekte hängt von der jeweiligen Zeitdauer der Maschinenherstellung und -nutzung ab. Während die Herstellung einer neuen Maschine nur eine kurze Zeitdauer benötigt, erfolgt die Nutzung meist als Gebrauchsgut über einen sehr langen Zeitraum. Die Investitionen sowie die damit verbundene Produktion und Beschäftigung steigen folglich nur einmalig, der Freisetzungseffekt besteht hingegen dauerhaft. ${ }^{60}$ Nur durch eine stetige Zunahme der Kapitalakkumulation kann der Freisetzungseffekt vollständig kompensiert werden. ${ }^{61}$

In Ergänzung zum traditionellen Maschinenherstellungsargument ist der gesamte Produktionsprozess der Vorprodukte des mit neuen Technologien produ-

\footnotetext{
${ }^{58}$ Bei einer vollständigen Kompensation liegt nach Marx (1872) überhaupt kein technischer Fortschritt vor, so dass ein Widerspruch entstünde.

${ }^{59}$ So beschreibt Vivarelli (2007, S.5): "Finally, the new machines can be implemented either through additional investments [...] or simply by substitution of the obsolete ones (scrapping). In the latter case - which is indeed the most frequent one - there is no compensation at all."

${ }^{60}$ Dabei ist anzumerken, dass sich die Diffusion und damit auch die Herstellung einer neuen Technologie in der Regel über einen langen Zeitraum erstreckt. Holwegler (2003, S.189) leitet daraus eine besonders hohe Bedeutsamkeit des Maschinenherstellungsargumentes ab. Allerdings vernachlässigt er bei seiner Argumentation die geschilderte Substitution alter Maschinen. Die Effekte werden bei einer Substitution aber nicht höher, sondern verteilen sich nur bezüglich des Zeitraumes.

${ }^{61}$ Nach Neisser (1942, S.70) entsteht hier ein offenes Wettrennen zwischen diesen Effekten: [...] a race between diplacement of labor through technological progress and reabsorption of labor through accumulation."
} 
zierten Gutes zu analysieren. Diese Betrachtung wird besonders bei der empirischen Input-Output-Analyse deutlich (siehe Kapitel 4.3). Eine Prozessinnovation diffundiert in der Regel nur dann, wenn sie eine kosteneffizientere Herstellung des Gutes ermöglicht. ${ }^{62}$ Somit verringern sich die benötigten Inputs bei der Herstellung für einen gegebenen Output. Wenn es sich bei der Einsparung nicht um den Faktor Arbeit handelt, werden andere Inputfaktoren - Kapital- oder andere Vorleistungsgüter - eingespart. Dies führt zu Produktions- und Arbeitseinsparungen in den Vorleistungssektoren, Freisetzungseffekte werden somit verstärkt. Ebenso können sich durch Prozessinnovationen benötigte Vorleistungen verändern, bspw. bei den benötigten Rohstoffen. Die Bereitstellung dieser Vorleistungen kann sich in ihrer Arbeitsintensität unterscheiden und somit die Freisetzung verstärken, bzw. kompensieren.

\subsubsection{Internationale Wettbewerbsfähigkeit und Außenhandel}

Ein weiterer wichtiger Wirkungsmechanismus auf die Produktion bzw. Beschäftigung ist die Erhöhung der internationalen Wettbewerbsfähigkeit durch den Einsatz neuer Technologien. Diese führt zu höheren Exporten und/oder einer Substitution von Importen und somit zu positiven Beschäftigungseffekten im Inland. Die Veränderungen des Außenhandels können dabei bei verschiedenen Gütern und Dienstleistungen auftreten (OECD 1994, S.131; OECD 1996, S.75).

1. Produktivitätsfortschritte durch Einsatz neuer Technologien können zu einer Erhöhung der preislichen Wettbewerbsfähigkeit der hiermit hergestellten Güter führen. Infolgedessen steigen die Weltmarktanteile der Anwender neuer Technologien. ${ }^{63}$

2. Durch Produktinnovationen bzw. Qualitätsverbesserungen kann das Exportvolumen ebenfalls signifikant angehoben werden (Holwegler 2003, S.359). Die Frage des Standorts der Herstellung dieser Güter ist von hoher Bedeutung, da schließlich gerade diese Produktmärkte bei einer Diffusion der neuen Technologie rasch wachsen und Beschäftigungsmöglichkeiten bieten (Edquist et al. 2001, S.97). Darüber hinaus lassen sich Nachteile einer Volkswirtschaft beim Qualitätswettbewerb nicht durch Währungsabwertung egalisieren. Es steht somit oftmals nicht die preisliche, sondern die so genannte technologische Wettbewerbsfähigkeit im Vordergrund.

3. Auch die Investitionsgüter für die Realisation des Einsatzes der neuen Technologie können ein mögliches Exportprodukt sein. Diese Exportmöglichkeit wird besonders dann zum Tragen kommen, wenn in anderen

\footnotetext{
${ }^{62}$ Ausnahmen sind besonders durch politische Eingriffe (z.B. Subventionen, Auflagen) denkbar.

${ }^{63}$ Die Senkung der Preise ist bei hohem internationalen Wettbewerb mit Niedriglohnländern wahrscheinlicher als die Verteilung der Produktivitätsfortschritte auf die Löhne (Holwegler, 2003; OECD 1982).
} 
Ländern die Technologiediffusion später erfolgt und die Maschinenherstellung im Inland bereits ausgereifter ist. Daneben können sich die bei einer Veränderung der Produktion neu benötigten Vorleistungen (z.B. Investitionsgüter) in ihrer inländischen Verfügbarkeit gegenüber den bisherigen Vorleistungsgütern unterscheiden. Folglich kann sich der inländische Anteil in der Wertschöpfungskette bei der Herstellung des Produktes verschieben.

$\mathrm{Ob}$ es zu den erhofften positiven Effekten im internationalen Wettbewerb kommt, ist von zahlreichen Faktoren abhängig. Schließlich werden in der Regel eine Vielzahl von Ländern vergleichbare Anstrengungen unternehmen, um eine starke Stellung auf dem Weltmarkt zu erreichen. Dabei wird meist eine frühzeitige Diffusion der Technologie („first-mover-advantage") als wichtig für den internationalen Wettbewerb angesehen (z.B. Brusoni et al. 2006; OECD 1994; Dosi et al. 1990). ${ }^{64}$ Häufig kann dadurch ein längerfristiger Innovationsvorsprung und Spezialisierungsvorteile (z.B. durch Skaleneffekte, spezifisch qualifizierte Arbeitskräfte) eines Landes für die F\&E und/oder die Produktion der betreffenden Technologie erreicht werden (u.a. Beise 2001; OECD 1996). Allerdings reicht die Betrachtung der zeitlichen Technologiediffusion meist nicht aus, um die Bewegungen im internationalen Güterhandel zu erklären. Stattdessen wird eine Vielzahl von angebots- und nachfrageseitigen sowie institutionellen Faktoren diskutiert, welche die Standortattraktivität eines Landes für die Entwicklung, Herstellung und Anwendung neuer Technologien beeinflussen (u.a. Nusser et al. 2007c; RWI 2005). Dies wird besonders in den Ansätzen der Innovationssysteme deutlich. Zudem führt die zunehmende Internationalisierung zu einer häufigeren räumlichen Trennung der einzelnen Schritte der Wertschöpfungskette eines Produkts (u.a. Amity/Wei 2006). Die Entwicklungs- und Produktionsschritte werden dort durchgeführt, wo die jeweils erforderlichen Rahmenbedingungen optimal sind. Dabei ist es bei Gütern mit hohem Preiswettbewerb sogar wahrscheinlich, dass in entwickelten Ländern die höhere Exportfähigkeit durch eine Verlagerung von Teilen der Wertschöpfungskette in billigere Länder erkauft werden muss (Vivarelli/Pianta 2000, S.6). Folglich gibt es aus Sicht der Kompensationsargumente keinerlei Garantie dafür, dass die positiven Effekte räumlich dort auftreten, wo sie eingespart wurden (David 1986).

Daneben wird häufig auf weitere Grenzen der Wirksamkeit dieses Wirkungsmechanismus hingewiesen. Selbst wenn eine stärkere Stellung eines Landes auf dem Weltmarkt erreicht werden kann, stößt die Erzielung von positiven Beschäftigungseffekten auf Grenzen. So kann die Veränderung in der Zahlungsbilanz durch Exportüberschüsse (langfristig) zu einer Aufwertung der Währung und Zinseffekte führen. Dies hätte als Gegeneffekt eine negative Wirkung auf

${ }^{64}$ Diese Bedeutung kommt auch in den bekannten Theorien des „technology gap“ Theorie (Posner 1961) und des „Product Cycle“(Vernon 1966) zum Ausdruck. 
die preisliche Wettbewerbsfähigkeit und Außenhandelsbilanz einer Nation. Außerdem stellt eine starke Exportlastigkeit zentraler Sektoren auch ein potenzielles Risiko dar, da eine sehr hohe Abhängigkeit vom Weltmarkt besteht. ${ }^{65}$ Darüber hinaus sind die Kompensationswirkungen auf die Beschäftigung aus globaler Sicht möglicherweise gering. Während einzelne Länder Beschäftigungsgewinne erzielen können, trifft dies global gesehen nicht zwingend zu (Vivarelli/Pianta 2000). ${ }^{66}$ Zwar ist es weitgehend anerkannt, dass technischer Fortschritt langfristig auf internationaler Ebene kein Nullsummenspiel darstellt, sondern positive Effekte z.B. über eine steigende Weltnachfrage und produktion auslöst (u.a. Hagemann 1985). Inwiefern es zu diesen globalen Beschäftigungsgewinnen kommt, hängt aber davon ab, ob Qualitätsverbesserungen und Preissenkungen zu einer steigenden Weltnachfrage führen (Edquist et al. 2001). Hierfür sind analog zum Kaufkraftkompensationsargument die Preis- und Einkommenselastizitäten auf weltweiter Ebene von Bedeutung.

Trotz dieser Einschränkungen, hat die mit der Herstellung und dem Einsatz von neuen Technologien verbundene internationale Wettbewerbsfähigkeit eine zentrale Rolle für die wirtschaftlichen Auswirkungen. Schließlich nimmt die internationale wirtschaftliche Verflechtung weiter zu. Die Effekte müssen sich dabei nicht zwingend direkt in der Außenhandelsbilanz auswirken. Der grenzüberschreitende Austausch von Waren und Dienstleistungen vollzieht sich in abnehmendem Maße durch den traditionellen Außenhandel, dafür verstärkt durch grenzüberschreitende Tätigkeiten multinationaler Unternehmen (Döhrn et al. 2008). Dabei muss sich die zusätzliche inländische Produktion nicht in höheren Exporten oder geringeren Importen dieses Gutes niederschlagen, wenn der inländische Markt im Vergleich zu anderen Märkten stark wächst, z.B. durch Erhöhung der Konsumnachfrage oder inländische Weiterarbeitung eines Gutes.

\subsubsection{Fazit}

Zusammenfassend entstehen vielfältige Wirkungen durch neue Technologien auf die Produktion und Beschäftigung einer Volkswirtschaft. Insgesamt können sowohl Produktions- als auch Beschäftigungseffekte einer Technologie sektoral und regional sehr unterschiedlich ausfallen und sich zwischen Technologien deutlich unterscheiden. Die Effekte für die Produktion und Beschäftigung können vor allem aus zwei Gründen voneinander differieren. Erstens wenn der arbeitsfreisetzende Effekt hoch ausfällt, zweitens falls Veränderungen in der inländischen Produktion sich auf Sektoren mit besonders hoher oder niedriger Arbeitsintensität konzentrieren.

\footnotetext{
${ }^{65}$ Als unbestritten gilt allerdings, dass eine offene Volkswirtschaft den Einsatz neuer Technologien nicht verhindern sollte, da sonst erhebliche außenwirtschaftliche Einbußen zu erwarten sind (Hagemann 1985).

${ }^{66}$ Dies verdeutlicht, wie stark die Beschäftigungswirkungen von der Analyseebene abhängen.
} 
Die verschiedenen theoretischen Sichtweisen unterscheiden sich besonders darin, welchen Kompensationsmechanismen eine hohe Bedeutung zugesprochen wird. Während nach neoklassischer Sichtweise eine Absenkung der Faktor- und Güterpreise zu einer Kompensation der freigesetzten Arbeitskräfte führen, sind aus (Post-)Keynesiansischer Sicht die Entwicklung der Nachfrage und damit zusammenhängende institutionelle Bedingungen entscheidend. In der klassischen Sichtweise werden besonders Anpassungsreaktionen auf der Angebotsseite, vor allem der Kapitalakkumulation, hohe Bedeutung beigemessen.

Daneben kann den einzelnen Wirkungsmechanismen aber auch je nach Technologie eine unterschiedliche Bedeutung zukommen. Neben Unterschieden beim arbeitsfreisetzenden Charakter zeigen sich beispielsweise unterschiedliche Verhältnisse von Produkt- und Prozessinnovationen zwischen Technologien (Vivarelli/Pianta 2000). Ein anderes Beispiel sind Unterschiede bei der Kapitalintensität, welche für die Rolle des Maschinenherstellungsarguments wichtig sein kann. Eine konkrete Untersuchung welche Wirkungszusammenhänge für die Biotechnologie von hoher Bedeutung sind, wird nach einer Einführung in die Biotechnologie in Kapitel 3.3 erörtert. 
Sven Wydra - 978-3-631-75392-7

Downloaded from PubFactory at 01/11/2019 05:32:25AM

via free access 


\section{Charakterisierung und Entwicklung der Biotechnologie}

\subsection{Einführung}

\subsubsection{Begriffsklärung}

Der Biotechnologie ${ }^{67}$ wird seit einiger Zeit große Aufmerksamkeit zuteil; sie gilt als wichtige zukünftige Schlüssel- oder Querschnittstechnologie (z.B. Roobeck 1995; Reiss/Koschatzky 1997). Dabei ist es bisher keinesfalls eindeutig und einheitlich, wie diese Technologie definiert wird. ${ }^{68}$ So zählen bspw. Brink et al. (2004) zehn unterschiedliche Definitionen auf, die in wichtigen Veröffentlichungen allein seit Anfang der 1980er Jahre für die Biotechnologie verwendet wurden. Die OECD bemüht sich dabei seit einigen Jahren eine einheitliche Definition vorzugeben, welche die Vergleichbarkeit von Studien und Statistiken verbessern soll. ${ }^{69}$ In einer überarbeiteten Version im Rahmenwerk zur Erhebung von biotechnologischen Statistiken wird die Biotechnologie wie folgt definiert: „Biotechnologie ist die Anwendung von Wissenschaft und Technik auf lebende Organismen, Teile von ihnen, ihre Produkte oder Modelle von ihnen zwecks Veränderung von lebender oder nichtlebender Materie zur Erweiterung des Wissensstandes, zur Herstellung von Gütern und zur Bereitstellung von Dienstleistungen“"(OECD 2005a, S.9). ${ }^{70}$

In Einklang mit vielen anderen neueren Definitionen wird bei der oben genannten OECD-Definition nur die ,moderne Biotechnologie“ in Betracht gezogen (Brink et al. 2004). Diese biotechnologischen Methoden und Verfahren zeichnen sich durch eine starke Basierung auf wissenschaftlichen Fortschritten aus, im Gegensatz zu traditionellen biotechnologischen Verfahren, wie z.B. Herstellung und Konservierung von Käse oder Bier. Die Einschränkung auf moderne Verfahren soll der Tatsache Rechnung tragen, ,dass sich das öffentliche Interesse vor allem deshalb der Biotechnologie zuwendet, weil ein ausgeprägtes Innovations- und Wachstumspotenzial sowie neue, zukunftsgerichtete Aufgabenstellungen diese moderne Schlüsseltechnologie auszeichnen" (Statistisches Bundesamt 2005, S.9).

${ }^{67}$ In der Literatur wird nicht immer von der „Biotechnologie“ gesprochen, sondern z.B. von „biotechnologischen Methoden, Prozessen, Produkten und Dienstleistungen“ (Nusser et al. 2007a). Im Folgenden wird meist einfachhalber nur von der „Biotechnologie“ gesprochen, es sei denn die genannten Unterscheidungen sind für den konkreten Sachverhalt von Bedeutung.

${ }^{68}$ Dabei zeigen sich Parallelen zu anderen Technologien wie z.B. IKT. Auch dort dauerte es eine sehr lange Zeit bis sich ein relativ einheitlicher Begriffsgebrauch etabliert hat (Arundel 2003, S.16).

${ }^{69} \mathrm{Zu}$ den Problematiken der Verwendung von unterschiedlichen Definitionen siehe Kapitel 4.2 .

${ }^{70}$ Die deutsche Übersetzung basiert auf biotechnologie.de (2008). 


\subsubsection{Historische Entwicklung}

Der Unterschied zwischen den modernen und traditionellen Verfahren der Biotechnologie wird in der geschichtlichen Entwicklung der Biotechnologie deutlich. Dabei wird in der Regel zwischen drei Generationen unterschieden, die sich durch wichtige wissenschaftliche Entdeckungen oder erstmaligen Anwendungen abgrenzen lassen (z.B. Roobeck 1995):

Die erste Generation der Biotechnologie bezieht sich auf herkömmliche Techniken, die bereits seit Jahrtausenden zur Herstellung und Konservierung von Nahrungsmitteln (z.B. Käse, Brot) und Getränken (z.B. Bier, Wein) eingesetzt werden. Als Organismen dienten hier vor allem Bakterien, Hefe oder Pilze, um gewünschte Prozesse (z.B. Gärung) zu erreichen (Renneberg 2006). Dabei wurden deren Eigenschaften von Organismen eher zufällig genutzt und basierten nicht auf wissenschaftlichen Fortschritten (Roobeck 1995).

Die zweite Generation ist durch wissenschaftliche und technische Fortschritte (z.B. in der Molekularbiologie) Anfang des 20. Jahrhunderts geprägt (Brink et al. 2004). Diese ermöglichten es, verstärkt biologisch-basierte Produktionsprozesse für industrielle Zwecke einzusetzen, z.B. zur Waschmittelproduktion, Fruchtsaftproduktion oder auch der Textil- und Lederindustrie (Heneric 2006, S.40). Aber auch Vitamine und Glycerin ließen sich nun fermentativ herstellen. Dennoch verlor im 20. Jahrhundert die Biotechnologie zu Gunsten der chemischen Synthese an Bedeutung. Gründe hierfür werden zum einen in der Verschiebung der Rohstoffbasis gesehen (Marschall 2005). Das zunehmende Angebot an günstigen fossilen Rohstoffen und die Verknappung landwirtschaftlicher Erzeugnisse machten chemische Verfahren attraktiver, da sich diese häufig besser zur Nutzung fossiler Rohstoffe eignen. Zum anderen war die Biotechnologie nur bei einigen wenigen Anwendungen die günstigste Produktionsmethode, häufig war sie aber zeitaufwändiger, komplizierter in ihrer Anwendung und nicht zuverlässig bezüglich des erwünschten Ergebnisses in der Phase der Biotransformation (Peter 2002, S.66). Die chemische Synthese baute hingegen bereits zu dieser Zeit stärker auf wissenschaftlichen Erkenntnissen auf (Dominguez-Lacasa 2005, S. 54).

Die tatsächliche Wandlung der Biotechnologie zu einer wissensbasierten Technologie erfolgte erst mit der dritten Generation, der modernen Biotechnologie (Roobeck 1995). 1973 gelang es Stanley Cohen und Frederick Boyer zum ersten Mal, ein fremdes Gen gezielt in einen fremden Organismus zu übertragen und dort neu zu kombinieren (Orsenigo 1989). Diese DNA-Rekombinationstechnik ermöglicht die direkte Manipulation genetischen Materials und erlaubt die Produktion von Organismen mit gewünschten Eigenschaften. Als wichtige weitere wissenschaftliche Erfolge gelten die Erfindungen neuer Techniken zur Sequenzierung von Proteinen und DNA im Jahre 1977 sowie die Entschlüsselung des menschlichen Genoms im Humangenomforschungs-projekt (McKelvey 1996). Insgesamt bildete sich mittlerweile eine Vielzahl von biotechnischen Me- 
thoden und Verfahren heraus, die auf neuen wissenschaftlichen Erkenntnissen aufbauen.

\subsubsection{Wissenschafts- und Technikvielfalt der Biotechnologie}

Die Biotechnologie ist folglich keine eindeutig abgrenzbare Technologie, sondern besteht aus einer Gruppe von Techniken (Roobeck 1995; Wieland 2003). Häufig werden mehrere dieser Techniken simultan zur Problemlösung eingesetzt (Pisano 2006a). Diese Technikvielfalt spiegelt sich auch in der Definition der OECD wider, welche die obige verbale Definition durch eine exemplarische Aufzählung verschiedener biotechnologischer Verfahren ergänzt. Diese resultierende Liste (vgl. Tabelle 1) soll bei statistischen Erhebungen den befragten Unternehmen Orientierungshilfe geben, um sich entsprechend einordnen zu können. Sie erhebt dabei bewusst keinen Anspruch auf Vollständigkeit, da bislang immer neue Forschungsfelder entstanden sind (OECD 2005a).

Tabelle 1: Übersicht über verschiedene Biotechniken

\begin{tabular}{|c|c|}
\hline DNA & $\begin{array}{l}\text { DNA: Genomik, Pharmakogenetik, Gensonden, DNA- } \\
\text { Sequenzierung/-Synthese/-Amplifikation, Gentechnik, } \\
\text { RNA }\end{array}$ \\
\hline $\begin{array}{l}\text { Proteine und andere } \\
\text { Moleküle }\end{array}$ & $\begin{array}{l}\text { Sequenzierung, Synthese und Veränderung von Proteinen } \\
\text { und Peptiden (einschließlich hochmolekularer Hormone); } \\
\text { Identifikation von Zellrezeptoren; verbesserte Darrei- } \\
\text { chungsformen für hochmolekulare Wirkstoffe (beispiels- } \\
\text { weise mit Glykol oder bestimmten Lipiden); Proteomik }\end{array}$ \\
\hline $\begin{array}{l}\text { Zell- und Gewebe- } \\
\text { kultur sowie } \\
\text { Tissue-Engineering }\end{array}$ & $\begin{array}{l}\text { Zell- und Gewebekultur, Tissue-Engineering, Hybridisie- } \\
\text { rung, Zellfusion, Vakzine und Immunstimulanzien, Emb- } \\
\text { ryo-Kultivierung }\end{array}$ \\
\hline $\begin{array}{l}\text { Methoden der Bio- } \\
\text { verfahrenstechnik }\end{array}$ & $\begin{array}{l}\text { Fermentationen in Bioreaktoren, Bioverfahren, biologi- } \\
\text { sches Bleichen, biologische Zellstoffgewinnung, biologi- } \\
\text { sche Laugung, biologische Entschwefelung, biologische } \\
\text { Umweltsanierung und biologische Filtration }\end{array}$ \\
\hline $\begin{array}{l}\begin{array}{l}\text { Subzelluläre Orga- } \\
\text { nismen }\end{array} \\
\end{array}$ & Gentherapie, virale Vektoren \\
\hline Bioinformatik & $\begin{array}{l}\text { Erstellung von Datenbanken mit Genomen oder Protein- } \\
\text { sequenzen; Modellierung komplexer biologischer Vor- } \\
\text { gänge }\end{array}$ \\
\hline $\begin{array}{l}\text { Nanobiotechnolo- } \\
\text { gie }\end{array}$ & $\begin{array}{l}\text { Anwendung von Werkzeugen und Verfahren der Nano- } \\
\text { und Mikrosystemtechnik zur Herstellung von Hilfsmitteln } \\
\text { für die Erforschung biologischer Systeme sowie Anwen- } \\
\text { dungen }\end{array}$ \\
\hline
\end{tabular}

Quelle: OECD 2005a 
Dabei zeigt sich insbesondere, dass die Gentechnik, welche teilweise als Synonym zur Biotechnologie betrachtet wird, nur eine Teilmenge hiervon darstellt. Diese befasst sich mit der Charakterisierung, Isolierung, Neukombination und Vervielfältigung des Erbmaterials, der DNA (Kinkel et al. 2008).

Fortschritte in den Biotechniken kommen aus unterschiedlichen Wissenschaftsdisziplinen. Hierzu zählen vor allem die Biologie, Chemie, Physik, Nanowissenschaft und -technologien, Medizin, Material- und Werkstoffwissenschaften sowie Informations- und Kommunikationswissenschaften und technologien (Kinkel et al. 20082006). Die Biotechnologie ist demnach nicht unabhängig von anderen Forschungsgebieten und Technologien, sondern hängt eng mit diesen zusammen (OTA 1991; Roobeck 1995). Insbesondere in dieser Überschneidung der Forschungsbereiche werden häufig die Potenziale der Biotechnologie gesehen (Freeman 2003, S.11; Kapitel 3.2.1).

Im Folgenden wird zunächst die Entwicklung der Biotechnologie zu einer stark wissensbasierten Technologie in einem kurzen historischen Überblick verdeutlicht. Im Anschluss daran wird in Kapitel 3.1.5 anhand ausgewählter Indikatoren die bisherige Bedeutung der Biotechnologie in der Forschung und Anwendung skizziert.

\subsubsection{Bedeutung der Biotechnologie in F\&E und Anwendung}

Die beschriebenen Fortschritte in der Biotechnologie haben zu einem Bedeutungsgewinn dieser Technologie in der Wissenschaft geführt. Für die Nachzeichnung von Entwicklungen der angewandten Forschung und technischen bzw. experimentellen Entwicklung eignen sich Patentindikatoren (Kapitel 4.2.3.1). Die Entwicklung der jährlichen Patente zeigt eine weltweite Verachtfachung zwischen 1980 und 2005, für Deutschland immerhin eine Verfünffachung (siehe Abbildung 4). Die Entwicklung ist dabei dynamischer als für die jeweiligen gesamten Patente.

Seit Mitte der 1990er Jahre ist der Anteil der Biotechnologie an allen Patenten eher konstant und nimmt in der EU25 und weltweit gut 5\% ein (vgl. Tabelle 2). Auf einen starken Patenanstieg erfolgte ein deutlicher Rückgang der Biotechnologiepatente zwischen 2000 und 2004. Dies dürfte eine Folge der Börsenkrise zu Beginn des Jahrtausends sein (Nusser 2007b) sowie in einer Verschärfung der Kriterien für die Patenterteilung in der Biotechnik liegen (OECD 2008a). Ein positiveres Bild zeigen der Anteil und die Entwicklung bei den Publikationen. Dieser bibliometrische Indikator eignet sich zur Abbildung der Entwicklung im Bereich der wissenschaftlichen Forschung. Weltweit beträgt der Anteil der Biotechnologie an den gesamten Publikationen im Zeitraum 2002-2004 rund 14\%. 


\section{Abbildung 4: Entwicklung der Biotechnologie-Patente (Index 1980=100)}

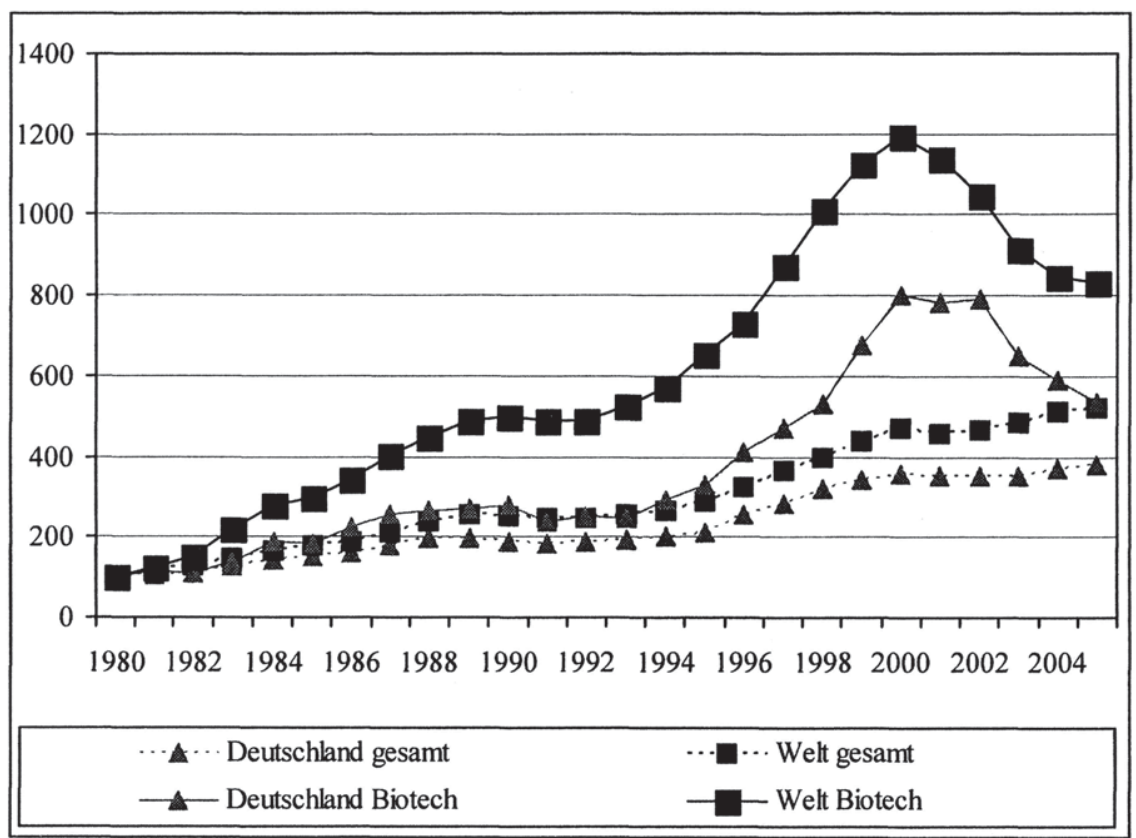

Quelle: Datenbank OECD-STATS 2008

Tabelle 2: Anteile der Biotechnologie an den gesamten Patenten und Publikationen im Zeitraum 1995 bis 2004

\begin{tabular}{|c|c|c|c|c|c|c|}
\hline & \multicolumn{2}{|c|}{ Welt } & \multicolumn{2}{|c|}{ EU25 } & \multicolumn{2}{c|}{ USA } \\
\hline & Patente & Publikationen & Patente & Publikationen & Patente & Publikationen \\
\hline $\begin{array}{c}\mathbf{1 9 9 5 -} \\
\mathbf{1 9 9 7}\end{array}$ & $7 \%$ & $11 \%$ & $5 \%$ & $12 \%$ & $11 \%$ & $15 \%$ \\
\hline $\begin{array}{c}\mathbf{1 9 9 9 -} \\
\mathbf{2 0 0 1}\end{array}$ & $8 \%$ & $13 \%$ & $6 \%$ & $14 \%$ & $11 \%$ & $16 \%$ \\
\hline $\begin{array}{c}\mathbf{2 0 0 2}- \\
\mathbf{2 0 0 4}\end{array}$ & $6 \%$ & $13 \%$ & $5 \%$ & $14 \%$ & $8 \%$ & $17 \%$ \\
\hline
\end{tabular}

Quelle: Reiss et al. 2007

Infolge der dabei erzielten wissenschaftlichen Erfolge bildeten sich rein auf Biotechnologie fokussierte Unternehmen (Kernbiotechnologieunternehmen), die vor allem biotechnologisches Wissen bereitstellen (Technologieproduzenten). Daneben stiegen vermehrt große, diversifizierte Unternehmen in der biotechnologi- 
schen F\&E oder Produktion ein. ${ }^{71}$ Eine quantitative Darstellung der Bedeutung und Entwicklung ist allerdings nur schwer möglich, da die Datenlage für wirtschaftliche Kenngrößen für die Biotechnologie sehr eingeschränkt ist. Diese Problematik wird detailliert in Kapitel 4.2.2 erörtert. Die seit jüngerer Vergangenheit einheitlich erhobenen Daten für die so genannte „Biotechnologieindustrie" zeigen, dass im Jahr 2007 ca. 550 Unternehmen mit knapp 30.000 Mitarbeitern direkt der Biotechnologie zurechenbar sind (vgl. Anhang Tabelle 31; Kapitel 4.2.2). Auch wenn diese Daten einigen statistischen Einschränkungen unterliegen, lässt sich aber zumindest folgern, dass es wenig Hinweise dafür gibt, dass von den reinen Biotechnologieunternehmen eine große gesamtwirtschaftliche Bedeutung ausgeht. Stattdessen ist ein bedeutender Einfluss eher in den verschiedensten Anwendersektoren, in denen vor allem die Ergebnisse der biotechnologischen Forschung eingehen, zu erwarten.

\subsubsection{Anwendungsfelder der Biotechnologie}

Diese Techniken und Methoden der Biotechnologie werden in verschiedensten Anwendungen und industriellen Bereichen eingesetzt. In allen Anwendungsfeldern der Biotechnologie kommen eine Vielzahl der in Tabelle 1 skizzierten Biotechniken zum Einsatz. Dabei zeichnet sich jedes Anwendungsfeld durch ein spezifisches Set an Methoden und Verfahren aus; die einzelnen verwendeten Techniken können aber in den Anwendungsfeldern identisch oder ähnlich sein. ${ }^{72}$ Ein Beispiel für den breiten Anwendungscharakter einzelner Techniken ist der Einsatz rekombinanter DNA im Pharma- und Agrarsektor (Roobeck 1995). Deutliche Unterschiede zwischen den Anwendungsfeldern können vor allem im jeweiligen Einsatzzweck (z.B. Kostenreduktion, höhere Qualität, geringere Umweltbelastung) liegen. Abbildung 5 gibt einen Überblick, in welchen Sektoren biotechnologische Methoden hauptsächlich eingesetzt werden. Die Biotechnologie wird in einigen Industriesektoren angewendet, in der Landwirtschaft und vereinzelt im Dienstleistungssektor (Gesundheitsdienstleistungen, Forschung \& Entwicklung, Abfallentsorgung).

\footnotetext{
${ }^{71}$ In Deutschland dauerte die Kommerzialisierung der modernen Biotechnologie relativ lange. So standen Ende der 1980er Jahre 245 US-amerikanischen Biotech-Unternehmen nur 15 deutsche Unternehmen gegenüber (Lange 2006). Die „Initialzündung“ für den Aufschwung der Biotechnologieindustrie in Deutschland wird dem 1995 gestarteten Bio-Regio Wettbewerb zugeschrieben (Lange 2006; Peter 2002). Zielsetzung dieses Wettbewerbs war die Verknüpfung biotechnologischer Forschung mit der Umsetzung ihrer Ergebnisse. ${ }^{71}$ Dieses Programm führte zu einer hohen Dynamik in der Gründerszene.
}

${ }^{72}$ Für eine Übersicht siehe Reiss et al. (2006, S.37). 


\section{Abbildung 5: Anwendersektoren der Biotechnologie}

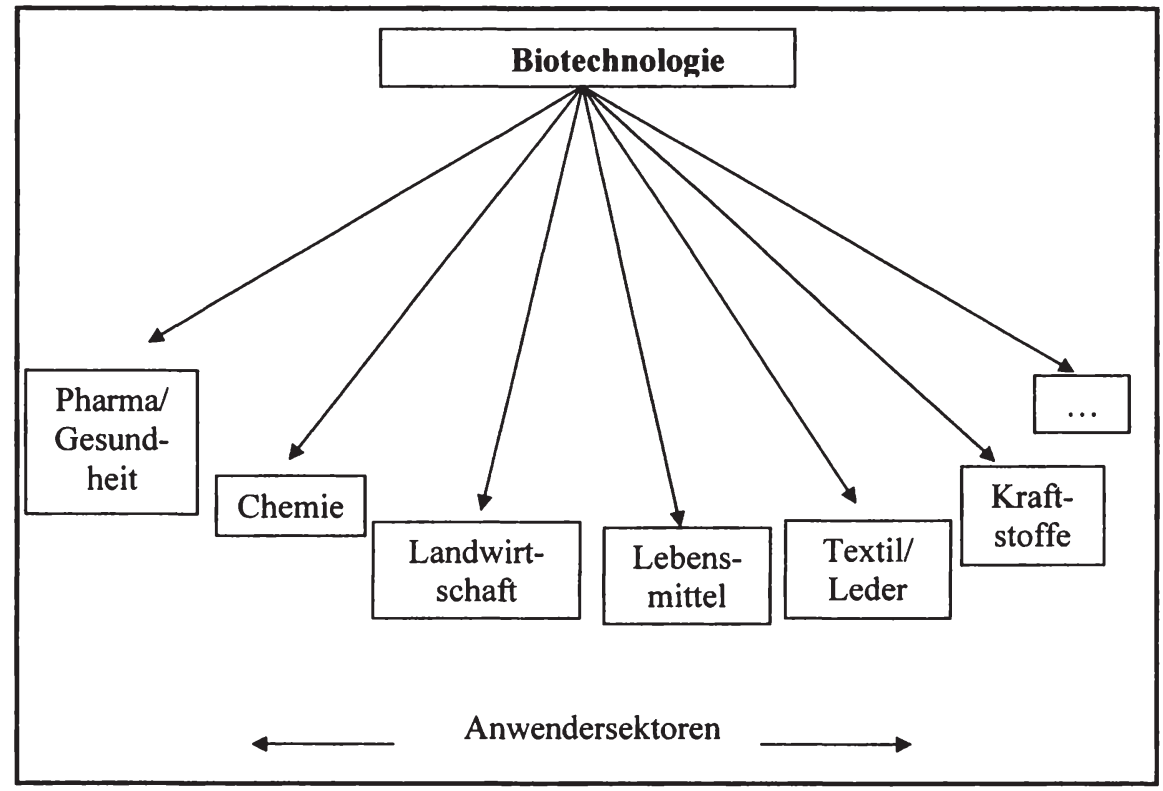

Quelle: Eigene Darstellung

Im Folgenden wird ein kurzer Überblick über die wichtigsten Anwendungen und der jeweiligen Einsatzzwecke der Biotechnologie gegeben. Dabei wird zwischen vier Anwendungsgebieten unterschieden:

- Pharmazeutika und Gesundheit (rote Biotechnologie)

- Industrielle Anwendungen (weiße Biotechnologie)

- Agro-Food Bereich (grüne Biotechnologie)

- Umweltbiotechnologie (graue Biotechnologie)

\subsubsection{Pharmazeutika und Gesundheit}

Die pharmazeutische Industrie stellt den derzeit am weitesten entwickelten und ökonomisch bedeutsamsten Anwendungsbereich der Biotechnologie dar (Kinkel et al. 2008; Nusser et al. 2007a; Peter 2002). Biotechnologische Methoden sind $\mathrm{zu}$ einem wichtigen Bestandteil sowohl in der Forschung und Entwicklung als auch in der Produktion von Arzneimitteln geworden und haben dort zu einer deutlichen Veränderung der Prozesse geführt. ${ }^{73}$ Zuvor dominierten strukturchemische Überlegungen bei der Wirkstoffsuche, es wurden in Frage kommende chemische Komponenten analysiert und per Tests versucht, deren Eigenschaften

${ }^{73}$ Für eine detaillierte Betrachtung von Biopharmazeutika siehe Kapitel 6.4. 
herauszufinden (Hopkins et al. 2007). Diese Vorgehensweise ist somit stark empirisch geprägt und von Zufallstreffern bestimmt. Neue wissenschaftliche Erkenntnisse (z.B. in Genetik, Physiologie, Biochemie) ermöglichen zusammen mit der Nutzung biotechnologischer Methoden hingegen eine Fokussierung auf genetische Ursachen und biotechnischer Eigenschaften von Organismen bei der Wirkstoffsuche für Krankheiten. Sie verändern dadurch die traditionelle Wirkstoffsuche und ermöglichen auch die Entwicklung völlig neuer Wirkstoffklassen, z.B. Antikörper (Henderson et al. 1999). Auch für die Produktion von Pharmazeutika (z. B. Insulin, Interferone, Antikörper, Hormone, Antibiotika) und eine Reihe von Impfstoffen (z. B. gegen Hepatitis B) werden biotechnologische Methoden verwendet. Sie sind bei Biopharmazeutika häufig die einzige Produktionsalternative, um die erforderliche Menge und Qualität zu erreichen. Folglich kann der Einsatz der Biotechnologie in der Pharmaindustrie vielfältig sein. In Abbildung 6 ist eine beispielhafte Wertschöpfungskette von (Bio-)Pharmazeutika skizziert. Neben dem Einsatz biotechnologischer Methoden in der fast kompletten Wertschöpfungskette bei Biopharmazeutika kommt die Biotechnologie bei chemischen-synthetisierten Pharmazeutika vor allem im „Forschungs-Entdeckungsstadium“ zum Einsatz.

\section{Abbildung 6: Wertschöpfungskette von (Bio-)Pharmazeutika}

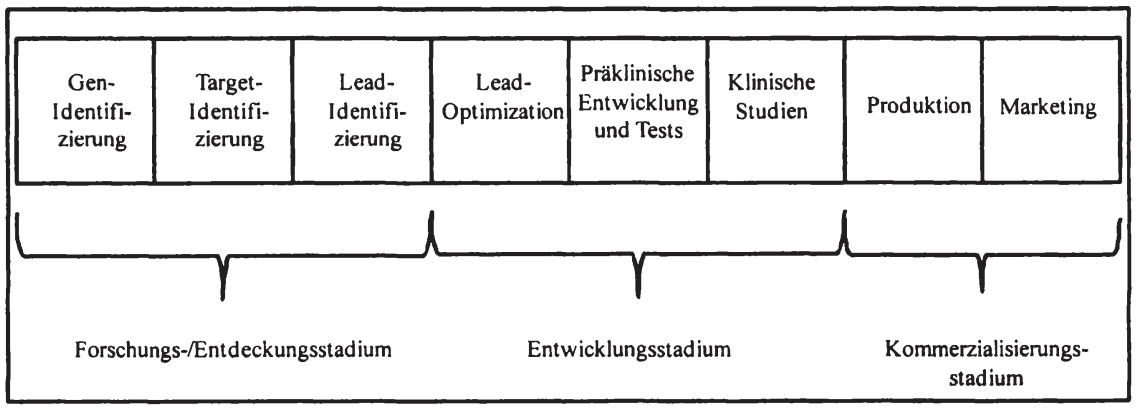

Quelle: Hine/Kapeleris 2006

Daneben werden biotechnologische Methoden auch zur Diagnose von Krankheiten (molekularer Diagnostik) eingesetzt. Dazu tragen die Kenntnis der Sequenzen des Erbguts des Menschen, wichtiger Nutztiere und wichtiger Krankheitserreger sowie die verschiedenen Ansätze zur Funktionsaufklärung der Gene (Genomik, Proteomik etc.) bei (Kinkel et al. 2008). Beispiele sind Immun- und DNA-Diagnostika zur Diagnose von Infektionskrankheiten, zur Krebsfrüherkennung oder zur Identifizierung von Erberkrankungen. Im Gesundheitsbereich ermöglicht die Biotechnologie neue Therapieprinzipien, wie z. B. die Gentherapie, Zelltherapien, das „Tissue Engineering“ oder die Regenerative Medizin sowie neuartige Möglichkeiten der Arzneimittelverabreichung (Kinkel et al. 2008). 
Bisher befinden sich viele dieser Verfahren allerdings noch in der Entwicklung (Reiss et al. 2006).

\subsubsection{Industrielle Anwendungen}

Bei der industriellen Biotechnologie werden biotechnologische Prozesse zur Herstellung einer Vielzahl verschiedenster industrieller Produkte eingesetzt. Dabei werden vor allem fermentative oder biokatalytische (z.B. enzymatische) Verfahren verwendet. Diese biotechnischen Verfahren können (konventionelle) mechanische, chemische, physikalische Verfahrensschritte ersetzen oder ergänzen (Kinkel et al. 2008). So haben biokatalytische Prozesse häufig eine höhere Spezifität und laufen bei geringerem Druck und bei geringerer Temperatur sowie geringerem Materialeinsatz als chemische Prozesse ab (OECD 2008b; Hoppenheidt et al. 2005). Hieraus ergeben sich verschiedene Vorteile, z.B. Einsparung bei Material oder Energie, eine umweltschonendere Produktion etc. (siehe Kapitel 6.3). Daneben können die biotechnologisch hergestellten Produkte Qualitätsvorteile (z. B. Reinheit) gegenüber konventionell hergestellten Produkten aufweisen. Teilweise sind sie sogar die einzige Produktionsalternative, mit der sich bestimmte Produkte - z. B. chirale organische Chemikalien oder Joghurt und Käse mit traditioneller Biotechnologie - wirtschaftlich herstellen lassen. Biotechnische Verfahren eignen sich dabei besonders für die Umwandlung von Naturstoffen. Sie werden daher von der Entwicklung des zunehmenden Wechsels der Produktion von fossilen auf nachwachsende Rohstoffe (z.B. Zucker, Getreide) begünstigt. Durch die effizientere Nutzung trägt die Biotechnologie aber auch selbst wiederum zu einem wirtschaftlich rentablen Rohstoffwechsel bei (Nusser et al. 2007b). Zum Beispiel können Biokunststoffe anstatt traditioneller Kunststoffe auf fossiler Rohstoffbasis eingesetzt werden oder Biokraftstoffe(-energie) fossile Kraftstoffe oder Energieträger ersetzen (z.B. Bioethanol $^{74}$ ).

Die Anwendung biotechnologischer Methoden findet dabei für sehr unterschiedliche Produkte statt, von billigen Chemikalien in sehr großen Mengen (z.B. Bulkchemikalien) bis hin zu hochpreisigen Spezialprodukten. Von Bedeutung sind die Anwendungen der industriellen Biotechnologie vor allem in der chemischen Industrie, Lebensmittel- und Getränkeindustrie, sowie bei der Textilveredelung, der Lederherstellung und der Zellstoff- und Papierproduktion (OECD 1998,2001; Dechema 2004; Hüsing et al. 1998; Nusser et al. 2007b). Daneben existieren bisher vereinzelte Anwendungen im Bergbau, in der Metallindustrie etc. (Warhurst 1995; IBTF 2004; Nusser et al. 2007b). Eine prototypische Wertschöpfungskette zeigt Abbildung 7. Biotechnologische Verfahren

74 Technologisch denkbar, aber bisher nicht im kommerziellen Einsatz ist die biotechnologische Produktion von Biodiesel, Biobutanol, mikrobielle Brennstoffzellen und Biowasserstoff (OECD 2008d). 
werden häufig zur Herstellung für Zwischenprodukte (z.B. Biomaterialien) eingesetzt und gehen nur indirekt in das Endprodukt für den Konsumenten ein. Ausnahmen sind z.B. biotechnologische Kosmetikprodukte, Biokraftstoffe oder Nahrungsmittel.

\section{Abbildung 7: Einsatz der industriellen Biotechnologie in verschiedenen Wertschöpfungsketten}

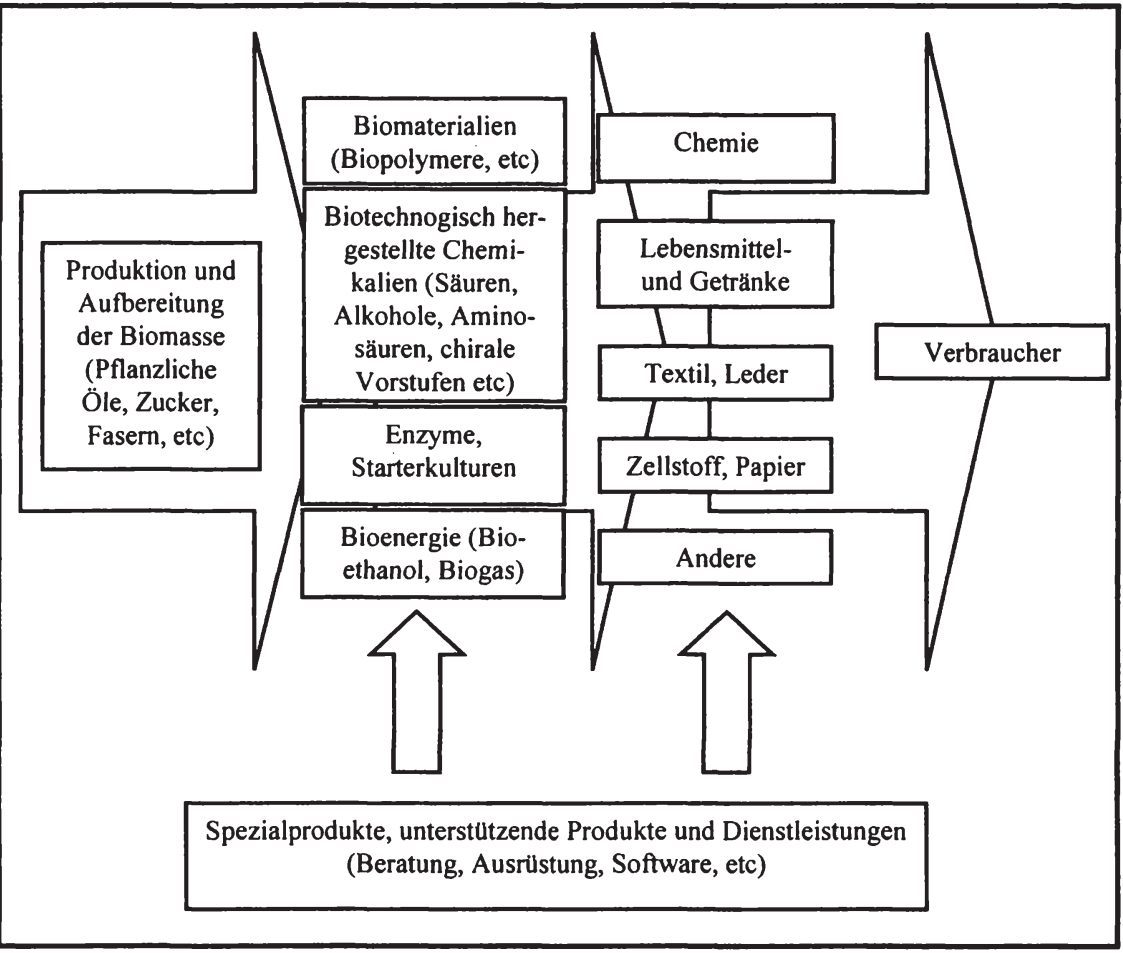

Quelle: Leicht abgeänderte Darstellung von Kinkel et al. (2008) und Kern/ Enzing (2003)

Bisher ist die Verbreitung der Biotechnologie in den genannten Anwendersektoren begrenzt. Für die Zukunft wird von vielen Experten aber ein deutlicher Anstieg erwartet (ColognePaper 2007; Nusser et al. 2007b; Dechema 2004; IPTS 2007). Hauptgründe hierfür sind die oben genannten Vorteile in Verbindung mit der technologischen Weiterentwicklung und günstige Rahmenbedingungen durch die Verteuerung der chemischen Alternativen infolge der steigenden Preise und abnehmenden Verfügbarkeit fossiler Rohstoffe. 


\subsubsection{Landwirtschaft und Lebensmittel (Agro-Food)}

Der Einsatz der Biotechnologie im Agro-Food Bereich umfasst Anwendungen in der Landwirtschaft, der Forstwirtschaft, dem Gartenbau, der Tierproduktion, Fischerei und Aquakultur sowie der Lebensmittel- und Getränkeherstellung. Dabei werden diese Anwendungen in der Regel der "Grünen Biotechnologie" zugeordnet. ${ }^{75}$ Eine viel diskutierte Rolle nimmt die gentechnische Veränderung der Nutztiere und -pflanzen ein. Dabei werden gentechnisch veränderte Organismen eingesetzt, deren genetisches Material unter natürlichen Bedingungen oder durch Kreuzen bzw. durch eine natürliche Rekombination nicht vorkommt (Heneric 2006, S.49). Daneben ist die markergestützte Selektion (MAS) von hoher Bedeutung (Arundel 2002). Diese nutzt molekulare Marker um gewünschte genetische Eigenschaften für Züchtungsprogramme bei Nutzpflanzen und -tieren zu untersuchen.

Die Züchtungsziele sind bei der markergestützten Züchtung und bei gentechnisch veränderten Organismen sehr ähnlich (Kinkel et al. 2008). Häufig wird das Ziel verfolgt, den Pflanzen oder Tieren erwünschte Eigenschaften (traits) zu geben oder diese auszuschließen. Dabei wird zwischen „input traits“ und „output traits“ unterschieden. Unter ,input traits“ werden anbautechnische Vorteile zusammengefasst. Beispiele hierfür sind widerstandsfähigere Pflanzen mit z.B. Herbizid- oder Insektenresistenz. „Output traits“ spiegeln eine qualitative oder quantitative Verbesserung der Pflanze oder des Erzeugnisses des Nutztieres wider. Die quantitative Verbesserung kann in einer Erhöhung des Ertrags und der Leistung liegen, die Verbesserung der Qualität des pflanzlichen oder tierischen Produkts kann z.B. in einem erhöhten Gehalt an wertgebenden Inhaltsstoffen, in einer besseren Verarbeitungsmöglichkeit oder einer längeren Haltbarkeit, bestehen. Ein weiterer Einsatzzweck ist die Beeinflussung der Fortpflanzungsfähigkeit und Fortpflanzung (z. B. Erhöhung der Fortpflanzungsrate bei züchterisch besonders wertvollen Nutztieren). Darüber hinaus werden auch in der Tier- und Pflanzenproduktion biotechnische Produktionsverfahren eingesetzt, so z. B. biobzw. gentechnisch hergestellte Veterinärpharmazeutika oder Futtermittelzusätze.

Abbildung 8 verdeutlicht den Einsatz der Biotechnologie in der Agro-Food Wertschöpfungskette. Die moderne Biotechnologie kommt vor allem in der Primärproduktion zum Einsatz, ebenso in der Lebensmittelherstellung durch die Nutzung von Enzymen sowie zur Überprüfung der Lebensmittelqualität (z.B. für Tests auf Salmonellen) (IPTS 2007).

Die bisherige Diffusion der Biotechnologie in der Landwirtschaft ist sehr unterschiedlich zwischen einzelnen Ländern. Während bspw. in einigen Ländern der Anbau von Mais mit gentechnisch veränderten Organismen (GMO) bereits in sehr hohem Ausmaß erfolgt - in USA liegt der Anteil an der gesamten Mais-

\footnotetext{
${ }^{75}$ Bei der Lebensmittel- und Getränkeherstellung bestehen erhebliche Überschneidungen zur industriellen Biotechnologie, eine konkrete Abgrenzung ist hier nur schwer möglich.
} 
produktion im Jahr 2005 über 50\% - ist dies in anderen Ländern nicht der Fall (IPTS 2007). In Deutschland liegt der Anteil bei gentechnisch verändertem Mais im gleichen Jahr unter 0,1\%. (IPTS 2007). Deutlich weiter verbreitet ist z.B. die markergestützte Selektion. Hier ist davon auszugehen, dass fast alle Pflanzenzüchtungsunternehmen in Deutschland diese Techniken bereits verwenden (Nusser et al. 2007a; Arundel 2002). ${ }^{76}$

\section{Abbildung 8: Biotechnologie in der Wertschöpfungskette im Agro-Food Bereich}

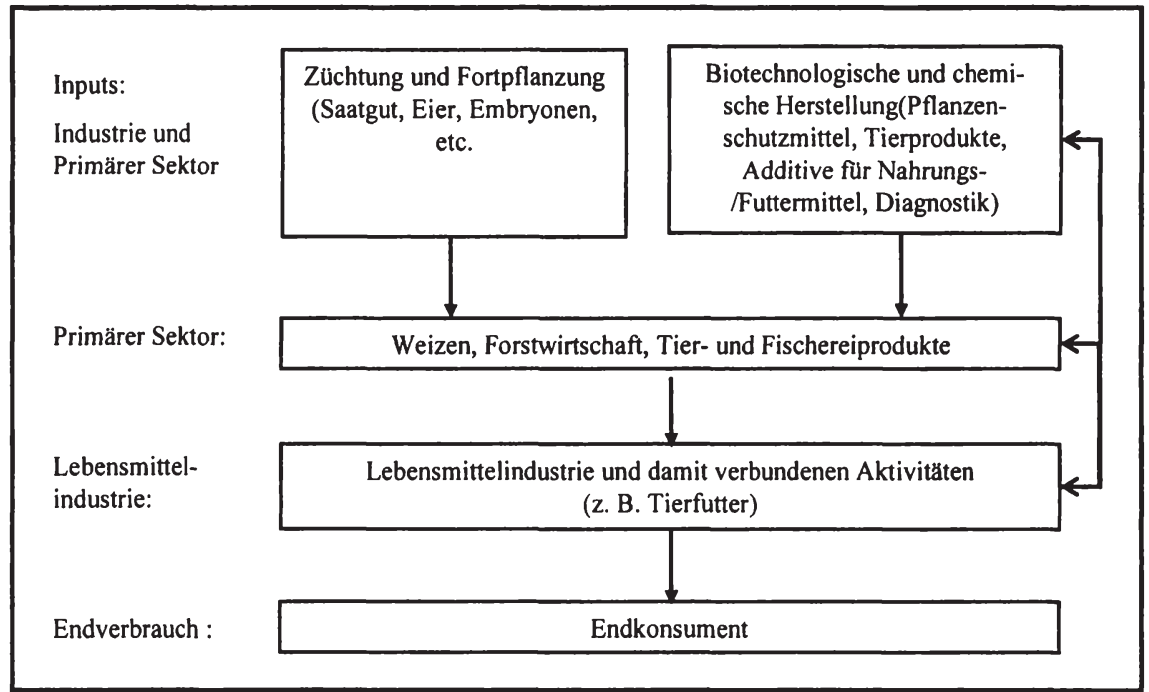

Quelle: IPTS (2007)

\subsubsection{Umweltbiotechnologie}

Neben möglichen umweltschonenden Effekten bei den genannten Anwendungen werden biotechnische Prozesse auch direkt im nachsorgenden Umweltschutz angewendet (Kinkel et al. 2008; Reiss et al. 2006). So werden Mikroorganismen und Enzyme zur Reinigung von Wasser, Luft oder Böden eingesetzt. Insbesondere in der Wasser- und Abwasserbehandlung und der Behandlung von festen Abfällen und Reststoffen (z.B. Kompostierung) sind biotechnische Verfahren von Bedeutung. In der Abluftbehandlung kommen vor allem Biofilter und Biowäscher zur Entfernung von Geruchsstoffen und toxischen Substanzen zum Ein-

${ }^{76}$ Allerdings ist hierbei nicht bekannt, welcher Anteil der Pflanzen- und Getreideproduktion mit markergestützter Selektion (MAS) erfolgt (IPTS 2007). 
satz. In der Landwirtschaft sind biotechnische Verfahren u.a. zum Abbau von Schadstoffen in kontaminierten Böden von Bedeutung. Insgesamt wird die Bedeutung der modernen Biotechnologien für diese Umweltschutzanwendungen aber eher gering eingeschätzt (Reiss et al. 2006).

\subsection{Biotechnologie als General Purpose Technology}

In den folgenden beiden Kapiteln 3.2 und 3.3 wird das theoretische Konzept der GPT und die geschilderten Wirkungszusammenhänge für Produktions- und Beschäftigungseffekte neuer Technologien konkret für die Biotechnologie diskutiert. Ziel der Betrachtung aus diesen zwei theoretischen Perspektiven ist es einen Überblick $\mathrm{zu}$ den technologische Charakteristika, dem Innovations/Diffusionsprozess und den gesamtwirtschaftlichen Folgewirkungen der Biotechnologie zu geben. Dieses Vorgehen soll die Spezifizierung der empirischen Untersuchung und eine Einordnung deren Ergebnisse in den Gesamtkontext ermöglichen.

Zunächst wird der Frage nachgegangen, ob sich das Konzept der GPTs zur Charakterisierung der Biotechnologie und ihrer wirtschaftlichen Effekte eignet. Dabei wird zuerst erörtert, inwieweit die Biotechnologie die generellen Charakteristika einer GPT erfüllt. Darauf aufbauend wird analysiert, inwiefern die bisherige Entwicklung der (modernen) Biotechnologie Parallelen und Unterschiede zu bisherigen GPTs aufweist. Schließlich kann sich nur im Zeitablauf zeigen, wie sich die Technologie in ihrem institutionellen und ökonomischen Umfeld tatsächlich entwickelt und ob die Charakteristika einer GPT tatsächlich erfüllt werden. ${ }^{77}$

\subsubsection{Erfüllung der Charakteristika einer GPT?}

Bei der Einordnung einer Technologie anhand der genannten Kriterien, stellt sich die Frage, mit welcher Methodik die Erfüllung eines Kriteriums gemessen oder beurteilt werden kann. Während einige Autoren die Analyse von Patententwicklungen für eine Identifikation einer GPT nutzen (Moser/Nicholas 2004; Youtie et al. 2008), wird diese von Lipsey et al. (2005) als zu eng und teilweise nicht unbedingt adäquat betrachtet. So spiegeln sich besonders die Bedeutung der Technologie in Anwendersektoren nicht unbedingt in Patenten und deren Zitate wider (Lipsey et al. 2005, S.109). Im Folgenden wird analog zu Lipsey et al. (2005) und anderen Autoren (Freeman 1995; Russell 1999; Ruttan 2001) eine breitere Bewertungsgrundlage auf Basis stilisierter Fakten zur Beurteilung der

\footnotetext{
${ }^{77}$ Als häufiges Beispiel einer Technologie, die trotz hoher Erwartungen bisher nur begrenzte Diffusion und Auswirkungen aufweisen konnte, gilt die Atomkraft. Gründe liegen vor allem in der mangelnden öffentlichen Akzeptanz und hohen Kapitalkosten (Freeman 2003; Lipsey et al. 2005).
} 
Biotechnologie herangezogen. Dabei werden die einzelnen vier GPT-Kriterien (Kapitel 2.2.3.2) für die Biotechnologie nacheinander erörtert.

Das erste Kriterium betrifft die (potenziellen) Verbesserungen und Leistungssteigerungen der GPT in ihrer zeitlichen Entwicklung, durch welche sich im Ergebnis Kosteneinsparungen in bestehenden Anwendungen sowie neue Anwendungsmöglichkeiten der Technologie ergeben. Für die Biotechnologie wird ein solches Entwicklungspotenzial bisher besonders in der pharmazeutischen Industrie und der Landwirtschaft gesehen (z.B. Arundel 2003; Lipsey et al. 2005). So werden von dem zunehmenden Verständnis der genetischen und molekularen Ursachen von Krankheiten in der pharmazeutischen Forschung Medikamente für bisher kaum heilbare Krankheiten, neue Diagnoseverfahren und Therapiemethoden (z.B. Stammzellentherapie) erhofft (IPTS 2007, S.39). In der Landwirtschaft würde beispielsweise über die Idee, Pflanzen und Tiere als Produktionssystem für die Herstellung von Arzneimittel, anderen medizinischen Produkten, Chemikalien, Kraftstoffe oder Plastik zu verwenden, der produzierende Sektor eine zunehmende Anzahl an Sektoren beeinflussen, die zunächst kaum von diesen biotechnologischen Methoden berührt waren (Lipsey et al. 2005, S.212). Bisher zeigen sich vor allem über die Veränderungen von Eigenschaften bei Pflanzen (z.B. Widerstandsfähigkeit) und Lebensmittel (,functional food") neue Anwendungsmöglichkeiten im Agro-Food-Bereich. Kritiker verweisen aber hingegen auf das bisher eingeschränkte Potenzial der Biotechnologie zur Kostensenkung (Freeman 1995; Wonglimpiyarat 2008). Insbesondere zeigt sich seit langem eine fehlende Kostenüberlegenheit der Biotechnologie in den Sektoren, bei denen die Kosten einen besonders hohen Wettbewerbsfaktor einnehmen, z.B. bei Bulkchemikalien, Nahrungsmitteln, Kraftstoffe etc. (Freeman 1995, S.16; Nusser et al. 2007b). Auch Effizienzgewinne in der Pharmaforschung und Landwirtschaft bleiben bisher teilweise hinter den Erwartungen zurück (Hopkins et al. 2007; Ruttan 2001).

Das zweite Kriterium betrifft die Reichweite der Technologie. Eine GPT wird in vielen Bereichen der Wirtschaft eingesetzt. Zur Erfüllung dieses Kriteriums bestehen für die Biotechnologie durchaus Hinweise. Sie weist mit ihren Anwendungsmöglichkeiten in einer Reihe von Sektoren einen deutlichen Querschnittscharakter auf. Die Biotechnologie findet im primären (Landwirtschaft, Fischerei), sekundären (Chemie, Pharma etc.) und tertiären Sektor (z.B. Gesundheit, Bildung, Forschung) Anwendung. Allerdings weisen einige Autoren darauf hin, dass die bisher erreichte Reichweite enger als das Potenzial ist und die bekannte potenzielle Reichweite im Vergleich zur IKT recht gering ist (Arundel 2003; Freeman 2003; IPTS 2007). Schließlich konzentriert sich der bisherige direkte Einfluss der Biotechnologie doch weitgehend auf Industriesektoren, die mit „lebenden Organismen" arbeiten. Die Verbreitung in Sektoren, wie z.B. Stahlindustrie, Elektrotechnik, Automobilbau und vielen Dienstleistungssektoren, ist gering. Folglich befindet sich ein Großteil - mit Ausnahme 
des Bereichs Pharma/Gesundheit - der Anwendungsfelder (z.B. Chemie, Landwirtschaft, Nahrungsmittel) in Sektoren, die zumindest im Bezug auf Beschäftigung gesamtwirtschaftlich in den letzten Jahrzehnten an Bedeutung verlieren (Buttel 1995, S.36). Inwieweit diese fehlende Beschäftigungsdynamik einiger wichtiger Anwendersektoren die Bedeutung der Biotechnologie schmälert, wird analog zur Debatte über die Bedeutung der Industrie für eine Volkwirtschaft kontrovers diskutiert. ${ }^{78}$ Auch Sektoren mit geringen Beschäftigungsanteilen können bspw. über einen hohen Bezug von Vorleistungen aus anderen Sektoren oder technologischen Spillover-Effekten und Produktivitätsgewinnen, erheblich zum gesamtwirtschaftlichen Wohlstand und zur Wachstumsdynamik beitragen. Zudem ist es denkbar, dass die langfristige potenzielle Anwendungsreichweite der Biotechnologie höher ist als bisher erkennbar (Lipsey et al. 2005; Roobeck 1995). So vermuten Lipsey et al. (2005, S.211), dass analog zu bisherigen GPTs die Anwendungsmöglichkeiten der Biotechnologie erst deutlich werden, wenn sie perfektioniert wird und über längere Zeit in verschiedenen Anwendungen Einsatz findet. Insbesondere durch das Zusammenspiel mit anderen Technologien - siehe viertes GPT-Kriterium - sehen einige Autoren es für möglich an, dass sich der Einfluss der Biotechnologie sich auch auf den Bereich der „toten Materie" erweitert (z.B. Roobeck 1995).

Das mit dem zweiten Kriterium nah verwandte dritte Kriterium der Vielfalt an Nutzungsmöglichkeiten besagt, dass die GPT zu unterschiedlichen Zwecken in einer Vielzahl verschiedenartiger Produkte und Prozesse zum Einsatz kommt. Für vielfältige Nutzungsmöglichkeiten der Biotechnologie sprechen einige Indizien. Wie in Kapitel 3.1.5 dargestellt, erfüllen die verschiedenen Biotechniken in den einzelnen Anwendungsfeldern teilweise unterschiedliche Zwecke. So dient die Biotechnologie im Bereich Pharma als Forschungsmethode zur Ursachenforschung und Entwicklung von Therapeutika, in industriellen Prozessen zur Herstellung von Gütern, zur Umweltschonung durch Abfall-, Luft- und Wasserbehandlung und in der Landwirtschaft zur Züchtung neuer Pflanzensorten und Veränderung von Pflanzeneigenschaften (Heneric 2006, S.27; McKelvey 2007, S.607). Auch lässt sich eine hohe Vielfalt bei den Produkten beobachten, die mit biotechnologischen Methoden entwickelt und/oder hergestellt werden. So kommt die Biotechnologie in der Pharmazie in unterschiedlichen Krankheitsgebieten zum Einsatz, auch in den heterogenen Chemie- und Lebensmittelindustrien finden sich in recht verschiedenen Produktgruppenbereichen Einsatzfelder der Biotechnologie (IPTS 2007). Etwas skeptischer gegenüber der Vielfalt sind Freeman (1995) und Arundel (2003), die diese mehr aus Sicht der Tangierung von Produktionsfaktoren betrachten und hier die Nutzungsmöglichkeiten der Biotechnologie im Vergleich zur IKT als sehr gering

\footnotetext{
${ }^{78}$ Für Diskussionen zur Biotechnologie siehe Buttel (1995) und Otero (1995). Für eine allgemeine Diskussion über die gesamtwirtschaftliche Bedeutung industrieller Sektoren siehe z.B. Kalmbach et al. (2003), Nusser et al. (2007c).
} 
ansehen. „Information technology [...] operates through substitution of a given production factor (e.g. labour) and has been able to penetrate almost all products and processes of human activity. There is no process that has not been or cannot be modified by the use of information technology [...] This does not hold true for biotechnology" (Freeman 1995, S.20). Die Biotechnologie beeinflusst die Produktionsfaktoren weniger direkt. Zum Beispiel sind beim Faktor Arbeit Tätigkeitsfelder außerhalb des technischen Bereichs (z.B. Verwaltung, Finanzierung, Marketing, Verkauf) nicht direkt von der Biotechnologie tangiert.

Das vierte Kriterium einer GPT betrifft die hohen Komplementaritäten zu anderen Technologien und zum technologischen System insgesamt. Durch Spillovereffekte entstehen neue Möglichkeiten für profitable Investitionen in neue Produkte und Prozesse. Komplementaritäten der Biotechnologie werden insbesondere mit den IKT und der Nanotechnologie diskutiert (Kern/Enzing 2003). Auch Skeptiker eines Erfolgs der Biotechnologie sehen gerade in der Verbindung mit den IKT den bedeutendsten Potenzialbereich der Biotechnologie (Freeman 2003, S.11; Buttel 1995, S. 40). Auf der einen Seite wird die Forschung und Anwendung der Biotechnologie durch die IKT unterstützt, z.B. durch Computer-Ausstattung in Laboratorien und Produktionsstätten oder der Bioinformatik, welche die Bearbeitung einer Vielzahl an genetischen Informationen ermöglicht (Freeman 2003, S.12). Auf der anderen Seite beeinflusst die Biotechnologie die IKT bspw. bei der Entwicklung von Biochips. ${ }^{79}$ Die Verbindungsmöglichkeit der Bio- mit der Nanotechnologie besteht durch die Größe von Nanopartikeln, die mit Biomolekülen auf der Oberfläche und in Zellen interagieren können (Kinkel et al. 2008). Mögliche Anwendungen sind die Arzneimittelverabreichung mittels Nanopartikeln oder Systeme zur Detektion von biologischen und chemischen Kampfstoffen oder Umweltgiften (Kinkel et al. 2008). Weitere Komplementaritäten werden bei der Diskussion der Konvergenz der Spitzentechnologien über das Zusammenwachsen der vier Forschungsfelder Nano-, Bio- und Informationstechnologie sowie der Kognitionswissenschaften (NBIC) diskutiert (z.B. Rocco/Bainbridge 2003; Roco/Montamagno 2004). Hierbei ist allerdings noch unklar, inwieweit die zum Teil sehr visionären Ideen in tatsächliche Aktivitäten umgesetzt werden (Beckert et al. 2006).

Die horizontalen Spillover-Effekte zwischen den Anwendersektoren, die Bresnahan und Trajtenberg (1995) als zentral für eine GPT ansehen (Kapitel 2.2.3.2), werden für die Biotechnologie gering eingeschätzt (Swann et al. 1998; Kern/Enzing 2003; Luukonnen/Palmberg 2007). Während bspw. in der Computerindustrie ein gemeinsames Endprodukt (z.B. Computer) für eine Vielzahl von Sektoren erzeugt wird und Netzwerkeffekte erreicht werden können, erfolgt die Entwicklung in der Biotechnologie häufig separat für einzelne Anwendersekto-

${ }^{79}$ Allerdings haben sich bislang die hohen Erwartungen an diese Innovationen noch nicht erfüllt (Soete 2006). 
ren. Zwar werden häufig ähnliche Techniken verwendet, die Bedürfnisse an das Produkt oder Verfahren unterscheiden sich zwischen den Anwendersektoren "[...] the distinction broadly holds that biotechnology firms depend on existing companies in their own sector of application for their market and for the joint development of their products. Links have tended to run vertically downstream to user industries for each sector rather than between sectors, with little technological interdependence between sectors" (Swann et al. 1998, S.231). Die Lerneffekte zwischen Anwendersektoren als wichtige Komponente des technologischen Fortschritts bleiben demnach eher gering.

Zusammenfassend zeigt sich, dass für alle vier Kriterien zwar Indizien zur Erfüllung bestehen, diese aber teilweise auf Zukunftserwartungen aufbauen. Eine abschließende Bewertung kann zum jetzigen Zeitpunkt nicht stattfinden. Zudem zeigen sich im Vergleich zur IKT deutliche Begrenzungen des zumindest bisher bekannten möglichen Potenzials. Als größte Einschränkungen bei diesen Kriterien sind die bisher fehlenden Durchbrüche bei Kosteneinsparungen und die Begrenzungen des direkten Einflusses auf die Wirtschaft durch die Biotechnologie zu nennen. Die Bedeutung dieser Einschränkungen wird je nach Konzept etwas unterschiedlich beurteilt. Im Konzept der technoökonomischen Paradigmen von Freeman und Perez (1988) führen diese Einschränkungen zu deutlichen Fragezeichen dahingehend, ob die Biotechnologie die Kriterien erfüllen kann (Freeman 1995/2003; Nightingale/Martin 2004). Nach Lipsey et al. (1998/2005) müssen GPTs tendenziell geringere Anforderungen erfüllen und können unterschiedlich hohe Potenziale für Wachstums- und Produktivitätssteigerungen haben. Besonders für potenziell zukünftige GPTs halten sie die Anlegung von strengen Kriterien als nicht korrekt. „It is far easier to identify some emerging technologies as potential GPTs than to rule out others" (Lipsey et al. 2005, S.110). Inwiefern sich die Biotechnologie tatsächlich zu einer GPT entwickelt, hängt nach diesem Konzept entscheidend davon ab, wie sich das institutionelle, soziale und ökonomische Umfeld (,Faciliating Structure“) im Zusammenspiel mit der Technologie entwickelt. Die bisherige Entwicklung, Gründe hierfür und mögliche zukünftige Veränderungen werden im folgenden Kapitel erörtert.

\subsubsection{Diffusion und Entwicklung der Biotechnologie}

\subsubsection{Bisherige kommerzielle Entwicklung}

Analog zur Beurteilung der GPT-Kriterien fällt die Einschätzung, in welchem Entwicklungsstadium sich die Biotechnologie aktuell befindet, nicht leicht. Bisherige Einordnungen sehen die Biotechnologie zumeist in einer frühen Entwicklungsphase, sie lassen dabei aber zumeist offen, auf welchem Niveau (z.B. Reichweite der Anwendungen) sich die Biotechnologie in ihrer Diffusionsphase zukünftig bewegen kann (Luukonnen/Palmberg 2007; Hirooka 2006; Freeman 1995). Insgesamt wird die Biotechnologie mittlerweile aber kritischer bezüglich 
ihres Potenzials diskutiert (z.B. Hopkins et al. 2007). So zeigen bspw. Veröffentlichungen im Zeitablauf von Freeman eine zunehmende Skepsis gegenüber der Realisierung des Potenzials der Biotechnologie (Freeman/Perez 1988, Freeman 1995, Freeman 2003). Dabei berufen sich die Kritiker insbesondere darauf, dass viele frühere Prognosen und Einschätzungen über baldige neue Anwendungen und Vorteile durch die Biotechnologie bisher nicht eingetroffen sind. "Numerous new applications were being forecast during the 1970s and early 1980s, but many of these hopes have not been fulfilled" (Freeman 2003, S. 9). Am meisten Skepsis zeigt sich bei der engen Betrachtung auf der Unternehmensebene, die besonders Akteure in der Pharmabranche erfasst (McKelvey 2008; Pisano 2006b). So konnte nach bisherigen Erkenntnissen die lange Zeit erhoffte Steigerung der Forschungsproduktivität der Pharmaunternehmen durch die Biotechnologie bis heute nicht erreicht werden (Hopkins et al. 2007, siehe Kapitel 6.4). ${ }^{80}$ Ebenso blieb die Entwicklung reiner Biotechnologieunternehmen bei Umsätzen und Profitabilität deutlich hinter den Erwartungen zurück (Pisano 2006b).

Diesen skeptischen Meinungen stehen die optimistischen Argumente der Kriteriums-Diskussion gegenüber und den Erfahrungen zu bisherigen GPTs, welche die lange Zeitdauer bis zur Realisierung der Diffusion und Potentiale betonen. Russell (1999) hebt beispielsweise hervor, dass auch beim elektronischen Computer erst nach zwei Generationen durch die Einführung des Mikroprozessors und integrierten Schaltkreisen Kostersparnisse deutlich wurden und auch für die Biotechnologie ein ähnlicher Fall eintreten kann. In den folgenden Kapiteln werden mögliche Erklärungen für diese bisherige Entwicklung im Kontext des GPT-Konzeptes diskutiert. Dabei werden drei Erklärungsansätze voneinander getrennt: ${ }^{81}$

- Erstens ist es denkbar, dass durch überzogene Erwartungen an die Biotechnologie sowie oder durch Messungsprobleme die Entwicklung der Biotechnologie unterschätzt wird.

- Zweitens können technologische Charakteristika und die Einordnung des technologischen Entwicklungsstadiums inhaltliche Erklärungen für bisherige kommerzielle Enttäuschungen liefern.

\footnotetext{
${ }^{80}$ Ruttan (1999) berichtet von ähnlichen Entwicklungen in der Landwirtschaft. Auch hier zeigt sich eine verhaltene Steigerung der Erträge je Hektar, obwohl durch die Einfuhrung von biotechnologischen Methoden hohe Steigerungen erwartet wurden.

${ }^{81}$ Diese Trennung in verschiedene Erklärungsansätze erfolgt in Anlehnung an ähnliche Thesen zum Solowschen Produktivitätsparadoxon. Die Diskussion des Solowschen Produktivitätsparadoxon geht um die Frage, wie der lange Zeit beobachtbare, scheinbare Widerspruch zwischen der zunehmenden Investitionstätigkeit bei IKT und dem geringen Produktivitätswachstum erklärt werden kann (siehe z.B. Melzig-Thiel 2000, Hagemann et al. 2001).
} 
- Drittens sind zahlreiche Anpassungsfriktionen beim Zusammenspiel der Biotechnologie mit institutionellen, sozialen und ökonomischen Umfeldfaktoren denkbar.

\subsubsection{Hohe Erwartungshaltung und schwierige Messbarkeit von Erfolgen}

Eine Teilerklärung für die bisherigen kommerziellen Enttäuschungen wird in unrealistisch hohen Erwartungen in die Biotechnologie gesehen (Hopkins et al. 2007; Barben 2007). Als eine Ursache hierfür gilt, dass viele Akteure, welche große Erwartungen in die Biotechnologie verbreitet haben, selbst deutlich abhängig von den rosigen Zukunftssausichten waren (Nightingale/Martin 2004, S.587). ${ }^{82}$ Schließlich ist ein Teil der Beweggründe der staatlichen Förderungen oder die Finanzierung durch mögliche Kapitalgeber auf die Erwartung über hohe zukünftige Erträge mit biotechnologischen Prozessen, Produkten oder Dienstleistungen begründet. Unterstützt wurde diese Erwartungshaltung von frühen Erfolgen einiger Biotechnologieunternehmen, wie z.B. Amgen oder Genentech, die zu unrealistisch hohen Erwartungen in der öffentlichen Meinung geführt haben (Hopkins et al. 2007). Dieser frühe Erfolg, den andere Biotechnologieunternehmen in Folge nicht erzielen konnten, wird von einigen Autoren als das Ernten einiger weniger „low hang fruits" bzw. atypische Beispiele für den Beitrag der Biotechnologie zu einigen Forschungsproblemen angesehen (Hopkins et al. 2007, S.584). So weicht die Art der Anwendung und Fragestellung biotechnologischer Methoden mittlerweile deutlich von denen mit den bisherigen Techniken behandelten Fragestellungen ab (IPTS 2007). Die moderne Biotechnologie wird demnach für viel komplexere Probleme eingesetzt, die sich bisher der Möglichkeit der Forschung entzogen (siehe Kapitel 6.4; Pisano 2006b). Der potenzielle Produktivitätsfortschritt der Biotechnologie kann nicht mit der zunehmenden Komplexität der Probleme mithalten und mögliche qualitative Verbesserungen durch die Biotechnologie werden verdeckt. (Hopkins et al. 2007, S.584) Ohne die Biotechnologie wäre demnach die Pharmaindustrie in eine starke Krise geraten (Tait 2007).

Folglich sind erstens sehr hohe Erwartungen in die Biotechnologie gesetzt worden und zweitens werden tatsächliche Erfolge der Biotechnologie möglicherweise nicht in einer steigenden Produktivität erkennbar. Diese Aspekte finden sich auch im Konzept der GPT wieder. Es wird davor gewarnt, zu hohe kurzfristige Erwartungen in die Technologie zu setzen und einen „technologi-

\footnotetext{
${ }^{82}$ Wonglimpiyarat (2008) verdeutlicht, dass die unterschiedliche Perspektive der Akteure stark zu den verschiedenen Einschätzungen eines revolutionären Einflusses der Biotechnologie beiträgt. Während aus mikroökonomischen und technologischen Gesichtspunkten die Biotechnologie durchaus revolutionär sein kann (z.B. durch Ermöglichung personalisierter Medizin), muss dies nicht unbedingt auf einer makroökonomischer Ebene der Fall sein, wenn bestimmte Kriterien einer GPT oder Basistechnologie nicht erfült sind.
} 
schen Superoptimismus“ zu entwickeln, ,which tends to ignore the hard economic realities of costs, profitability and size and consumer acceptance of entirely new products" (Freeman 1995, S.8). Daneben sind nach Lipsey et al. (1998/2005) die Effekte einer Technologie entscheidend abhängig von den bisher implementierten Technologien und es kommt nur unwahrscheinlich zu höheren Produktivitätswachstumsraten bei neuen GPTs. Bereits der chemischen Synthese, für welche die Biotechnologie häufig ein Substitut bildet, werden die Eigenschaften einer GPT zugesprochen (Rosenberg 1998; Chandler 2005). So konnten hohe Produktivitätserfolge erreicht und für viele Produkte und Prozesse effektive Forschungs- und Herstellungsmethoden entwickelt werden. Allein die Fortsetzung einer Wachstumsentwicklung ist demnach eine große Herausforderung für die Biotechnologie.

\subsubsection{Technologische Charakteristika und Entwicklungsstadium}

Wie im ersten Kriterium der GPT deutlich wird, entwickelt eine GPT ihre Effizienzpotenziale und Anwendungsmöglichkeiten erst im Laufe der Evolution der Technologie. Ungelöste technologische Probleme bei der Biotechnologie sind deshalb nicht grundsätzlich als bedenklich für die weitere Entwicklung einzustufen. Es zeigen sich aber einige Besonderheiten bei der biotechnologischen Forschung, welche die technologische Weiterentwicklung sowie die Generierung ökonomischer Effekte vor große Herausforderungen stellen.

Als wichtiges Charakteristikum, welches die Biotechnologie von einigen anderen bedeutenden Technologien, wie z.B. den IKT, unterscheidet, ist die stärkere Abhängigkeit von wissenschaftlicher Forschung und deren Durchbrüche (Luukonnen/Palmberg 2007, S.332; Kern/Enzing 2003, S.12; Ruttan 2002, S.19). Weitgehend unbestritten ist, dass bedeutende Fortschritte in der Vergangenheit erzielt wurden. Dennoch wird häufig festgestellt, dass die Kenntnisse über biologische Prozesse - wie z.B. die Funktionsweise von Molekülen im menschlichen Körper - immer noch eingeschränkt sind. Zudem ist unklar, zu welchem Grad die technologischen Möglichkeiten bislang ausgeschöpft sind und wie lange weitere Entdeckungen brauchen werden (Kern/Enzing 2003, S.12; Pisano 2006a). Ein wichtiger Grund für Schwierigkeiten bei der Forschung sowie der Entwicklung von Anwendungen wird in der komplexen Kombination der Vielzahl von Techniken und wissenschaftlichen Disziplinen (u. a. Biochemie, Bioverfahrenstechnik, Bioinformatik, Biophysik) gesehen, die bei der Biotechnologie zur Lösung notwendig sind (Amir-Aslani/Negassi 2006, S.77). Beispielsweise werden für die Erhöhung der Effizienz von Biochemikalien die Integration von Biochemie, Mikrobiologie und chemischer Prozesstechnologie benötigt (Buttel 1995). Analog wird bei Forschungsprozessen für neue Wirkstoffe in der Pharmaindustrie eine Vielzahl von neuen Techniken kombiniert. Diese Integration der verschiedenen Wissensbasen wird in der Biotechnologie als be- 
sonders schwierig angesehen, da nur eine simultane Lösung verschiedenster Probleme den Forschungserfolg bzw. die rentable Anwendung ermöglicht (Pisano 2006a). Im Vergleich zu anderen Technologien, wie den IKT, wird für die Biotechnologie eine geringere Modularität festgestellt. Während sich bei den IKT einige hochkomplexe F\&E-Probleme in relativ unabhängige Teilprobleme aufteilen und bearbeiten lassen, um dann in einem späteren Schritt zusammengefügt zu werden, ist das bei der Biotechnologie nicht der Fall. Diese Schwierigkeiten behindern die Integration der Biotechnologie in die Prozesssysteme (Cooper 1994; Pisano 2006a). So stellen Kern und Enzing (2003) im Vergleich zwischen IKT und Biotechnologie fest: "ICT shows much stronger tendencies towards integration, i.e. ICT applications tend increasingly and relatively easy to become integral parts of broader integrated systems. Such integrative tendencies are still very modest for biotechnology and it can be questioned whether it will ever reach such a state." (Kern/Enzing 2003, S.12).

Selbst wenn wichtige Teilschritte im Forschungsprozess durch die Biotechnologie produktiver gestaltet werden können, bedeutet dies nicht, dass der gesamte F\&E-Prozess effektiver wird. Stattdessen sind komplementäre Innovationen für eine effektive Nutzung der Technologie notwendig (Bresnahan/Trajtenberg 1995). Beispielsweise ermöglichen in der Pharmaindustrie genom- und proteombasierte Methoden die Identifizierung neuer und einer größeren Anzahl von „Targets“ (Nightingale/Martin 2004). Allerdings können diese Verfahren kaum in späteren Phasen des F\&E-Prozesses eingesetzt werden. Gerade hier entstehen aber zusätzliche Herausforderungen durch die Zunahme der identifizierten „Targets“. So entstehen Informationsüberlast, zusätzliche statistische Qualitätskontrollen etc. und Probleme bei der Targetvalidierung, da das Wissen über jedes einzelne „Target“" aufgrund der deutlich größeren Vielzahl geringer ist (Booth/Zemmel 2004). Weitere Umsetzungsprobleme der biotechnologischen Fortschritte im F\&E-Prozess können in der Phase der klinischen Studien auftreten, welche auf die Nützlichkeit und Nebenwirkungen abzielen. Diese haben einen signifikanten Anteil am Zeit- und Kostenaufwand bei der Arzneimittelentwicklung. Allerdings sind die Fortschritte in diesen Bereichen hinsichtlich einer höheren Effizienz begrenzt, bzw. wird durch Besonderheiten der Biotechnologie zusätzlich erschwert (Nightingale/Martin 2004). Ein Beispiel ist die Notwendigkeit von zusätzlichen Immunologie-Prüfungen in klinischen Studien. Selbst wenn es gelingt bestimmt Abschnitte des F\&E-Prozesses durch die Biotechnologie effizienter zu gestalten, ist die Wirkung auf die Veränderung des Gesamtprozesses der Entwicklung und Anwendung neuer Medikamente gering (Pisano 2006b). Entsprechende Fortschritte sind erst bei komplementären Innovationen in anderen Prozessen möglich. 


\subsubsection{Kosten- und zeitintensive Anpassungsprozesse}

Neben technologischen Fortschritten ist ein komplementärer Anpassungsprozess von ökonomischen, sozialen und institutionellen Faktoren für eine erfolgreiche Diffusion der Biotechnologie sowie der Realisierung ökonomischer Effekte notwendig. Analog zu früheren GPTs verläuft dieser Anpassungsprozess bei der Biotechnologie aber nicht reibungslos, sondern ist zeit- und kostenintensiv. Mit dem zunehmendem Reifegrad der Biotechnologie rücken dabei immer stärker Faktoren für die Kommerzialisierung biotechnologischer Produkte und für die Anwendung biotechnologischer Verfahren in Produktionsprozessen in den Vordergrund (Arundel 2000; Luukonnen/Palmberg 2007). Allerdings wird die „Distanz" zwischen fundamentalen wissenschaftlichen Fortschritten und der Anwendung in neuen Produkten und Prozessen bei der Biotechnologie als besonders groß eingestuft (Kern/Enzing 2003; Luukonnen/Palmberg 2007; IPTS 2007). Im Folgenden werden die von Lipsey et al. $(1998,2005)$ hervorgehobenen Faktoren Humankapital, Organisationsstruktur, Infrastruktur und institutionelle Faktoren einschließlich der Politik im Bezug auf ihr Wechselspiel mit der Biotechnologie erörtert.

\section{Humankapital ${ }^{83}$}

Gegenüber den bisherigen qualifikatorischen Erfordernissen bei konventionellen Prozessen sind bei der Biotechnologie eine stärkere Interdisziplinarität des Wissens und stärkere Kenntnisse, z.B. in den Bereichen der Biologie und Biochemie, notwendig (Freeman 2003, S.11). Dieses erforderliche biotechnologische Know-how verbreitete sich bisher in vielen Unternehmen recht langsam. Zudem führten die mangelnden Kenntnisse zu einem geringen Bewusstsein für biotechnologische Alternativen und zu Berührungsängsten. Viele Entscheidungsträger aber auch Forschungsabteilungen in etablierten Unternehmen bestanden lange Zeit vorwiegend aus Chemikern und Ingenieuren, die in ihrer Ausbildung mit den Neuerungen der Biotechnologie noch nicht vertraut gemacht wurden und die skeptisch gegenüber den Potenzialen der konkurrierenden Technologie standen (Freeman 2003, S.11; Nusser et al. 2007a, S.110). Dies führte zu einer deutlichen Verlangsamung oder dem kompletten Verzicht der Einführung biotechnologischer Methoden in Unternehmen. Zwar hat die biotechnologische Wissensbasis vieler Unternehmen der pharmazeutischen und chemischen Industrie mittlerweile zugenommen, allerdings kann dies bisher nicht für alle Anwendersektoren beobachtet werden (Pflaum et al. 2008). Für die zukünftige Entwicklung in

${ }^{83}$ Das Institut IPTS sieht hierin den entscheidenden Engpass für die Diffusion der Biotechnologie: „The limits to growth in these fields of technology are not set by the availability of natural resources but by access to verified scientific knowledge and specialised skills." (IPTS 2002a, S.38). Dieser Aussage ist einschränkend entgegenzuhalten, dass die Verfügbarkeit nachwachsender Rohstoffe ebenfalls nicht als gesichert angesehen werden kann (siehe Kapitel 5.2.3.2). 
Deutschland wird im Zuge des möglichen Fachkräftemangels vor allem ein quantitativer Mangel an qualifizierten Arbeitskräften befürchtet (Kriegesmann et al. 2005; Colognepaper 2007). Folglich sind auch zukünftig zeitliche Verzögerungen bei der Verbreitung der Biotechnologie aufgrund fehlenden Humankapitals nicht ausgeschlossen und hohe Kosten für die Qualifizierung der Fachkräfte möglich.

\section{Infrastruktur}

Trotz der hohen Bedeutung des Humankapitals sind auch erhebliche infrastrukturelle Voraussetzungen beim Einsatz der Biotechnologie notwendig. Der biotechnische Prozess erfordert häufig eine andere Produktionsanlagenkonzeption als der bestehende Prozess, für den die Anlage bereits existiert. Zudem besteht in der industriellen Produktion häufig ein Netzwerk an Verfahren, in welche sich biotechnische Prozesse nicht ohne weiteres einfügen lassen. Ein Großteil der chemischen Produktion, besonders die von Grundchemikalien, wird an wenigen zentralen Verbundstandorten hergestellt, die meist auf synthetische Rohstoffe ausgerichtet sind. Bei diesen Systemen sind Produktionsbetriebe, Energie- und Abfallströme, Logistik und Infrastruktur eng miteinander vernetzt und Mengenströme von Haupt- und Nebenprodukten weitestgehend optimiert. Diese über Jahrzehnte gewachsenen kapitalintensiven Verbundstrukturen erschweren die Diffusion der Biotechnologie in der industriellen Produktion (Marschall 2005, Drumm 2005, Herrera 2004). Der Aufbau neuer passender Infrastrukturen erfordert erhebliche Investitionen in neue Kapitalanlagen. Falls ein Rohstoffwechsel stattfindet, ist zusätzlich die Integration von nachwachsenden Rohstoffen bei der Lagerung und dem Transport nötig (Nusser et al. 2007b; Hüsing et al. 2003; Gaisser et al. 2002).

\section{Organisationsstruktur}

Sehr intensiv werden notwendige Veränderungen von Organisationsformen und Geschäftsmodellen für die Biotechnologie diskutiert (u.a. Tait 2007; AmirAslami/Negassi 2006; Hopkins et al. 2007; McKelvey 2008). So stellt beispielsweise Hopkins et al. (2007) für die Entwicklung der Biotechnologie in der Pharmaindustrie fest: ,[...] the introduction of many gene and cell based biological drugs will require novel manufacturing technologies, changes in the organisation of clinical work, innovative service models and new regulatory environments" (Hopkins 2007, S.585). Es zeigten sich bereits in der Vergangenheit deutliche Veränderungen bei den Industriestrukturen und Vernetzungen der Akteure. Dabei steht besonders die Rolle kleiner „Start-up“ Biotechnologieunternehmen im Pharma- und z.T. im Agrarsektor im Mittelpunkt sehr zahlreicher Untersuchungen (McKelvey 2007). Bis in die 1980er Jahre war die Forschung in den Pharmaunternehmen fast ausschließlich ,inhouse“ orientiert. Seit den 1980er Jahren zeigen sich deutliche Veränderungen bei der Arbeitsteilung in der 
Forschung (Gambardella 1995). Mittlerweile investieren die Pharmaunternehmen einen erheblichen Anteil ${ }^{84}$ ihrer Forschungsaufwendungen in externe Partnerschaften mit Universitäten und kleinen spezialisierten Biotechnologieunternehmen (Dolata 2007; Ruttan 2001). Die Vorteile dieser Biotechnologieunternehmen liegen in der sehr spezifischen Fokussierung auf bestimmte Biotechniken, ihrer meist starken Anbindung zur Universitätsforschung gekoppelt mit ihrer Anwendungsorientierung (Ruttan 2002). Allerdings haben sich diese Biotechnologieunternehmen entgegen früherer Erwartungen - mit Ausnahme einiger weniger früher Unternehmenserfolge von Amgen und Genentech - bisher im Pharmabereich nicht zu vollintegrierten Unternehmen entwickelt, welche die gesamte Wertschöpfungskette von Forschung bis zur Produktion und der Vermarktung abdecken (Ruttan 2001, S.403). Die hohen regulatorischen Hürden für die Neuzulassung von Produkten mit sehr hohem Zeit- und Kapitalaufwand ${ }^{85}$ sowie die etablierten Strukturen zur Vermarktung von Arzneimitteln führten zu hohen Eintrittsbarrieren für die kleineren Unternehmen (Ruttan 2001; Tait 2007). Infolgedessen hat sich bisher eine vertikale Arbeitsteilung zwischen groBen Pharmaunternehmen - die alle Wertschöpfungsstufen abdecken - und kleinen spezialisierten Biotechnologieunternehmen herausgebildet (Kranich 2007, S.45). Somit zeigte sich bisher trotz der breiteren Akteurskonstellation auch eine gewisse Konstanz in der Industriestruktur mit der Dominanz großer multinationaler Unternehmen und einem Geschäftsmodell, das stark auf „BlockbusterProdukte" konzentriert ist (Tait 2007). Die Eignung dieser Organisationstruktur der Pharma- und Biotechnologieindustrie für die langfristige Entwicklung der Biotechnologie wird allerdings sehr in Frage gestellt (Amir-Aslani/Negassi 2006; Pisano 2006b; McKelvey 2008; Tait et al. 2007). So stehen nach Pisano (2006a) der vertikale Forschungsprozess sowie die notwendige Integration von verschiedenen Techniken der bisherigen Fragmentierung mit vielen Kleinunternehmen entgegen. Diese bilden "[...] islands of expertise that impede the integration of critical knowledge" (Pisano 2006a, S.116). McKelvey (2008) und Tait et al. (2007) sehen durch Umfeldentwicklungen (z.B. regulative Veränderungen, Veränderungen der Nachfrage) zunehmenden Druck auf die Akteure der Pharmaindustrie ihre Organisation und Geschäftsmodelle zu verändern. Es besteht in der Literatur aber keine Einigkeit, wie geeignete industrielle Organisationsstrukturen aussehen sollten. Pisano (2006a) sieht bspw. eine stärkere Rolle der etablierten Pharmaunternehmen als notwendig an, während McKelvey (2008) und Tait et al. (2007) aufgrund der starken Veränderungen im Umfeld eine stärkere Ausdifferenzierung und Veränderung der verschiedenen Geschäftsmodelle als

\footnotetext{
${ }^{84}$ Zum Beispiel lag der Anteil der externen Forschungsaufwendungen in Deutschland im Jahr 2006 bei 33\% (Stifterverband Wissenschaft 2008).

${ }^{85}$ Dabei wird besonders die Funktions- und Passfähigkeit von Venture Capital als wichtige Finanzierungsquelle für diese Unternehmen diskutiert (Luukonnen/Palmberg 2007; Pisano 2006b).
} 
geeignet einschätzen. ${ }^{86}$ Solche Veränderungen konnten bereits in der Agrarbiotechnologie beobachtet werden (Tait et al. 2007). Dort fanden Abtrennungen der agrobiotechnologischen Einheiten von Pharmagroßunternehmen mit erheblichen Konzentrationsprozessen statt (Blank 2008; Tait 2007; Chataway et al. 2006; Ruttan 2001).

Zusammenfassend haben sich in der Vergangenheit einige Veränderungen in den Organisationsformen (z.B. Arbeitsteilung in der Pharmaindustrie) ergeben. Gerade im Hinblick auf die zunehmende Kommerzialisierung biotechnologischer Produkte scheinen allerdings noch Veränderungen im organisatorischen Umfeld notwendig und wahrscheinlich. Nur wenn profitable Geschäftsmodelle gefunden werden können, die zu einem effektiven Forschungsprozess führen, wird es zu weitreichenden Innovations- und Diffusionserfolgen kommen. Dies gilt nicht nur für die hier diskutierte Pharmaindustrie, sondern mit zunehmender Diffusion auch für andere Anwendersektoren der Biotechnologie. So ist z.B. die Rolle und die Art der Geschäftsmodelle kleiner forschender Biotechnologieunternehmen in der viel stärker kosten- und zumeist an höheren Produktionsvolumen orientierten Chemie nach Aussagen von Experten bisher offen.

\section{Akzeptanz}

Ebenfalls eine hohe Bedeutung für die Entwicklung einer potenziellen GPT nimmt die soziale und politische Akzeptanz der neuen Technologie ein (Russell 1999; Freeman 2003; Lipsey 2005; Ruttan 2001; Bauer 1995; Barben 2007). So entstehen durch die Einführung neuer Technologien öffentliche Debatten, die häufig Rückwirkungen auf die Technologieentwicklung haben. Zum einen hat die öffentliche Akzeptanz eine direkte Bedeutung für die Nachfrage nach Produkten, die mit der neuen Technologie hergestellt wurden, aber auch für die Angebotsseite, z.B. für die Attraktivität für Nachwuchsforscher sich auf diesem Gebiet zu betätigen (Allansdottir 2002). Zum anderen bildet sie einen Einflussfaktor für die Politik. Die Biotechnologie ruft durch ihre direkte Bedeutung für das menschliche Leben und die Umwelt, durch die Manipulation von Organismen, große Kontroversen hervor (Russell 1999). "New biotechnology is distinguished from other major technologies of the $20^{\text {th }}$ century by the fact that its impacts on the quality of life, its human and social consequences, are arriving earlier and may go deeper than macro-economic impacts measured by productivity, investment or GDP growth." (OECD 1989, S.9). Die Unsicherheit über mögliche unbeabsichtigte Wirkungen der Biotechnologie ist dabei groß. Besonders Ängste über unerwünschte Langzeiteffekte auf Menschen, Pflanzen und Tiere im Ökosystem rufen öffentlichen Widerstand hervor (Freeman 2003, S.10). Zwar ist die öffentliche Akzeptanz nicht durchwegs negativ. Insbesondere bei

${ }^{86}$ Allerdings zweifelt Pisano (2006b) an, ob die verschiedenen Akteure der Biotechnologie genug Anreize haben, eine aus technologischer Sicht notwendige Organisationsstruktur zu etablieren, oder ob Partialinteressen diese Entwicklung dauerhaft beeinträchtigen. 
Anwendungen, bei denen sich ein medizinischer Nutzen oder eine Verringerung von Umweltbelastungen zeigt, ist sie sogar ein förderlicher Faktor (Allansdottir 2002). Insgesamt hat die geringe öffentliche Akzeptanz die Entwicklung der Biotechnologie jedoch deutlich beeinträchtigt. "There is little doubt that public protests, working through political and extra-legal channels, have slowed - for better or for worse - the evolution of the biotechnological GPT." (Lipsey et al. 2005, S.214).

\section{Politik}

Die Politik nimmt insgesamt eine vielfältige Rolle in der Entwicklung der Biotechnologie ein (Russell 1999; Arantes-Oliviera 2007; Barben 2007; Ruttan 2001). Sie stellt ein wichtiger Förderer der F\&E in der Biotechnologie, aber auch ihrer Kommerzialisierung dar. Ihre finanzielle Unterstützung (Förderprogramme, Steuererleichterungen etc.) ist aufgrund des hohen Finanzierungsaufwandes über eine längere Zeitperiode in der Biotechnologie für die Unternehmen besonders wichtig (Fier/Heneric 2005, S.7). Zudem sind öffentliche Forschungseinrichtungen und Universitäten sehr wichtige Akteure in der wissenschaftlichen Forschung der Biotechnologie (Ruttan 2002). Daneben beeinflusst die Politik zentrale Rahmenbedingungen, deren Wirkungen für die Entwicklung der Biotechnologie vielfältig und schwer bestimmbar sind. Deutlich wird dies beim Einfluss auf die Möglichkeiten der Akteure, aus den eigenen Innovationen selbst zu profitieren (Heller/Eisenberg 1998). Die Möglichkeit der Patentierung bildet in diesem Bereich einen Hauptanreiz für die riskante und unabwägbare Forschung. Eine Einschränkung der Patentierungsmöglichkeit würde für viele Akteure den Anreiz nehmen, sich in diesem Bereich in der Forschung zu betätigen. Allerdings kann die Gewährung „breiter“ Patentrechte einen negativen Effekt auf die Rate des technischen Fortschritts haben, wenn sie die Erforschung alternativer Anwendungen einer patentierten Erfindung verhindert (Dosi et al. 2003). Dies ist besonders der Fall, wenn fundamentale Techniken und Wissen betroffen sind, wie z.B. bei Genen oder dem Patent für ein genetisch verändertes Tier. Wenn wissenschaftliche Fortschritte sukzessive auf der Originalerfindung aufbauen, kann die Gewährung breiter Eigentumsrechte hinderlich für die weitere Entwicklung sein.

Darüber hinaus gehören viele der bisher wichtigsten Anwendungsfelder der Biotechnologie (z.B. Gesundheit, Landwirtschaft, Nahrungsmittelproduktion, Umwelt) zu den am stärksten regulierten Sektoren in einer Volkswirtschaft. Preise, Angebot und Nachfrage sowie Sicherheits- und Zulassungsvorschriften werden entscheidend durch staatliche Regelungen beeinflusst. Aufgrund der besonderen Eigenschaften sind zudem häufig spezifische Regelungen für die Biotechnologie notwendig (z.B. Gentechnikgesetz, Stammzellengesetz). Auch hier können erhebliche Zusatzkosten, z.B. zusätzlicher Forschungsaufwand zur Fest- 
stellung der Sicherheit der Produkte und Zeitverzögerungen bei Rechtsunklarheiten entstehen.

\subsubsection{Fazit}

Wenn man das evolutorisch geprägte GPT-Konzept von Lipsey et al. (1998/2005) akzeptiert, lassen sich für die Biotechnologie einige Schlussfolgerungen ziehen. Die geschilderten Herausforderungen und Probleme stehen einer schnellen Diffusion der Biotechnologie und der Realisierung ihrer Potentiale entgegen. Allerdings zeigen historische Beispiele von GPTs, dass ,revolutionäre" Technologien oft eine große Zeitspanne benötigen, bis sich komplementäre technologische und organisationale Entwicklungen vollziehen und die Technologie sich durchsetzt. Dabei können hohe Anpassungskosten entstehen. "These complementary technical and organisational innovations in surrounding infrastructure can then take decades to be generated which means that new technologies are often subject to large and increasing development costs" (von Tunzelmann 1995, S.5).

Diese Überwindung der Anpassungsprobleme ist aber keinesfalls zwingend und einige Skeptiker bezweifeln eine solche für die Biotechnologie. So können die existierenden Hemmnisse zu einer negativen Dynamik des Innovations- und Diffusionsprozesses führen und den Entwicklungspfad der Biotechnologie beeinträchtigen. Fehlende erkennbare Erfolge und Nutzen der neuen Technologie führen zu einer geringen Akzeptanz und Förderung, welche die Anstrengungen $\mathrm{zu}$ Verbesserungen einer Technologie verringern. So vermutet Freeman: "The failure to achieve major cost breakthroughs in industry, the public fears and opposition, and the still relatively narrow range of applications all interact to make biotechnology an unlikely candidate for the role of a general purpose technology capable of lifting the entire world economy onto a new plane of high growth" (Freeman 2003, S.11).

Trotz dieser noch offenen Entwicklung ergeben sich aus dem Konzept der GPTs interessante Folgerungen für die Wirkung der Biotechnologie. Im Rahmen dieser Arbeit sind besonders Unterschiede bei den Auswirkungen von Technologien von Interesse. Ein wichtiger Grund für mögliche Unterschiede ist das Verhältnis der neuen zur existierenden Technologie. Gerade hier zeigen sich bei vielen Anwendungsgebieten alternative Technologien, die sich teilweise noch selbst in der Weiterentwicklung befinden. Der relative Vorteil der Biotechnologie wäre demnach eher geringer. Zudem zeigt sich die Bedeutung der Biotechnologie nach Lipsey et al. (2005) nicht zwingend in höheren Wachstumsraten der Produktivität, sondern vielmehr durch neue Impulse zur Aufrechterhaltung des Wachstumsprozesses. Allein z.B. der Ausgleich der abnehmenden Möglichkeiten durch konventionelle Technologien neue Arzneimittel für bisher kaum 
behandelbare Krankheiten zu entwickeln, wäre demnach als sehr bedeutender wirtschaftlicher Beitrag der Biotechnologie zu werten.

\subsection{Wirkungsmechanismen für Produktions- und Beschäftigungswirkun- gen der Biotechnologie}

Während diese GPT-Diskussion wichtige Aspekte über die mögliche langfristige Entwicklung der Biotechnologie und deren zentralen Einflussfaktoren verdeutlicht, ist eine konkretere Betrachtung einzelner Wirkungszusammenhänge für Abschätzungen zu induzierten Produktions- und Beschäftigungsänderungen nötig. In Kapitel 2.3 wurden wichtige Wirkungszusammenhänge der Produktionsund Beschäftigungseffekte neuer Technologien dargestellt und mögliche Unterschiede zwischen Technologien hervorgehoben. Im Folgenden sollen diese Aspekte kurz für die Biotechnologie diskutiert werden, um die Wirkungszusammenhänge zu konkretisieren, die für eine empirische Untersuchung der Biotechnologie im Fokus stehen sollten. Allerdings wird, wie bereits erläutert, die Biotechnologie mit unterschiedlichen Anwendungszwecken in sehr unterschiedlichen Sektoren eingesetzt. So unterscheiden sich die wichtigen Anwendersektoren (Pharma, Chemie, Landwirtschaft, Gesundheit, Textil etc.) zum Teil erheblich in der Rolle von Innovationen (z.B eigene Herstellung vs. Fremdbezug, Produkt vs. Prozessinnovationen), Nachfrage (z.B. Preiselastizitäten, Sättigungsgrenzen), Standortfaktoren, Marktstrukturen und institutionellen Rahmenbedingungen (Dewick et al. 2006; Nusser et al. 2007a). ${ }^{87}$ Die Auswirkungen der Biotechnologie können sich demnach in einzelnen Anwendungsfeldern erheblich unterscheiden. Deshalb empfiehlt sich die Untersuchung der direkten Technologiewirkungen eher auf der Meso- als auf der Makroebene. Eine Erörterung der Wirkungsmechanismen für die Biotechnologie in Summe trägt dennoch zur Charakterisierung der Biotechnologie bei, indem Gemeinsamkeiten und Unterschiede zwischen Anwendungsfeldern verdeutlicht werden. Dabei werden im Folgenden die in den Kapiteln 2.3.1 bis 2.3.5 erläuterten Wirkungszusammenhänge für die Biotechnologie diskutiert.

\section{Produktivitätswirkung von Prozessinnovationen}

Die unmittelbare Freisetzung von Arbeitern durch den Einsatz der Biotechnologie scheint von einer eher geringen Bedeutung zu sein. Die ökonomische Vorteilhaftigkeit ergibt sich nur begrenzt aus der direkten Einsparung von Arbeitskräften, vielmehr stehen je nach Sektor andere (ökonomische) Vorteile (z.B. Einsparung von Rohstoffen, neue Produkte) im Vordergrund (Nusser et al. 2007a). Allgemein drücken sich die Vorteile der Biotechnologie nur in wenigen

${ }^{87}$ Dewick et al. $(2004,2006)$ unterscheiden in ihrer Untersuchung zur Auswirkung der Biotechnologie deshalb explizit die Sektoren nach technologischen Charakteristika, aufbauend auf der bekannten sektoralen Taxonomie von Pavitt (1984). 
Anwendungsfeldern in einer höheren Produktivität aus, am ehesten trifft dies für einige Anwendungen in der Landwirtschaft und verschiedenen industriellen Sektoren (z.B. Chemie, Papier, Textil) zu (Das 2007, IPTS 2002b).

\section{Produktinnovationen}

Bei der Betrachtung von Produktinnovationen wird die schwierige Abgrenzung der Art von Innovationen deutlich. Einige Innovationen sind recht deutlich als Prozessinnovationen (z.B. Bioethanol statt fossiler Kraftstoffe) zu identifizieren, das Produkt bleibt identisch oder sehr ähnlich. Häufig führen aber biotechnologische Verfahren zu veränderten Funktionalitäten, die in einigen Fällen einen deutlichen Zusatznutzen erbringen, in anderen Fällen vom Anwender gar nicht als vorteilhaft angesehen werden. Beispielsweise ist die Eigenschaft der Bioabbaubarkeit in einigen Produkten (z.B. Verpackungen) erwünscht, bei anderen Produkten (z.B. Automobil-Interieur) aber nachteilhaft. Darüber hinaus gibt es biotechnologische Produkte, die auf anderem Wege gar nicht herstellbar sind (z.B. viele Biopharmazeutika). Für die wirtschaftliche Auswirkungen ist dagegen von hoher Bedeutung, ob es sich um eine additive oder substitutive Produktinnovation handelt. Dies gilt sowohl aus Sicht der Endnachfrage als auch aus sektoraler Sicht (Kapitel 2.3.2). Diese Problematik lässt sich aber aus einer Perspektive für die gesamte Biotechnologie kaum beantworten. In den Kapiteln 6.3 und 6.4 wird diese Thematik für die Anwendungsfelder Fein-/Spezialchemikalien und Biopharmazeutika näher diskutiert.

\section{Kaufkraftkompensation}

Das Kaufkraftkompensationsargument kann nur dann eintreten, wenn Kosteneinsparungen durch die neue Technologie erzielt werden können. Analog zum Freisetzungseffekt ist dieses Ziel bei der Biotechnologie nicht so sehr im Fokus, wie es bei anderen Technologien der Fall ist. Während Kostensenkungen in einigen Anwendungsfeldern sicherlich ein Ziel des Biotechnologieeinsatzes darstellt (z.B. bestimmte Chemikalien), sind in anderen Feldern eher Kostenerhöhungen (z.B. Bioethanol, Biopharmazeutika) zu erwarten (siehe Kapitel 6). Es entstehen durch die Biotechnologie demnach gegenläufige Effekte auf die Kaufkraft, die auf unterschiedlichem Wege (z.B. Substitutionseffekt, Einkommenseffekt) die Gesamtwirtschaft beeinflussen. Bspw. werden zusätzliche Produktionskosten bei Bioethanol über staatliche Regelungsquoten (Biokraftstoffquote) zunächst auf den Produzenten überwälzt. Die höheren Kosten werden vermutlich zu einem Großteil in höheren Preisen weitergegeben. Da die Preiselastizität für Kraftstoffe eher gering ist, wird vor allem der Einkommenseffekt bei den Haushalten zum Tragen kommen. 


\section{Internationale Wettbewerbsfähigkeit und Außenhandel}

Durch die Diffusion der Biotechnologie können sich auf verschiedenen Stufen der Wertschöpfungskette Veränderungen im Außenhandel ergeben. Die Außenhandelsbilanz kann sich infolge

- der preislichen Wettbewerbsfähigkeit der Biotechnologie anwendenden Industrien aufgrund besserer Prozesse (z. B. geringerer Energie- und Ressourcenverbrauch),

- qualitativen Wettbewerbsfähigkeit der Biotechnologie anwendenden Industrien bei biotechnologischen Produktinnovationen und

- der qualitativen Wettbewerbsfähigkeit der Hersteller von biotechnologischen Prozessen und Produkten

verändern. Die Bedeutung des internationalen Wettbewerbs ist in der Biotechnologie sehr hoch. Die bedeutenden Anwenderindustrien Chemie und Pharma gehören in Deutschland zu den am stärksten mit dem Ausland verflochtenen Sektoren (Statistisches Bundesamt 2008c). Zudem bemüht sich eine Vielzahl von Ländern jeweils eine inländische „Biotechnologieindustrie“ $\mathrm{zu}$ etablieren und die Diffusion der Biotechnologie in Prozesse und Produkte zu fördern (Reiss et al. 2003).

\section{Maschinenherstellung und sektoraler Strukturwandel}

Das Maschinenherstellungsargument stellt sich bei der Biotechnologie unterschiedlich zu den IKT dar. Die IKT können, allerdings mit zunehmender Einschränkung, als Investitionsgut betrachtet werden (ACIL Tasman/Innovation Dynamics 2006a; OECD 2006a). Hingegen sind neue technologische Fortschritte bei der Biotechnologie weniger in produktiveren Maschinen und anderen Investitionsgütern verkörpert, als sie in veränderten Prozessen, Vorgehensweisen oder in veränderten Inputs (z.B. neues/verbessertes Enzym) für darauf aufbauende Prozesse auftreten. Die Biotechnologie unterscheidet sich von anderen Technologien vor allem durch ihre besonders starke Basierung auf F\&E. Dennoch werden für die Anwendung der Biotechnologie häufig spezielle Anlagen benötigt, die vor allem in kapitalintensiven Industrien (z.B. Chemie) mit erheblichen Investitionen verbunden sind. Hier stellt sich die schwierige Frage, ob die Investitionen zusätzlich oder substitutiv zu Investitionen in bisherige Technologien erfolgen. Insofern verliert das klassische Maschinenherstellungsargument nicht völlig an Bedeutung, kann aber etwas abgeändert werden. Neben Investitionen in Sachkapital sind erhebliche Aufwendungen in F\&E notwendig, zudem ist häufig ein Rohstoffwechsel mit der Diffusion der Biotechnologie verbunden. Entscheidend für die Wirkung des Maschinenherstellungsarguments ist die Frage, inwiefern sich der neue Vorleistungsbedarf durch unterschiedliche Arbeitsund Importintensitäten vom bisherigen unterscheidet. 


\section{Fazit}

Zusammenfassend zeigen sich für die wirtschaftlichen Auswirkungen der Biotechnologie wenig Hinweise für eine starke Produktions-/Produktivitätsschere, zumindest die direkten Freisetzungseffekte sind gering. Dies würde für eine geringe Divergenz zwischen Produktions- und Beschäftigungseffekten der Biotechnologie sprechen. Allerdings sind auch strukturelle Effekte zu betrachten. Werden bei der Anwendung der Biotechnologie Vorleistungen mit einer höheren Arbeitsintensität benötigt (z.B. Biomasse statt Öl), entstehen positive gesamtwirtschaftliche Beschäftigungsimpulse bei möglicherweise gleichbleibender Produktion. Die Richtung der Produktions- und Beschäftigungseffekte wird zudem stark durch die Importintensität der Vorleistungen sowie Veränderungen im Außenhandel durch die internationale Wettbewerbsfähigkeit beeinflusst. Die möglichen Nettowirkungen dieser indirekten strukturellen Effekte sind argumentativ kaum zu erfassen und erfordern eine quantitative Untersuchung. Welche Methodik dafür geeignet ist, wird im nächsten Kapitel erörtert. 
Sven Wydra - 978-3-631-75392-7

Downloaded from PubFactory at 01/11/2019 05:32:25AM

via free access 


\section{Messung von wirtschaftlichen Effekten der Biotechnologie}

\section{1 Überblick zur Messung wirtschaftlicher Effekte von Technologien}

Die theoretische Analyse hat gezeigt, dass die wirtschaftlichen Auswirkungen von neuen Technologien nicht eindeutig sind. Es sind vielmehr empirische Untersuchungen notwendig, um die Gesamtwirkungen bestimmen zu können (Grupp 1997; Vivarelli 2007). Die empirische Messung steht allerdings vor großen Herausforderungen. Schließlich steht keine allgemein anerkannte Methode zur Abschätzung der wirtschaftlichen Wirkungen von Technologien zur Verfügung (Kalmbach/Kurz 1992, S.11). Die Hauptschwierigkeit jeglicher Analyse besteht in der Berücksichtigung der Vielzahl von gleichzeitig wirkenden Einflussfaktoren auf die Produktion und Beschäftigung. Es besteht keine Möglichkeit, den Einfluss von technologischen Veränderungen auf die Beschäftigung direkt - losgelöst von den Veränderungen bei anderen Marktfaktoren - zu messen (Kalmbach 1989, S.14; Melzig-Thiel 2000, S.120). Die bisherigen empirischen Studien versuchen diese Zusammenhänge auf sehr unterschiedlichen Aggregationsebenen (Mikro, Meso, Makro) zu erfassen und messen damit gleichzeitig verschiedene Sachverhalte:

Die mikroökonomische Ebene eignet sich besonders für die Analyse der direkten, kurzfristigen Beschäftigungswirkungen, da auf Unternehmensebene neue Technologien eingeführt und eingesetzt werden (Pianta 2005, S.7; RWI 2005, S.51). Allerdings werden indirekte Effekte, die nicht in diesen Unternehmen auftreten, in solchen Analysen nicht berücksichtigt. Dies kann zu erheblichen Fehlschlüssen bei Verallgemeinerung der Ergebnisse auf der Makroebene führen (Matzner 1990, S.23; Kalmbach et al 1989, S.15). Einerseits kann z.B. eine hohe Freisetzung von Arbeitskräften darin begründet sein, dass sich der Wertschöpfungsanteil des betrachten Unternehmens durch die Einführung der neuen Technologie reduziert hat. Jedoch steigt damit der Vorleistungsanteil und erhöht die Beschäftigung in anderen Unternehmen. Andererseits ist es wahrscheinlich, dass Erfolge von Unternehmen durch den Einsatz neuer Technologien auf Kosten anderer Unternehmen, die auf den Einsatz bisher verzichten, gehen. Die negativen Effekte bei diesen Unternehmen werden aber auf der Mikroebene häufig nicht betrachtet, der Nettoeffekt wird in diesem Falle überschätzt. Folglich ist die Betrachtung einzelner Unternehmen nur sehr bedingt geeignet, um die Wirkung der Biotechnologie zu untersuchen.

Zur Ermittlung der Nettoeffekte ist stattdessen eine Berücksichtigung der indirekten Effekte sowie längerfristig wirkender Anpassungsprozesse auf der Makroebene nötig (RWI 2005, S.42). Die Probleme auf der Makroebene liegen allerdings in der Schwierigkeit, die sich teilweise überlagernden Effekte zu erfassen. Häufig ist es dabei kaum mehr möglich, den konkreten Bezug der Er-

gebnisse zur Technologie herzustellen und zu veranschaulichen (Fröhlich et al. 1992, S.193). Annahmen über gesamtwirtschaftliche Prozesse dominieren häu- 
fig die spezifischen Wirkungen von Technologien (IPTS 2002a, S.49). Deshalb heben einige Autoren die Vorzüge der Mesoebene hervor (z.B. Pianta 2005). Auf dieser Ebene lassen sich sektorale Unterschiede und zumindest ein Teil der indirekten Effekte berücksichtigen. Dies gilt insbesondere für die sektorale Nachfrage, die ein wichtiger Faktor für die Auswirkungen von technologischem Fortschritt ist (Pianta 2005). Die kompensatorischen Wirkungen von Preis- und Mengeneffekten in anderen Sektoren, technologische Spillovereffekte oder Nachfrageveränderungen durch Einkommenseffekte etc. können auf der sektoralen Ebene jedoch nicht betrachtet werden (Cyert/Mowery 1988, S.94).

Aufgrund der unterschiedlichen verwendeten Methoden auf den einzelnen Aggregationsebenen ist es schwierig, die Ergebnisse zueinander in ein Verhältnis zu setzen, noch lässt sich eine Analyseebene grundsätzlich der anderen vorziehen. Vielmehr ist zu berücksichtigen, dass die einzelnen Konzeptionen unterschiedliche Sachverhalte messen. Letzteres wird nach Kalmbach häufig nicht explizit genug hinterfragt. „Thus, it is frequently not different answers to the same problem that are the cause of dispute, but the fact that researchers attempt to answer quite different questions - a circumstance they do not always realise “88 (Kalmbach 1992, S.70). Ansätze, die ausschließlich die Unternehmensoder sektorale Ebene betrachten, können zwar wichtige Erkenntnisse für die ökonomischen Auswirkungen neuer Technologien liefern, aber eignen sich allein nur bedingt für Aussagen zu gesamtwirtschaftlichen Effekten.

Deshalb kommt im Rahmen dieser Arbeit ein Modell in Betracht, welches gesamtwirtschaftliche Effekte berücksichtigt. Diese gesamtwirtschaftlichen Modelle können - zu unterschiedlichem Grade - indirekte Effekte durch Rückwirkungen mit anderen Marktfaktoren erfassen. (ACIL Tasman/ Innovation Dynamics 2006a, S.36) Da allerdings technologische und sektorale Besonderheiten für die Wirkung der Biotechnologie einen wichtigen Einfluss auf die Ergebnisse haben und auch im Mittelpunkt dieser Arbeit stehen, ist ein möglichst hoher konkreter Technikbezug zur Biotechnologie sehr wichtig. Dies muss in der konkreten Modellwahl berücksichtigt werden. Deshalb wird zunächst die Datenlage und Aussagekraft existierender Indikatoren für biotechnologische Aktivitäten erörtert.

\subsection{Indikatoren für die wirtschaftliche Bedeutung der Biotechnologie}

\subsubsection{Relevanz der Technologiecharakteristika für die Indikatorenbildung}

Die Bemühungen, geeignete und international vergleichbare Indikatoren für biotechnologische Aktivitäten und ihrer Auswirkungen zu entwickeln, haben in

\footnotetext{
88 Ähnliches stellen Brusoni et al. (2006) bei einer Übersicht über Studien zu wirtschaftlichen Erträgen und Effekten durch Innovationen fest.
} 
den vergangenen Jahren zugenommen. ${ }^{89}$ Die OECD hat ein Rahmenwerk entworfen, mit dem Ziel, Leitlinien für die Erhebung von informationsgerechten und international vergleichbaren offiziellen Daten aufzustellen (OECD 2005a). Denn technikspezifische Indikatoren bilden eine wichtige Grundlage für die Bewertung des biotechnologischen Innovationssystems und einen Anhaltspunkt, ob forschungspolitische Ziele erreicht werden können (Arundel 2003; Carlsson et al. 2002). Kausale Zusammenhänge zwischen Faktoren sowie indirekte Effek$\mathrm{te}^{90}$ lassen sich allerdings kaum über Indikatoren abbilden, so dass sie für sich alleine genommen die gesamtwirtschaftlichen Effekte der Biotechnologie nicht erfassen können.

Die Eignung von bestimmten Indikatoren zur Messung von technologischen Aktivitäten und Auswirkungen kann je nach Eigenschaften der untersuchten Technologie variieren (Patel/Pavitt 1995, S.20). Kennziffern, die für einige Technologien geeignet sind, können bei anderen zu systematischen Über- oder Unterschätzungen führen. So eignen sich bspw. Indikatoren für F\&E-Aktivitäten in stark wissensbasierten Technologien (z.B. Chemie, Biotechnologie) deutlich besser als in produktions- und informationsbasierten Technologien (Mechanik, Software etc.) für die Messung des Innovationsprozesses. Schließlich nehmen formale Forschungsaktivitäten in stark wissensbasierten Technologien eine wichtigere Rolle für die Generierung neuer Innovationen ein (Patel/Pavitt 1995, S.21). Ebenso sind je nach Wirkungsmechanismen der Technologien unterschiedliche Indikatoren für die Messung möglicher Effekte von hoher Bedeutung (Produktivitätseffekte, Veränderung von Wertschöpfungsketten etc.). Ana$\log$ zur Theorie werden auch bei der Messung häufig Parallelen zwischen Biotechnologie und IKT vor dem Hintergrund diskutiert, inwiefern Messkonzepte für die IKT auf die Biotechnologie übertragen werden können (Arundel 2003; ACIL Tasman/Innovation Dynamics 2006a). In der Biotechnologie und den IKT wird jeweils ein bedeutender Teil der Effekte in den Anwenderbranchen erwartet, die nicht rein über eine Analyse des technologieproduzierenden Sektors selbst abgedeckt werden können. ${ }^{91}$ Beide Technologien umfassen dabei ein weites Spektrum an Techniken und Aktivitäten, die sich in ihrer Neuigkeit, Innovativität und möglichen Wachstumsimpulsen unterscheiden können (Brink et al. 2004, S.31). Folglich ist für beide Technologien eine Unterscheidung in

${ }^{89}$ Für die Diskussion von Indikatoren für die Biotechnologie, siehe z.B. Arundel (2000, 2003), Brink et al. (2004), OECD (2005a), Reiss et al. (2005), Rose/McNiven (2007).

90 Z.B. kann ein verstärkter Einsatz von biotechnologischen Methoden zu einer Erhöhung der Nachfrage von Biomasse führen, mögliche Veränderungen in Preis und Menge bspw. in der Landwirtschaft können aber nicht durch Indikatorenbetrachtung kausal der Biotechnologie zugeschrieben werden.

${ }^{91}$ Die OECD stellt hierzu genauer fest: „As with ICT, most of the impacts of biotechnology will be due to its diffusion and adoption by 'user' firms and from social and environmental effects that are difficult to measure in economic terms" (OECD 2006a, S.3). 
ihre Anwendungsfelder, im besten Falle auch nach konkreten Techniken, wichtig.

Neben diesen Parallelen zwischen den beiden Technologien existieren aber einige Faktoren, die eine Übertragbarkeit der Messkonzepte und die Messung der Biotechnologie allgemein, deutlich gegenüber IKT erschweren. Dabei werden meist folgende Gründe genannt (OECD 2004, 2006a; ACIL Tasman/Innovation Dynamics 2006a):

- Die explizite Separierung eines technologieproduzierenden Sektors ist schwieriger als bei den IKT.

- Die fehlende Möglichkeit, die Biotechnologie als „Prozesstechnologie“ physisch anhand von Investitionsströmen zu erfassen, erschwert die Messung und die Eignung von methodischen Konzeptionen bei denen Investitionen ein wichtiger Bestandteil bildet. ${ }^{92}$ Schließlich sind Investitionen i. d. R. kompatibel zu anderen statistischen Erhebungen (z.B. Volkswirtschaftliche Gesamtrechnung).

- Die allgemeine Schwierigkeit, die biotechnologisch hergestellten Güter zu erkennen und statistisch zu erfassen.

Diese Schwierigkeiten bei der Messung biotechnologischer Aktivitäten werden zusammen mit der aktuellen Datenverfügbarkeit zur wirtschaftlichen Bedeutung der Biotechnologie, im Folgenden näher erörtert. Dabei dient Abbildung 9 als vereinfachter Ausgangspunkt der Betrachtung, quasi als Idealvorstellung zur Abgrenzung von Akteuren und Indikatoren. Der Schwerpunkt der Betrachtung liegt dabei auf der Erörterung der Verfügbarkeit und der Aussagekraft von Indikatoren für die Anwendung der Biotechnologie (oberste Ebene), da diese Indikatoren eine wichtige Grundlage für eine Modellierung der wirtschaftlichen Effekte bilden.

Zunächst wird die statistische Erfassung der Hauptakteure (institutionelle Erfassung) diskutiert. ${ }^{93}$ Diese bilden eine zentrale Grundlage für die Erfassung von Indikatoren für die Biotechnologie. Im Anschluss werden statistische Indikatoren für die Biotechnologie erörtert. Dabei werden besonders die Kompatibilität und Möglichkeiten der Integration in die amtliche Statistik diskutiert. Diese wäre bedeutend für eine gute räumliche und zeitliche Vergleichbarkeit sowie wei-

92 So fällt die Anwendung des neoklassischen „Growth-Accounting“ Ansatzes, der für die gesamtwirtschaftliche Bedeutung der IKT von hoher Bedeutung ist, für die Analyse der Biotechnologie weg. (ACIL Tasman/ Innovation Dynamics 2006a, S.58).

${ }^{93}$ Bei der institutionellen Erfassung werden Unternehmen, die schwerpunktmäßig ähnliche Produkte erzeugen, zu Wirtschaftszweigen zusammengefasst. Die funktionelle Erfassung betrachtet ähnliche Produkte in Produktklassen (für Definitionen siehe Greulich 2001). In der amtlichen Statistik wird dabei versucht die institutionelle und funktionelle Erfassung aufeinander abzustimmen, wobei einige Probleme und Zurechnungsfragen bestehen (Greulich 2001). Diese zusätzliche Problematik wird im Folgenden aber nicht weiter betrachtet. 
tergehende Analysen durch Verknüpfung zu bestehenden Datenbeständen (z.B. Produktivitätskennziffern, Input-Output-Tabellen). Dabei steht zunächst die Erfassung von biotechnologisch hergestellten Produkten und Dienstleistungen (funktionale Erfassung) im Vordergrund. Danach werden weitere Indikatoren diskutiert, die zur Messung des technologischen Wandels durch die Biotechnologie und Diffusion in der Anwendung dienen. Abschließend wird auf Indikatoren und Untersuchungen eingegangen, die Hinweise zu wirtschaftlichen Auswirkungen der Diffusion der Biotechnologie geben.

\section{Abbildung 9: Rahmen zur Erfassung der Akteure und Indikatoren für die Biotechnologie}

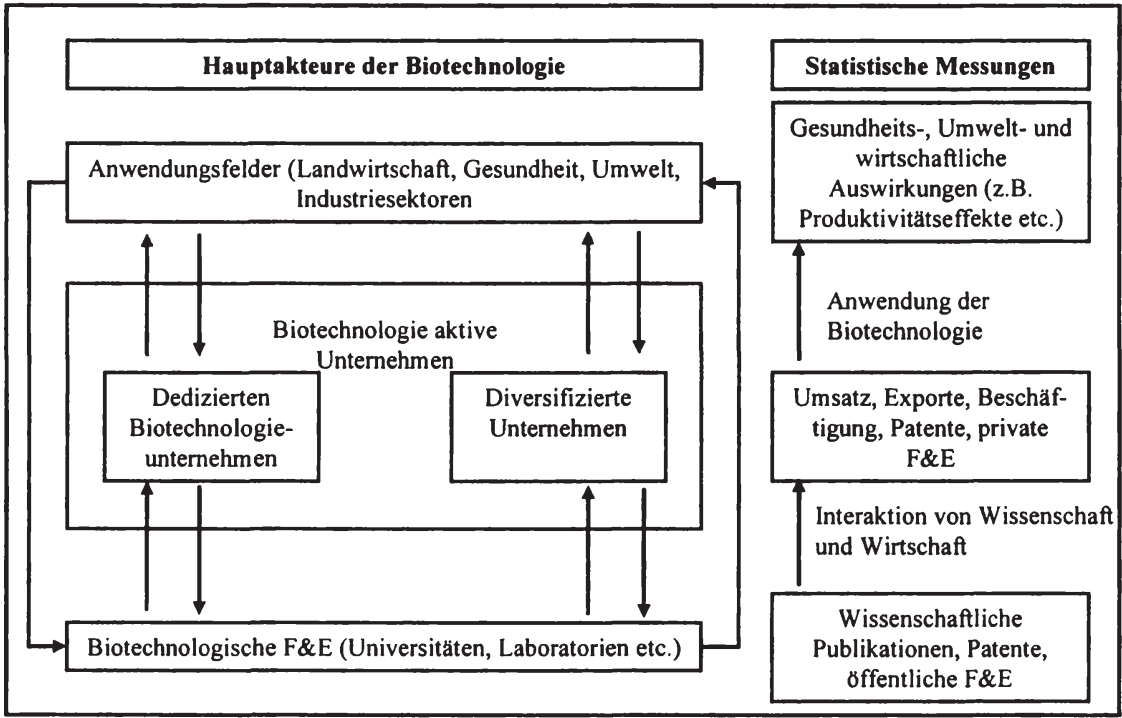

Quelle: Eigene Darstellung in Anlehnung an Lim/Choi (2006)

\subsubsection{Messung des "Biotechnologiesektors"}

\subsubsection{Erfassung der Biotechnologieunternehmen}

„The statistical challenge is to identify firms within these sectors that are wholly reliant on biotechnology or which include biotechnology among other activities" (Arundel et al. 2006, S.4). Besonders schwierig ist die Identifikation der Unternehmen, die nicht nur die Biotechnologie, sondern gleichzeitig auch andere Technologien (z.B. Chemie) erforschen und nutzen (Arundel 2000, S.89). Zwar gibt es in vielen Ländern private und öffentliche Initiativen, die versuchen, biotechnologieaktive Unternehmen zu identifizieren und relevante Merkmale (Mit- 
arbeiterzahl, F\&E-Ausgaben etc.) zu erheben (z.B. OECD 2006b). Bei den Untersuchungen entstehen aber verschiedene Abgrenzungs- und Erhebungsfragen, die deren Vergleichbarkeit erschwert. Sie unterscheiden sich insbesondere in der Definition von Biotechnologie und in der Zurechnungsfrage, wann ein Unternehmen als aktiv in der Biotechnologie gilt (Rose 2000). In den letzten Jahrzehnten wurde eine große Vielzahl von Definitionen verwendet (Brink et al. 2004). Erst in jüngerer Vergangenheit setzt sich die in Kapitel 3.1.1 beschriebene Definition der OECD mehr und mehr durch (OECD 2006b).

Für die Frage, wann ein Unternehmen aktiv in der Biotechnologie ist, schlägt die OECD (2005a) als Lösung dabei folgende Klassifizierung vor: Unternehmen, welche mindestens eine dieser Biotechniken in der listenbasierten Definition (siehe Tabelle 1 in Kapitel 3.1.3) zur Produktion von Gütern, Bereitstellung von Dienstleistungen und/oder für die Forschung und Entwicklung nutzen, gelten als biotechnologieaktive Unternehmen. Diese Klassifizierung bedeutet, dass einige interessante Akteure nicht erfasst werden:

- Es werden Unternehmen nicht berücksichtigt, die biotechnologisch hergestellte Zwischenprodukte verwenden, aber nicht selbst biotechnologische Prozesse einsetzen oder erforschen. Beispiele hierfür sind Anwender von biotechnologisch hergestellten Enzymen in der Leder- oder Textilbranche, die aber selbst keine eigenen biotechnologischen Verfahren anwenden. Allerdings können wirtschaftliche Effekte gerade hier auftreten, beispielsweise durch eine kostengünstigere Produktion mithilfe des Enzymeinsatzes. Die Erfassung dieser Unternehmen wäre zur Analyse der ökonomischen Effekte der Biotechnologie wichtig, bisher erscheinen aber die Schwierigkeiten der Abgrenzung und Erfassungsmöglichkeit dieser Unternehmen und relevanter Indikatoren unüberwindlich. Schließlich sind biotechnologische Produkte für den Nutzer häufig gar nicht als solche erkennbar (ACIL Tasman/Innovation Dynamics 2006b, S.49).

- Es werden keine Anbieter von Investitionsgütern für die Biotechnologie erfasst. Wirtschaftliche Effekte zunehmender Investitionen in die Biotechnologie können daher unzureichend untersucht werden.

- Ebenfalls unberücksichtigt bleiben öffentliche Akteure. Diese spielen aber vor allem in der Forschung und Entwicklung eine wichtige Rolle (siehe Kapitel 3.2.2.4).

Die biotechnologieaktiven Unternehmen werden konkreter zwischen Kernbiotechnologieunternehmen, deren vorrangige Tätigkeit in diesen genannten biotechnologischen Aktivitäten liegt, und diversifizierten Unternehmen unterschieden (OECD 2005a, S.7). Bei letzteren liegt die Tätigkeit nicht schwerpunktmäßig in der Biotechnologie, sie wenden aber biotechnologische Verfahren zum Zwecke der Eingliederung neuartiger bzw. wesentlich verbesserter Produkte oder Herstellungsprozesse an. Beispiele hierfür sind große Pharma-, Che- 
mie- oder Saatgutunternehmen. Bei den bisherigen statistischen Erhebungen sind deutlich mehr Informationen über die Kernbiotechnologieunternehmen verfügbar. In den meisten Studien werden sie sogar ausschließlich betrachtet (z.B. Ernst\&Young 2008; Kriegesmann et al. 2005). Die Gründe hierfür liegen in den Schwierigkeiten die diversifizierten Unternehmen zu erfassen und der schwierigen Abgrenzung der Biotechnologie innerhalb dieser Unternehmen von anderen Aktivitäten. (Brink et al. 2004) Umfragen zeigen, dass diese Unternehmen viele relevante Daten (Umsatz, F\&E-Aufwand etc.) nicht eindeutig ihren Aktivitäten in der Biotechnologie zuordnen können (Reiss et al. 2006; Rose/McNiven 2007). So werden z.B. für Deutschland aktuell für die diversifizierten Unternehmen als einzige Merkmale die Anzahl der Unternehmen und die Mitarbeiteranzahl erhoben (biotechnologie.de 2008). Für die Kernbiotechnologieunternehmen sind darüber hinaus die F\&E-Ausgaben, Umsätze, Größenstrukturen etc. verfügbar (OECD 2006b). Wichtige volkswirtschaftliche Kennzahlen wie die Bruttowertschöpfung, die Investitionen und Daten zum Außenhandel fehlen aber in vielen Ländern (u.a. Deutschland) auch hier.

Neben diesen Erhebungsschwierigkeiten wird eine Integration der biotechnologieaktiven Unternehmen in die amtliche Statistik durch den Querschnittscharakter der Biotechnologie erschwert. Die Unternehmen sind aufgrund der oben beschriebenen Anwendungsbreite der Biotechnologie in vielen verschiedenen existierenden Sektoren zu finden und kaum zu isolieren (Rammer et al. 2006). „Thus biotechnology cannot be considered as an industrial sector but rather as set of technologies based upon advances in the life sciences, and this makes the task of constructing a NACE based definition almost impossible" ${ }^{94}$ (Arundel et al. 2006, S.5).

Darüber hinaus ist eine Unterscheidung der Unternehmen nach Biotechnologieaktivitäten -z.B. zwischen technologieproduzierenden und technologieanwendenden Unternehmen - schwierig. Die OECD unterscheidet bei biotechnologischen Aktivitäten zwischen Grundlagen- und angewandter Forschung, Kommerzialisierung (z.B. Lizenzierung), Nutzung für Produkt oder Prozessentwicklung, Nutzung für die Produktion von Gütern und Dienstleistungen. Dabei entsteht die Problematik, dass große Unternehmen häufig sowohl in der Anwendung als auch in der Forschung verbesserter Methoden aktiv sind. Die obere und zweite Ebene der Abbildung 9 lassen sich in diesem Fall nicht voneinander unterscheiden.

\subsubsection{Erfassung der Biotechnologie in der Produktionsstatistik}

Die Erfassung der Biotechnologie in der Produktionsstatistik könnte Aufschluss über die Aktivitäten der Bereitstellung sowie Anwendung biotechnologischer Methoden geben. Analog würde eine Erfassung in der Güterklassifikation des

${ }^{94}$ NACE steht für das europäische Wirtschaftszweigklassifikationssystem. 
Außenhandels Aussagen über die internationale Wettbewerbsfähigkeit und Arbeitsteilung ermöglichen. Bei der Nutzung von biotechnologischen Methoden in der Güterproduktion bildet diese allerdings häufig nur eine Prozessalternative (z.B. zur Herstellung von Vitaminen), aber nicht die einzige (Brink et al. 2004; Flaschel/Sell 2005). Aus offiziellen Produktionsgruppenstatistiken ist in der Regel nicht erkennbar, mit welcher Technologie die Produkte hergestellt wurden. Auch neue Produktinnovationen durch die Biotechnologie werden meist nicht separat erfasst. Hierzu wäre die Bildung neuer Produktionsgruppen in den offiziellen Statistiken notwendig, um Menge und Wert des neuen Gutes isoliert von anderen Gütern zu messen. Da dies, wenn überhaupt, mit einer deutlichen Zeitverzögerung stattfinden kann, sind sie bis dahin in bestehende Produktionsgruppenklassifikationen integriert und schwierig von anderen Produkten zu isolieren. Die Schwierigkeiten der praktischen Umsetzung werden auch hier durch den Querschnittscharakter der Biotechnologie verstärkt (Brink et al. 2004, S.31). Durch die sehr unterschiedlichen Anwendungen der Biotechnologie ist eine Vielzahl von Produktionsgütern tangiert, die bereits bestimmten Wirtschaftssektoren zugeordnet sind. Zudem nehmen die Anwendungsgebiete der Biotechnologie im Zeitablauf voraussichtlich deutlich zu (z.B. Polymere), die Abgrenzung der Biotechnologie anhand von Produktionsgruppen wird daher weiter erschwert. ${ }^{95}$ Zusammenfassend zeigt sich, dass über Produktions- und Unternehmensstatistiken direkt bisher nur unzureichend Schlüsse auf die Diffusion und deren Auswirkung gezogen werden können. Eine andere Art von Indikatoren, die wichtige Hinweise für Zukunftsprojektionen geben können, sind Innovations- und Diffusionsindikatoren. Diese werden im Folgenden diskutiert.

\subsubsection{Technologischer Fortschritt und Diffusion der Biotechnologie}

\subsubsection{Indikatoren für den technologischen Wandel}

„A major difficulty in the empirical analysis of the relationship between technology and employment is the lack of a satisfactory measure of technical change" (OECD 1996, S.137). Dabei gestaltet es sich schwierig, geeignete Output-Indikatoren zu finden, die das Ergebnis des Innovationsprozesses erfassen. In der empirischen Analyse dieser Arbeit (siehe Kapitel 5) wird versucht, über

\footnotetext{
${ }^{95}$ Um dieser Dynamik und der Humankapitalintensität der Biotechnologie Rechnung zu tragen schlagen Brink et al. (2004) eine konzeptuelle Matrix zur Analyse der Biotechnologie vor. Dabei sollen auf der einen Achse die einzelnen Felder der Wissensbasis, auf der anderen Achse die Produktionsseite durch die einzelnen Anwendersektoren erfasst werden. Dadurch können neue Anwendungsfelder der einzelnen Wissensbasis erfasst und der unterschiedliche Einfluss bestimmter Techniken oder einer Wissensbasis in einzelnen Anwendungsfeldern explizit berücksichtigt werden. Allerdings handelt es sich hierbei nicht um ein Konzept für spezielle Indikatoren, sondern eher um einen Analyserahmen, der die Vergleichbarkeit und Einordnung von Studien ermöglichen soll.
} 
einen Diffusionsindikator gekoppelt mit spezifischen Informationen für einzelne Anwendungsfelder den technologischen Wandel abzubilden. Um einen breiteren Überblick über mögliche Indikatoren zu geben, werden im Folgenden kurz mit F\&E-Ausgaben/Personal sowie Patente zwei traditionelle Innovationsindikatoren für die Biotechnologie diskutiert: ${ }^{96}$

\section{Messung der F\&E-Aktivitäten}

Mögliche Indikatoren für die Messung von F\&E-Aktivitäten sind zum Beispiel das F\&E-Personal oder die verbundenen Ausgaben. Solche F\&E-Indikatoren sind für Forschungsbereiche oder Technologien geeignet, in denen Entdeckungen und Entwicklungen in spezialisierten Forschungs- und Entwicklungsnetzwerken in öffentlichen und privaten Institutionen stattfinden. Hinzu kommt die Praktikabilität eines solchen F\&E- Indikators durch die internationale Vergleichbarkeit und in seiner vergleichsweise guten Kompatibilität zu anderen Wirtschaftszweigdaten (Sirilli 1998, S.6). Die Grenzen dieses Innovationsindikators liegen allerdings unbestritten darin, dass er nur den Input von Innovationsaktivitäten messen kann, nicht aber deren Erfolg (Smith 2001). Daneben ist der Indikator für Innovationen bei Technologien mit geringen formalen F\&EAktivitäten (z.B. Software) oder nicht-technologischen Innovationsaktivitäten (z.B. Organisationsinnovation) weniger geeignet. ${ }^{97}$

Für die Biotechnologie werden F\&E-Ausgaben/-Personal dennoch häufig als wichtiger Indikator eingestuft, da Fortschritte stark auf wissenschaftlichen Erkenntnissen der öffentlichen und privaten Forschung aufbauen (z.B. Luukonnen/ Palmberg 2007). Besonders die Kernbiotechnologieunternehmen zeichnen sich durch eine sehr hohe Forschungsintensität aus. Eine vollständige empirische Erhebung der F\&E-Aktivitäten in der Biotechnologie ist aber schwierig (ACIL Tasman/ Innovation Dynamics 2006a; Hetmeyer et al. 1995). Die Zurechnung der F\&E-Aktivitäten zu Technologien in vielen Ländern ist mit bisherigen statistischen Erhebungen nicht abgedeckt (Brink et al. 2004; Enzing et al. 2007a).

\section{Patente}

Einen stärkeren Output-Charakter wird Patenten als Innovationsindikator zugesprochen, da sie als kommerziell verwertbares Ergebnis von Innovationsprozessen entstehen.$^{98}$ Daneben existiert eine systematische, zuverlässige und sich über sehr lange Zeiträume erstreckende Datenerhebung. Mit gewissen Einschränkun-

\footnotetext{
${ }^{96}$ Ein weiterer Indikator der häufig für die Messung von biotechnologischen Aktivitäten verwendet wird, sind Publikationen (Reiss et al. 2007, Kapitel 3.1.4). Dieser Indikator wird nicht näher dargestellt, da er sich besonders für die Messung von Forschungsaktivitäten, aber weniger zur Messung des „Outputs“ der Innovationen eignet.

97 Zusätzliche Erhebungsprobleme existieren bei kleinen Unternehmen, da F\&E hier häufig nicht in statistisch abgrenzbaren Organisationseinheiten stattfindet (Patel/Pavitt 1995).

${ }^{98}$ Für eine Übersicht siehe Basberg (1987) und Grupp (1997).
} 
gen lässt sich auch die Biotechnologie abgrenzen und ihre Entwicklung nachvollziehen (Kapitel 3.1.4). ${ }^{99}$ Allerdings haben Patente nur eine eingeschränkte Aussagefähigkeit über die Höhe der wirtschaftlichen Bedeutung und den zukünftigen Erfolg von neuen Technologien (Kalmbach 1992, S.171). Der Marktwert zwischen den Patenten kann sich deutlich unterscheiden, viele Patente werden gar nicht in marktfähige Produkte umgesetzt (Sirilli 1998, S.8). Daneben unterscheiden sich Sektoren und Technologien in ihrer Kodifizierbarkeit und in der Art der Innovationen. Patente können deshalb je nach Bereich eine unterschiedliche Aussagekraft haben (Brink et al. 2004, S.35). Für die Messung des Innovationsoutputs in der Biotechnologie stellen Patente meist einen wichtigen und häufig verwendeten Indikator dar. Patente haben in der Biotechnologie eine bedeutsame Schutzfunktion vor Wettbewerbern und geben Unternehmen die Möglichkeit über den Verkauf der Patentrechte Lizenzeinnahmen zu kassieren oder mögliche zukünftige Einnahmen zu signalisieren, um Investoren für weitere Investitionen zu finden. Die Möglichkeit der Patentierung bildet somit in diesem Bereich einen Hauptanreiz für die riskante und unabwägbare Forschung (Dosi et al. 2003).

Zusammenfassend spricht für eine Verwendung dieser traditionellen Innovationsindikatoren die Basierung der Biotechnologie auf formale F\&E-Prozesse. Die Datenverfügbarkeit ist zudem vergleichsweise gut. Allerdings umfassen die Indikatoren nur einen Teil des Innovationsoutputs. Einige Dimensionen und Ergebnisse des Innovationsprozesses, wie z.B. implizites Wissen, Verbreitung in der Produktion oder Veränderung der Wertschöpfung können nicht abgebildet werden. Sie sind somit nur in der Lage bestimmte Aspekte des biotechnologischen Fortschritts zu erfassen. Durch die zunehmende Bedeutung der Biotechnologie in der Anwendung steigt allerdings der Bedarf an zusätzlichen Indikatoren. So stellt Arundel (2000) fest: „As the economic impact of biotechnology expands from research to applications, a different set of statistics are required in order to provide the type of statistics gathered for IT" (Arundel 2000, S.90).

\subsubsection{Indikatoren für die Diffusion in Anwendersektoren}

Indikatoren zur Diffusion der Biotechnologie in der Anwendung existieren bisher nur begrenzt und sind häufig anwendungsspezifisch. ${ }^{100}$ Beispiele sind der Anteil von Biopharmazeutika in der Wirkstoffzulassung, die Flächenanteile des Anbaus von Pflanzen mit gentechnisch modifizierten Organismen (GMO) oder das Volumen (z.B. in Tonnen) von eingesetzten Enzymen in der Produktion.

\footnotetext{
${ }^{99}$ Brink et al. (2004) weisen allerdings darauf hin, dass die internationale Vergleichbarkeit der Klassifikationen bei den Patentämtern eingeschränkt ist und nicht alle Bereiche der Biotechnologie erfasst werden.

${ }^{100}$ Eine ausführliche Analyse von vorhandenen und wünschenswerten Indikatoren findet sich bei Reiss et al. (2006).
} 
Insgesamt stellt ETEPS (2005) aber eine recht unterschiedliche internationale Datenverfügbarkeit und -qualität für die verschiedenen Anwendersektoren fest.

Eine Möglichkeit, die Diffusion der Biotechnologie in der Anwendung auf aggregierter und monetärer Ebene zu erfassen und zu projizieren, ist die Ermittlung der Höhe der gesamten Umsätze, die mit der Biotechnologie verbunden sind. ${ }^{101}$ Dieses Konzept der „Biotechnology-related sales“ (BRS) orientiert sich nah an der Produktionsstatistik. Da die Biotechnologie nicht direkt über die Produktionsstatistik abgrenzbar ist, wird expertengestützt der jeweilige Anteil der Biotechnologie am Umsatz- oder Produktionswert der Produktionsgruppen oder Sektoren geschätzt (OECD 1998). Die OECD (1998) unterscheidet in einer älteren Untersuchung im Jahr 1998 bspw. zwischen drei Kategorien:

- Umsatz mit neuen Produkten, deren Entwicklung direkt auf Anwendungen der neueren Biotechnologie zurückzuführen ist (z.B. genmanipuliertes Saatgut, Biopharmazeutika),

- Umsatz mit Produkten, deren Herstellung auf verbesserte Anwendungen durch den Einsatz moderner Biotechnologie basiert. Das Endprodukt selbst wird dabei nicht verändert (z.B. rekombiniertes Insulin),

- Umsatz mit Produkten, für deren Herstellung verbesserte Prozesse genutzt werden, die auf Produkten der modernen Biotechnologie basieren (u.a. Enzym-gesteuerte Prozesse) und deren Wert durch den Einsatz dieser neuen Produkte steigt.

Dabei entstehen allerdings erhebliche Abgrenzungs- und Zurechnungsfragen. ${ }^{102}$ Besonders bei der dritten genannten Kategorie ist diese Abgrenzung schwierig, inwieweit die Produktumsätze tatsächlich dem Einfluss der Biotechnologie zugerechnet werden können. Bei der zweiten Kategorie stellt sich die Frage, ob der gesamte an die Biotechnologie geknüpfte Umsatz auch tatsächlich der Biotechnologie zugeordnet werden kann. ${ }^{103}$ Schließlich bildet der Einsatz von biotechnologischen Prozessen und Produkten neben anderen Technologien nur ein Teil des Inputs zur Produktion eines Gutes (IPTS 2007). In der Regel findet aus Praktikabilitätsgründen eine volle Zurechnung statt, da der jeweilige Biotechnologieanteil an einem Produkt kaum ermittelt werden kann. Dabei wird

101 Für die Nutzung dieses Konzeptes zur Messung der Diffusion der Biotechnologie siehe OECD (1998), Deke (2004), McKinsey (2006), Menrad et al. (2003), Nusser et al. (2007a), IPTS (2007). Allerdings unterscheiden sich die Studien in der Erhebungsmethodik (z.B. Aggregationsebene, Datenquellen) und sind daher nur eingeschränkt miteinander vergleichbar.

${ }^{102}$ Ein weiterer Kritikpunkt sind mögliche Doppelzählungen. Während einige Produkte Güter in der Endnachfrage darstellen, gehen andere als Vorprodukte in Güterproduktionen ein. Dies kann insbesondere bei Aufsummierung ein mögliches Konsistenz-Problem in der Marktbeschreibung geben. Diese Aufsummierung wird allerdings von den meisten Studien aus diesem Grund vermieden (z.B. Nusser et. al. 2007a; IPTS 2007).

${ }^{103} \mathrm{Vgl}$. Kapitel 4.3.3 für eine genauere Diskussion über Zurechnungsfragen von Effekten einer Technologie. 
die Annahme getroffen, dass die Bedeutung der Biotechnologie für den Herstellungsprozess in den einzelnen Sektoren bzw. Produktbereichen annähernd gleich ist (Deke 2004, S.16). Dieses Vorgehen unterschätzt tendenziell die relative Bedeutung der Biotechnologie in der Pharmaindustrie, bei welcher der Biotechnologie häufig eine große Bedeutung für die Umsatzerzielung zugesprochen wird, während in der Lebensmittelindustrie biotechnologische Prozessschritte nur eher einen kleinen Teil des Produktionsprozesses bei großem Produktionsvolumen ausmachen (Arundel 2002; Menrad et al. 2003).

Dennoch ist dieser Indikator bisher am besten geeignet die Verbreitung der Biotechnologie über mehrere Anwendungsfelder hinweg zu messen. Er wird im empirischen Teil dieser Arbeit verwendet. Dabei kommt die genannte Kritik bezüglich der Vergleichbarkeit zwischen Anwendersektoren weniger zum Tragen, da die dritte Kategorie für den beabsichtigten Zweck nicht relevant ist und aus der Diffusionshöhe nicht allein die Bedeutung oder Effekte abgeleitet werden. Stattdessen wird dieser Indikator jeweils mit spezifischen Informationen zu den Auswirkungen verknüpft. Welche spezifische Informationen sich dafür eignen, wird im folgenden Abschnitt erörtert.

\subsubsection{Wirtschaftliche Effekte der Biotechnologie}

Während die in den beiden vorigen Teilkapiteln geschilderten Indikatoren wichtige Hinweise auf die Diffusion der Biotechnologie geben, sagen sie nur wenig über mögliche wirtschaftliche Veränderungen aus. Hierfür sind für einzelne Wirkungsmechanismen (z.B. Produktivität) konkrete Indikatoren notwendig, Rose und McNiven (2007) sprechen von „Impact“-Indikatoren.

Vorschläge für solche Indikatoren finden sich bei Reiss et al. (2006) und Rose und McNiven (2007). Tabelle 3 zeigt die Vorschläge von Rose und McNiven (2007). Diese Indikatoren spiegeln allerdings eher einen Idealfall wider, als eine bald realisierbare Datenbasis. So ist z.B. offen, wie die skizzierten Probleme der Zurechenbarkeit und notwendigen Verknüpfungen mit amtlichen Statistiken gelöst werden können.

Dabei sind sowohl die makro- als auch die mikroökonomischen Indikatoren dieser Übersicht bedeutsam für gesamtwirtschaftliche Modelle. So geben Veränderungen der Preisstrukturen und die Substitution von Inputs (z.B. Rohstoffveränderung, Materialeinsatz etc.) wichtige Hinweise für strukturelle Veränderungen und das Ausmaß einzelner Wirkungsmechanismen (z.B. Kostenreduktion). Die bisherige Verfügbarkeit von solchen „Impact"-Indikatoren für die Biotechnologie ist aber bisher gering und am wenigsten fortgeschritten (Reiss et al. 2006). Als international führend gelten nach Expertenmeinungen die Erhebungen des Statistischen Amtes in Kanada. Dort werden beispielsweise Kostenstrukturen für die biotechnologische Produktion oder deren Effekte (z.B. Kostenreduzierung, Produktionserhöhung) in amtlichen Umfragen erhoben (Statistics Canada 2005; 2007). 
Wichtige Hinweise für mögliche Effekte können technoökonomische Studien geben. Diese Studien (u.a. Patel et al. 2006; Reiss et al. 2007) analysieren z.B. die Ökobilanzen (fossiler Energiebedarf, $\mathrm{CO}_{2}$ Bilanz etc.) oder die Machbarkeit und Wirtschaftlichkeit von bestimmten biotechnologischen Verfahren auf Basis von Herstellungskostenvergleichen. Diese können Informationen gerade für zukünftige Einschätzungen der Auswirkungen (z.B. Produktivitätseffekte, Strukturveränderungen auf Basis neuer Wertschöpfungsketten) geben. Insgesamt lässt sich eine Zunahme an geeigneten Studien feststellen, die aber verglichen mit anderen Bereichen (z.B. erneuerbaren Energien) immer noch als gering einzuschätzen ist. Dabei ist die Möglichkeit zur Verallgemeinerung der Ergebnisse jeweils zu prüfen, da häufig nur bestimmte Produktionsverfahren unter spezifischen Annahmen betrachtet werden. Die Abdeckung der Anwendungsbereiche der Biotechnologie ist zudem recht unterschiedlich. Während vergleichsweise viele Untersuchungen für Bioethanol durchgeführt wurden, gibt es für viele andere Bereiche deutlich weniger Anhaltspunkte. Dies trifft vor allem für Anwendungsfelder mit einer Vielzahl unterschiedlicher biotechnologischer Produkte zu, z.B. Fein-/Spezialchemikalien.

Zusammenfassend befindet sich die Datenerhebung und Indikatorenentwicklung für die Biotechnologie in einem frühen Stadium. ${ }^{104}$ Für eine bessere Datenverfügbarkeit sind zusätzliche Datenerhebungen, Änderungen in den Klassifikationen der amtlichen Statistik sowie Verknüpfungen von Datenbeständen (z.B. Volkswirtschaftliche Gesamtrechnung) notwendig. Rose und McNiven (2007) sehen Änderungen in den Produktgruppen- und Außenhandelsklassifikationen und den Einbezug weiterer Sektoren mit Bezug zur Biotechnologie (z.B. öffentlicher Sektor) in die nationalen Erhebungen als am dringlichsten an. Zum jetzigen Zeitpunkt sind die Analysemöglichkeiten und der Einsatz bestimmter Methoden zur ökonomischen Bedeutung der Biotechnologie auf jeden Fall eingeschränkt (ACIL Tasman/Innovation Dynamics 2006a).

${ }^{104}$ Ein Teil der zukünftigen Datenverfügbarkeit hängt aber auch sicherlich von der zukünftigen Bedeutung und Wahrnehmung der Biotechnologie ab. So stellt Arundel (2003) im Vergleich der Biotechnologie zur IKT fest, dass Indikatoren relativ schnell der Entwicklung von IKT-Anwendungen gefolgt sind. Die vergleichsweise schwache Datenlage sieht er deshalb auch zum Teil als Ausdruck der vergleichsweise geringen Bedeutung der Biotechnologie. 
Tabelle 3: „Impact"-Indikatoren für die Biotechnologie

\begin{tabular}{|c|c|}
\hline Kategorien & Indikatoren \\
\hline \multicolumn{2}{|c|}{ Makroökonomische Auswirkungen } \\
\hline $\begin{array}{l}\text { Produktivitätsveränderun- } \\
\text { gen durch innovative Pro- } \\
\text { dukte und Prozesse }\end{array}$ & - Sektorale Produktivitätsindizes \\
\hline Wirtschaftswachstum & $\begin{array}{l}\text { - Nationale und sektorale Wachstumsraten } \\
\text { - Wachstumsraten von Biotechnologieunterneh- } \\
\text { men vs. anderen Unternehmen }\end{array}$ \\
\hline $\begin{array}{l}\text { Sektorale Strukturverän- } \\
\text { derungen }\end{array}$ & $\begin{array}{l}\text { - Umsatzanteile der Biotechnologie je Sektor } \\
\text { - F\&E-Ausgaben für Biotechnologie je Sektor } \\
\text { - Konzentrationsmaße für Umsatz, Beschäftigung } \\
\text { und F\&E } \\
\text { - Veränderung und Wachstum einzelner Sektoren }\end{array}$ \\
\hline $\begin{array}{l}\text { Veränderungen im } \mathrm{Au}- \\
\text { Benhandel }\end{array}$ & $\begin{array}{l}\text { - Veränderung der Import-/Exportstruktur } \\
\text { - Import-/Exportindikatoren für Sektoren und Gü- } \\
\text { ter }\end{array}$ \\
\hline \begin{tabular}{|l|} 
Veränderungen auf dem \\
Arbeitsmarkt
\end{tabular} & $\begin{array}{l}\text { - Aufteilung nach Berufsgruppen } \\
\text { - Bildungsstand und Art der Berufsausbildung }\end{array}$ \\
\hline \multicolumn{2}{|c|}{ Mikroökonomische Auswirkungen } \\
\hline $\begin{array}{l}\text { Veränderungen in der Un- } \\
\text { ternehmenslandschaft }\end{array}$ & $\begin{array}{l}\text { - Anzahl von Unternehmensgründungen, } \\
\text {-schließungen, -fusionen, -übernahmen } \\
\text { - Unternehmenscharakteristika: Alter, Eigentümer } \\
\text { (privat, öffentlich) } \\
\text { - Unternehmensentwicklung: Venture Capital, Li- } \\
\text { zensierungen, Kooperationen, Produktneuheiten }\end{array}$ \\
\hline $\begin{array}{l}\text { Veränderungen bei Prei- } \\
\text { sen und Kostenstrukturen }\end{array}$ & $\begin{array}{l}\text { - Preise der biotechnologischen Produkte und de- } \\
\text { ren Substitute } \\
\text { - Kostenstrukturen von Biotechnologieunterneh- } \\
\text { men vs. anderen Unternehmen }\end{array}$ \\
\hline Substitution von Inputs & - Inputstruktur bei Rohstoffen, Energie etc. \\
\hline $\begin{array}{l}\text { Veränderungen in der } \\
\text { Wettbewerbsfähigkeit }\end{array}$ & $\begin{array}{l}\text { - Marktanteil und -veränderungen von Biotechno- } \\
\text { logieunternehmen } \\
\text { - Zeitreihen für Unternehmensentwicklungen und } \\
\text {-charakteristika }\end{array}$ \\
\hline $\begin{array}{l}\text { Veränderungen der Rah- } \\
\text { menbedingungen für die } \\
\text { Wettbewerbsfähigkeit }\end{array}$ & $\begin{array}{l}\text { - Geographischer Standort } \\
\text { - Distanzmaße }\end{array}$ \\
\hline
\end{tabular}

Quelle: Rose/McNiven (2007) 


\subsection{Input-Output-Analyse für die wirtschaftlichen Effekte neuer Techno- logien}

Die geschilderte eingeschränkte Datenverfügbarkeit hat neben den zu erfassenden gesamtwirtschaftlichen Wirkungsmechanismen (Kapitel 3.3) erhebliche Implikationen für eine geeignete Modellwahl und -spezifizierung zur Abbildung der Effekte. Im Rahmen dieser Arbeit wird ein Input-Output-(I-O) Modellansatz verwendet. Die Eignung eines solchen Ansatzes, aber auch die Schwächen und Einschränkungen im Vergleich zu anderen Modellsträngen für eine Abbildung der wirtschaftlichen Effekte der Biotechnologie, werden in Kapitel 4.3.4 diskutiert. Zunächst erfolgt eine Darstellung der I-O-Analyse in ihren Grundzügen und der Analysemöglichkeit von Auswirkungen neuer Technologien.

\subsubsection{Darstellung der Input-Output-Analyse}

Die I-O-Analyse geht auf die Arbeiten von Wassily Leontief aus den 1930er Jahren zurück. „Die Input-Output-Analyse ist eine Methode der systematischen Quantifizierung der Wirkungsbeziehungen zwischen den verschiedenen Sektoren eines komplexen ökonomischen Systems“" (Leontief 1966, Übersetzung nach Iten et al. 2005, S.185). Dabei wird in der Regel zwischen deskriptiven I-OTabellen und den analytisch eingesetzten I-O-Modellen unterschieden (Fleissner et al. 1993; Miller/Blair 1985). Diese werden im Folgenden näher dargestellt.

\subsubsection{Input-Output-Tabelle}

Die Datengrundlage der I-O-Analyse bilden I-O-Tabellen. Diese erfassen die quantitativen Güterströme (Waren und Dienstleistungen) zwischen den Sektoren in einer Volkswirtschaft und dem Ausland in einer bestimmten Periode. Es handelt sich im Prinzip um eine Ergänzung zur Volkswirtschaftlichen Gesamtrechnung (VGR), bei der die Produktionsseite und die Verwendungsseite der VGR zusammengeführt werden (Stahmer et al. 2000). Die Tabellen zeigen die Produktion von Waren und Dienstleistungen und ihre Verwendung auf (siehe Abbildung 10).

Auf der Produktionsseite werden auf der niedrigsten Aggregationsebene des Statistischen Bundesamtes aktuell 71 produzierende Wirtschaftssektoren unterschieden. Jeder dieser produzierenden Wirtschaftssektoren wird mit seinen Input- und Outputströmen in monetären Einheiten (z.B. Mio. $€$ ) erfasst. Die Zeilen der Tabelle enthalten die Lieferungen der einzelnen Produktionssektoren an die Abnehmerbereiche, die sich zwischen Vorleistungsnachfrage und Endnachfrage unterscheiden lassen. Die Vorleistungsnachfrage erfolgt von Produktionssektoren, die Güter zur weiteren Verarbeitung nachfragen. Bei der Endnachfrage werden die Güter und Dienstleistungen ihrer letzten Verwendung zugeführt. Dazu gehören der Konsum der privaten Haushalte und des Staates sowie die Investitionsnachfrage und die Auslandsnachfrage (Exporte). 


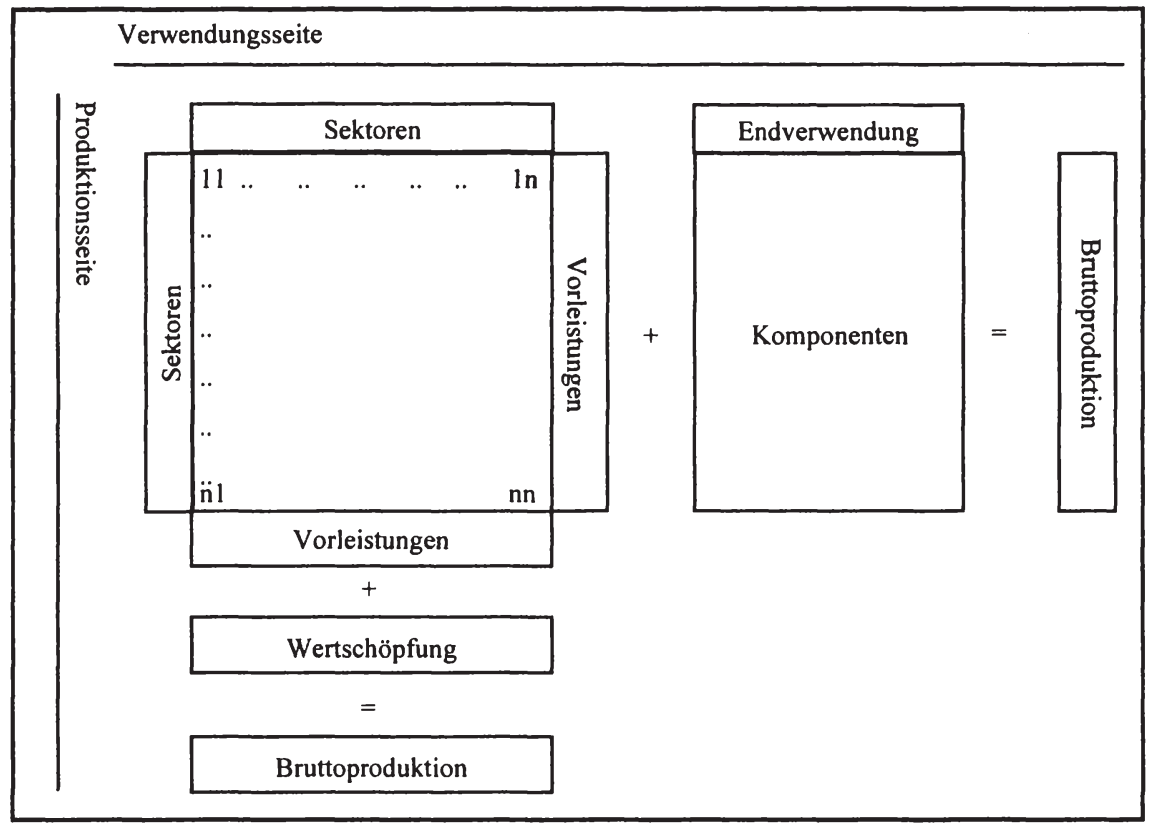

Quelle: Eigene Darstellung in Anlehnung an Iten et al. (2005)

Betrachtet man die I-O-Tabelle spaltenweise, so erkennt man den Inputbedarf der einzelnen Sektoren für die eigene Produktionstätigkeit. Dieser Inputbedarf besteht aus den benötigten Vorleistungen von den eigenen und anderen Sektoren sowie den primären Inputs. Letztere setzen sich aus der Entlohnung für Arbeit und Kapital im Produktionssektor zusammen und bilden die Bruttowertschöpfung der Sektoren. Diese lässt sich genauer in Abschreibungen, der Differenz aus Produktionssteuern und Subventionen, dem Einkommen aus Unternehmertätigkeit und Vermögen sowie dem Einkommen aus unselbständiger Arbeit unterscheiden. Definitionsgemäß ist die Zeilen- und Spaltensumme - die Bruttoproduktion -des jeweiligen Sektors identisch. Den Kern der I-O-Tabelle bildet die Verflechtungsmatrix, welche die Vorleistungsverflechtungen zwischen den Produktionssektoren abbildet.

I-O-Tabellen stellen somit eine Deskription der existierenden Verflechtungen für zurückliegende Jahre dar, sind aber keine Analyse dieser Verflechtungen. Für die Beantwortung analytischer Fragestellungen, muss man über die rein deskriptive Auswertung von I-O-Tabellen hinausgehen. Hierfür sind in der Vergangenheit zahlreiche Modelle entwickelt worden. 


\subsubsection{Grundformen des Input-Output-Modells}

Bei den Modellansätzen wird meist zwischen drei Begriffspaaren unterschieden (Holub/Schnabl 1994):

- Mengen- und Preismodelle,

- offene und geschlossene Modelle,

- statische und dynamische Modelle.

Das Mengenmodell ${ }^{105}$ stellt den funktionalen Zusammenhang zwischen der Endnachfrage und der Produktion bzw. den primären Inputs her. Es dient der Ermittlung von direkten und indirekten Produktionseffekten bei einer vorgegebenen Endnachfragekonstellation. Preisänderungen und deren Auswirkungen werden in Mengenmodellen nicht berücksichtigt, eine mögliche Preis-MengenDynamik kann folglich nicht analysiert werden (Nathani 2003, S.26). Bei Preismodellen stehen hingegen die Güterpreise in funktionaler Abhängigkeit zu den Preisen der primären Inputs und Vorleistungsgüter. Dadurch lassen sich die Auswirkungen von veränderten Kosten, z.B. Löhne und Gehälter oder Importe, auf die Produzentenpreise quantifizieren. Allerdings sind dafür die Mengen konstant, Mengenanpassungsreaktionen auf Preisveränderungen können nicht betrachtet werden.

Offene Modelle sind dadurch gekennzeichnet, dass mindestens eine Größe des Modells als exogen betrachtet wird und nicht in einem interdependenten Verhältnis mit den anderen Größen steht. So wird bspw. häufig die Endnachfrage oder der Außenhandel als exogen betrachtet und unabhängig von anderen Modellgrößen vorgegeben (z.B. Leontief/Duchin 1986). In einem geschlossenen Modell sind hingegen alle Endnachfragegrößen und auch die primären Inputs durch gegenseitige Rückkoppelungen endogenisiert.

In statischen I-O-Tabellen werden als Variablen nur Stromgrößen für eine Periode verwendet. Es werden nur die für die aktuelle Produktion in einer bestimmten Periode benötigten Güterströme und primären Inputs, betrachtet. Diese Modelle erlauben somit keine Analyse des zeitlichen Verlaufs bestimmter Variablen. Es werden weder zeitliche Verschiebungen der Nachfrage (lead bzw. lagBeziehungen) noch der Kapazitätseffekt der Investitionen berücksichtigt. Dynamische Modelle berücksichtigen hingegen zeitliche Zusammenhänge zwischen Variablen und können daher Entwicklungen der Variablen im Zeitablauf analysieren. Mittels Reaktionsgleichungen werden die Variablen verschiedenen zeitlichen Perioden zugeordnet. Wie in Kapitel 5.2.2 begründet wird, erfolgt im Rahmen dieser Arbeit aber die Betrachtung eines statischen offenen Mengenmodells, das im Folgenden näher erläutert wird.

${ }^{105}$ Dabei werden die Mengeneinheiten meist nicht als physische Einheiten, sondern als in Geldeinheiten bewertete Wertgrößen gemessen. Für die genauen Annahmen, die hierfür getroffen werden, siehe Holub/Schnabl (1994, S.92). 


\section{Statische offene Mengenmodelle}

Mit dem statischen offenen Mengenmodell lassen sich die sektoralen Outputs zur Produktion der Vorleistungsnachfrage aus anderen Produktionssektoren und der exogenen Endnachfrage bestimmen. Dabei stellt jeder Sektor nur ein homogenes Gut her, unter Einsatz einer homogenen Produktionstechnologie. ${ }^{106}$ Der Output $x_{i}$ eines Sektors i ist dabei die Summe der Lieferungen von Gütern und Dienstleistungen an alle industriellen Sektoren $\mathrm{j}\left(z_{i j}\right)$ und die Lieferungen an die Endnachfrage $y_{i}$.

$$
x_{t}=\sum_{j=1}^{n} z_{i j}+y_{i} \text { mit } i, j=1, . ., n
$$

Die grundlegende Annahme des Modells dabei ist, dass ein proportionales Verhältnis zwischen dem In- und Output eines Sektors besteht. Es wird dabei eine linear-limitationale Produktionsfunktion unterstellt - auch Leontief-Produktionsfunktion genannt - welche die Substitution von Inputs ausschließt. Mit dieser Annahme werden konstante Skalenerträge und die Unabhängigkeit der Vorleistungskoeffizienten vom Faktoreinsatz unterstellt. Formal ergibt sich dadurch (unter Vernachlässigung der Primärinputs) ein Inputkoeffizient $a_{i j}$ der den Bedarf eines Sektors $\mathrm{j}$ an Vorleistungen des Sektors i zur Produktion einer Mengeneinheit des eigenen Outputs $x$, beschreibt.

$$
a_{i j}=\frac{z_{i j}}{x_{j}}
$$

Jede Erhöhung oder Verringerung der Produktion von Sektor j um $x_{j}$ Einheiten würde demnach eine Erhöhung bzw. Verringerung des Inputs an Zwischengut $\mathrm{i}$ um $a_{i j}{ }^{*} x_{j}$ Einheiten erfordern.

Die Grundgleichung des statischen offenen Mengenmodells lässt sich mit der $n^{*} n$ Matrix der Inputkoeffizienten $A=\left(a_{i j}\right)$, dem Endnachfragevektor $y=\left(y_{i}\right)$ und dem Outputvektor $x=\left(x_{t}\right)$ in Matrixnotation darstellen:

$$
A x+y=x
$$

Durch Auflösung dieser Gleichung nach $x$ erhält man

$$
x=(I-A)^{-1} y
$$

Dabei stellt I die Einheitsmatrix dar. Die inverse Matrix $C=(I-A)-1$, die auch als Leontief-Inverse bezeichnet wird, ist das Kernstück der modellgestützten Auswertung eines statischen offenen I-O-Modells. Ihre Elemente $\mathrm{c}$ geben an, um wie viele Einheiten der Output des Sektors i steigen muss, damit Sektor j eine

\footnotetext{
${ }^{106}$ Die Bedingung der Gleichung ist auch dann erfüllt, wenn verschiedene Güter und verschiedene Technologien zu deren Herstellung existieren, allerdings nur, wenn der Produktund Prozess-Mix konstant sind (Nathani 2003, S.27). Siehe Kapitel 4.3.2 für mögliche Einflussfaktoren von Veränderungen des Produkt- und Prozess-Mixes.
} 
weitere Einheit für die Endnachfrage bereitstellen kann. Hiermit sind die mit der Veränderung der Endnachfrage verbundenen direkten und auf vorgelagerten Produktionsstufen angestoßenen indirekten Produktionseffekte in der gesamten Volkswirtschaft ermittelbar.

\subsubsection{Erweiterungen des Input-Output-Modells}

Dieses Grundmodell ist dabei in der Vergangenheit vielfach erweitert worden (Holub/Schnabl 1994). So können bspw. andere Größen mit diesem Modell verknüpft werden, um Aussagen über zusätzlich interessierende Sachverhalte treffen zu können. Beispiele hierfür sind die Integration von Variablen zu Umweltbelastungen, ausgewählter physischer Energieinputs oder der Beschäftigung. Für solche Verknüpfungen gibt es eine Vielzahl von Möglichkeiten (Holub/Schnabl 1994, S.140 ff.). Die Verknüpfung kann zum einen rekursiv erfolgen, d.h. an den Ergebnisvektor des sektoralen Outputs wird eine zusätzliche Größe angehängt, die selbst aber nicht den Output beeinflusst. Zum anderen hängt bei Modellen mit Rückkoppelungen der Ergebnisvektor des Outputs auch von der zusätzlichen Größe ab, d.h. es besteht ein wechselseitiger Zusammenhang. Im Rahmen dieser Arbeit ist dabei die Erweiterung des Modells um die Beschäftigung von Relevanz, deshalb wird diese im Folgenden kurz dargestellt. Dabei wird der am häufigsten verwendete Fall der linearen, rekursiven Verknüpfung betrachtet.

Für jeden Sektor $\mathrm{j}$ wird dabei ein Arbeitskoeffizient bestimmt. Dieser gibt an, wie viel Arbeitsmenge $B S_{j}$ (z.B. Arbeitsstunden) für die Erstellung einer Einheit des produzierten Gutes $X$ in Sektor $\mathrm{j}$ benötigt wird. Im Fall der Annahme von homogenen Arbeitskräften ergibt sich der Arbeitskoeffizient $b_{j}$ aus

$$
b_{j}=\frac{B S_{j}}{X_{j}}
$$

Die Aufhebung der Annahme sektoral homogener Arbeitskräfte erfolgt in der Regel durch die Differenzierung nach Qualifikationen. Dabei lässt sich eine $r^{*} n$ Matrix $L$ definieren, die für $r$ Qualifikationen und $n$ Sektoren die Arbeitskoeffizienten ausweist. Die einzelnen Elemente $z_{i j}$ geben an, wie viele Arbeitskräfte im Sektor $\mathrm{j}$ in der Qualifikation i zur Erstellung einer Einheit des Outputs $X$, benötigt werden. Sie bestimmen sich aus

$$
z_{\imath}=\frac{Z_{\jmath}}{X}
$$

$Z_{i j}$ gibt dabei die Anzahl der Arbeitskräfte mit der Qualifikation i an, die für den Output $x$, des Sektors j benötigt werden. Die Matrix L lautet somit 


$$
L=\left(\begin{array}{cccc}
\frac{Z_{11}}{X_{1}} & \frac{Z_{12}}{X_{2}} & \cdots & \frac{Z_{1 n}}{X_{n}} \\
\cdot & \cdot & \\
\cdot Z_{r 1} & \frac{Z_{r 2}}{X_{1}} & \ldots & \frac{Z_{m}}{X_{n}}
\end{array}\right)
$$

Folglich erhöht (verringert) sich bei jeder zusätzlich (weniger) produzierten Einheit des Sektors j der Arbeitskräftebedarf der Qualifikation i um $z_{i j}$ Arbeitskräfte.

Diesem zusätzlichen Erkenntnisgewinn gegenüber dem Grundmodell, wie viele Arbeitskräfte und mit welcher Qualifikation zur Produktion benötigt werden, stehen zusätzlich zu treffende Annahmen gegenüber (Holub/Schnabl 1994). Die proportionale Verknüpfung des Arbeitskräftebedarfs mit der jeweiligen Produktionsmenge unterstellt eine konstante Arbeitsproduktivität, unabhängig davon, für welchen anderen Sektor oder für welche Endnachfrage produziert wird. Analog zur Produktionsmengenbestimmung werden mit der Proportionalitätsannahme die Substituierbarkeit mit anderen Inputfaktoren sowie Skaleneffekte in Abhängigkeit der Produktionsmenge ausgeschlossen. Darüber hinaus wird angenommen, dass es zu keiner zeitlichen Verzögerung des Arbeitskräfteeinsatzes kommt und keine Rückwirkungen von Arbeitsmarktgrößen auf die Produktion ausgehen.

Eine andere Möglichkeit der Modellmodifikation betrifft die Änderung von kritischen Annahmen (z.B. Linearitätsannahme; siehe auch Kapitel 4.3.4) der Grundvariante des statisch offenen Mengenmodells. Prinzipiell ist eine Vielzahl von Modifikationen des Grundmodells denkbar, die auf einen Teil dieser Annahmen verzichten (Rose 1984; Ten Raa 2006). Ein Beispiel hierfür stellt die Teilendogenisierung der Endnachfrage dar, bei der im Gegensatz zum geschlossenen Modell, nicht alle Endnachfragekomponenten endogenisiert werden. So kann über einkommensabhängige Konsum- und Investitionsfunktionen das Grundmodell um die keynesianische Multiplikatortheorie erweitert werden (Knottenbauer 2000, S.142). Weitere Zielsetzungen von Modifikationen können z.B. in der Aufhebung der Linearitätsannahme oder in der Berücksichtigung der Auswirkungen von Preisveränderungen liegen. Für die vorliegende Arbeit ist die Annahme der Konstanz der I-O-Koeffizienten im Zeitablauf als kritisch zu bewerten. Denn gerade durch die Wirkungen der Biotechnologie lassen sich Veränderungen in den Produktionsprozessen und damit verbundenen sektoralen Strukturen erwarten. Deshalb wird im Folgenden vertieft erörtert, wann es zu Veränderungen der Koeffizienten kommt und inwiefern diese sich prospektiv analysieren lassen. 


\subsubsection{Bestimmungsgründe für die Änderung von Koeffizienten}

Grundsätzlich führen eine Reihe von Faktoren zu Veränderungen von Inputkoeffizienten einer I-O-Tabelle. In der Literatur existieren mehrere Klassifikationen bezüglich des Einflusses von Faktoren auf Input-Koeffizienten (u.a. Rose 1984; Groenberg 1980). In Anlehnung an Groenberg (1980) wird hier zwischen unmittelbaren und mittelbaren Ursachen unterschieden. Zu den unmittelbaren Ursachen gelten der Produkt-Mix und der Prozess-Mix (Carter 1970). Der ProduktMix ergibt sich daraus, dass in dem Aggregationsniveau der existierenden I-OTabellen, ein Sektor mehr als ein Gut herstellt. Jeder Sektor zeichnet sich dabei zu einem bestimmten Zeitpunkt mit einem gewissen Produkt-Mix von Gütern aus. So umfasst der Chemiesektor sowohl rohstoffintensive, billige Grundchemikalien, als auch hochwertige Fein-/Spezialchemikalien mit hoher Wertschöpfung. Diese Güter unterscheiden sich häufig in ihrem Herstellungsprozess und den benötigten Vorleistungen. Die Inputkoeffizienten spiegeln demnach den gewogenen Durchschnitt der Vorleistungen dieser Güter wider. Wenn sich im Zeitablauf die Anteile dieser hergestellten Güter (Produkt-Mix) verändern, ändert sich somit auch die durchschnittliche Vorleistungsstruktur des Sektors, selbst wenn die Produktionstechnologien der einzelnen Güter gleich bleiben. Deshalb wird der Produkt-Mix auch als Aggregationsphänomen bezeichnet (Richter 1991, S. 113; Nathani 2003, S.32). Bei einer Disaggregation in EinGüter-Sektoren tritt er nicht auf. Stattdessen würde sich die Nachfrage bei diesen Sektoren ändern, die jeweiligen Relationen des Input-Bedarfs aber gleich bleiben.

Ein Prozess-Mix entsteht durch unterschiedliche Herstellungsverfahren eines Produkts. Viele Werkstoffe (z.B. Metalle, Glas, Papier) können z.B. auf Basis verschiedener Rohstoffe mit unterschiedlichen Verfahren und Vorleistungsinputs hergestellt werden (Nathani 2003, S.32). Die I-O-Koeffizienten eines Gutes spiegeln demnach nicht nur einen Prozess, sondern eine Kombination von Prozessen wider. Eine Veränderung der Anteile einzelner Prozesse (Prozess-Mix) bei der Produktion in einem Sektor führt folglich zu einer Veränderung der Inputkoeffizienten.

Empirisch sind Änderungen des Produkt-Mix und Prozess-Mix schwer voneinander zu trennen und hängen voneinander ab. Bspw. kann eine Veränderung des Prozess-Mix in einem Sektor zu einer Veränderung der Vorleistungen nach Produkten anderer Sektoren führen und somit deren Produkt-Mix verändern (Holub/Schnabl 1994). Einflussfaktoren für den Produkt- und Prozess-Mix sind die mittelbaren Ursachen. Dabei kann zwischen folgenden - sich teilweise gegenseitig beeinflussenden ${ }^{107}$ - mittelbaren Ursachen unterschieden werden:

- Technischer Fortschritt

${ }^{107}$ Beispielsweise können steigende Skalenerträge und die Veränderung der relativen Preise Folge des technischen Fortschritts sein, müssen dies aber nicht zwingend. 
- Veränderungen der relativen Preise

- Skalenerträge und veränderte Kapazitätsauslastungen

- Veränderungen der politischen und wirtschaftlichen Rahmenbedingungen

Die Auswirkungen des technischen Fortschritts in Form von Prozessinnovationen hängen entscheidend von seiner Art ab (siehe Kapitel 2.3.1). Im Fall eines Hicks-neutralen Fortschritts erhöht sich die Grenzproduktivität aller Inputs in gleichem Maße, d.h. die Inputstruktur bleibt identisch. Solch eine neutrale Wirkung wird häufig Lerneffekten, z.B. organisatorischen Verbesserungen, zugesprochen. Bei faktorgebundenem technischem Fortschritt sinkt hingegen der Bedarf nach einigen Inputs - z.B. Energie und Rohstoffe bei materialeffizienteren Verfahren - während andere Produktionsfaktoren komplementär sein können. Durch die Diffusion einer solchen Prozessinnovation ändert sich unmittelbar der Prozess-Mix in einem Sektor und gegebenenfalls der Produkt-Mix in vorgelagerten Sektoren. Produktinnovationen führen meist zu einer Veränderung des Produkt-Mix. Sowohl durch additive als auch substitutive Produktinnovationen ändern sich die Anteile einzelner Produkte an der Gesamtproduktion des Sektors.

Die Veränderung von relativen Preisen von End- oder Vorleistungsgütern kann zur Substitution zwischen Gütern und Produktionsprozessen führen. Unterschieden wird dabei zwischen einer Substitution auf Basis kurzfristiger Produktionsfunktionen oder aufgrund des Einflusses auf die Technologiewahl vor einer neuen Investitionsentscheidung (Johansen 1972; Richter 1991). Während kurzfristig die Substitutionsmöglichkeiten von Technologien und Prozessen meist begrenzt sind, sind langfristig Änderungen durch Preisverschiebungen wahrscheinlich (Faber et al. 2007, S.27).

Skalenerträge lösen häufig eine Veränderung in den Vorleistungen aus, da bei zusätzlicher Produktion sich vor allem Inputs mit Fixkostencharakter unterproportional ändern. Zudem können Größendegressionseffekte zu einer größeren Rentabilität und schnelleren Diffusion bestimmter Verfahren mit spezifischen Inputkoeffizienten führen (Nathani 2003). Ähnliche Auswirkungen haben Schwankungen in der Kapazitätsauslastung. So führt zum Beispiel eine Ausweitung der Produktion bei einer nicht ausgelasteten Produktionsanlage zu einem unterproportionalen Zuwachs der Investitionen.

Ebenso kann sich eine Veränderung der Rahmenbedingungen, innerhalb derer produziert wird, auf den Produkt- und Prozess-Mix auswirken. Hierbei ist insbesondere an Politikmaßnahmen, wie z.B. Umweltauflagen oder Subventionen, zu denken. Ein Beispiel hierfür ist die in einigen Ländern praktizierte Subvention von Bioenergie, die zu einer größeren Vorleistungsnachfrage nach landwirtschaftlichen Produkten führt. Neben diesen politischen Rahmenbedingungen können Engpässe auf der Angebotsseite bei den benötigten Inputs und Ressourcen zu Anpassungsreaktionen der Unternehmen führen. Diese Engpässe können 
z.B. durch demographische Veränderungen oder Präferenzen bezüglich Arbeit und Freizeit ausgelöst werden.

Zusammenfassend gibt es eine Reihe von Einflussfaktoren, die zu Veränderungen von I-O-Koeffizienten führen können. Der technologische Fortschritt spielt dabei eine sehr wichtige Rolle. ${ }^{108} \mathrm{Da}$ im Rahmen dieser Arbeit eine exante Schätzung der Veränderung von Koeffizienten durch die Biotechnologie durchgeführt wird, werden mögliche Verfahren hierfür dargestellt und ihre Eignung für die Biotechnologie diskutiert.

\subsubsection{Zukünftige Technologieentwicklung im Input-Output Modell}

\subsubsection{1 Übersicht zu Methoden der Projektion}

In der Vergangenheit wurde eine Vielzahl an Verfahren für die Projektion von IO Koeffizienten entwickelt. Die Ansätze zur Projektion lassen sich nur schwierig klassifizieren, da es meist keine genau festgeschriebene Vorgehensweise gibt. Im Folgenden wird in Anlehnung an Richter (1991) und Faber et al. (2007) zunächst zwischen den „formalen Ansätzen“ und den so genannten „pragmatischen Ansätzen“" unterschieden.

Bei formalen Ansätzen werden entweder mechanistisch oder auf Basis bestimmter Vergangenheitsinformationen meist komplette Koeffizientenmatrizen in die Zukunft projiziert. Dabei kommen häufig einfache Fortschreibungsverfahren $^{109}$, z.T. aber auch sehr komplexe Schätzfunktionen zum Einsatz. Letztere versuchen bspw. die Wirkungen von Preisveränderungen oder Sättigungsgrenzen von Gütern in der Nachfrage, zu berücksichtigen. Ein Hauptkritikpunkt bei diesen Ansätzen ist die fehlende explizite Betrachtung von technologischen Veränderungen. Diese werden nur als sich im Zeitablauf verändernde Parameter behandelt. Es ist allerdings nicht möglich, zukünftige technologische Pfade allein aus Vergangenheitstrends zu bestimmen (Faber et al. 2007, S.26). Deshalb sind sie für die Betrachtung der Auswirkung der Biotechnologie nicht geeignet.

Pragmatische Ansätze ermöglichen hingegen die Betrachtung neuer, innovativer Technologien. Die Idee dieser Ansätze ist es, die zukünftige Entwicklung von Koeffizienten auf Basis von Expertenwissen zu bestimmen (Richter 1991; Nathani 2003). Dabei steht in der Regel der Einfluss der technologischen Entwicklung im Vordergrund. Leontief selbst zeigte sich sehr optimistisch hinsicht-

${ }^{108}$ Bei der hier nicht im Fokus stehenden ex-post Analyse mit I-O-Modellen wird kritisiert, dass Substitutionseffekte und technischer Fortschritt sich nicht voneinander unterscheiden lassen (Carter 1970).

${ }^{109}$ Ein bekanntes Beispiel für die zwischenzeitlich sehr verbreiteten mechanistischen Methoden bildet der RAS-Ansatz (Rose 1984, Pan 2006). Hierbei werden für die einzelnen Sektoren die absoluten Werte für Input- und Outputgüter für die Zukunft projiziert. Auf dieser Basis werden die I-O-Koeffizienten proportional auf Basis der zukünftigen Input- sowie Outputverhältnisse angepasst. 
lich der Möglichkeiten, rein durch das Wissen über die Technologien viele Hinweise für die Erstellung von I-O-Tabellen zu bekommen. "Es gibt eigentlich keinen Grund, warum die I-O-Koeffizienten nicht vollständig von unten, von den Informationen der Ingenieure, von den Planungsunterlagen der Prozesse und den Erfahrungen der Produktion abgeleitet werden sollten" (Leontief 1966, S.26, Übersetzung nach Richter 1991, S.231). Auch zur Projektion von I-O-Koeffizienten wird das Wissen von Experten über technologische Prozesse als großes Reservoir für nützliche Hinweise betrachtet. Schließlich sind einige Informationen zu den Charakteristika einzelner Technologien bereits häufig einige Zeit vor der Kommerzialisierung bzw. der breiten Diffusion bekannt (Richter 1991, S.232). Literatur- und Experteneinschätzungen sind auch für zukünftige Entwicklungen, z.B. der Technologiediffusion oder die Struktur des Kapitalstockes, eine mögliche Informationsquelle (Nathani 2003, S.35). Beispielsweise sieht es Tilanus (1968) als Idealvorstellung an, in die I-O-Analyse möglichst viele Informationen über technologische, aber auch über andere Entwicklungen in den Sektoren, in die Projektion mit einzubeziehen. Die Abbildung der neuen Technologie kann dabei in einem neuen, eigenen Sektor oder einem bestehenden Sektor als Veränderung des Prozess-Mix in den I-O-Tabellen erfolgen.

\subsubsection{Vorgehensweise bei pragmatischen Ansätzen}

Bei den pragmatischen Ansätzen gibt es bei der Verwendung der verschiedenen Informationsquellen keine festen Regeln, solange die Konsistenz des verwendeten I-O-Modells gewahrt bleibt (Nathani 2003, S.36). Die verschiedenen Vorgehensweisen in der Literatur lassen sich aber anhand einiger Kriterien einordnen. Im Folgenden werden zunächst zentrale Arbeitsschritte und verschiedene Möglichkeiten der genauen Ausgestaltung erläutert. Im Anschluss wird konkreter die Berücksichtigung zukünftiger Weiterentwicklungen der betrachteten Technologie diskutiert. Hierin unterscheiden sich einzelne Studien mit pragmatischen Ansätzen grundlegend voneinander.

\section{Arbeitsschritte}

In vielen Studien lassen sich zwei zentrale Arbeitsschritte voneinander unterscheiden. Erstens ist die Technologie zu charakterisieren und ihre Vorleistungsstruktur zu ermitteln. Zweitens ist die zeitliche Diffusion der Technologie zu bestimmen. Diese Arbeitsschritte werden im Folgenden näher betrachtet:

Bei der Charakterisierung der Technologie werden in der Regel verschiedenste Informationsquellen verwendet. Dazu zählen insbesondere Fallstudien, technoökonomische Studien und Hintergrundinformationen aus der Literatur, ex-post Daten und Expertengespräche. ${ }^{110}$ Allerdings ist die Gewinnung ausrei-

${ }^{110}$ Bei Studien, welche ökologische Effekte in die Analyse mit einbeziehen, wird häufig versucht, Ingenieursdaten direkt mithilfe eines physikalischen I-O-Modells zu integrieren oder 
chender technologischer Informationen für die Bestimmung der I-OKoeffizienten alles andere als trivial und mit vielen Herausforderungen verbunden. Dabei zeigen sich bei der Verwendung von Fallstudien oder technoökonomischen teilweise unterschiedliche Vorteile und Probleme als bei der Verwendung von Expertenaussagen:

Fallstudien oder technoökonomische Studien geben für viele Technologiebereiche wichtige Anhaltspunkte für die direkten Effekte der Technologie. Allerdings werden bspw. technoökonomische Betrachtungen nicht mit der aus Sicht des I-O-Modells relevanten Fragestellungen durchgeführt. Dadurch wird oftmals nur auf bestimmte Tendenzen (z.B. zur Vorleistungsstruktur) hingewiesen, ohne dass ein quantitatives Ausmaß deutlich wird (Kalmbach et al. 1989, S.297). Oder die technologischen Informationen sind nicht in dem aus der Input-OutputModellsicht notwendigen Detaillierungsgrad verfügbar (Kalmbach et al. 1989). So ist die Unterscheidung von Studien zu Kostenstrukturen einzelner Prozesstechnologien häufig deutlich aggregierter als die Sektorengliederung in den I-OTabellen. Ein Beispiel hierfür sind Kostenanteile für Hilfs- und Betriebsmittel oder Investitionskosten, die meist nicht genauer spezifiziert werden. Zudem sind die technologischen Informationen bei Fallstudien häufig nicht vollständig, d.h. es sind z.B. nicht für alle Sektoren Auswirkungen bei der Anwendung einer neuen Technologie bekannt.

Der Einsatz von Expertengesprächen hat den Vorteil, dass Auskünfte hinsichtlich der spezifischen Fragestellung erhalten werden können. Aber auch hier ist es nicht immer einfach, valide Aussagen auf der benötigten Aggregationsebene zu bekommen (Richter 1991). Die Experten sind häufig nur in der Lage für bestimmte (Teil-)Anwendungen verlässliche Auskunft zu einer Technologie zu geben. Bei Fragen auf einer höher aggregierten Ebene entsteht das Problem, dass nicht nur die Technologie in den anderen Anwendungen weniger bekannt ist, sondern auch die Anteile der Anwendungen am Gesamtaggregat dem Befragten unklar sind. Dies führt leicht zu verzerrten Schätzungen (Richter 1991, S.234). Darüber hinaus können Fachleute häufig nur über Teilaspekte der untersuchten Inputstrukturen Auskunft geben, bspw. nur über bestimmte Materialeinsätze. Komplementäre Koeffizientenänderungen, z.B. bei Primärinputs oder anderen Vorleistungsvektoren sowie die Wahrung der Konsistenz und Vollständigkeit der Tabellen müssen durch den Modellierer selbst berücksichtigt werden (Richter 1991). Miernyk (1975) bemängelt zudem die mögliche Uneinigkeit der Experten bezüglich ihrer Einschätzung der zukünftigen Entwicklung und die Unsicherheit, ob die Experten alle relevanten Faktoren für die Entwicklung der Koeffizienten berücksichtigen. Häufig besteht zudem die Herausforderung, dass die relevanten Experten häufig aus den Naturwissenschaften oder dem Ingeni-

daran zu koppeln (Faber et al. 2007, S.23). Für eine Integration, siehe z.B. Idenburg (1993), für Kopplungen mit Stoffstrommodellen, siehe z.B. Nathani (2003), Paulus (2005). 
eursbereich kommen, weshalb deren Sichtweise auf die Technologie von der IO-Logik des Ökonomen differiert. So fasst Duchin die Zusammenarbeit zwischen Ökonomen und Ingenieuren zur Generierung von I-O Koeffizienten mit folgenden Worten zusammen: „The collaboration is not easy, however, as the two professions have different conceptions of what constitutes important questions, the level of detail appropriate for an inquiry about technology, and the degree of accuracy to be required in quantitative descriptions" (Duchin 1998, S.46).

Aufgrund dieser Unvollständigkeiten und Nachteile einzelner Datenquellen bietet sich häufig die Nutzung mehrerer Datenquellen für die Bestimmung der I-O-Koeffizienten an. Dabei ist für die Übersetzung technologischer Informationen in Koeffizienten neben der Abgrenzung der untersuchten Technologie die Zurechnung möglicher Effekte zu klären (Kalmbach et al. 1989). Die Frage ist dabei, inwiefern wirtschaftliche Veränderungen tatsächlich auf die betrachtete Technologie zurückzuführen sind. Viele Anwendungen sind nur durch den Einsatz mehrerer komplementärer Technologien möglich. Allein können viele Technologien bestimmte Aufgaben nicht lösen. Beispielsweise ist für einen breiten Einsatz für die auf der Informationstechnologie aufbauenden Industrieroboter in der Montage die Kombination mit der Sensortechnik notwendig. ${ }^{111}$ Aus Gründen der Praktikabilität findet meist implizit eine volle Zurechnung der Effekte (z.B. Vorleistungsveränderungen) auf die Technologie statt (z.B. Kalmbach et al. 1989; Edler 1990).

Für die Bestimmung der Diffusion der neuen Technologie bestehen analog zu den I-O-Koeffizienten mehrere Möglichkeiten. Häufig wird die Technologiediffusion exogen festgelegt. Dies erfolgt entweder durch die Annahme einer vollständigen Diffusion der Technologie in den betrachteten Anwendungsbereichen oder durch Experteneinschätzungen, politische Ziele etc.. Sofern die Technologie im Zeitablauf und nicht nur für einen Endpunkt betrachtet wird, ist der Diffusionsverlauf zu bestimmen. Im einfachsten Fall wird eine gleichmäßige Diffusion angenommen (z.B. Leontief/Duchin 1986). Eine andere Möglichkeit ist die Verwendung von S-förmigen Diffusionskurven. Häufig werden dabei logistische oder Gompertz-Kurven verwendet (Pan 2006, S.208). Diese bauen weniger auf einer theoretisch fundierten Grundlage, als vielmehr auf dem in vielen Fällen empirisch zu beobachtenden S-förmigen Verlauf der Technologiediffusion auf (Dewick et al. 2006; Rogers 1995; Pan 2006; Holwegler 2003). Der Zeitverlauf der Diffusion wird exogen vorgegeben, schwierig ist dabei die Bestimmung der benötigten Parameter dieser Kurve. Es sind sowohl die Zeitdauer des

\footnotetext{
111 Streng genommen ist allein die Entwicklung der betrachteten Technologie häufig von technologischen Entwicklungen in anderen Bereichen abhängig. Bspw. ist die Entwicklung der Industrieroboter nicht allein auf Fortschritte in der Informationstechnik zurückzuführen, sondern auch auf Verbesserungen in der Mechanik. Diese Zurechnungsproblematik ist allerdings noch schwieriger lösbar als bei der Anwendung (Kalmbach et al. 1989, S.295).
} 
Diffusionsprozesses als auch die Asymptoten der Funktion festzulegen (Richter 1991, S.237). Eine andere häufig eingesetzte Methode ist die direkte Koppelung der Technologiediffusion an den sektoralen Kapitalstock (Carter 1970; Pan 2006; Edler 1990). Bei diesen Vintage-Ansätzen enthält der neueste Kapitaljahrgang das aktuelle technologische Wissen. Die neue Technologie diffundiert nach und nach über neue Investitionen. Die Bestimmung dieser Investitionen in die „Best-Practice-Technologie“ erfolgt bspw. über die Koppelung des Kapitalstocks an die Produktionsmengen (Pan 2006).

Diese genannten Ansätze zur Diffusion gehen explizit von einer Superiorität der neuen Technologie aus. Dies ist aus theoretischen Überlegungen nicht unproblematisch, da eine „Best-Practice-Technologie“ nur in Kenntnis der jeweiligen Faktorpreisrelationen bestimmt werden kann. Bei Ausblendung des Preissystems und einer reinen Mengenmodellierung, wie es häufig der Fall ist, wird diesem Sachverhalt nicht ausreichend Rechnung getragen (Kalmbach et al. 1989, S.52). Einige Ansätze modellieren deshalb explizit den Entscheidungsprozess der Technologiewahl, indem alternative Technologiemöglichkeiten über den Preismechanismus miteinander in Wettbewerb stehen (z.B. Duchin et al. 1994). Häufig sind diese Modellierungen an anderen Stellen limitiert, da die Komplexität durch die Modellierung dieses Entscheidungsprozesses deutlich zunimmt.

\section{Berücksichtigung technologischer Weiterentwicklungen}

Ein bedeutender Unterschied zwischen den einzelnen pragmatischen Ansätzen besteht darin, ob zukünftige mögliche Veränderungen in der Technologie selbst berücksichtigt werden. Dabei wird in der Regel zwischen den Vorgehensweisen der „Best-Practice-Methode“ und in Abgrenzung hierzu stärker auf Expertenschätzungen beruhenden Ansätzen unterschieden (z.B. Rose 1984; Kalmbach et al. 1989; Edler 1990; Richter 1991; Faber et al. 2007). Die genaue Abgrenzung dieser beiden Ansätze in der Literatur ist allerdings nicht ganz einheitlich, die folgenden Beschreibung der beiden Vorgehensweisen orientiert sich an Kalmbach et al. (1989) und Edler (1990). Demnach beinhaltet die „Best-PracticeMethode" eine ausgiebige Nutzung von Expertenwissen zur Charakterisierung der Technologie, eine zukünftige Weiterentwicklung der Technologie wird allerdings ausgeschlossen. ${ }^{112}$

Die Ausgangshypothese der „Best-Practice-Methode“ ist, dass die heute fortschrittlichste Technologie die Durchschnittstechnologie von morgen wird. Folglich wird analysiert, wie sich die Inputstrukturen eines Sektors verändern, wenn die als fortschrittlichste bekannte Methode der Standard im Sektor wird

112 Richter (1991) setzt z.B. hingegen die „Best-Practice-Methode“ mit Vintage-CapitalModellen gleich. Nach dieser engen Abgrenzung würde der hier vorgestellte Ansatz von Kalmbach et al. (1989) nicht zu der Best-Practice-Methode gehören, da die neue Technologie dort unabhängig von den Investitionen diffundiert. 
(Richter 1991). Da es in der Regel Zeit benötigt, bis die fortschrittlichste Technologie weiter im Sektor diffundiert, ergibt sich die Inputstruktur eines Sektors als Durchschnitt der Vorleistungsbezüge von Unternehmen mit fortschrittlichen und Unternehmen mit weniger fortschrittlichen Methoden. Für die Analyse ist der Unterschied zwischen der „Best-Practice-Technologie“ (BPT) und bisherigen Technologien zu bestimmen. Im Folgenden werden zwei Studien zu diesem Ansatz dargestellt. Interessant ist hierbei die unterschiedliche Vorgehensweise aufgrund der unterschiedlich weiten Technologieabgrenzung und Aggregationsebene.

In der Teilstudie von Edler (1990), im Rahmen der so genannten MetaStudie $^{113}$, werden die Beschäftigungswirkungen der Diffusion von Industrierobotern untersucht. Dies erfolgt anhand eines dynamischen I-O-Modells für den Betrachtungszeitraum von 1980 bis 1995 . In das I-O Modell wird zur genaueren Analyse ein eigener Sektor für die Herstellung von Robotern integriert. Als Ausgangspunkt für die Bestimmung der Vorleistungskoeffizienten dient aufgrund der Ähnlichkeit der Sektor Maschinenbau, der auf Basis von Expertenschätzungen modifiziert wird. Bei der Anwendung von Industrierobotern werden elf verschiedene Einsatzgebiete (Beschichten, Punktschweissen usw.) in elf Anwendersektoren (u.a. Maschinenbau) betrachtet. Für die elf Einsatzgebiete werden der Kapitalbedarf und die Vorleistungsstruktur je installierten Roboter in monetären Werten berechnet. Auf Basis der technoökonomischen Literatur wird die Verteilung der Industrieroboter und deren Einsatzgebiete auf die Anwendersektoren für den ex-post Zeitraum zugeordnet. Die Diffusion der Industrieroboter für den ex-ante Zeitraum ist an das Produktionsvolumen der Sektoren gekoppelt. Bei der Projektion der Koeffizienten wird gemäß der Best-PracticeMethode eine technologische Weiterentwicklung der Industrieroboter ausgeschlossen. Andere technologische Entwicklungen werden ebenfalls nicht betrachtet, d.h. die I-O-Matrizen werden auf dem damaligen aktuellsten Stand von 1984 eingefroren. Hintergrund dieser eher realitätsfernen Annahme für die restlichen Input-Koeffizienten ist der Vorteil, Veränderungen der Koeffizienten eindeutig auf die Industrieroboter zuschreiben zu können.

Ein deutlich breiteres Technologiespektrum untersuchen Kalmbach et al. (1989), indem die Arbeitsmarktwirkungen der Mikroelektronik betrachtet werden. Der Untersuchungszeitraum umfasst die Jahre von 1980 bis 2000. Analog zur Studie von Edler wird ein Teil des Betrachtungszeitraums ex-post simuliert und die Technik im Startjahr 1980 eingefroren. Aufgrund der technologischen Breite der Mikroelektronik und ihrer Anwendung in allen Wirtschaftssektoren wird bei der Bestimmung der BPT ein pragmatisches Vorgehen gewählt. Da die

${ }^{113}$ Die META-Studie versucht anhand verschiedener Ansätze zu klären, inwieweit und unter welchen Bedingungen arbeitssparende oder arbeitsschaffende Effekte durch den technischen Fortschritt entstehen (RWI 2005). Die Bezeichnung „META“ soll die angestrebte Integration von Makro- und Mikrountersuchungen zum Ausdruck bringen. 
Aufspaltung von Hersteller- und Anwendersektoren aufgrund des breiten Spektrums deutlich schwieriger ist, werden sie nicht wie bei Edler explizit getrennt, sondern für die betroffenen Sektoren (z.B. Maschinenbau) direkt Nettoeffekte bestimmt. Bei der Technologieanwendung wird zwischen Auswirkungen der auf Informationstechnik aufbauenden Büromittel und den darüber hinaus gehenden programmgesteuerten Arbeitsmittel (z.B. programmgesteuerte Fertigungstechniken) unterschieden. Bei den (elektronischen) Büromitteln werden auf der Basis bestimmter Diffusions- und Branchenindikatoren alle Sektoren drei verschiedenen Anwendertypen zugeordnet. Für diese Anwendertypen wird jeweils eine BPT mit dazugehörigen Auswirkungen auf I-O- und ProduktivitätsKoeffizienten bestimmt. Bei den programmgesteuerten Arbeitsmitteln werden nur für ausgewählte Sektoren (z.B. Druckgewerbe, Gesundheitssektor) eine BPT und ihre Auswirkungen ermittelt. Die Bestimmung der Koeffizienten für die BPT erfolgte über Fallstudien, Expertenaussagen, Auswertung von Zukunftsprognosen und Analysen von ex-post Entwicklungen. Die Technologiediffusion wird linear im Zeitablauf modelliert, d.h. die jeweilige Koeffizientenprojektion für das Ende des betrachteten Zeitraums vollzieht sich in konstanten Schritten.

Die Einsetzbarkeit und Realitätsnähe der „Best-Practice-Methode“ ist aufgrund der fehlenden Berücksichtigung von Lerneffekten eingeschränkt. Der Zukunftshorizont kann beim Ansatz der BPT nur relativ begrenzt bleiben. Formal gesagt nur solange, wie der Abstand der BPT zur Durchschnittstechnologie Bestand hat (Richter 1991, S.239). Der „Best-Practice-Ansatz“ ist dagegen weniger geeignet, wenn sich die betrachtete Technologie noch in einem sehr frühen Entwicklungsstadium befindet und zum Zeitpunkt der Betrachtung gegenüber der Durchschnittstechnologie aktuell keine oder nur geringe Vorteile aufweist. ${ }^{114}$ Erst bei einer Verbesserung ist eine Diffusion der Technologien zu erwarten. Gerade dieser Aspekt trifft zumindest für einige Anwendungsfelder der Biotechnologie (z.B. Bulkchemikalien) zu. Die notwendigen Verbesserungen können die Auswirkungen der Biotechnologie aber erheblich verändern. Beispielsweise beeinflussen neue Einsparungsmöglichkeiten an Rohstoffen oder Verbesserungen im Konversionsprozess die Kosten- und Vorleistungsstruktur zur Herstellung der biotechnologischen Produkte.

Andere Ansätze zur Projektion berücksichtigen hingegen auch technologische Weiterentwicklungen. Allerdings steigt der Grad an Unsicherheit, den eine solche Schätzung mit sich bringt. Zur Bestimmung der Weiterentwicklung werden häufig Expertenschätzungen eingesetzt. Bei Technologien mit relativ

\footnotetext{
114 Darüber hinaus werden die Auswirkungen der BPT auch von der jeweiligen arbeitsorganisatorischen Lösung beeinflusst (Kalmbach et al. 1989, S.44). Auch hier ist in der Regel die Festlegung auf eine, meist bereits praktizierte organisatorische Lösung notwendig. Dies ist aus theoretischer Sicht nicht unproblematisch, schließlich zeigen die GPT-Konzepte, dass für eine effiziente Nutzung solcher Technologien erst eine neue organisatorische Lösung gefunden werden muss (Kapitel 2.2.3.2).
} 
guter Datenlage ist auch der Einsatz von Lernkurven denkbar. Im Folgenden werden drei Beispiele aufgezeigt, die einen guten Überblick über das Spektrum möglicher Vorgehensweisen geben.

Leontief und Duchin (1986) untersuchen anhand eines dynamischen I-OModells die zukünftigen Auswirkungen der Computer-unterstützten Automatisierung auf die Beschäftigung in den USA. Die Projektion umfasst dabei den Zeitraum von 1980 bis 2000 . Es werden dabei sämtliche Vorleistungs- und Nachfragekoeffizienten für die Sektoren „Roboters“, „Computers“ und „Office Equipment" untersucht. Zusätzlich werden für jeweils drei ausgewählte Anwendersektoren die Vorleistungs- und Nachfragekoeffizienten unter Einfluss der Automatisierung bestimmt. Neben diesen Koeffizienten werden zusätzlich die Auswirkungen auf die sektorale Arbeitsproduktivität und die Nachfrage nach bestimmten Berufsgruppen ermittelt. Die Bestimmung der technologischen Auswirkungen erfolgte über Fallbeispiele aus technoökonomischen Studien, Vergangenheitsentwicklungen und Expertengesprächen. Bei der Projektion der Koeffizienten werden ausgehend vom Jahr 1980 die Veränderung für die Jahre 1990 und 2000 aufgrund einer weiteren exogen festgelegten Diffusion, aber auch Verbesserungen der existierenden Techniken zur Computer-unterstützten Automatisierung, berücksichtigt. Die Wirkungen von potenziellen neuen Techniken werden hingegen nicht in die Untersuchung mit einbezogen.

Faber et al. (2007) untersuchen anhand eines dynamischen I-O-Modells die technologische Entwicklung und deren wirtschaftlichen und ökologischen Folgen der jeweiligen Entwicklung für die Niederlande im Jahr 2030. Dabei steht nicht nur eine bestimmte Technologie im Fokus. Stattdessen versuchen die Autoren explizit die Entwicklung von potentiell stark entwickelnden Technologien zu erfassen, von denen ökonomische und ökologische Effekte erwartet werden können. Zunächst wird ein Referenzszenario bestimmt, um die zukünftigen Auswirkungen bereits diffundierender Technologien zu erfassen. Diese Effekte werden anhand einer Trendexpolation der Entwicklung der Input-Koeffizienten von 1995 bis 2000 auf das Jahr 2030 fortgeschrieben. Anschließend wird anhand von Literatur und Expertengesprächen eine Liste mit 134 zukünftig relevanten Technologien erstellt und $\mathrm{zu}$ zwölf Technologieclustern zusammengefasst. ${ }^{115}$ Für diese Technologiecluster werden Expertengespräche über die Veränderung auf die I-O-Koeffizienten - ohne Kapitalkoeffizienten - auf Sektorebene für das Jahr 2030 im Vergleich zum Referenzszenario durchgeführt. Dabei werden im Gegensatz zu anderen Ansätzen den Experten vier unterschiedliche Szenarien zur Bewertung vorgegeben, die sich entscheidend in ihren umweltpolitischen Rahmenbedingungen untereinander und vom Referenzszenario unterscheiden. Unterschiedliche Rahmenbedingungen werden somit explizit erfasst.

\footnotetext{
${ }^{115}$ Bei fünf dieser Technologiecluster sind biotechnologische Methoden enthalten, vor allem im Agrar- und Chemiebereich. Gesundheitstechnologien werden aufgrund ihrer eher geringen Relevanz für Emissionen vernachlässigt.
} 
Von besonderer Bedeutung im Rahmen dieser Arbeit ist der Ansatz von Dewick et al. (2006), da hier neben der Informationstechnologie und Nanotechnologie konkret die Biotechnologie betrachtet wird. Das Ziel der Untersuchung ist die Bestimmung der Auswirkungen dieser drei Technologien auf die I-OKoeffizienten im Jahr 2020 und 2050 für die EU, China und USA als Ausgangsbasis einer nicht näher spezifizierten Modellierung. Die Vergleichsbasis bildet im Gegensatz zu Faber et al. (2007) die gegenwärtige I-O-Struktur des Jahres 2000. Die Auswirkungen der Informationstechnologie werden auf Basis von Vergangenheitswerten unter der Annahme eines logistischen Diffusionsverlaufs mit eintretender Marktsättigung projiziert. Für die Bio- und Nanotechnologie werden für fünf der 32 Modellsektoren Expertengespräche durchgeführt und dabei die jeweils zwei am potentiell stärksten beeinflussten Vorleistungskoeffizienten abgefragt. Kapitalkoeffizienten werden nicht betrachtet. Diese Änderungen der Koeffizienten für die fünf Sektoren werden unter Anwendung der sektoralen Taxonomie von Pavitt (1984) jeweils für Sektoren des gleichen Innovationstyps in identischer Höhe übertragen. ${ }^{116}$ Dabei erfolgten für die Biotechnologie 45 semiquantitative Experteninterviews anhand einer 5-er (2020) bzw. 7-er Skala (2050), wie und aus welchem Grunde sich die Input-Koeffizienten verändern (z.B. starker Zuwachs, keine Veränderung, starker Rückgang). Die häufigste Antwort wird dabei als Ergebnis verwendet und über eine einfache Formel in einer prozentualen Veränderung der Koeffizienten umgerechnet. Um Verzerrungen aufgrund der unterschiedlichen Expertise der Befragten zu vermeiden, wird über die Selbsteinschätzung der Befragten bezüglich ihrer Kenntnisse der Technologie und der befragten Sektoren versucht, Abweichungen von Expertisengruppen zu ermitteln. Dabei werden keine Unterschiede festgestellt. Insgesamt leistet die Studie eine breite Abdeckung der Anwendungsfelder der Biotechnologie. Allerdings ist die Bestimmung der Koeffizienten über die direkte Abfrage in Expertengesprächen kritisch zu sehen. Diese häufig verwendete Methodik bringt Probleme mit sich, da die Experten vermutlich weder alle, noch die gleichen Faktoren berücksichtigen, welche die Entwicklung der Koeffizienten beeinflussen. Auch die einfache Umrechnungsformel der qualitativen Einschätzungen kann zu einer Verzerrung der Ergebnisse führen. Daneben bleibt die Wirkung der Technologien relativ ungenau umschrieben. Die Annahmen zur Diffusion und zur technologischen Weiterentwicklung lassen sich nicht explizit voneinander unterscheiden.

\footnotetext{
${ }^{116}$ Abweichend hiervon wurden Ergebnisse für den Sektor Gesundheitsdienstleistungen in schwächerem Maße für andere Sektoren der „Supplier Dominated Services“ übertragen, da bspw. im Gaststättengewerbe signifikant weniger Anwendungen der Biotechnologie zu erwarten sind (Dewick et al. 2006). Dabei stellt sich allerdings die Frage, ob dies nicht auch für das Versicherungsgewerbe und andere Sektoren, für die bisher keinerlei biotechnologischen Anwendungen bekannt sind, gelten müsste.
} 
Diese verschiedenen Beispiele aus der Literatur zeigen wichtige Aspekte auf, die für die Wahl der geeigneten methodischen Vorgehensweise ausschlaggebend sind. Eine bedeutende Rolle spielen die Anwendungsbreite und die Messbarkeit der zu betrachtenden Technologie. „Berechnungen werden umso schwerer, je weniger sich die Anwendung einer neuen Technik bereits durchgesetzt hat, je weniger sich die Herstellung als eigenständiger Wirtschaftszweig abgrenzen lässt und je weniger einschlägige trennscharfe statistische Daten vorliegen" (Klauder 1986, S.10). Dies führt zu Unterschieden in der Detailliertheit der Betrachtungen und getroffenen Annahmen. Dabei ist aber eine möglichst detaillierte und disaggregierte Technologiebetrachtung sinnvoll, da sich ansonsten erhebliche Verzerrungen in den Ergebnissen ergeben können. Daneben eignen sich Technologien aufgrund ihrer Charakteristika unterschiedlich für bestimmte Abbildungsmöglichkeiten im I-O-Modell. Für Technologien, bei denen das Wissen stark an den Kapitalstock gebunden ist (z.B. Industrieroboter), kann die Bestimmung der Vorleistungsstruktur und Diffusion anhand der Betrachtung der Kapitalanlagen erfolgen. Für andere Technologien (z.B. im Energiebereich) ist eine physikalische Messung möglich und über eine Kombination mit monetären Daten die Koppelung oder Integration in das I-O-Modell durchführbar (z.B. Meade 1995; Nathani 2003). Bevor hier eine Diskussion über einen geeigneten Projektionsansatz für die Biotechnologie erfolgt, werden aber zunächst die Vorteile und Nachteile einer solchen Verbindung der Technologieprojektion mit der I-O-Analyse diskutiert. Die methodischen Stärken und Schwächen haben Implikationen für geeignete Anpassungen bei der Vorgehensweise.

\subsubsection{Stärken und Schwächen der Input-Output-Analyse}

Ein Vergleich eines solchen pragmatischen Ansatzes der Technologieprojektion mit dem I-O-Modell und Ansätzen mit anderen gesamtwirtschaftlichen Modellen ist nur begrenzt möglich. Sowohl für das I-O-Modell als auch für andere Modellansätze gibt es verschiedene Varianten und Erweiterungen, die darauf abzielen, die jeweiligen Schwächen zu vermindern. ${ }^{117}$ Eine Gegenüberstellung einiger Grundcharakteristika des I-O-Modells zu anderen gesamtwirtschaftlichen Modellen verdeutlicht aber einige Vorteile, in der die Ansatzwahl für diese Arbeit begründet ist. Daneben können aber auch spezifische Problembereiche der I-O-Analyse aufzeigt werden, die nach Möglichkeit durch Erweiterungen des Modells zu minimieren sind oder zumindest bei der Interpretation berücksichtigt werden müssen (Rose 1995). Für einen Vergleich bieten sich besonders die Allgemeinen Gleichgewichtsmodelle (AGE) und makroökonometrischen

\footnotetext{
117 Insbesondere können in Allgemeinen Gleichgewichtsmodellen (AGE) und makroökonometrischen Modellen auch I-O-Tabellen integriert werden (Rose 1995). Allerdings ist die Berücksichtigung von Veränderungen der I-O-Koeffizienten schwieriger aufgrund der geringeren Modellflexibilität.
} 
Modelle an, da sie neben der I-O-Analyse häufig als die wichtigsten gesamtwirtschaftlichen Modellfamilien eingeschätzt werden (z.B. Pfaffenberger 1995; Zeddies 2006).

\subsubsection{Vergleich zu anderen gesamtwirtschaftlichen Modellen}

Die Vorteile der I-O-Analyse liegen vor allem in der hohen Flexibilität (Holub/Schnabl 1994, S.609; Zeddies 2006, S.14). Es lassen sich vergleichsweise gut verschiedenste quantitative, aber auch qualitative Informationen (z.B. Expertenaussagen) in das Modell integrieren. Dieser Vorteil ist bei der Projektion von technologischen Veränderungen wichtig, da hierfür selten hinreichend quantitatives Informationsmaterial auf benötigter Aggregationsebene zur Verfügung steht und eine „Bottom-Up“-Schätzung sinnvoll ist. Das I-O-Modell ermöglicht dabei eine sektoral differenzierte Betrachtung technologischen Fortschritts und die Analyse gesamtwirtschaftlicher Auswirkungen über Produktionsbeziehungen zwischen Sektoren. (Cyert/Mowery 1988; Mettelsiefen/Barens 1987; Kalmbach et al. 1989). Eine solche Vorgehensweise ist mit komplexen makroökonometrischen oder AGE-Modellen meist nur schwer machbar (Zeddies 2006). ${ }^{118}$ Der Datenbedarf ist wegen der Vielzahl der betrachteten Zusammenhänge (z.B. Wirkung von relativen Preisen) dort deutlich höher. Dabei werden für die Kalibrierung meist ex-post Daten verwendet, so dass sich Zukunftsprojektionen recht stark auf Beobachtungen aus der Vergangenheit stützen. Dieses Vorgehen ist dann kritisch zu sehen, wenn eine geeignete Datenbasis hierfür fehlt und/oder deutliche Strukturbrüche durch die neue Technologie zu erwarten sind (Nathani 2003).

Daneben lässt sich das I-O-Grundmodell um weitere Variablen und Zusammenhänge flexibel erweitern und kann daher an den jeweiligen Analysezweck geeignet angepasst werden (Holub/Schnabl 1994; Rose 1995). Ein Grund hierfür ist die vergleichsweise hohe Robustheit gegenüber exogenen Impulsen (z.B. große Veränderungen der Nachfrage). Bei den komplexeren Modellen kann die Vielzahl von Modellzusammenhängen zu einer Modelldynamik führen, die möglicherweise wenig plausible Ergebnisse zur Folge hat (Holub/Schnabl 1994). ${ }^{119}$ Zugleich lassen sich die Ergebnisse aufgrund der Simultanität der einzelnen Effekte bei AGE oder makroökonometrischen Modellen schwerer deuten und bestimmten Effekten (z.B. Nachfrageänderung) zuordnen. Die einfachen

118 Beispielsweise lassen sich die für AGE zentralen Ausgangspreise sowie biotechnologiespezifische Preiselastizitäten für die verschiedenen Teilmärkte kaum bestimmen. Eine Ausnahme bildet der bezüglich Mengen und Preise statistisch gut erfasste Agrarsektor, für den bereits Analysen zu den wirtschaftlichen Auswirkungen von genmodifizierten Pflanzen mithilfe eines AGE-Modells durchgeführt wurden (z.B. Das 2007). Darüber hinaus führt die OECD (2008d) eine Analyse für Bioethanol mit einem AGE-Modell durch.

${ }^{119}$ Zudem entstehen häufig Konsistenz- und Kalibrierungsprobleme (Pfaffenberger 1995). 
sektoralen Reaktionsfunktionen des I-O-Modells lassen sich ökonomisch einfacher interpretieren.

Ein häufig genannter Nachteil der Anwendung der I-O-Analyse liegt in der Annahme konstanter Koeffizienten im Grundmodell, die unabhängig von Zeit-, Technologie- und Preisfaktoren bestimmt werden (Pfaffenberger et al. 1995, S.25). Das Modell basiert dabei auf Daten von Stichjahren. Bei Simulationen muss deshalb angenommen werden, dass dieses Jahr ein typisches Abbild der Wirtschaftsstrukturen vermittelt (Iten et al. 2005, S.189). Die Konstanz im Zeitablauf gilt für die Verflechtungsstruktur, als auch für den Anteil der Arbeit und der Wertschöpfung an der sektoralen Bruttoproduktion (Holub/Schnabl 1994). Die fixen Koeffizienten unterstellen eine linear-limitationale Produktionsfunktion, die eine Veränderung des Produktionsprozesses (z.B. durch Faktorsubstitution) nicht zulässt. Insbesondere die fehlende Reagibilität der Mengen auf die Preise, wie sie beispielsweise in AGE-Modellen erfasst werden, wird dabei häufig kritisiert (Pfaffenberger 1995, S.27; Zeddies 2006, S.11). ${ }^{120}$ Diese Annahme der Konstanz von I-O-Koeffizienten im Grundmodell hat zur Folge, dass ihre Eignung insbesondere für mittel- bis langfristige Zukunftsprojektionen kritischer als bei AGE und makroökonometrischen Modellen gesehen wird, welche stärker funktionale Abhängigkeiten abbilden (Pfaffenberger et al. 1995, S.25; Zeddies 2006, S.12). Schließlich hat die Diskussion in Kapitel 4.3.2 gezeigt, dass verschiedenste Einflussfaktoren die Koeffizienten verändern können.

Diese kritische Annahme des I-O-Modells - bzw. deren Bedeutung - lässt sich aber durch folgende Schritte in der Vorgehensweise abschwächen. Wenn ein Nettovergleich zwischen zwei Technologien erfolgt, ist der verzerrende Effekt durch die vermutlich bedingt passende I-O-Matrix für den Betrachtungszeitraum geringer als im Vergleich zu einer Berechnung der Bruttobeschäftigung für eine Technologie oder einen Sektor einzustufen. Denn häufig unterscheiden sich zwei Technologien bei vielen I-O-Koeffizienten eher geringfügig, der verzerrende Effekt tritt bei beiden Technologien auf und kürzt sich bei einer Nettobetrachtung raus. Bei den Vorleistungs- und Inputstrukturen, in denen sich die Technologien unterscheiden, können über die oben erläuterte Projektion des technologischen Fortschritts entsprechende Anpassungen erfolgen (Faber et al. 2007). Die Annahme der linear-limitationalen Produktionsfunktion wird demnach für diese betrachteten Vektoren aufgehoben. Dies gilt aber meist nicht für weitere Vorleistungsrunden, z.B. den Vorleistungen eines Lieferantensektors. Diese werden zwar im Ergebnis erfasst, die entsprechenden I-O-Koeffizienten können aber häufig aufgrund des hohen Aufwandes nur begrenzt durch technoökonomische Informationen an den Untersuchungsgegenstand angepasst werden. Auswirkungen dieser weiteren Vorleistungsrunden auf das Gesamtergebnis

\footnotetext{
${ }^{120}$ Deren Einbezug durch Einführung preisabhängiger Koeffizienten ist grundsätzlich möglich, aber mit stark steigender Komplexität, Stabilitätsproblemen und zunehmenden Datenbedarf verbunden.
} 
sind aber in der Regel gering (Holub/Schnabl 1994, S.612). Wenn eine konkrete Fallprüfung keine Hinweise auf eine Ausnahme zu dieser Feststellung ergibt, ist der verzerrende Effekt durch die fixe I-O-Struktur als gering einzustufen.

Neben diesen Einschränkungen werden Teile der Kompensationsmechanismen unzureichend im I-O-Grundmodell abgebildet (Pianta 2005). So fehlt die Betrachtung einer möglichen höheren Güterendnachfrage (Kaufkraftkompensation), die durch geringere Preise oder höhere Löhne infolge der Produktivitätssteigerung entstehen können. Hier sind Erweiterungen an das I-O-Modell denkbar (z.B. gekoppeltes Nachfragesystem), häufig wird aber versucht, über einfache Annahmen (z.B. alleinige Betrachtung einer Einkommenssteigerung) diese Zusammenhänge zu berücksichtigen. Darüber hinaus wird im Gegensatz zu anderen Modellen keine Knappheit der Ressourcen berücksichtigt. Es wird von unterausgelasteten Kapazitäten ausgegangen, die beliebig erhöht werden können. Auch hier sind Erweiterungen im I-O-Modell denkbar. Allerdings sind hierfür aufwändige Einzelfallbetrachtungen notwendig, die meist nur in begrenztem Ausmaß erfolgen können. ${ }^{121}$

\subsubsection{Allgemeine Einschränkungen bei Modellösungen}

Über diese spezifischen Vor- und Nachteile der einzelnen Modellarten hinaus, zeigen sich einige allgemeine Grenzen und Herausforderungen von gesamtwirtschaftlichen Modellen bei der Analyse wirtschaftlicher Effekte neuer Technologien. Ein Nachteil entsteht durch den relativ hohen Aggregationsgrad (siehe Kapitel 4.1). Dieser ist allerdings in den Modellen notwendig, um die gesamte Volkswirtschaft erfassen zu können (Pfaffenberger 1995). Zwar können bestimmte Teilbereiche der Volkswirtschaft einer genaueren Analyse unterzogen werden, dies ist allerdings aufgrund des Aufwandes, Datenbedarfs und insbesondere der Wahrung der Konsistenz nicht für alle Bereiche möglich. Stattdessen müssen stets spezifische Annahmen auf Basis von Beobachtungen aus der Vergangenheit getroffen werden, unter denen die zukünftige Entwicklung modelliert wird. „So sind volkswirtschaftliche Modelle immer unter dem Blickwinkel zu sehen, dass sie mögliche Entwicklungen unter speziellen Annahmen und unter Zugrundelegung vergangener empirischer Beobachtungen aufzeigen wollen" (Pfaffenberger 1995, S.40).

${ }^{121}$ Ein weiteres Argument, dass gegen die Nutzung der I-O-Analyse zur Erfassung der wirtschaftlichen Potenziale der Biotechnologie hervorgebracht wird, ist die fehlende Möglichkeit, die Biotechnologie als nur eine Aktivität zu behandeln und einem Sektor zuordnen zu können (ACIL Tasman/Innovation Dynamics 2006a). Obwohl die letztere Feststellung - wie in Kapitel 4.2.2 näher erörtert wurde - an sich richtig ist und die Abbildung der Biotechnologie erschwert, so spricht es nicht grundsätzlich gegen die I-O-Analyse, sondern nur für eine Untersuchung der einzelnen relevanten Sektoren, wie es für andere Modelle auch nötig ist. 
Eine weitere Herausforderung für alle gesamtwirtschaftlichen Modelle ist die konkrete Abbildung des technischen Fortschritts. Besonders aus Sicht der Evolutorik ist die Abbildung des Innovations- und Diffusionsprozesses neuer Technologien meist stark vereinfacht (Pianta 2000; Petit 1995; Welsch 2006; Pan 2006). Der Diffusionsverlauf einer neuen Technologie wird meist exogen vorgegeben und dynamische Prozesse der technologischen Entwicklung nicht adäquat berücksichtigt. ${ }^{122}$ Häufig wird dabei das Verhältnis zwischen Diffusion und Produktivität auf neoklassischen Annahmen basierend modelliert. Wie die Diskussion über GPTs allerdings zeigte (Kapitel 2.2.3.2), sind sowohl zeitliche Verzögerungen möglicher Produktivitätsgewinne („Time-Lags)“ als auch Lerneffekte infolge einer steigenden Verbreitung der Technologie möglich (Petit 1995, S.387). ${ }^{123}$ Folglich wird das zeitliche Auftreten ökonomischer Effekte in den Modellierungen möglicherweise falsch eingeschätzt. Daneben werden Produktinnovationen in aller Regel unzureichend von Simulationsmodellen erfasst (Petit 1995, S.392). Besonders die Kausalitäten für die Entstehungen neuer Sektoren und die Nachfrageentwicklungen (z.B. Sättigungen nach Gütern) können nur rudimentär abgebildet werden.

Darüber hinaus sind viele Modelle national ausgerichtet und beschränken sich auf geschlossene Volkswirtschaften. Zwar werden internationale Handels- und Kapitalverkehrsströme berücksichtigt, sie werden aber häufig nicht endogen im Modell erklärt (Pfaffenberger 1995). Diese mangelnde Berücksichtigung der Standort- und Außenhandelseffekte schränkt die Aussagekraft der Modelle ein (Zeddies 2006). Es werden Teile der ökonomischen Effekte weitestgehend ausgeblendet und es sind systematische Verzerrungen in der Bewertung von volkswirtschaftlichen Potenzialen von Technologien möglich. Die Diffusion von Technologien mit eher geringer Arbeitsproduktivität wird bei der Betrachtung einer geschlossenen Volkswirtschaft rechnerisch tendenziell höhere Beschäftigungseffekte haben als eine Technologie mit einer hohen Arbeitsproduktivität. In einer offenen Volkswirtschaft ist eine hohe Produktivität durch kontinuierliche Produkt- und Prozessinnovationen allerdings ein wichtiger Faktor für die internationale Wettbewerbsfähigkeit. Technologien mit einer hohen Produktivitätssteigerung werden daher eher zu einer positiven Entwicklung der Außenhandelsströme und Direktinvestitionen beitragen.

122 Dies gilt auch für die dynamische I-O-Analyse, bei der sich die Charakteristik der Dynamik auf die zeitliche Entwicklung der Investitionen und nicht auf technologische Entwicklung bezieht. So stellt Drejer fest: "[...] Leontief's 'dynamic' input-output analysis was only a more complex method for comparative statics than traditional static input-output analysis [...]" (Drejer 1999, S.5).

${ }^{123}$ So stellen Patel und Pavitt bezüglich der Entwicklung der IKT fest: „[...] one reason for the apparent mismatch between best-practice information technology and the average-practice productivity is the time-consuming and costly process of learning that accompies the diffusion of information technology“" (Patel/ Pavitt 1995, S.33). 
In jüngerer Vergangenheit haben die Bemühungen, Innovations- und Diffusionsprozesse (z.B. Guerrieri/Padoan 2007; Köhler 2005; Pan 2006; Bernardes et al. 2006) sowie Entwicklungen des Außenhandels (z.B. Lehr et al. 2008; IPTS 2002a) in gesamtwirtschaftlichen Modellen abzubilden, deutlich zugenommen. Allerdings bezieht sich nur ein Teil dieser Ansätze auf technologiespezifische Innovations- und Diffusionsprozesse und bildet diese nur unter recht restriktiven Annahmen ab (Guerrieri/Padoan 2007).

Zusammenfassend wurden in diesem Kapitel 4 die verschiedenen Aspekte einer empirischen Analyse der gesamtwirtschaftlichen Auswirkungen der Biotechnologie betrachtet. In Kapitel 4.1 zeigte sich, dass für die Erfassung der indirekten wirtschaftlichen Effekte einer Technologie ein gesamtwirtschaftliches Modell notwendig ist, die direkten Effekte hingegen aber besser auf einem niedrigeren Aggregationsniveau betrachtet werden können. Deshalb bietet eine möglichst disaggregierte - Technologieprojektion gekoppelt mit einem gesamtwirtschaftlichen I-O-Modell eine interessante Möglichkeit zur Analyse wirtschaftlicher Auswirkungen von neuen Technologien. Zwar bestehen einige Einschränkungen bei der Erfassung der gesamtwirtschaftlichen Folgewirkungen, da makroökonomische Rückkoppelungseffekte im Vergleich zu makroökonometrischen oder AGE-Modellen kaum erfasst werden. Einige Kritikpunkte (z.B. fixe Koeffizienten im Zeitablauf) können aber durch eine geeignete Vorgehensweise abgemildert werden. Für einen Einsatz spricht aber die hohe Flexibilität, welche die Nutzung verschiedener technoökonomischer Informationen (z.B. Expertenaussagen) ermöglicht. Dies ist gerade für die Betrachtung der Biotechnologie ein großer Vorteil, da die Verfügbarkeit geeigneter Daten zu wirtschaftlichen Größen, die für komplexe Modelle benötigt werden, als gering einzustufen ist (Kapitel 4.2). Zugleich besteht erheblicher Forschungsbedarf über die Art und Weise der Auswirkungen der Biotechnologie, welcher bei dieser Vorgehensweise vergleichsweise gut sichtbar wird.

Bei einer solchen Abschätzung zukünftiger Auswirkungen der Biotechnologie erscheint ein pragmatischer Ansatz als geeignet. Dieser Ansatz ermöglicht die Betrachtung neuer, innovativer Technologien und damit eine plausiblere Beschreibung zukünftiger Entwicklungen. Bei den pragmatischen Ansätzen ist die Berücksichtigung technologischer Weiterentwicklungen als sinnvoll zu erachten. Die theoretischen Ansätze der Innovations- und Diffusionsforschung und die Betrachtung der (potenziellen) Anwendungen der Biotechnologie zeigen die Bedeutung der technologischen Weiterentwicklung für die Diffusion und Effekte der Biotechnologie. In vielen Anwendungsfeldern befindet sich die Biotechnologie noch in einem Entwicklungsstadium, eine Best-Practice-Technologie lässt sich zum aktuellen Zeitpunkt gar nicht definieren. Dabei ist natürlich zu beachten, dass eine solche Projektion der Koeffizienten mit erheblichen Unsicherheiten belastet ist. Allgemein muss festgehalten werden, dass es sich bei einer projektiven Schätzung der Koeffizienten immer nur um einen „best-guess“ 
handeln kann (Petit 1995, S.384). Um zumindest die Willkür bei der Bestimmung der Koeffizienten gering zu halten, ist eine möglichst breite Datenbasis heranzuziehen.

Insgesamt kann aber gefolgert werden, dass eine Technologieprojektion gekoppelt mit einem I-O-Ansatz geeignet ist, wichtige Erkenntnisse für die gesamtwirtschaftlichen Auswirkungen der Biotechnologie zu erhalten. Die empirische Umsetzung eines solchen Ansatzes steht im Mittelpunkt der folgenden Abschnitte dieser Arbeit. 


\section{Empirische Vorgehensweise}

\subsection{Ziel der Untersuchung}

Im Mittelpunkt der folgenden Untersuchungen stehen die Effekte der Diffusion und Weiterentwicklung der Biotechnologie auf die Produktion und Beschäftigung in Deutschland. Dabei erscheint eine Analyse mit einem hinreichend großen Zukunftshorizont sinnvoll. Schließlich ist die Abschätzung der Effekte einer Technologie dann interessant, wenn sie einen hohen Diffusionsgrad im Rahmen ihrer Anwendungsmöglichkeiten erreicht. Wie oben erörtert wurde (siehe Kapitel 3.2.2), ist die Diffusion der Biotechnologie allerdings ein sehr zeitintensiver Prozess. Insbesondere in Sektoren, in denen sich die Biotechnologie noch in einem vermutlich frühen Stadium befindet, wie in der Chemie, werden die Diffusion und deren Auswirkungen erst mittel- bis langfristig wirksam. Deshalb wird in Einklang mit Studien, die wichtige Informationen über die Diffusion und mögliche Effekte der Biotechnologie als Grundlage zur Abschätzung liefern, das Jahr 2020 als Zeithorizont definiert. ${ }^{124}$ Allerdings weisen solche Zukunftsprojektionen erhebliche Unsicherheiten auf. Um eine konsistente Vorgehensweise sicherzustellen, werden im Rahmen dieser Arbeit Szenarien erstellt. Sie sind keine Prognosen über einen sich in Zukunft einstellenden Zustand, sondern liefern vielmehr eine Wenn-Dann-Aussage über einen möglichen Entwicklungspfad unter bestimmten Bedingungen dar. „Zentrales Erkenntnisinteresse derartiger Projektionsanalysen ist eine systematische Identifikation und Analyse der Auswirkungen, die bei der Einführung oder Modifikation von technischen Systemen entstehen können" (Walz et al. 2001, S.51).

In der Untersuchung stehen nicht die Abbildung bestimmter makroökonomischer Wirkungsmechanismen (z. B. Zinseffekte), sondern technologiespezifische Struktureffekte im Vordergrund. Hierfür kommt die in Kapitel 4 abgeleitete „Bottom-Up"-Betrachtung der technologischen Impulse gekoppelt mit einem IO-Modellansatz (Top-Down) zur Erfassung der ökonomischen Folgewirkungen zum Einsatz. Die „Bottom-Up“-Technologiebetrachtung ist notwendig, da direkte Effekte auf einer Mikro- oder Mesoebene sichtbar und für die Biotechnologie sehr anwendungsfeldspezifisch sind (siehe Kapitel 3.3). Die ökonomischen Impulse der Biotechnologie werden dabei in ausführlichen Fallstudien bestimmt. Hier kommt eine Kombination aus verschiedenen Informationsquellen (u.a. Experteninterviews, technoökonomische Studien) zum Einsatz. Bei der Bestimmung der Impulse der Biotechnologie werden sowohl die Diffusion als auch technologische Weiterentwicklungen berücksichtigt. Die Betrachtung erfolgt auf einer Mesoebene, bei der die gesamte Wertschöpfungskette der biotechnologi-

${ }^{124}$ Diese Zeitspanne ist dabei geringer als bei anderen Untersuchungen zu Veränderungen von Input-Koeffizienten für die Biotechnologie. Untersuchungen von Faber et al. (2007) oder Dewick et al. (2006) haben ein Zeithorizont von 2030 bzw. 2050. 
schen Produkte betrachtet wird. Zur Berechnung der Nettoeffekte der Biotechnologie wird für jedes Anwendungsfeld jeweils ein eigener I-O-Sektor für die Biotechnologie als auch für die substituierte Technologie (z.B. chemische Polymere) bestimmt. Die Berechnung indirekter wirtschaftlicher Effekte erfolgt „Top-Down“ mit einem statisch-offenen I-O-Modell.

Die genaue Vorgehensweise für die empirische Analyse ist in Abbildung 11 skizziert und wird in den folgenden Abschnitten näher dargestellt. Zunächst werden die Anwendungsfelder der Biotechnologie ausgewählt, für die eine empirische Analyse erfolgt. Im Anschluss muss geklärt werden, inwiefern und auf welche Art und Weise die in Kapitel 2.3 beschriebenen Wirkungsmechanismen in der Untersuchung für die betrachteten Anwendungsfelder berücksichtigt werden können. Auf dieser Basis werden mehrere Szenarien für die Entwicklung und Diffusion der Biotechnologie erstellt. Für diese Szenarien erfolgt die Bestimmung der ökonomischen Impulse der Biotechnologie durch die Auswertung von technoökonomischen Informationen aus der Literatur und der mit einem Fragebogenleitfaden gestützten Expertenbefragung. Die gesamtwirtschaftlichen Folgewirkungen dieser Impulse werden anschließend in den Modellanalysen simuliert.

\section{Abbildung 11: Vorgehensweise zur Ermittlung gesamtwirtschaftlicher Effekte der Biotechnologie}

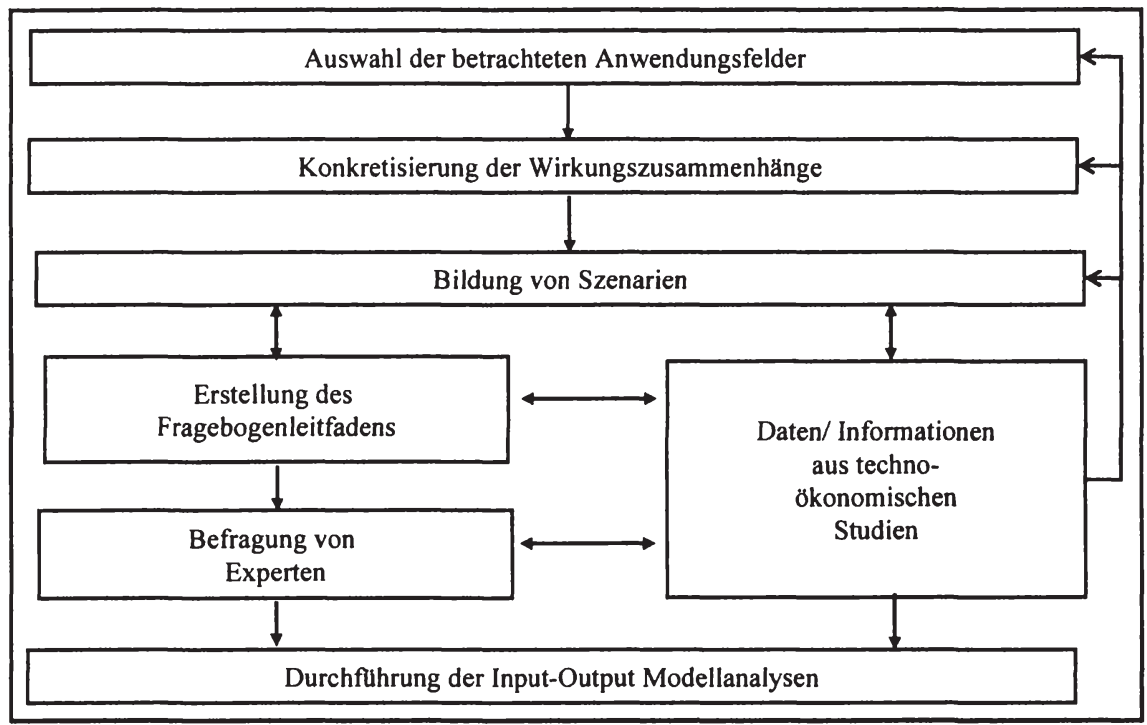

Quelle: Eigene Darstellung 


\subsection{Methodische Konzeption}

\subsubsection{Auswahl der betrachteten Anwendungsfelder}

Zunächst muss eine Auswahl getroffen werden, in welchen Feldern die Effekte der Biotechnologie untersucht werden. Wünschenswert wäre natürlich die Untersuchung der Auswirkungen der Biotechnologie in allen Anwendungsfeldern. Dies ist aber bei einer Betrachtung einer solchen Querschnittstechnologie nur begrenzt möglich. Schließlich sprechen die Heterogenität einzelner Anwendungen, die unterschiedliche sektorale Einbettung, das unterschiedliche Entwicklungs-/Diffusionsstadium in einzelnen Anwendungsbereichen sowie Unterschiede zwischen den einzelnen Biotechniken für eine disaggregierte Vorgehensweise. Gleichzeitig besteht für viele Anwendungsfelder ein hohes Informationsdefizit bezüglich den wirtschaftlichen Auswirkungen der Biotechnologie. Deshalb ist im Rahmen dieser Arbeit eine Begrenzung auf Fallbeispiele notwendig.

Die geeignete Auswahl der Fallbeispiele hängt entscheidend von der spezifischen Fragestellung ab. Das Ziel dieser Analyse ist die Untersuchung der verschiedenen Wirkungsmechanismen (z.B. Produktivität, Nachfrage, Struktureffekte), durch welche die Biotechnologie die Produktion und Beschäftigung in Deutschland beeinflusst. Dabei sollen anhand der verschiedenen Anwendungsfelder insbesondere auch Unterschiede in der Wirkungsweise und Bedeutung der Biotechnologie analysiert werden. Schließlich liegt hier die Bedeutung der Biotechnologie als Querschnittstechnologie im Fokus. Es werden deshalb eher heterogene Anwendungsfelder gewählt. Die gewählten Fallbeispiele unterscheiden sich dabei in ihrer vorrangigen Art der Innovation (Produkt- vs. Prozessinnovation), den Aktivitäten bei denen Biotechnologie eingesetzt wird (z.B. F\&E), dem Bedarf an Biomasse als Rohstoff, der Datenverfügbarkeit und ihrem Entwicklungsstadium. Es wird folglich versucht, der bestehenden Anwendungsvielfalt der Biotechnologie in unterschiedlichen Kontexten Rechnung zu tragen. Dabei werden tendenziell die Felder aus den unterschiedlichen Bereichen ausgewählt, bei denen die Datenverfügbarkeit vergleichsweise geeignet erscheint. Gleichzeitig sollen sich die ausgewählten Fallbeispiele durch ihre mögliche wirtschaftliche Bedeutung auszeichnen, d.h., die betrachteten Techniken und Produkte stellen keine Nischen dar.

Infolge dieser Gründe werden die vier Anwendungsfelder Bioethanol, Biopolymere, biotechnologisch hergestellte Fein-/Spezialchemikalien und Biopharmazeutika untersucht. In Tabelle 4 sind die verschiedenen Charakteristika dieser Fallbeispiele ersichtlich. Die Fallbeispiele Bioethanol und Biopolymere dienen besonders zur Untersuchung der strukturellen Veränderungen durch die Biotechnologie, die durch einen Rohstoffwechsel - Biomasse statt fossile Rohstoffe - entstehen. Bei Bioethanol steigen dabei die gesamtwirtschaftlichen Kosten infolge der höheren Herstellungskosten, bei Biopolymeren sind unter günstigen Rahmenbedingungen Kostensenkungen möglich. Das Beispiel der Biotech- 
nologie Fein-/Spezialchemikalien weist einige Charakteristika einer klassischen Prozessinnovation auf, verdeutlicht aber auch die schwierige Abgrenzung von Produkt- und Prozessinnovationen. Das vierte Fallbeispiel der Biopharmazeutika eignet sich zur Analyse der Wirkung von Produktinnovationen, welche durch die Biotechnologie durchaus möglich sind. Zudem nehmen Biopharmazeutika und Fein-/Spezialchemikalien bezüglich der Bereitstellung und Anwendung von biotechnologischem Wissen und Verfahren eine Zwischenstellung ein. Einerseits werden direkt in unternehmenseigenen F\&E-Abteilungen biotechnische Methoden, Prozesse oder Produkte erforscht und weiterentwickelt. Andererseits wird die Biotechnologie dort auch zur Produktion eingesetzt, beispielsweise von bestimmten Wirkstoffen (z. B. Insulinproduktion mit gentechnisch veränderten Mikroorganismen). Die wirtschaftlichen Effekte setzen sich folglich aus mehreren biotechnologischen Aktivitäten zusammen. ${ }^{125}$

Tabelle 4: Ausgewählte Anwendungsfelder der Biotechnologie

\begin{tabular}{|l|l|l|l|}
\hline Bezeichnung & $\begin{array}{l}\text { Anwender- } \\
\text { sektor }\end{array}$ & $\begin{array}{l}\text { Biotechnologi- } \\
\text { sche (Haupt-) } \\
\text { Aktivitäten }\end{array}$ & $\begin{array}{l}\text { Hypothesen zum Einfluss } \\
\text { der Biotechnologie }\end{array}$ \\
\hline Bioethanol & Kraftstoff & $\begin{array}{l}\text { Anwendung in } \\
\text { Produktion }\end{array}$ & $\begin{array}{l}\text { frühe Diffusionsphase, Pro- } \\
\text { zessinnovation, hoher Bio- } \\
\text { massebedarf (pro t), steigen- } \\
\text { de Kosten }\end{array}$ \\
\hline Biopolymere & Chemie & $\begin{array}{l}\text { Anwendung in } \\
\text { Produktion }\end{array}$ & $\begin{array}{l}\text { sehr frühe Diffusionsphase, } \\
\text { eher Prozessinnovation, ho- } \\
\text { her Biomassebedarf (pro t), } \\
\text { Kostensenkungen }\end{array}$ \\
\hline $\begin{array}{l}\text { Biotechnologi- } \\
\text { sche Fein-/Spe- } \\
\text { zialchemikalien }\end{array}$ & Chemie & $\begin{array}{l}\text { F\&E (teilweise) } \\
+ \text { Anwendung } \\
\text { in Produktion }\end{array}$ & $\begin{array}{l}\text { mittlere Diffusionsphase, } \\
\text { Prozess-/Produktinnovation, } \\
\text { geringer Biomassebedarf } \\
\text { (pro t), Kostensenkungen }\end{array}$ \\
\hline $\begin{array}{l}\text { Biopharmazeu- } \\
\text { tika }\end{array}$ & Pharma & $\begin{array}{l}\text { F\&E + Anwen- } \\
\text { dung in Produk- } \\
\text { tion }\end{array}$ & $\begin{array}{l}\text { frühe/mittlere Diffusions- } \\
\text { phase, Produktinnovation, } \\
\text { kein Biomassebedarf, stei- } \\
\text { gende Kosten }\end{array}$ \\
\hline
\end{tabular}

Quelle: Eigene Darstellung

${ }^{125}$ Eine Disaggregation nach diesen verschiedenen Aktivitäten ist aufgrund der fehlenden statistischen Abgrenzungsmöglichkeiten nicht möglich (siehe Kapitel 4.2.2). Deshalb werden die Veränderungen der Sektoren nur netto in Erscheinung treten und einige interessante Aspekte, wie z.B. eine Produktionszunahme von Entwicklern neuer Enzyme, nicht explizit erkenntlich. 
Wenngleich ein bedeutender Anteil der Reichweite der Biotechnologie mit diesen Fallstudien abgedeckt wird, werden einige Anwendungsfelder der Biotechnologie damit nicht untersucht. ${ }^{126}$ Dies gilt zum einen für potenzielle Anwendungen, die sich bislang erst in einer frühen F\&E-Phase befinden und deren Diffusion bis zum Jahr $2020 \mathrm{sehr}$ offen ist (z.B. Stammzellentherapie). Zum anderen können im Rahmen dieser Arbeit neben den kleineren Feldern, wie Textil, Papier/Zellstoff oder Umweltbiotechnologie, der größere Agro-Food-Bereich nicht vertieft betrachtet werden. Besonders diese fehlende Betrachtung des Agro-Food-Bereichs ist einer Vielzahl von Schwierigkeiten bei der empirischen Analyse geschuldet, die für diesen Bereich in Summe auftreten. Die geringe Datenverfügbarkeit, die schwierige Abgrenzung der modernen Biotechnologie, starke regulative Einflüsse und die schwierige Berücksichtigung der Qualitätsänderung von Produkten erschweren eine Quantifizierung erheblich. Einige dieser Probleme treten auch in den untersuchten Anwendungsfelder (v.a. Biopharmazeutika) auf, allerdings nicht alle davon gleichzeitig. Die Begrenzung auf diese Fallbeispiele bedeutet allerdings eine erhebliche Einschränkung für die Aussagekraft der empirischen Analyse bezüglich der Frage der gesamtwirtschaftlichen Auswirkungen der Biotechnologie. Zum einen können auf Basis einzelner Beispiele nur begrenzt Aussagen über die hier im Fokus stehenden Wirkungsmechanismen der Biotechnologie (bspw. bezüglich Arbeitsproduktivität, Veränderung von Wertschöpfungsketten, Kapitalintensivierung) getroffen werden. Verzerrende Aussagen treten besonders dann ein, wenn die nicht untersuchten Teilbereiche sich in ihrer Wirkungsweise von den untersuchten Teilbereichen deutlich unterscheiden. Ein Beispiel wären sehr hohe Kosteneinsparungen, die in den untersuchten Anwendungsfeldern nicht auftreten. Dieser mögliche Sachverhalt wird in Kapitel 6.5 erörtert. Zum anderen ist bei diesem Vorgehen keine Abschätzung über das Ausmaß der gesamtwirtschaftlichen Bedeutung der Biotechnologie möglich. So lassen sich z.B. die direkten Effekte nicht für den kompletten Sektor der chemischen Industrie bestimmen. Gleiches gilt für die indirekten Effekte in Vorleistungssektoren. Beispielsweise kann für den Agrarsektor nicht die gesamte Höhe der Nachfrageveränderung geschätzt werden, die sich durch die Umstellung der Rohstoffbasis infolge der Einführung der Biotechnologie in den Anwendungsfeldern ergibt.

\subsubsection{Erfassung und Modellierung der Wirkungszusammenhänge}

In diesem Kapitel wird erörtert, welche Wirkungszusammenhänge und auf welcher Art und Weise im Rahmen dieser Arbeit berücksichtigt werden. In der folgenden Tabelle 5 erfolgt zunächst eine Zusammenfassung aus Sicht der in Kapi-

\footnotetext{
${ }^{126}$ Dabei kann die ungefähre Höhe des Anteils der untersuchten Anwendungsfelder bei der Reichweite nicht geschätzt werden, da bei den nicht betrachteten Anwendungsfelder zu wenig Informationen über die Reichweite der Biotechnologie zur Verfügung stehen.
} 
tel diskutierten Wirkungszusammenhänge. Zur Verdeutlichung der berücksichtigten Zusammenhänge werden dabei Struktureffekte vom Maschinenherstellungsargument und dem Argument der internationalen Wettbewerbsfähigkeit getrennt.

Tabelle 5: Berücksichtigung der Wirkungsmechanismen in der empirischen Analyse

\begin{tabular}{|c|c|}
\hline $\begin{array}{l}\text { Wirkungsmechanis- } \\
\text { men }\end{array}$ & Art der Berücksichtigung \\
\hline Freisetzungseffekte & $\begin{array}{l}\text { Grundsätzlich über Produktivitätsvergleich erfasst, } \\
\text { mögliche zeitliche Verschiebungen (time-lags) der } \\
\text { Produktivität werden nicht berücksichtigt. }\end{array}$ \\
\hline $\begin{array}{l}\text { Nachfrage nach Pro- } \\
\text { duktinnovationen }\end{array}$ & $\begin{array}{l}\text { Relevanz v.a. für Biopharmazeutika. Hierfür werden } \\
\text { zwei alternative „Maximalrechnungen“ durchgeführt: } \\
\text { 1. Abbildung des Endnachfragerweiternden Effekts, } 2 . \\
\text { Entgegenrechnung höherer Gesundheitskosten }\end{array}$ \\
\hline $\begin{array}{l}\text { Steigerung internatio- } \\
\text { nale Wettbewerbsfä- } \\
\text { higkeit }\end{array}$ & $\begin{array}{l}\text { Exogene Abbildung der Effekte über Experteninter- } \\
\text { views und Literatur. Dabei ergeben sich wenig Hinwei- } \\
\text { se für deutliche Unterschiede zwischen Biotechnologie } \\
\text { und Substitut. Die Auswirkungen von größeren Ver- } \\
\text { schiebungen werden in Sensitivitätsrechnungen be- } \\
\text { trachtet. }\end{array}$ \\
\hline $\begin{array}{l}\text { Kauf } \\
\text { tions }\end{array}$ & $\begin{array}{l}\text { Einbezug unter Annahme einer Preissenkung und Nut- } \\
\text { zung von Preis-/Einkommenselastizitäten aus der Lite- } \\
\text { ratur. Die alternative Möglichkeit der Kompensation } \\
\text { über höhere Löhne werden implizit (ohne Distributi- } \\
\text { onseffekte) in der Sensitivitätsrechnung mit durch- } \\
\text { schnittlichem Konsumvektor angenommen. Mögliche } \\
\text { Profitänderungen sowie Veränderungen der Sparquote } \\
\text { werden nicht betrachtet. }\end{array}$ \\
\hline $\begin{array}{l}\text { Maschine } \\
\text { lungsargu }\end{array}$ & $\begin{array}{l}\text { Keine Endogenisierung und Betrachtung des Endnach- } \\
\text { frageerweiternden Effekts der Investitionen. Verände- } \\
\text { rung der durchschnittlichen Investitionshöhe/-struktur } \\
\text { werden aber in den Fallstudien betrachtet und fließen } \\
\text { in die Struktureffekte mit ein. }\end{array}$ \\
\hline $\begin{array}{l}\text { Struktureffekte } \\
\text { (veränderte Beschäf- } \\
\text { tigungs- und Import- } \\
\text { intensitäten) }\end{array}$ & $\begin{array}{l}\text { Unterschiedliche Beschäftigungs- und Importintensitä- } \\
\text { ten im I-O-Modell zeichnen Struktureffekte nach, die } \\
\text { sich durch Veränderungen der Wertschöpfungsketten } \\
\text { (z.B. Rohstoffwechsel) ergeben. }\end{array}$ \\
\hline
\end{tabular}

Quelle: Eigene Darstellung 
Viele Argumente können grundsätzlich in die Betrachtung einfließen, aber es müssen vereinfachende Annahmen getroffen werden. Bspw. werden nur bestimmte mögliche Wirkungsweisen auf der Nachfrageseite berücksichtigt. Im Folgenden werden die Wirkungszusammenhänge aus Modellsicht (Inputkoeffizienten, Nachfrage) dargestellt und jeweils die Datenerhebung sowie die Modellierung diskutiert. Anhand dessen kann auf die in Tabelle 5 dargestellte Berücksichtigung der Wirkungsmechanismen geschlossen werden. Zunächst erfolgt dabei die Bestimmung der Höhe der Diffusion sowie der möglichen Unterschiede der Inputkoeffizienten der Biotechnologie zu den bisherigen Produktionsprozessen. Im Anschluss werden die verschiedenen Effekte auf die Nachfragehöhe und -struktur dargestellt. Bei der Nachfrage wird zwischen einzelnen Nachfragekomponenten (z.B. Endnachfrage) unterschieden.

\section{Diffusion und Inputkoeffizienten}

Die Bestimmung möglicher Diffusionspfade einer Technologie ist mit erheblichen Unsicherheiten verbunden. Dies gilt besonders für die Biotechnologie, da neben der Entwicklung möglicher wirtschaftlicher Einflussfaktoren auch weiterhin hohe Unsicherheiten bei der technologischen Wirkungsweise (z.B. Nebeneffekte bei Medikamenten) und Akzeptanz bestehen. Da eine Modellierung für das Jahr 2020 und nicht im Zeitablauf erfolgt, ist keine Diffusionskurve, sondern ein möglicher Diffusionsgrad bis zum Jahr 2020 zu bestimmen. Hierzu werden Hypothesen zur möglichen Verbreitung auf Basis von Studien in Expertengesprächen diskutiert. Eine Ausnahme bildet Bioethanol. Hier bestimmt sich die Diffusion aus der Annahme der Erreichung aktueller politischer Ziele. Als Diffusionsindikator wird der Wertanteil der biotechnologischen Produktion im Anwendungsfeld verwendet, dessen Stärken und Schwächen in Kapitel 4.2.3.2 diskutiert wurden.

Die Diffusion der Biotechnologie kann den Prozess-Mix des Anwendersektors auf drei unterschiedliche Arten verändern (Faber et al. 2007). Die explizite Herausstellung dieser unterschiedlichen Effekte ist wichtig, ${ }^{127}$ da sie unterschiedlichen Wirkungsmechanismen von Technologien auf Produktion und Beschäftigung zuzuordnen sind. Es werden sowohl die Produktivitätssteigerungen als auch verschiedene sektorale Struktureffekte abgebildet.

- Erstens führt eine Effizienzsteigerung im Sinne der Totalen Faktorproduktivität zu einem geringeren Bedarf an allen Inputs bei einem gegebenen Output oder zu einem höheren Output bei gegebenen Inputs. Für die Bestimmung der Inputkoeffizienten wird dabei ersterer Fall betrachtet, letzterer Fall kann sich nur in Kombination mit einer Nachfrageerhöhung ergeben. Die Steigerung der Totalen Faktorproduktivität kann insbesonde-

${ }^{127}$ Zudem ist zu vermuten, dass sich durch die differenzierte Betrachtung der Einzeleffekte furr die Experten einfacher zu beantwortende Fragen ableiten lassen. 
re dann eintreten, wenn die Biotechnologie den Produktionsprozess grundlegend ändert. Als prototypisches Beispiel gilt die Produktion von Riboflavin (Vitamin B2). Während dieses Vitamin lange Zeit in sechs chemischen Schritten hergestellt wurde, ist mittlerweile durch den Einsatz biotechnologischer Methoden nur noch ein Verarbeitungsschritt notwendig. Durch diese Vereinfachungen des Prozesses sinkt der Bedarf an allen Produktionsfaktoren (IPTS 2007; Hoppenheidt et al. 2005). Infolge dieser Produktivitätssteigerungen können Kostensenkungen und Nachfragerhöhungen eintreten.

- Zweitens kann eine Anwendung der neuen Technologie auch zu einem geringeren Bedarf an einem spezifischen Input führen, z.B. einem niedrigeren Energiebedarf durch den Einsatz von Enzymen. Auch hier sind als Folge Kostensenkungen und Nachfrageerhöhungen möglich.

- Eine dritte Möglichkeit bildet die Substitution von Vorleistungsmaterialien, z.B. der Einsatz von nachwachsenden Rohstoffen statt Erdöl. Hier treten keine weitere Folgewirkungen (z.B. Kostensenkungen) auf, es handelt sich um reine Struktureffekte. ${ }^{128}$

Dabei ist zu beachten, dass sich in einigen Anwendungsfeldern (z.B. Bioethanol, Biopharmazeutika) auch der Bedarf an einzelnen oder allen Inputs erhöhen kann. Daneben kommt es beim Einsatz der Biotechnologie nicht selten simultan zur Realisierung aller drei geschilderten Wirkungszusammenhänge. Bspw. kann der Einsatz von Enzymen zu einem geringeren Rohstoffbedarf bei gleichzeitigem Wechsel der Rohstoffbasis führen, aber auch mit weniger Produktionsstufen den Gesamtprozess verbessern.

Die benötigte Vorleistungsfrage einer neuen Technologie kann sich dabei von dem Produkt-Mix der Zuliefersektoren unterscheiden. Die Inputkoeffizienten der Zuliefersektoren, welche den Durchschnitt der Produktionsprozesse widerspiegeln, sind in einem solchen Falle zur Abbildung der Wertschöpfungsprozesse nur bedingt geeignet. Deshalb wäre die spezifische Untersuchung der gesamten Wertschöpfungsketten ideal, um diese Veränderungen zu berücksichtigen. Allerdings sind die Möglichkeiten der Untersuchung von weiteren Veränderungen in Vorleistungsstufen aufgrund des hohen Aufwandes begrenzt. Meist wird hiervon komplett abstrahiert, da die Auswirkungen auf das Gesamtergebnis in der Regel recht gering einzuschätzen sind (Holub/Schnabl 1994, S.612). Allerdings trifft die geringe Bedeutung nicht generell $\mathrm{zu}$, deshalb sollte eine solche Abstrahierung sorgfältig für den Einzelfall geprüft werden (Walz 2002). Für die betrachteten Anwendungsfelder dieser Arbeit ergeben sich aber nur wenige Hinweise, dass sich die benötigten Vorleistungsgüter deutlich vom Produkt-Mix des dazugehörigen Vorleistungssektors unterscheiden. Beispielsweise fällt die

${ }^{128}$ Diese Aussage trifft nur zu, wenn die monetäre Höhe beim Einsatz der beiden Rohstoffe gleich ist. Falls sie unterschiedlich ist, trifft der erste oder zweite Fall zu. 
Nachfrage nach Kapitalgütern bei der biotechnologischen Produktion quantitativ kaum ins Gewicht. Eine andere Einschätzung ergibt sich nur für den Sektor Landwirtschaft. Dieser Sektor hat eine sehr hohe Bedeutung als Zulieferer für den Einsatz der Biotechnologie bei der Herstellung von Ethanol und Biopolymeren. So führt der Einsatz der Biotechnologie in der Chemie zu einer erhöhten Nachfrage nach nachwachsenden Rohstoffen (z.B. Weizen). Diese zusätzliche Nachfrage in der Landwirtschaft unterscheidet sich aber vermutlich von der bisherigen durchschnittlichen Produktionsstruktur der Landwirtschaft, in die auch die arbeitsintensive Viehhaltung und Sonderkulturen eingehen. Die deshalb notwendigen Anpassungen der Inputkoeffizienten werden in der Fallstudie zu Bioethanol (Kapitel 6.1) erläutert.

\section{Konsumnachfrage}

Die Auswirkungen technologischen Wandels auf die Konsumnachfrage wurden in der Theorie im Rahmen der Diskussion über Produktinnovationen und dem Kaufkraftkompensationsargument erörtert (Kapitel 2.3). Die Abbildung von Produktinnovationen stellt eine schwierige Herausforderung für die Modellierung dar, so auch in der I-O-Analyse (Petit 1995; Pianta 2005). Besonders die Möglichkeit der Abbildung des Endnachfrageerweiternden Effekts von Produktinnovationen ist schwierig. Im Rahmen des verwendeten Modells wäre die exogene Festlegung einer Erhöhung recht willkürlich, hätte aber gleichzeitig einen sehr großen Effekt auf die Ergebnisse. Deshalb werden in den Szenarienrechnungen Produktinnovationen nur mit einer substitutiven Wirkung modelliert. ${ }^{129}$ Die Höhe der Produktinnovationen wird aus der Diffusion der Biotechnologie und dem geschätzten Gesamtwachstum des Sektors abgeleitet. Als Ausgleich zur steigenden Nachfrage der Produktinnovationen wird in einer „Gegenbilanzierung" eine sinkende Nachfrage des privaten Konsums nach anderen Gütern angenommen. Folglich werden nur Strukturveränderungen durch Produktinnovationen betrachtet. Um die Bedeutung dieser restriktiven Annahmen zu untersuchen, wird für Biopharmazeutika - dem einzigen untersuchten Anwendungsfeld in dem additive Produktinnovationen von hoher Relevanz sind - eine detaillierte Sensitivitätsrechnung durchgeführt, bei der von einer additiven Wirkung ausgegangen wird.

Eine grundsätzlich ähnliche Vorgehensweise erfolgt bei Prozessinnovationen: Produktivitätserhöhungen durch Prozessinnovationen können im Rahmen des Kaufkraftkompensationsarguments unterschiedliche Folgewirkungen haben. Neben steigenden Unternehmensprofiten und möglichen Folgewirkungen ist besonders eine Nachfrageveränderung aufgrund von Lohn- oder Preisänderungen denkbar. In der Realität wird es vermutlich zu einem Ineinandergreifen der ver-

129 Analog gehen z.B. Nathani (2003), Edler (1990), Walz et al. (2001), Neuwahl et al. (2008), Leontief/Duchin (1986) vor. 
schiedenen Wirkungsmechanismen kommen (Nathani 2003). ${ }^{130}$ Dabei sind auch Konsumänderungen durch mögliche Verschiebungen in der Einkommensverteilung denkbar. Diese Vielfältigkeit der Nachfrageeffekte kann im Rahmen eines I-O-Modells nur eingeschränkt oder mit sehr hohem Aufwand modellendogen abgebildet werden. Da dieses grundlegende Problem auch nicht im Mittelpunkt der vorliegenden Arbeit steht, soll lediglich eine vereinfachende Abschätzung der Effekte vorgenommen werden, die aber dennoch einer plausiblen Wirkungskette folgt und die Größenordnung und Richtung der Effekte erfasst. Dabei werden alle Senkungen bzw. Erhöhungen des monetären Gesamtinputbedarfs als Kostensenkung bzw. -erhöhung interpretiert. ${ }^{131}$ Die Folgeeffekte dieser Veränderungen der Kostenstrukturen werden als Erhöhung (Senkung) der Endnachfrage aufgrund sinkender (höherer) Preise dargestellt. Zur Abschätzung der Wirkungen der Preisänderungen werden Preiselastizitäten und Einkommenselastizitäten der Nachfrage berücksichtigt. Dies gilt allerdings nur für die inländische Nachfrage. So führen bspw. Preissenkungen, die durch Exporte im Ausland realisiert werden, annahmegemäß zu keiner höheren Nachfrage im Inland.

Die zusätzlichen (bzw. geringeren) Konsumausgaben werden mittels Preiselastizitäten für den Substitutionseffekt und eines marginalen Konsumvektors für den Einkommenseffekt berechnet. Bei dem marginalen Konsumvektor wird nicht der bisherige durchschnittliche Konsum angenommen, sondern versucht Sättigungsgrenzen $\mathrm{zu}$ berücksichtigen. $\mathrm{Da}$ für Deutschland keine geeigneten Werte in der Literatur zu Preis- und Einkommenselastizitäten für Produktgruppen existieren, wurden Schätzwerte von Neuwahl et al. (2007) für die EU-15 verwendet. Diese nur für hoch aggregierte Produktgruppen (z.B. Landwirtschaft, Kraftstoffe) verfügbaren Elastizitäten wurden für die jeweils zugehörigen I-OSektoren als identisch angenommen (Anhang Tabelle 32). Die Modellierung der Konsumnachfrage ist in Abbildung 12 zusammengefasst. Die in den Szenariomodellierungen nicht berücksichtigten additiven Produktinnovationen und Profite sind dabei kursiv dargestellt.

\footnotetext{
${ }^{130}$ Carter (1990) schlägt zudem vor, bei der Analyse der Folgeeffekte von Innovationen zwischen Sektoren zu unterscheiden, die Kostensenkungen aufgrund ihres Wettbewerbsumfelds als Preissenkungen weitergeben müssen und solchen, die damit eine höhere Wertschöpfung erzielen können. Diese Methode ist bisher jedoch nicht empirisch umgesetzt worden, da die benötigten Daten nur schwierig zu gewinnen sind (Nathani 2003, S.87).

${ }^{131}$ Wie bereits erläutert, können mögliche (eingesparte) Folgekosten durch Auswirkungen auf die Gesundheit der Bevölkerung oder ökologische Folgen dabei nicht berücksichtigt werden. Diese Kosten sind bislang keinesfalls absehbar und werden kontrovers diskutiert (siehe u.a. Rosenberg-Yunger et al. 2008; IPTS 2007).
} 


\section{Abbildung 12: Modellierung der Auswirkungen von Produkt-/Prozess- innovationen auf die Nachfrage}

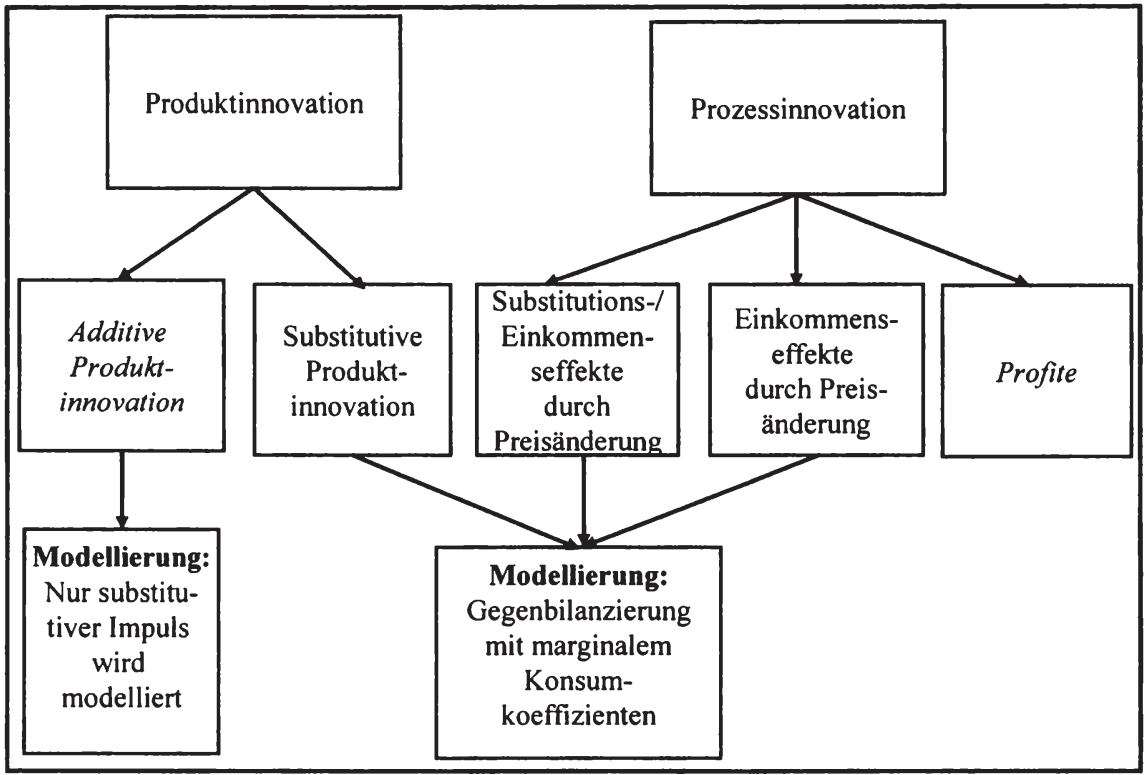

Quelle: Eigene Darstellung

In Sensitivitätsrechnungen wird diese gewählte Variante des Konsumvektors überprüft. Als Alternative wird die in der Literatur (z.B. Nathani 2003; Edler 1990) häufig verwendete einfache Variante eines vollkommenen preisunelastisches Nachfrageverhaltens angewandt und dabei ein Konsumvektor unterstellt, der dem Durchschnitt aus den aktuellsten I-O-Tabellen entspricht. In anderen Worten wird jeglicher Mehr- oder Minderkonsum nach dem bisherigen Durchschnitt der privaten Endnachfrage auf die Sektoren verteilt.

\section{Staatsnachfrage}

Die Staatsnachfrage kann im Rahmen verschiedener Fiskaleffekte von der Diffusion der Biotechnologie tangiert sein. Veränderungen der Staatseinnahmen sind, z.B. bei einer Unterstützung der Diffusion durch Steuerbefreiungen, bei Mehrwertsteuern durch Mengenänderungen oder durch staatlich finanzierte Mehrkosten für biotechnologische Medikamente, denkbar. Aus der hier verfolgten gesamtwirtschaftlichen Perspektive ${ }^{132}$ werden diese fiskalischen Effekte un-

132 Der genaue Fördermechanismus und die Vorteilhaftigkeit bestimmter Förderalternativen stehen somit nicht im Blickpunkt dieser Arbeit. 
ter dem Aspekt des Budgeteffekts diskutiert (z.B. Zeddies 2006): Förderbedingte Steuerausfälle oder Mehrausgaben für Biopharmazeutika müssen beim Staat durch Steuermehreinnahmen oder -minderausgaben an anderer Stelle kompensiert werden. Diese führen jeweils analog zu Preiseffekten zu geringeren privaten Konsumausgaben. Weitere fiskalische Veränderungen, wie z.B. veränderte Einnahmen aus der Mehrwertsteuer, werden nicht betrachtet. Schließlich ergeben sich kaum Hinweise, dass diese Effekte eine wichtige Rolle für die Höhe der Auswirkungen der Biotechnologie haben.

\section{Investitionsnachfrage}

Die Investitionsnachfrage wird in verschiedenen Studien endogenisiert, um das Maschinenherstellungsargument empirisch zu überprüfen (z.B. Edler 1990, Kalmbach et al. 1989). In der vorliegenden Arbeit werden die Investitionen allerdings vereinfachend als exogen angenommen. Hierfür sind mehrere Gründe ausschlaggebend:

- Erstens existieren bei der sehr aufwändigen Endogenisierung der Investitionen im I-O-Modell erhebliche Stabilitätsprobleme (Nathani 2003), die zu wenig plausiblen Ergebnissen führen können.

- Zweitens gibt es in der amtlichen Statistik für Deutschland keine Kapitalstockmatrizen, welche die sektoralen Lieferbeziehungen von Investitionen wiedergeben. Eine eigene Schätzung wäre mit erheblichen, zusätzlichen Annahmen verbunden.

- Drittens erscheint die Unterschätzung des zusätzlichen Nachfrageeffekts durch die Investitionen begrenzt zu sein, da die Erörterung in Kapitel 3.3 wenig Hinweise für ein hohes gesamtwirtschaftliches Ausmaß zusätzlicher Investitionen durch die Biotechnologie gab. Eine direkte Substitution von Arbeit durch Kapital infolge des Einsatzes der Biotechnologie scheint von geringerer Relevanz zu sein (Nusser et al. 2007a). Vielmehr stehen den Investitionen in Anlagen für die Biotechnologie vermutlich häufig entfallene Investitionen für konventionelle Technologien gegenüber. Schließlich gibt es auch wenig Hinweise dafür, dass der Kapitalstock durch die Biotechnologie schneller erneuert wird, stattdessen vollzieht sich die Diffusion eher langsam (Nusser et al. 2007c).

Für die einzelnen Fallstudien werden die im dritten Punkt genannten Zusammenhänge genauer erörtert und exogen bestimmt, inwiefern sich Implikationen für zusätzliche Investitionen und/oder eine veränderte Investitionsstruktur aus der Nutzung der Biotechnologie ergeben. Bei der Modellierung wird der Nachfrageeffekt berücksichtigt, indem eine durchschnittliche jährliche Höhe des Investitionsvolumens auf Basis von Literatur und Expertenaussagen ermittelt wird 
und als Nachfrageimpuls im I-O-Modell simuliert wird. ${ }^{133}$ Der Effekt der Investitionen wird allerdings nicht als Erweiterung der Endnachfrage betrachtet, sondern die Investitionsvolumina fließen in die Kostenbetrachtung zur Bestimmung von kompensatorischen Nachfrageimpulsen mit ein. Da wie oben erläutert, keine Kapitalstockmatrizen existieren, sondern in den I-O-Tabellen nur die liefernden Sektoren der Investitionen mit entsprechenden Volumen aufgelistet sind, müssen zusätzliche Annahmen für die sektorale Aufteilung der Investitionen getroffen werden. ${ }^{134}$ Auf Basis von Informationen aus der Literatur und Expertengesprächen wird analog zu den I-O-Tabellen zwischen Anlagen- und Bauinvestitionen unterschieden. Für die Anlagen- und Bauinvestitionen wird der Durchschnitt der Investitionslieferungen zugrunde gelegt und leicht modifiziert. So wurden Sektoren, die offenkundig keine Rolle als Investitionslieferant für die relevanten Sektoren spielen (z.B. der Sektor „Möbel, Schmuck, Musikinstrumente, Sportgeräte, Spielwaren u.Ä.") ausgeschlossen. Bei den entfallenen Investitionen für die ersetzten Produkte wird angenommen, dass diese zumeist in in bereits bestehenden Anlagen produziert werden. Folglich werden für die Substitute nicht die Investitionskosten für eine Neuanlage angesetzt, sondern nur jährliche Abschreibungen. Diese ergeben sich hauptsächlich aus den deutschen I-O-Tabellen für das Jahr 2005.

\section{Exportnachfrage}

Die Exportnachfrage und die Importe nach biotechnologischen Produkten sowie deren Vorleistungen werden ebenfalls exogen durch Literaturanalysen und Expertenbefragungen bestimmt. Mögliche Veränderungen in der Wettbewerbsfähigkeit durch den Einsatz der Biotechnologie werden in Sensitivitätsrechnungen analysiert. Hingegen ist eine kausale modellendogene Bestimmung der Standortentscheidungen und Exportnachfrage in einem nationalen Modell, welches im Rahmen dieser Arbeit verwendet wird, nicht möglich. Hierfür wäre neben einer genaueren Differenzierung des internationalen Handels mit einzelnen Ländern auch die Bestimmung der Diffusion und Auswirkungen der Biotechnologie für diese Länder notwendig (Kalmbach et al. 1989, Kapitel 4.3.4.2).

\subsubsection{Bildung von Szenarien}

\subsubsection{Wahl der Szenarien}

Als Grundlage für die Modellrechnungen werden Szenarien definiert. Die Szenariotechnik ist eine Methode zur konsistenten Analyse zukünftiger Ent-

\footnotetext{
${ }^{133}$ Für ein analoges Vorgehen siehe z.B. Nusser et al. (2007d) und Wicke (2006).

134 Im Gegensatz zur oben erläuterten Endogenisierung der Investitionen, sind diese Schätzungen und Annahmen nicht für alle Sektoren der Volkswirtschaft, sondern jeweils nur für das spezifische Anwendungsfeld und jeweilige Substitut, notwendig.
} 
wicklungen. Szenariobetrachtungen sind besonders bei komplexen Systemen mit vielen Einflussfaktoren und hohen Unsicherheiten hinsichtlich der künftigen Entwicklung der beschriebenen Einflussfaktoren angebracht. Sie sind im Gegensatz zur Prognose aber keine Vorhersagen über wahrscheinliche, zukünftige Entwicklungen, sondern stellen Bündel von konsistenten Annahmen zu Umfeldveränderungen, Entwicklungstrends und Maßnahmen dar (Gausenmeier et al. 1997). Dabei werden häufig mehrere alternative Szenarien entwickelt. Für diese einzelnen Szenarien bzw. Annahmebündel werden im Rahmen dieser Arbeit die Produktions- und Beschäftigungseffekte der Biotechnologie simuliert.

Die Art und Weise, wie der Untersuchungsgegenstand bei einer Szenarioanalyse abgebildet (modelliert) wird und welche beeinflussenden Kausalitäten zwischen den Einflussfaktoren berücksichtigt werden, entscheidet wesentlich über den notwendigen Aufwand bei der Szenarioentwicklung. Bei der Abbildung des Untersuchungsgegenstandes wird im Allgemeinen zwischen dem Gegenstandsbereich und dem Umfeldbereich unterschieden (Gausenmeier et al. 1997). Der Gegenstandsbereich ist der im Rahmen der Szenarienanalyse eigentlich interessierende Teil der Realität. In dieser Studie ist dies die Bereitstellung und Anwendung der Biotechnologie in den unterschiedlichen Anwendungsfeldern und die daraus resultierenden wirtschaftlichen Effekte. Der Umfeldbereich repräsentiert zunächst den „Rest der Welt" und ist das Komplement des Gegenstandsbereichs. Eine umfassende Anwendung der Szenariotechnik würde die Berücksichtigung unterschiedlicher Ausgestaltungen des Umfeldbereichs erfordern, d.h. z.B. die Bestimmung politischer, wirtschaftlicher und sozialer Einflussfaktoren für einzelne Szenarien (Paulus 2005). Allerdings sind die Möglichkeiten der Berücksichtigung dieser Einflussfaktoren des Umfeldbereichs unter Wahrung der internen Konsistenz begrenzt, zumal der Zusammenhang zwischen diesen Faktoren auf erheblichen zusätzlichen Annahmen beruht. Zudem können für die Berechnung des Nettoeffektes überlagernde Entwicklungen störend wirken, da der Effekt der Technologie nicht mehr isoliert betrachtet werden kann, sondern möglicherweise von anderen Faktoren dominiert wird (Kalmbach et al. 1989, Neuwahl et al. 2008). Deshalb wird der Umfeldbereich auf die wesentlichen Randbedingungen reduziert, die für den Einsatz und das Ausmaß der Effekte der Biotechnologie von Bedeutung sind und für alle Szenarien eine identische Entwicklung des gesamtwirtschaftlichen Umfelds unterstellt. ${ }^{135}$

Der Schwerpunkt der Szenariobetrachtung liegt aber auf dem Gegenstandsbereich, also den Auswirkungen der Biotechnologie auf Produktion und Beschäftigung. Hierbei ist die Betrachtung der Entwicklung der Faktoren, die sich entscheidend auf Diffusion, Weiterentwicklung und wirtschaftlichen Effekte der

${ }^{135}$ Für den Versuch und die Diskussion über die Verbindung unterschiedlicher Szenarien für den Umfeldbereich mit einer I-O-Analyse siehe Wilting et al. (2008). 
Biotechnologie auswirken, von Bedeutung. ${ }^{136}$ Dabei werden alternative Annahmen für zentrale Einflussfaktoren angenommen. ${ }^{137}$ Insgesamt werden drei Szenarien sowie einige Sensitivitätsrechnungen erstellt (Abbildung 13). Diese werden im Folgenden kurz charakterisiert. Die im Einzelnen getroffenen Szenarienannahmen des Umfeld- und Gegenstandsbereiches werden anschließend in Kapitel 5.2.3.2 dargestellt.

\section{Abbildung 13: Vorgehen der Szenariobildung}

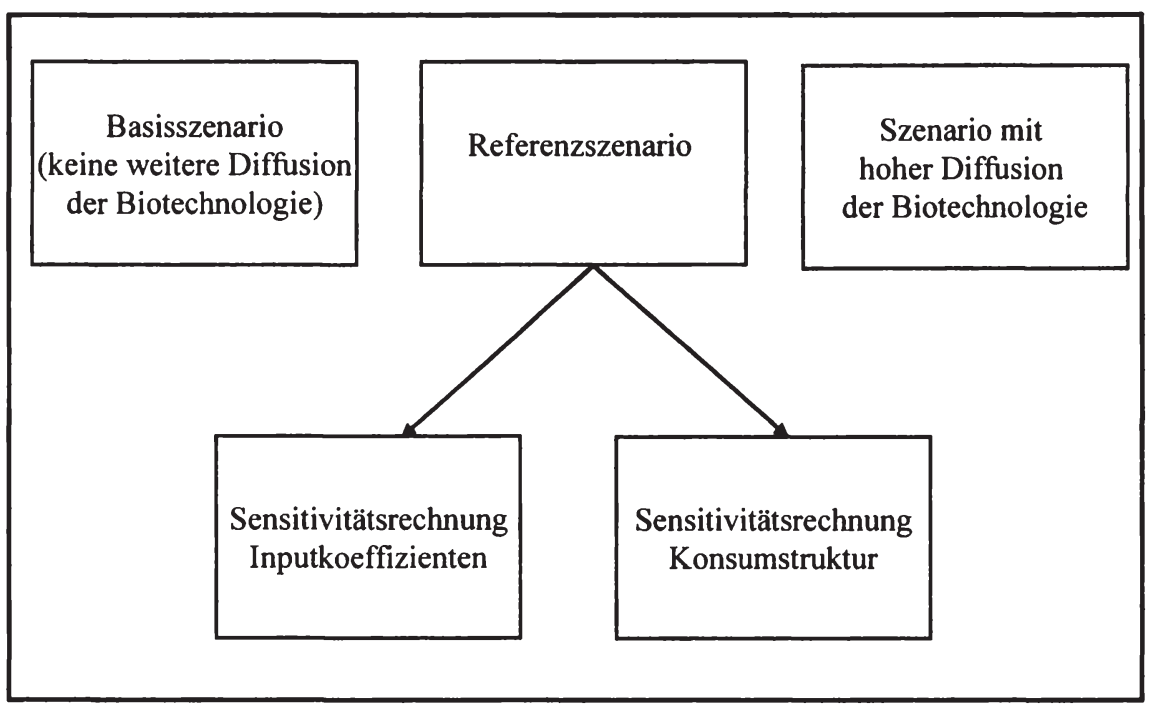

Quelle: Eigene Darstellung

\section{Basisszenario}

Um mögliche Nettoeffekte in den Szenarien zu bestimmen, ist ein Vergleichs$\mathrm{maßstab}$ für diese Zukunftsbilder notwendig. Hierfür wird das Basisszenario gebildet. Gemäß dem Untersuchungsziel der Effekte durch die Diffusion und Weiterentwicklung der Biotechnologie bis zum Jahr 2000 wird dabei keine weitere Biotechnologiediffusion angenommen. Da dies eine recht unrealistische An-

${ }^{136}$ Für die substituierten Konkurrenztechnologien werden keine unterschiedlichen Entwicklungspfade betrachtet, sondern eine Referenzentwicklung, die in den Szenarien nur durch die unterschiedlichen Rohstoffpreise differiert. Da in den meisten Anwendungsfeldern die Konkurrenztechnologie als recht ausgereift gilt, sind nur geringe Anpassungen für das Jahr 2020 notwendig.

${ }^{137}$ Insbesondere in der im Rahmen dieser Arbeit erfolgenden Befragung ergäbe sich sonst das Problem, dass der Experte nur in begrenztem Maße vorgegebene Szenarioüberlegungen berücksichtigen kann. 
nahme darstellt, ist das Basisszenario als Analyseinstrument und nicht als Zukunftsbild mit eigenständigem Interpretationswert zu betrachten. ${ }^{138}$ Es dient als Vergleichsmaßstab dazu, die Höhe der Substitutionseffekte (z.B. zusätzliche Mehrausgaben an Gesundheitskosten durch Biopharmazeutika) zu bestimmen und mögliche Größenordnungen der Veränderungen durch die Biotechnologie gegenüber dem heutigen Stand der Technik zu verdeutlichen. Abgesehen von der Biotechnologiediffusion gelten dabei die gleichen Grundannahmen wie im Referenzszenario.

\section{Referenzszenario}

Das Referenzszenario soll eine „Business as Usual“-Entwicklung widerspiegeln. Es wird eine gewisse Diffusion der Biotechnologie unterstellt, die ohne besondere zusätzliche Anstrengungen zur Beeinflussung der Technologieentwicklung eintreten kann. Der Term „Referenz" bezieht sich demnach auf die biotechnologische Entwicklung. Der Vergleich von Referenz- zu Basisszenario soll es ermöglichen, wirtschaftliche Substitutionseffekte (z.B. Ersetzung der Rohstoffbasis) zu identifizieren, die durch die biotechnologische Entwicklung und nicht durch andere Faktoren beeinflusst werden.

\section{Szenario mit hoher Diffusion (HD-Szenario)}

Unter günstigen Rahmenbedingungen (z.B. hoher Ölpreis, politischen Fördermaßnahmen) ist eine höhere Diffusion der Biotechnologie als im Referenzszenario denkbar. Um die Auswirkungen einer schnelleren Verbreitung der Biotechnologie für die inländische Produktion und Beschäftigung zu untersuchen, wird deshalb das Szenario „Hohe Diffusion“ (HD-Szenario) gebildet. In diesem Szenario werden günstigere Entwicklungen im Gegenstandsbereich gegenüber dem Referenzszenario angenommen. Dies führt annahmegemäß sowohl zu einer höheren Diffusion als auch einem größeren technologischen Fortschritt (z.B. höhere Energiekosteneinsparung) der Biotechnologie. Schließlich sind aufgrund von Größenskaleneffekten sowie Innovationseffekten höhere Effizienzgewinne im Szenario mit einer hohen Diffusion zu erwarten (Faber et al. 2007; Kapitel 2.2.3). Die konkrete Parameteranpassung wird in den Fallstudien explizit erwähnt (Kapitel 6).

\section{Sensitivitätsrechnungen}

Bei den gewählten Szenarien ist zu beachten, dass sich auch unter konkret definierten Annahmen für ein Szenario, Bandbreiten und Unsicherheiten für die zu wählenden Modellparameter ergeben. Für wichtige Modellparameter werden in Sensitivitätsberechnungen Ober- und Untergrenzen für mögliche Werte gebildet und deren Effekte modelliert. Alle anderen Parameter werden identisch zum Re-

${ }^{138}$ Für eine analoge Vorgehensweise siehe Neuwahl et al. (2007). 
ferenzszenario gewählt. Dabei werden für alle Anwendungsfelder die Auswirkungen von alternativen Annahmen für die Diffusion, Kostenhöhe und Außenhandel und der oben erläuterten Alternativvariante der Konsumstruktur berechnet. Darüber hinaus erfolgt je nach Anwendungsfeld noch eine spezifische Sensitivitätsrechnung, z.B. die Annahme einer alternativen Kostenstruktur bei Bioethanol.

\subsubsection{Szenarioannahmen}

Für diese einzelnen Szenarien werden spezifische Annahmen getroffen. Die jeweiligen Annahmen gelten für alle Anwendungsfelder, um eine möglichst konsistente Betrachtung der Effekte zu gewährleisten. Daneben sind zu einem kleinen Teil fallspezifische Annahmen notwendig. Dies betrifft vor allem spezifische Politikmaßnahmen (z.B. gesetzliche Festschreibung einer Bioethanolquote), welche in den konkreten Fallstudien in Kapitel 6 erörtert werden. Im Folgenden werden die fallübergreifenden Annahmen dargestellt. Als Ausgangsjahr für alle Berechnungen wird dabei das Jahr 2005 gewählt, da sich auch die aktuellsten deutschen I-O-Tabellen auf dieses Jahr beziehen.

\section{Gesamtwirtschaftliche Annahmen}

Die folgenden gesamtwirtschaftlichen Annahmen stellen den Umfeldbereich dar und sind für alle Szenarien gleich. Es werden dabei für diejenigen Parameter explizite Annahmen getroffen, die für das Ergebnis von besonderem Interesse sind:

- Um eine Überschätzung der Beschäftigungseffekte möglichst zu vermeiden, werden Produktivitätszuwächse für die an das Modell gekoppelte Beschäftigungskoeffizienten berücksichtigt. Auf Basis von Produktivitätskennziffern zwischen dem Jahr 1995 und 2005 werden Produktivitätsfortschritte auf die Zeitperiode 2005-2020 fortgeschrieben. ${ }^{139}$

- Beim Produktionsvolumen wird eine partielle Projektion für die betrachteten Anwendungsfelder durchgeführt. Grundlage für die unterstellten Wachstumsraten zwischen dem Jahr 2005 und 2020 bilden Marktprojektionen, die mit einer Vergangenheitsfortschreibung verglichen werden.

139 Diese Schätzung wurde auf Basis der einheitlich methodisch erhobenen deutschen I-OTabellen des Jahres 1995 und 2005 durchgeführt. Für die Berücksichtigung der Realpreisentwicklung wurden Preisindizes der Volkswirtschaftlichen Gesamtrechnung verwendet (Statistisches Bundesamt 2008a). In ganz wenigen Ausnahmefällen (z.B. Unternehmensbezogene Dienstleistungen) hätten sich bei der Produktivitätsfortschreibung erhebliche Produktivitätsverluste geben. Da dies wenig plausibel ist, wurde hier von einer konstanten Produktivität bis zum Jahr 2020 ausgegangen. 
- Die Bevölkerungszahl in Deutschland wird als relativ konstant angenommen. Dies impliziert u.a. einen in etwa gleich bleibenden Nahrungsmittelverbrauch (siehe unten).

Daneben ist zu klären, wie die I-O-Matrix für das Jahr 2020 formuliert wird. Die Auswirkungen der technologischen Entwicklung außerhalb der Biotechnologie sowie nicht-unmittelbar technisch bedingt Einflüsse auf Koeffizientenveränderungen können, analog zu einer Vielzahl anderer Studien, im Rahmen dieser Arbeit nicht berücksichtigt werden. Neben dem hohen Erstellungsaufwand entstehen bei der Matrizenbestimmung für die Zukunft (z.B. durch Trendexpolationen) erhebliche zusätzliche methodische Schwierigkeiten. Bspw. ließe es sich kaum abgrenzen, ob Teile der Effekte der Biotechnologie durch die Matrizenbestimmung bereits integriert sind (Wilting et al. 2008).

Eine Produktions- und Beschäftigungsprojektion für die komplette Gesamtwirtschaft erfolgt in den Szenarien demnach nicht. ${ }^{140}$ Sie stellen damit kein realistisches Gesamtbild der Produktions- und Beschäftigungssituation im Jahr 2020 dar. Wie in Kapitel 4.3.4 erörtert wurde, ist für die hier erfolgende Nettobetrachtung der verzerrende Effekt durch die eher starren Annahmen aber als begrenzt zu erwarten. Zumal in den Aspekten, in denen deutliche Unterschiede (z.B. Herstellungskosten) zwischen der Biotechnologie und den $\mathrm{zu}$ ersetzenden Technologien zu erwarten sind, genauere Betrachtungen und Anpassungen erfolgen.

Schließlich bilden diese Unterschiede den Gegenstand der Szenariobetrachtung. Neben der Herleitung der I-O-Koeffizienten in den Fallstudien werden die für einige Anwendungsfelder zentralen Inputpreise in den Szenarien unterschieden. So ist der Einsatz der Biotechnologie in den Anwendungsfeldern Bioethanol und Biopolymere und zu kleinerem Teil bei Fein-/Spezialchemikalien eng mit der Substitution von fossilen Rohstoffen (z.B. Öl) durch nachwachsende Rohstoffe (z.B. Weizen, Zuckerrübe) verknüpft. Eine entscheidende Rolle für die Rentabilität dieser Ersetzung spielen allerdings die Preise der Rohstoffe selbst, da sie einen hohen Anteil mit bis zu über $50 \%$ an den Herstellungskosten ausmachen (siehe Kapitel 6.1 und 6.2). Gerade in jüngerer Vergangenheit zeigen sich bei allen dieser Rohstoffe sehr starke Schwankungen mit zwischenzeitlich hohen Anstiegen. Daneben ist für die Bestimmung der inländischen Wertschöpfung im Landwirtschaftssektor zu klären, ob überhaupt ein ausreichendes Angebot für die Nachfrage erfolgen kann. Für die konsistente Bildung von Szenarien ist für alle diese Aspekte vorrangig die langfristige Entwicklung von Interesse. Dabei werden unterschiedliche Annahmen für das HD-

${ }^{140} \mathrm{Da}$ die berechneten Produktivitätssteigerungen ähnlich wie die Wachstumsraten der Produktion liegen - wobei nicht für alle Sektoren explizit Wachstumsraten hergeleitet wurden handelt es sich gegenüber dem Ausgangsjahr 2005 tendenziell um beschäftigungsneutrale Umfeldannahmen. 
Szenario gegenüber dem Referenzszenario getroffen. Das als Vergleichsmaßstab geltende Basisszenario entspricht in den folgenden Annahmen dem Referenzszenario und wird nicht explizit weiter erwähnt.

\section{Ölpreis}

Die historische Entwicklung des Ölpreises ist von unterschiedlichen Phasen geprägt (WBA 2007). In den 1970er Jahren kam es nach einer langen Periode niedriger Energiepreise zu einem ersten starken Preisanstieg (1.Ölkrise), Anfang der 1980er Jahre folgte ein weiterer starker Preisanstieg (2.Ölkrise). Danach sanken die Preise allerdings wieder deutlich ab und verblieben bis zum Anfang des laufenden Jahrzehntes auf einem niedrigem Niveau (Casamassima et al. 2007). Seit 2005 stieg der Preis allerdings erheblich an, zwischenzeitlich auf über $140 \$ / \mathrm{bbl}$ im Juli 2008 (IEA 2008). Allein der durchschnittliche Rohölpreis von $72 \$ / b b l$ im Jahr 2007 stellt ungefähr eine Verfünffachung gegenüber den Rohölpreisen Mitte der 1990er Jahre dar (OECD/FAO 2008). Für die aktuellen Schwankungen werden vielfältige Ursachen diskutiert. Als Hauptgründe gelten neben dem starken Nachfrageanstieg in Ostasien z. B. auch Engpässe durch Ausfall von Förderkapazitäten, OPEC-Beschlüsse sowie Spekulationsgeschäfte (WBA 2007; EWI/Prognos 2006). ${ }^{141}$ Viele Langfristprognosen aus den Jahren 2005 bis 2007 kommen allerdings zu der Einschätzung, dass - ausgehend von Ölpreisen zwischen 60-70 US\$/bbl - mittelfristig nicht mit einem weiteren Anstieg der realen Ölpreise zu rechnen ist (WBA 2007; Casamassima et al. 2007). Schließlich werden bei langfristig höheren Ölpreisen mit einer Dämpfung des Nachfragewachstums (z.B. Substitution durch Biomasse) und erheblichen Ausweitungen des Ölangebots (z.B. durch Rentabilität der Förderung nichtkonventioneller Öle) gerechnet. Gegen einen deutlichen Preisrückgang sprechen vor allem die weiterhin zunehmende Erdölnachfrage in den Entwicklungs- und Schwellenländern und die höheren Förderkosten der noch verbliebenen konventionellen bzw. nicht-konventionellen Erdölreserven. Übersichten des Wissenschaftlichen Beirates für Agrarpolitik (WBA 2007) und Casamassima et al. (2007) zu verschiedenen Langfristprognosen zeigen, dass Referenzprognosen nahezu keinen realen Preisanstieg projizieren und sich in einer Bandbreite von 40-60 US\$/bbl (Realpreise 2005) für das Jahr 2020 bewegen. Hochpreisvarianten einzelner Projektionen liegen bei 70-80 US\$/bbl (z.B. European Commission 2004; EIA 2007). Allerdings liegen die jüngsten Prognosen aus dem Jahr 2008 deutlich über diesen Werten. Die IEA (2008) prognostiziert in ihrem neu-

${ }^{141}$ Insgesamt wird der Ölpreis von einer Vielzahl von angebotsseitigen Faktoren (verfügbare Reserven und Ressourcen, technischer Fortschritt, Angebotselastizitäten, Investitionsverhalten, OPEC-Politik), nachfrageseitigen Faktoren (Bevölkerungs- und BIP-Wachstum, Ölintensität der Produktion, Nachfrageelastizitäten, Substitutionsmöglichkeiten, Wetter/Klima) und sonstigen Faktoren (Umwelt-/Klimapolitik, Krisen und Kriegen, Naturkatastrophen, Marktspekulationen) beeinflusst (EWI/Prognos 2006). 
esten „World Energy Outlook“ für das Jahr 2020 über 105 US\$/bbl in Realpreisen von 2007 , nominal liegt der Wert bei über 150 US\$/bbl.

Die großen Schwankungen in den Projektionen erschweren die Festlegung von Szenariowerten. Das Referenzszenario lehnt sich an die Prognosen mit eher konstanten Realpreisentwicklungen des Ölpreises an. Hieraus. ergibt sich eine Preisfestsetzung von 70 US $\$ / b b l$ in Realpreisen von 2005. Für das HD-Szenario wird der genannte Wert der IEA verwendet, in Realpreisen von 2005 liegt dieser bei ca. $100 \mathrm{US} \$ / \mathrm{bbl}$. Schließlich wird, zumindest für die Bereiche Bioethanol und Biopolymere, ein hoher Ölpreis als ein zentraler Faktor für die Entwicklung und Diffusion der Biotechnologie angesehen (z.B. Crank et al. 2004; Nusser et al. 2007b). Allerdings ist dabei nicht direkt der absolute Ölpreis entscheidend, als vielmehr das relative Preisverhältnis zu den nachwachsenden Rohstoffen, die häufig für biotechnologische Prozesse notwendig sind. Die Festlegung jener Preise wird in den folgenden Teilabschnitten beschrieben.

\section{Exkurs: Angebotsrestriktionen in der Landwirtschaft}

Da die Zusammenhänge und Entwicklungen im Landwirtschaftssektor komplex und vielschichtig sind, werden diese zunächst kurz dargestellt. Dabei steht nicht nur die Frage der Preise für nachwachsende Rohstoffe im Vordergrund, sondern auch, ob das notwendige Angebot an nachwachsenden Rohstoffen überhaupt im Inland erfolgen kann. Denn gerade die Annahme des I-O-Modells von fehlenden Engpässen auf der Angebotsseite, wird für den Landwirtschaftssektor in der Literatur kritisch bewertet (z.B. WBA 2007). ${ }^{142}$ So wird ein erheblicher Nachfrageanstieg nach landwirtschaftlichen Produkten erwartet, das mögliche Angebot ist in diesem Sektor aber durch die verfügbaren Agrarflächen begrenzt (SRU 2007). Deshalb wird beabsichtigt, analog zu anderen I-O-Analysen für landwirtschaftliche Produkte (z.B. Neuwahl et al. 2008; Wicke 2006), diese Engpässe und Anpassungserfordernisse durch zusätzliche exogene Annahmen zu berücksichtigen. Im Folgenden werden kurz einige Kernaspekte der Angebots- und Nachfrageentwicklung skizziert. Im Anschluss wird erörtert, inwieweit diese Entwicklungen im Rahmen der Arbeit erfasst werden und es werden die Preise für nachwachsende Rohstoffe für die Szenarien bestimmt.

In jüngerer Vergangenheit werden nach Abbau von Überschussproduktion in der Landwirtschaft Flächen- und Nutzungskonkurrenzen beim Ackerbau diskutiert (z.B SRU 2007, WBA 2007, WI/RWI 2008). Dabei stehen der Nahrungsmittelproduktion eine steigende Nachfrage für energetische (z.B. Bioethanol, Biogas) und stoffliche Nutzung (z.B. Biopolymere, Schmierstoffe) dieser Pflanzen gegenüber. Bei diesen verschiedenen Verwendungen wird häufig der gleiche

\footnotetext{
${ }^{142}$ Grundsätzlich ist eine Vielzahl von Möglichkeiten von Angebotsrestriktionen (z.B. fehlende Kapitalakkumulation, Materialienengpässe) denkbar. Die Untersuchung potenzieller Restriktionen ist aber in der Regel nur eingeschränkt möglich und wird hier auf den für diese Arbeit zentralen Landwirtschaftssektor beschränkt.
} 
Rohstoff benötigt (z.B. Weizen für Ethanol und Nahrungsmittel) oder es erfolgt eine indirekte Konkurrenz über Zugriff auf die gleiche Ackerbaufläche. Diese Zusammenhänge sind in Abbildung 14 erfasst.

\section{Abbildung 14: Flächen- und Verwendungskonkurrenzen von pflanzlichen Erzeugnissen}

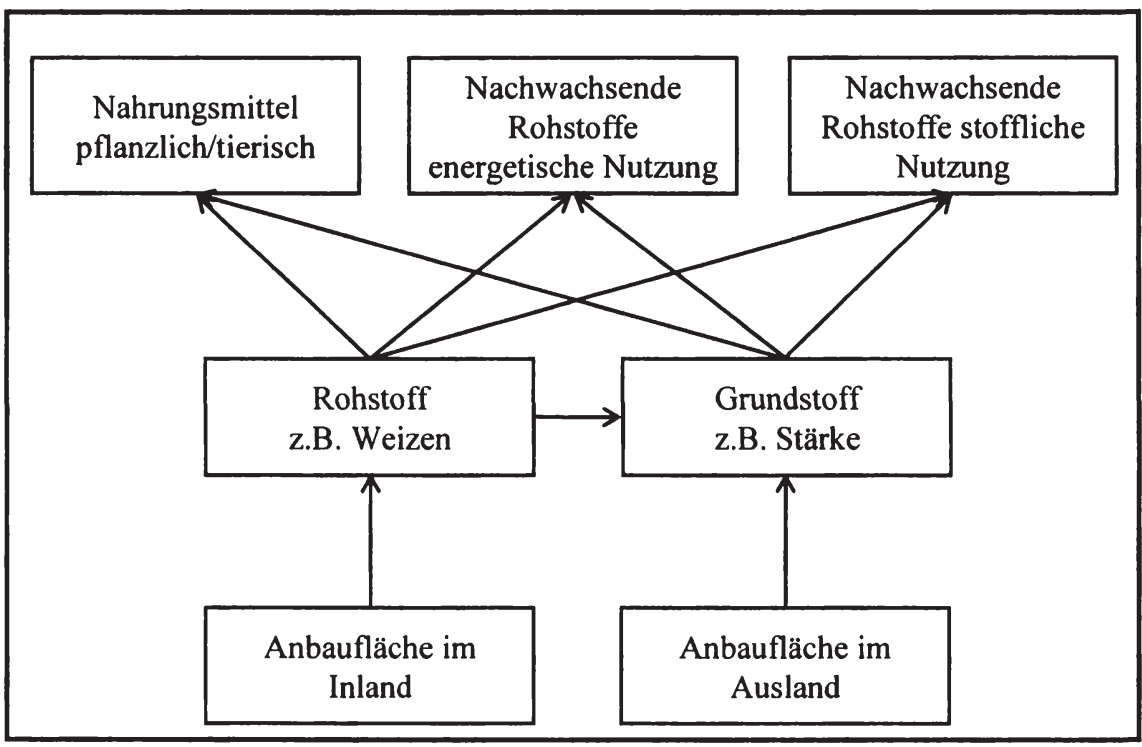

Quelle: Abgeänderte Darstellung von WI/RWI (2008)

Aufgrund dieser vielfältigen Zusammenhänge müssen alle Verwendungen betrachtet werden, um zu klären, ob genug Flächen für die Herstellung von Bioethanol und Biopolymeren im Inland zur Verfügung stehen. Zunächst werden die stoffliche und energetische Nutzung von nachwachsenden Rohstoffen betrachtet und im Anschluss die Verwendung für Nahrungsmittel sowie die Anbaufläche im Inland analysiert.

Die für stoffliche und energetische Verwendungen genutzte Fläche hat in den vergangenen Jahren deutlich zugenommen. ${ }^{143}$ Eine Abschätzung des zukünftigen Flächenbedarfs ist mit großen Schwierigkeiten verbunden, da es sehr viele unterschiedliche Märkte gibt und deren Entwicklungen teilweise stark von der

${ }^{143}$ Der inländische Anbau nachwachsender Rohstoffe ist von ca. 290.000 ha im Jahr 1993 auf über 2 Mio. ha (17\% der Ackerfläche) im Jahr 2007 gestiegen (FNR 2007). Es werden aber auch bereits jetzt große Mengen an nachwachsenden Rohstoffen importiert. Im Jahr 2006 wurden bspw. ungefähr 66 Prozent der für industrielle oder stoffliche Zwecke genutzten nachwachsenden Rohstoffe importiert (Rösch et al. 2008). 
politischen Förderung abhängen. Studien auf Basis einer breiten Marktanalyse des Fachverbands Nachwachsender Rohstoffe schätzen unter Berücksichtigung von steigenden Flächenverträgen ${ }^{144}$ in Szenarien einen inländischen Flächenbedarf von 1,5 Mio. ha im Jahr 2004 auf ca. 2,8 bis 3,5 Mio. ha für den Anbau nachwachsender Rohstoffe (ohne Stroh) im Jahr 2020 (Nusser 2007d; WI/RWI 2008). ${ }^{145}$

Bei den Nahrungsmitteln ist in Deutschland bei einer in etwa gleichbleibenden Bevölkerungszahl und Verbrauch pro Kopf eine ungefähr stabile Nachfrage bis zum Jahr 2020 zu erwarten (Thrän et al. 2005). Bei weiteren Flächenertragssteigerungen würde somit Fläche frei werden, die für andere Verwendungen zur Verfügung stehen würde. Weitere Veränderungen bei der landwirtschaftlichen Flächennutzung sind durch Ausdehnungen der Bewirtschaftung von brach liegenden Agrarflächen oder der Erschließung bislang anderweitig genutzter Flächen (z.B. Wald, Viehland) denkbar (WBA 2007, S.181). Allerdings ist die ausdehnbare Fläche durch Flächenansprüche für den Naturschutz (z.B. flächendeckende Biotopvernetzung) und dem zunehmenden Flächenbedarf von Siedlung und Verkehr eingeschränkt (Rösch et al. 2008). Die Annahmen einzelner Studien zur Einschätzung des Flächenpotenzials für nachwachsende Rohstoffe bis 2020 unterscheiden sich allerdings bezüglich der Flächenertragsentwicklung, dem Selbstversorgungsgrad für Nahrungsmittel, der Beachtung von Naturschutzbelangen sowie den Anteilen von Brachflächen und ökologischer Landwirtschaft. Gemäß einer Übersicht des Sachverständigenrats für Umweltfragen (SRU 2007) liegen die „Business as Usual“-Szenarien von vier weit verbreiteten Studien zwischen 2 und 5,6 Mio. ha verfügbare Fläche für nachwachsende Rohstoffe, ohne dass absolut gesehen eine Flächenkonkurrenz entsteht.

Somit ergeben sich erhebliche Unsicherheiten bei Angebot und Nachfrage von Flächen zur Produktion von nachwachsenden Rohstoffen. In einigen Analysen wird eine deutlich stärkere Zunahme der Nachfrage gegenüber dem Angebotspotenzial festgestellt (z.B. WI/RWI 2008; SRU 2007; IFEU 2007). In anderen Betrachtungen liegt das theoretische Angebotspotenzial über dem projizierten Nachfragebedarf, allerdings ist auch hier bereits eine gewisse Zunahme der

144 Zwar ist teilweise umstritten, inwieweit sich Ertragssteigerungen fortsetzen können (Bringezu/Schütz 2008). Die meisten quantitativen Abschätzungen in der Literatur für das Jahr 2020 liegen aber für die meisten Pflanzen mit Ertragssteigerungen von 1-2\% pro Jahr in einem ähnlichen Bereich (WI/RWI 2008; Thrän et al. 2005; Nusser et al. 2007d). Diesen Größenordnungen an Flächensteigerungen liegen den in diesem Kapitel genannten Flächenzahlen für das Jahr 2020 zugrunde.

${ }^{145}$ Diesen Zahlen liegen Annahmen von hohen zusätzlichen Importen zugrunde. Insgesamt wird diese Entwicklung besonders durch die Nachfrage nach Biodiesel und der Verstromung aus nachwachsenden Rohstoffen insbesondere aus inländischem Maisanbau) getrieben (WI/RWI 2008). Für die im Rahmen dieser Arbeit relevanten nachwachsenden Rohstoffe Weizen und Zuckerrübe ist die zusätzliche Flächennachfrage geringer. 
Importe meist schon berücksichtigt (z.B. Nusser et al. 2007d; Oertel 2007). ${ }^{146}$ Folglich ist durch die Nachfrage von nachwachsenden Rohstoffen von einer $\mathrm{Zu}$ nahme der Importe dieser Rohstoffe auszugehen. Daneben ist auch langfristig eine Verdrängung des inländischen Anbaus anderer Agrargüter (v.a. Nahrungsmittel) denkbar. Durch die weltweite Verschiebung von Angebot und Nachfrage von Agrargütern können gleichzeitig auch Preissteigerungen eintreten. ${ }^{147}$

\section{Annahmen zur Verfügbarkeit inländischer Agrarflächen}

Die Gültigkeit der Annahme des I-O-Modells einer fehlenden Ressourcenknappheit ist demnach nicht aufrecht zu erhalten und wird in Studien mit I-OModellen auf verschiedene Art und Weise (z.B. Preiserhöhungen, Importannahmen) berücksichtigt. ${ }^{148}$ Im Rahmen dieser Arbeit sollen die Folgewirkungen auf die Landwirtschaft auf folgende Weise erfasst werden:

- In den Fallstudien sind teilweise Importannahmen enthalten. Allerdings spielt hier die internationale Wettbewerbsfähigkeit zusätzlich eine wichtige Rolle, daher variiert die Importquote bei Bioethanol und Biopolymeren. ${ }^{149}$

${ }^{146}$ Dabei ist einschränkend zu beachten, dass diese Potenzialstudien nur eine begrenzte Aussagekraft über tatsächliche Nutzungskonkurrenzen haben. Eine Nutzungskonkurrenz kann auch dann bestehen, wenn theoretisch gesehen genug Flächen vorhanden sind (z.B. Nusser et al. 2007d; WI/RWI 2008; WBA 2007). Falls die industrielle Nutzung nachwachsender Rohstoffe durch Preisentwicklungen oder Politikanreize (z.B. Transferzahlungen, Energieeinspeisegesetz) rentabel wird, dürfte der Agrarsektor die Erzeugung der Rohstoffe über das errechnete Potenzial hinweg zu Lasten der Nahrungsmittelproduktion ausdehnen (WBA 2007; SRU 2007). Allerdings ist die Thematik für die Modellierung dieser Arbeit nur dann relevant, wenn sich dadurch die Inlandsproduktion bei Bioethanol und Biopolymere ändert. Es liegen aber keine konkreten Hinweise vor, die für eine starke Abweichung bei der Außenhandelsbilanz sprechen.

${ }^{147}$ Dabei sind viele Verschiebungen in der internationalen Verteilung bei der Produktion von Nahrungsmitteln und nachwachsenden Rohstoffen denkbar. Für ausfuhhrliche Diskussionen siehe SRU (2007) und WBA (2007).

${ }^{148}$ Neuwahl et al. (2008) berechnen bei einer Untersuchung der Beschäftigungseffekte von Biokraftstoffen in einem zusätzlichen angekoppelten agrarwirtschaftlichen Modell die Preisauswirkungen der gestiegenen Nachfrage nach landwirtschaftlichen Erzeugnissen. Diese Preissteigerungen werden, abzüglich von Preisrückgängen bei Kuppelprodukten und Erdöl durch Substitutionsprozesse, als konsummindernde Nachfrage im I-O-Modell simuliert. Wicke (2006) rechnet bei ihrer Analyse der Beschäftigungseffekte von Biokraftstoffen in Argentinien, die durch Produktivitätssteigerungen in der Landwirtschaft freigesetzten Arbeitskräfte der Nachfrage nach Biomasse, entgegen. Diese Vorgehensweise trägt der Feststellung Rechnung, dass nur teilweise eine zusätzliche Beschäftigung durch eine höhere Nachfrage nach landwirtschaftlichen Gütern entsteht. Preiserhöhungen der Produkte oder Importe finden aber annahmegemäß nicht statt.

${ }^{149}$ Dabei liegen die angenommen Importquoten ungefähr in einer ähnlichen Höhe der Studien, auf deren Basis die gesamte Nachfrage von nachwachsenden Rohstoffen geschätzt wird. 
- Ein Teil der landwirtschaftlichen Produktion für die biotechnologischen Anwendungen findet auf Konkurrenzflächen statt. Um eine ungefähre Größenordnung abzuleiten, werden folgende Werte zugrunde gelegt. In zwei „Business as Usual“-Szenarien des WI/RWI (2008) wird ein Anstieg des Bedarfs an Fläche für nachwachsende Rohstoffen zwischen 2005 und 2020 um 1,8 Mio. ha bzw. 2 Mio. ha auf 3,35 bzw. 3,55 Mio. ha projiziert. Das Angebot an Fläche für nachwachsende Rohstoffe liegt bei mehreren Studien, die vom Sachverständigenrat für Umweltfragen diskutiert und als plausibel eingeschätzt werden, bei rund 3 Mio ha (SRU 2007). Dies sind knapp 1,5 Mio. ha. mehr als im Jahr 2005. Demnach können ungefähr 70 bzw. $80 \%$ der zusätzlichen Nachfrage durch inländischen Anbau gedeckt werden. Der Wert von $80 \%$ wird für das Referenzszenario genutzt, der Wert von $70 \%$ für das HD-Szenario, da hier eine deutliche höhere inländische Produktion von Biopolymeren und Bioethanol stattfindet und die Konkurrenz in einem solchem Fall zunimmt. Der verbleibende Anteil ${ }^{150}$ der zusätzlichen Nachfrage nach landwirtschaftlichen Produkten verdrängt die Produktion anderer Agrarprodukte, die nun aus Importen zu decken sind. In Sensitivitätsrechnungen werden alternative Annahmen überprüft.

- Preiserhöhungen für die in biotechnologischen Prozessen eingesetzten nachwachsenden Rohstoffe werden im folgenden Abschnitt diskutiert. Andere Folgeeffekte (z.B. höhere Kosten von Nahrungsmitteln und deren Folgen) können nicht berücksichtigt werden. Dieser Preiseffekt wird zumindest teilweise durch die dämpfenden Impulse auf den Ölpreis ausgeglichen, der durch die Substitution von fossilen Produkten durch biotechnologische Produkte entsteht.

\section{Preise für nachwachsende Rohstoffe}

Ähnlich wie beim Ölpreis hängen die Preise für nachwachsende Rohstoffe von vielfältigen Einflussfaktoren ab. Insgesamt zeigt sich hier langfristig ein relatives stabiles Preisniveau mit kurzfristig hohen Schwankungen. So stiegen die Preise in den Jahren 2006 und 2007 erheblich an. Dieser Anstieg wird vor allem auf geringe Erträge aufgrund von Witterungsverhältnissen, dem Anstieg des Ölpreises, der erhöhten Nachfrage nach Biokraftstoffen und der Zunahme der Nachfrage nach Nahrungsmitteln zurückgeführt (OECD 2008c). Während einige Einflüsse eher vorübergehender Natur sind (z.B. Witterungsverhältnisse) wird von anderen Faktoren (z.B. weltweite Nahrungsmittelnachfrage) ein dauerhafter Einfluss auf die Preise erwartet. Die OECD und FAO $(2007,2008)$ gehen deshalb in ihren Projektionen davon aus, dass die Rohstoffpreise zwar im Vergleich

${ }^{150}$ Ausgenommen hiervon ist die Produktion von Biopolymeren und Bioethanol, die aus Reststoffen (z.B. Lignocellulose) erfolgt (Kapitel 5.2.3.2). 
zum hohen Niveau des Jahres 2007 zurückgehen werden, allerdings dauerhaft über den Preisen vor dem Jahr 2006 bleiben werden. Diese Entwicklung zeigt sich z.B. für den Rohstoff Weizen, der in dieser Arbeit von besonderem Interesse ist. Vor dem Jahr 2006 galt ein Preis für nachwachsende Rohstoffe aus Weizen von $100 \mathrm{E} / \mathrm{t}$ als plausibler Durchschnittswert (WBA 2007), der in vielen bisherigen Studien auch verwendet wird (z.B. Henniges 2007; Concawe 2006; Thrän et al. 2005). ${ }^{151}$ Etwas höhere Preise werden von IFEU (2007) mit ca. 110 $€ / t$ und von Neuwahl et al. (2008) mit $124 € / t$ angesetzt, deutlich höhere Preise unterstellt der Wissenschaftliche Beirat für Agrarpolitik (WBA 2007) mit $180 € / \mathrm{t}$. ${ }^{152}$ Im Rahmen dieser Arbeit wird analog zur Projektion von OECD und FAO (2008) für die weltweiten Weizenpreise eine reale Steigerung von $20 \%$ gegenüber dem Zeitraum Anfang 2002 bis Mitte 2006 unterstellt und somit 120 $\epsilon / t$ für Weizen im Referenzszenario angenommen. ${ }^{153} \mathrm{Sehr}$ ähnliche Projektionen von OECD und FAO (2008) lassen sich für Zucker und Stroh ableiten, weshalb vereinfacht ebenfalls $20 \%$ gegenüber den Werten von Concawe (2006) und Henniges (2007) unterstellt wird. ${ }^{154}$

Um zu untersuchen, wie sich ein günstiges Rohstoffpreisverhältnis auf die Effekte der Biotechnologie auswirken, werden für das HD-Szenario keine deutlich differierenden Preisannahmen getroffen. Dieses Szenario soll die Möglichkeit eines Anstiegs des Ölpreises gegenüber den Preise für nachwachsende Rohstoffe widerspiegeln. Allerdings ist eine Annahme eines höheren Ölpreises bei gleich bleibenden Preisen für nachwachsende Rohstoffe allein aufgrund der Produktionsbeziehungen nur begrenzt plausibel. ${ }^{155}$ Höhere Ölpreise führen zu steigenden Kosten in der Agrarproduktion, z.B. für das Betreiben landwirtschaftlicher Maschinen oder für Dünger und Pestizide (OECD/FAO 2008). Deshalb werden im HD-Szenario die Preise für nachwachsende Rohstoffe um die höheren Energiepreise bei der Produktion angepasst. Dabei wird in Anlehnung zu Concawe

${ }^{151}$ Für die Verwendung von Weizen für industrielle Zwecke wird in der Regel kein Qualitätsweizen benötigt, Futterweizen reicht hierfür aus (Thrän et al. 2005).

${ }^{152}$ Die Studien unterscheiden sich dabei beim Zukunftshorizont. Allerdings weisen viele Studien keinen expliziten Betrachtungszeitpunkt aus, so dass ein Vergleich nur eingeschränkt möglich ist. Zudem wird meist nicht ausgewiesen, ob es sich um reale oder nominale Preise handelt.

${ }^{153}$ Diese Steigerung entspricht auch der Sensitivitätsberechnung von OECD und FAO (2008), bei dem ein Ölpreis unterstellt wird, wie er im Rahmen des Referenzszenarios dieser Arbeit verwendet wird.

154 Henniges (2007) untersucht dabei nur Ethanol aus Zuckerrüben, aber nicht aus Lignocellulose. Sehr ähnliche Steigerungsraten, wie die beschriebenen $20 \%$, verwendet auch die GEMIS-Datenbank.

${ }^{155}$ Zudem ist eine stärkere Koppelung der Preise für fossile und nachwachsende Rohstoffe durch Substitutionsmöglichkeiten denkbar. Diese stehen aber nicht im Mittelpunkt dieser Arbeit. Vielmehr ist es Ziel des HD-Szenarios die Wirkungen eines durchaus denkbaren veränderten relativen Preisverhältnisses der Rohstoffe zu untersuchen. 
(2006) der Einfluss des Ölpreises auf die Preise für nachwachsende Rohstoffe auf $5 \%$ geschätzt.

\subsubsection{Bestimmung der ökonomischen Impulse}

Für diese erstellten Szenarien werden jeweils die ökonomischen Impulse der Biotechnologie als Grundlage für die Modellrechnungen bestimmt. Die möglichen Informationsquellen über die Diffusion und Eigenschaften der Biotechnologie wurden in Kapitel 4.3.3.2 erörtert. Dabei wurde festgestellt, dass in der Regel eine Mischung mehrerer Informationsquellen sinnvoll ist. Einzelne Quellen können meist nur über einen Teil des benötigten Informationsbedarfs Auskunft geben. Für sich alleine genommen sind sie häufig unvollständig beim abzubildenden Produktions- und Technikbereich bzw. den Informationen über die Technologiewirkung. Im Rahmen dieser Arbeit nehmen aufgrund der eingeschränkten Datenlage Expertengespräche eine bedeutende Rolle bei der Ergänzung, Verallgemeinerung und Bestätigung von Literaturinformationen und Indikatoren ein.

Die Bestimmung der Parameter für die Biotechnologie bringt zum Teil erhebliche Herausforderungen mit sich. Für Bioethanol und, mit Einschränkungen für Biopolymere ist die Verfügbarkeit von disaggregierten Informationen über Kostenstrukturen und Vorleistungsgüter als vergleichsweise gut einzustufen. $\mathrm{Zu}$ gleich ist das Substitut relativ klar definierbar, schließlich handelt es sich eher um eine Prozessinnovation. Dagegen werden bei Biopharmazeutika und Fein/Spezialchemikalien Produkte hergestellt für die erstens kaum repräsentative disaggegierte Informationen vorliegen und zweitens für einige Produkte keine vergleichbaren, konventionellen Verfahren zur wirtschaftlichen Herstellung existieren. Deshalb erfolgt die Betrachtung stärker in einem sektoralen Vergleich mit der Frage, inwiefern sich der Inputbedarf der bisherigen Sektoren (Pharmazeutika, Fein-/Spezialchemikalien) durch die Produkt- und Prozessinnovationen verändert.

Bei der Bestimmung der Koeffizienten für die Substitute stellt sich die Problematik, dass zum einen auf einer disaggregierten Basis zum Teil recht wenige Informationen zur Verfügung stehen. Zum anderen liegen Daten aus I-OTabellen für Deutschland auf einem höheren Aggregationsniveau vor. Ein Beispiel hierfür ist das Anwendungsfeld Biopolymere. Das Substitut der chemisch hergestellten Polymere bildet ein Teil des I-O-Sektors Chemie. Polymere benötigen aber je Werteinheit deutlich mehr Energie als chemische Produkte im Durchschnitt. Um einen I-O-Sektor für Polymere zu bilden, wird der Chemiesektor deshalb soweit wie möglich mit technoökonomischen Informationen (z.B. Kostenstrukturen, Experteneinschätzungen) und Informationen von stärker disaggregierteren I-O-Tabellen aus anderen Ländern (z.B. USA, Großbritannien) 
angepasst. Diese Mischung der Informationsquellen erfolgt für die anderen Anwendungsfelder analog.

\section{Erstellung des Fragebogenleitfadens}

Für die Ermittlung der biotechnologiespezifischen Koeffizienten in der Expertenbefragung wird ein semi-strukturierter Fragebogenleitfaden entwickelt. Einen beispielhaften Überblick mit den wichtigsten Kernfragen gibt Tabelle 6. Dabei ist ein möglichst einheitliches Vorgehen für die zu untersuchenden Anwendungsfelder sinnvoll, um die Nachvollziehbarkeit der gewählten Werte zu erhöhen und Verzerrungen durch unterschiedliche Erhebungsmethoden zu vermeiden. Da aber nicht für alle I-O-Koeffizienten größere, spezifische Änderungen durch die Biotechnologie zu erwarten sind, ist nur die Untersuchung von relevanten Koeffizienten zweckmäßig. Die relevanten Wirkungen werden durch eine Literaturanalyse über verfügbare Studien und Daten sowie vorab durchgeführten, offenen Experteninterviews bestimmt. ${ }^{156}$ Insgesamt ist der Fragebogenleitfaden für die einzelnen Anwendungsbereiche aber recht ähnlich. Variationen ergeben sich bei Biopharmazeutika, um zusätzlich die Bedeutung der Biotechnologie im F\&E-Prozess zu erfassen. Für einige Teilaspekte ließen sich in der Literatur Abschätzungen bzw. sehr konkrete Anhaltspunkte über das quantitative Ausmaß der Effekte finden (z.B. Energieeinsparungspotenzial). Da sich die Informationen meist auf bestimmte Verfahren beziehen und einigen Annahmen unterliegen, wurden diese in den Expertengesprächen diskutiert. ${ }^{157}$

Da implizite Annahmen der Experten über mögliche relevante Entwicklungen sich von den getroffenen Szenarioannahmen unterscheiden können, werden zu Beginn der Gespräche die wichtigsten Informationen hierzu erläutert. Zudem werden Unterschiede in der Wirkung unter den alternativen Annahmen (z.B. höherer Ölpreis) des Szenarios mit „, hoher Diffusion“ mit den Fachleuten erörtert.

\footnotetext{
${ }^{156}$ Für die offenen Expertengespräche und Pre-Test Interviews wurden Experten des Fraunhofer ISI und externe Experten bei einschlägigen Fachkonferenzen befragt.

${ }^{157}$ Der Vorteil dieses gestützten Vorgehens im Vergleich zu einer offenen Befragung liegt in der besseren Einschätzung der möglichen Dimensionen der Effekte. Außerdem wird die Hemmschwelle des Befragten bezüglich der Abgabe eines Schätzwertes reduziert. Nachteile können in der Beeinflussung des Befragten liegen. In der Regel ist aufgrund des Phänomens der sozialen Erwünschtheit mit einer tendenziellen Zustimmung des Experten zur These zu rechnen.
} 
Tabelle 6: Auszug aus dem Fragebogenleitfaden für Biopolymere

\begin{tabular}{|c|c|}
\hline Informationsbedarf & Beispiel Biopolymere \\
\hline Inputveränderung & $\begin{array}{l}\text { - Rohstoffersetzung (nachwachsende Rohstoffe statt Petrochemie) } \\
\text { - zukünftige Rohstoffeinsparung } \\
\text { - Veränderung Arbeitskräftebedarf } \\
\text { - Veränderung Kapitalbedarf } \\
\text { - Veränderung Transportkosten } \\
\text { - Veränderung Abfall, Energie, Entsorgung etc. }\end{array}$ \\
\hline Nachfrage & $\begin{array}{l}\text { - Neue Anwendungsmöglichkeiten? Ersetzung anderer Materialien } \\
\text { (ausserhalb Polymere)? } \\
\text { - Auslandsnachfrage: Weltmarktstellung Deutschlands }\end{array}$ \\
\hline Diffusion & $\begin{array}{l}\text { - Aktuelle und zukünftige Verbreitung } \\
\text { - Entscheidende Faktoren }\end{array}$ \\
\hline
\end{tabular}

Quelle: Eigene Darstellung

\section{Expertenabfrage}

Insgesamt wurden 39 Interviews mit dem Fragebogenleitfaden durchgeführt, damit für alle Anwendungsbereiche ein breiteres Meinungsbild erfasst wird und kritische Werte nicht auf einzelne Expertenaussagen beruhen. Je nach Wissensstand des Experten wurden in den jeweiligen Interviews ein oder zwei der betrachteten Anwendungsfelder abgedeckt. Eine Liste der befragten Unternehmen und Institutionen ist im Anhang (Tabelle 33) zu finden. Die Expertengespräche wurden vor allem in den Bereichen Biopolymere, Fein-/Spezialchemikalien und Biopharmazeutika durchgeführt. Dort ist noch eine geringe Informationsbasis vorhanden. Für den bereits recht gut dokumentierten Bereich Bioethanol wurde die Expertenbefragung auf einige zentrale mögliche Entwicklungen (z.B. Nahrungsmittelkonkurrenz, Nutzung von Lignocellulose) begrenzt. Insgesamt wurde ein breites Spektrum an Akteuren befragt, um einen möglichen Antwort-Bias gering zu halten. Beispielsweise sind überzogene Erwartungen von Unternehmen, deren Geschäftsmodell allein auf die Biotechnologie ausgerichtet ist, denkbar (Hopkins et al. 2007). Die Gruppe der Interviewten setzt sich deshalb aus Vertretern von diversifizierten Großunternehmen mit Aktivitäten in der Biotechnologie, auf Biotechnologie spezialisierte F\&E-Dienstleister, Forschungs- 
einrichtungen und Universitäten, Anlagenbau, Verbände und Unternehmen ohne eigene Biotechnologie-Aktivitäten, zusammen. Am häufigsten wurden Personen aus diversifizierten Großunternehmen befragt, da hier ein guter Überblick zum Vergleich der Biotechnologie zu konventionellen Methoden zu erwarten ist. Dabei konnten fast alle großen Unternehmen, bei denen Biotechnologieaktivitäten in den betrachteten Anwendungsfeldern in Deutschland bekannt sind, befragt werden. ${ }^{158}$

\subsubsection{Input-Output-Modellrechnungen}

Aus der Ableitung der ökonomischen Impulse folgen für jeden Anwendungsbereich und jedes Szenario die Inputkoeffizienten und die Nachfrage. Mithilfe des I-O-Modells werden über Leontief-Inverse die Vorleistungsproduktion ermittelt. Das verwendete I-O-Modell basiert auf den I-O-Tabellen der Version „Inländische Produktion und Einfuhren" des Statistischen Bundesamtes für das Jahr 2005 und ist der Gruppe der statischen, offenen Leontief-Modelle zuzuordnen. Darin wird die deutsche Volkswirtschaft in 71 produzierenden Sektoren und sechs verschiedene Endnachfragesektoren unterteilt, wobei die Lieferung von Waren und Dienstleistungen zwischen den produzierenden Sektoren sowie von diesen an die Endnachfragesektoren abgebildet werden. Zudem werden für die produzierenden Sektoren die Primärinputs (Bruttowertschöpfung) und Beschäftigungskoeffizienten je Mio. $€$ angegeben. Das in Kapitel 4.3.1.2 diskutierte Modul der Modellerweiterung um die Beschäftigungskoeffizienten kann somit direkt mit Daten aus der amtlichen Statistik verknüpft werden.

${ }^{158}$ Als Abgleich hierzu dienten entsprechende Unternehmenslisten von Dechema (2004) und Mietzsch (2008). 
Sven Wydra - 978-3-631-75392-7

Downloaded from PubFactory at 01/11/2019 05:32:25AM

via free access 


\section{Auswirkungen der Biotechnologie in ausgewählten Anwendungsfeldern}

Im Folgenden werden die wirtschaftlichen Auswirkungen der Biotechnologie in den ausgewählten Anwendungsfeldern anhand verfügbarer Daten, Literatur und Experteninterviews diskutiert und Modellparameter für die verschiedenen Szenarien abgeleitet. Anschließend erfolgen jeweils die Modellberechnungen. Dabei ist die Analyse für die vier Anwendungsfelder mit leichten Abweichungen (v.a. Biopharmazeutika) jeweils folgendermaßen aufgebaut.

- Beschreibung der Anwendung

- aktuelle und mögliche zukünftige Diffusion

- internationaler Wettbewerb und Außenhandel

- Höhe und Struktur (z.B. Rohstoffe, Energie) der Herstellungskosten zur Bestimmung der Inputkoeffizienten

- Zusammenfassung der Szenariowerte

- Berechnung der Produktions- und Beschäftigungseffekte

- Durchführung der Sensitivitätsanalyse (z.B. für Diffusion, Außenhandel)

Bei den Produktions- und Beschäftigungseffekten ist zu beachten, dass in den Szenarien keine Bruttobetrachtung der jeweiligen gesamten biotechnologischen Produktion oder Beschäftigung erfolgt, sondern Nettoeffekte unter Abzug von substituierter Produktion.

\subsection{Bioethanol}

Fermentativ produziertes Bioethanol wird seit langem zur Herstellung von alkoholischen Getränken und für industrielle Zwecke (z.B. als Grundchemikalie, Lösungs- oder Desinfizierungsmittel usw.) verwendet. Durch die stark gestiegene Nachfrage nach erneuerbaren Energie- und Kraftstoffquellen und - zu einem kleineren Teil - durch die technologische Weiterentwicklung der Biotechnologie auf der Angebotsseite ist die Produktion von Bioethanol als Kraftstoff in jüngerer Zeit deutlich angestiegen (Walter et al. 2008). Deshalb wird im Folgenden nur der Einsatz als Kraftstoff betrachtet, da besonders hier eine zusätzliche Diffusion durch die politische Unterstützung erwartet wird. Die Ersetzung von konventionellen Kraftstoffen durch Ethanol stellt eine Prozessinnovation dar, funktional bestehen zwischen diesen Produkten nur geringe Unterschiede. Bei dieser Prozessinnovation steht keine Effizienzsteigerung oder Kostenersparnis im Vordergrund, sondern in erster Linie der Rohstoffwechsel und damit verbundene mögliche Vorteile, wie z.B. eine nachhaltigere Rohstoffnutzung oder zusätzliche Einkommensperspektiven für die inländische Landwirtschaft.

Bisher erfolgt die Ethanolherstellung in Deutschland vorwiegend auf Basis von Getreide (Weizen, Mais, Roggen) und z.T. auf Zuckerrüben. ${ }^{159}$ Eine Mög-

159 In der Literatur werden für diese Herstellung häufig der Begriff „Biokraftstoffe der ersten Generation“ verwendet, die Herstellung aus Lignocellulose wird als „Biokraftstoffe der zwei- 
lichkeit zur Verbesserung der Rohstoffsituation wird in der Erschließung von lignocellulosehaltiger Biomasse als Rohstoff für Bioethanol und andere biotechnologische Produkte gesehen (Patel et al. 2006). Als Vorteil der lignocellulosehaltigen Biomasse gegenüber der Verwendung zucker- und stärkehaltiger Substrate gelten niedrige Rohstoffkosten. Daneben sind die Einsparungsmöglichkeiten fossiler Energie bei der Herstellung tendenziell höher und die Nutzbarkeit einer Vielzahl von Quellen zur Rohstoffgewinnung möglich, z.B. aus Reststoffen (IFEU 2007). ${ }^{160}$ Der Flächenbedarf fällt daher geringer aus und damit auch die Konkurrenz zu Lebensmittel- und Futtermittelanwendungen. Im Folgenden wird vorrangig die Produktion von Lignocellulose aus Stroh betrachtet, da sich die Produktion aus $\mathrm{Holz}$ als bedeutende Alternative in starker Verwendungskonkurrenz zur Strom- und Wärmegewinnung befindet (Widmann/Remmele 2008). Allerdings bestehen noch erhebliche technologische Probleme. So wird u.a. ein kosteneffizienterer, ressourcenschonender Aufschluss der Lignocellulose, um die enthaltenden Zucker nutzbar zu machen sowie die wirtschaftliche Abtrennung des daraus entstehenden Produkts, als notwendig angesehen (IFEU 2007; Nusser et al. 2007d). Im Gegensatz zur Ethanolproduktion aus Stärke (z.B. Weizen) sind zusätzliche vorgelagerte technische Verfahren zur Auftrennung des mit der Cellulose verbundenen Pektin, Lignin und Hemicellulosen nötig. Für die Wirtschaftlichkeit des Prozesses ist der Konversionsprozess der Biomasse von hoher Bedeutung, also dort, wo die Biotechnologie direkt zum Einsatz kommt (Lynd et al. 2008). Daneben ist die hochwertige Verwertung bzw. Kreislaufführung der im Prozess anfallenden Kuppelprodukte (z. B. Lignin, Schlempen) und die Integration sämtlicher Teillösungen in einen Gesamtprozess notwendig (Mussatto et al. 2006). Deshalb bestehen Unsicherheiten, ob und wann die Verwendung von Lignocellulose kommerziell erfolgreich sein wird (Patel et al. 2006). ${ }^{161}$ Zukunftsbetrachtungen zu Auswirkungen von Bioethanol auf Flächenbedarf, Agrarpreise und ökonomische Effekte differieren deutlich in ihren Annahmen und Projektionen zum Rohstoffeinsatz von Lignocellulose. Die in dieser Arbeit befragten Experten sind analog zu einer Expertenbefragung des IFEU-Instituts (IFEU 2007, S.112) optimistisch, dass es bis zum Jahr $2020 \mathrm{zu}$ einer kommerziellen Verwendung kommen wird. Deshalb wird in der folgenden Betrachtung der wirtschaftlichen Auswirkungen des Einsatzes von Ethanol Lignocellulose als möglicher Rohstoff mit einbezogen.

ten Generation" bezeichnet. Neben der Nutzung von Lignocellulose bestehen noch Forschungsbemühungen für andere Rohstoffe, z.B. Algen (OECD 2008d).

${ }^{160}$ Dabei kommen prinzipiell Durchforstungsholz, feste Siedlungsabfälle, landwirtschaftliche Reststoffe (z. B. Stroh, Maiskolben, Bagasse), industrielle Produktionsrückstände sowie Industriepflanzen (Einjahrespflanzen, Kurzumtriebshölzer) in Betracht, die nicht zugleich als Lebens- oder Futtermittel verwendet werden (Peters 2006).

${ }^{161}$ Bislang sind einige Pilotanlagen in Betrieb genommen worden (IFEU 2007, S.109). 


\subsubsection{Diffusion}

Die weltweite Produktion von Bioethanol hat sich vom Jahr 2000 bis zum Jahr 2007 verdreifacht auf 28,6 Mio. Tonnen (OECD 2008d). Der Anteil an den Ottokraftstoffen beträgt mittlerweile energetisch ca. 2\% (Walter et al. 2008). Weltweit sind Brasilien und die USA führend in der Ethanolherstellung (vgl. Tabelle 7). In Deutschland ist der Markt für Bioethanol bei Kraftstoffen durch staatliche Unterstützung 2004 entstanden und hat sich von wenigen Tonnen Produktion auf $226.000 \mathrm{t}$ im Jahr 2005 und $461.000 \mathrm{t}$ im Jahr 2007 erhöht (FNR 2008).

\section{Tabelle 7: Weltweite Produktion von Bioethanol im Jahr 2007}

\begin{tabular}{|l|c|c|}
\hline Land & in Mio. Liter & in Mio. Tonnen \\
\hline USA & 26500 & 14,55 \\
\hline Brasilien & 19000 & 10,44 \\
\hline Europäische Union & 2253 & 1,24 \\
(Deutschland) & $(837)$ & $(0,46)$ \\
\hline China & 1840 & 1,01 \\
\hline Kanada & 1000 & 0,55 \\
\hline Indien & 500 & 0,22 \\
\hline Sonstige Länder & 1017 & 0,56 \\
\hline Welt insgesamt & 52009 & 28,57 \\
\hline
\end{tabular}

Quelle: OECD 2008d; FNR 2008

Die zukünftige Diffusion von Bioethanol ergibt sich aus der gesamten Verwendung von Ottokraftstoffen (Benzin) und dem Anteil von Bioethanol. Während der Anteil von Bioethanol an Ottokraftstoffen in den vergangenen Jahren zugenommen hat, geht der deutsche Benzinverbrauch insgesamt seit Anfang der 1990er Jahre zurück. So sank er bspw. von 28,8 Mio. t im Jahr 2000 auf 21,3 Mio. $t$ im Jahr 2007 (MWV 2008). Ursachen hierfür liegen vor allem im niedrigeren Verbrauch bei Neufahrzeugen und in der Verschiebung des PKWBestands hin zu Dieselfahrzeugen (Henke 2005). Hinzu kommt eine Abnahme an gefahrenen Kilometern aufgrund der Preiszunahme. Nach Auffassung des Mineralölwirtschaftsverbandes wird sich dieser Trend in den nächsten Jahren verstärkt fortsetzen. Im Jahr 2020 soll der Verbrauch nur noch 15,6 Mio. t betragen (MWV 2006). Die zukünftige Verbreitung von Bioethanol in Deutschland hängt entscheidend von politischen Maßnahmen ab, da eine Kostenwettbewerbsfähigkeit von Ethanol zu Ottokraftstoff bislang nicht absehbar ist. In vielen Ländern wird mit verschiedenen Politikmaßnahmen (z.B. Steuerbefreiung, Quotenfestlegung, Importzölle) die Produktion von Bioethanol unterstützt (OECD 2008d). Argumente für die politische Unterstützung sind die Einschätzung, dass Bioethanol eine sinnvolle ökologische Alternative zur fossilen Kraftstoffproduk- 
tion ist und eine Anbaualternative für den rückläufigen Flächenbedarf in der Nahrungsmittelproduktion und damit zusätzliche Einnahmequellen und Beschäftigungsmöglichkeiten in der Landwirtschaft bietet (Enzing et al. 2007b; OECD 2008d). ${ }^{162}$ Darüber hinaus tragen alternative Kraftstoffe zur Versorgungssicherheit im Verkehr bei.

Seit Ende des Jahres 2005 zeigte sich aber zunehmende Kritik (z.B. OECD 2008c). Der Hauptgrund hierfür lag in der starken Zunahme der Nahrungsmittelpreise und -engpässe, die zum Teil auf die starke Zunahme der konkurrierenden Landnutzung für Biokraftstoffe zurückgeführt wird (OECD 2008c). Auch der klimapolitische Nutzen der Biokraftstoffe wird zunehmend in Frage gestellt (z.B. Searchinger et al. 2008). ${ }^{163}$

Infolge dieser Probleme wurden die Politikmaßnahmen und anvisierten Ziele in einigen Ländern reduziert oder abgeändert. Ende 2008 erfolgte zudem eine Abänderung der für die derzeitige Biokraftstoffpolitik maßgebliche EURichtlinie 2003/30/EG zur Förderung von Biokraftstoffen im Verkehrssektor (Euractiv 2008). Bislang galt eine sukzessive Steigerung des Mindestanteils von Biokraftstoffen an allen verkauften Kraftstoffen bis $10 \%$ des Heizwertes im Jahr 2020 als Ziel. Die Abänderung sieht vor, dass 4/10 dieses 10\%-Zieles aus Biokraftstoffen, die nicht mit Nahrungsmittel konkurrieren (z.B. aus Lignocellulose oder auf Basis von Wasserstoff bzw. Elektroantrieben), erreicht werden muss. Die nationalen Pläne Deutschlands liegen sogar darüber. So wird im „Integrierten Energie- und Klimaprogramm" der Bundesregierung (Bundesregierung 2007) für die Biokraftstoffbeimischung für das Jahr 2020 eine Beimischungsquote von $14 \%$ angestrebt. ${ }^{164}$ Allerdings wurden auch hier zumindest für die kommenden Jahre die Beimischungsziele reduziert (BMU 2008). Langfristig sollen der Bestimmungsquote die eingesparten Treibhausgase zugrunde gelegt werden. Wie diese häufigen Abänderungen zeigen, ist die Realisierung der politischen Ziele von einer Reihe von Umfeldentwicklungen abhängig. Entscheidend werden die Auswirkungen auf Nahrungsmittelkonkurrenz und Umwelt so-

\footnotetext{
${ }^{162}$ Dabei ist davon auszugehen, dass ein Großteil der verbundenen Arbeitsplätze in ländlichen Regionen entsteht (OECD 2008d). Dies betrifft neben der Agrarwirtschaft auch die Weiterverarbeitung. Dieser aus regionaler Sichtweise bedeutende Punkt wird in der hier erfolgten Gesamtbetrachtung nicht weiter berücksichtigt.

${ }^{163}$ Klimapolitischer Schaden durch die Biokraftstoffe wird vor allem dann befürchtet, wenn die Agrarproduktion andernorts flächenmäßig ausgedehnt wird (z.B. Abholzung von Wäldern) oder auf vorhandenen Ackerflächen intensiviert wird (z.B. höherer Einsatz von Stickstoff). In diesem Falle werden vermehrt Kohlendioxid und Lachgas emittiert (OECD 2008d, WBA 2007).

${ }^{164}$ Neben dieser direkt auf die Verwendung bezogenen Förderung bestehen weitere Maßnahmen wie F\&E-Förderung zur Entwicklung von Biokraftstoff-Technologien mit höherer Kosteneffizienz, Förderung von Investitionen in Biokraftstoffanlagen und der Förderung der Produktion von Energiepflanzen (OECD 2008d). Trotz dieser Maßnahmen ist eine Diffusion oberhalb der festgesetzten Quoten unwahrscheinlich.
} 
wie deren öffentlichen Wahrnehmung sein. Daneben ist die relative Preisentwicklung von Ethanol und Benzin sowie anderen alternativen Kraftstoffen von Bedeutung. Bei relativ niedrigen Ölpreisen wird die Beimischung von Ethanols, welches in diesem Fall deutlich teurer ist, schwieriger zu rechtfertigen sein.

Für die Szenarien wird eine Umsetzung der politischen Ziele angenommen. Dabei wird im Referenzszenario entsprechend dem EU-Beschluss von 2008 ein Biokraftstoffanteil von $6 \%$ aus bisher gebräuchlichen Rohstoffen unterstellt. $\mathrm{Zu}$ sätzlich wird als Beitrag zur Erfüllung der Restquote aus alternativen Kraftstoffen weiter 1,5\% aus Lignocellulose angenommen. Der resultierende Anteil von Lignocellulose zur Ethanolherstellung entspricht Einschätzungen von Neuwahl et al. (2008). Für das HD-Szenario werden die ambitionierteren Pläne der deutschen Regierung von insgesamt $14 \%$ zu Grunde gelegt. ${ }^{165}$ Da eine Zunahme der Produktion vor allem auf Lignocellulose denkbar ist, steigt der Anteil im Diffusions-Szenario auf 33\%. ${ }^{166}$ Die restliche Produktion erfolgt in Anlehnung an Potenzialschätzungen des Fachverbands für Nachwachsende Rohstoffe (FNR) jeweils zu $85 \%$ durch Weizen und zu $15 \%$ Zucker. Bei einem Gesamtkraftstoffverbrauchs von 15,6 Mio. t Benzin im Jahr 2020 (MWV 2006) entsteht, unter Berücksichtigung der unterschiedlichen Heizwerte, ${ }^{167}$ eine inländische Nachfrage an Ethanol von 1,91 Mio. t (Referenzszenario) bzw. 3,56 Mio. t (HD Szenario). Abzüglich der bereits im Jahr 2005 produzierten Menge von knapp 0,23 Mio. $t$ ergeben sich die in Tabelle 8 aufgeführten Werten für die Berechnungen.

\section{Tabelle 8: Diffusion von Bioethanol in den Szenarien}

\begin{tabular}{|l|c|c|}
\hline & Referenzszenario & HD-Szenario \\
\hline Diffusion im Jahr 2020 in Mio. $\mathrm{t}$ & 1,91 & 3,56 \\
\hline Diffusion im Jahr 2005 in Mio. $\mathrm{t}$ & 0,23 & 0,23 \\
\hline $\begin{array}{l}\text { Differenz 2020-2005 in Mio. } \mathrm{t} \\
\text { (Berechnungswert) }\end{array}$ & 1,68 & 3,33 \\
\hline
\end{tabular}

Quelle: Eigene Berechnungen, FNR 2008

${ }^{165}$ Neben Bioethanol fällt der nicht biotechnologisch produzierte Biodiesel als Dieselersatz unter diesen Regelungen zu den Biokraftstoffen. Für die Szenarien wird eine gleichmäßige Aufteilung der Biokraftstoffquote angenommen, Ethanol und Biodiesel haben folglich jeweils einen Anteil von 7,5 bzw. 14\% an Benzin oder Diesel.

${ }^{166}$ Dies entspricht einem Alternativszenario von Neuwahl et al. (2008) mit der geringsten Verbreitung von Lignocellulose.

${ }^{167}$ Die Beimischung von Ethanol zum Ottokraftstoff stellt unter energetischen Gesichtspunkten eine Verdünnung dar und führt zum Mehrverbrauch. So ist für die gleiche Fahrleistung mit $1 \mathrm{t}$ Benzin eine Ethanolmenge von 1,63 t notwendig (FNR 2008). 


\subsubsection{Internationaler Wettbewerb und Außenhandel}

$\mathrm{Da}$ es bei Bioethanol kaum qualitative Unterschiede gibt, ist im internationalen Wettbewerb besonders dessen Preis entscheidend. Durch die preiswerten Rohstoffe, niedrigen Lohn- und Investitionskosten sowie langjähriger Erfahrung ist die Produktion in Brasilien international am günstigsten. Die Gründe hierfür liegen besonders in den geringen Rohstoffkosten, die klimatischen Bedingungen ermöglichen die Nutzung von Zuckerrohr. Daneben werden hohe Skaleneffekte durch große Anbauflächen erreicht und die Arbeits- und Investitionskosten sind geringer als bspw. in Deutschland (Thrän et al. 2005). Die Unterschiede bei den Herstellungskosten werden auf bis zu 0,43 €/l Ethanol geschätzt (Meyer et al. 2007). Auch im Vergleich zu den USA oder den asiatischen Ländern hat die Herstellung in Deutschland Kostennachteile (z.B. Rohstoffkosten), allerdings in geringerem Ausmaß. Innerhalb der EU sind die Kostenunterschiede dagegen eher gering, meist wird vereinfachend von identischen Herstellungskosten ausgegangen (z.B. Thrän et al. 2005; Concawe 2006; OECD 2008d). Grundsätzlich implizieren diese Kostenunterschiede einen Anreiz für einen internationalen Handel von Bioethanol (Henniges 2007). ${ }^{168}$ Für Brasilien wird daneben ein vergleichsweise großes Ausdehnungspotenzial beim Angebot von Bioethanol festgestellt (Henniges 2007) und die Regierung beabsichtigt einen Ausbau der Produktion und der Exportstellung Brasiliens auf dem Weltmarkt (OECD 2008d). Bisher ist der internationale Handel recht gering und beträgt $9 \%$ der weltweiten produzierten Ethanolmenge (OECD 2008d, S.17). Der Hauptgrund hierfür liegt in relativ hohen Importzöllen ${ }^{169}$ und beschränkenden Importkontingenten in vielen Ländern, über deren Abbau noch keine Einigung erzielt wurde (Meyer et al. 2007). ${ }^{170}$ Für die zukünftige inländische Produktion und der deutschen Außenhandelsbilanz bei Bioethanol ist folglich die Entwicklung der Importzölle entscheidend sowie die Bestrebungen anderer Länder die Produktion von Ethanol über den heimischen Bedarf hinaus auszubauen. Das Ausmaß des Handels ist demnach bisher unsicher. Aufgrund der hohen Importtarife erwarten die OECD und die FAO (2008) in einem „Baseline“-Szenario nur einen Handelszuwachs, der in etwa proportional zum Produktionswachstum verläuft. Unter diesen Annahmen ist mit einem signifikanten Anstieg der Importquoten nicht zu rechnen.

\footnotetext{
${ }^{168}$ Dagegen gilt ein Ethanolimport aus den USA als unwahrscheinlich. Zwar sind die Produktionskosten dort günstiger, bisher kann allerdings kaum die eigene Nachfrage gedeckt werden, die Transportkosten wären aufgrund der Produktion fernab der Küsten besonders hoch und die bisher bestehenden Importzölle nivellieren die Kostenunterschiede (Henniges 2007; Nusser et al. 2007d).

${ }_{169}$ Bei Bioethanol beträgt der Importzoll der EU für nichtdenaturiertes Bioethanol 19,20 $\mathrm{E} / \mathrm{hl}$ (Meyer et al. 2007). In den USA liegt der Importzoll für Bioethanol bei $15 € / \mathrm{hl}$.

${ }^{170}$ Zudem würden sich Kostenunterschiede weiter erhöhen, wenn die starke Subventionierung der inländischen Produktion in vielen Ländern (z.B. durch Energiepflanzenprämie in Deutschland) reduziert werden würde.
} 
Die Importquote in Europa betrug im Jahr 2006 ca. 20\%. In Deutschland lag sie mit 33\% sogar etwas höher (WI/RWI 2008; OECD 2008d). Allerdings wurden seitdem die inländischen Produktionskapazitäten deutlich erhöht (WI/RWI 2008), sodass auch bei zunehmender Produktionsmenge ein deutlicher Anstieg der Importquote eher unwahrscheinlich ist. ${ }^{171}$ Nach Angaben des IWR (Internationales Wirtschaftsforum Regenerative Energien) ergeben die im Jahr 2007 bestehenden oder im Bau befindlichen Anlagen 1,34 Mio. t Jahreskapazitäten, weitere 0,13 Mio t. Jahreskapazitäten befinden sich in Planung (IWR 2007). Im Referenzszenario wird deshalb von einer stabilen Importquote für beide Szenarien von $30 \%$ ausgegangen. ${ }^{172}$ Dieser Wert liegt etwas unter den Annahmen (40\%) von Nusser et al. (2007d) und innerhalb der Bandbreite (20-50\%) der Schätzungen von WI/RWI (2008).

\subsubsection{Herstellungskosten für Bioethanol}

Bioethanol wird für die Herstellung von Ottokraftstoffen in dreierlei Weise verwendet. Der größere Anteil (ca. 79\%) wird zur Erzeugung des Kraftstoffzusatzes ETBE (Ethyltertiärbutylether) eingesetzt. Dies substituiert MTBE auf Basis von Methanol (Kemnitz 2008). Ein kleinerer Anteil (ca. 20\%) von Ethanol wird dem Kraftstoff beigemischt. Ethanol als Reinkraftstoff wird in Deutschland dagegen kaum verwendet, das steuerlich begünstigte E85 (Ottokraftstoff mit einem Anteil von $85 \%$ Ethanol) hat ein Anteil von unter 1\%. Da sich dies voraussichtlich bis zum Jahr 2020 nicht ändern wird, werden keine eigenen Distributionslinien und Zapfsäulen an Tankstellen für Ethanol notwendig (Schöpe/Brischkat 2006). Der Kostenvergleich von Ethanol und Benzin kann sich daher auf die Herstellung beschränken.

Für die Herstellung von Bioethanol liegen eine Reihe von Kostenbetrachtungen in der Literatur vor (z.B. Henniges 2007; Concawe 2006; IFEU 2007; GEMIS-Datenbank). Diese untersuchen die aktuelle Kostensituation, aber auch teilweise mögliche zukünftige Entwicklungen. Die fermentative Produktion von Bioethanol gilt als relativ ausgereift, durch eine Reihe möglicher Verbesserungen besteht aber noch etwas Potenzial zur Kostenreduzierung. ${ }^{173}$ Insgesamt zei-

${ }^{171}$ Der Importanteil ist dabei rückläufig von 100\% im Jahr 2004 und etwa 39\% im Jahr 2005 (WI/RWI 2008).

172 Die höhere Produktionsmenge im HD-Szenario könnte aufgrund der begrenzten Flächenverfügbarkeit für eine höhere Importquote sprechen, allerdings liegt der Hauptzuwachs gegenüber dem Referenzszenario bei der Ethanolproduktion mit Lignocellulose, welches weniger Flächenkonkurrenzproblematik unterliegt.

${ }^{773}$ Diese beinhalten z.B. die Implementierung von modernen Prozesstechnologien und Automatisierung, intensivere Nutzung von Kuppelprodukten oder deren Verringerung, energieeffizientere Entsorgung von Abfallprodukten, Erhöhung der Energieeffizienz in der gesamten Prozesskette, Reduzierung der Prozessschritte, Diversifizierung, Vermeidung von Kontamination etc. (Patel et al. 2006, S.14). 
gen sich bei den Kostenstudien aber deutliche Unterschiede für die gesamten Herstellungskosten und bei den einzelnen Kostenarten (z.B. Rohstoffkosten). Zum einen gibt es bei der Erzeugung von Bioethanol unterschiedliche Alternativen. So haben die Unternehmen Auswahl beim verarbeitenden Rohstoff, des Energieträgers und bei der Verwertung der Nebenprodukte (Schöpe/Brischkat 2006). Zum anderen unterscheiden sich die Studien beim Zeit- und Ortsbezug, technischen Parametern zu den Verfahren (z.B. physischer Energiebedarf), Annahmen zu Preisen von Vorprodukten (z.B. Rohstoffkosten), Anlagengröße und besonders auch in der Methode der Kostenbilanzierung. Letztere differieren bspw. bei den für Kapitalkosten unterstellten Abschreibungszeiträumen und Zinsraten, den einbezogenen variablen und fixen Kosten (Reparaturkosten, „Overhead“, Versicherung, Personalkosten) sowie in der teilweisen Berücksichtigung eines Gewinnaufschlags. Das IFEU-Institut (IFEU 2007) kommt bei einem Vergleich einiger weit verbreiteter Studien zu Kostenbetrachtungen allein durch methodenbedingten Unterschiede in den Nettoproduktionskosten auf eine Bandbreite von $590 € / \mathrm{t}$ bis $830 \mathrm{\epsilon} / \mathrm{t}$ für Ethanol aus Mais. ${ }^{174}$ Die unmittelbare Vergleichbarkeit der Ergebnisse von Studien ist demnach eingeschränkt. Um eine möglichst konsistente Datenbasis für die quantitative Berechnung zu erhalten, ${ }^{175}$ werden die wichtigen Parameter anhand eines Datensatzes bestimmt. Hierfür werden Angaben von Concawe (2006) verwendet. ${ }^{176}$ Gründe hierfür liegen in einer konsistenten Betrachtung der drei Rohstoffe (Weizen, Zuckerrübe, Lignocellulose) und der expliziten Angabe von Rohstoff- und Ölpreisen. Ergänzend werden wichtige Hinweise aus anderen Studien für eine genauere Unterscheidung einzelner Kostenblöcke (bspw. Overhead) für die Bestimmung der I-O-Koeffizienten verwendet. Im Folgenden werden die einzelnen Kostenarten und die Umsetzung in I-O-Koeffizienten konkretisiert.

\section{Rohstoffkosten und -bedarf}

Einen hohen Kostenanteil von oftmals über 50\% haben die eingesetzten Rohstoffe (z.B. Concawe 2006). In diesen Rohstoffkosten ist bereits der Transport der Biomasse zur Konversionsanlage enthalten. Diese Kosten variieren allerdings je nach betrachteter Biomasseart, bei Lignocellulose sind sie recht niedrig. Die Rohstoffkosten (einschließlich Lieferung zur Anlage) ergeben sich dabei aus dem Preis je $t$ Biomasse, dem Einsatzverhältnis der benötigten Menge Biomasse je $t$ Ethanol und zu einem kleinen Teil aus den Transportkosten. Zukünf-

174 Hierzu wurden identische Annahmen zu Stoffströmen (z.B. Energiebedarf) und Preisen (Rohstoffkosten etc.) unterstellt (IFEU 2007, S. 68).

${ }^{175}$ Dies gilt besonders für die Annahmen zu technischen Parametern, wie die Rohstoffausbeute (z.B. $t$ Ethanol je $t$ Weizen) oder Zusatzkosten für Herstellung des Kuppelprodukts, und den Annahmen zu zukünftigen technologischen Verbesserungen.

${ }^{176}$ Diese europäische Studie untersucht den Einsatz alternativer Kraftstoffe im Verkehrssektor hinsichtlich Energiebedarf, Treibhausgasemissionen und Produktionskosten. 
tige Änderungen für die Rohstoffausbeute, dem Einsatzverhältnis von Biomasse zu Ethanol, werden kaum erwartet. Eine Ausnahme bildet Lignocellulose, für die eine Effizienzsteigerung ( $t$ Ethanol je $t$ Biomasse) erreichbar erscheint (Hamelinck/Faaij 2006). ${ }^{177}$ Deutlich größere Auswirkungen für die zukünftigen Kosten haben hingegen die Preise für Biomasse. Wie in Kapitel 5.2.3.2 erörtert, wird langfristig ein Preisanstieg gegenüber dem Niveau der Preise bis zum Jahr 2005 erwartet. ${ }^{178}$ Auf Basis der Einsatzverhältnisse von Concawe (2006) ergeben sich die Rohstoffkosten für die verschiedenen Szenarien. Diese liegen bei Zuckerrübe zwischen 404 und $424 € / t$, bei Weizen zwischen 422 und $442 € / t$ und bei Lignocellulose zwischen 170 und $187 € /$ t.

Wie in Kapitel 5.2.3.2 erläutert wurde, kann nicht davon ausgegangen werden, dass genug inländische Fläche zur Rohstoffproduktion ohne Nutzungskonkurrenzen zur Verfügung steht. Der zusätzliche Flächenbedarf durch die Verwendung von Bioethanol ist im Anhang (Tabelle 34) dargestellt. Unter der Annahme von Bandbreiten für Ertragssteigerungen aus der Literatur ${ }^{179}$ ergeben sich für Weizen ein anteiliger Flächenbedarf von 2,8-3,0\% im Referenzszenario und 3,4-3,6\% im HD-Szenario gemessen an der aktuell bewirtschafteten Agrarfläche. Dabei wird analog zu anderen Studien (z.B. Nusser et al. 2007d; WI/RWI 2008) von keiner Flächenkonkurrenz bei Lignocellulose ausgegangen, da es auf den zur Nahrungserzeugung oder NAWARO-Produktion genutzten Getreideflächen als Kuppelprodukt gewonnen werden kann. ${ }^{180}$

Unter den in Kapitel 5.2.3.2 erläuterten Annahmen ergibt sich folgende Höhe für die freie Fläche (Tabelle 9). Der Anteil der Ethanolproduktion auf konkurrierenden Flächen berechnet sich aus dem Anteil von Weizen und Zuckerrüben an der Ethanolproduktion (80 bzw. 67\%) und dem Anteil der Weizen und Zuckerrübenproduktion auf Konkurrenzflächen (20 bzw. 30\%). Der Anteil an Konkurrenzfläche ist im HD-Szenario mit $20 \%$ höher als im Referenzszenario mit $16 \%$. Für die substituierte Fläche wird davon ausgegangen, dass in entsprechender Höhe andere landwirtschaftliche Erzeugnisse aus dem Ausland importiert werden.

${ }^{177}$ Z.B. gehen Hamelinck/Faaij (2006) von einer langfristigen (ca. Jahr 2025) Steigerung der Effizienz von 38 auf 52\% gegenüber dem Jahr 2005 aus.

${ }_{178}$ Daneben sind die Rohstoffkosten im Zeitverlauf geographischen und kurzfristigen Schwankungen unterworfen. Die OECD (2008d) kommt bei einem Vergleich der Produktionskosten für die Jahr 2004 bis 2007 sogar zu dem Schluss, dass der Kostenunterschied zwischen Benzin und Ethanol ansteigt, da die gestiegenen Rohstoffkosten für Ethanol in den Jahren 2006 und 2007 den höheren Ölpreis für Benzin überkompensieren.

${ }_{179}$ Quantitative Abschätzungen in der Literatur für den Ertrag von Weizen in Deutschland liegen in eher ähnlichen Größenordnungen mit Einschätzungen von 1,3\% (WI/RWI 2008), 1,7\% (Thrän et al. 2005) und 1,8\% (Nusser et al. 2007d). Bei Zuckerrübe belaufen sich die Einschätzungen zwischen 1,0 (Thrän et al. 2005) und 1,4\% (Nusser et al. 2007d).

${ }^{180} \mathrm{Sehr}$ langfristig sind durch die verschiedenen möglichen Verwendungszwecke aber auch hier Nutzungskonkurrenzen denkbar (Oertel 2007). 
Tabelle 9: Anteil der Ethanolproduktion auf konkurrierenden Flächen

\begin{tabular}{|l|c|c|}
\hline & $\begin{array}{c}\text { Referenz- } \\
\text { szenario }\end{array}$ & $\begin{array}{c}\text { HD- } \\
\text { Szenario }\end{array}$ \\
\hline $\begin{array}{l}\text { Anteil Ethanolproduktion aus Weizen/ Zucker- } \\
\text { rübe in \% }\end{array}$ & $80 \%$ & $67 \%$ \\
\hline $\begin{array}{l}\text { Anteil Ethanolproduktion aus Weizen/ Zucker- } \\
\text { rübe auf Konkurrenzflächen in \% }\end{array}$ & $20 \%$ & $30 \%$ \\
\hline $\begin{array}{l}\text { Anteil gesamte Ethanolproduktion auf Konkur- } \\
\text { renzflächen in \% }\end{array}$ & $16 \%$ & $20 \%$ \\
\hline
\end{tabular}

Quelle: Eigene Berechnungen

Darüber hinaus ist eine Anpassung der I-O-Koeffizienten notwendig. Während für diese Importgüter der Durchschnittsvektor der I-O-Tabelle für Landwirtschaft angenommen werden kann, ist dies für die Produktion von nachwachsenden Rohstoffen nur bedingt realistisch. Allgemein gilt die pflanzliche Produktion als weniger arbeitsintensiv als andere Teilbereiche (z.B. Viehhaltung) (WBA 2007; Nusser et al. 2007d; Steininger et al. 2008). Deshalb wird der Arbeitskoeffizient auf Basis von Informationen der Landwirtschaftsstatistik des BMVEL (2007) über Arbeitszeiten je Fläche um 1/3 gegenüber dem Durchschnitt der Landwirtschaft reduziert. ${ }^{181}$ Auch die Arbeitsentgelte werden entsprechend angepasst, ebenso wie der Vorleistungsvektor für Nahrungsmittel. Dieser ist für die Pflanzenproduktion kaum relevant. Angaben von Steininger et al. (2008) zu den Produktionskosten für einzelne nachwachsende Rohstoffe lassen höhere Vorleistungen aus Investitionen für Maschinen und landwirtschaftliche Erzeugnisse (Saatgut, Dünger). Die geringeren Vorleistungen aus dem Nahrungsmittelsektor werden auf diese beiden Sektoren entsprechend aufgeteilt. ${ }^{182}$

Die in den Rohstoffkosten bereits inbegriffenen Transportkosten hängen hauptsächlich von der Dichte des verwendeten Rohstoffes und der Transportentfernung $a b$. Vergleichsweise hohe Transportkosten ergeben sich für Lignocellulose. Hauptgrund hierfür ist die geringe Dichte im Vergleich zu anderen Rohstoffen (Hess et al. 2007). Die Transportkosten nehmen zusammen mit den Sammel- und Lagerkosten den Hauptanteil bei den Rohstoffkosten für Lignocellulose ein (Bohlmann 2006; Hess et al. 2007). Es stellt sich dabei die Frage, ob überhaupt genug Rohstoffe aus einer Entfernung, bei der ein LKW-

\footnotetext{
${ }^{181}$ Steininger et al. (2008) weisen für Österreich den fast identischen Wert aus.

182 Daneben sehen Steininger et al. (2008) bei der Biomasseproduktion besondere Einsparmöglichkeiten durch den Einsatz von mehrjährigen Kulturen sowie eine verstärkte Inanspruchnahme von überbetrieblichen, kapitalintensiven Leistungen. Eine Berücksichtigung dieser möglichen Kostenunterschiede zwischen der Produktion von Nahrungsmitteln und der industriell verwendeten Biomasse erfolgt im Rahmen der vorliegenden Arbeit allerdings nicht, da kein ausreichendes Datenmaterial vorliegt.
} 
Transport $(<100 \mathrm{~km})$ rentabel ist, für größere Anlagen bereitgestellt werden können (WBA 2007; Schmitz 2005). Die logistischen Herausforderungen sind erheblich und die Infrastruktur müsste auf die zunehmende Verkehrsbelastung im Umfeld der Anlagen ausgerichtet werden (WBA 2007). Insgesamt unterscheiden sich einzelne Studien (z.B. Thrän et al. 2005; IFEU 2007; GEMIS 2008) für die Transportkosten für alle Rohstoffe recht deutlich, die Bedeutung für die Gesamtproduktionskosten ist allerdings gering (IFEU 2007). Im Folgenden werden Daten der Datenbank GEMIS (2008) verwendet, da hier eine explizite Unterscheidung einzelner Rohstoffe vorliegt. Die durchschnittlichen Transportkosten bei einer Transportentfernung von $50 \mathrm{~km}$ für Weizen $3,95 € / \mathrm{t}$ Bioethanol und für Zucker aufgrund des höheren Rohstoffbedarfs 16,7 €/t. Für Stroh werden $100 \mathrm{~km}$ Transport angesetzt, da ein größerer Radius für die Sammlung der Rohstoffe notwendig ist. Die angesetzten Kosten betragen $11 \mathrm{€/t}$.

\section{Kuppelprodukte}

Bei der Herstellung von Bioethanol entstehen je nach Rohstoff und Herstellungsverfahren unterschiedliche Kuppelprodukte, für die jeweils meist mehrere Verwendungszwecke bestehen. Bei Weizen zur Stärkeproduktion für Ethanol fällt vor allem Getreideschlempe an. Getrocknet wird sie häufig als Eiweißfuttermittel DDGS („Distiller's Dried Grains with Solubles“) verwendet, welches Sojaschrot ersetzen kann (Schöpe/Brischkat 2006). ${ }^{183}$ Eine alternative aber grundsätzlich eher weniger lukrative Verwertungsmöglichkeit für die Schlempe würde sich durch den Einsatz als Co-Substrat in Biogasanlagen zur Energiegewinnung (Strom, Wärme) ergeben (Concawe 2006). Für die Szenarien wird erstere Alternative angenommen und unterstellt, dass dabei aus dem Ausland importiertes Sojaschrot ersetzt wird. Dieses Vorgehen liegt im Einklang zur Literatur (z.B. Schöpe/Brischkat 2006), schließlich liegt die Importquote für Sojaschrot bei ca. 80\% (BMELV 2007). Ebenso kann aus Zuckerrübe entsprechendes Futtermittel gewonnen werden, wenn auch in geringerem Umfang. Bei Lignocellulose entsteht als Kuppelprodukt Lignin. Für die zusätzlichen Erträge ist hier ebenfalls entscheidend, ob sich eine ertragreichere stoffliche Nutzung von Lignin findet oder sie energetisch verwendet wird. In den quantitativen Berechnungen wird analog zu der vorwiegenden Einschätzung in der Literatur, (z.B. Hamelinck et al. 2005; IFEU 2007; Concawe 2006) die für diesen Zeithorizont wahrscheinlichere energetische Verwendung angenommen. Diese Ersparnis wird direkt mit dem Energiebedarf verrechnet (Concawe 2006).

183 Allerdings stellt sich die Frage, ob das zusätzliche DDGS zu bestehenden Marktpreisen verkauft werden kann. Schließlich entsteht ein erhebliches Zusatzangebot an diesen Produkten. So geht beispielsweise Concawe (2006) bei einer Biokraftstoffquote von 5,75\% für die EU von einer 9\%-Substitution des Ölsaaten-Futtermarktes durch DDGS bei Ethanol aus. 


\section{Investitionskosten}

Die Investitionskosten je $t$ Ethanol unterscheiden sich je nach Anlagengröß ${ }^{184}$, Rohstoff und verwendeter Biokonversionstechnologie. Hinzu kommen in der Regel lokale Faktoren, so sind bspw. unterschiedliche Aufwendungen für infrastrukturelle Einrichtungen notwendig, die ebenfalls in die erforderlichen Investitionskosten einfließen. Besonders hoch liegen die Investitionskosten bei Lignocellulose. Es sind zusätzliche Anlagenteile, so beispielsweise zur Vorbehandlung und Hydrolyse sowie zur (energetischen) Nutzung des abgetrennten Lignins erforderlich. Deshalb werden die Investitionskosten um das 2 bis 2,5fache im Vergleich zu Bioethanol aus Weizen eingeschätzt (Concawe 2006; Bohlmann 2006). Ob diese Differenz bis zum Jahr 2020 reduziert werden kann, hängt stark vom Erreichen von Lerneffekten ab. Potenziale zur Senkung von Investitionskosten werden z.B. durch eine zunehmende Prozessintegration (bspw. einer Durchführung der Hydrolyse und Fermentation im selben Reaktor) und dadurch weniger benötigten Reaktoren erwartet (IFEU 2007).

Neben den reinen Investitionskosten für die Ethanolanlage werden in Kalkulationen $\mathrm{zu}$ zahlende Zinsen berücksichtigt. Die verwendeten Werte von Concawe gehen von einem jährlichen Zinssatz von $8 \%$ und einer rechnerischen Betriebsdauer der Anlage von etwa 14 Jahren aus. Hieraus resultieren Kapitalkosten in Höhe von $12 \%$ der Investitionskosten. $40 \%$ hiervon entfallen für Zinszahlungen, $60 \%$ sind tatsächliche Investitionskosten: diese Werte werden durch die jährlich produzierte Ethanolmenge dividiert. Die resultierenden Investitionskosten liegen in den verschiedenen Szenarien bei Zuckerrübe zwischen 49 und $54 € / \mathrm{t}$, bei Weizen zwischen 64 und $70 € / \mathrm{t}$ und bei Lignocellulose zwischen 149 und $157 € /$ t. Diese werden nach Angaben von Thrän et al. (2005) und Henniges (2007) auf $15 \%$ für Gebäude und $85 \%$ für Maschinen und Inventar aufgeteilt. ${ }^{185}$

\section{Energie- und Hilfsstoffe}

Für die Produktion von Bioethanol sind einige Hilfs-/Betriebsstoffe (z.B. Phosphorsäure, Ammoniak, Schwefelsäure) sowie der Einsatz von Energie (Dampf, Elektrizität) notwendig. Die Kosten sind recht stark an den Ölpreis gekoppelt (Concawe 2006). Bei Lignocellulose stellen bisher die Enzymkosten zur Vorbehandlung der Biomasse einen bedeutenden Anteil an den Verarbeitungskosten dar. Im großtechnischen Maßstab betragen diese bisher ca. 150 €/t Ethanol, im Labormaßstab konnten sie bereits auf $20 \mathrm{E} / \mathrm{t}$ Ethanol reduziert werden (IFEU 2007). Die Verringerung dieser Kosten stellt eine wichtige Vorrausetzung für

\footnotetext{
${ }^{184}$ Eine Vergleichsrechnung des IFEU (2007) für eine sehr kleine Anlage mit $17.000 \mathrm{t}$ und einer $200.000 \mathrm{t}$ Anlage zeigt einen Unterschied der Investitionskosten je $\mathrm{t}$ Bioethanol um den dreifachen Wert.

${ }^{185}$ Für eine genauere Spezifizierung der Investitionskosten für einzelne Sektoren der I-OTabelle wurde die in Kapitel 5.2.2 beschriebene Vorgehensweise gewählt.
} 
die wirtschaftliche Produktion dar, wird aber auch bis zum Jahr 2020 als realisierbar angesehen (Dornburg et al. 2007).

Insgesamt sind die Kosten für Energie- und Hilfsstoffe stark vom Ölpreis abhängig und differieren daher im Referenz- und HD-Szenario deutlich (siehe Tabelle 10 und Tabelle 11). Diese Kosten werden anhand von Angaben von Taheripour et al. (2008) und Toro (2006) zu 40\% chemischen Hilfsstoffen und zu $60 \%$ dem Energieeinsatz zugerechnet, wobei 1/10 der Energie dem Wasserverbrauch zuzuordnen ist (Henniges 2007).

\section{Arbeits- und sonstige Kosten}

Weitere Kosten werden in einer Pauschalberechnung erfasst. Hierzu wird in Kostenbetrachtungen in der Regel ein Prozentsatz auf die Investitionskosten angesetzt (IFEU 2007). Concawe (2006) setzt hierfür einen Kostenanteil von 4,5\% an. Diese Kosten werden in dieser Arbeit analog zu Neuwahl et al. (2008) für Zucker und Weizen im Verhältnis von $75 \%$ Arbeitskosten zu $25 \%$ „operating costs" aufgeteilt. Die sehr viel höheren Kosten für Lignocellulose werden im umgekehrten Verhältnis $25 \mathrm{zu} 75 \%$ verrechnet. Die angesetzten direkten Arbeitskosten für Herstellung von Ethanol sind folglich gering. Detaillierte Schätzungen über die Ermittlung des verbundenen Arbeitsaufwands von Henniges (2007) liegen sogar knapp darunter. Die Zurechnung der verbleibenden „operating costs" (Reparatur- und Instandhaltung, Overhead) zu einzelnen I-OSektoren erfolgt auf Basis von Schätzungen für I-O-Koeffizienten in den USA von Urbanchuk (2007) und von Hayashi et al. (2008) für Japan. Ein wichtiger Kostenblock (ca. 30\%) wird dabei dem Sektor „Bauinstallations- und sonstige Bauarbeiten" für Reparatur- und Instandhaltung der Konversionsanlage zugerechnet.

\subsubsection{Zusammenfassende Darstellung der Szenariowerte}

Die aus diesen Angaben resultierenden Kosten auf Basis der verschiedenen Rohstoffe werden in Tabelle 10 und Tabelle 11 für die beiden Szenarien zusammengefasst. Durch Gewichtungen der Rohstoffe mit den jeweiligen Anteilen an der Produktionsmenge lässt sich jeweils ein Durchschnittskostenvektor für die Bioethanolproduktion errechnen, der für die I-O-Modellierung verwendet wird. Für die Produktionskosten ergeben sich durchschnittlich $516 €$ je $t$ Ethanol im Referenzszenario und $562 € / \mathrm{t}$ Ethanol im HD-Szenario. Im HD-Szenario führen die höheren Ölpreise zu steigenden Kosten von Bioethanol, wenngleich in geringerem Maße als bei Benzin. Neben den Agrarkosten hängen auch die Energiekosten der Weiterverarbeitung sowie der Bau der Anlage vom Ölpreis ab (OECD 2008d; WBA 2007; Concawe 2006). Die Kosten für Bioethanol steigen im HD-Szenario für die einzelnen Rohstoffe zwischen 10 (Lignocellulose) und $15 \%$ (Weizen) durch die höheren Annahmen für den Ölpreis. Diese Steigerung 
entspricht auch den Ergebnissen für ähnliche Sensitivitätsanalysen des Wissenschaftlichen Beirats für Agrarpolitik (WBA 2007) und der OECD (2008d). ${ }^{186}$

Daneben sind noch Lerneffekte zu berücksichtigen, da die Werte von Concawe (2006) einem kürzeren Zeithorizont unterliegen und daher geringere Lerneffekte enthalten. Für Weizen und Zucker werden lediglich Lerneffekte bei den Investitionen (10\% Einsparung) angenommen. Bei der noch stärker in der technologischen Entwicklung befindlichen Herstellung von Lignocellulose wird im Referenzszenario eine Kosteneinsparung von $10 \%$ gegenüber den Werten von Concawe (2006) angenommen. Basis hierfür bilden Werte von GEMIS (2008). Aufgrund der höheren Verbreitung werden in Anlehnung an Neuwahl et al. (2008) weitere Einsparungen von 12,5\% im HD-Szenario geschätzt. Allerdings liegen auch unter diesen Annahmen die Kosten für Ethanol aus Lignocellulose höher als aus Weizen und Zuckerrübe. Hier sind die zugrunde gelegten Annahmen von Concawe (2006) vergleichsweise zurückhaltend. Einige andere Betrachtungen halten hingegen erhebliche Kosteneinsparungen für möglich (z.B. Lynd 1996; Osborne 2007; Hamelinck et al. 2005). ${ }^{187}$ Kostenbetrachtungen unterliegen aber besonders großen Unsicherheiten aufgrund der bisher fehlenden kommerziellen Produktion im großvolumigen Maßstab.

Die angesetzten Durchschnittskosten in den Szenarien liegen trotz der vergleichsweise höher angesetzten Rohstoffkosten und Ölpreise eher im mittleren Bereich der existierenden Bandbreiten in der Literatur (IFEU 2007). Dies erscheint plausibel, da der inländische Kapazitätsausbau vorrangig in größeren Anlagen mit den wirtschaftlich rentabelsten Verfahren stattfindet.

\footnotetext{
${ }^{186}$ Beispielsweise geht der WBA (2007) bei einer 80\%-igen Steigerung der Energiepreise von einer Kostenerhöhung von 0,10 auf $0,13 € /$ khw (ca. $25 \%$ ) aus.

${ }^{187}$ Für Europa sieht eine häufig zitierte Studie Hamelinck/Faaij (2006) bis 2015-2020 ein Potenzial von $415 € / t$, längerfristig (ca. 2025) sogar ein Potenzial von bis zu $230 € / \mathrm{t}$ Ethanol. Kostenunterschiede gegenüber den deutlich höheren Werten von Concawe (2006) liegen bei den Investitions- und Fixkosten, für welche Hamelinck/Faaij (2006) deutliche Einsparpotenziale erwarten. Auch für die Rohstoffkosten erwarten sie erhebliche Einsparungen, diese sind aber bei Concawe (2006) für den Ausgangspunkt geringer, so dass diese Differenz der Studien durch die Zukunftsprojektion von Hamelinck/Faaij (2006) nur ausgeglichen wird.
} 
Tabelle 10: Kosten für Bioethanol im Referenz-Szenario $(€ \text { je } t)^{*}$

\begin{tabular}{|l|c|c|c|c|}
\hline Kostenart & Weizen & $\begin{array}{c}\text { Zuckerrü- } \\
\text { ben }\end{array}$ & $\begin{array}{c}\text { Lignocell } \\
\text { ulose }\end{array}$ & $\begin{array}{c}\text { Durch- } \\
\text { schnitt** }\end{array}$ \\
\hline Kosten je t Rohstoff & 120 & 32 & 53 & - \\
t Rohstoff je t Ethanol & 3 & 13 & 4 & - \\
Rohstoffkosten je t Ethanol & $\mathbf{4 0 4}$ & $\mathbf{4 2 2}$ & $\mathbf{1 8 7}$ & $\mathbf{3 5 5}$ \\
$\ldots$...darunter Transportkosten & 4 & 17 & 12 & 8 \\
\hline Chemische Hilfs- & & & & \\
/Betriebsstoffe & $\mathbf{2 2}$ & $\mathbf{3 2}$ & $\mathbf{2 7}$ & $\mathbf{2 4}$ \\
Energie & $\mathbf{3 3}$ & $\mathbf{4 7}$ & $\mathbf{4 0}$ & $\mathbf{3 7}$ \\
Arbeit & $\mathbf{2 0}$ & $\mathbf{1 6}$ & $\mathbf{2 9}$ & $\mathbf{2 2}$ \\
Overhead & $\mathbf{7}$ & $\mathbf{5}$ & $\mathbf{8 7}$ & $\mathbf{2 6}$ \\
Zinskosten & $\mathbf{4 2}$ & $\mathbf{3 2}$ & $\mathbf{9 3}$ & $\mathbf{5 3}$ \\
Investitionskosten & $\mathbf{6 3}$ & $\mathbf{4 8}$ & $\mathbf{1 3 9}$ & $\mathbf{7 9}$ \\
Kuppelprodukterlöse & $-\mathbf{1 1 2}$ & $\mathbf{- 7 1}$ & - & $-\mathbf{7 9}$ \\
\hline Gesamtkosten & $\mathbf{4 7 9}$ & $\mathbf{5 3 2}$ & $\mathbf{6 0 3}$ & $\mathbf{5 1 6}$ \\
\hline
\end{tabular}

Quelle: Eigene Berechnungen, Datenbasis: siehe verschiedene Textstellen

* Hervorgehobene Werte gehen direkt in die Gesamtkostenrechnung ein

**Die Durchschnittskosten ergeben aus den Anteilen der Rohstoffe an der zusätzlichen Ethanolproduktion

Tabelle 11: Kosten für Bioethanol im HD-Szenario ( $\in$ je $t)^{*}$

\begin{tabular}{|l|c|c|c|c|}
\hline Kostenart & Weizen & $\begin{array}{c}\text { Zuckerrü- } \\
\text { ben }\end{array}$ & $\begin{array}{c}\text { Lignocell } \\
\text { ulose }\end{array}$ & $\begin{array}{c}\text { Durch- } \\
\text { schnitt** }\end{array}$ \\
\hline Kosten je t Rohstoff & 126 & 33 & 55 & - \\
t Rohstoff je t Ethanol & 3 & 13 & 3 & - \\
Rohstoffkosten je t Ethanol & $\mathbf{4 2 5}$ & $\mathbf{4 4 2}$ & $\mathbf{1 7 0}$ & $\mathbf{3 3 4}$ \\
...darunter Transportkosten & 4 & 17 & 12 & 8 \\
\hline Chemische Hilfs-/Betriebsstoffe & $\mathbf{4 0}$ & $\mathbf{4 4}$ & $\mathbf{3 0}$ & $\mathbf{3 7}$ \\
Energie & $\mathbf{6 0}$ & $\mathbf{6 6}$ & $\mathbf{4 6}$ & $\mathbf{5 6}$ \\
Arbeit & $\mathbf{2 2}$ & $\mathbf{1 8}$ & $\mathbf{2 8}$ & $\mathbf{2 3}$ \\
Overhead & $\mathbf{7}$ & $\mathbf{6}$ & $\mathbf{8 3}$ & $\mathbf{3 5}$ \\
Zinskosten & $\mathbf{4 4}$ & $\mathbf{3 3}$ & $\mathbf{8 9}$ & $\mathbf{5 9}$ \\
Investitionskosten & $\mathbf{6 5}$ & $\mathbf{5 0}$ & $\mathbf{1 3 3}$ & $\mathbf{8 8}$ \\
Kuppelprodukterlöse & $\mathbf{- 1 1 7}$ & $\mathbf{- 7 5}$ & - & $-\mathbf{7 0}$ \\
\hline Gesamt & $\mathbf{5 4 5}$ & $\mathbf{5 8 7}$ & $\mathbf{5 7 8}$ & $\mathbf{5 6 2}$ \\
\hline
\end{tabular}

Quelle: Eigene Berechnungen, Datenbasis: siehe verschiedene Textstellen

* Hervorgehobene Werte gehen direkt in die Gesamtkostenrechnung ein

**Die Durchschnittskosten ergeben aus den Anteilen der Rohstoffe an der zusätzlichen Ethanolproduktion 


\section{Input-Output-Koeffizienten der substituierten Güter}

Zur Bestimmung der gesamtwirtschaftlichen Effekte ist neben dieser Bestimmung der Ethanolkosten und der darauf basierenden Berechnungen der I-OVektoren eine analoge Parameterschätzung für die zu substituierenden Güter notwendig. Für die Berechnung der durch Bioethanol substituierten Güter ist zu beachten, dass im Ausgangsjahr 2005 bereits 226.000 t Bioethanol aus Getreide produziert werden. Da nur geringe Produktionsverbesserungen für Getreide angenommenen werden, wird diese Menge in der Quantifizierung nicht weiter betrachtet. Die restliche Menge Ethanol ersetzt Benzin. Hieraus folgt, dass im Referenzszenario knapp 6,4\% (1 Mio. t) Benzin des Ottokraftstoffverbrauchs aus Benzin und im HD-Szenario ca. 13,1\% (2,04 Mio. t) Benzin ersetzt wird. Da die Importe von Benzin in der Vergangenheit meist durch leicht höhere Exporte ausgeglichen werden (z.B. BAFA 2008), wird beim Substitut Benzin von einer Inlandsproduktion der gesamten Menge ausgegangen.

Wichtigster Kostenfaktor für Benzin ist der Ölpreis. Während im Referenzszenario für den Ölpreis von $70 \$ / \mathrm{bbl}$ die Benzinkosten ohne Steuern auf Basis von WBA (2007) auf $0,45 € / l$ geschätzt werden, erfolgt im HD-Szenario eine Anpassung aufgrund des höheren Ölpreises. Dabei werden die Produktionskosten für Benzin in Abhängigkeit vom Ölpreis auf Basis von Angaben des Mineralölwirtschaftsverbandes (MWV 2008) geschätzt. ${ }^{188}$ Eine Regression der realen logarithmierten Importkosten in Abhängigkeit der beobachteten realen logarithmierten Rohölpreise (in €/l) im Zeitraum zwischen 1991 und 2007 ermöglicht die Berechnung von Elastizitäten zwischen den Kosten bzw. Preisen. Unter Annahme der zeitlichen Konstanz der berechneten Elastizitäten und der inländischen Wertschöpfung wird für den Ölpreis von $100 \$ / b b l$ im HD-Szenario Benzinkosten von $0,60 € / 1$ ermittelt. ${ }^{189}$ Für die Inputstruktur wird der Sektor „Kokereierzeugnisse, Mineralölerzeugnisse, Spalt- und Brutstoffe“ leicht modifiziert. Neuwahl et al. (2008) schätzen für Europa den Anteil des Sektors der Öllieferung für Benzin bei einem Ölpreis von $58 € / \mathrm{bbl}$ auf $68 \%$. Auf Basis dieser Werte und des errechneten Zusammenhangs zwischen Ölpreis und Benzin ergeben sich Vorleistungsanteile von 70 bzw. 76\% für den Sektor "Erdöl, Erdgas, DL für Erdöl-, Erdgasgewinnung" am Produktionswert von Benzin. Die weiteren Inputfaktoren werden im gleichen Verhältnis wie die Mineralölherstel-

${ }^{188}$ Zur Schätzung wurden die Ölpreise von $\$ / b b l$ in $€ /$ auf Basis von Wechselkursangaben der OECD-STATS Datenbank umgerechnet. Die nominalen Preise wurden auf Basis von Angaben der Volkswirtschaftlichen Gesamtrechnung (Statistisches Bundesamt 2008a) auf das Jahr 2000 deflationiert. Dieses Vorgehen zur Bestimmung der Benzinkosten ist an Steininger et al. (2008) angelehnt, dort werden für Österreich die Benzinpreise auf Basis von Ölpreisen geschätzt.

${ }_{89}$ Die Elastizität beträgt in den Regressionsrechnungen $86 \%$. Zu den Importkosten für Öl hinzu werden für die Berechnung der Benzinkosten entsprechend dem Durchschnitt der letzten Jahr 9c/l für weitere Kosten (u.a. Verarbeitungskosten) angesetzt (MWV 2008). 
lung insgesamt angenommen, wegen fehlender Angaben für eine geeignete Disaggregation. Da die Herstellungskosten für Ethanol höher als für Benzin sind, entstehen insgesamt Mehrbelastungen durch die gesetzlich festgelegte Beimischung von Ethanol von 245 Mio. $€$ im Basisszenario und 218 Mio. $€$ im HD-Szenario. ${ }^{190}$ Diese Mehrausgaben werden durch sinkende private Konsumausgaben an anderer Stelle kompensiert.

\section{Produktion und Substitution von Ethanol und Benzin}

Die aus den Annahmen resultierenden Nachfrageimpulse sind in Tabelle $12 \mathrm{zu}-$ sammengefasst. Die Werte beziehen sich dabei auf die gesamte resultierende Produktion. Die inländische Produktion lässt sich mittels mit den in Klammern angegebenen Importquoten ableiten. Beispielsweise fallen im Referenzszenario $30 \%$ Ethanolproduktion von 869 Mio. $€$ im Ausland an, bei den substituierten Produkten findet nur Inlandsproduktion statt. Allerdings ist dabei zu beachten, dass Öl als zentrales Vorleistungsgut für Benzin fast ausschließlich importiert wird. Insgesamt ergibt sich ein Produktionswert von ca. 1 Mrd. $€$ im Referenzszenario und ca. 2,1 Mrd. $€$ im HD-Szenario.

Tabelle 12: Szenarienannahmen für die Produktion von Bioethanol im Jahr 2020 (in Mio. €)

\begin{tabular}{|l|c|c|c|c|}
\hline & \multicolumn{2}{|c|}{ Referenzszenario } & \multicolumn{2}{c|}{ HD-Szenario } \\
\hline & Ethanol & Benzin & Ethanol & Benzin \\
\hline Kraftstoffproduktion & $869(30 \%)$ & $624(0 \%)$ & $1872(30 \%)$ & $1654(0 \%)$ \\
\hline $\begin{array}{l}\text { Kuppelprodukt } \\
\text { (Futtermittel) }\end{array}$ & $133(30 \%)$ & $133(100 \%)$ & $232(30 \%)$ & $232(100 \%)$ \\
\hline Konsumveränderung & 0 & $245(0 \%)$ & 0 & $218(0 \%)$ \\
\hline $\begin{array}{l}\text { Gesamter Produktions- } \\
\text { wert }\end{array}$ & 1002 & 1002 & 2104 & 2104 \\
\hline
\end{tabular}

Quelle: Eigene Berechnungen. Die Werte in Klammern geben den Anteil der Importe an.

\subsubsection{Produktions- und Beschäftigungseffekte}

Die Auswirkungen der Diffusion von Bioethanol auf Produktion und Beschäftigung sind in Abbildung 15 und Abbildung 16 ersichtlich. Dabei ergibt sich eine positive inländische Nettoproduktion mit knapp 500 Mio. $€$ im Referenzszenario und knapp 1100 Mio. $€$ im HD-Szenario gegenüber dem als Vergleichsmaßstab dienenden Basisszenario. Die Beschäftigungsbilanz fällt mit knapp $3000 \mathrm{Er}-$

${ }^{190}$ Fiskalische Effekte, z.B. durch Veränderungen bei Mineralöl- oder Mehrwertsteuer, werden nicht betrachtet (siehe Kapitel 5.2.2). Sie sind als gering einzuschätzen. 
werbstätigen im Referenzszenario und ca. 9300 Erwerbstätigen im HD-Szenario ebenfalls positiv aus. Die Produktions- und Beschäftigungssalden nehmen jeweils einen ähnlichen Anteil an der Gesamtwirtschaft ein. ${ }^{19}$

Die positiven Effekte treten allerdings weniger in der Kraftstoffproduktion selbst, sondern in sehr vielen der 71 I-O-Sektoren auf (Anhang Tabelle 36 bis Tabelle 39). Dies spiegelt sich auch in den höher aggregierten Bereichen in den folgenden Abbildungen wider. Entscheidend hierfür sind Unterschiede bei den Importen zwischen der Produktion von Ethanol und Benzin. Entscheidend für diese sektorübergreifenden positiven Produktions- und Beschäftigungseffekte in beiden Szenarien sind aber die deutlich geringeren Importe für die benötigten Vorleistungen für Bioethanol im Vergleich zu Benzin. Während die inländische Produktion für Bioethanol aus einheimischen Pflanzen erfolgt, ist bei Benzin die quantitative Bedeutung des vollständig importierten Erdöls sehr hoch. Daher findet bei Benzin ein geringer Anteil der Wertschöpfung im Inland statt.

Die größten Effekte treten erwartungsgemäß im Landwirtschaftssektor auf. Aufgrund der hohen Arbeitsintensität schlägt sich dies besonders in der Beschäftigungsbilanz nieder, die Mehrbeschäftigung beträgt ca. 3000 (Referenzszenario) bzw. 5000 (HD-Szenario) Personen. Ein deutlich höherer Produktions- und Beschäftigungseffekt ergibt sich im HD-Szenario bei den Dienstleistungen. Im Referenzszenario resultiert durch die hohe Kostendifferenz je Produktionseinheit zwischen Ethanol und Benzin eine deutliche private Konsumminderung. Da ein besonders großer Anteil des privaten Konsums auf Dienstleistungen entfällt, ist der Nettoproduktionseffekt nur gering. Im HD-Szenario ist dieser negative Effekt anteilsmäßig kleiner, die positiven Effekte auf den Produktionswert für Dienstleistungen überwiegen deshalb stärker. Deshalb sind auch die gesamten Produktions- und Beschäftigungseffekte überproportional größer als im Referenzszenario. In den Industriesektoren ergeben sich hingegen in beiden Szenarien kaum Veränderungen, hier heben sich die Impulse von Bioethanol und Benzin gegenseitig auf.

${ }^{191}$ Die Größeneinordnung dieser Effekte ist allerdings schwierig, da ein geeigneter Maßstab hierfür fehlt. Wenn man die direkte Beschäftigung in der Mineralölindustrie mit ca. 17000 Erwerbstätigen im Jahr 2005 zum Vergleich nimmt (Statistisches Bundesamt 2006), erscheinen die Zuwächse beachtlich. 
Abbildung 15: Nettoproduktionswirkungen durch zusätzliche Diffusion von Bioethanol im Vergleich zum Basisszenario (in Mio. $€$ )

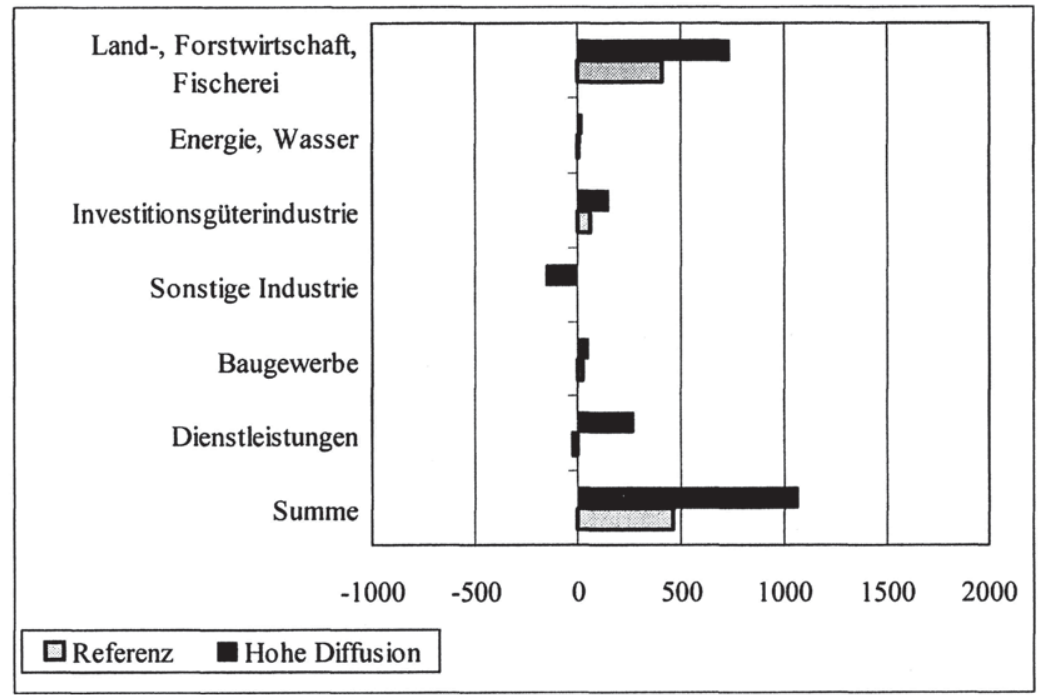

Quelle: Eigene Berechnungen

Abbildung 16: Nettobeschäftigungswirkungen durch zusätzliche Diffusion von Bioethanol im Vergleich zum Basisszenario (in Erw.)

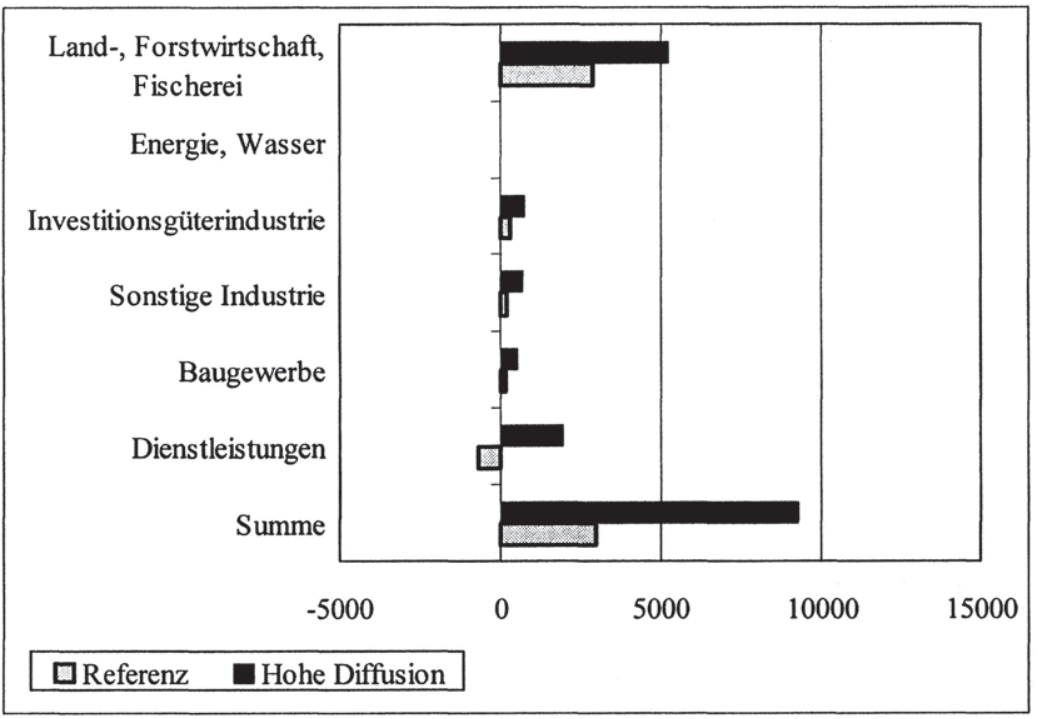

Quelle: Eigene Berechnungen 


\subsubsection{Sensitivitätsanalyse}

Aufgrund der Unsicherheit einzelner Kostenparameter und divergierender Annahmen für die Literatur, werden für die wichtigen Variablen Sensitivitätsanalysen durchgeführt. Es werden dabei für die Diffusion, Importquote für Ethanol, Verdrängungseffekte bei der Landwirtschaftsfläche und der Kosten- und Konsumstruktur Bandbreiten für die Variablenwerte aus der Literatur abgeleitet. ${ }^{192}$ In Tabelle 13 sind der Referenzwert sowie mögliche niedrige und hohe Ausprägungen der Variablen ersichtlich.

\section{Tabelle 13: Sensitivitätsannahmen für Bioethanol}

\begin{tabular}{|l|l|l|l|l|}
\hline \multicolumn{1}{|c|}{ Variable } & $\begin{array}{c}\text { Refe- } \\
\text { renz }\end{array}$ & $\begin{array}{c}\text { Nied- } \\
\text { rig }\end{array}$ & Hoch & \multicolumn{1}{|c|}{ Quelle/Anmerkung } \\
\hline $\begin{array}{l}\text { Diffusion (in \% Ener- } \\
\text { giegehalt an Otto- } \\
\text { kraftstoff) }\end{array}$ & $7,25 \%$ & $3,25 \%$ & $10 \%$ & $\begin{array}{l}\text { Niedrig: Aktuelle Höchst- } \\
\text { grenze der erlaubten Beimi- } \\
\text { schung (BMU 2008); } \\
\text { Hoch: Bis Oktober 2008 be- } \\
\text { stehende EU-Regelung }\end{array}$ \\
\hline Importquote & $30 \%$ & $0 \%$ & $40 \%$ & $\begin{array}{l}\text { Niedrig: Annahme vollstän- } \\
\text { diger Inlandsproduktion; } \\
\text { Hoch: Obere Bandbreite aus } \\
\text { Literatur (z.B. Nusser et al. } \\
\text { 2007a) }\end{array}$ \\
\hline $\begin{array}{l}\text { Agrar- } \\
\text { Konkurrenzfläche } \\
\text { (in\%) }\end{array}$ & $16 \%$ & $0 \%$ & $\begin{array}{l}\text { Niedrig: Annahme keiner } \\
\text { Flächensubstitution (z.B } \\
\text { Schöpe/Brischkat 2006); } \\
\text { Hoch: Annahme doppelter } \\
\text { Flächensubstitution }\end{array}$ \\
\hline Kostenstruktur & $\begin{array}{l}\text { 32\% } \\
\text { Hauptunterschied liegt im höheren Rohstoffkostenan- } \\
\text { teil bei GEMIS (76\%) gegenüber dem Referenzszena- } \\
\text { rio(58\%) }\end{array}$ \\
\hline Konsumstruktur & $\begin{array}{l}\text { Konsumstruktur mit preisunelastischem Nachfragever- } \\
\text { halten und Durchschnittsvektor der I-O-Tabelle 2005 }\end{array}$ \\
\hline
\end{tabular}

Quelle: Eigene Berechnungen

${ }^{192}$ Keine Sensitivitätsanalysen finden für die Kostenhöhe statt. Die Durchführung von Sensitivitätsanalysen ist problematisch, da im I-O-Modell höhere Kosten auch in eine höhere Produktion und eine höhere Beschäftigung übersetzt werden (Wicke 2006). Dies ist häufig aber nur begrenzt plausibel. Z.B. ist es unwahrscheinlich, dass ein Anstieg der Weizenpreise um $10 \%$ zu einer proportional höheren Beschäftigung führt. 
Das Ergebnis für die Sensitivitätsanalyse der Nettoproduktionseffekte ist in Abbildung 17 dargestellt. Es werden nur die Nettoergebnisse für die Produktion und Beschäftigung betrachtet, die Sensitivitätswerte werden dabei in Parametervariation zum Referenzwert $(=100 \%)$ dargestellt. Bspw. stellt die Annahme einer Importquote von $40 \%$ den 1,33-fachen Wert (=133\%) gegenüber dem Referenzwert von $30 \%$ dar. Für diese Erhöhung der Importquote ergibt sich ein Nettoproduktionseffekt von ca. 200 Mio. $€$, unter der Annahme, dass alle anderen Parametern identisch zum Referenzszenario sind. Bei einer Importquote von $0 \%$ liegt der Nettoproduktionswert bei knapp 1300 Mio. $€$. Folglich wirkt sich eine Zunahme der Importquote negativ auf die Nettoproduktion aus, die Nettoeffekte bleiben innerhalb der gesamten Bandbreite positiv. Eine Ausnahme bei dieser Darstellung bilden Konsum- und Kostenstrukturveränderungen. Da hier keine Höhe der Parametervariation angegeben werden kann, werden diese Werte auf der x-Achse wie der Referenzwert bei $100 \%$ angegeben. Der Wert für die Kostenstruktur in Abbildung 17 sagt beispielsweise, dass die Verwendung der alternativen Kostenstruktur auf Basis der GEMIS-Datenbank für die Schätzung der IO-Koeffizienten zu einer Nettoproduktion von ca. 570 Mio. $€$ führt und damit höher als im Referenzszenario liegt. Die Differenz ist aber nicht sehr groß, das Ergebnis ist im Bezug auf die Kostenstruktur als eher robust einzuschätzen, da weitere alternative Kostenstrukturen in der Literatur (z.B. Henniges 2007, IFEU 2007) nur geringfügig von den beiden verwendeten Vektoren abweichen.

Abbildung 18 zeigt die Sensitivitätsanalyse für die Nettobeschäftigungseffekte. Die Produktions- und Beschäftigungseffekte bleiben insgesamt in allen Sensitivitätsrechnungen positiv. Die geringeren Importquoten in der Wertschöpfungskette bei Bioethanol im Vergleich zu Benzin überlagern andere Effekte deutlich. Bei den Sensitivitätsrechnungen für die einzelnen Variablen (z.B. Diffusion) zeigen sich die erwarteten Richtungsänderungen. Eine reine Erhöhung der Diffusion führt zu deutlich höheren Produktions- und Beschäftigungswirkungen. Dabei ist zu beachten, dass im Gegensatz zum HDSzenario alle sonstigen Parameter konstant gehalten werden. In ähnlicher Stärke beeinflussen die Importquote die Ergebnisse. Nur marginale Unterschiede ergeben sich durch die alternative Konsumstruktur des Minderkonsums und der Höhe der konkurrierenden Agrarfläche. Die sektorale Struktur bleibt in allen Sensitivitätsrechnungen sehr ähnlich zum Referenzszenario. 
Abbildung 17: Nettoproduktionswirkungen für Bioethanol in Sensitivitätsrechnungen im Vergleich zum Basisszenario (in Mio. $€$ )

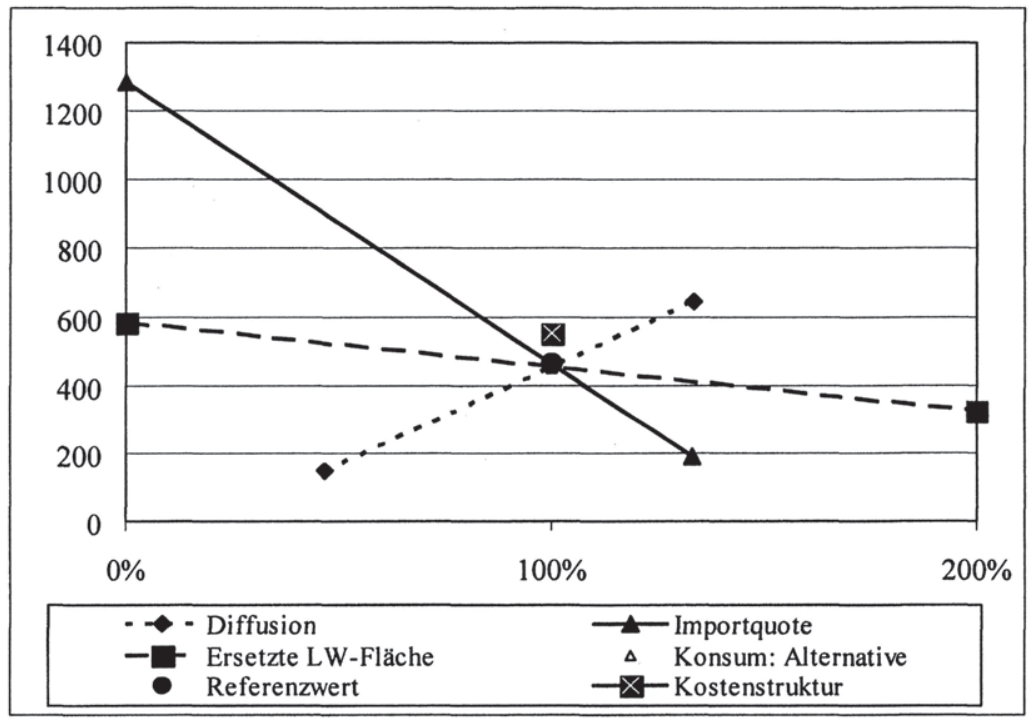

Quelle: Eigene Berechnungen

Abbildung 18: Nettobeschäftigungswirkungen für Bioethanol in Sensitivitätsrechnungen im Vergleich zum Basisszenario (in Erw.)

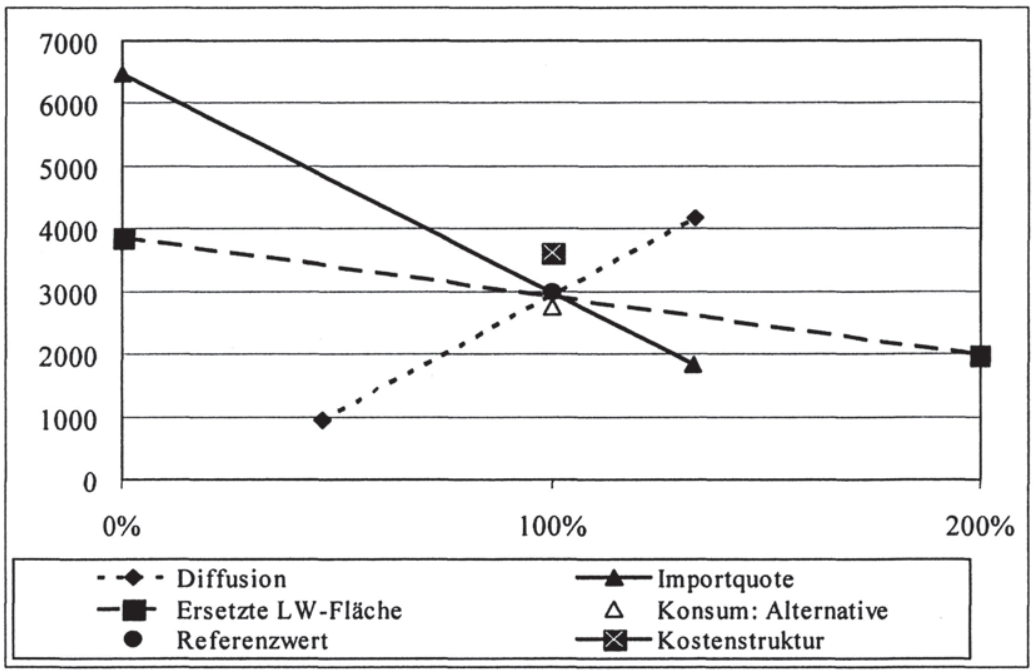

Quelle: Eigene Berechnungen 


\subsection{Biopolymere}

Im Bereich der (Bio-)Polymere ist die aktuelle Nutzung der Biotechnologie gering, aber mittel- bis langfristig werden große Zukunftspotenziale gesehen. Polymere - häufig werden die Begriffe Plastik und Kunststoffe synonym verwendet - gehören aufgrund der geringen Herstellungskosten und breiten Anwendungsmöglichkeiten zu den meist verwendeten Materialien der Welt (u.a. Beucker/Marscheider-Weidemann 2007; IPTS 2007). Der Term Biopolymere wird in der Literatur für unterschiedliche Arten von Polymeren verwendet (IPTS 2007). So werden teilweise Polymere, die auf Biomasse aufbauen, Polymere die biotechnologisch hergestellt werden, aber auch für Polymere die biologisch abgebaut werden können, als Biopolymere definiert (Enzing et al. 2007b). Zwar gibt es deutliche Überschneidungen bei diesen Abgrenzungen, sie sind aber nicht identisch. So sind biotechnologisch hergestellte Polymere in der Regel aus nachwachsenden Rohstoffen und meistens biologisch abbaubar, aber nicht immer. Zudem existieren mittlerweile auch petrochemische Polymere (z.B. Ecoflex der BASF), die biologisch abbaubar sind. Im Rahmen dieser Arbeit ist die biotechnologische Herstellung für die Verwendung des Begriffs Biopolymere entscheidend. Dabei werden vereinfachend biotechnologisch hergestellte Polymere auf Basis nachwachsender Rohstoffe mit chemisch hergestellten Polymeren auf fossiler Rohstoffbasis verglichen, da die anderen möglichen Fälle quantitativ nur von sehr geringer Bedeutung sind.

Im Fokus der Analyse stehen dabei Polymere für Bulk-Anwendungen, das heißt Anwendungen mit hohem Produktionsvolumen. Über $90 \%$ der hergestellten Polymere werden in hoher Menge, bei bislang sehr geringen Preisen ( 1 bis 2 $€$ pro kg) hergestellt (Patel et al. 2006). Bei Biopolymeren in BulkAnwendungen bestehen in der Regel ähnliche Funktionalitäten wie bei Standardpolymeren. Die Konkurrenz ergibt sich folglich über den Preis, der wegen geringen Profitmargen eng an den Produktionskosten liegt (Crank et al. 2004, S.123). Somit wird der quantitativ mit großem Abstand bedeutendere Teil der Prozessinnovationen betrachtet.

\subsubsection{Diffusion}

Die Höhe der Diffusion und Produktion von Biopolymeren wird aus dem Wachstum der Polymerproduktion und dem Anteil von Biopolymeren abgeleitet. Deutschland produzierte im Jahr 2005 mit 18 Millionen Tonnen ca. 9\% des weltweiten Kunststoffaufkommens von 224 Mio t (PlasticsEurope 2007). Die Plastikproduktion verzeichnete in den vergangenen Jahrzehnten größere Wachstumsraten als andere Gruppen von Bulkmaterialen und es wird erwartet, dass sich dieser Trend bis 2020 fortsetzt (Crank et al. 2004, S.5). Polymere werden nach dieser Einschätzung zunehmend Glas und zu einem kleineren Teil Stahl ersetzen können. Die Interviewpartner sehen allerdings kaum völlig neue An- 
wendungsmöglichkeiten für Polymere, auch nicht durch mögliche Veränderungen der Funktionalitäten auf Basis biotechnologischer Methoden. Projektionen für das Wachstum in Europa bis zum Jahr 2020 liegen zwischen 1,5 und 2,3\% p.a. (Phylipsen et al., 2002; Chateau et al., 2005; Patel et al. 2006). ${ }^{193}$ In den Szenarioanalysen werden analog zu Phylipsen et al. (2002) ein Wachstum bis zum Jahr 2020 von 2\% p.a. angesetzt. Dies entspricht einem absoluten Produktionsvolumen von 24 Mio. t.

Der Einsatz von Biopolymeren ist in vielen Anwendungsgebieten der Polymere denkbar. Der wichtigste Einsatzbereich liegt, wie bei konventionellen Polymeren, bei Verpackungen und wird es voraussichtlich bleiben (Oertel 2007; Müssig/Carus 2007). ${ }^{194}$ Ein relativ hoher Einsatz von Biopolymeren wird im Automobilbereich (v.a. Innenausstattung) erwartet. Das direkte Substitutionspotenzial von petrochemischen Erzeugnissen ist allerdings insgesamt begrenzt, da bislang für einige Anwendungen, z.B. in vielen Bereichen des Bausektors, keine denkbare biotechnologische Lösung existiert. Crank et al. (2004, S.193) schätzen in einer Detailanalyse für einzelne Polymere auf Basis nachwachsender Rohstoffe - sowohl biotechnologisch als auch chemisch produziert - ein maximales Substitutionslevel von einem Drittel der petrochemischen Polymere. Die bisherige Verbreitung der Biopolymere ist deutlich geringer. Das Volumen in Europa wird im Jahr 2005 auf knapp $45.000 \mathrm{t}$ im Jahr geschätzt, dies entspricht $0,13 \%$ der europäischen Polymer-Produktion (Enzing et al. 2007b). In den USA und Japan wird der Anteil auf 0,45\% geschätzt.

Die zukünftige Verbreitung von Biopolymeren wird unter bestimmten Bedingungen recht positiv eingeschätzt. Faktoren, wie Regulierungen zum Klimaschutz, Bestrebungen einer nachhaltigen Entwicklung und steigende Ölpreise wirken sich positiv auf die Entwicklung aus (Enzing et al. 2007b). ${ }^{195}$ So gibt es nach Eindruck der befragten Experten eine Vielzahl von Ankündigungen von Unternehmen zu geplanten Produktionsaufnahmen. Allerdings sind die Diffusion und ökonomischen Wirkungen von einer Reihe von Einflussfaktoren abhängig. Sehr hohe Bedeutung für die zukünftige Verbreitung kommt dabei den relativen Rohstoffkosten der nachwachsenden Rohstoffe gegenüber den fossilen Rohstoffen zu. Technologische Unsicherheiten bestehen aber z.B. darin, ob die Umwandlung von Lignocellulose so gelingt, dass sie den Anforderungen der weiterverarbeitenden Sektoren entspricht (Phylipsen et al. 2002, S.91). Unsicherheiten bestehen auch auf der Nachfrageseite, z.B. inwiefern Konsumenten

\footnotetext{
${ }^{193}$ Die Studien betrachten dabei verschiedene Szenarien. Die angegebene Bandbreite bezieht sich auf das jeweilige Basis- bzw. „Medium“-Szenario dieser Studien.

${ }^{194}$ Die größten spezifischen Einsatzfelder von Kunststoffen liegen in Deutschland im Bereich der Verpackungen (33\%), in der Bauwirtschaft (23,5\%), im Automobilbereich (9\%) sowie in der Elektronik (7,5\%) (VKE 2007).

${ }^{195}$ Für ausführlichere Diskussionen zur Diffusion bei Biopolymeren, siehe Crank et al. (2004) und Beucker/Marscheider-Weidemann (2007).
} 
biobasierte Chemieprodukte aufnehmen (Crank et al. 2004). Als häufige Diffusionshürde gelten schließlich die erwähnten hohen Investitionskosten in neue Produktionsstätten. Dabei zeigt sich das Problem des Erreichens einer kritischen Masse. So lange sich nicht Wertschöpfungsketten auf Basis von Biopolymeren etabliert haben, sind Investitionen in große Anlagen sehr riskant. Aber erst wenn solche Anlagen und Produktionsvolumen existieren, werden Lern- und Skaleneffekte und infolge Kostenwettbewerbsfähigkeit sowie weitere Vorteile (z.B. etablierte Entsorgungswege) erreicht. Die Politik übt bisher eher einen indirekten Einfluss aus, z.B. über die Beeinflussung von Preisen und Verfügbarkeit von Biomasse, Förderung von F\&E (v.a. zur Nutzbarkeit von Lignocellulose), Harmonisierung von Standards oder Unterstützung der Infrastruktur zur Abfallentsorgung (Crank et al. 2004, S.181, ECCP 2001). Eine direkte Festschreibung von Quoten (z.B. Biokraftstoffe) oder finanzielle Unterstützung der Verwendung (z.B. Energieeinspeisegesetz bei Bioenergie) existiert für Biopolymere bislang nicht.

Die meisten Projektionen für die zukünftige Verbreitung der Biopolymere beziehen sich auf nachwachsende Rohstoffe insgesamt, wobei den hiervon biotechnologisch hergestellten Polymeren mit Abstand die positivsten Wachstumsaussichten eingeräumt werden (Beucker/Marscheider-Weidemann 2007) und voraussichtlich einen absoluten Großteil ausmachen werden. Biotechnologisch hergestellte Polymere gelten auch als zweite Generation von Kunststoffen aus nachwachsenden Rohstoffen (Fraunhofer ISI 2006). Sie können besser als die bisher gebräuchlichen thermoplastischen Stärken und Cellulose(-acetate) an spezifische Einsatzbedingungen angepasst werden und besitzen daher ein größeres Spektrum an Einsatzmöglichkeiten (Beucker/Marscheider-Weidemann 2007). Die europäische Studie ProBip (Crank et al. 2004) projiziert für den Anteil der Biopolymeren an allen Polymeren - bei Fortsetzung aktueller politischer Unterstützungen - einen Wert von $2,5 \%$, in einem optimistischen Fall 4,3\%. ${ }^{196}$ Andere Projektionen mit absoluten Tonnen oder Produktionswertangaben liegen verhältnismäßig leicht höher (z.B. Müssig/Carus 2007, Roland Berger 2007). Die befragten Experten schätzen die Diffusion mehrheitlich bei ca. $5 \%$ ein, allerdings gab es auch ein paar pessimistischere Einschätzungen. Bei günstigen relativen Preisen für nachwachsende Rohstoffe im Vergleich zu Öl sehen einige Experten einen Anstieg auf 10\% für möglich an. Auch hier gibt es aber ein paar skeptischere Meinungen. ${ }^{197}$ Deshalb wird für das Referenzszenario ein Wert von

\footnotetext{
${ }^{196}$ Dabei werden die Bedingungen für das Eintreten des optimistischen Falls nicht genauer spezifiziert.

${ }^{197}$ Begründungen hierfür liegen in der benötigten Zeit bis entsprechende Kapazitäten aufgebaut werden, in den Preisstrategien der Hersteller von konventionellen Polymeren, die teilweise zu Preisen unterhalb der Herstellungskosten verkaufen - dies ist aufgrund der teilweisen Gewinnung als Abfallprodukt möglich - sowie zum Teil in der Einschätzung, dass das bisherige Öl-NAWARO Preisverhältnis nicht für eine Vorteilhaftigkeit ausreicht.
} 
$4 \%$, für das $\mathrm{HD}$-Szenario von $8 \%$ einer Produktion von knapp über $24 \mathrm{Mio}$. $\mathrm{t}$ Polymeren festgelegt. Daraus ergeben sich die Tabelle 14 dargestellten Mengen an zusätzlicher Diffusion.

Tabelle 14: Diffusion von Biopolymeren in den Szenarien

\begin{tabular}{|l|c|c|}
\hline & Referenzszenario & HD-Szenario \\
\hline Diffusion im Jahr 2020 in Mio. t & 0,98 & 1,96 \\
\hline Diffusion im Jahr 2005 im in Mio. t & 0,01 & 0,01 \\
\hline $\begin{array}{l}\text { Differenz 2020-2005 in Mio. t } \\
\text { (Berechnungswert) }\end{array}$ & 0,97 & 1,95 \\
\hline
\end{tabular}

Quelle: Eigene Berechnungen, Datenbasis für Jahr 2005: Enzing et al. (2007b)

\subsubsection{Internationaler Wettbewerb und Außenhandel}

International variieren die Aktivitäten hinsichtlich F\&E und Aufbau von Produktionskapazitäten zwischen verschiedenen Ländern. Allgemein werden besonders in den USA, China und Japan Politikmaßnahmen zur Ersetzung der Petrochemie durch Biomasse durchgeführt (Enzing et al. 2007b). Zudem gehören die USA und China zu den größten Anbietern von nachwachsenden Rohstoffen (Enzing et al. 2007b). Die Exporteure der Grundstoffe haben dabei gute Vorrausetzungen, direkt in die Produktion von Biopolymeren einzusteigen (Beucker/Marscheider-Weidemann 2007). Eine ähnliche Entwicklung wie beim Öl, welches als Rohstoff über weite Distanzen transportiert wird, ist für Biopolymere unwahrscheinlicher, da die Transportkosten für nachwachsende Rohstoffe deutlich höher sind. Folglich würde es zu einem Handel der fertiggestellten Polymere oder deutlich weiterverarbeiteten Zwischenprodukten, aber weniger zu einem Austausch der zu bearbeitenden Rohstoffe kommen. Deutschland würde demnach eher Nettoimporteuer werden. Allerdings zeigt sich aus technologischer Sicht eine deutlich höhere Wettbewerbsfähigkeit Deutschlands. Die Auswertung der Patentanmeldungen für biotechnisch hergestellte Polymere zeigen, dass diese in den letzten Jahren stagniert und Deutschland mit 10\% den dritten Platz hinter den USA (40\%) und Japan (14\%) einnimmt (Beucker/Marscheider-Weidemann 2007). Die befragten Experten bestätigen diese Pro- und Kontra-Argumente und gehen mehrheitlich von einem stabil bleibenden weltweiten Produktionsanteil Deutschlands aus, ohne größere Unterschiede zwischen Biopolymeren und ölbasierten Polymeren. Für die Szenarioberechnungen werden deshalb keine Unterschiede bei der Außenhandelsbilanz im Vergleich zu ölbasierten Polymeren angenommen. Allerdings ist in diesem Fall zu beachten, dass es zu einem erheblichen Bedarf an nachwachsenden Rohstoffen kommt. 


\subsubsection{Herstellungskosten für Biopolymere}

Die Herstellung von Biokunststoffen unterscheidet sich von konventionellen Kunststoffen besonders in der Rohstoffbasis. Je nach Biokunststoff werden unterschiedliche Agrarrohstoffe (z. B. Zuckerrüben, Getreide) für die Herstellung benötigt, die dann über Zwischenprodukte (z. B. Milchsäure) durch Polymerisation zu Biopolymeren weiterverarbeitet werden. Der biotechnologische Prozess (Fermentation) findet dabei meist bei der Produktion für die Zwischenprodukte statt. Die Polymerisation hingegen ist ein chemischer Prozess. In der Wertschöpfungskette klassischer chemischer Kunststoffe werden die Rohstoffe hingegen von der Mineralöl- und der petrochemischen Industrie bezogen. Aus den Vorprodukten werden die Endprodukte (z.B. Kunststoffverpackungen, Spritzgussteile) gefertigt, wobei diese letzten Fertigungsschritte für petrochemische Kunststoffe und Biokunststoffe vergleichbar sind und hauptsächlich in der Kunststoffverarbeitenden Industrie stattfinden (Beucker/Marscheider-Weidemann 2007). Diese Schritte können demnach bei einem Vergleich vernachlässigt werden ( $\mathrm{Pa}$ tel et al. 2006). Nach der Gebrauchsphase können die Biopolymere biologisch abgebaut, thermisch verwertet oder theoretisch auch recycelt werden.

Bei den (Bio-)Polymeren sind im Gegensatz zu Bioethanol zwischen verschiedenen Polymerklassen zu unterscheiden. Die bisher wichtigsten Biopolymere für Bulk-Anwendungen sind Polylactide (PLA), Polyhydroxyalkanoate (PHA) und 1,3-Propanediol (BIO-PDO), aber auch weitere Polyester und Polyamide befinden sich in der fortgeschrittenen Forschung (Enzing et al. 2007b; Crank et al. 2004). ${ }^{198}$ Für die Szenarienberechnungen werden die ersten drei genannten Klassen berücksichtigt. Für die Herstellung dieser Biopolymere sind verschiedene Rohstoffe denkbar, z.B. Weizen, Zucker, Mais, aber auch Glycerin. Für Deutschland erscheint der Einsatz von Weizen nach Expertenaussagen am wahrscheinlichsten, deshalb wird die Betrachtung auf diesen Rohstoff fokussiert. ${ }^{199}$ Daneben ist die Verarbeitung von Lignocellulose für Biopolymere nach Ansicht der Mehrheit der Experten zu erwarten. Für die Berechnungen wird aus Konsistenzgründen ein gleicher Anteil an der Produktion wie bei Bioethanol unterstellt, d.h. 20\% im Referenzszenario und 33\% im HD-Szenario.

Im Gegensatz zu Bioethanol existieren deutlich weniger Angaben zu Kosten und deren Strukturen in der Literatur, so dass diese Informationen durch direkte Vergleiche zu ölbasierten Polymeren, die auf Expertenaussagen beruhen, ver-

\footnotetext{
${ }^{198}$ Eine alternative Möglichkeit, die im Folgenden nicht näher betrachtet wird, ist die geplante Produktion von Dow Chemicals und Braskem von biobasierten Ethylen in Brasilien (Blauuw et al. 2008). Ethylen ist eine Basischemikalie, die z.B. zu dem Kunststoff Polyethylen weiterverarbeitet werden kann. Es werden somit quasi identische Polymere zur chemischen Vorgehensweise erzeugt. Eine solche Produktion in Deutschland ist aufgrund der schlechteren Rohstoffposition nach Expertenaussagen mittelfristig eher unwahrscheinlich.

199 Dieses Vorgehen ist analog zu Müssig/Carus (2007) und WI/RWI (2008), die ebenfalls eine vollständige Produktion von Biopolymeren aus Weizen annehmen.
} 
vollständigt werden. Eine Schwierigkeit beim Kostenvergleich liegt darin, dass Kostenstrukturen stark von der Skalengröße abhängen (Mühlbauer 2006). Bisher werden Biopolymere zumeist aber nur in Pilotanlagen produziert und die bisherigen kommerziell betriebenen Anlagen sind vergleichsweise klein. Allerdings ist auch nicht zu erwarten, dass biotechnologische Anlagen eine ähnliche Größe wie chemische Polymere erreichen. Nach Aussagen von Experten wird bei Biopolymeren der Großteil der möglichen Skaleneffekte bereits bei einer geringeren Produktionsmenge im Vergleich zu Standardpolymeren erreicht (Dornburg 2004). Ursächlich hierfür sind zunehmende Transportkosten und Logistikherausforderungen bei zusätzlichen Produktionsmengen wegen des dezentralen Anfalls von Biomasse. Deshalb wird für die Kostenbetrachtung eine Anlagengröße von $100.000 \mathrm{t}$ zugrunde gelegt. Diese Größe liegt über den meisten bisherigen Anlagen, aber unter denen von vielen ölbasierten Polymeren.

\section{Rohstoffkosten}

Die Rohstoffkosten nehmen bei ölbasierten Massenpolymeren mit ungefähr 50\% bei größeren Anlagen einen erheblichen Kostenanteil ein (Buchholz 2006; Müssig/Carus 2007). Da sich die Biopolymere und ölbasierten Polymere bei der Herstellung besonders in der Rohstoffbasis unterscheiden, ist das Verhältnis der relativen Rohstoffkosten entscheidend für die Vorteilhaftigkeit (Crank et al. 2004, S.134). Bisher liegen nach Expertenaussagen die Rohstoffkosten bei Biopolymeren insgesamt auf einem ähnlichen Niveau. ${ }^{200}$ Bei genauer Betrachtung unterscheiden sich die Kosten zwischen den Biopolymerklassen aufgrund des unterschiedlichen Rohstoffbedarfs deutlich. Während bei PLA die Einsatzverhältnisse zwischen 2,0 und 2,4 t Weizen je t Biopolymere geschätzt werden (Dornburg 2004; Patel et al. 2006; Müssig/Carus 2007), liegt der entsprechende Rohstoffbedarf bei PHA bei bis zu $5 \mathrm{t}$ Weizen. Technologische Verbesserungsmöglichkeiten im Vergleich zu den den heutigen Möglichkeiten bei der Ausbeute von Rohstoffen werden von den befragten Experten aber nur bedingt gesehen. Die Verfahren (z.B. Fermentation von Milchsäure für PLA) sind bereits recht fortgeschritten. Das Potenzial für zukünftige Rohstoffeinsparungen ist daher limitiert. So gehen auch Patel et al. (2006) von sehr geringen Veränderungen in den Einsatzverhältnissen bei Rohstoffen aus. Aus diesen Einsatzverhältnissen und den angenommenen Weizenpreisen ergeben sich für die Rohstoffkosten Bandbreiten von ca. $290 € / \mathrm{t}$ im Referenzszenario für PLA bis zu $630 € / \mathrm{t}$ im HDSzenario für PHA. Bei Lignocellulose sind die Kosten geringer und werden auf knapp die Hälfte angesetzt. Die Transportkosten und Kuppelprodukte ${ }^{201}$ sind aufgrund der gleichen betrachteten Rohstoffbasis ähnlich zu Bioethanol. Unter-

\footnotetext{
${ }^{200}$ Diese Aussagen beziehen sich dabei auf Preisverhältnisse für das Referenzszenario.

201 Weitere Kuppelprodukte in späteren Verarbeitungsschritten (z.B. Polymerisation) fallen nach Aussagen von Experten hingegen kaum an.
} 
schiede zwischen den einzelnen Biopolymeren sowie zu Bioethanol ergeben sich aufgrund der unterschiedlichen Höhe des Rohstoffbedarfs.

Große Ähnlichkeiten zu Bioethanol ergeben sich beim Flächenbedarf für den Anbau der Rohstoffe (vgl. Anhang Tabelle 35). Er fällt unter den Annahmen in den Szenarien, gemessen an der aktuellen landwirtschaftlichen Fläche, mit ca. 1,8 - 2\% im Referenzszenario und ca. 3,0-3,2\% im HD-Szenario aber etwas geringer aus. Der Anteil an Konkurrenzfläche ist aufgrund identischer Annahmen zum Einsatz von Lignocellulose gleich (vgl. Tabelle 15) mit 16\% im Referenzszenario und $20 \%$ im HD-Szenario.

\section{Tabelle 15: Anteil der Produktion von Biopolymeren auf konkurrierenden Flächen}

\begin{tabular}{|l|c|c|}
\hline & $\begin{array}{c}\text { Referenz- } \\
\text { szenario }\end{array}$ & HD-Szenario \\
\hline Anteil Biopolymerproduktion aus Weizen in \% & $80 \%$ & $67 \%$ \\
\hline $\begin{array}{l}\text { Anteil Biopolymerproduktion aus Weizen auf } \\
\text { Konkurrenzflächen in \% }\end{array}$ & $20 \%$ & $30 \%$ \\
\hline $\begin{array}{l}\text { Anteil gesamte Biopolymerproduktion auf Kon- } \\
\text { kurrenzflächen in \% }\end{array}$ & $16 \%$ & $20 \%$ \\
\hline
\end{tabular}

Quelle: Eigene Berechnungen

\section{Investitionskosten}

Die kapitalintensive Produktion mit hohen Produktionsmengen von Polymeren erfordert erhebliche Investitionskosten. ${ }^{202}$ Die Investitionskosten je t Biopolymere hängen analog zu Bioethanol von der Anlagenkonzeption (Anlagengröße, Rohstoff, Biokonversionstechnologie) ab, wobei die Anlagengröße aufgrund des Fixkostencharakters eine zentrale Rolle einnimmt. Bei großen Anlagen unterscheiden sich die Investitionskosten zu den ölbasierten Polymeren nach Aussagen der Experten kaum. Bisher werden die Investitionskosten aufgrund des Entwicklungsrückstandes gegenüber den recht optimierten ölbasierten Polymeranlagen etwas höher geschätzt. Darüber hinaus kann die Verwendung von Biomasse zu einem größeren Bedarf an Lagerhallen und Reaktoren führen. Allerdings stellt die Biotechnologie durch die seichteren Produktionsbedingungen (niedrigere Temperaturen und Drücke) tendenziell niedrigere Ansprüche an Regelungs- und Sicherheitstechniken und hat hierdurch einen gewissen Preis-

${ }^{202}$ In vielen Fällen ist nicht nur eine Umstellung der Anlage für die direkte Produktion des betrachteten Biomaterials notwendig, sondern setzt auch Investitionen in die vorherigen Zwischenprodukte voraus. Folglich ist das tatsächliche Investitionsvolumen durch eine Diffusion höher, da bisherige Anlagen für die Zwischenproduktion ersetzt werden müssen. Eine quantitative Abschätzung ist aufgrund mangelnder verfügbarer Daten zu diesen Investitionen nicht möglich. 
vorteil für die Unternehmen (Hoppenheidt et al. 2005; Wagner et al. 2005). Ana$\log$ zu Bioethanol sind Investitionskosten auf Basis von Lignocellulose im Vergleich zu traditionellen Rohstoffen nach Einschätzungen der Experten aufgrund zusätzlich benötigter Analgenteile als deutlich höher einzuschätzen. Angaben für die Höhe der Investitionskosten finden sich in der Literatur nur bedingt (z.B. Meiß et al. 2003; Crank et al. 2004). Für die Szenarien ergeben sich auf Basis dieser Literatur, Expertengesprächen unter Berücksichtigung der höheren Investitionskosten für Lignocellulose Durchschnittswerte von ca. $180 € / \mathrm{t}$ (Referenz) bzw. $195 € /$ t (HD-Szenario).

Eine besondere Rolle kann die Entwicklung zu so genannten Bioraffinerien einnehmen. Diese Konzepte stehen in Anlehnung an die Erdölraffineriekonzepte ${ }^{203}$ und versuchen die komplex vernetzten und historisch gewachsenen Strukturen der Kohle- und Erdölchemie auf nachwachsende Rohstoffe übertragen. Anstatt in gesonderten Anlagen die Produktion von Biopolymeren oder Bioethanol durchzuführen, sollen nachwachsende Rohstoffe in einer integrierten Produktion in eine umfangreichen Produktpalette von Kraftstoffen, aber auch Chemikalien und Futtermitteln umgewandelt werden. Das Ziel ist es, verschiedene Stoff- und Energieströme auf möglichst optimale Art so miteinander zu verbinden, dass bei geringem Energieaufwand hohe Produktmengen hergestellt werden. Die Frage bei Bioraffinerien ist, ob sich analog zu Erdölraffinerien, aus einer begrenzten Anzahl von Primärchemikalien eine Vielzahl von Produkten herstellen lässt. Hierfür müssen viele Synthesebäume sowie entsprechend effiziente Mikroorganismen und/oder Enzymsysteme entwickelt werden. Die befragten Experten halten eine Kommerzialisierung solcher Bioraffinerien bis zum Jahr 2020 für wahrscheinlich, allerdings erst in einem Anfangsstadium. „Bottom-Up“-Berechnungen von Dornburg (2004) für eine „einfache“ Bioraffinerie für die Produktion von PLA zeigen, dass die multifunktionale Nutzung deutlich zu einer Verringerung des Bedarfs fossiler Energie beiträgt, sie aber nur zu vernachlässigbaren Kostenreduzierungen führt (Dornburg 2004, S.145). Für die Szenarien werden deshalb keine zusätzlichen Effizenzverbesserungen durch die mögliche Einführung von Bioraffinerien angenommen.

\section{Energiekosten}

Aus ökologischen Gesichtspunkten werden Biopolymeren und anderen biotechnischen Produkten Potenziale zur Einsparung von Stoffströmen und fossiler Energie zugesprochen (Hoppenheidt et al. 2005). Energieeinsparungen können nicht nur bei der biotechnologischen Produktion selbst, sondern in der gesamten Wertschöpfungskette auftreten. Energiebilanzen für Biopolymere kommen allerdings nicht zu einheitlichen Ergebnissen. So hängt der kumulierte Energie-

${ }^{203}$ Nach Oertel (2007) ist die Bezeichnung „Bioraffinerie“ nicht ganz präzise, da nicht im klassischen Sinne destilliert wird; sie hat sich aber als Bezeichnung durchgesetzt. 
verbrauch für die Produktion einer Tonne Biopolymere insbesondere von den Flächenerträgen beim Rohstoffanbau, der Art und Verwendung der Nebenprodukte, der Art der Entsorgung, vom Wirkungsgrad der Konversionstechnologie, aber auch entscheidend vom zu substituierenden chemischen Vergleichsprodukt ab (Deimling et al. 2007; IFEU 2007). Einzelne Herstellungsverfahren sind sogar energieintensiver als bei fossilen Kunststoffen (Oertel 2007). Die meisten Untersuchungen zeigen allerdings deutliche Energieeinsparungen, vor allem für die Produktionsverfahren, die wirtschaftlich erscheinen und wahrscheinlich zum Einsatz kommen werden (Patel et al. 2006, S.159 f.). Der Grund für relativ hohe Energieeinsparungen liegt in der Tatsache, dass die Herstellung von konventionellen Polymeren sehr energieintensiv ist. Ergebnisse von Crank et al. (2004) und Patel et al. (2006) kommen bei der Betrachtung verschiedenster Herstellungsweisen - unter Einbezug von Verbesserungspotenzialen - bei der Nutzung von Stärke (z.B. Weizen) zu einem durchschnittlichen Einsparpotenzial beim Bedarf von fossiler Energie von ungefähr $30 \%{ }^{204}$ Bei der Nutzung von Lignocellulose können die Einsparungen noch höher liegen. Die befragten Experten halten insgesamt Einsparungsmöglichkeiten von 30\% für plausibel, dieser Wert wird daher für das Referenz- und HD-Szenario verwendet.

\section{Entsorgungskosten}

Für Polymere sind verschiedene Entsorgungswege denkbar: Während für die meisten ölbasierten Kunststoffe die Möglichkeiten des stofflichen Recyclings, der thermischen Verwertung sowie der Deponierung bestehen, können viele biotechnologisch hergestellte Polymere darüber hinaus durch mikrobiellen $\mathrm{Ab}$ bau in Kompostierungsanlagen verwertet werden. Die Bedeutung dieser Bioabbaubarkeit von Polymeren wird seit langem kontrovers diskutiert. Mögliche Vorteile liegen in der Schonung der Umwelt und potenziell geringeren Entsorgungskosten (Oertel 2007). Allerdings ist die Eigenschaft der Bioabbaubarkeit gar nicht immer erwünscht, da für viele Verwendungszwecke der Kunststoff auf lange Zeit erhalten bleiben soll. Die Kompostierung wird auch nicht von allen Experten als generell die geeignetste Verwertung von Polymeren gesehen, da z.B. eine Energiegewinnung über eine thermische Verwertung möglich wäre. Bisher ist die Kompostierung eher wenig verbreitet. Bislang enthalten die meisten Biopolymere aus technischen Gründen petrochemische Additive (z.B. Flammschutzmittel) und sind damit nach der Bioabfall- und Düngemittelverordnung von der Kompostierung ausgeschlossen (Fraunhofer ISI 2006). Bei gesetzlichen Änderungen ist es durchaus denkbar, dass sich die Entsorgung von Biopolymeren im Durchschnitt gegenüber den ölbasierten Polymeren zukünftig unterscheiden wird. Da allerdings auch ölbasierte Polymere mit biologischer Ab-

${ }^{204}$ Auch IPTS bestätigt diese Einsparpotenziale bei einer Analyse der einschlägigen Literatur für Biopolymere (IPTS 2007). 
baubarkeit (z.B. Ecoflex von BASF) existieren, kann diese unterschiedliche Verwertung aber nicht direkt auf einen technologischen Unterschied zurückgeführt werden und wird von vielen Experten als Nutzungsstrategiethema, weniger als technologische Frage betrachtet. Deshalb wird für die Szenarien angenommen, dass die Biopolymere und das ersetzte Substitut gleich entsorgt werden und keine Unterschiede bei den damit verbundenen Kosten entstehen.

\section{Arbeitskosten}

Bei den direkt für die Herstellung benötigten Arbeitskräften gibt es nach Meinung aller Experten zwischen Biopolymeren und ölbasierten Polymere keine Unterschiede. Zwar liegen die Arbeitsmenge und -kosten bisher für Biopolymere je $t$ höher, dies liegt aber an der geringen Anlagengröße. So werden kaum zusätzliche Arbeitskräfte für eine $100.000 \mathrm{t}$ Anlage im Vergleich zu einer $10.000 \mathrm{t}$ Anlage benötigt. Da bereits ein sehr hoher Automatisierungsgrad vorliegt, ist höchstens mit sehr geringen Einsparungen an Arbeitskräften und -kosten bis zum Jahr 2020 zu rechnen.

\section{Hilfs- und Betriebsstoffe}

Einen größeren Kostenblock bilden hingegen die Hilfs- und Betriebsstoffe (z.B. Aceton). Angaben in der Literatur gehen von bis zu 670 €/t (Meiß et al. 2003) aus, im optimierten Zustand ist aber von geringeren Kosten auszugehen. Deshalb werden in den Szenarioberechnungen maximal knapp $600 € / t$ (für PLA und PHA) angesetzt. Im Vergleich zu ölbasierten Polymeren bestehen nach Aussagen einiger Experten bisher noch leichte Nachteile, die bis 2020 voraussichtlich ausgeglichen werden können.

\section{Sonstige Kosten}

Weitere Kosten entstehen für Reparatur und Instandhaltung, die ungefähr 3\% der Gesamtkosten betragen und in den Overheadkosten enthalten sind. Dieser Prozentsatz ist konsistent zu den Annahmen für Bioethanol und liegt leicht (um ca. 1 Prozentpunkt) unter Schätzungen von Mühlbauer (2006). Darüber hinaus ist die Einführung der Biopolymere sehr F\&E-intensiv (Crank et al. 2004). Zwar ist davon auszugehen, dass bis 2020 dieser Kostenanteil reduziert werden kann, zumindest ein Teil (ca 10\%) ist dennoch den verbleibenden Overhead-Kosten zuzurechnen. Die geringen restlichen Overheadkosten werden gemäß dem durchschnittlichen I-O-Chemievektor verteilt.

\subsubsection{Zusammenfassende Darstellung der Szenariowerte}

Aus den dargestellten Kostenbestandteilen lässt sich erkennen, dass die Unterschiede zwischen Biopolymeren und petrochemischen Polymeren zukünftig als gering erwartet werden. Bislang sind die Kosten von Biopolymeren noch höher, 
allerdings hat sich dieser Unterschied in den vergangenen Jahren verringert (Oertel 2007; Kaeb 2006). Schätzungen in der Literatur und der befragten Experten liegen mit ca. 2-3 $€ / \mathrm{kg}$ für viele Biopolymere ungefähr beim 1,5 bis 2fachen Preis als für ölbasierten Polymere in Massenanwendungen (Oertel 2007; Müssig/Carus 2007). ${ }^{205}$ Dabei werden diese höheren Kosten auf die Entwicklungskosten und den erwähnten fehlenden Skaleneffekten aufgrund kleiner Anlagen zurückgeführt (Enzing et al. 2007b; Carole et al. 2004). Der Bau größerer Anlagen und die Erzielung von Lerneffekten können zukünftig zu deutlichen Kosteneinsparungen führen, gleichzeitig werden ölbasierte Polymere bei steigenden Ölpreisen deutlich teurer. Für das Jahr 2020 erwarten die meisten befragten Experten bei dem im Referenzszenario angenommenen Preisverhältnis zwischen Öl und nachwachsenden Rohstoffen eine Kostenwettbewerbsfähigkeit der Biopolymere, die Kosteneinsparungen würden aber nur im geringen Prozentbereich liegen. Höhere Kostenersparnisse werden bei relativ steigenden Ölpreisen gesehen.

Auf ein analoges Ergebnis lassen die in geringem Umfang existierenden Kostenanalysen schließen, die jeweils ähnliche Ergebnisse aufweisen. Die konkreten gesamten Herstellungskosten werden den Angaben von Patel et al. (2006) ${ }^{206}$ entnommen und mit den eigenen Annahmen zu den Rohstoffkosten angepasst. Da die Ergebnisse von Patel et al. (2006) nur unmittelbar auf die Gesamt- und Rohstoffkosten schließen lassen, wurden die Daten um die oben erläuterten Kostenblöcke durch Aussagen der Experten und Angaben aus anderen Kostenstudien ergänzt (Meiß et al. 2003; Müssig/Carus 2007; Hagen 2000; Crank et al. 2004). Zur Berechnung der Durchschnittskosten für die I-O-Analyse werden für alle drei Biopolymerklassen aus Basis von Expertenaussagen gleiche Anteile an der Produktion angesetzt. Zwar unterscheiden sich PLA, PHA und PDO in ihren Kosten, sind aber gegenseitig nur begrenzt technisch substituierbar. Insgesamt betragen die Durchschnittskosten ca. 1280 €/t (vgl. Tabelle 16). Die Rohstoffkosten sind dabei geringer wie bei Bioethanol und betragen knapp unter $400 € / \mathrm{t}$.

${ }^{205}$ Noch geringere Unterschiede sehen Müssig und Carus (2007, S.16). Demnach liegt z.B. der Unterschied von PLA (ca. 1,40 Euro/kg) zu Polypropylen (ca. 1,10 Euro/kg) unter dem Faktor 1,3.

${ }^{206}$ Patel et al. (2006) untersuchen eine Vielzahl von einzelnen Verfahren für die verschiedenen Arten von Biopolymeren. Für weitergehende Berechnungen verwenden sie jeweils ein aktuelles und ein zukünftiges Verfahren für PLA, PHA und PDO. In der vorliegenden Arbeit wird angenommen, dass im Referenzszenario ein Drittel, im HD-Szenario zwei Drittel der Biopolymere mit diesen zukünftigen Verfahren hergestellt werden, der übrige Anteil mit dem aktuellen Verfahren. Erschwerend für die Übertragbarkeit der Berechnungen von Patel et al. (2006) ist der niedrig angesetzte Ölpreis von $25 € / \mathrm{bbl}$. Die Kostenstrukturen für Biopolymere und Polymere werden auf den Ölpreis des Referenzszenarios so angepasst, dass das relative Preisverhältnis zwischen Biopolymeren und ölbasierten Polymeren sich leicht zugunsten der Biopolymere verschiebt. Die sich daraus ergebenden Kostenverhältnisse entsprechen den Expertenmeinungen sowie zusätzlichen Simulationen von Patel et al. (2006) für höhere Ölpreise. 
$\mathrm{Zu}$ beachten ist, dass die Berechnungen für PLA, PHA und PDO auf der alleinigen Betrachtung von Weizen als Rohstoff beruhen. Für die Produktion von Biopolymeren aus Lignocellulose stehen kaum spezifische Daten zur Verfügung. Lediglich bei den Rohstoffkosten ist eine Anpassung möglich. Diese werden durch die höher anzunehmenden Investitionskosten ausgeglichen. Die Anpassungen werden nur in der Durchschnittskostenbetrachtung ersichtlich. Folglich ergeben sich die Rohstoff-, Investitions- und Zinskosten nicht direkt aus dem Durchschnitt der Werte für die drei Polymerklassen.

Tabelle 16: Kosten für Biopolymere im Referenz-Szenario (in $€$ je $t$ )

\begin{tabular}{|l|c|c|c|c|}
\hline Kostenart & PLA & PHA & PDO & $\begin{array}{c}\text { Durch- } \\
\text { schnitt }\end{array}$ \\
\hline Kosten je t Rohstoff & 120 & 120 & 120 & \\
t Rohstoff je t Biopolymer & 2 & 5 & 3 & \\
Kosten je t Biopolymer & $\mathbf{2 8 8}$ & $\mathbf{6 0 0}$ & $\mathbf{4 0 8}$ & 393 \\
$\ldots$..darunter Transportkosten & 3 & 6 & 4 & 4 \\
\hline Chemische Hilfs- & & & & \\
/Betriebsstoffe & $\mathbf{5 3 9}$ & $\mathbf{4 8 0}$ & $\mathbf{2 1 4}$ & $\mathbf{4 1 1}$ \\
Energie & $\mathbf{1 7 8}$ & $\mathbf{1 4 7}$ & $\mathbf{7 1}$ & 132 \\
Arbeit & 35 & $\mathbf{3 5}$ & $\mathbf{3 5}$ & $\mathbf{3 5}$ \\
Overhead & 190 & $\mathbf{1 2 0}$ & $\mathbf{1 1 0}$ & $\mathbf{1 4 0}$ \\
Zinskosten & $\mathbf{1 0 8}$ & $\mathbf{1 1 4}$ & $\mathbf{9 1}$ & $\mathbf{1 2 1}$ \\
Investitionskosten & $\mathbf{1 6 2}$ & $\mathbf{1 7 2}$ & $\mathbf{1 3 7}$ & $\mathbf{1 7 9}$ \\
Kuppelprodukterlöse & $\mathbf{- 8 6}$ & $\mathbf{- 1 8 0}$ & $-\mathbf{1 2 2}$ & $\mathbf{- 1 3 0}$ \\
\hline Gesamt & $\mathbf{1 . 4 1 4}$ & $\mathbf{1 . 4 8 8}$ & $\mathbf{9 4 3}$ & $\mathbf{1 . 2 8 2}$ \\
\hline
\end{tabular}

Quelle: Eigene Berechnungen, Datenbasis: siehe verschiedene Textstellen

* Hervorgehobene Werte gehen direkt in die Gesamtkostenrechnung ein

Durch den höheren Ölpreis steigen die Kosten für Biopolymere im HDSzenario. Angaben in der Literatur zur Abhängigkeit der Biopolymere vom Ölpreis existieren kaum. Die Schätzungen der befragten Experten liegen aber einstimmig bei $20 \%$. Aus Angaben von Müssig/Carus (2007, S.92) lässt sich für PLA ebenfalls eine Abhängigkeit in Höhe von $20 \%$ ableiten. Dieser Kostenerhöhung stehen Lerneffekte mit zunehmender Diffusion gegenüber. ${ }^{207}$ Insgesamt ergeben sich Durchschnittskosten von 1340 E/t (vgl. Tabelle 17).

${ }^{207}$ Diese werden auf Basis von Angaben von Patel et al. (2006) für PDO auf 12\% und für PLA auf 5\% der Prozesskosten geschätzt, für PHA ergeben sich keine Änderungen. 
Tabelle 17: Kosten für Biopolymere im HD-Szenario (in $€$ je t)

\begin{tabular}{|c|c|c|c|c|}
\hline Kostenart & PLA & PHA & PDO & $\begin{array}{l}\text { Durch- } \\
\text { schnitt }\end{array}$ \\
\hline $\begin{array}{l}\text { Kosten je t Rohstoff } \\
\text { t Rohstoff je t Biopolymer } \\
\text { Kosten je t Biopolymer } \\
\text {....darunter Transportkosten }\end{array}$ & $\begin{array}{c}126 \\
2 \\
302 \\
3\end{array}$ & $\begin{array}{c}126 \\
5 \\
630 \\
6\end{array}$ & $\begin{array}{c}126 \\
3 \\
403 \\
4\end{array}$ & $\begin{array}{c}408 \\
4\end{array}$ \\
\hline $\begin{array}{l}\text { Chemische Hilfs- } \\
\text { /Betriebsstoffe } \\
\text { Energie } \\
\text { Arbeit } \\
\text { Overhead } \\
\text { Zinskosten } \\
\text { Investitionskosten } \\
\text { Kuppelprodukterlöse }\end{array}$ & $\begin{array}{c}585 \\
242 \\
33 \\
181 \\
107 \\
161 \\
-98\end{array}$ & $\begin{array}{c}535 \\
205 \\
34 \\
117 \\
117 \\
175 \\
-205\end{array}$ & $\begin{array}{c}213 \\
89 \\
31 \\
96 \\
83 \\
124 \\
-139\end{array}$ & $\begin{array}{c}444 \\
179 \\
33 \\
131 \\
129 \\
193 \\
-136\end{array}$ \\
\hline Gesamt & 1.513 & 1.608 & 899 & 1.340 \\
\hline
\end{tabular}

Quelle: Eigene Berechnungen, Datenbasis: siehe verschiedene Textstellen

* Hervorgehobene Werte gehen direkt in die Gesamtkostenrechnung ein

\section{Input-Output-Koeffizienten der substituierten Güter}

Für die Kostenstrukturen der substituierten ölbasierten Polymere ist die frei zugängliche Datenlage ebenfalls deutlich eingeschränkt. ${ }^{208}$ Eine zentrale Rolle bei den Kosten spielt der Ölpreis. Schätzungen zum Einfluss des Ölpreises auf die Kosten von Polymeren lagen in den Expertengesprächen zwischen 60 und $80 \%$. Nach Angaben von Buchholz (2006) beträgt der Anteil des Rohölpreises bei 43 bis 50\%. Allerdings liegt der Ölpreis und damit auch dessen Anteil an den Kosten vermutlich deutlich unter dem für das Referenzszenario angenommen Wert. ${ }^{209}$ Für die Modellierung erscheint die untere Grenze der Expertenschätzungen von $60 \%$ deshalb als plausibel. Ausgehend von verschiedenen Literaturangaben, die Preise von ca. $1200 € / \mathrm{t}$ bei einem Ölpreis von ca. $60 \$ / \mathrm{bbl}$ angeben

${ }^{208}$ Zudem wird in der Literatur darauf hingewiesen, dass sich auch Kostenstrukturen der konventionellen Polymere bspw. Bei der Ölpreisabhängigkeit unterscheiden (Müssig/Carus 2007). Allerdings kommen bei den betrachteten Biopolymeren die Ersetzung von Polymeren in Betracht (Polyethylen, Polytrimethylene, Polystyrol), die sich hierbei kaum unterscheiden (Crank et al. 2004; Oertel 2007)

${ }^{209}$ Analysen von Nordhoff et al. (2007) zur zeitlichen Entwicklung des Ölpreises und Preisindizes für Kunststoffe für die vergangenen Jahre lassen auf Werte von ca. $60 \%$ schließen. Allerdings ist zu beachten, dass historische Preisveränderungen auf vielen Faktoren beruhen. Neben den Kosten für Erdöl, prägten die Verfügbarkeit von Vorprodukten, Preise für substitutive Materialien, Einkommensentwicklung und die eigene Preiselastizität die Preisentwicklung (Patel et al. 2006). 
(Nordhoff et al. 2007; Oertel 2007), ergeben sich bei höheren Ölpreisen Kosten von $1320 € / \mathrm{t}$ im Referenzszenario und knapp $1640 € / \mathrm{t}$ im HD-Szenario. Folglich sind die Kosten für ölbasierte Polymere im HD-Szenario höher als für Biopolymere. Diese Kostenersparnis wird als Konsumsteigerung infolge von Einkommenssteigerungen durch Preisrückgänge modelliert (Kapitel 5.2.2).

Für die Bestimmung der I-O Koeffizienten der ersetzten ölbasierten Polymere wird als Basis der Chemiesektor gewählt. Zwar umfasst dieser Sektor Polymere, allerdings auch noch weitere heterogene Produktgruppen. Deshalb wird der Sektor auf Basis zusätzlicher Informationen modifiziert. Die Ergebnisse der Expertenbefragung geben auch für die Modellierung der ölbasierten Polymere konkrete Anhaltspunkte. Zum Teil bestehen Unterschiede (z.B. Transportkosten), zum Teil Übereinstimmungen (z.B. Hilfs- und Betriebsstoffe, Arbeitskosten) zwischen den beiden betrachteten Polymergruppen. Insgesamt sind die Kosten für Energie, Mineralölerzeugnisse und Vorleistungen aus der Chemie selbst höher als für die gesamte Chemie anzusetzen. I-O-Tabellen für Großbritannien und die USA, die in einer niedrigeren Aggregationsebene vorliegen, weisen für Mineralöl um den Faktor 1,5 höhere Werte aus. Deshalb wird in den Szenarien ebenfalls der Faktor um 1,5 erhöht und durch eine entsprechende Herabsetzung der übrigen Koeffizienten ausgeglichen. Die Zeilensumme der I-O-Koeffizienten bleibt demnach konstant.

\section{Produktions- und Substitutionswerte für die Szenarien}

Die aus den geschilderten Annahmen resultierende Produktion und Substitution von Biopolymeren und ölbasierten Polymeren ist in Tabelle 18 zusammengefasst. Durch die Produktion von Biopolymere werden 1.406 Mio. $€$ (Referenzszenario) bzw. 3.464 Mio. $€$ an Produktion substitutiert. Aufgrund der geringeren Kosten ist der Produktionswert bei Biopolymeren etwas geringer als bei ölbasierten Polymeren. Durch die Exporte wird zudem nur ein Teil der Kosteneinsparung im Inland wirksam, die Konsumveränderung tritt zu einem anderen Teil im Ausland (33\%) auf. Die Importquoten für die (Bio-)Polymerproduktion beträgt jeweils $0 \%$. Denn der Vergleich findet für inländische produzierte Polymere statt und es werden keine Außenhandelsunterschiede zwischen den Gütern angenommen. 
Tabelle 18: Szenarienannahmen für die Produktion von Biopolymeren im Jahr 2020 (in Mio. €)

\begin{tabular}{|l|c|c|c|c|}
\hline & \multicolumn{2}{|c|}{ Referenzszenario } & \multicolumn{2}{c|}{ HD-Szenario } \\
\hline & $\begin{array}{c}\text { Biopolyme- } \\
\text { re }\end{array}$ & Polymere & Biopolymere & Polymere \\
\hline $\begin{array}{l}\text { Polymerproduktion } \\
\text { für Inlandsnachfrage }\end{array}$ & $785(0 \%)$ & $809(0 \%)$ & $1642(0 \%)$ & $2007(0 \%)$ \\
\hline $\begin{array}{l}\text { Polymerproduktion } \\
\text { für Auslandsnachfrage }\end{array}$ & $458(0 \%)$ & $471(0 \%)$ & $958(0 \%)$ & $1171(0 \%)$ \\
\hline $\begin{array}{l}\text { Kuppelprodukt (Fut- } \\
\text { termittel) }\end{array}$ & $126(0 \%)$ & $126(100 \%)$ & $286(0 \%)$ & $286(100 \%)$ \\
\hline Konsumveränderung & $37(33 \%)$ & - & $578(33 \%)$ & - \\
\hline $\begin{array}{l}\text { Gesamt- } \\
\text { Produktionswert }\end{array}$ & 1406 & 1406 & 3464 & 3464 \\
\hline
\end{tabular}

Quelle: Eigene Berechnungen. Die Werte in Klammern geben den Anteil der Importe an.

\subsubsection{Produktions- und Beschäftigungseffekte}

Die Produktionseffekte der Diffusion von Biopolymeren sind in Abbildung 19 dargestellt. Die Nettoproduktionseffekte sind im Referenzszenario leicht positiv mit knapp $300 \mathrm{Mio}$. $€$, im HD-Szenario hingegen leicht negativ mit minus 50 Mio. $€$. Im HD-Szenario sind die Produktionseffekte in der Chemieindustrie durch den geringeren Vorleistungsbedarf an chemischen Zwischenprodukten sowie einem geringeren direkten Produktionswert durch Kosteneinsparungen deutlich negativ. Sie überkompensieren dabei die positiven Effekte in der Landwirtschaft. Im Referenzszenario ist der Effekt in der Chemieindustrie schwächer ausgeprägt, da die Einsparungen bei Kosten und chemischen Zwischenprodukten gering ausfallen.

Der Beschäftigungseffekt (vgl. Abbildung 20) ist dagegen in beiden Szenarien leicht positiv. Dies liegt an den hohen Arbeitsintensitäten in den Sektoren mit Nettoproduktionswirkungen (z.B. Landwirtschaft, verschiedene Dienstleistungssektoren) und den niedrigeren Arbeitsintensitäten bei den Sektoren mit Produktionsverlusten (z.B. im Energiebereich). Zudem sind die Effekte in der Chemieindustrie recht neutral, da der direkte Arbeitskräftebedarf bei Biopolymeren und Polymeren als ähnlich ermittelt wurde und der Arbeitskoeffizient gering ist. Diese Zusammenhänge wirken sich besonders im HD-Szenario stark aus. Absolut liegt die Nettobeschäftigung bei ca. 4.000 Erwerbstätigen im Referenzszenario und knapp 7.000 im HD-Szenario. Die insgesamt eher positive wirtschaftliche Bilanz lässt sich auf eine niedrigere Importquote bei den Vorleistungen in 
der Wertschöpfungskette der Biopolymere im Vergleich zu konventionellen Polymeren zurückführen. ${ }^{210}$

\section{Abbildung 19: Nettoproduktionswirkungen durch zusätzliche Diffusion von Biopolymeren im Vergleich zum Basisszenario (in Mio. $€$ )}

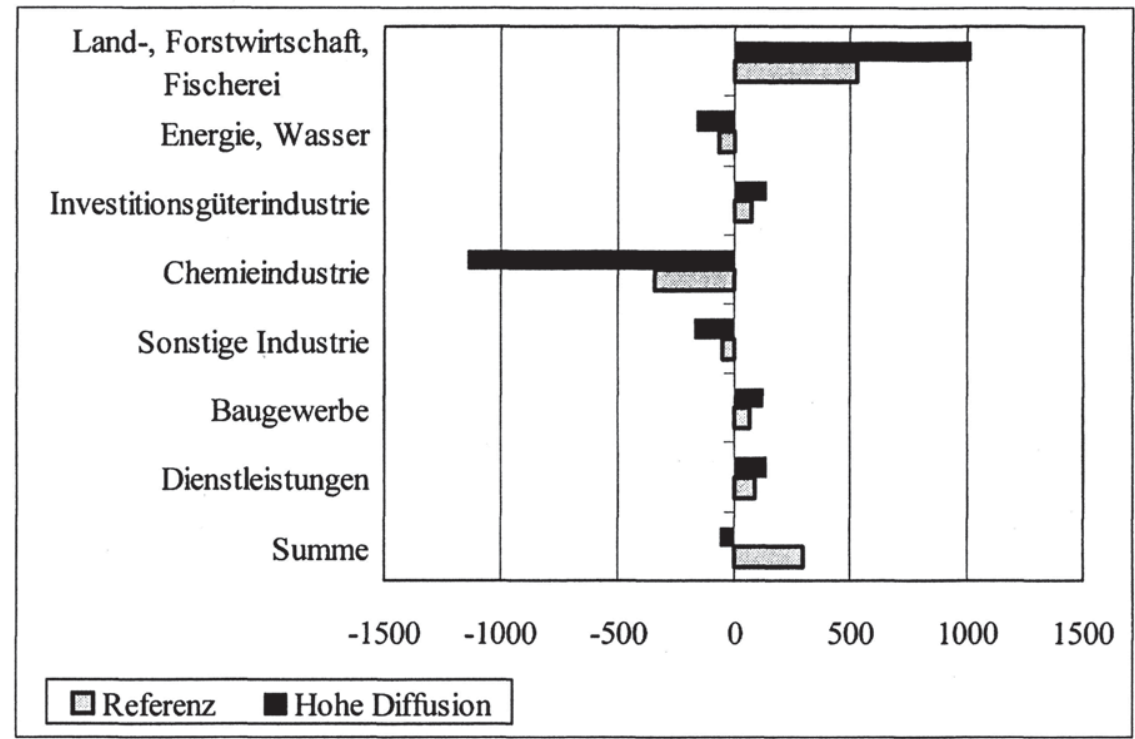

Quelle: Eigene Berechnungen

Die Effekte sind dabei sektoral recht unterschiedlich verteilt (vgl. Anhang Tabelle 40 bis Tabelle 43). Die größten Nettoeffekte ergeben sich im Bereich der Landwirtschaft mit 500 Mio. $€$ bzw. 1.000 Mio. $€$ höherer Produktion. Die leicht positiven Effekte bei den Dienstleistungen beruhen zu einem bedeutenden Anteil auf dem Mehrkonsum infolge von Kosteneinsparungen. Die ebenfalls positiven Effekte im Baugewerbe entstehen durch die höheren Investitionen sowie Reparatur-/Instandhaltungskosten. Negative Effekte treten in der Chemieindustrie, aber auch z.B. im Bereich Energie/Wasser auf. Diese Verluste in den Energiesektoren sind Folge der direkten Energieeinsparung bei der Produktion.

210 Die zusätzliche Beschäftigung ist im Vergleich zu den direkten Beschäftigten in der Polymerindustrie mit ca. 90.000 Beschäftigten im Jahr 2005 (Statistisches Bundesamt 2006) eher moderat einzustufen. Allerdings ist erneut auf die eingeschränkte Vergleichbarkeit dieser gesamtwirtschaftlichen Nettoeffekte mit der direkten, sektoralen Beschäftigung hinzuweisen. 


\section{Abbildung 20: Nettobeschäftigungswirkungen durch zusätzliche Diffusion von Biopolymeren im Vergleich zum Basisszenario (in Er- werbstätige)}

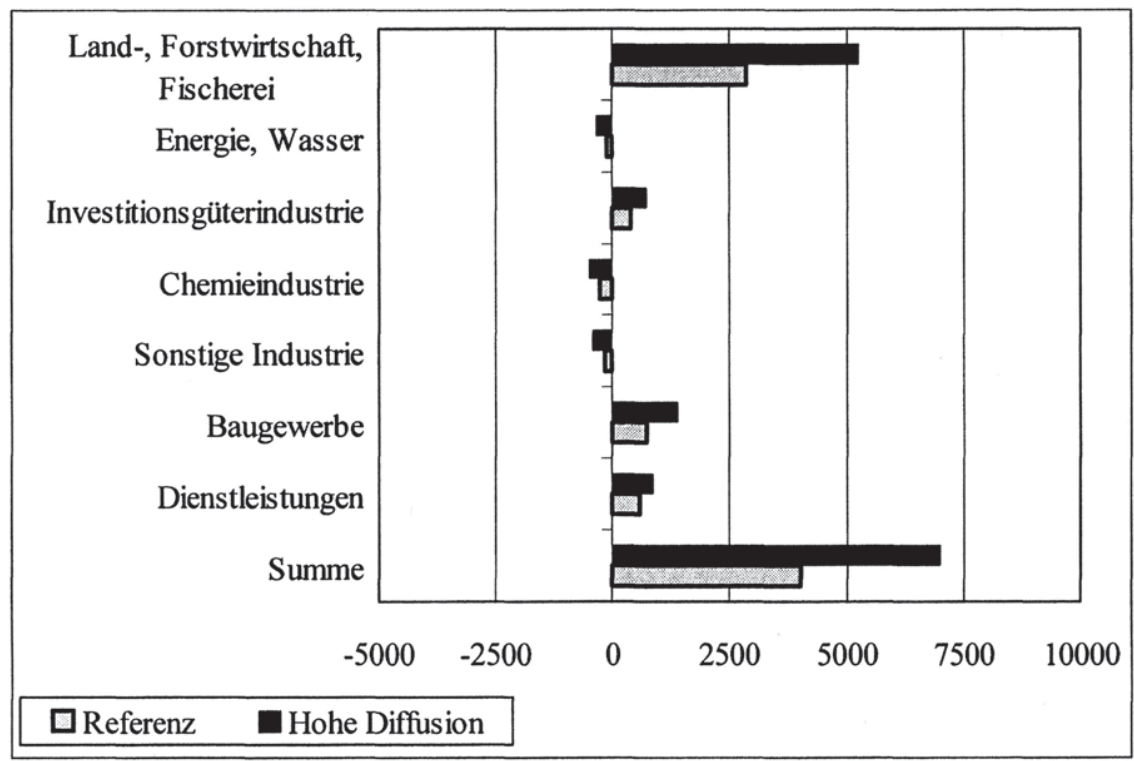

Quelle: Eigene Berechnungen

\subsubsection{Sensitivitätsanalyse}

Auf Basis der Literatur und Expertengespräche wurden für wichtige Variablen Werte für die Sensitivitätsberechnungen bestimmt (Tabelle 19). Da nur wenig Hinweise für zusätzliche Exportsteigerungen bestehen, wird die mögliche Erhöhung der Inlandsproduktion moderat auf 1.506 Mio. $€$ (+10 Prozentpunkte) angesetzt. Auf die Berechnung einer alternativen Kostenstruktur wird hier verzichtet. Für Biopolymere liegen nur wenige alternative Schätzwerte für die Kostenstruktur vor und diese unterscheiden sich nur so geringfügig von den hier verwendeten Werten, so dass der Unterschied nahe null liegen würde. 
Tabelle 19: Sensitivitätsannahmen für Biopolymere

\begin{tabular}{|l|c|c|c|l|}
\hline \multicolumn{1}{|c|}{ Variable } & $\begin{array}{c}\text { Refe- } \\
\text { renz }\end{array}$ & Niedrig & Hoch & \multicolumn{1}{|c|}{ Quelle/Anmerkung } \\
\hline Diffusion in \% & $4 \%$ & $3 \%$ & $7 \%$ & $\begin{array}{l}\text { Bandbreite aus Experten- } \\
\text { gesprächen }\end{array}$ \\
\hline $\begin{array}{l}\text { Inlandsproduktion } \\
\text { (inkl. Kuppelprodukt) } \\
\text { in Mio. } €\end{array}$ & 1369 & 862 & 1506 & $\begin{array}{l}\text { Niedrig: Annahme einer } \\
\text { ausgeglichenen Handelsbi- } \\
\text { lanz; Hoch: Eigene An- } \\
\text { nahme auf Basis Experten- } \\
\text { gespräche }\end{array}$ \\
\hline $\begin{array}{l}\text { Agrar- } \\
\text { Konkurrenzfläche } \\
\text { (in\%) }\end{array}$ & $16 \%$ & $0 \%$ & $32 \%$ & $\begin{array}{l}\text { Niedrig: Annahme keiner } \\
\text { Flächensubstitution (z.B } \\
\text { Schöpe/Brischkat 2006); } \\
\text { Hoch: Annahme doppelter } \\
\text { Flächensubstitution }\end{array}$ \\
\hline Konsumstruktur & $\begin{array}{l}\text { Konsumstruktur mit preisunelastischem Nachfragever- } \\
\text { halten und Durchschnittsvektor der I-O-Tabelle 2005 }\end{array}$ \\
\hline
\end{tabular}

Quelle: Eigene Berechnungen

Die Ergebnisse für die Nettoproduktion und -beschäftigung in den Sensitivitätsanalysen sind in Abbildung 21 und Abbildung 22 zusammengefasst. Die Effekte bleiben in den meisten Rechnungen positiv und ihre Verteilung auf einzelne Sektoren sehr ähnlich. Die sehr hohe Steigung der Geraden „Inländische Produktion" zeigt aber, dass sowohl Produktions- als auch Beschäftigungseffekte sehr sensitiv auf eine Veränderung der Außenhandelsbilanz reagieren. Im Falle einer neutralen Außenhandelsbilanz bei Biopolymeren (Inlandsproduktion = $67 \%$ ) sind Produktion und Beschäftigung sogar negativ. Die Diffusionshöhe beeinflusst vor allem die Nettobeschäftigung, bleibt aber für die angenommenen Unter- und Obergrenzen im positiven Bereich. Die alternative Annahme zur Konsumstruktur führt hingegen zu keinen Unterschieden, da schließlich nur geringe Veränderungen des Endkonsums im Referenzszenario auftreten. Ebenso ergeben sich keine größeren Auswirkungen durch alternative Annahmen für die Höhe der konkurrierenden Agrarfläche. 


\section{Abbildung 21: Nettoproduktionswirkungen für Biopolymere in Sensitivi-}

tätsrechnungen im Vergleich zum Basisszenario (in Mio. $€$ )

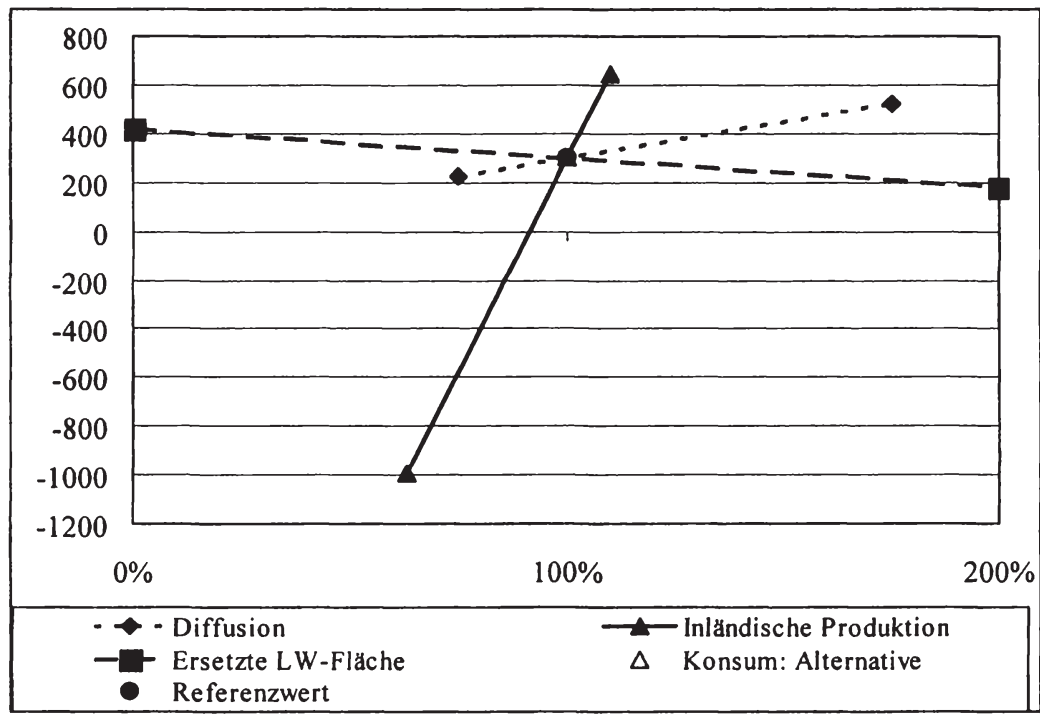

Quelle: Eigene Berechnungen

Abbildung 22: Nettobeschäftigungswirkungen für Biopolymere in Sensitivitätsrechnungen im Vergleich zum Basisszenario (in Erw.)

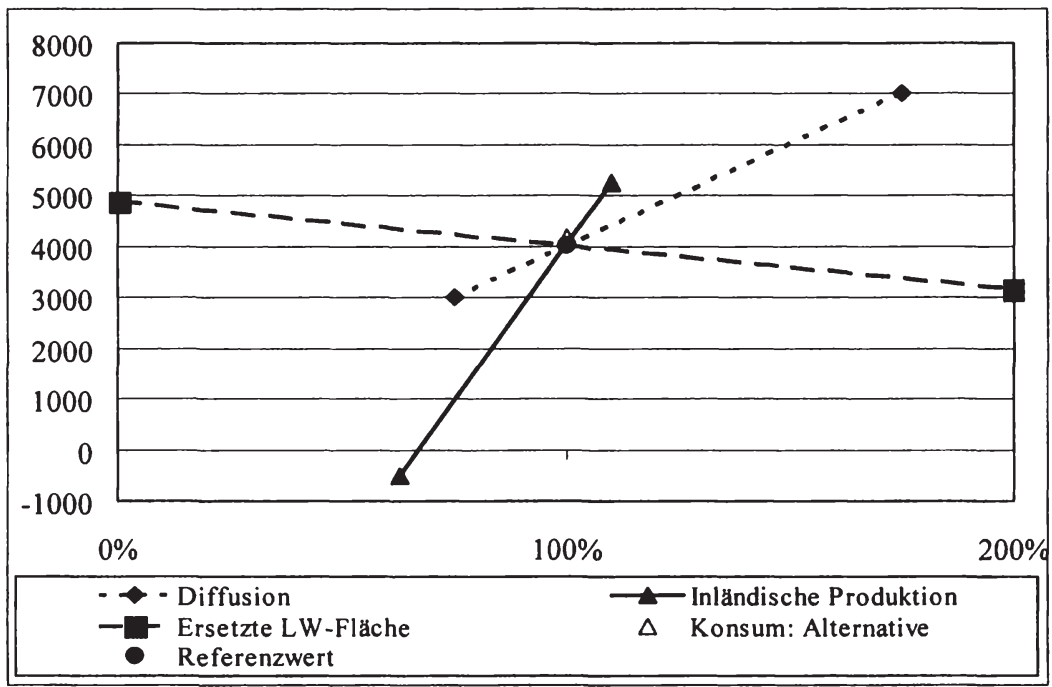

Quelle: Eigene Berechnungen 


\subsection{Fein-/Spezialchemikalien}

Fein-/Spezialchemikalien bilden den derzeitigen Schwerpunkt des Einsatzes biotechnischer Verfahren in der Chemie. Auch für den Zeithorizont bis zum Jahr 2020 werden vor allem in diesem Bereich der Chemie zusätzliche Anwendungen der Biotechnologie erwartet (von Armansperg/Patel 2007). Die Abgrenzung von Fein-/Spezialchemikalien ist in der Literatur aber nicht eindeutig (MarscheiderWeidemann/Hüsing 2004). Unter dem Begriff Feinchemikalien werden in der Regel komplexe Chemikalien verstanden, die in vergleichsweise geringen Mengen $\left(<10.000\right.$ t. p.a.) hergestellt werden. ${ }^{211}$ Spezialchemikalien zeichnen sich in der Regel durch besondere „Performance“ aus und umfassen Klebstoffe, Diagnostika, Desinfektionsmittel, Elektrochemikalien, Lebensmittelzusätze, Chemikalien für den Bergbau, Fotochemikalien, Spezialpolymere und Chemikalien zur Wasserbehandlung (Marscheider-Weidemann/Hüsing 2004). Im Folgenden werden Fein-/Spezialchemikalien zusammengefasst betrachtet, es handelt sich aber gegenüber den Biopolymeren und Bioethanol um ein deutlich heterogeneres Anwendungsfeld. Im Gegensatz zu Polymeren und anderen Bulkchemikalien werden Fein-/Spezialchemikalien mit geringem Produktionsvolumen, hohen Produktpreisen und hoher Wertschöpfung produziert (Patel et al. 2006, Gavrilescu/Chisti 2005). Sie werden als Zwischenprodukte für weitere chemische oder biotechnologische Reaktionen eingesetzt, als Zwischenprodukte an andere Sektoren (z.B. Pharma-, Textil-, Papier-, Zellstoffindustrie) geliefert oder auch als Endprodukte (z.B. Vitamine/ Antibiotika) verwendet.

Biotechnologische Verfahren bieten dabei durch ihre spezifischen Eigenschaften komparative Vorteile gegenüber "herkömmlicher Synthesechemie“ (z.B. hohe Spezifität, „Enantioselektivität“). Bspw. können Prozesse mit Enzymen häufig bei geringerem Druck und geringerer Temperatur sowie geringerem Materialeinsatz als chemische Prozesse ablaufen. Daneben ermöglichen biotechnologische Methoden über die Entwicklung von komplexeren Molekülen auch neue Wege zu bisher wenig erforschten Gebieten der Synthesechemie (OECD 2008b). Diese beschriebenen technischen Vorteile können zu verschiedenen Vorzügen (z.B. kosteneffiziente Produktion, neue Produkte) führen. Die Vorteile der biotechnologischen Methoden sind in diesem heterogenen Anwendungsfeld allerdings unterschiedlich und in der Regel nicht alle gleichzeitig erfüllt. Z.B. werden nicht immer chemische durch biotechnologische Verfahren aus Kostengründen ersetzt. So rechtfertigen in einigen Fällen bessere Funktionalitäten, Vorteile der Umweltverträglichkeit und Nachhaltigkeit etwas höhere Kosten und Preise der biotechnologischen Produkte, die Ersetzung (Patel et al.

${ }^{211}$ Eine eindeutige (statistische) Abgrenzung von Fein-/Spezialchemikalien in der Produktionsstatistik liegt allerdings nicht vor (Marscheider-Weidemann/Hüsing 2004). Im Folgenden wird die Gruppierung der amtlichen Produktionsstatistik des Verbands der chemischen Industrie (VCI) verwendet. 
2006). Im Gegensatz zu Biopolymeren und Bioethanol handelt es sich nur teilweise um Prozessinnovationen, aber auch um Produktinnovationen. Die Bedeutung der neuen Produkte und eine mögliche Produktionsausweitung werden im folgenden Teilkapitel mit erörtert, die getrennte Betrachtung der Kosten erfolgt in Kapitel 6.3.3. Abschließend wird die jeweilige quantitative Bedeutung abgeschätzt.

\subsubsection{Diffusion und internationaler Wettbewerb}

Im Jahr 2005 wurden in Deutschland Fein-/Spezialchemikalien im Wert von knapp 29 Mrd. $€$ (2007: 33 Mrd. €) hergestellt, der Anteil an der gesamten Chemieproduktion beträgt 25\% (Statistisches Bundesamt 2008a; VCI 2008). Auch zukünftig werden besonders Spezialchemikalien als Wachstumsmarkt der Chemie angesehen, während in vielen Bereichen der Grundstoffchemie Sättigungstendenzen zu erkennen sind (Dewick et al. 2004; Prognos 2002; CEFIC 2004). Für die Szenarien wird in Anlehnung an Prognos (2006) ein Wachstum von $2,1 \%$ angesetzt, der Produktionswert beträgt in diesem Fall rund $43 \mathrm{Mrd}$. $€$ im Jahr 2020.

Die bisherige Durchdringung der Biotechnologie bei Fein-/Spezialchemikalien ist uneinheitlich (Nusser et al. 2007c; von Armansperg/Patel 2007). Während sie z.B. bei der Herstellung von Geschmacks- und Geruchsstoffen bereits weit verbreitet sind, werden in anderen Segmenten der Spezialchemie auch in den nächsten 10 bis 15 Jahren eher geringfügig biotechnologische Prozesse eingeführt werden. Nach Aussagen der Experten sind viele chemische Produkte entweder biotechnologisch gar nicht machbar oder es ist sehr unwahrscheinlich, dass biotechnologische Verfahren deutliche Vorteile bieten können, die zu entsprechenden Anstrengungen führen. Für die Bereiche, bei denen ein Einsatz der Biotechnologie denkbar ist, kommen verschiedenste Diffusionsfaktoren zum Tragen. Ein wichtiger Faktor liegt in den Bemühungen der stärkeren Umstellung auf eine regenerative Rohstoffbasis. Diese Entwicklung begünstigt biotechnische Verfahren auch im Fein-/Spezialchemikalienbereich, da diese häufig Vorteile bei der Umwandlung von nachwachsenden Rohstoffen aufweisen (Nusser et al. 2007c). Der Einfluss des Preisverhältnisses zwischen fossilen auf nachwachsenden Rohstoffen auf die Diffusion der Biotechnologie ist nach Aussagen der Experten aber eher gering. Die Rohstoffkosten bilden bei Fein/Spezialchemikalien nur einen geringen Anteil. Stattdessen sind Veränderungen der Konsumnachfrage nach Produkten aus „Naturstoffen“, Politikmaßnahmen zur Förderung des Einsatzes nachwachsender Rohstoffe oder Nachhaltigkeitsüberlegungen von Unternehmen im Bezug auf die Endlichkeit fossiler Rohstoffe mögliche Gründe für die Rohstoffersetzung. Darüber hinaus ist die Erzielung wichtiger technologischer Fortschritte für die Diffusion der Biotechnologie von Bedeutung. Neben der kostengünstigen Entwicklung von Biokatalysatoren ist 
auch die Schnelligkeit der jeweiligen Entwicklung entscheidend. Diese Schnelligkeit Substanzen in großen Mengen auf den Markt zu bringen ist oft sogar wichtiger, als Produktionsverfahren zu entwickeln, die im Hinblick auf Kosten und Ressourcenverbrauch optimiert sind (Nusser et al. 2007d). Deshalb kommen bei der Etablierung des Produktionsprozesses überwiegend konventionelle Technologien zum Einsatz, die bereits vorrätig sind bzw. in kurzer Zeit mit absehbarem Erfolg an die Problemstellung adaptiert werden können (Nusser et al. 2007a). Die Experten sind aber optimistisch, dass die Biotechnologie mit zunehmendem Reifegrad in dieser Hinsicht konkurrenzfähiger wird, da die zur Verfügung stehenden Methoden und Verfahren weiter zunehmen. Ein weiteres zentrales Hemmnis, welches bereits in der Diskussion um die GPTs deutlich wurde (Kapitel 3.2), bilden die nötigen Neuinvestitionen, welche unter Unsicherheit der Rentabilität getroffen werden müssen. In der Regel ist bei der Einführung biotechnologischer Methoden in Unternehmen ab einem bestimmten Volumen der Bau von Neuanlagen notwendig, welche mit bereits abgeschriebenen Anlagen konkurrieren.

Insgesamt wird in der Literatur und von den befragten Experten bis zum Jahr 2020 ein Zuwachs beim Produktionsanteil der mithilfe der Biotechnologie hergestellten Güter erwartet. ${ }^{212}$ Für das Jahr 2005 gehen die befragten Experten fast einstimmig von einem Anteil von ca. 5\% aus. Dies entspricht anderen Schätzungen aus der Literatur (z.B. von Armansperg/Patel 2007; Nusser et al. 2007a). Zukünftige Projektionen liegen allerdings deutlich auseinander. Während von Armansperg und Patel (2007) einen Anteil von ca. 9\% für das Jahr 2015 projizieren, gibt der Verband für chemische Industrie (VCI) eine Schätzung für 2015 von ca. 19\% (VCI 2008) an. Die befragten Experten stufen solch hohe Schätzungen für zu optimistisch ein. Die deutliche Mehrheit erwartet einen Anteil von ca. $10 \%$ im Jahr 2020. Unter günstigen Rahmenbedingungen und technologischer Entwicklung werden etwas höhere Anteile erwartet. Auf Basis der Expertenmeinungen werden für die Szenarioberechnungen im Referenzszenario ein Anteil von 10\% angesetzt, im HD-Szenario ein Anteil von $15 \%$ des oben geschätzten Produktionswertes für das Jahr 2020 von knapp $43 \mathrm{Mrd}$. $€$ gewählt. Abzüglich der angenommen Diffusion für das Jahr 2005 (5\% am damaligen Produktionswert) ergeben sich die Berechnungswerte für die Szenarien (vgl. Tabelle 20). Diese betragen 2,7 Mrd. $€$ für das Referenzszenario und 4,8 Mrd. $€$ für das HD-Szenario.

\footnotetext{
${ }^{212}$ Analog der Erläuterung zu den „Biotechnology-related-sales“ in Kapitel 4.2.3.2 wird dabei ein Produkt als „,biotechnologisch hergestellt“ definiert, bei dem für mindestens ein Produktionsschritt gegenüber dem Vorprodukt ein biotechnologisches Verfahren angewendet wird.
} 
Tabelle 20: Biotechnologische Produktion von Fein-/Spezialchemikalien in den Szenarien

\begin{tabular}{|l|c|c|}
\hline & Referenzszenario & HD-Szenario \\
\hline Diffusion im Jahr 2020 in Mrd. $€$ & 4,3 & 6,4 \\
\hline Diffusion im Jahr 2005 im in Mrd. $€$ & 1,6 & 1,6 \\
\hline $\begin{array}{l}\text { Differenz 2020-2005 in Mrd. } € \\
\text { (Berechnungswert) }\end{array}$ & 2,7 & 4,8 \\
\hline
\end{tabular}

Quelle: Eigene Berechnungen

\section{Internationaler Wettbewerb und Außenhandel}

Im internationalen Wettbewerb ist die Stellung Deutschlands sehr ähnlich wie bei Polymeren. Die Außenhandelsverflechtung ist sehr hoch, im Jahr 2005 betrugen die Exporten knapp $23 \mathrm{Mrd} . €$ und die Importe ca. 12,5 Mrd. $€$. Inwiefern diese Stellung als Nettoexporteur auch bei biotechnologisch hergestellten Produkten erreicht werden kann, ist offen. Die meisten befragten Experten halten eine analoge Außenhandelsbilanz für die biotechnologische Herstellung und einen weiterhin konstanten Anteil an der weltweiten Produktion für möglich. Im Referenz- und HD-Szenario wird deshalb von einer konstanten und gleichen Außenhandelsbilanz wie bei konventionellen Fein-/Spezialchemikalien ausgegangen. Nach einigen Meinungen ist die oben erwähnte Diffusionshöhe der Biotechnologie bei Fein-/Spezialchemikalien sogar notwendig, um den Produktionsanteil in der Fein-/Spezialchemie zu halten. Gemäß diesen Meinungen würde bei geringen biotechnologischen Aktivitäten der Produktionsanteil Deutschlands sinken, da die Produktion standardisierter Güter nach Asien oder Osteuropa verlagert werden würde. Diese mögliche bessere relative Außenhandelsbilanz von biotechnologischen Produkten gegenüber den konventionellen Gütern wird in Sensitivitätsanalysen betrachtet.

\subsubsection{Bedeutung der Biotechnologie für Produktinnovationen}

Um die Auswirkungen der Biotechnologie in diesem Anwendungsfeld zu bestimmen, ist zusätzlich die Bedeutung neuer Produkte zu klären. Biotechnologische Verfahren werden nach Expertenaussagen häufig im "High-Value" Bereich eingesetzt, bei denen besondere Funktionalitäten von hoher Bedeutung sind. ${ }^{213}$ Auch zukünftig (Jahr 2020) schätzen einige Experten die Bedeutung der Biotechnologie bei den neu entwickelten Produkten deutlich höher als das Substitutionspotenzial bei bestehenden Produkten. Allerdings zeigt sich nach Aussagen

${ }^{213}$ In einigen Fällen ist es durch die Biotechnologie überhaupt erst möglich, ein Produkt auf dem Markt anzubieten, da es bei der chemischen Herstellung nicht zu wettbewerbsfähigen Preisen angeboten werden kann (IPTS 2007). 
von Experten bisher häufig eine begrenzte Nachfrage für veränderte Produkteigenschaften, weil der Anpassungsaufwand bei den Anwenderunternehmen meist hoch ist. ${ }^{214}$ Zudem stellt sich aus volkswirtschaftlicher Sicht die Frage, inwiefern diese neuen Produkte Potenzial für die Erschließung neuer Märkte und Anwendungsbereiche (additiv) haben oder andere Produkte (substitutiv) stärker verdrängen. Vorteile für den Anwender (Verbraucher bzw. Weiterverarbeiter) werden vor allem durch höhere Qualität oder Sicherheit (z.B. geringere Entzündlichkeit von Materialien) der Produkte gesehen. Allerdings werden sich die Anwendungsfelder von Fein-/Spezialchemikalien auch zukünftig durch die Biotechnologie nach Expertenaussagen nur bedingt verändern und weiter vorrangig in den bisherigen Bereichen bleiben. Ebenso zeigten sich in den Interviews wenig Hinweise, dass Güter aus anderen Sektoren stärker durch biotechnologische Produkte bei Fein-/Spezialchemikalien ersetzt werden. Einige Experten sehen verstärkte Anwendungsmöglichkeiten im „Haushaltsbereich“. Insgesamt werden die Märkte demnach nur inkrementell durch neue Produkte beeinflusst, es kommt zu keinen größeren strukturellen Veränderungen. Verschiebungen in der Nachfrage oder Kosteneinsparungen in späteren Teilen der Wertschöpfungskette (z.B. durch Einsatz neuartiger Enzyme im Textil, Leder- oder Papierbereich) werden von den Experten nur begrenzt gesehen. Für die Szenarioberechnungen wird deshalb von keiner additiven Produktinnovation aus Sektorsicht ausgegangen, die neuen biotechnologischen Produkte ersetzen dabei bisherige chemische Produkte.

\subsubsection{Herstellungskosten}

Im Gegensatz zu den obigen Beispielen von Bioethanol und Biopolymeren ist bei diesem heterogenen Feld die Bestimmung eines eigenen Produktionsvektors auf Basis von detaillierten Kostenanalysen nicht möglich. Stattdessen werden die I-O-Koeffizienten über einen Vergleich zu der bisherigen Struktur für Fein/Spezialchemikalien ermittelt. Die Herstellungskosten stehen dann im Vordergrund, wenn ein bestehendes, bisher chemisch hergestelltes Produkt durch biotechnologische Verfahren bei gleich bleibenden oder ähnlichen Funktionalitäten ersetzt wird. So ermöglicht die Ersetzung der oft komplexen und mehrstufigen chemischen Synthese durch den Einsatz von Biokatalysatoren (v.a. Enzyme) Ressourcen- und Energieeinsparungen gegenüber der klassischen chemischen Produktion. Im Idealfall können mehrere chemische Syntheseschritte durch eine geringe Anzahl an biotechnologischen Verfahrensschritten ersetzt werden. In diesen Fällen - prominente Beispiele hierfür sind Riboflavin, ACA7, Cephalosporin, Lysin - kommt es zu umweltfreundlichen Substitutionen, ver-

\footnotetext{
${ }^{214}$ Hierzu sind nach Expertenaussagen nur die großen Chemiefirmen als Anwender in der Lage, während Anbieter häufig darin bemüht sind, die Funktionalitäten möglichst der Chemie anzugleichen.
} 
bunden mit hohen Kosteneinsparungen (IPTS 2007, Hoppenheidt et al. 2005; Festel et al. 2004). Die Kosteneinsparungen kommen durch einen geringeren Inputbedarf von fast allen Produktionsfaktoren (v.a. Investitions- und Rohstoffkosten) zustande, d.h. die Gesamteffizienz des Prozesses steigt. Im Folgenden wird genauer erörtert, ob sich der Einsatz einzelner Produktionsinputs stärker durch die Diffusion der Biotechnologie verändert oder ob sie durch beschriebene Erhöhungen, im Sinne der totalen Faktorproduktivität, in ähnlichem Ausmaß eingespart werden können.

\section{Rohstoffkosten}

Die Rohstoffkosten spielen auch bei biotechnologisch hergestellten Fein/Spezialchemikalien eine bedeutende Rolle. Allerdings sind diese etwas geringer als bei Bioethanol und -polymeren und unterscheiden sich in ihrer Zusammensetzung. Bei Fein-/Spezialchemikalien werden diese Kosten vor allem vom Material- und Arbeitsaufwand bei den Zulieferern bestimmt, welche die fossilen bzw. nachwachsenden Rohstoffe zunächst verarbeiten. Der tatsächliche Kostenanteil der Rohstoffe am Endprodukt selbst ist meistens gering, kann aber bei den heterogenen Produkten unterschiedlich ausfallen. Eine Schätzung des Durchschnittsanteils auf Basis von disaggregierten Angaben in der Literatur und/oder Expertenaussagen ist mangels ausreichender Datenverfügbarkeit und Vielzahl von Produkten nicht möglich. Für die Berechnung des I-O-Koeffizienten wird deshalb eine vereinfachende aggregierte Berechnung angewendet. Für das Jahr 2004 wird für nachwachsende Rohstoffe ein Anteil an den gesamten Rohstoffen für die Chemie von $10,4 \%$ bei einem Produktionswert von ca. 1 Mrd. $€$ geschätzt (von Armansperg 2006). Unter der Annahme, das Produkte, bei denen nachwachsende Rohstoffe eingesetzt werden, ebenfalls einen Anteil von 10,4\% bei einem gesamten Produktionswert von 133 Mrd. $€$ (Statistisches Bundesamt 2008c) einnehmen, haben nachwachsende Rohstoffe einen durchschnittlichen Kostenanteil von knapp 7\% am Endprodukt. ${ }^{215}$ Für biotechnologische Verfahren werden allerdings nicht generell nachwachsende Rohstoffe als Ausgangsstoffe eingesetzt, sondern zum (kleineren) Teil auch fossile Rohstoffe. ${ }^{216}$ Von Armansperg und Patel (2007) schätzen einen Rohstoffwechsel in $80 \%$ der eingesetzten Verfahren. Diese Größenordnung wurde in den Expertengesprächen weitestgehend bestätigt und wird auch zukünftig in diesem Bereich eingeschätzt. Folglich wird ein Inputkoeffizient von 5,5\% des Produktionswertes für den Sektor Landwirtschaft angenommen. Bei den nachwachsenden Rohstoffen werden

${ }^{215}$ Detailliertere Angaben von Reineke und von Armansperg (2007, S.316) für einzelne Segmente von Farben und Lacke, welche einen wichtigen Teilsektor von Fein-/Spezialchemikalien bilden, lassen auf einen sehr ähnlichen Wert für den Kostenanteil (ca. 8\%) von nachwachsenden Rohstoffen schließen.

216 Nach Expertenaussagen wird derjenige Rohstoff verwendet, welcher möglichst wenig Umwandlungsschritte zur Erstellung des gewünschten Endproduktes benötigt. 
für biotechnologische Chemikalien nicht nur Stärke aus Getreide oder Zucker eingesetzt, sondern u.a. auch Fette und Öle. Diese verschiedenen Rohstoffe unterscheiden sich deutlich in der Importquote, bspw. werden Palmöl oder Sojaöl gar nicht inländisch angebaut. Die quantitative Nutzung einzelner nachwachsender Rohstoffe bei biotechnologischen Verfahren kann aber nur schwer eingeschätzt werden. Deshalb wird für die Berechnungen vereinfachend die durchschnittliche Importquote für die stoffliche Nutzung von nachwachsenden Rohstoffen im Chemiesektor verwendet. ${ }^{217}$ Die verfügbaren Schätzungen für die Importquote im Jahr 2020 liegen zwischen 58\% (Nusser et al. (2007d) und 62\% (WI/RWI 2008), für die Szenarien wird das Mittel von $60 \%$ verwendet. Für die inländische Produktion wird analog zu den vorherigen Fallbeispielen eine Verdrängung des Anbaus anderer landwirtschaftlicher Produkte angenommen. Aufgrund der hohen Importquote beträgt der Anteil der konkurrierenden Flächen aber nur ca. $6 \%$ im Referenzszenario und $8 \%$ im HD-Szenario.

Kostenvorteile entstehen allerdings weniger durch den Wechsel der Rohstoffbasis, sondern durch mögliche Einsparungen beim Rohstoffbedarf. Die Reduktion von Prozessschritten führt vor allem zu einer Senkung des Volumens unerwünschter Nebenprodukte. Dies ist besonders für die Feinchemikalienproduktion von Bedeutung, die durch hohe so genannte E-Faktoren (Quotient aus kg Nebenprodukt pro kg erwünschtem Produkt) bei der chemischen Synthese gekennzeichnet ist. Es fallen daher vergleichsweise viele Abfälle und ein hoher Rohstoffbedarf an. Beide können durch den Einsatz der Biotechnologie gesenkt werden (Nusser et al. 2007b). ${ }^{218}$ Diese Effekte werden zumeist durch Annahmen zur Erhöhung der Gesamteffizienz (siehe Kapitel 6.3.4) in den Szenarien abgebildet. Da aber nach Expertenaussagen in der Rohstoffeinsparung einer der Haupteffekte liegt, wird eine spezifische Einsparung von 10\% angenommen. Die resultierende Gesamteinsparung liegt im Bereich von Experteneinschätzungen.

\section{Investitionskosten}

Neben der Rohstoffeinsparung kann die Reduzierung der notwendigen Verfahrensschritte zur Erzielung eines Produkts zu einer Senkung des Investitionsbe-

\footnotetext{
${ }^{217}$ Dieses Vorgehen überschätzt vermutlich den Importanteil, da vor allem die zumeist inländisch angebaute Stärke in Betracht für biotechnologischer Verfahren kommt.

${ }^{218}$ Ein Nachteil in der Produktion kann sich dadurch ergeben, dass die für die Produktion notwendigen Biokatalysatoren über den Aufbau von Biomasse des Produktionsstamms bei jedem Fermentationslauf neu hergestellt werden müssen. Dadurch werden Teile des eingesetzten Rohstoffs für die Bildung des Biokatalysators verbraucht, was sich nachteilig auf die Bilanz der erzeugten Produktmenge pro Einheit des Rohstoffs auswirkt (Hoppenheidt et al. 2004). Allerdings ergaben sich in der Literatur und den Expertengesprächen keine Hinweise dafür, dass dieser Nachteil quantitativ von hoher Bedeutung ist, da die Menge des Biokatalysators selbst, gering ist.
} 
darfs für Neuanlagen führen. Es werden bspw. weniger Kessel zur Fermentation benötigt. Zudem werden durch seichtere Produktionsbedingungen (niedrigere Temperaturen und Drücke) geringere Ansprüche an Regelungs- und Sicherheitstechniken gestellt. Die Ersparnisse liegen nach Schätzungen einiger Experten bei ca. $20-30 \%$. Einsparungen von $40-50 \%$, die in einigen Fallbeispielen erreicht wurden (Festel et al. 2004), sind im Durchschnitt nicht zu erwarten. Um diese Größenordnung von $20-30 \%$ in den Berechnungen abzubilden, werden analog zu den Rohstoffkosten neben Annahmen zur höheren Gesamteffizienz spezifische Einsparungen von $10 \%$ angesetzt.

\section{Abfallkosten}

Ein häufiges Argument für die Biotechnologie im Chemiebereich bildet die Umweltverträglichkeit (z.B. Hoppenheidt et al. 2004, Angrick 2006). Während chemische Verfahren häufig mit für die Umwelt problematischen Stoffen unter physikalisch anspruchsvollen, energieintensiven Reaktionsbedingungen durchgeführt werden, laufen biotechnische Verfahren dagegen unter vergleichsweise milden Bedingungen in wässrigem Milieu, bei niedrigen Temperaturen, Normaldruck und neutralem pH-Wert ab (Hoppenheidt et al. 2004). In der Folge sind viele biotechnische Verfahren ressourceneffizienter, risikoärmer und gesundheitsverträglicher. Allerdings ist zu beachten, dass nicht alle derzeit realisierten biotechnischen Verfahren bzw. Produkte unter Umweltgesichtspunkten vorteilhaft sind und auch zukünftig stets die umweltverträglichere Alternative bieten (Angrick 2006). ${ }^{219}$ Nachteile entstehen z.B. im häufig volumenmäßig höheren Abwasserverbrauch, da die Biotechnologie in wässrigen Lösungen arbeitet. Die meisten Experten sehen den Umgang mit höherem Abwasserverbrauch in der Regel aber als sehr optimiert an, so dass der zusätzliche Kostenaufwand hierfür gering ist. Häufig werden Standorte in Nähe zu Kläranlagen gewählt. Einige befragten Experten betonen aber besonders die erfolgte Optimierung der chemischen Verfahren. So sind besonders in großen, integrierten Anlagen die Abfallströme derart optimiert, dass hieraus zumeist wieder wertvolle Produkte gewonnen werden können. Ähnliches gilt für verschiedene Einsatzstoffe (bspw. Lösemittel) die in der Chemie bereits optimiert wurden, während dies für die Biotechnologie noch im geringeren Maße der Fall ist.

Insgesamt sieht aber eine deutliche Mehrzahl der befragten Experten Vorteile für die Biotechnologie, die sich auch in einer Reduktion der Abfallmengen und -kosten auswirken kann (OECD 2001). Eine Quantifizierung dieses Potenzials ist allerdings schwierig. Viele große Chemieunternehmen erfassen die Abfallströme nur zentral, weshalb eine Zurechnung zu einzelnen Prozessen oder Pro-

219 So stellt das Umweltbundesamt fest: „Auch wenn zahlreiche biotechnische Verfahren umweltentlastende Effekte zeigen, so wird es auf keinen Fall eine Unbedenklichkeitserklärung für die weiße Biotechnik im Allgemeinen geben. Vielmehr ist eine genaue Einzelfallbewertung notwendig“" (Angrick 2006, S.9). 
dukten nicht möglich ist (Marscheider-Weidemann/Hüsing 2004). Für die Berechnung der I-O Koeffizienten wird auf Basis einzelner Expertenmeinungen vereinfachend eine Halbierung des ohnehin geringen Kostenblocks für Dienstleistungen der Abfallwirtschaft angenommen.

\section{Energiekosten}

Daneben werden von der deutlichen Mehrheit der Experten auch im Bereich der Fein-/Spezialchemikalien die Möglichkeit zur Energieeinsparung durch die Biotechnologie gesehen. So gelten enzymatische Prozesse oftmals als weniger energieaufwändig wie chemisch katalysatorische Prozesse. Allerdings ist, wie bei Biopolymeren, die jeweilige Energiebilanz stark von verschiedenen Faktoren (u.a. konkrete Biomasse, Konversionsverfahren, Zielprodukt) abhängig. Im Gegensatz zu Biopolymeren ist der Anteil der Kosten für Energie deutlich geringer und damit auch die Anreize die Energiebilanz des biotechnologischen Verfahrens zu optimieren. Dies gilt besonders für den Bereich der Feinchemikalien mit geringen Tonnagen. Die meisten Schätzungen der Experten liegen deshalb bei ca. $20 \%$ und damit niedriger als im Bereich der Biopolymere oder anderen Bulkchemikalien (Patel et al. 2006).

\section{Arbeits- und sonstige Kosten}

Der quantitative Arbeitseinsatz ist bei beiden Technologien sehr ähnlich, die meisten Arbeitsschritte bleiben bei einer Umstellung auf die Biotechnologie gleich. Schließlich sind nach Expertenaussagen diejenigen biotechnologischen Verfahren, die einen höheren Arbeitseinsatz verursachen, zumindest im Inland nicht rentabel. Allerdings sinkt der Arbeitsbedarf bei der Reduktion von Prozessschritten. Änderungen ergeben sich auch beim benötigten Wissen der Arbeitskräfte, aus dem ein unterschiedlicher Qualifikationsbedarf der Unternehmen folgt. Allerdings führt dies nach Expertenaussagen nicht zu signifikant höheren Kosten. Für die Szenarien wird deshalb eine Einsparung aufgrund der höheren Gesamteffizienz angenommen.

Ähnliche Veränderungen zeigen sich auch bei sonstigen Kosten, z.B. für Hilfs- und Betriebsstoffe. Der Bedarf kann bei einer Reduzierung der Verfahrensschritte sinken. Bei den Berechnungen werden sie analog zu den Rohstoffkosten behandelt. Zu beachten ist allerdings, dass in Einzelfällen die Kosten für Hilfs- und Betriebsstoffe zum Nachteil der Biotechnologie ausfallen. So können bei den geringen Tonnagen die Enzymkosten relativ teuer sein.

\subsubsection{Zusammenfassende Darstellung der Szenariowerte}

Zusammenfassend sind Kostensenkungen vor allem durch eine höhere Gesamteffizienz zu erwarten. Bei den bereits genannten Fallbeispielen (Riboflavin, ACA-7, Cephalosporin, Lysin) liegen diese sogar bei ca. 40-50\% (IPTS 2007, 
Hoppenheidt et al. 2005; Festel et al. 2004). Diese Verfahren sind allerdings als Positivbeispiele zu werten, im Durchschnitt ist eine geringe Kostenersparnis zu erwarten. Zudem stehen längst nicht in allen Fällen die Einsparung von Kosten, sondern vielmehr Qualitätssteigerungen im Vordergrund. So liegen Expertenschätzungen für Kostensenkungen zumeist im Bereich von 10-15\%. Da einzelne Meinungen auch eher unter dieser Bandbreite als darüber liegen, wird für das Referenzszenario eine Einsparung von $10 \%$ angesetzt. Für das HD-Szenario werden Lerneffekte und technologische Fortschritte angenommen, die zu einer Einsparung von $15 \%$ führen.

Die Kostenstrukturen werden so aufgeteilt, dass zunächst die oben angenommen spezifischen Einsparpotenziale für Rohstoffe, Investitionen, Energie und Abfall berechnet und die restlichen Kosteneinsparungen als Senkung des Einsatzes aller Produktionsfaktoren berechnet werden (siehe Tabelle 21). Bei den bereits biotechnologisch hergestellten Produkten stellt sich zusätzlich die Frage, ob weitere Veränderungen im Produktionsprozess dieser Verfahren zu erwarten sind oder voraussichtlich keine weitere wirtschaftlichen (Struktur-)Effekte stattfinden. Hierbei zeigt sich keine einheitliche Expertenschätzung hinsichtlich des Ausmaßes der eingeschätzten Verbesserungsmöglichkeiten; nach Einschätzung einiger Experten hängt dies sehr stark vom Einzelfall ab. ${ }^{220}$ Schließlich unterscheiden sich die Produktionsverfahren in der Zeitdauer, in der sie angewendet werden und, damit verbunden, auch häufig im Reifegrad. Zudem ist der Druck zur Prozessoptimierung verschieden, da die Marktsegmente zu unterschiedlichem Grade preissensitiv sind. Insgesamt ergeben sich keine klaren Hinweise für deutliche Herstellungsverbesserungen bei bestehenden Produkten, deshalb wird nur die zusätzliche Diffusion in den Szenarien betrachtet.

\section{Input-Output-Koeffizienten der substituierten Güter}

Analog zu den Polymeren sind Fein-/Spezialchemikalien in den deutschen I-OTabellen nur im aggregierten Sektor Chemie ausgewiesen. Deshalb werden auf Basis von genaueren Angaben der Kostenstrukturstatistik sowie I-O-Tabellen für Großbritannien und USA die Koeffizienten leicht angepasst. ${ }^{221}$ Fein/Spezialchemikalien zeichnen sich demnach durch einen geringeren Energiever-

\footnotetext{
${ }^{220}$ So sind nach Expertenaussagen bei Aminosäuren kaum Kostensenkungen zu erwarten, weil der Ertrag nahe am theoretischen Maximum eingeschätzt wird. Zudem stellen Rohstoffkosten (v.a. Zucker), die in Zukunft eher unwahrscheinlich fallen werden, einen beträchtlichen Kostenanteil dar.

${ }^{221}$ Streng genommen ist die Eignung des Sektors Chemie aus den I-O-Tabellen für 2005 zur Beschreibung des Substituts eingeschränkt geeignet, da zu diesem Zeitpunkt die Biotechnologie teilweise in diesem Sektor diffundiert ist und in den Durchschnittsvektor des Substituts einfließt. Da aber die Unterschiede durch die Biotechnologie und deren Anteil am gesamten Sektor zu diesem Zeitpunkt gering sind, tritt keine signifikante Verzerrung der Koeffizienten des Substitutes ein.
} 
brauch (-50\%), geringere Investitionen (-10\%) und höheren Arbeitskostenanteil $(+10 \%)$ wie die Chemie im Durchschnitt aus. In Tabelle 21 sind zusammenfassend die aggregierte Grundlage für die I-O-Vektoren der substituierten Fein/Spezialchemikalien, als auch die Vektoren für die Biotechnologie im Referenz und HD-Szenario ersichtlich. Die Werte in der Tabelle summieren sich für die Biotechnologie wegen den geringeren Kosten auf 90\% (Referenzszenario) bzw. 85\% (HD-Szenario). Für die Modellberechnungen werden die I-O-Koeffizienten auf $100 \%$ normiert und die Kostensenkungen in Konsumsteigerungen weitergegeben. Durch die folgende Darstellung werden aber die angenommen Einsparungen bei bestimmten primären Inputs (z.B. Arbeit) und Vorleistungen (z.B. Abfall) deutlich.

Tabelle 21: I-O-Koeffizienten für Biotechnologie und Chemie in den Szenarien

\begin{tabular}{|l|c|c|c|}
\hline & $\begin{array}{c}\text { Biotechnolo- } \\
\text { gie Referenz- } \\
\text { szenario }\end{array}$ & $\begin{array}{c}\text { Biotechno- } \\
\text { logie HD- } \\
\text { Szenario }\end{array}$ & $\begin{array}{c}\text { Chemie } \\
\text { Substi- } \\
\text { tut }\end{array}$ \\
\hline Landwirtschaft & $5,5 \%$ & $5,3 \%$ & $0,0 \%$ \\
\hline $\begin{array}{l}\text { Sonstige Rohstoffkosten/Hilfs-, Be- } \\
\text { triebsstoffe }\end{array}$ & $46,1 \%$ & $42,6 \%$ & $58,2 \%$ \\
\hline Investitionskosten & $2,5 \%$ & $2,3 \%$ & $3,5 \%$ \\
\hline Abfall & $0,9 \%$ & $0,8 \%$ & $1,3 \%$ \\
\hline Energie & $1,2 \%$ & $1,1 \%$ & $1,8 \%$ \\
\hline F\&E-Vorleistungen & $0,7 \%$ & $0,7 \%$ & $0,5 \%$ \\
\hline Dienstleistungen & $17,4 \%$ & $17,4 \%$ & $17,4 \%$ \\
\hline Arbeit & $10,9 \%$ & $10,0 \%$ & $12,5 \%$ \\
\hline Sonstige Wertschöpfung (Gewinn etc.) & $4,8 \%$ & $4,8 \%$ & $4,8 \%$ \\
\hline Produktionskosten & $90,0 \%$ & $85,0 \%$ & $100,0 \%$ \\
\hline Kosteneinsparung (=>Mehrkonsum) & $10,0 \%$ & $15,0 \%$ & \\
\hline Gesamt & $100,0 \%$ & $100,0 \%$ & $100,0 \%$ \\
\hline
\end{tabular}

Quelle: Eigene Berechnungen, Datenbasis: Expertengespräche, Statistisches Bundesamt (2008c)

\section{Produktions- und Substitutionswerte für die Szenarien}

Für die zusätzliche Diffusion zwischen 2005 und 2020 und der (inländischen) Produktion von Biotechnologisch hergestellten Fein-/Spezialchemikalien ergeben sich die in Tabelle 22 zusammengefassten Annahmen. Der Mehrkonsum infolge der Kosteneinsparungen bei der biotechnologischen Herstellung in den Szenarien (270 bzw. 725 Mio. €) fällt zum Teil im Ausland an. 
Tabelle 22: Szenarienannahmen für die biotechnologische Produktion von Fein-/Spezialchemikalien im Jahr 2020 (in Mio. $€$ )

\begin{tabular}{|l|c|c|c|c|}
\hline & \multicolumn{2}{|c|}{ Referenzszenario } & \multicolumn{2}{c|}{ HD-Szenario } \\
\hline & $\begin{array}{c}\text { Biotechno- } \\
\text { logie }\end{array}$ & Chemie & $\begin{array}{c}\text { Biotechno- } \\
\text { logie }\end{array}$ & Chemie \\
\hline $\begin{array}{l}\text { Chemikalienproduktion } \\
\text { für Inlandsnachfrage }\end{array}$ & $1583(0 \%)$ & $1758(0 \%)$ & $2672(0 \%)$ & $3143(0 \%)$ \\
\hline $\begin{array}{l}\text { Chemikalienproduktion } \\
\text { für Auslandsnachfrage }\end{array}$ & $852(0 \%)$ & $947(0 \%)$ & $1439(0 \%)$ & $1693(0 \%)$ \\
\hline Konsumveränderung & $270(35 \%)$ & & $725(35 \%)$ & \\
\hline Gesamt-Produktionswert & 2705 & 2705 & 4836 & 4836 \\
\hline
\end{tabular}

Quelle: Eigene Berechnungen. Die Werte in Klammern geben den Anteil der Importe an.

\subsubsection{Produktions- und Beschäftigungseffekte}

Die Produktionseffekte der Diffusion der Biotechnologie bei Fein-/Spezialchemikalien sind in Abbildung 23 ersichtlich. Sowohl die Nettoproduktion als auch die Nettobeschäftigung sind negativ. Der Produktionsverlust beträgt ca. 500 Mio. $€$ im Referenzszenario und ca. 1.300 Mio. $€$ im HD-Szenario. Wegen der höheren Effizienz und der angenommenen vollständigen Weitergabe der Produktivitätsgewinne in niedrigeren Preisen sinken die direkte Bruttowertschöpfung und der Bedarf an eigenen Vorleistungen aus der Chemieindustrie. Die Konsumerhöhungen durch die Preissenkungen werden aufgrund des hohen Auslandsanteils der Nachfrage hingegen nur zum Teil inländisch wirksam. Sie reichen deshalb nicht aus, um die Produktionsverluste in der Chemie zu konsumieren. Im HD-Szenario fallen diese Verluste absolut und relativ auf das Produktionsvolumen gesehen höher aus, aufgrund der größeren Produktivitätssteigerungen bei der biotechnologischen Fein-/Spezialchemikalienproduktion. Dies verstärkt die genannten Wirkungen. 


\section{Abbildung 23: Nettoproduktionswirkungen durch zusätzliche Diffusion der Biotechnologie bei Fein-/Spezialchemikalien im Vergleich zum Basisszenario (in Mio. $€$ )}

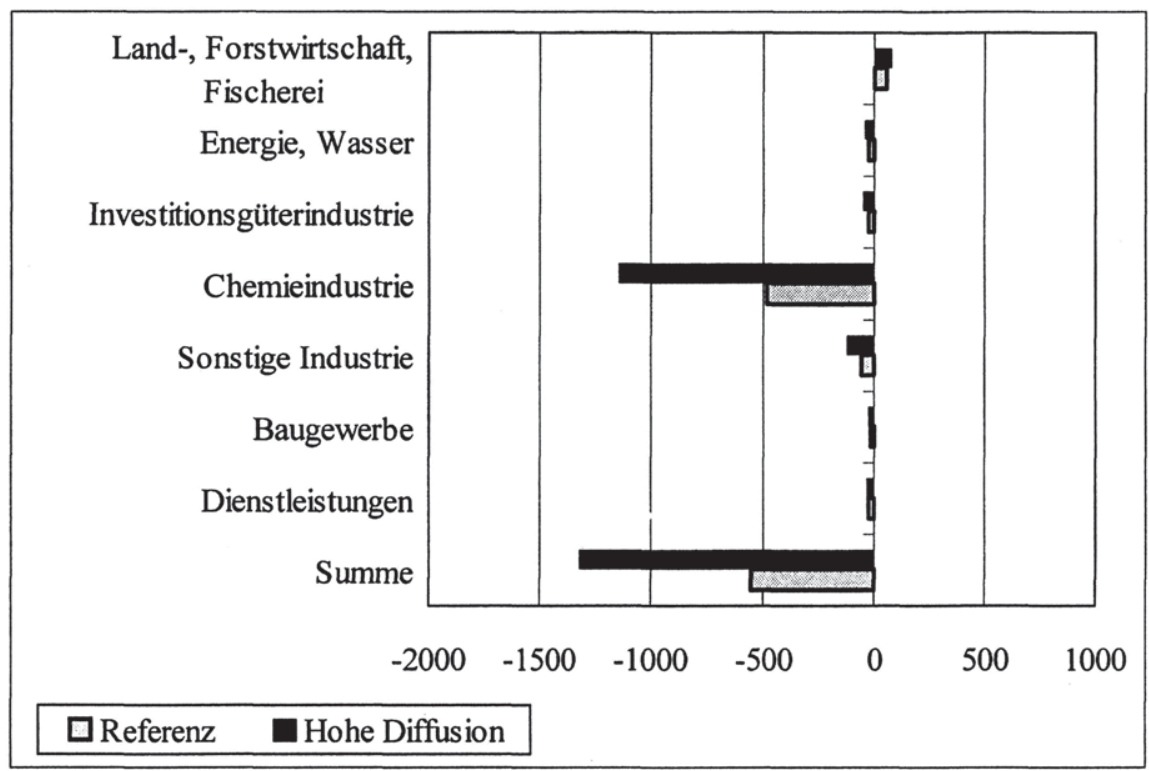

Quelle: Eigene Berechnungen

Bei der Nettobeschäftigung ergeben sich nur geringe Unterschiede zur Produktion (vgl. Abbildung 24). Der Beschäftigungsverlust beträgt knapp 600 Erwerbstätige im Referenzszenario und knapp 1500 Erwerbstätige im HD-Szenario. Auf sektoraler Ebene (vgl. Anhang Tabelle 44 bis Tabelle 47) wirken sich die erläuterten Zusammenhänge in einem Produktions- und Beschäftigungsrückgang in der Chemieindustrie selbst aus. Bei den sonstigen Industriesektoren zeigt sich vor allem ein Beschäftigungsrückgang mit ca. minus 200 Personen im Referenzszenario und knapp minus 500 Personen im HD-Szenario. ${ }^{222}$ Der Grund liegt im geringeren Bedarf bei der biotechnologischen Produktion von Vorleistungen aus anderen (inländischen) Industrien. In den anderen Bereichen sind die Effekte ebenfalls leicht negativ, mit Ausnahme des Landwirtschaftssektors.

222 Verglichen mit der Beschäftigung bei Fein-/Spezialchemikalien mit ca. 117.000 Erwerbstätigen im Jahr 2005 (Statistisches Bundesamt 2006) sind die Nettobeschäftigungsverluste marginal. Allerdings ist erneut auf die eingeschränkte Vergleichbarkeit dieser gesamtwirtschaftlichen Nettoeffekte mit der direkten, sektoralen Beschäftigung hinzuweisen. 
Abbildung 24: Nettobeschäftigungswirkungen durch zusätzliche Diffusion der Biotechnologie bei Fein-/Spezialchemikalien im Vergleich zum Basisszenario (in Erwerbstätige)

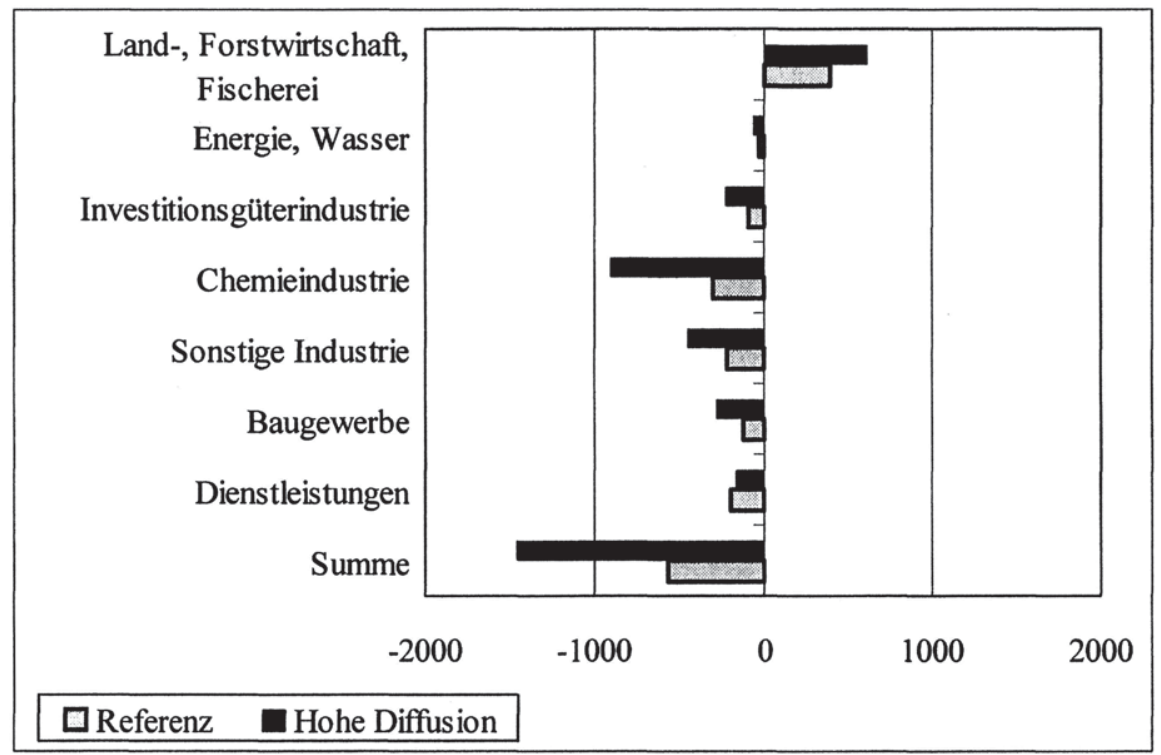

Quelle: Eigene Berechnungen

\subsubsection{Sensitivitätsanalyse}

Die aus der Literatur und Expertenmeinungen abgeleiteten Sensitivitätswerte sind in Tabelle 23 zusammengefasst. Aufgrund der fast einstimmigen Aussagen der Experten zur Diffusion und zum Außenhandel bzw. zur inländischen Produktion stellen diese Sensitivitätswerte Einzelmeinungen dar und liegen sehr nahe an den Referenzwerten. Die Sensitivitätsberechnungen führen zu recht unterschiedlichen Veränderungen der Produktions- und Beschäftigungseffekten (vgl. Abbildung 25 und Abbildung 26). Nur geringe Veränderungen ergeben sich durch alternative Diffusionsannahmen. Neben den vergleichsweise engen Bandbreiten bei den Variablen, ist die Sensitivität auf die Parameterveränderungen gering. Die Geraden verlaufen deshalb relativ flach. Infolgedessen bleiben für die Bandbreiten der Diffusion die Nettoproduktionseffekte in einem engen Korridor von ungefähr minus 300 bis minus 800 Mio. $€$. Die Nettoproduktionsbeschäftigung liegt in einem Korridor von knapp unter null bis minus $1000 \mathrm{Er}$ werbstätigen. Deutlich sensitiver ist die Variable der inländischen Produktion. Hier führen geringe Veränderungen der Variable zu deutlich abweichenden Ergebnissen von den Referenzwerten. Bspw. führt eine Erhöhung der inländischen 
Produktion um 10 Prozentpunkte über steigende Exporte zu neutralen Nettoproduktions- und positiven Beschäftigungseffekten. Im Gegensatz zu den vorigen beiden Anwendungsfeldern hat auch die Annahme des alternativen Konsumvektors deutliche Auswirkungen auf das Beschäftigungsergebnis. Bei der dabei angenommen preisunelastischen Nachfrage ergibt sich ein hoher Konsum von arbeitsintensiven Dienstleistungen. Die positive Nettobeschäftigung bei den Dienstleistungen überkompensiert die Verluste in der Chemieindustrie. Daher ergibt sich ein leicht positiver Beschäftigungseffekt. Die Nettoproduktion bleibt aber wie beim Referenzszenario negativ.

Tabelle 23: Sensitivitätsannahmen für die biotechnologische Produktion von Fein-/Spezialchemikalien

\begin{tabular}{|l|c|c|c|l|}
\hline Variable & Referenz & Niedrig & Hoch & \multicolumn{1}{|c|}{ Quelle/Anmerkung } \\
\hline Diffusion in \% & $10 \%$ & $8 \%$ & $12 \%$ & $\begin{array}{l}\text { Bandbreiten aus Exper- } \\
\text { tengesprächen }\end{array}$ \\
\hline $\begin{array}{l}\text { Inlandsproduktion } \\
\text { (in Mio. } € \text { ) }\end{array}$ & 2435 & 1583 & 2679 & $\begin{array}{l}\text { Niedrig: Annahme einer } \\
\text { ausgeglichenen Außen- } \\
\text { handelsbilanz; } \\
\text { Hoch: Eigene Annahme } \\
\text { auf Basis Expertengesprä- } \\
\text { che }\end{array}$ \\
\hline Konsumstruktur & $\begin{array}{l}\text { Konsumstruktur mit preisunelastischem Nachfrageverhal- } \\
\text { ten und Durchschnittsvektor der I-O-Tabelle 2005 }\end{array}$ \\
\hline
\end{tabular}

Quelle: Eigene Berechnungen

Insgesamt sind deshalb die im Referenz- und HD-Szenario ermittelten negativen Effekte zu relativieren. Die Robustheit der Ergebnisse ist geringer. Unter alternativen Annahmen sind auch positive Effekte möglich. Besonders wenn die Kostenersparnis durch den Einsatz der Biotechnologie mit einer höheren Wettbewerbsfähigkeit einhergehen würde, können neutrale bzw. positive Nettoeffekte entstehen Darüber hinaus ist zu beachten, dass die Modellierung der Fein/Spezialchemikalien als reine Prozessinnovation erfolgt, eine stärkere Wirkung als additive Produktinnovation kann die Effekte ebenfalls verändern. 
Abbildung 25: Nettoproduktionswirkungen für biotechnologische Produktion von Fein-/Spezialchemikalien in Sensitivitätsrechnungen

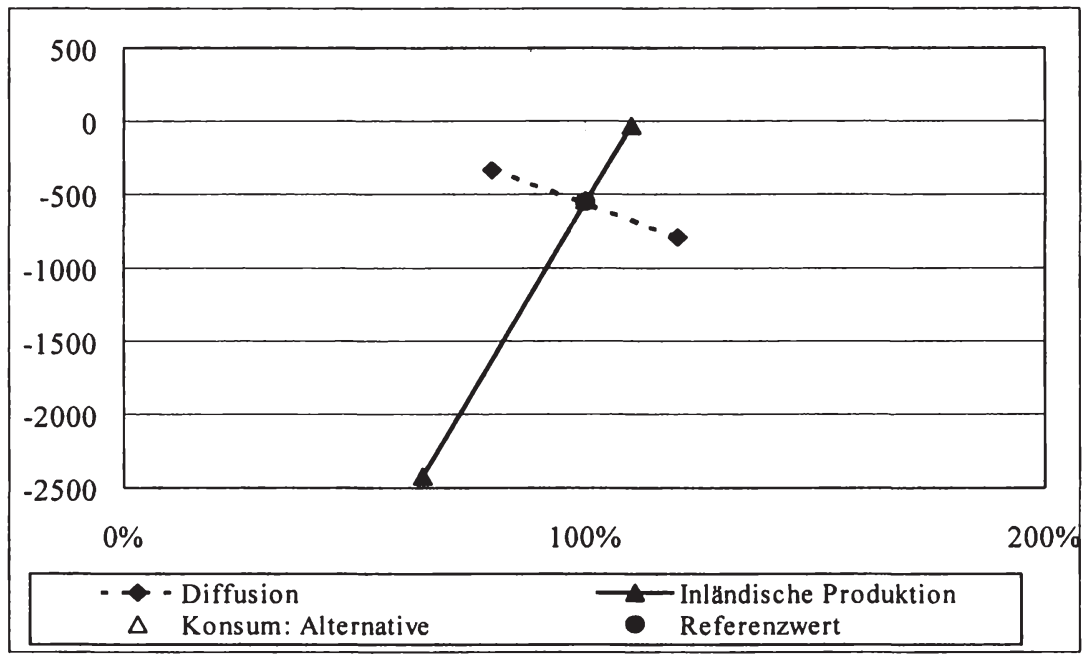

Quelle: Eigene Berechnungen

Abbildung 26: Nettobeschäftigungswirkungen für biotechnologische Produktion von Fein-/Spezialchemikalien in Sensitivitätsrechnungen

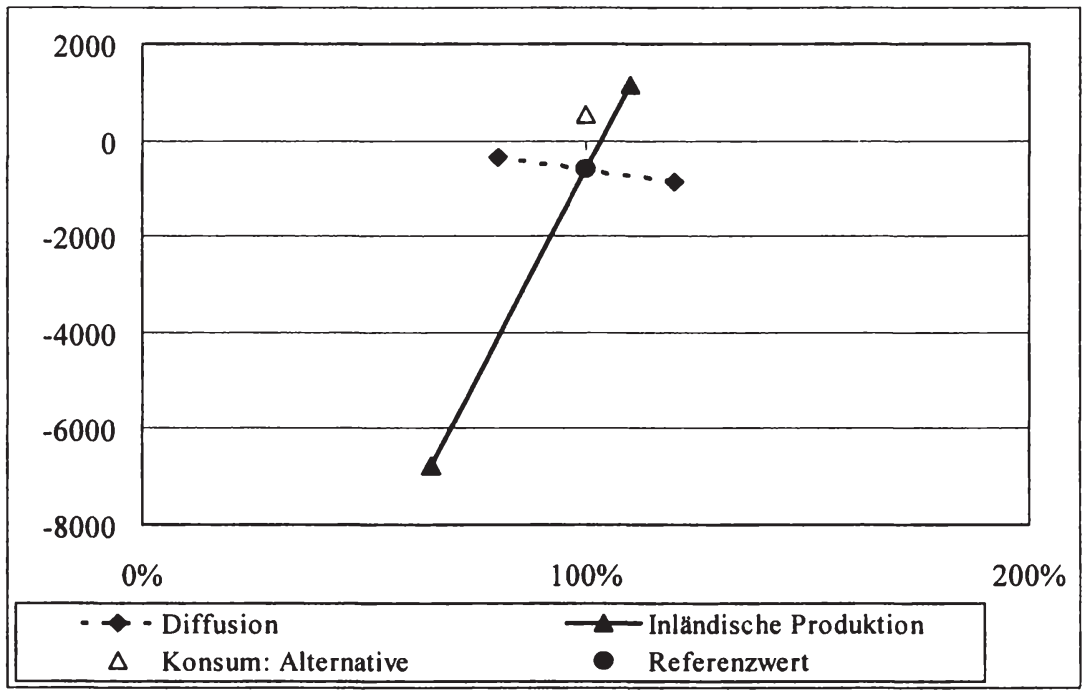

Quelle: Eigene Berechnungen 


\subsection{Biopharmazeutika}

Der große Einfluss der Biotechnologie in der Pharmaindustrie liegt zu einem wichtigen Teil in der Entwicklung neuer Wirkstoffe (Kapitel 3.1.5.1), den so genannten Biopharmazeutika. In der Literatur zeigen sich allerdings erhebliche Unterschiede bei der Definition und Zuordnung einzelner Wirkstoffe zu dieser Gruppe (z.B. Arundel/Mintzes 2004; BCG 2006); die Aussagen zur größenmäBigen Bedeutung von Biopharmazeutika differieren daher (siehe Kapitel 6.4.1) ${ }^{223}$ Im Rahmen dieser Arbeit wird in Anlehnung an Reiss et al. (2007) Biopharmazeutika als alle ,[...] recombinant products such as interferon, interleukin, growth factors; blood factors, hormones and other peptides and proteins; antibodies, immuntoxins, and immunoconjugates" (Reiss et al. 2007, S.45) definiert. Daneben wird die kleine Gruppe der rekombinanten Vakzine (Impfstoffe) mit einbezogen. ${ }^{224}$

Allerdings muss beachtet werden, dass der Vergleich zwischen Biopharmazeutika und chemischen Pharmazeutika (,small molecules") für den Einfluss der Biotechnologie in der Pharmaindustrie nur begrenzt aussagekräftig ist. Biotechnologische Methoden sind auch für die F\&E chemischer Wirkstoffe von immer höherer Bedeutung, bspw. für die Identifizierung neuer Wirkorte (Gaisser et al. 2005). Nach Expertenaussagen werden alle neuen Medikamente in einer oder mehreren Phasen des Entwicklungsprozesses mit biotechnologischen Methoden bearbeitet. Daneben werden biotechnologische Methoden teilweise auch zur Produktion von "small molecules“ eingesetzt (IPTS 2007, Arundel 2007). ${ }^{225}$ Dennoch zeigen sich einige Unterschiede zwischen Biopharmazeutika und „small molecules“, die in den folgenden Kapiteln erläutert werden. Diese Unterschiede sind von erheblicher wirtschaftlicher Relevanz und machen die Auswirkungen der Biotechnologie deutlich.

Wie in Kapitel 6.4.2 eingehend diskutiert wird, stellen Biopharmazeutika gegenüber den „small molecules“ eine Produktinnovation dar. Dabei zeigt sich besonders die Bedeutung der Biotechnologie für die F\&E neuer Produkte. Daneben bestehen Unterschiede in Kostenhöhe und -struktur der biotechnologischen

${ }^{223}$ Gründe hierfür sehen Arundel und Mintzes (2004, S.4) in der schnellen technologischen Weiterentwicklung in den Gesundheitswissenschaften sowie der zunehmenden Interdisziplinarität, welche die eindeutige Zuordnung zu Technologien erschwert. Daneben definieren einige Studien Biopharmazeutika als alle Wirkstoffe von „Biotechnologie-Unternehmen“, dieser Begriff ist aber ebenfalls recht unscharf.

${ }^{224}$ In der vorliegenden Fallstudie werden Vakzine mit einbezogen, da die meisten befragten Experten diese unter Biopharmazeutika subsumieren.

${ }^{225}$ Allerdings bestehen hier bisher keine Möglichkeiten die ökonomischen Auswirkungen der Nutzung der Biotechnologie zu bestimmen, da statistische Daten oder ein Überblick von Experten hierzu fehlen (Reiss et al. 2007; Arundel 2007). Der Anteil der biotechnologischen Produktion von „small molecules“ wird von Reiss et al. (2007) auf ungefähr 10-15\% bei steigender Tendenz geschätzt. 
und chemischen Produktion. Für die Bestimmung der wirtschaftlichen Auswirkungen ist deshalb sowohl die Betrachtung von additiven und/oder substitutiven Wirkungen neuer Produkte als auch von Effizienz- und Strukturunterschieden in der Forschung und Produktion notwendig.

\subsubsection{Diffusion und internationaler Wettbewerb}

Die Pharmaindustrie gehört zu den dynamischsten Branchen innerhalb des Verarbeitenden Gewerbe mit einem hohen Anteil der Bruttowertschöpfung am Produktionswert (ca. 35\%) (Statistisches Bundesamt 2008c). Der Produktionswert in der Pharmaindustrie lag im Jahr 2005 bei 22,7 Mrd. $€$ (2007: 26,2 Mrd. $€$ ), die Beschäftigung betrug ca. 113 Tsd. (2007:112 Tsd.) Erwerbspersonen (Statistisches Bundesamt 2008d). Zukünftig werden die Wachstumsperspektiven der Pharmabranche positiv eingeschätzt (z.B. Bräuninger et al. 2008; Nusser et al. 2007b). Als wichtiger Faktor auf der Angebotsseite gelten neue technologische Möglichkeiten (z.B. durch Biotechnologie). Auf der Nachfrageseite entstehen zunehmend neue Absatzmärkte in aufstrebenden Ländern (z.B. Asien) sowie ein höherer inländischer Bedarf aufgrund der alternden Bevölkerung (u. a. viele Patienten mit mehreren Krankheitsbildern) und der Zunahme chronischer Krankheiten (Nusser et al. 2007c; Bräuninger et al. 2007). Die Ableitung von möglichen Wachstumsraten ist dennoch schwierig. Für die Szenarien wird in Anlehnung an Prognos (2006) ein Wachstum der Pharmaindustrie von 2,1\% angesetzt.

Bevor die Bedeutung von Biopharmazeutika untersucht werden kann, ist zu klären, wie disaggregiert die Analyse erfolgt. In der Regel wird bei Pharmazeutika zwischen neu am Markt zugelassenen, patentgeschützten Substanzen (,New Molecular Entities"=NMEs) und Nachahmerprodukten mit identischen Substanzen für patentfreie Originalprodukte (Generika) unterschieden. Generika sind bei chemisch-synthetisierten Produkten aufgrund des kleinen Anteils der Produktionskosten und der eher geringen Forschungsausgaben erheblich günstiger. Auch bei Biopharmazeutika ist grundsätzlich ein wachsender „BiogenerikaMarkt" möglich, da einige biopharmazeutische „Blockbuster-Produkte“ in den nächsten Jahren den Patentschutz verlieren oder bereits patentfrei sind (IKB/Prognos 2007). Allerdings ist die Nachahmung deutlich schwieriger, da die komplizierten Moleküle nur mit Hilfe genveränderter Mikroorganismen oder Säugetierzellen hergestellt werden können. Bereits kleine Unterschiede im Herstellungsverfahren können dabei zu Änderungen in Molekülstruktur und Wirkung führen. Das Imitat ist dem Originalwirkstoff daher nur „sehr ähnlich“, aber nicht identisch (Dukes 2008). ${ }^{226}$ Deshalb werden von den Behörden bei den Zulassungsverfahren in der Regel, wie bei den neuen Wirkstoffen, klinische Studien verlangt. Dies erschwert die Einführung der Biogenerika und verringert die Preisdifferenz zum Originalprodukt erheblich. Aufgrund mangelnder Daten

${ }^{226}$ Deshalb wird statt Biogenerika häufig der Begriff „Biosimilars“ verwendet. 
können Biogenerika nicht explizit betrachtet, sondern nur implizit in der Diffusion und der Kostensituation von Biopharmazeutika bei den Expertengesprächen mitberücksichtigt werden. Aufgrund des geringen Preisunterschiedes zu den Originalprodukten wären die Auswirkungen einer sehr geringen bzw. starken Bedeutung von Biogenerika für die Ergebnisse aber jeweils eher gering einzuschätzen.

Bei der Untersuchung der Diffusion von Biopharmazeutika ist die Verbreitung in der Produktion sowie in der F\&E von Interesse. Daher sind mehrere Indikatoren zu unterscheiden. Diese hängen aber eng miteinander zusammen, schließlich ist z.B. die Verbreitung der Biopharmazeutika in klinischen Studien ein möglicher Frühindikator für die Entwicklung in der Produktion. Es werden dabei folgende Indikatoren analysiert:

- Anteil von Biopharmazeutika an den neu am Markt zugelassenen Substanzen

- Anteil der Biopharmazeutika an getesteten Substanzen in den klinischen Studien (als Frühindikator für die zukünftigen am Markt zugelassenen Substanzen)

- Anteil der Biopharmazeutika beim Umsatz von pharmazeutischen Produkten

Als zentraler Indikator für den Forschungsoutput gilt der Anteil von Biopharmazeutika an den neu am Markt zugelassenen Substanzen (NMEs = „New Molecular Entities"). Dabei unterscheiden sich die vorliegenden Berechnungen aufgrund der unterschiedlichen Definitionen von Biopharmazeutika. Nach OECD-Berechnungen ist der Anteil der Biopharmazeutika bei den neu zugelassenen NMEs in Europa und USA zwischen 1989 und 1998 stark gestiegen, ist aber seitdem eher konstant bei einem Anteil von ca. 15\% geblieben (Arundel 2007). Nach Reiss et al. (2007) lag der Anteil (allerdings ohne Vakzine) zwischen 1996 und 2005 in der EU bei ca. 10\%, in den USA bei $11 \%$. Anderen Berechnungen zufolge lag der Anteil in den vergangenen Jahren bei rund 20\% (z.B. BCG 2008), einige der befragten Experten gehen sogar von deutlich höheren Werten aus. Dabei ist insgesamt zu beachten, dass sich die absolute Zahl an zugelassenen Biopharmazeutika eher verhalten entwickelt hat. Die Bedeutung steigt demnach nur relativ zu „small molecules“, bei denen die Zulassungen rückläufig sind (Hopkins et al. 2007). Dies wird in Abbildung 27 deutlich. Bei den zugrunde liegenden Berechnungen von EFPIA (2008), betrug der durchschnittliche Anteil der Biopharmazeutika zwischen 1996 und 2005 rund $20 \%$. 


\section{Abbildung 27: Anzahl der jährlich weltweit eingeführten „New Molecular Entities"}

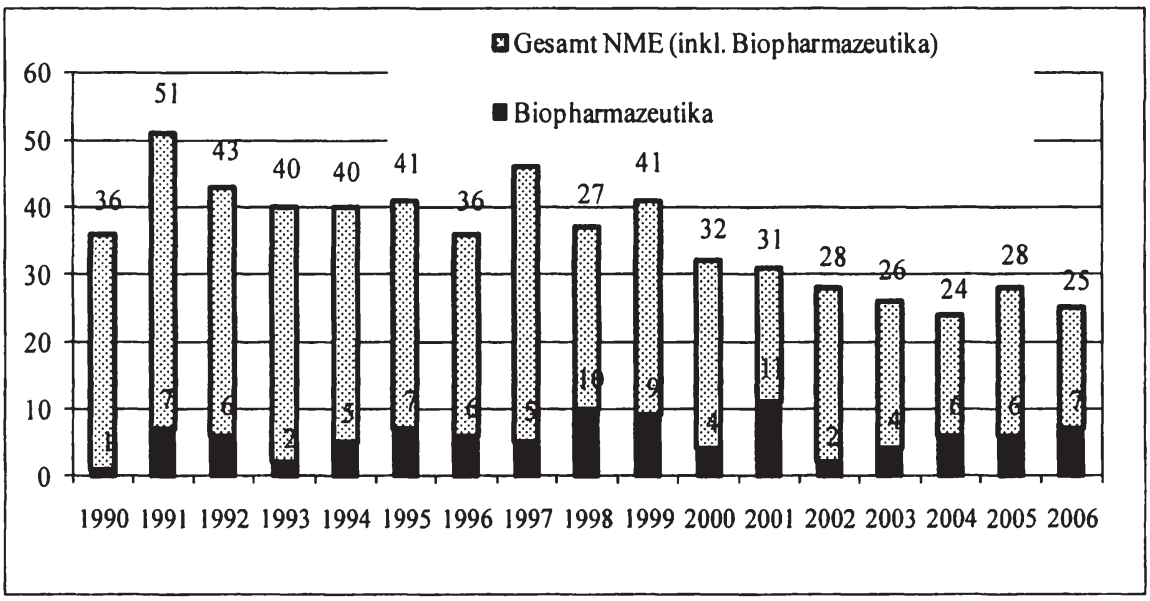

Quelle: EFPIA 2008

Als Anhaltspunkt für die mögliche zukünftige Entwicklung des Anteils der Biopharmazeutika an den NMEs gilt der Anteil der Biopharmazeutika an getesteten Substanzen in den klinischen Studien. Die biopharmazeutische „Forschungspipeline" deutet nach der OECD (Arundel 2007) auf eine ungefähre Verdoppelung der biopharmazeutischen NMEs pro Jahr im Zeitraum 2010 bis 2015 gegenüber dem Zeitraum 2000 bis 2006 bei einem leicht zunehmenden Anteil (18\% statt 14\%) an den gesamten NMEs hin. Reiss et al. (2007) stellt für die EU einen stabilen Anteil der Biopharmazeutika an klinischen Studien fest, in den USA ist der Trend sogar leicht negativ. Demnach würden nur deutlich höhere Zulassungsraten für Biopharmazeutika für eine Erhöhung des Marktanteils sprechen. Die befragten Experten sind etwas optimistischer. So wird teilweise bereits aktuell ein höherer Anteil angenommen, der zukünftig gehalten oder sogar ausgebaut werden kann.

Die Bedeutung der Biopharmazeutika beim Umsatz hat besonders seit Mitte der 1990er Jahre zugenommen. Nach Berechnungen von Reiss et al. (2007) ist der Umsatz in den Jahren 1996 bis 2005 mit 23\% p.a. in der Europäischen Union wesentlich dynamischer gewachsen als derjenige der gesamten Pharmazie (11\% p.a.). Der Anteil der Biopharmazeutika hat sich von knapp 4\% im Jahr 1996 auf 10\% im Jahr 2005 erhöht (Reiss et al. 2007). ${ }^{227}$ Dies liegt besonders

${ }^{227}$ Dabei zeigen sich keine erheblichen Schwankungen im Zeitablauf, wie es bei den zugelassenen neuen Molekülen pro Jahr der Fall ist. 
daran, dass der durchschnittliche Umsatz je Biopharmazeutika in der EU deutlich gestiegen ist. ${ }^{228}$ Zeitreihen für Biopharmazeutika in Deutschland liegen nur bedingt vor, zeigen aber zumindest für die vergangenen Jahre eine sehr ähnliche Tendenz. Nach Angaben des Verbands forschender Arzneimittelhersteller (VFA 2007,2008) lag der Umsatz (inklusive Vakzine) in Deutschland im Jahr 2005 bei rund $11 \%$ (2007 bei $14,2 \%) .{ }^{229}$ Die Expertenmeinungen bei der Einschätzung der zukünftigen Diffusion differieren deutlich stärker als bei den anderen Fallbeispielen. Dies lässt sich zum vermutlich kleineren Teil auf die differierenden Annahmen der Experten zu den bisherigen Anteilen der Biopharmazeutika an NME zurückführen. Bedeutender ist, dass die Diffusion der Biopharmazeutika durch einige zentrale Faktoren stark beeinflusst wird. Von der technologischen Seite aus ist dabei entscheidend, inwiefern es gelingt, erfolgreiche Therapien für weit verbreitete, bisher nur sehr eingeschränkt behandelbare Krankheiten (Krebs, Alzheimer etc.) zu entwickeln. Unsicherheiten ergeben sich auch bei der Nachfrage nach Pharmazeutika im Allgemeinen und Biopharmazeutika im Besonderen. Dabei sind vor allem die Kostendämpfungspolitik und in diesem $\mathrm{Zu}$ sammenhang stehende Regelungen von Bedeutung (OECD 2005b; Paris/Docteur 2008). Durch die Regelungen für Neuzulassungen von Arzneimitteln und Festlegung der Erstattungsbestimmungen (z.B. Einbezug in die Erstattung, Budgethöchstgrenzen für Ärzte oder Höchsterstattungspreise) wird die Diffusion neuer Arzneimittel und Anreize für zukünftige F\&E stark beeinflusst (OECD 2008e; OECD 2005b; Productivity Commission 2005). Biopharmazeutika können von Änderungen in der Regulierung in besonderem Maße betroffen sein, aufgrund der tendenziell höheren Therapiekosten, der vergleichsweise hohen Bedeutung von Neuzulassungen und ihrem häufigen Einsatz für kleine Patientengruppen liegen (Danzon/Furukawa 2006; OECD 2005b; Rosenberg-Yunger et al. 2008).

Für eine "Business as Usual"-Entwicklung, in der es zu keiner grundsätzlichen Veränderung der bisher eingeschlagenen Politik kommt, liegt der Durchschnitt der Expertenschätzung bei $24 \% .{ }^{230}$ Bei einer expansiveren Gesundheitspolitik, welche eine Finanzierung einer Vielzahl von neuen, häufig teuren Biotechnologie-Medikamenten ermöglicht sowie einer günstigen technologischen

${ }^{228}$ Der durchschnittliche Umsatz je Biopharmazeutika hat sich zwischen 1995 und 2005 verdreifacht und erreichte im Jahr 2005 einen Wert von 133 Mio. $€$ pro Jahr (Reiss et al. 2006).

${ }^{229} \mathrm{Zu}$ ähnlichen Ergebnissen kommt die Boston Consulting Group für Biopharmazeutika mit 12\% für 2006 und 15\% für das Jahr 2007 (BCG 2007,2008).

${ }^{230}$ Die Werte liegen innerhalb des Korridors der Schätzungen von Nusser et al. (2007a), die zwischen 18 und 40\% für Jahr 2020 betragen. Die Grundlage für diese Schätzungen bildet eine schriftliche Befragung von rund 70 Experten. 
Entwicklung liegt der Durchschnitt der Schätzungen bei $32 \%{ }^{231}$ Diese in den Szenarien geschätzten Zuwächse am Umsatzanteil liegen höher als es die Betrachtung der Anteile an neuen Produkten vermuten lässt. Begründet werdet kann dies nach Aussagen der Experten darin, dass es sich zumeist um teurere Produkte im Hochpreissegment handelt. Daneben diffundieren die relativ jüngeren, biopharmazeutischen Produkte stärker als „small molecules“, die Steigerung des Umsatzes je Produkt ist höher. Bei der angenommenen Steigerung des Produktionswertes von $2,1 \%$ p.a. müssten die jährlichen Wachstumsraten von Biopharmazeutika bei $7 \%$ bzw. 9\% liegen, eine Entwicklung, die zumindest in jüngerer Vergangenheit erreicht wurde (Reiss et al. 2007; VFA 2007, 2008). Deshalb wird gemäß den Expertenschätzungen für das Referenzszenario ein Anteil von $24 \%$ und für das HD-Szenario ein Anteil von 32\% für das Jahr 2020 angenommen. Abzüglich der Diffusion des Jahres 2005 von 2,5 Mrd. $€$ (VFA 2007) ergeben sich für das Referenzszenario ein zusätzlicher Produktionswert von 5,4 Mrd. $€$ und im HD-Szenario von 8,0 Mrd. $€$ (Tabelle 24). Die erwähnten Bandbreiten zwischen einzelnen Expertenmeinungen werden in den Sensitivitätsrechnungen berücksichtigt.

\section{Tabelle 24: Diffusion von Biopharmazeutika in den Szenarien}

\begin{tabular}{|l|c|c|}
\hline & Referenzszenario & HD-Szenario \\
\hline Diffusion 2020 in Mrd. $€$ & 7,9 & 10,5 \\
\hline Diffusion 2005 in Mrd. $€$ & 2,5 & 2,5 \\
\hline $\begin{array}{l}\text { Differenz 2020-2005 in Mrd. } € \\
\text { (Berechnungswert) }\end{array}$ & 5,4 & 8,0 \\
\hline
\end{tabular}

Quelle: Eigene Berechnungen, Datenbasis: Experteninterviews, VFA (2007)

\section{Internationaler Wettbewerb}

Die Pharmaindustrie gehört zu dem am stärksten internationalisierten Branchen. Es herrscht ein hoher Standortwettbewerb. Die Exportquote im betrug in Deutschland Jahr 2007 56\%, der Produktionsanteil am Weltmarkt ca. 7\% (VFA 2008). Seit den frühen 1990er Jahren zeigt sich aber ein deutlicher Bedeutungsverlust der deutschen Pharmaindustrie (Gaisser et al. 2005). Ein Hauptgrund lag in der mangelnden Gründungsdynamik von Biotechnologie-Unternehmen und der unzureichenden Anwendung von Biotechnologie in großen Unternehmen (Lange 2006; Peter 2002; Jürgens/Sablowski 2005). In jüngerer Vergangenheit sind aber wieder Anzeichen einer Erholung der Pharmaindustrie in Deutschland erkennbar (Bräuninger et al. 2008; Nusser et al. 2007c). So sind bei den F\&E-

231 Dabei ist wie bei Fein-/Spezialchemikalien zu beachten, dass die Diffusion der Biopharmazeutika nicht einheitlich ist. Mögliche Bedeutungsverschiebungen von Krankheitsbildern wurden von den Experten bei den Schätzungen berücksichtigt. 
Ausgaben, wissenschaftlichen Publikationen und Patenten absolut und relativ im internationalen Vergleich Zuwächse für Deutschland erkennbar (Nusser et al. 2007c; OECD 2006b, 2006c; Paris/Docteur 2008). Diese Feststellung trifft, soweit die Datenlage Schlüsse hierfür zulässt, auch für Biopharmazeutika zu (OECD 2006b, 2006c; Nusser et al. 2007a, 2007c). Zahlen für Anteile Deutschlands bei der Produktion von Biopharmazeutika liegen in der Literatur nicht vor, der weltweite Produktionsanteils Europas beträgt rund 30\% und damit ähnlich wie für die gesamte Pharmaindustrie (IPTS 2007). Bei der Zahl der spezialisierten Biotechnologieunternehmen in der Pharmaindustrie nimmt Deutschland im Jahr 2005 die dritte Stelle ein (OECD 2006b), bei den Produktionskapazitäten von Biopharmazeutika (in Liter Fermenter) liegt Deutschland im gleichen Jahr hinter den USA an zweiter Stelle (BCG 2006). Folglich bestehen einige positive Anzeichen für eine hohe internationale Wettbewerbsfähigkeit Deutschlands bei F\&E und Produktion von Biopharmazeutika.

Eine Verbesserung der internationalen Wettbewerbsfähigkeit hätte erhebliche positive Folgewirkungen für die inländische Wirtschaft, da die internationalen Absatzmärkte in der Pharmaindustrie ein großes Nachfragepotenzial bilden und von wenigen Standorten aus beliefert werden können. Allerdings unterliegt die Einschätzung einer deutlich positiven Entwicklung der Position Deutschlands bei Biopharmazeutika erheblichen Unsicherheiten, wie auch durch unterschiedliche Einschätzungen der Experten deutlich wurde. Schließlich zeigen sich in Deutschland neben den genannten Stärken auch einige Schwächen, z.B. bei der Venture-Capital-Finanzierung (Lange 2006; OECD 2006c). Deshalb wird für die Szenarien angenommen, dass sich die Außenhandelsbilanz für Biopharmazeutika analog zur bisherigen Bilanz für alle Pharmazeutika entwickelt. Die Wirkung einer deutlichen Steigerung der inländischen Produktion für den Weltmarkt wird in einer ausführlichen Sensitivitätsanalyse für das Referenzund das HD-Szenario betrachtet, die detaillierter als für die bisherigen Anwendungsfelder erfolgt.

\subsubsection{Bedeutung der Biotechnologie für Produktinnovationen}

Die Entwicklung neuer Produkte hat im Pharmasektor eine besondere Bedeutung, da über geistige Eigentumsrechte (v.a. Patente) eine langjährige Monopolstellung für das Produkt erreicht werden kann. Zudem besteht Bedarf nach verbesserten und neuen medizinischen Produkten zur Behandlung von Krankheiten (Patel et al. 2008; McKelvey 2008). Dabei lässt es sich aber schwer beurteilen, inwiefern neue Produkte additiv oder substitutiv zu anderen Arzneimitteln eingesetzt werden.

Aus technologischer Sicht sprechen einige Argumente für die Neuartigkeit und einen tendenziellen additiven Impuls der Produkte. So sehen zum einen die befragten Experten die Biopharmazeutika hauptsächlich als Erweiterung der 
„small molecules“ an. Die Einsatzbereiche der Biotechnologie sehen sie vorrangig dort, wo man chemisch schwer eingreifen und ähnliche Wirkung erzielen kann, z.B. über die Bindung an bestimmten Rezeptoren oder der Ersetzung von fehlenden Stoffen/Proteinen im Körper. So entstehen durch die Biotechnologie neue „Wirkstoffklassen“, wie monoklonale Antikörper oder rekombinante Proteine. Bei Einsatzgebieten, bei denen sowohl biotechnologische als auch chemische Lösungen denkbar sind, wird nach Meinung der Experten die chemische Variante bevorzugtes Interesse erhalten, da zumindest bei der Produktion erhebliche Kostenvorteile für die Chemie existieren. Zudem ist tendenziell bei nicht lebensnotwendigen Arzneimitteln die Akzeptanz chemischer Präparate höher, da sie in Form von Tabletten eingenommen werden können, während Biopharmazeutika bisher über Spritzen verabreicht werden müssen.

Darüber hinaus werden biopharmazeutische Wirkstoffe in aller Regel auch nur biotechnologisch hergestellt, da sie nicht mit anderen Methoden in der erforderlichen Menge wirtschaftlich und in notwendiger Qualität produzierbar sind. ${ }^{232}$ Als klassisches Beispiel gilt die Produktion von Insulin. Dies kann zwar auch nicht-biotechnologisch aus Tieren ${ }^{233}$ gewonnen werden, dort können aber erhebliche Verunreinigungen und dadurch Infektionsgefahren auftauchen und die Produktion im nachgefragten Volumen wäre kaum machbar (IPTS 2007). Nach einstimmiger Expertenmeinung gilt diese Vorteilhaftigkeit der biotechnischen Produktion für den großen Teil der Biopharmazeutika. Eine Ausnahme bilden einige Peptide, wenn sie aus kurzen Aminosäurenketten bestehen. Nach Expertenaussagen erhöhen sich möglicherweise die Größengrenzen von chemisch hergestellten Peptiden, demnach würde auch für komplexere Aminosäuren die chemische Herstellung in Betracht kommen. Diese Gruppe nimmt aber einen sehr kleinen Anteil an Biopharmazeutika ein (La Merie 2008). Auch sind zukünftig keine größeren Änderungen zu erwarten. ${ }^{234}$

Inwiefern es tatsächlich zu einer additiven Wirkung biopharmazeutischer Produkte am Markt kommt, hängt entscheidend vom Zusammenspiel dieser technologischen Möglichkeiten mit der Nachfrage und den regulativen Komponenten ab. Die oben genannten staatlichen Regelungen beeinflussen neben dem relativen Anteil der Biopharmazeutika auch entscheidend das absolute Wachstum und damit die Höhe der Umsätze neuer Produkte. Aus Nachfragesicht haben Biopharmazeutika besonders bei den Krankheitsbildern (v.a. Stoffwechselerkrankungen, Krebserkrankungen, Immun- und Infektionskrankheiten) eine hohe

${ }^{232}$ Der Grund hierfür liegt darin, dass der Wirkstoff aus lebenden Organismen besteht, welcher mit der traditionellen chemischen Synthese nur schwer zu erreichen ist.

${ }^{233}$ Die Gewinnung von Insulin aus Tieren erfolgt aus der Bauchspeicheldrüse geschlachteter Rinder und Schweine.

${ }^{234}$ Für die Betrachtung der Herstellungskosten (Kapitel 6.4.3) wird deshalb von einer vollständigen biotechnologischen Herstellung von biopharmazeutischen Wirkstoffen ausgegangen. 
Bedeutung ${ }^{235}$, bei denen das Marktvolumen groß ist, die Wachstumsraten in jüngerer Vergangenheit vergleichsweise hoch waren und auch zukünftig hoch eingeschätzt werden (Nusser et al. 2007c; IKB/Prognos 2007). Hieran schließt sich die konkrete Diskussion an, ob die Biopharmazeutika besonders zur Erfüllung von „unmet clinical needs“ beitragen, das heißt, einen therapeutischen $\mathrm{Zu}$ satznutzen für Krankheiten haben, wo ein Bedarf nach wirksameren Produkten besteht (Hopkins et al. 2007; Arundel/Mintzes 2004). Bisherige Vergleichsuntersuchungen zum therapeutischen Zusatznutzen zeigen leichte Vorteile der Biopharmazeutika. Bspw. wird deren Anteil von „me-too“ Produkten etwas geringer eingeschätzt (Arundel 2007; Arundel/Mintzes 2004; Joppy et al. 2005). Allerdings betonen die Autoren die Unsicherheit, ob sich diese Vorteilhaftigkeit fortsetzt oder der Zusatznutzen bspw. neuer Antikörper oder Proteine sich abschwächt.

Insgesamt ergeben sich aber Hinweise für eine zumeist additive Wirkung biopharmazeutischer Produkte im Pharmasektor, dies wird in einigen Expertengesprächen auch bestätigt. Für die Szenarioberechnungen wird angenommen, dass Biopharmazeutika in dem Umfang "small molecules“ ersetzen, bei dem der Anteil auch beim zusätzlichen Wachstum des Pharmasektors stabil bleibt. Die zusätzliche Diffusion ist hingegen additiv. In anderen Worten wird unterstellt, dass pharmazeutische Produkte auch unabhängig von der Biotechnologie ein Wachstumspotenzial von $2,1 \%$ p.a. hätten, aber in Höhe dieses Zuwachses von Biopharmazeutika ersetzt werden. Das Wachstum der Biopharmazeutika, welches über dieses Durchschnittswachstum von 2,1\% p.a. hinaus geht, wird als zusätzlicher Impuls betrachtet. Die Aufteilung der oben berechneten zusätzlichen Diffusion von 5,4 Mrd. $€$ im Referenzszenario und $8 \mathrm{Mrd}$. $€$ im HD-Szenario in additive und substitutive Impulse ist in Tabelle 25 ersichtlich.

Tabelle 25: Szenarienannahmen für die Wirkung von Produktinnovationen bei Biopharmazeutika

\begin{tabular}{|l|c|c|}
\hline & Referenzszenario & HD-Szenario \\
\hline $\begin{array}{l}\text { Ersetzung von chemischen Pharma- } \\
\text { zeutika in Mrd. } €\end{array}$ & 0,9 & 0,9 \\
\hline $\begin{array}{l}\text { Additiver Nachfrageimpuls durch } \\
\text { Produktinnovationen in Mrd. } €\end{array}$ & 4,5 & 7,1 \\
\hline Summe (zusätzliche Diffusion) & 5,4 & 8.0 \\
\hline
\end{tabular}

Quelle: Eigene Berechnungen

${ }^{235}$ Die Bedeutung wird dabei an Umsatzanteilen und Anteilen an Substanzen in der klinischen Phase gemessen (BCG 2008). Allerdings ist zu beachten, dass bei den großen Bereichen der Herz-/Kreislauferkrankungen und Erkrankungen des Nervensystems Biopharmazeutika bisher eher eine geringe Rolle spielen. 
Eine Erhöhung des Umsatzes in der Pharmaindustrie führt allerdings zu höheren Gesundheitsausgaben, wenn diese nicht an anderer Stelle infolge des Einsatzes der Biotechnologie eingespart werden können. ${ }^{236}$ Beispielsweise können neue therapeutische Behandlungsmöglichkeiten Aufenthalte in Krankenhäusern ersetzen oder die Art der medizinischen Intervention verändern. Einzelfallstudien zeigen (z.B. IPTS 2007; BCG 2006), dass in manchen Fällen Gesundheitskosten durch Biopharmazeutika eingespart werden können, während in anderen Fällen zusätzliche Kosten entstehen. Aggregierte Betrachtungen existieren bisher kaum. Rosenberg-Yunger et al. (2008, S.364) sehen in Summe bisher keine eindeutigen Hinweise für Einsparungen durch die Biotechnologie an anderen Stellen des Gesundheitssystems. Für die quantitativen Berechnungen wird deshalb vereinfachend von keinen Veränderungen an anderen Stellen des Gesundheitssystems ausgegangen. Die höheren Gesundheitskosten bestimmen sich demnach durch die Höhe des zusätzlichen Absatzes für biopharmazeutische Produkte.

Zusammenfassend stellen die Annahmen des starken additiven Charakters der Biopharmazeutika mit steigenden Gesundheitsausgaben auf der einen Seite sowie die Modellierung als Substitut für die Gesamtwirtschaft durch die konstante Endnachfrage auf der anderen Seite gewisse Extremannahmen dar. Diese werden zum Teil dadurch relativiert, dass auch für die zusätzliche Produktion der bisherige Durchschnitt des Exportüberschusses angesetzt wird. Folglich führt nur ein Teil der Biopharmazeutika zu Mehrausgaben im Inland. In den Sensitivitätsrechnungen wird zudem die Bedeutung dieser Annahmen für die Ergebnisse überprüft.

\subsubsection{Effizienz in Forschung und Herstellung}

Neben der Entwicklung verbesserter Therapeutika wurden in der Vergangenheit häufig Hoffnungen in eine ressourceneffizientere F\&E durch die Biotechnologie gesetzt (Hopkins et al. 2007). Der F\&E-Prozess für neue Wirkstoffe in der Pharmazie ist sehr komplex und aufwändig. Er erstreckt sich über die Wirkstoffsuche hin zur präklinischen Phase (Tests der biologischen Wirkung und Sicherheit an Tieren) und den drei klinischen Phasen, bei denen Wirksamkeit, Sicherheit und Nebenwirkungen an kranken und gesunden Menschen überprüft werden. Danach erfolgt die Entscheidung von einer staatlichen Behörde, ob das

\footnotetext{
${ }^{236}$ Allgemein gilt der Zusammenhang zwischen technischem Fortschritt und Gesundheitsausgaben als sehr komplex (Productivity Commission 2005). Selbst wenn in manchen Fällen der technische Fortschritt die Kosten für ein Medikament oder eine Therapie verringert, können die Gesamtkosten bei einer hohen Preiselastizität steigen (Rosenberg-Yunger et al. 2008, S.360). Zudem sind die Rückwirkungen zwischen Gesundheitsausgaben und technischem Fortschritt zu beachten. Eine geringe Zahlungsbereitschaft für neue Therapeutika wird die Anreize für die Unternehmen, in F\&E zu investieren, verringern (OECD 2008e; McKelvey 2008).
} 
Medikament zugelassen wird. ${ }^{237}$ Insgesamt beträgt die Dauer des F\&E-Prozess von der Wirkortsuche bis zur Zulassung ca. 8 bis 12 Jahre und auf eine zugelassene Substanz kommen in der Regel 5.000 bis 10.000 ursprünglich in der Wirkstoffsuche identifizierte Substanzen (OECD 2006c).

Die Effizienz des Forschungsprozesses wird mit der so genannten Forschungsproduktivität, dem F\&E-Aufwand für neue NME, gemessen (Booth/ Zemmel 2004). Eine einfache Berechnung der Forschungsproduktivität erfolgt anhand der Division der in einem Jahr neu zugelassen NMEs durch die gesamten F\&E-Aufwendungen in diesem Zeitraum. ${ }^{238}$ Hier zeigt sich bereits seit den 1970er Jahren, dass die Forschungsproduktivität im Trend abnimmt (Booth/Zemmel 2004; CRA 2004; Suresh/Basu 2008). Die F\&E-Ausgaben haben in der Vergangenheit kontinuierlich stark zugenommen und sich seit Anfang der 1990er Jahre bis zum Jahr 2007 in Europa vervierfacht und in den USA verfünffacht (EFPIA 2008). Allerdings stagniert die Zahl der jährlichen NMEs, seit Ende der 1990er Jahre ist sie sogar weiter gesunken (vgl. Abbildung 27). Zwar ist es bei den stark zunehmenden Forschungsaufwendungen zunächst wenig verwunderlich, dass der Ertrag nicht in gleichem Maße zunimmt, aufgrund von zeitlichen Verzögerungen bis sich der Forschungsaufwand in Erfolge und Erträge niederschlägt (Hopkins et al. 2007; Lipsey et al. 2005; Kapitel 3.2), die abnehmende Tendenz erstreckt sich aber über einen langen Zeitraum. ${ }^{239}$

Eine deutlich aufwändigere Berechnungsmethode besteht in der Auswertung von Unternehmensdaten, bei denen der für die einzelnen Phasen des F\&EProzesses getätigten Aufwand für die Entwicklung eines neuen Wirkstoffes (NME) berechnet wird. Diese werden im Abstand von mehreren Jahren vom universitären „Tufts Center for the Study of Drug Development" durchgeführt. Diese Berechnungen sind allerdings besonders dahingehend umstritten, ob alle berechneten Kosten kausal dem Forschungsaufwand (z.B. Opportunitätskosten) zugeschrieben werden können (Dickson/Gagnon 2004). Dieser Indikator eignet sich aber zumindest für den relativen Vergleich zwischen Biopharmazeutika und „small molecules“. Die durchschnittlichen F\&E-Investitionen je erfolgreicher Zulassung für eine Substanz wird nach dieser Berechnungsmethode für das Jahr 2001 auf ca. 800 Mio. \$ angesetzt, im Jahr 1987 waren es noch rund 300 Mio. \$ (DiMasi et al. 2003). Eine explizite neuere Untersuchung nur für Biopharmazeutika von DiMasi und Grabowski (2007) zeigt etwas höhere Kosten für Bio-

${ }^{237} \mathrm{Zu}$ den F\&E-Ausgaben wird häufig noch die so genannte 4.Phase hinzugezählt, den "PostMarketing" Studien. Bei diesen Studien wird geprüft, ob bei bereits auf dem Markt befindlichen Medikamenten Nebenwirkungen auftreten.

${ }^{238}$ Hopkins et al. (2007) sehen die Aussagekraft dieses Indikators aufgrund möglicher quantitativen Veränderungen in der Struktur der F\&E-Inputs sowie der Beobachtung, dass sich Profite und Umsätze sehr unterschiedlich auf einzelne Therapeutika verteilen, beeinträchtigt.

${ }^{239}$ Ein direkter Vergleich zwischen Biopharmazeutika und ,small molecules“ ist nicht möglich, da lange Zeitreihen für F\&E-Ausgaben speziell für Biopharmazeutika nicht existieren. 
pharmazeutika, bei einer Fortschreibung früherer Kostensteigerungen für „small molecules" stellen sie jedoch geringere Kosten für Biopharmazeutika fest (DiMasi/Grabowski 2007). Ein signifikanter Unterschied zwischen Biopharmazeutika und „small molecules“ lässt sich demnach nicht ableiten.

Die Ableitung von Experteneinschätzungen zu diesem Vergleich und der möglichen zukünftigen Entwicklung ist aufgrund der Vielzahl von Faktoren, die den F\&E-Prozess beeinflussen, sehr komplex. So werden in der Diskussion über den Einfluss der Biotechnologie nicht eindeutige Wirkungszusammenhänge, sondern sich gegenseitig überlagernde Entwicklungen diskutiert. Unterschiede im Forschungsaufwand können sich durch die verschiedenen Aufwendungen für einzelne Moleküle oder in den unterschiedlichen Abbruchsraten für Wirkstoffe im F\&E-Prozess ergeben. Vor allem bei letzterem werden möglichen Vorteile der Biotechnologie diskutiert. Während nur ca. $20 \%$ der chemischen Wirkstoffe, die in die klinischen Phasen kommen, auch die Zulassung erreichen, sind es bei den Biopharmazeutika ca. 30\% (DiMasi/Grabowski 2007, S.472). Die Experten schätzen die Zahlen geringer ein, das relative Verhältnis zueinander aber ähnlich. ${ }^{240}$ So ermöglicht die Kenntnis der molekularen Prozesse im Körper durch biotechnologische Methoden die Medikamentenentwicklung auf Basis von Ursache-Wirkungszusammenhängen. Ein weiterer, aber bislang eher nur theoretischer Vorteil liegt darin, dass bei Biopharmazeutika häufig die Ersetzung von körpereigenen Substanzen stattfindet, wobei geringe Verträglichkeitsprobleme auftreten bzw. zielgenauer die erwünschte Wirkung erreicht wird (DiMasi/ Grabowski 2007). ${ }^{241}$ Deshalb ist die Abbruchsrate besonders in der ersten klinischen Phase, bei der die Verträglichkeit der Substanzen und die Wirkung auf Körperfunktionen (z.B. Herz-Kreislauf, zentrales Nervensystem) im Vordergrund stehen, eher gering. Die befragten Experten erwarten diesen prinzipiellen Vorteil der niedrigeren Abbruchsrate auch für die Zukunft, allerdings in geringerem Ausmaß. So wird vermutet, dass der starke Anstieg an biopharmazeutischen Forschungsprojekten in der prä-klinischen Phase und den klinischen Phasen mit einer zunehmenden Zahl an Wirkstoffkandidaten mit geringerer Erfolgswahrscheinlichkeit einhergeht. Verstärkend könnten die regulatorischen Anforderungen bei der Zulassung und Kostenerstattung zunehmen, da sich mit steigender Anzahl von Therapeutika mit ähnlicher Wirkung der Wettbewerb untereinander verstärkt.

Ein weiterer Vorteil der Biopharmazeutika kann in der Bildung von Subpopulationen in den klinischen Phasen anhand genetischer Informationen (z.B. mit Biomarkern) bestehen, für die eine höhere Wirksamkeit des Therapeutikums

240 Auch in der Literatur weichen die Schätzungen teilweise von den angegeben Werten ab (siehe z.B. Hopkins et al. 2007).

${ }^{241}$ In der Praxis reicht das Wissen über das Gesamtsystem allerdings nicht unbedingt aus und es kann ebenfalls zu Verträglichkeitsproblemen kommen. Ein Beispiel ist die Antikörpertherapie bei Brustkrebs mit Herceptin. Hier können Störungen am Herzmuskel auftreten. 
besteht. In eine ähnliche Richtung gehen Bestrebungen einer stärker individualisierten Arzneimitteltherapie, bei welcher Arzneimittel für unterschiedliche genetische Klassen entwickelt werden (Patel et al. 2008). Der Forschungsaufwand je NME könnte sich dabei durch die Reduzierung von Patientengruppen in klinischen Studien sowie über niedrigere Abbruchsraten durch die Verbesserung der Verträglichkeit und die Fokussierung der Medikamente auf bestimmte Patientengruppen erhöhen. Die Mehrzahl der befragten Experten stuft diesen technologischen Vorteil und dessen Verbreitung als bedeutend ein, einige davon sehen dabei aber nur einen begrenzten Einfluss auf die absolute und/oder relative Forschungsproduktivität, da z.B. die Bildung von Subpopulationen durch Biomarker auch bei „small molecules" eingesetzt werden. ${ }^{242}$ Für eine stärker individualisierte Arzneimitteltherapie müsste sich das regulatorische Umfeld ändern, z.B. bei den Anforderungen an Größen von Patientengruppen in den klinischen Studien. Allgemeiner gesagt können nur bei der Verbindung von technischen, regulatorischen sowie organisatorischen Veränderungen im F\&E-Prozess und der Gesundheitsversorgung die möglichen Potenziale der Biotechnologie ausgeschöpft werden (Kapitel 2.2.3.2; Patel et al. 2008; Amir-Aslani/Negassi 2006; Hopkins et al. 2007; Nightingale/Martin 2004; Tait et al. 2007).

Auf der Gegenseite können vergleichsweise höhere Kosten für Biopharmazeutika dadurch entstehen, dass sie für komplexere Krankheitsbilder entwickelt werden, die aufwändigere klinische Prüfungen erfordern (Gaisser et al. 2005). Dieses Argument wird von vielen befragten Experten als sehr bedeutend angesehen. Zudem können leicht höhere Kosten durch zusätzlich notwendige Immunitätsprüfungen entstehen. ${ }^{243}$

Wie diese verschiedenen Argumente zeigen, ist die zukünftige Entwicklung in Höhe und im Vergleich zur Chemie eher offen. ${ }^{244}$ Zukünftig wird von der deutlichen Mehrheit der Experten bis zum Jahr 2020 eine weitere Zunahme des Forschungsaufwandes für NMEs eingeschätzt. Als Gründe hierfür werden die $\mathrm{Zu}$ nahme der notwendigen Grundlagenforschung ${ }^{245}$ durch die Erforschung immer

${ }^{242}$ Geringe Produktivitätseffekte sehen auch Hopkins et al. (2007). Zudem wird darauf hingewiesen, dass die Technologieanwendung selbst recht kostenintensiv ist.

${ }^{243}$ Bei Biopharmazeutika handelt es sich nicht um eine identische Substanz, wie bei „small molecules", sondern sie werden über die Herstellung spezifiziert. Deshalb sind bei Biopharmazeutika immunologische Reaktionen denkbar.

${ }^{244}$ Es zeigen sich auch kaum Hinweise für einen geringeren Technologiereifegrad der Biotechnologie, der für eine relativ steigende Ressourceneffizienz sprechen würde. Die Experten sehen aufgrund der langen Zeitperiode der Anwendung der Biotechnologie in der Pharmaindustrie kaum einen Entwicklungsrückstand gegenüber der Chemie. Zugleich werden die zukünftigen Herausforderungen für Chemie und Biotechnologie als ähnlich eingestuft.

${ }^{245}$ Offen ist dabei die exakte Arbeitsteilung zwischen Unternehmen und Universitäten für diesen möglichen zusätzlichen Forschungsaufwand. Nach Einschätzungen von Experten werden aber zumindest Teile dieser möglichen Kosten für die Unternehmen anfallen, da sie für das Wissen der Universitäten Lizenzgebühren o.ä. bezahlen werden müssen. 
komplexerer Krankheiten sowie steigenden Kosten für klinische Studien infolge zunehmender regulatorischen Anforderungen gesehen. ${ }^{246}$ Einen signifikanten Unterschied zwischen der Forschungsproduktivität bei Biopharmazeutika und „small molecules“ erwarten die Experten hingegen nicht. Der Effekt der Biopharmazeutika wird nach den Aussagen viel mehr in einem zunehmenden Anteil der Biopharmazeutika bestehen (siehe Kapitel 6.4.1), weniger in einer unterschiedlichen Forschungsproduktivität. ${ }^{247}$ Damit zusammenhängend ist der mögliche qualitative Beitrag der Biopharmazeutika zur Erforschung komplexere Krankheiten zu beachten. Hopkins et al. (2007, S.584) stellen hierzu fest: "Quantitative declines in productivity may hide very real qualitative improvements, as the pharmaceutical industry tackles increasingly difficult diseases."

Zusammenfassend bestehen keine Hinweise für deutliche Unterschiede in der Forschungsproduktivität als auch bei den F\&E-Aufwendungen an den gesamten $\operatorname{Kosten}^{248}$, deshalb wird für die Szenarien eine konstante Entwicklung gegenüber den substituierten „small molecules“ angenommen.

\section{Herstellung von Biopharmazeutika}

Der Anteil der Produktionskosten an den gesamten Ausgaben ist in der Pharmaindustrie vergleichsweise gering, allerdings nicht vernachlässigbar. Schätzungen liegen bei rund 25\% (Reinhard 2001; Basu et al. 2008). Zudem zeigen sich bei der Herstellung und der damit verbundenen Kostenhöhe und -struktur erhebliche Unterschiede zwischen Biopharmazeutika und "small molecules“" ${ }^{249}$ Fast alle Experten bestätigen, dass die Herstellungskosten für Biopharmazeutika deutlich höher sind. Der Herstellungsprozess für Biopharmazeutika ist sehr komplex und anfällig für kleine Veränderungen. Neben diesen direkten technologischen Un-

${ }^{246}$ Dabei wird von einigen Experten allerdings nur eine begrenzte Zunahme des Forschungsaufwandes vermutet, da der Investitions- bzw. Finanzierungsbereitschaft der Akteure Grenzen gesetzt sind (McKelvey 2008). In diesem Falle würde die abnehmende Forschungsproduktivität eher zu einer Verringerung an NMEs führen.

${ }^{247}$ Einige Experten sehen die zukünftige Entwicklung der „small molecules“ optimistischer, aufgrund der möglichen Synergien die zwischen Biotechnologie und „small molecules“ bestehen. Auch in einem solchen Fall würde ein wichtiger Impuls durch die Biotechnologie kommen, wenn auch nicht in Form von Biopharmazeutika. Deshalb wird für die Szenariobetrachtung diese Entwicklungsmöglichkeit nicht explizit berücksichtigt.

${ }^{248}$ Die Forschungsintensität von Biotechnologie-Unternehmen ist aktuell noch deutlich höher. Dies liegt aber daran, dass der Anteil der sich noch in der F\&E-Phase befindenden Produkte in Relation zu dem bereits am Markt kommerzialisierten Produkte besonders hoch ist und die Aufwendungen für Produktion und Vermarktung sowie Umsatzerträge demnach gering sind. Dieses Verhältnis wird sich vorrausichtlich bis zum Jahr 2020 verschoben haben.

${ }^{249}$ Dabei ist zu beachten, dass sich die Herstellungskosten je nach Produkt - auch in der reinen Chemie - deutlich voneinander unterscheiden können. Insbesondere wurde von Experten auf Unterschiede zwischen Krankheitsklassen hingewiesen. Diese Unterschiede konnten nicht explizit untersucht werden, mögliche Verschiebungen wurden aber von den Experten bei den Schätzungen berücksichtigt. 
terschieden beim Herstellungsprozess unterliegen diese auch den verschiedenen regulatorischen Rahmenbedingungen. Unterschiede bestehen insbesondere im sogenannten Arzneimittelrechtlichem Validierungsfenster. So ist der Nachweis, dass der Prozess immer in der gleichen Reinheit stattfindet bei allen Produktionsschritten zu erbringen, während dies bei chemischen Produkten nur für die letzten Bearbeitungsschritte zutrifft. Dies erhöht bspw. die Anforderungen an die Ausstattung und Qualitätskontrollen. Die höheren Herstellungskosten der biopharmazeutischen Produktion sehen die Experten dabei zu einem großen Teil über alle Produktionsfaktoren verteilt an. Im Folgenden werden kurz die Kostenunterschiede näher dargestellt, die Annahmen für die Szenariorechnungen werden im Anschluss erläutert.

\section{Investitionskosten}

Deutliche Mehrkosten werden von den Experten bei den Investitionskosten gesehen. Die benötigte Zeit, bis eine neue Anlage aufgebaut werden kann, wird als ungefähr doppelt so lange eingestuft. Es werden mehr klassifizierte Reinräume für die Produktion und zusätzliche Ausstattungen vor allem für die Aufreinigung (Downstreaming) benötigt.

\section{Kosten für Vorprodukte und Hilfsstoffe}

Veränderungen bei den Material-/Hilfsmittelkosten zeigen sich sowohl in der Höhe als auch in der Struktur. Im Unterschied zu „small molecules" werden bei der Produktion nach Expertenaussagen keine chemischen Vorstufen eingekauft, sondern die komplette Produktion findet ohne chemische Synthesevorstufen statt, da nicht standardisierte, sondern sehr spezifische Zwischenstufen notwendig sind. Als leichtes Indiz hierfür kann auch die Entwicklung der I-OKoeffizienten der Pharmaindustrie in der jüngeren Vergangenheit gesehen werden, die durch eine Zunahme bei der biopharmazeutischen Produktion geprägt war. Im Gegensatz zu den anderen betrachteten Anwendungsfeldern liegen für die Pharmaindustrie eigene I-O-Koeffizienten für Deutschland vor. Die Entwicklung der Vorleistungskoeffizienten zeigt zwischen dem Jahr 2000 und den aktuellsten Tabellen im Jahr 2005 eine deutlich abnehmende Bedeutung von Vorprodukten aus der sonstigen chemischen Industrie (Statistisches Bundesamt 2008c). Allerdings ist bei Biopharmazeutika dafür die Nachfrage nach teureren Hilfsmitteln (z.B. Harze) deutlich höher. Zusätzlich fallen im Gegensatz zu chemischen Produkten erheblich höhere Kosten für die Gefriertrocknung und Kühlung der Produkte an, da die Wirkstoffe aus lebenden Organismen bestehen.

\section{Arbeitskosten}

Einigkeit der Experten besteht auch darin, dass der Arbeitsaufwand bei der biotechnologischen Produktion höher liegt. So wird sowohl ein höherer Anteil an hochqualifiziertem Personal als auch mehr Personal benötigt. Dies betrifft 
zum einen die Qualitätsüberwachung, bei der in der Regel mehr Personen notwendig sind. Zum anderen werden auch im betrieblichen Mittelmanagement mehr Ingenieure und Techniker benötigt.

\subsubsection{Zusammenfassende Darstellung der Szenariowerte}

Für die Beurteilung der Kostensituation für die Szenarien sind mögliche zukünftige Entwicklungen bei der biopharmazeutischen Herstellung zu beachten. Einigkeit bei den Experten besteht darin, dass zukünftig die Herstellungsprozesse weiter optimiert werden können. Fortschrittmöglichkeiten werden bspw. in der Verbesserung von (Hoch-) Expressionssystemen gesehen, indem Reinigungsschritte und Ausbeute erhöht werden können. ${ }^{250}$ Die Meinungen über das mögliche Ausmaß der Optimierungsmöglichkeiten differieren allerdings. Während die eine Hälfte eine deutliche Optimierung erwartet, sieht die andere Hälfte nur einen geringe Optimierungsmöglichkeit, die als „Business as Usual“ betrachtet wird. Eine Begrenzung liegt in den erwähnten regulatorischen Anforderungen, die wenig Spielräume für Veränderungen offen lassen, und für die es nach Aussagen der Experten keine Anzeichen für grundlegende Änderungen gibt. Ähnlicher sind die Expertenaussagen zu der zukünftigen Produktionskostendifferenz zwischen Biopharmazeutika und „small molecules“, da ein Teil der Experten mit sehr optimistischen Aussagen bei Kostensenkungen diesen Vorteil auch in der Chemie sieht.

Insgesamt ist es aufgrund der Unterschiede einzelner (bio)pharmazeutischer Produkte und ihrer eingeschränkten Vergleichbarkeit nur schwer möglich die Kostendifferenzen zu quantifizieren. Um die Folgeeffekte berechnen zu können, müssen allerdings Annahmen getroffen werden, inwiefern der ermittelte Produktionswert für Biopharmazeutika auf Preis- und Mengensteigerungen zurückgeführt werden kann. Der Anteil der Herstellungskosten an den Gesamtkosten bei „small molecules" wird auf Basis von Reinhard (2001) und Basu et al. (2008) auf rund $25 \%$ geschätzt. Für die Herstellungskosten bei Biopharmazeutika wird auf Basis von Expertenaussagen vereinfachend die Annahme getroffen, dass diese für biopharmazeutische Produkte doppelt so hoch wie für „small molecules" sind. Wie der Vergleich des Herstellungsprozesses zeigte, ist sowohl eine Zunahme für die Investitionskosten, den Personalkosten ${ }^{251}$ und den Materialkosten zu erwarten. Zudem findet bei den Materialien eine Verschiebung von Vorleistungen aus der Chemieindustrie hinzu anderen Vorleistungen, vor allem aus der Pharmaindustrie selbst, statt. Bei den Berechnungen wird angenommen,

${ }^{250}$ Fernere zukünftige Möglichkeiten werden bspw. in der Produktion der Biopharmazeutika in transgenen Tieren und Pflanzen gesehen. Eine signifikante Verbreitung dieser Herstellungsmethoden bis 2020 wird von den Experten allerdings nicht erwartet.

251 Bei den Personalkosten für die Produktion wird angenommen, dass diese, analog zum Anteil der Produktionskosten an den Gesamtkosten, 25\% am gesamten Arbeitsentgelt betragen. 
dass sich in beiden Szenarien diese Kostenarten jeweils um einen solchen Prozentsatz zunehmen, dass sich die Herstellungskosten insgesamt um 25 Prozentpunkte erhöhen. ${ }^{252}$ Für die substituierten Pharmaprodukte wird der separat in den I-O-Tabellen ausgewiesene Pharmasektor zugrunde gelegt. ${ }^{253}$ Daraus folgen die in Tabelle 26 aufgelisteten Kostenstrukturen. Die Werte für Biopharmazeutika summieren sich wegen den höheren Kosten auf 125\%. Für die Modellberechnungen werden die I-O-Koeffizienten auf $100 \%$ normiert und die Kostensteigerungen in Konsumsenkungen weitergegeben. Durch die folgende Darstellung werden aber der angenommene Mehrbedarf bei bestimmten primären Inputs (z.B. Arbeit) und Vorleistungen (z.B. sonstige Industrie) deutlich. ${ }^{254}$

Tabelle 26: Input-Output-Struktur für Biopharmazeutika (in \%)

\begin{tabular}{|l|c|c|}
\hline & Biopharmazeutika & Pharma - Substitut \\
\hline Chemievorleistungen & $5,0 \%$ & $10,8 \%$ \\
\hline Vorleistungen sonstige Industrie & $38,1 \%$ & $21,5 \%$ \\
\hline F\&E-Dienstleistungen & $12,7 \%$ & $10,2 \%$ \\
\hline $\begin{array}{l}\text { Vorleistungen sonstige Dienstleis- } \\
\text { tungen }\end{array}$ & $17,1 \%$ & $16,0 \%$ \\
\hline Investitionen & $5,7 \%$ & $3,3 \%$ \\
\hline Arbeitsentgelte & $34,4 \%$ & $26,3 \%$ \\
\hline $\begin{array}{l}\text { Sonstige Wertschöpfung (Nettobe- } \\
\text { triebsüberschuss, Gütersteuern) }\end{array}$ & $11,9 \%$ & $11,9 \%$ \\
\hline Produktionskosten & $125,0 \%$ & $100,0 \%$ \\
\hline Mehrkosten (=> Minderkonsum) & $-25,0 \%$ & $0,0 \%$ \\
\hline Produktionswert & $100,0 \%$ & $100,0 \%$ \\
\hline
\end{tabular}

Quelle: Eigene Berechnungen

\section{Produktions- und Substitutionswerte für die Szenarien}

Für die Diffusion und die inländische Produktion von Biopharmazeutika ergeben sich für das Jahr 2020 die in Tabelle 27 zusammengefassten Annahmen. Nur ein Teil der zusätzlichen biopharmazeutischen Produkte in Höhe von knapp 5 Mrd. $€$ im Referenzszenario und knapp 7,5 Mrd. $€$ substituiert andere pharma-

${ }^{252}$ Diese Kostenberechnung ist für die beiden Szenarien gleich, da es sehr unsicher ist, ob Lern-/Skaleneffekte erreicht werden können (siehe oben).

${ }^{253}$ Analog zu Fein-/Spezialchemikalien ist der Pharmasektor aus den I-O-Tabellen für 2005 zur Beschreibung des Substituts eingeschränkt geeignet, da zu diesem Zeitpunkt die Biotechnologie teilweise im Sektor diffundiert war und in den Durchschnittsvektor des Substituts einfließt. Da aber die Unterschiede durch die Biotechnologie und deren Anteil am gesamten Sektor zu diesem Zeitpunkt gering waren, tritt keine signifikante Verzerrung der Koeffizienten des Substitutes ein.

${ }^{254}$ Die deutliche Zunahme bei der sonstigen Industrie beruht nicht auf den angenommenen Mehrkosten, sondern auch auf der Ersetzung von chemischen Vorleistungen. 
zeutische Produkte (ca. 1 Mrd. $€$ ), der Rest führt zu höheren Ausgaben und damit zu Konsumminderungen an anderer Stelle. ${ }^{255}$ Ein Teil der Konsumeinbußen (23\%) wird durch die Exporte im Ausland wirksam.
Tabelle 27: Szenarienannahmen für Biopharmazeutika im Jahr 2020 (in Mio. €)

\begin{tabular}{|l|c|c|c|c|}
\hline & \multicolumn{2}{|c|}{ Referenzszenario } & \multicolumn{2}{c|}{ HD-Szenario } \\
\hline & $\begin{array}{c}\text { Biopharma- } \\
\text { zeutika }\end{array}$ & $\begin{array}{c}\text { Pharma- } \\
\text { Substitut }\end{array}$ & $\begin{array}{l}\text { Biopharma- } \\
\text { zeutika }\end{array}$ & $\begin{array}{c}\text { Pharma- } \\
\text { Substitut }\end{array}$ \\
\hline $\begin{array}{l}\text { Pharmaproduktion für } \\
\text { Inlandsnachfrage }\end{array}$ & $3618(0 \%)$ & $673(0 \%)$ & $5430(0 \%)$ & $673(0 \%)$ \\
\hline $\begin{array}{l}\text { Pharmaproduktion für } \\
\text { Auslandsnachfrage }\end{array}$ & $1338(0 \%)$ & $249(0 \%)$ & $2008(0 \%)$ & $249(0 \%)$ \\
\hline Konsumveränderung & - & $4034(23 \%)$ & - & $6516(23 \%)$ \\
\hline $\begin{array}{l}\text { Gesamt- } \\
\text { Produktionswert }\end{array}$ & 4956 & 4956 & 7438 & 7438 \\
\hline
\end{tabular}

Quelle: Eigene Berechnungen. Die Werte in Klammern geben den Anteil der Importe an.

\subsubsection{Produktions- und Beschäftigungseffekte}

Zunächst werden in Abbildung 28 die Produktionswirkungen der Diffusion von Biopharmazeutika dargestellt. Bei der Produktion ist der Nettoeffekt positiv mit ca. 2,3 Mrd. $€$ im Referenzszenario und über 3,8 Mrd. $€$ im HD-Szenario. Dieser Effekt ist zu einem bedeutenden Teil auf den Exportüberschuss zurückzuführen. Der positive Effekt zeigt sich dabei ausschließlich in der Pharmaindustrie selbst und entsteht durch den zusätzlichen Nachfrageimpuls, der aus sektoraler Sicht additiv ist. Negative Effekte zeigen sich bei den Dienstleistungen durch die geringeren Konsumausgaben. Für die anderen Sektoren liegen die Nettoproduktionseffekte nahe null. Auch der in der obigen Analyse festgestellte geringere Vorleistungsbedarf aus der Chemieindustrie macht sich in der Nettobetrachtung kaum bemerkbar. Zwar ist der Bedarf in der biopharmazeutischen Produktion niedriger als in der konventionellen Pharmaproduktion, er wird allerdings durch den deutlichen Zuwachs bei Biopharmazeutika wieder ausgeglichen.

${ }^{255}$ Bei der Modellierung der sinkenden Konsumnachfrage wird angenommen, dass die Kostensteigerungen von $25 \%$ über höhere Preise auch wiederum zu einer etwas - gemäß den angenommen Preiselastizitäten - geringeren Nachfrage nach anderen Pharmazeutika führen. Der deutlich größere Anteil wird aber als Einkommensminderung modelliert, bei denen nur die Einkommenselastizitäten bei der Verteilung der Konsumsenkung berücksichtigt wird. Schließlich wären hohe Effekte durch Preiselastizitäten bei diesem stark regulierten Sektor wenig plausibel. 


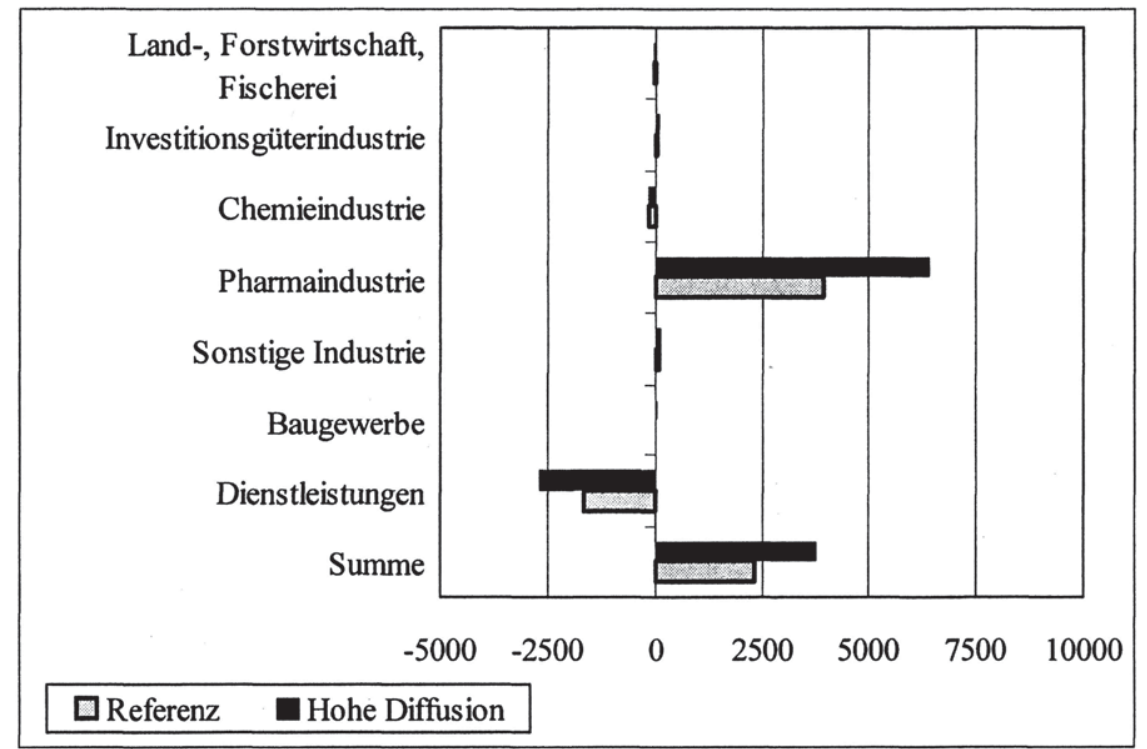

Quelle: Eigene Berechnungen

Die Nettobeschäftigungseffekte der Diffusion von Biopharmazeutika sind hingegen leicht negativ mit knapp minus 11.000 Erwerbstätigen im Referenzszenario und ca. minus 18.000 im HD-Szenario (vgl. Abbildung 29). Der Grund liegt in der deutlich geringeren Arbeitsintensität in der Pharmaindustrie gegenüber den Dienstleistungen. Die negativen Effekte bei den Dienstleistungen überkompensieren die zusätzliche Beschäftigung in der Pharmaindustrie deutlich. Zwar zeichnet sich die Pharmaindustrie im Vergleich zu vielen anderen Industriesektoren durch einen hohen Anteil an Bruttowertschöpfung sowie an Arbeitsentgelten aus, der Arbeitskoeffizient ist allerdings recht gering. Die hohe Entlohnung lässt auf gut bezahltes, hoch qualifiziertes Personal schließen. Für viele Dienstleistungssektoren lässt sich der Umkehrschluss feststellen. Folglich liegt eine Teilerklärung der negativen Beschäftigungsbilanz hierin, dass viele niedrig qualifizierte Jobs zugunsten weniger höher qualifizierter Jobs wegfallen. ${ }^{256}$

${ }^{256}$ Dieser Aspekt wird besonders in einer vereinfachenden Zusatzberechnung deutlich, bei der die durchschnittlichen Anteile von einzelnen Qualifikationsgruppen (kein Abschluss, berufliche Ausbildung, Meister/Techniker oder gleichwertiger Fachschulabschluss, Akademiker) auf Basis des Mikrozensus des Jahres 2004 für die einzelnen I-O-Sektoren unterstellt werden. Nach dieser Berechnung ist die Nettobeschäftigung bei den Akademikern in beiden Szenarien positiv, bei den drei Gruppen mit geringerem Qualifikationsniveau dagegen stark negativ. 


\section{Abbildung 29: Nettobeschäftigungswirkungen durch zusätzliche Diffusion von Biopharmazeutika im Vergleich zum Basisszenario (in Erwerbstätige)}

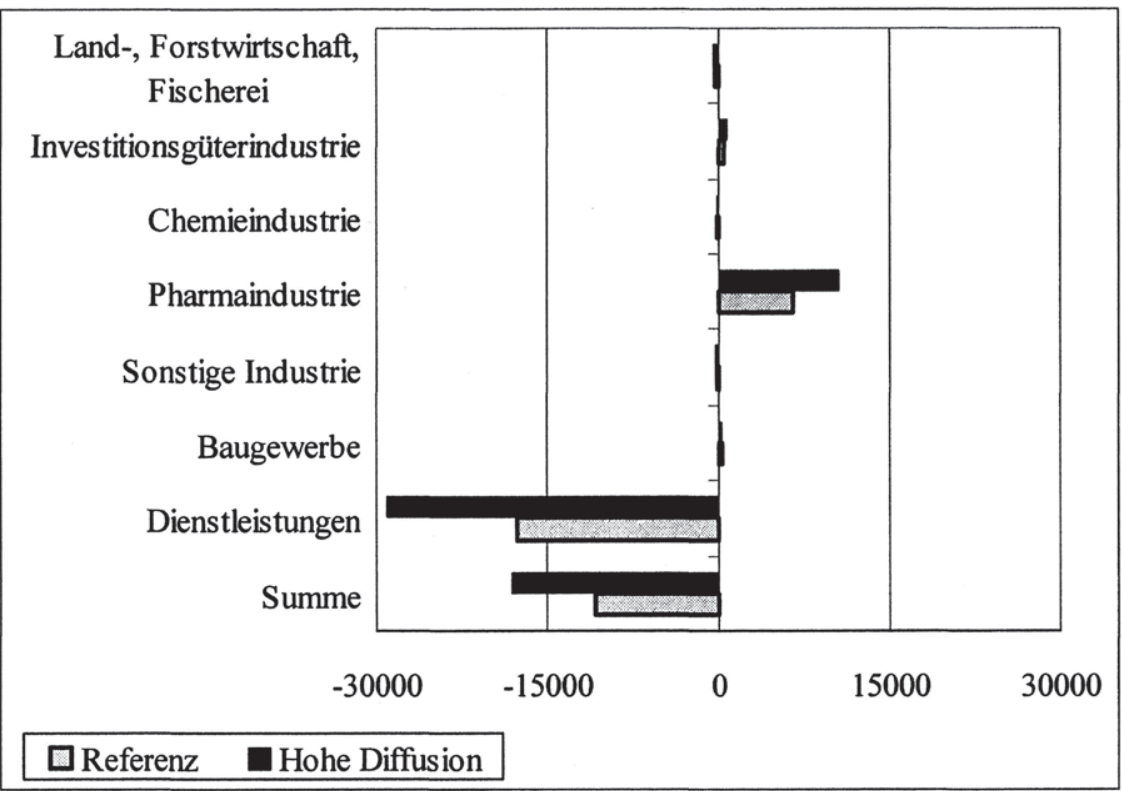

Quelle: Eigene Berechnungen

Vergleiche zwischen dem Referenz- und dem HD-Szenario für Produktion und Beschäftigung zeigen, dass die gesamten Nettoeffekte in der Pharmaindustrie und Dienstleistungen um ca. $40 \%$ höher im HD-Szenario liegen. Dies entspricht ungefähr dem höheren unterstellten Produktionswert, die Effekte nehmen also recht proportional zu. Die sektorale Betrachtung zeigt, dass ein Großteil der Effekte sich auf wenige Bereiche bzw. Sektoren beschränkt (vgl. Anhang Tabelle 48 bis Tabelle 51 ).

\subsubsection{Sensitivitätsanalyse}

Bei diesen Szenarioergebnissen für Biopharmazeutika ist die hohe Unsicherheit bei den Annahmen zu der Wirkungsweise der Produktinnovationen und dem Außenhandelsbilanzergebnis zu beachten. Auch bei Produktinnovationen im

Dieser beschriebene Effekt könnte tatsächlich noch etwas höher ausfallen, da in den Expertengesprächen deutlich wurde, dass die Qualifikationsstruktur bei der biopharmazeutischen Produktion höher ist als in der in dieser Berechnung verwendete Durchschnitt der Pharmaindustrie. 
Pharmasektor ist eine Steigerung der Endnachfrage durch die Befriedigung nicht-gesättigter Nachfrage denkbar. ${ }^{257}$ Der Exportsaldo kann sich, wie in Kapitel 6.4.1 dargestellt, bei diesen Produkten sehr deutlich verändern. Zugleich werden Produktinnovationen und die internationale Wettbewerbsfähigkeit häufig als zentrale Kompensationsmechanismen angesehen (siehe Kapitel 2.3), von denen eine hohe Wirkung auf Produktion und Beschäftigung ausgehen kann. Allerdings werden gesamtwirtschaftlich additiv wirkende Produktinnovationen und eine stärkere Weltmarktposition Deutschland im Referenz- und HDSzenario nicht berücksichtigt. Sie würden einen deutlichen exogenen Eingriff in die Modellberechnungen darstellen, der bei den anderen Fallbeispielen nicht erfolgt.

Stattdessen werden alternative Annahmen im Folgenden gesondert ausgewiesen, aber ausführlicher als die anderen Sensitivitätsrechnungen dargestellt. Dabei wird in einer Rechnung angenommen, dass die gesamten Produktinnovationen additiv wirken und zu einer Erhöhung der Endnachfrage führen. In der anderen Rechnung wird von einer Steigerung der Exporte ausgegangen. Diese Steigerung findet in gleicher Höhe wie die zusätzliche Inlandsnachfrage ca. 4 Mrd. $€$ im Referenzszenario bzw. 6,5 Mrd. $€$ statt. Der Vergleich mit den Szenariorechnungen in Tabelle 28 verdeutlicht die Auswirkungen bei einem gesamtwirtschaftlich additiven Impuls der Produktinnovationen sowie höheren Exporten. Die Nettoproduktionswerte fallen in beiden Alternativrechnungen mehr als doppelt so hoch aus. Z.B. beträgt die zusätzliche inländische Produktion ca. 5,5 Mrd. $€$ bei additiven Produktinnovationen und 5,2 Mrd. $€$ bei höheren Exporten, unter ansonsten gleichen Rahmenbedingungen wie im Referenzszenario. Die Nettobeschäftigungsimpulse werden bei den additiven Produktinnovationen im Gegensatz zu den Szenariorechnungen sogar deutlich positiv (z.B. plus 19.000 im Referenzszenario). ${ }^{258}$ Bei den höheren Exporten bleiben die Beschäftigungseffekte hingegen weiter leicht negativ. Wegen der Konsumminderung im Inland fällt die Beschäftigung bei den Dienstleistungen stark negativ aus (z.B. minus 12.000 im Referenzszenario). Zusammenfassend kann ein Inkraftreten der beiden betrachteten Kompensationsmechanismen die Produktions- und $\mathrm{Be}$ schäftigungseffekte der Biopharmazeutika erheblich verändern.

${ }^{257}$ Ein solcher Fall ist vor allem bei einer gleichzeitigen Einkommenssteigerung in einer Volkswirtschaft vorstellbar.

${ }^{258}$ Verglichen mit der Beschäftigung bei Pharmazeutika von ca. 113.000 Erwerbstätigen im Jahr 2005 (Statistisches Bundesamt 2006) sind die Nettobeschäftigungsverluste beachtlich. Allerdings ist erneut auf die eingeschränkte Vergleichbarkeit dieser gesamtwirtschaftlichen Nettoeffekte mit der direkten, sektoralen Beschäftigung hinzuweisen. 
Tabelle 28: Nettoeffekte für Produktion und Beschäftigung bei alternativen Annahmen für Produktinnovationen und Exporte im Vergleich zum Basisszenario

\begin{tabular}{|l|c|c|c|c|c|c|}
\hline \multirow{2}{*}{$\begin{array}{l}\text { Nettoproduktion } \\
\text { (in Mio. } \boldsymbol{~} \text { ) }\end{array}$} & \multicolumn{2}{|c|}{$\begin{array}{c}\text { Szenariorech- } \\
\text { nung }\end{array}$} & \multicolumn{2}{c|}{$\begin{array}{c}\text { Additive Pro- } \\
\text { duktinnovation }\end{array}$} & \multicolumn{2}{c|}{ Hohe Exporte } \\
\cline { 2 - 7 } & $\begin{array}{c}\text { Refe- } \\
\text { renz }\end{array}$ & HD & $\begin{array}{c}\text { Refe- } \\
\text { renz }\end{array}$ & HD & $\begin{array}{c}\text { Refe- } \\
\text { renz }\end{array}$ & HD \\
\hline Summe & 2310 & 3761 & 5536 & 9091 & 5223 & 8084 \\
\hline Dienstleistungen & -1627 & -2680 & 934 & 1551 & -1118 & -1924 \\
\hline Baugewerbe & 24 & 35 & 72 & 114 & 57 & 84 \\
\hline Sonstige Industrie & 100 & 126 & 509 & 801 & 330 & 468 \\
\hline Pharmaindustrie & 3940 & 6404 & 3948 & 6417 & 5950 & 9386 \\
\hline Chemieindustrie & -161 & -167 & -135 & -124 & -126 & -115 \\
\hline $\begin{array}{l}\text { Investitionsgüterin- } \\
\text { dustrie }\end{array}$ & 58 & 84 & 201 & 319 & 150 & 220 \\
\hline $\begin{array}{l}\text { Land-, Forstwirt- } \\
\text { schaft, Fischerei }\end{array}$ & -24 & -39 & 7 & 12 & -20 & -34 \\
\hline $\begin{array}{l}\text { Nettobeschäftigung } \\
\text { (in Erwerbstätige) }\end{array}$ & $\begin{array}{c}\text { Refe- } \\
\text { renz }\end{array}$ & HD & $\begin{array}{c}\text { Refe- } \\
\text { renz }\end{array}$ & HD & $\begin{array}{c}\text { Refe- } \\
\text { renz }\end{array}$ & HD \\
\hline Summe & -10847 & -18156 & 19202 & 31503 & -812 & -3262 \\
\hline Dienstleistungen & -17604 & -28999 & 9139 & 15196 & -12605 & -21581 \\
\hline Baugewerbe & 266 & 387 & 797 & 1264 & 631 & 929 \\
\hline Sonstige Industrie & -161 & -374 & 1766 & 2811 & 670 & 859 \\
\hline Pharmaindustrie & 6575 & 10681 & 6588 & 10704 & 9912 & 15633 \\
\hline Chemieindustrie & -141 & -146 & -118 & -108 & -110 & -101 \\
\hline $\begin{array}{l}\text { Investitionsgüterin- } \\
\text { dustrie }\end{array}$ & 472 & 720 & 955 & 1518 & 911 & 1371 \\
\hline $\begin{array}{l}\text { Land-, Forstwirt- } \\
\text { schaft, Fischerei }\end{array}$ & -255 & -425 & 74 & 117 & -220 & -373 \\
\hline
\end{tabular}

Quelle: Eigene Berechnungen

\section{Weitere Sensitivitätsrechnungen}

Neben diesen detaillierten Zusatzberechnungen werden analog $\mathrm{zu}$ den anderen Fallbeispielen Sensitivitätsrechnungen für die Diffusion, Inlandsproduktion und Konsumstruktur durchgeführt. Die aus der Literatur und Expertenmeinungen abgeleiteten Sensitivitätswerte sind in Tabelle 29 zusammengefasst.

Die Sensitivitätsrechnungen in Abbildung 30 und Abbildung 31 zeigen, dass die Ergebnisse für die Produktions- und Beschäftigungseffekte in der Höhe erheblich von einzelnen Parametern abhängen. Allerdings bleiben für alle Werte die Nettoproduktionswirkungen positiv, während die Nettobeschäftigungseffekte 
hingegen stets negativ sind. Es zeigen sich dabei einige Unterschiede bei der Wirkungsrichtung der Variablen. Eine höhere Diffusion führt zu zunehmend positiven Produktionseffekten, bei gleichzeitig immer negativeren Beschäftigungseffekten. Diese Beobachtung stimmt mit den obigen beiden Szenarien überein. Bei den Beschäftigungseffekten dominieren die negativen Impulse des Konsumausfalls nach arbeitsintensiven Dienstleistungen, bei den Produktionseffekten hingegen die zusätzliche Produktion (bio-)pharmazeutischer Produkte. Dagegen steigen bei zunehmender inländischen Produktion durch Exportüberschüsse die Nettoproduktion und -beschäftigung. Die Effekte der höheren inländische Produktion sind dabei stark positiv, da es hier annahmegemäß zu zusätzlichen Mehrausgaben und folglich zu einer Konsumminderung im Ausland kommt, die keine Rückwirkungen auf das Inland haben. Auffällig ist zudem, dass bei einer ausgeglichenen Handelsbilanz, welche durch die untere Bandbreite widergespiegelt wird, die Nettoproduktionswirkungen nur bei 500 Mio. $€$ liegen. Der im Referenzszenario festgestellte Nettoproduktionsüberschuss ist folglich fast allein auf die Exportannahme zurückzuführen.

Moderaten Einfluss auf die Ergebnisse hat der gewählte Konsumvektor. Der alternative, preisunelastische Konsumvektor führt zu einer etwas geringeren inländischen Produktion, aber vor allem zu einer geringeren Beschäftigung. Bei dieser Konsumalternative sinkt die Nachfrage nach Dienstleistungen deutlich stärker als beim Konsumvektor des Referenzszenarios. Deutliche Abweichungen der sektoralen Effekte zeigen sich in den Sensitivitätsrechnungen zum Referenzszenario nur insofern, dass die Produktions- und Beschäftigungswirkungen in der Pharmaindustrie und bei Dienstleistungen sehr stark auf Parameterveränderungen reagieren.

Tabelle 29: Sensitivitätsannahmen für die Biopharmazeutika

\begin{tabular}{|l|c|c|c|l|}
\hline Variable & Referenz & Niedrig & Hoch & Quelle/Anmerkung \\
\hline Diffusion in \% & $24 \%$ & $16 \%$ & $40 \%$ & $\begin{array}{l}\text { Bandbreiten aus Nus- } \\
\text { ser et al. (2007a) }\end{array}$ \\
\hline $\begin{array}{l}\text { Inlandsproduktion (in } \\
\text { Mio. } € \text { ) }\end{array}$ & 4956 & 3860 & 6810 & $\begin{array}{l}\text { Niedrig: Annahme } \\
\text { keiner Exportüber- } \\
\text { schüsse; } \\
\text { Hoch: Annahme der } \\
\text { Verdoppelung der Ex- } \\
\text { portüberschüsse }\end{array}$ \\
\hline Konsumstruktur & $\begin{array}{l}\text { Konsumstruktur mit preisunelastischem Nachfragever- } \\
\text { halten und Durchschnittsvektor der I-O-Tabelle 2005 }\end{array}$ \\
\hline
\end{tabular}

Quelle: Eigene Berechnungen 
Abbildung 30: Nettoproduktionswirkungen für Biopharmazeutika in Sensitivitätsrechnungen (in Mio. $€$ )

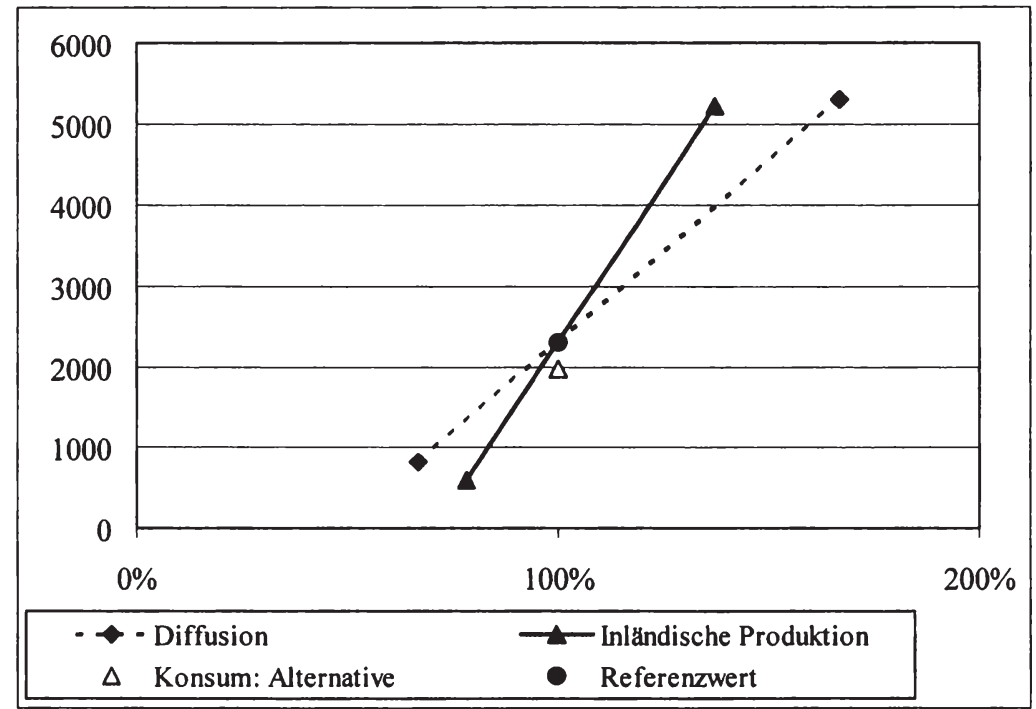

Quelle: Eigene Berechnungen

Abbildung 31: Nettobeschäftigungswirkungen für Biopharmazeutika in Sensitivitätsrechnungen (in Erwerbstätige)

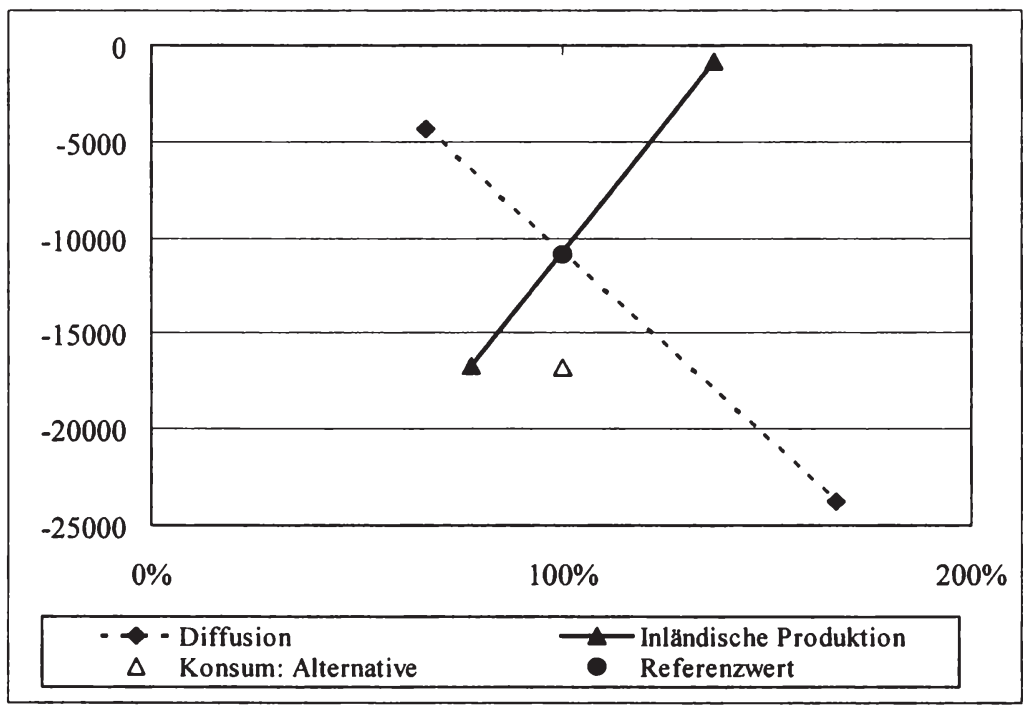

Quelle: Eigene Berechnungen 


\subsection{Zusammenfassende Ergebnisse und Diskussion der Modellberechnun- gen}

Im Folgenden werden die Ergebnisse zusammengefasst und diskutiert. Tabelle 30 gibt zunächst eine Kurzübersicht über die die Kernergebnisse.

\section{Tabelle 30: Zusammenfassung der Ergebnisse und Wirkungsmechanismen in den Fallbeispielen}

\begin{tabular}{|c|c|c|c|c|}
\hline & Ethanol & Biopolymere & $\begin{array}{l}\text { Fein-/Spezial- } \\
\text { chemikalien }\end{array}$ & $\begin{array}{c}\text { Biopharma- } \\
\text { zeutika }\end{array}$ \\
\hline 芯 & $\begin{array}{l}\text { positive Produk- } \\
\text { tions- und Be- } \\
\text { schäftigungs- } \\
\text { effekte }\end{array}$ & $\begin{array}{l}\text { zumeist positive } \\
\text { Produktions- } \\
\text { und Beschäfti- } \\
\text { gungseffekte }\end{array}$ & $\begin{array}{l}\text { negative Produk- } \\
\text { tions- und Be- } \\
\text { schäftigungs- } \\
\text { effekte }\end{array}$ & $\begin{array}{l}\text { positive Produk- } \\
\text { tionseffekte; } \\
\text { negative* Be- } \\
\text { schäftigungseffe } \\
\text { kte }\end{array}$ \\
\hline 总 & $\begin{array}{l}\text { Strukturelle Ef- } \\
\text { fekte durch Roh- } \\
\text { stoffwechsel; } \\
\text { leichter Rück- } \\
\text { gang Konsum- } \\
\text { nachfrage; } \\
\text { keine direkten } \\
\text { Freisetzungsef- } \\
\text { fekte }\end{array}$ & $\begin{array}{l}\text { Strukturelle Ef- } \\
\text { fekte durch Roh- } \\
\text { stoffwechsel; } \\
\text { zusätzliche Kon- } \\
\text { sum-nachfrage } \\
\text { durch Preissen- } \\
\text { kung; } \\
\text { keine direkten } \\
\text { Freisetzungsef- } \\
\text { fekte; }\end{array}$ & $\begin{array}{l}\text { Zusätzliche } \\
\text { Konsumnachfra- } \\
\text { ge durch Preis- } \\
\text { senkung; } \\
\text { geringe direkte } \\
\text { Freisetzungsef- } \\
\text { fekte }\end{array}$ & $\begin{array}{l}\text { Rückgang Kon- } \\
\text { sum-nachfrage } \\
\text { da höhere } \\
\text { Gesundheitskos- } \\
\text { ten; } \\
\text { sehr positive di- } \\
\text { rekte Effekte } \\
\text { durch Produkt- } \\
\text { innovation }\end{array}$ \\
\hline 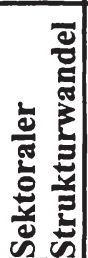 & $\begin{array}{l}\text { starke Zunahme } \\
\text { Landwirtschaft; } \\
\text { Industrie- und } \\
\text { Dienstleistungs- } \\
\text { sektoren eher } \\
\text { gleichbleibend }\end{array}$ & $\begin{array}{l}\text { starke Zunahme } \\
\text { Landwirtschaft; } \\
\text { leichte Zunah- } \\
\text { men im Bauge- } \\
\text { werbe und } \\
\text { Dienstleistungen }\end{array}$ & $\begin{array}{l}\text { starker Rück- } \\
\text { gang Chemiein- } \\
\text { dustrie; } \\
\text { leichte Zunah- } \\
\text { men in Land- } \\
\text { wirtschaft }\end{array}$ & $\begin{array}{l}\text { starke Zunah- } \\
\text { men in Pharma- } \\
\text { industrie; } \\
\text { starker Rück- } \\
\text { gang bei Dienst- } \\
\text { leistungen }\end{array}$ \\
\hline
\end{tabular}

Quelle: Eigene Darstellung

*bei Annahme additiver Produktinnovationen sind die Beschäftigungseffekte positiv

\section{Einordnung der Ergebnisse}

Insgesamt zeigen sich für die Anwendungsfelder jeweils unterschiedliche Nettoeffekte für Produktion und Beschäftigung. Positive Effekte ergeben sich bei Bioethanol und Biopolymeren, aufgrund der höheren inländischen Wertschöpfung bei der Verwendung von nachwachsenden anstatt fossilen Rohstoffen. Bei 
Fein-/Spezialchemikalien sind die Effekte eher negativ, hier können die direkten Freisetzungswirkungen nicht durch andere Effekte kompensiert werden. Bei Biopharmazeutika ergeben sich keine eindeutigen Ergebnisse; sie sind stark von der Modellierung der Produktinnovationen (substitutiv vs. additiv) abhängig. Zugleich zeigt sich hier die größte Differenz zwischen den Produktions- und Beschäftigungsimpulsen durch große Unterschiede der relevanten Sektoren bei den Arbeitsintensitäten.

Die Beurteilung der Größenordnung dieser Effekte ist aufgrund des Fehlens geeigneter Vergleichsmaßstäbe schwierig. ${ }^{259}$ Insgesamt lässt sich folgern, dass in diesen wichtigen Anwendungsfeldern eher wenig Anzeichen für immense Nettoimpulse der Biotechnologie auf Produktion und Beschäftigung deutlich werden. Diese Erkenntnis ist insofern wenig überraschend, als dass gegenläufige Effekte stattfinden und auch für andere Technologien (z.B. Mikroelektronik) in der Literatur wenig eindeutige Effekte festgestellt werden konnten (z.B. Kalmbach et al. 1989; Edler 1990). Zudem liegen, abgesehen von Biopharmazeutika, die Diffusionserwartungen bis zum Jahr 2020 und damit die Höhe der Impulse eher im niedrigen Prozentbereich, gemessen an der jeweiligen Größe des betrachteten Sektors. Nach Aussagen der Experten ist eine weitere Steigerung der Diffusion über diesen Zeithorizont hinaus denkbar.

Generell sind die jeweiligen Nettoproduktions- und -beschäftigungseffekte aufgrund des größeren Volumens im HD-Szenario in fast allen Fällen stärker ausgeprägt als im Referenzszenario. Die Effekte sind zwar nicht unbedingt proportional zum Diffusionsvolumen, aber meist nicht sehr weit davon entfernt. ${ }^{260}$ Für einzelne Sektoren fallen die Unterschiede teilweise etwas stärker aus. Dies ergibt sich meist aus der etwas differierenden Kostensituation aufgrund der verschiedenen Rohstoffpreise und Lerneffekten im HD-Szenario. Daraus resultieren Unterschiede bei den Konsumimpulsen.

\footnotetext{
${ }^{259}$ Wenn man die Nettoeffekte vereinfachend mit der aktuellen direkten Beschäftigung in den Anwendersektoren vergleicht, erscheinen die Nettoeffekte bei Biopolymeren und Fein/Spezialchemikalien moderat, für Biopharmazeutika und Bioethanol als eher hoch. Allerdings führt dieser Vergleich zu einer Überschätzung der Bedeutung der Nettoeffekte, da die restliche Wertschöpfungskette beim Vergleichswert nicht betrachtet wird.

${ }^{260}$ Eine größere Bandbreite der zukünftigen Entwicklungspfade hätte es vermutlich bei stärker ausdifferenzierteren Szenarien (z.B. Variation von politischen Rahmenbedingungen) ergeben. Diese lassen sich aber kaum konsistent in Expertengespräche abbilden, noch lassen die technologischen Informationen aus der Literatur eine solche Zuordnung zu einzelnen Szenarien zu. Bspw. sind die Bandbreiten eher den unsicheren technologischen Entwicklungen zuzuordnen als zurechenbaren Szenariofaktoren.
} 


\section{Robustheit der Ergebnisse und zentrale Einflussfaktoren}

Um die Unsicherheit der Ausprägung zentraler Parameter und ihren Auswirkungen abzubilden, werden umfangreiche Sensitivitätsrechnungen durchgeführt. ${ }^{261}$ Die Sensitivitätsrechnungen hinsichtlich Diffusion, Inlandsproduktion und einigen anwendungsspezifischen Variablen (z.B. Konkurrenzfläche in der Landwirtschaft) zeigen eine starke Abhängigkeit der Ergebnisse von der gewählten Ausprägung der Variablen (z.B. Außenhandelsbilanz). Das Vorzeichen für die Nettoproduktion und -beschäftigung ändert sich allerdings nur in vereinzelten Fällen (siehe z.B. Fein-/Spezialchemikalien). Für die Einflussfaktoren allgemein kann aus den Szenario- und Sensitivitätsergebnissen gefolgert werden, dass besonders der Außenhandel die Ergebnisse zentral beeinflusst. Schließlich ändert sich dadurch die Höhe der inländischen Produktion von biotechnologischen Gütern. Für die Szenarioberechnungen reichten die Hinweise aber nicht aus, Annahmen für den Außenhandel zu rechtfertigten, die deutlich von den Substitutgütern abweichen. Folglich kommt der mögliche Wirkungsmechanismus der Steigerung der internationalen Wettbewerbsfähigkeit nicht in den Szenariomodellierungen, sondern nur in den Sensitivitätsrechnungen zum Tragen. Allerdings können sich gerade bei der Einführung neuer Technologien durchaus gröBere Verschiebungen im internationalen Gütermarkt ergeben. Diese hätten einen zentralen Einfluss auf die Ergebnisse.

Die Technologiespezifizierung (z.B. Kostenstruktur) und die Annahmen zur Konsumstruktur haben hingegen nur einen moderaten Effekt. Schließlich bleibt die Höhe der Nachfrage nach den biotechnologischen Produkten jeweils gleich; es wird nur die sektorale Struktur der Produktion beeinflusst. Die Bedeutung für den Gesamteffekt ist bei solchen sektoralen Verschiebungen eher gering.

\section{Bedeutung direkter und indirekter Effekte}

In allen betrachteten Anwendungsfeldern sind die direkten Effekte durch den Einsatz der Biotechnologie gering. Lediglich bei Fein-/Spezialchemikalien lassen sich im Rahmen von Effizienzsteigerungen, z.B. durch Reduktion von Prozessschritten, eine Einsparung von Arbeitskräften erzielen. In der Produktion von Ethanol und Biopharmazeutika deutet sich hingegen sogar eine etwas höhere Arbeitsintensität an. Im Anwendungsfeld Biopharmazeutika ergeben sich etwas verstärkende direkte wirtschaftliche Veränderungen durch die aus sektoraler Sicht additive Wirkung der Produktinnovationen. Diese höhere Bedeutung der direkten Effekte liegt in einer Linie mit der allgemeinen Einschätzung, dass die Biotechnologie in der Pharmaindustrie ihren größten Einfluss hat und voraussichtlich auch zukünftig haben wird. Dabei ist allerdings die hohe Bedeutung der getroffenen Annahme, dass keine stärkeren Politikmaßnahmen zur Dämpfung

${ }^{261}$ Dabei ist zu beachten, dass durch die Variation nur einzelner Parameter keine Berechnungen für komplett andere Annahmenbündel durchgeführt wurden, z.B. eine Entwicklung die zu hohen Herstellungskosten, geringer Diffusion und schlechter Außenhandelsbilanz führt. 
bei Arzneimittelausgaben eingeführt werden, zu beachten. Falls eine Finanzierung auf der Nachfrageseite nicht erfolgt, bleibt die Wirkung der Produktinnovationen und damit das Wachstum des Sektors aus.

Insgesamt werden die vorliegenden Ergebnisse aber insbesondere von den indirekten Wirkungen bestimmt. In den Feldern Bioethanol und Biopolymeren kommt der Veränderung der Rohstoffbasis eine wichtige Rolle zu. Die Ergebnisse hängen dabei zentral von der Modellierung der Folgewirkungen im Landwirtschaftssektor ab. Für die Berechnungen in den Szenarien wurde angenommen, dass teilweise eine Ersetzung bisheriger landwirtschaftlicher Aktivitäten erfolgt. Allerdings fällt die Auswirkung dieser Restriktion des inländischen Angebots in den Sensitivitätsrechnungen gering aus. In den Anwendungsfeldern Biopharmazeutika und Fein-/Spezialchemikalien werden die Nettoergebnisse stärker von den positiven oder negativen Konsumnachfrageimpulsen beeinflusst. Sie nimmt in den Szenarien eine hohe Bedeutung ein, da sie zum absoluten Großteil im Inland und annahmegemäß im vollen Umfang der Kostenbelastungen/-ersparnisse stattfindet. Zudem unterscheidet sich die Produktionsstruktur für die Güter der Konsumnachfrage sehr erheblich von den in den Anwendungsfeldern betrachteten Produktionsprozessen. Die Ergebnisse bestätigen damit die zentrale Bedeutung der Reaktion der Nachfrage für die gesamtwirtschaftliche Bedeutung neuer Technologien.

Im Zusammenhang mit der Bedeutung dieser indirekten Effekte zeigen sich in den Fallbeispielen interessante strukturelle Effekte. So tritt ein bedeutender Teil der Nettoimpulse im Landwirtschaftssektor und im Dienstleistungssektor auf. Die Gründe hierfür liegen ebenfalls im Wechsel auf die nachwachsende Rohstoffbasis sowie der starken Veränderung der Dienstleistungsnachfrage bei Konsumsteigerungen/-senkungen. Folglich findet zwar der direkte Einsatz der Biotechnologie zum Großteil in der Industrie statt, die Folgewirkungen können sich aber stärker im primären und tertiären Sektor zeigen. Insgesamt sind die strukturellen Veränderungen in den Fallstudien verglichen mit den Nettoeffekten bei der jeweiligen Gesamtproduktion und -beschäftigung recht groß. Dabei konzentrieren sich diese strukturellen Veränderungen jeweils auf wenige Sektoren. Schließlich beschränken sich auch die Unterschiede zwischen den Wertschöpfungsprozessen der Biotechnologie und den substituierten Wertschöpfungsprozessen zumeist auf wenige I-O-Koeffizienten.

Eine sich aus diesen Aspekten zum Teil bereits beantwortete, aber dennoch andersgelagerte Frage, ist die Bedeutung der Biotechnologie selbst für die Ergebnisse. ${ }^{262}$ Die Biotechnologie wird im Kontext des Einsatzes von nachwachsenden Rohstoffen häufig eher als eine Umsetzungsmöglichkeit angesehen, als dass sie solch große wirtschaftliche Vorteile ermöglicht, die eine Rohstoffsubsti-

${ }^{262}$ Diese Bedeutung der Biotechnologie kann sich schließlich direkter, als auch indirekter Natur (z.B. über Kosten) ergeben. 
tution erzwingt. Rose und McNiven stellen fest: "[...] particularly in the industrial sector, biotechnology may only be a tool in a more fundamental process consisting of progressively substituting many existing petroleum based applications with applications based on new and different use of biomass, a renewable resource" (Rose/McNiven 2007, S.214). Die Diffusion wird stark politisch gesteuert und die wirtschaftlichen Effekte von den Kosten und Verfügbarkeit der Rohstoffe dominiert.

Die Unterschiede in den Konversionsverfahren (Biotechnologie vs. Chemie) sind eher von untergeordneter Bedeutung für die wirtschaftlichen Auswirkungen. Diese Feststellung kann sich allerdings in Zukunft ändern, wie die Betrachtung von Lignocellulose als Ausgangsbasis für Ethanol gezeigt hat. Die Kostenwettbewerbsfähigkeit hängt hier deutlich stärker von Fortschritten in Prozessverfahren ab, die Abhängigkeit von Rohstoffkosten und potenziellen Problemen bei der Verfügbarkeit wären deutlich geringer. Bei den beiden Anwendungsfeldern Biopharmazeutika und Fein-/Spezialchemikalien ist die Bedeutung der Biotechnologie für die berechneten Effekte bereits jetzt deutlich höher einzuschätzen. Hier führt der Einsatz biotechnologischer Methoden stärker zu veränderten Eigenschaften der Produkte sowie bei Fein-/Spezialchemikalien häufig $\mathrm{zu}$ einer Produktionsvereinfachung. Fortschritte im technologischen Wissen selbst spielen hier eine zentrale Rolle für die Diffusion der Biotechnologie und deren Auswirkungen.

\section{Kritische Würdigung der Modellrechnungen}

Modellrechnungen über Effekte neuer Technologien in der Zukunft sind von Unsicherheiten und Vereinfachungen gekennzeichnet. Die möglichen Auswirkungen hiervon auf die Ergebnisse der Modellierungen werden im Folgenden kurz diskutiert. Die Einordnung der empirischen Ergebnisse in die Theorie und die Ableitung des Forschungsbedarfs erfolgen in Kapitel 7.

In der empirischen Untersuchung ist die Eingrenzung auf einige wenige Anwendungsfelder notwendig, da die Datenbasis für die Biotechnologie sehr eingeschränkt ist. Die Betrachtung von Fallbeispielen limitiert selbstverständlich die möglichen Aussagen zu den gesamtwirtschaftlichen Effekten der Biotechnologie. Dies betrifft besonders die gesamte Höhe der Effekte. Mögliche Unterschiede in der Struktur sowie Art und Weise der wirtschaftlichen Effekte der Biotechnologie in einzelnen Anwendungsbereichen werden dagegen über die Auswahl von recht unterschiedlichen Anwendungsfeldern herausgearbeitet. Viele der hier nicht betrachteten potenziellen Anwendungsfelder bis zum Jahr 2020 lassen sich tendenziell hinsichtlich der Art ihrer Effekte den untersuchten Anwendungsfeldern zuordnen:

- Die Wirkungen der biotechnologischen Herstellung anderer Bulkchemikalien auf Basis von nachwachsenden Rohstoffen sind vermutlich ähnlich wie bei Biopolymeren. Der Anteil von nachwachsenden Rohstoffen ist in 
vielen Fällen als ähnlich oder etwas höher einzustufen (Patel et al. 2006; Expertenaussagen), zugleich ist die Kostenwettbewerbsfähigkeit für die Diffusion notwendig, da bisher keine direkte finanzielle Subvention (z.B. durch Steuererleichterungen) für diese Produkte erfolgt.

- Der Einsatz der Biotechnologie in der Pflanzenzüchtung hat einen ähnlichen Effekt wie bei Fein-/Spezialchemikalien, da hauptsächlich Veränderungen in der Produktivität (Das 2007) und den Produkteigenschaften zu erwarten ist. Dies trifft in geringerer Intensität auch auf industrielle Anwendungen im Textil-, Papier-, Lederbereich zu (Nusser et al. 2007d).

- Bestimmte medizinische Anwendungen (z.B. Diagnostik) sind in ihrem Effekt dem Bereich der Biopharmazeutika zuzuordnen. Sie ermöglichen neue Behandlungsalternativen. Zugleich ist eine Finanzierung der zusätzlichen Gesundheitsausgaben notwendig.

Schwieriger ist eine Aussage über einige in der Diskussion befindlichen sehr neuartigen Anwendungen der Biotechnologie, wie z.B. der Stammzellentherapie im Bereich Gesundheit, zu treffen. Diese konnten im Rahmen der Arbeit nicht untersucht werden. $\mathrm{Zu}$ unsicher sind zukünftige Entwicklungen sowie mögliche Implikationen auf die Wirtschaftsstrukturen (Wertschöpfungskette, indirekte Wirkungen auf andere Sektoren, Substitution anderer Produkte, etc.). Auch für die Umweltbiotechnologie fällt ein Vergleich zu anderen Anwendungsfeldern schwer, diesem Feld wird aber eine geringere Bedeutung zugesprochen (Reiss et al. 2006).

Daneben ergeben sich Einschränkungen bei den erfassten Wirkungszusammenhängen (Kompensationseffekten). In Kapitel 4.3.4 wurden bereits ausführlich die Limitationen diskutiert, die mit einem I-O-Modellansatz verbunden sind. Insgesamt ist eine ausführlichere Bestimmung der Folgen für die Agrarmärkte, der inländischen Nachfrage und dem Außenhandel sowie deren möglichen Rückwirkungen auf die Diffusion und Entwicklung der Biotechnologie als wünschenswert anzusehen. ${ }^{263}$ Jeder einzelne Aspekt hätte für eine genauere Untersuchung eine sehr aufwändige Modelllösung und teilweise kaum zu deckenden Datenbedarf benötigt. So fehlen besonders für eine stärkere Abbildung der technologischen Entwicklung (z.B. Diffusionsmodell, Bestimmung von Lerneffekten) verfügbare Daten, die eine halbwegs plausible Bestimmung möglich gemacht hätten.

${ }^{263}$ Bereits in Kapitel 3.3 wird eine geringere Bedeutung der fehlenden Endogenisierung der Investitionen festgestellt. Hier ergaben sich auch in den Fallbeispielen wenige Hinweise für einen grundlegend anderen Effekt. Besonders in den Bereichen Biopolymere und Fein/Spezialchemikalien wird von vielen Experten betont, dass Neuinvestitionen für die Einsetzung biotechnologischer Verfahren erhebliche Konkurrenznachteile gegenüber bereits bestehenden und abgeschriebenen Chemie-Anlagen haben. Stattdessen werden die Chancen der Umstellung auf biotechnologische Verfahren eher bei der Tätigung von Ersatzinvestitionen gesehen. 
$\mathrm{Zu}$ beachten ist hierbei, dass die Schwierigkeit der Abbildung bestimmter Wirkungsmechanismen, die Ergebnisse der einzelnen Anwendungsfelder unterschiedlich beeinflussen können. ${ }^{264}$ Besonders die Erfassung der Wirkungen von neuen oder qualitativ deutlich verbesserten Produkten ist nur eingeschränkt möglich. So werden bei Biopharmazeutika und in einer Sensitivitätsrechnung für Fein-/Spezialchemikalien die Produktionssteigerungen im Zuge neuer Innovationen durch einen geringeren Konsum nach anderen Produkten ausgeglichen. Die alternative Rechnung bei Biopharmazeutika mit einer gesamtwirtschaftlich vollständig additiven Wirkung der Produktinnovationen verdeutlicht, wie zentral die Ergebnisse von der Modellierungsweise von Produktinnovationen abhängen. Folglich ist es denkbar, dass bei Biopharmazeutika und Fein-/Spezialchemikalien die Produktions- und Beschäftigungswirkungen in den Szenariorechnungen unterschätzt werden.

\footnotetext{
${ }^{264}$ Daneben sind gerade für das Anwendungsfeld Biopharmazeutika die Szenarioannahmen vermutlich am kritischsten zu sehen. So wird selbst bei dem als Vergleich für die Nettoeffekte dienenden Basisszenario, ohne eine weitere Diffusion der Biotechnologie von einer Steigerung der Produktivität und des Wachstums ausgegangen. Aus technologischer Sicht lässt sich diese Annahme in Frage stellen. Zumindest der Ressourcenaufwand je neu zugelassenes Produkt wird ohne die Fortentwicklung biotechnologischer Methoden vermutlich weiter zunehmen. Folglich sind die Annahmen für das Basisszenario recht optimistisch. Eine Festlegung alternativer Werte für das Jahr 2020 konnte im Rahmen dieser Arbeit nicht erfolgen. Weder sind in der Literatur Hinweise für alternative Werte zu finden noch war es in den Expertengesprächen möglich, einen solchen hypothetischen Fall mit quantitativem Ergebnis zu diskutieren, da einstimmig von einer höheren Diffusion ausgegangen wird.
} 


\section{Schlussbemerkung}

\section{Ausgangsfrage}

Die Biotechnologie wird häufig als eine sehr bedeutende Technologie des 21.Jahrhunderts angesehen, die ein hohes Potenzial für die Entwicklung neuer oder verbesserter Prozesse, Produkte und Dienstleistungen hat. Sie kommt dabei in vielen verschiedenen Sektoren (u.a. Landwirtschaft, Lebensmittel, Pharmazie, Umwelttechnik, Chemie) zum Einsatz. In einer Vielzahl von Ländern werden deshalb erhebliche öffentliche und private Forschungsinvestitionen in die Biotechnologie getätigt, in der Erwartung neben positiven Effekten für Gesundheit und Umwelt auch wirtschaftliche Erfolge zu erzielen. Allerdings sind Impulse neuer Technologien auf gesamtwirtschaftliche Größen, wie Produktion und Beschäftigung, nicht eindeutig, sondern es entstehen gegenläufige Effekte. Dabei können erhebliche Unterschiede zwischen einzelnen Technologien eintreten, da sie sich in ihren Charakteristika und Rahmenbedingungen unterscheiden, welche für die Auswirkungen von hoher Bedeutung sind. Fundierte Aussagen über aktuelle und mögliche zukünftige wirtschaftliche Folgewirkungen der Biotechnologie sind deshalb nur durch eine spezifische empirische Analyse möglich. In bisherigen Untersuchungen zur wirtschaftlichen Bedeutung der Biotechnologie standen aber meist entweder nur die alleinige Betrachtung so genannter Kernbiotechnologieunternehmen - die zu einem Großteil nur Anbieter biotechnologischer Verfahren sind - oder Bruttogrößen (z.B. Produktionsanteil) in den Anwenderindustrien im Fokus. Wirtschaftliche Veränderungen, die durch eine Diffusion und Weiterentwicklung der Biotechnologie auf Produktion und Beschäftigung entstehen können, sind hingegen bislang kaum untersucht worden. Die empirischen Erkenntnisse sowohl über direkte Wirkungen in den Anwenderindustrien als auch über indirekte Wirkungen in vorgelagerten Zuliefersektoren sind gering. An dieser Stelle setzt die vorliegende Arbeit an.

Das Ziel dieser Arbeit ist die Analyse von möglichen zukünftigen Produktions- und Beschäftigungseffekten der Biotechnologie. Hierfür erfolgte eine Ableitung relevanter Wirkungsmechanismen aus der Theorie, die Erörterung der Verfügbarkeit und Aussagekraft verschiedener Daten(-quellen) und eine quantitative empirische Analyse für ausgewählte Anwendungsfelder der Biotechnologie. Die empirische Analyse erfolgt anhand der Verknüpfung einer „BottomUp“-Technologiebetrachtung gekoppelt mit einem gesamtwirtschaftlichen „TopDown"-Modell. Dieses Vorgehen ermöglicht auf Basis wichtiger Wirkungszusammenhänge die Auswirkungen der Biotechnologie auf die Produktion und Beschäftigung quantitativ zu modellieren.

\section{Implikationen aus der Theorie für die Auswirkungen der Biotechnologie}

Zunächst wurden in dieser Arbeit einige zentrale Begriffe eingeführt und erklärt. Im Anschluss wird ein Überblick über die theoretische Forschung zu den öko- 
nomischen Auswirkungen neuer Technologien gegeben. Dabei wurde für verschiedene Theorieansätze (Neoklassik, Post-Keynesianismus, Evolutorik) dargestellt, wie neue Technologien bzw. technischer Fortschritt spezifiziert werden, welche Rolle sie für die wirtschaftliche Entwicklung einnehmen und in welcher Art und Weise (z.B. Produktivität, strukturelle Wirkungen) sie sich auf die Gesamtwirtschaft auswirken. In allen Theorieansätzen haben neue Technologien eine hohe Bedeutung für die gesamtwirtschaftliche Entwicklung. Allerdings können die Unterschiede zwischen Technologien bei den Auswirkungen in den meisten Ansätzen jeweils nur bedingt erklärt und bestimmte gesamtwirtschaftliche Wirkungsmechanismen von neuen Technologien berücksichtigt werden. Bspw. drückt sich im neoklassischen Grundmodell technischer Fortschritt in einer Produktivitäts- und Produktionserhöhung aus, differenzierte Einflüsse (z.B. strukturelle Veränderungen) werden nicht erfasst. Die verschiedenen Theorieansätze erlauben dabei unterschiedliche Betrachtungsweisen. Während die Evolutorik wichtige Aufschlüsse über den Innovations- und Diffusionsprozess einer Technologie gibt, lassen sich nur sehr eingeschränkt empirisch falsifizierbare Ursache-Wirkungszusammenhänge ableiten und eine systematische gesamtwirtschaftliche Analyse der Effekte von Technologien durchführen. Konkretere formale Untersuchungsmöglichkeiten bieten die Neoklassik oder der Postkeynesianismus, der technische Fortschritt wird aber nur bedingt spezifiziert.

$\mathrm{Zu}$ einer konkreten Charakterisierung der Biotechnologie in langfristiger Perspektive ist das evolutorische „General-Purpose-Technologies"-Konzept (GPT) von Lipsey et al. (1998/2005) geeignet. Es trägt dazu bei, auf einer Makroebene die Bedeutung der Innovationsdynamik, das zeitlich differenzierte Auftreten makroökonomischer Impulse und die hierfür relevanten Faktoren (z.B. Institutionelle Rahmenbedingungen, Komplementarität zu existierenden Technologien) für die Biotechnologie zu verdeutlichen. Neben der besseren Einordnung der Ergebnisse der späteren quantitativen empirischen Umsetzung unterstützt diese Perspektive auch die Spezifizierung der Annahmen für die Szenarien der quantitativen Modellierung (z.B. Faktoren für Diffusion, Lerneffekte). Es lässt sich allerdings zum jetzigen Zeitpunkt nicht eindeutig klären, ob die Biotechnologie alle vier Kriterien einer GPT (Entwicklungspotenzial, Reichweite, Nutzungsvielfalt, Komplementaritäten) erfüllt. Es bestehen zwar für alle vier Kriterien Indizien zur Erfüllung; diese bauen aber teilweise auf Zukunftserwartungen auf. Zudem bestehen im Vergleich zu den IKT deutliche Begrenzungen des zumindest bisher bekannten möglichen Potenzials. Als größte Einschränkungen sind die bisher fehlenden Durchbrüche bei Kosteneinsparungen und die Begrenzungen des direkten Einflusses auf die Wirtschaft durch die Biotechnologie zu nennen. So ist die Reichweite der Nutzung im Vergleich zu den IKT bei der Biotechnologie begrenzt. Bislang zeigen sich vor allem Nutzungsmöglichkeiten in der Landwirtschaft und einigen Industriesektoren, aber nur in wenigen Dienst- 
leistungsbereichen. In dynamischer Perspektive zeigen sich analog zur historischen Betrachtung früherer GPTs die Bedeutung von zusätzlichen komplementären Innovationen und Lerneffekten sowie Anpassungsprobleme im organisatorischen und institutionellen Umfeld. Dabei kann die bislang eher hinter den Erwartungen zurückgebliebene kommerzielle Entwicklung durch diese Anpassungsprobleme, zusammen mit technologischen Besonderheiten und möglichen Fehleinschätzungen der tatsächlich realisierten Effekte nachgezeichnet werden. Denkbar wäre allerdings auch, dass durch das Zusammenspiel von ungünstigen Rahmenbedingungen sowie einigen Limitationen der Biotechnologie (z.B. geringes Kostensenkungspotenzial) das Einschlagen eines Technologiepfads möglich ist, der dauerhaft zu einer eingeschränkten Bedeutung der Biotechnologie führt.

Trotz dieser noch offenen Entwicklung ergeben sich aus dem Konzept der GPT interessante Implikationen für die wirtschaftlichen Auswirkungen der Biotechnologie. Diese sind neben den Eigenschaften der GPT besonders von der Ausprägung komplementärer Faktoren (z.B. Humankapital) und dem Potenzial bisher angewendeter Technologien abhängig und müssen sich nicht in hohen Produktivitätswachstumsraten widerspiegeln. Bspw. gilt bereits die Chemie, die für viele Anwendungen eine Konkurrenztechnologie zur Biotechnologie darstellt, als eine GPT, die Anfang/Mitte des 20. Jahrhunderts zu vielen neuen Anwendungen und Produktivitätsfortschritten führte. Ein durchaus hoch einzuschätzender Beitrag der Biotechnologie kann nun darin liegen, in bestehenden Anwendersektoren die Effizienz und die Vielfalt der eingesetzten Methoden zu erhöhen, welche ansonsten relativ ausgereizt wären. Der Nutzengwinn würde in einem höheren Produktivitätsniveau und zusätzlichen bzw. verbesserten Produkten bestehen, das Produktivitätswachstum könnte aber durchaus geringer als in der Vergangenheit ausfallen.

Allerdings lässt sich auf Basis dieses sehr offen gestalteten GPT-Konzepts keine biotechnologiespezifische quantitative Analyse der Produktions- und Beschäftigungswirkungen durchführen. Hierfür eignet sich ergänzend die Diskussion der Wirkungszusammenhänge der Freisetzungs- und Kompensationsdebatte für die Biotechnologie, besonders als Basis für die empirische Untersuchung. Bei der Darstellung der Freisetzungs- und Kompensationsdebatte zu den Beschäftigungseffekten neuer Technologien wird der lang anhaltende Disput über die Frage deutlich, ob und unter welchen Bedingungen mögliche Kompensationsmechanismen wirken. Jüngere Arbeiten zu diesem Thema bieten insbesondere Ergänzungen dazu, unter welchen institutionellen Voraussetzungen die Mechanismen wirksam werden und wie zeitlich und räumlich differenziert die Effekte auftreten können. Daneben kann sich die Bedeutung einzelner Wirkungszusammenhänge (z.B. arbeitsfreisetzender Charakter) zwischen verschiedenen Technologien deutlich unterscheiden. Diese Feststellungen bestätigen sich bei der konkreten Betrachtung der Biotechnologie, die in sehr heterogenen Anwen- 
dungsfeldern zu verschiedenen Einsatzzwecken eingesetzt werden. Dabei können neben rein wirtschaftlichen Motiven (z.B. neue Produkte, höhere Produktivität) auch die Verbesserung der Gesundheit, die Umweltschonung oder eine nachhaltige Ressourcennutzung im Fokus der jeweiligen Anwendung stehen. Deshalb können sich die relevanten wirtschaftlichen Wirkungsmechanismen zwischen Anwendungen unterscheiden und bedürfen einer spezifischen empirischen Betrachtung. Dennoch lassen sich zumindest in der Tendenz einige Besonderheiten der Biotechnologie herausarbeiten. Die direkte Arbeitsfreisetzung und damit zum Teil verbundene Folgewirkungen (z.B. Kaufkraftkompensation) zeigen sich bei der Biotechnologie nur für wenige Anwendungen. Eine stärkere Bedeutung kommt hingegen Produktinnovationen und strukturellen Wirkungen über veränderte Produktionsprozesse (v.a. bei den eingesetzten Rohstoffen) zu. Damit einhergehend kann sich der inländische Anteil an der Wertschöpfungskette, je nach Verfügbarkeit der Rohstoffe, einer Volkswirtschaft deutlich verändern.

\section{Empirische Messbarkeit wirtschaftlicher Effekte der Biotechnologie}

Für die Wahl eines geeigneten empirischen Ansatzes stellt sich zunächst die Frage der Datenverfügbarkeit. Darin wird gerade für die Biotechnologie ein entscheidender Engpass gesehen, welcher detaillierte Analysen zu wirtschaftlichen Effekten erschwert. Wichtige Gründe für die eingeschränkte Datenverfügbarkeit für diese Prozesstechnologie mit hohem Querschnittscharakter sind konzeptionelle Schwierigkeiten bei der Messung geeigneter Größen (z.B. Produktivitätswirkung). Die Diffusion und mögliche technologische Fortschritte in der Biotechnologie sind im Gegensatz zu anderen Technologien (z.B. Windenergie, Teile der IKT) weniger an spezifische, langlebige Kapitalgütern gebunden. Eine geeignete Messung über Investitionsindikatoren ist daher kaum möglich. Ähnlich schwierig ist eine separate Erfassung biotechnologischer Aktivitäten auf Unternehmensbasis sowie die Erfassung biotechnologisch hergestellter Güter in der Produktionsstatistik. Die meisten Güter sind nicht eindeutig auf ihre Produktionsverfahren zurückzuführen. Die Biotechnologie ist häufig nur eine mögliche Prozessalternative für bestehende Produkte, und völlig neue Güter durch die Biotechnologie werden, wenn überhaupt, nur mit erheblicher zeitlicher Verzögerung isoliert in der Produktionsstatistik erfasst. Aufgrund dieser grundlegenden Schwierigkeiten lässt sich die Biotechnologie weder in einem Wirtschaftszweig zusammenfassen noch ist für existierende Wirtschaftszweige der Anteil der Biotechnologie ersichtlich.

\section{Vorgehensweise bei der empirischen Untersuchung}

Infolge dieser Probleme ist das statistische Ausgangsmaterial für mögliche Modelllösungen erheblich eingeschränkt. In dieser Arbeit wird ein flexibler Ansatz gewählt, welcher den Einbezug verschiedenster Informationen (u.a. Experten- 
meinungen) und einige Freiheitsgrade für die Berücksichtigung von fallspezifischen Zusammenhängen erlaubt. Hierzu bietet sich ein so genannter ,pragmatischer Ansatz" der Technologieprojektion gekoppelt mit einem I-O-Modell an. Dabei wird das statische I-O Grundmodell an denjenigen Stellen erweitert und angepasst, wo die Ergebnisse zentral beeinflusst werden (z.B. zentrale Entwicklungen im Agrarsektor).

Um die Auswirkungen der Biotechnologie in unterschiedlichen Kontexten zu ermitteln und dem Querschnittscharakter der Biotechnologie möglichst gerecht zu werden, wird die vorliegende Untersuchung für vier recht heterogene Anwendungsfelder durchgeführt. Die ausgewählten Felder Bioethanol, Biopolymere, biotechnologisch hergestellte Fein-/Spezialchemikalien und Biopharmazeutika unterscheiden sich größtenteils beim Einsatzzweck der Biotechnologie (z.B. neue Produkte, Rohstoffersetzung), Reifegrad und Diffusion der biotechnologischen Anwendungen sowie in den sektoralen Spezifika.

Für eine möglichst konkrete Untersuchung der Wirkungsweise der Biotechnologie, werden die Einflussfaktoren auf die Veränderung der I-O-Koeffizienten (Technologiediffusion, Ersetzung von Vorleistungsgütern, Einsparung bei Produktionsfaktoren usw.) analytisch getrennt und für alle betrachteten Anwendungsfelder der Biotechnologie spezifisch bestimmt. Die technoökonomischen Informationen werden dabei in detaillierten Fallstudien durch eine breite Abdeckung der Fachliteratur und 39 Expertengespräche abgeleitet. Für die Durchführung der Modellberechnungen der Produktions- und Beschäftigungswirkungen der Biotechnologie werden für das Jahr 2020 mehrere Szenarien entworfen (Basisszenario als Vergleichsmaßstab, Referenzszenario und Szenario mit hoher Diffusion). Die Szenarien unterscheiden sich dabei in den Annahmen zu den Preisen wichtiger Rohstoffe, der technologischen Entwicklung und zentralen politischen Rahmenbedingungen (z.B. gesetzlich festgelegte Beimischung von Bioethanol). Mithilfe von Sensitivitätsanalysen wurden die Robustheit und die Folgewirkungen der getroffenen Annahmen untersucht.

\section{Empirische Ergebnisse und Kritische Würdigung}

Die Ergebnisse der Modellrechnungen wurden bereits in Kapitel 6.5 zusammengefasst und ausgiebig diskutiert. Sie lassen sich in Kürze wie folgt beschreiben: Nach Einschätzungen aus der Literatur und Experten wird für alle Anwendungsfelder eine deutliche Zunahme der Verbreitung biotechnologischer Methoden und Verfahren bis zum Jahr 2020 erwartet. Der Anteil der mithilfe der Biotechnologie entwickelten und/oder hergestellten Produkte am jeweiligen Produktionswert wird aber wohl begrenzt bleiben. Die abgeleiteten Anteile für die Szenarien liegen meist unter 20\%, bei Biopharmazeutika sind sie mit bis zu $32 \%$ etwas höher. Zusätzlich muss für die Pharmaindustrie beachtet werden, dass biotechnologische Methoden zunehmend auch die F\&E auch von chemisch-synthetisierten Therapeutika (,small molecules") unterstützen. 
Die Unterschiede zwischen den Wertschöpfungsprozessen der Biotechnologie und den substituierten Wertschöpfungsprozessen zeigen sich hauptsächlich in einigen wenigen Parametern (z.B. eingesetzter Rohstoff, Produktivität). Infolgedessen bleibt auch das absolute Ausmaß der Nettoeffekte in den Modellrechnungen eher moderat. Die Nettoeffekte sind dabei im Szenario mit hoher Diffusion stärker positiv bzw. negativ ausgeprägt als im Referenzszenario, die jeweiligen Vorzeichen ändern sich mit Ausnahme der Nettoproduktion bei Biopolymeren nicht. Positiv sind die berechneten Effekte bei Bioethanol und zumeist bei Biopolymeren aufgrund der höheren inländischen Wertschöpfung durch die Ersetzung importierter fossiler Rohstoffe durch zumeist inländisch hergestellte nachwachsende Rohstoffe. Bei Fein-/Spezialchemikalien sind die Ergebnisse für Produktion und Beschäftigung negativ. Bei Biopharmazeutika ergeben sich keine eindeutigen Ergebnisse: Zum einen differieren Nettoproduktionseffekte und Nettobeschäftigungseffekte sehr deutlich aufgrund großer Unterschiede der relevanten Sektoren bei den Arbeitsintensitäten. Zum anderen sind die Ergebnisse sehr stark von der Modellierung der Produktinnovationen (substitutiv vs. additiv) abhängig. Alternative Modellierungen zur Basisrechnung bei Biopharmazeutika zeigen, dass wenn einer der beiden Kompensationsmechanismen der additiven Produktinnovationen oder der Erzielung zusätzlicher Exportüberschüsse wirksam wird, die inländischen Produktions- und Beschäftigungseffekte deutlich positiver ausfallen.

Folglich lässt sich kein eindeutiger Schluss auf die Wirkungsrichtung der Biotechnologie für Produktion und Beschäftigung ziehen. Vielmehr scheint sich der jeweilige Einsatzzweck der Biotechnologie (z.B. Rohstoffersetzung, Kostenersparnis, neue Produkte) zusammen mit spezifischen Rahmenbedingungen (z.B. Flächenverfügbarkeit in der Landwirtschaft, Regelungen zur Kostendämpfung im Gesundheitssystem) entscheidend auf das jeweilige Ergebnis auszuwirken. Die einzelnen Freisetzungs- und Kompensationsmechanismen kommen in den Fallbeispielen dabei unterschiedlich zum Tragen. Beispielsweise sind bei Bioethanol und Biopolymeren Struktureffekte (Arbeitsintensitäten, Importintensitäten) von hoher Bedeutung, aufgrund der unterschiedlichen Rohstoffbasis im Vergleich zum Substitut. Hingegen ergeben sich bei Biopharmazeutika die Folgewirkungen stärker durch die Nachfrage. Die Sensitivitätsrechnungen zeigen besonders die Bedeutung der Annahmen zum Außenhandel und zur Diffusion der Biotechnologie für die Ergebnisse. Die Annahmen über Kosten- und Konsumstruktur haben dagegen eine geringere Bedeutung für die Nettoeffekte von Produktion und Beschäftigung. Die alternativen Berechnungen mit plausibel erscheinenden Ober- und Untergrenzen führen aber nur in wenigen Fällen zu einem Wechsel des Vorzeichens. In der Regel erscheinen die Kernaussagen der Modellergebnisse als robust.

Die strukturellen Veränderungen in den Fallstudien sind, verglichen mit den eher geringen Nettoeffekten bei der jeweiligen Gesamtproduktion und -beschäf- 
tigung, recht groß. Dabei konzentrieren sich diese Veränderungen jeweils auf sehr wenige Sektoren. Ein sehr bedeutender Teil der wirtschaftlichen Effekte der Biotechnologie tritt nicht direkt in den Anwendungsfeldern, sondern in anderen Bereichen der Wirtschaft (v.a. Landwirtschaftssektor und im Dienstleistungssektor) auf. Die Gründe hierfür liegen ebenfalls im Wechsel auf die nachwachsende Rohstoffbasis sowie der starken Veränderung der Dienstleistungsnachfrage bei Konsumsteigerungen/-senkungen. Folglich findet zwar der direkte Einsatz der Biotechnologie zum Großteil in der Industrie statt, die Folgewirkungen können sich aber stärker im primären und tertiären Sektor zeigen.

Eine Analyse, die sich allein auf direkte Wirkungen in den Anwendungsfeldern beschränkt, greift folglich zu kurz. Die Verbindung der technoökonomischen „Bottom-Up"-Betrachtung mit dem gesamtwirtschaftlichen I-O-Modell ermöglicht die Untersuchung dieser bedeutenden indirekten Effekte und die Analyse der unterschiedlichen Wirkungen in den einzelnen Anwendungsfeldern. Deshalb erscheint diese Kombination als geeigneter Ansatzpunkt, die gesamtwirtschaftlichen Auswirkungen der Biotechnologie zu untersuchen.

Bei der Gegenüberstellung und Einordnung dieser empirischen Ergebnisse zu den erläuterten Theorien zeigen sich zudem mehrere interessante Aspekte. Die allgemeine Diskussion der Biotechnologie im Kontext der Freisetzungs- und Kompensationseffekte sowie die empirische Untersuchung für die vier Anwendungsfelder zeigen, dass die ökonomischen Impulse sehr anwendungsspezifisch sind. Es können nur begrenzt Aussagen mit allgemeinerem Charakter zur Biotechnologie getroffen werden. Daher stellt sich die Frage, inwiefern eine Betrachtung der gesamten Biotechnologie als Analysegegenstand überhaupt sinnvoll ist. Hier liefert die theoretische Grundlage der GPT interessante Einsichten, indem die anwendungsübergreifenden Charakteristika und Technologieentwicklungen sowie Diffusionsfaktoren der Biotechnologie erkennbar werden. Außerdem wird in Expertengesprächen deutlich, dass die technologische Basis für die sehr unterschiedlichen Anwendungen große Ähnlichkeiten aufweist. Grundlegende technologische Fortschritte in der Biotechnologie können demnach verschiedensten Anwendungen zu Gute kommen. Sie müssen aber stärker auf den Anwendungszweck hin weiterentwickelt werden als es bspw. für viele Bereiche der IKT der Fall ist.

Allerdings können in der empirischen Analyse nur begrenzt Auswirkungen, die durch komplett neue Anwendungsmöglichkeiten entstehen, sowie SpilloverEffekte, die durch Komplementaritäten mit anderen Technologien auftreten, einbezogen werden. Dies liegt an der schwierigen Zurechenbarkeit zur Biotechnologie, der hohen Unsicherheit hinsichtlich der tatsächlichen Realisierbarkeit dieser radikalen Neuerungen und der schwer erfassbaren endnachfragsteigernden Wirkung von Produktinnovationen. Lipsey et al. (1998/2005) heben aber in ihrem GPT-Konzept die Bedeutung gerade dieser Aspekte hervor. Insofern werden die Potenziale der Biotechnologie wohl unterschätzt. Eine eher entgegenge- 
setzte Auswirkung haben mögliche Anpassungsprozesse, wie sie in einigen theoretischen Ansätzen diskutiert werden (z.B. temporäre Produktivitätsverluste, Nachfrageausfälle). Solche Anpassungsfriktionen sind bei der Biotechnologie durchaus zu beobachten. Sie können zu einem temporären Ausfall von Kompensationsmechanismen führen (z.B. Investitionen, Konsum) und haben daher eine dämpfende Wirkung auf ihre gesamtwirtschaftlichen Impulse. Aus innovationsökonomischer Sicht wären gerade die Rückwirkungen dieser Entwicklungen auf die Technologieentwicklung und -diffusion von Interesse. Diese Rückwirkungen konnten allerdings nicht explizit erfasst werden, stattdessen wurden drei unterschiedliche Fälle der Entwicklung mit modellexogenen Annahmen zur technologischen Entwicklung (keine Diffusion, Referenz, Hohe Diffusion) miteinander verglichen. Daneben wäre für die Beurteilung der gesamtwirtschaftlichen Wirkungen der Biotechnologie die Betrachtung aller potenziellen Anwendungsfelder wünschenswert. Eine qualitative Diskussion zeigt aber zumindest wenig Hinweise für prinzipiell unterschiedliche strukturelle Wirkungen. Sie lassen sich von der Art und Weise der Auswirkungen tendenziell jeweils zu einem der untersuchten Fallbeispiele zuordnen. Demnach können die Modellierungen zwar keine Aussagen zu der absoluten Höhe der gesamtwirtschaftlichen Effekte der Biotechnologie liefern, sie bilden aber zumindest den Querschnittscharakter der Biotechnologie einigermaßen ab.

Deshalb sind die insgesamt eher als neutral einzustufenden Effekte für die Produktions- und Beschäftigung in den Modellberechnungen vor folgenden Hintergründen zu sehen: Die Entwicklung der nicht betrachteten Einflussfaktoren (z.B. technologische Spillovereffekte, kostenintensive Anpassungsprozesse) kann die gesamtwirtschaftlichen Folgewirkungen der Biotechnologie verändern. Aber auch bei neutralen strukturellen Wirkungen kann die Biotechnologie eine wichtige Bedeutung für Produktion und Beschäftigung haben. Aus Sicht einer konkreten Volkswirtschaft kann der Einsatz biotechnologischer Methoden und Prozesse notwendig sein, um die internationale Wettbewerbsfähigkeit in den entsprechenden Sektoren zu erhalten. Ansonsten ist die Verlagerung der F\&E und Produktion sowie die damit verbundene Beschäftigung in andere Länder möglich. Darüber hinaus wären durch die erhofften positiven Effekte auf eine geringere Umweltverschmutzung, nachhaltige Rohstoffnutzung und die Gesundheit neben der Erfüllung anderer gesellschaftspolitischer Ziele auch Rückwirkungen auf die Wirtschaft denkbar.

\section{Zukünftiger Forschungsbedarf}

Aus diesen genannten Einschränkungen der vorliegenden empirischen Untersuchung lässt sich direkt ein Teil des zukünftigen Forschungsbedarfs ableiten. $\mathrm{Zu}$ nächst ist aber auf Forschungsbedarf in der Theorie hinzuweisen. Für eine Verbindung der zu einem bedeutenden Teil komplementären Erkenntnisse der theoretischen Ansätze gibt es bislang keine (in sich geschlossene) Konzepte, die eine 
geeignete Grundlage für eine quantitative und technologiespezifische empirische Analyse geben. Aus inhaltlicher Sicht besteht vor allem Weiterentwicklungsbedarf hinsichtlich des Erklärungsgehalts möglicher Entwicklungen im Außenhandel, die mit der Technologieentwicklung und -diffusion einhergehen. Daneben wäre eine stärkere Berücksichtigung und Herausarbeitung von möglichen technologischen Unterschieden als Fundament für die Empirie wichtig. Aus empirischer Sicht könnte eine stärkere dynamische Analyse der Innovations/Diffusionsprozesse und der gesamtwirtschaftlichen Entwicklung (z.B. Rückkoppelungsprozesse) einen zusätzlichen Beitrag zur Erklärung möglicher Entwicklungspfade der Biotechnologie und deren Folgewirkungen geben. Für eine tiefergehende Analyse möglicher gesamtwirtschaftlicher Implikationen wäre bspw. die Koppelung mit einem Agrarökonomischen Modell und/oder eines Nachfragesystems zur Ableitung der Effekte auf der Konsumseite sinnvoll. Wie die Sensitivitätsberechnungen zeigen, haben die Ausprägungen dieser Faktoren zumindest teilweise deutlichen Einfluss auf die Ergebnisse.

Zudem erfordert eine einfachere und detaillierte Erfassung der ökonomisch relevanten Impulse der Biotechnologie eine größere Datenbasis. Es kann aber bezweifelt werden, ob sich die Datenlage in näherer Zukunft grundlegend ändert. Der besseren Erfassung in der amtlichen Statistik stehen die oben erläuterten Probleme, wie z.B. die Abgrenzung zu anderen lang existierenden Wirtschaftsaktivitäten, entgegen. Zwar werden zukünftig vermutlich verstärkt Bemühungen zur statistischen Erfassung durchgeführt. Aufgrund des weiter zunehmenden Querschnittscharakters der Biotechnologie mit neuen Anwendungen und stärkerer Konvergenz mit anderen Technologien werden ihre Wirkungen aber weiterhin schwer erfassbar sein. Deshalb werden begleitende technoökonomische Betrachtungen, wie z.B. die Verbindung verschiedener Informationsquellen mit dem Input-Output-Modell, auch in Zukunft von hoher Bedeutung sein, um das Potenzial und die Effekte der Biotechnologie abschätzen zu können. 
Sven Wydra - 978-3-631-75392-7

Downloaded from PubFactory at 01/11/2019 05:32:25AM

via free access 


\section{Literaturverzeichnis}

ACIL Tasman; Innovation Dynamics (2006a): A proposed Methodology to identify Biotechnology's Contribution to the Australian Economy. Volume 1 - Main report, Final Report prepared for the Department of Industry, Tourism and Resources.

ACIL Tasman; Innovation Dynamics (2006b): A proposed Methodology to identify Biotechnology's Contribution to the Australian Economy. Volume 2 - Appendices, Final Report prepared for the Department of Industry, Tourism and Resources.

Aghion, P.; Howitt, P. (1992): A Model of Growth through Creative Destruction. In: Econometrica Vol. 60, S. 323-351.

Aghion, P.; Howitt, P. (1996): Research and Development in the Growth Process. In: Journal of Economic Growth, Vol.1, S. 49-73.

Aghion, P.; Howitt, P. (1998): On the Macroeconomic Effects of Major Technological Change. In: Helpman, E. (Hrsg.): General Purpose Technologies and Economic Growth MTT Press, Cambridge, MA, S. 121-144.

Allansdottir, A.; Bonaccorsi, A.; Gambardella, A.; Mariani, M.; Orsenigo, L.; Pammolli, F.; Riccaboni, M. (2002): Innovation and Competitiveness in European Biotechnology. In: Enterprise Papers, Nr. 7, European Commission.

Amir-Aslani, A.; Negassi; S. (2006): Is Technology Integration the Solution to Biotechnology's Low Research and Development Productivity?. In: Technovation, Vol. 26, S. 273-582.

Amiti, M.; Wei, S.-J. (2006): Service Offshoring, Productivity and Employment: Evidence from the US. In: CEPR Discussion Paper, No. 5475.

Angrick, M. (2006): Vorwort. In: Dubbert, W.; Heine, T.: Weiße Biotechnologie -Ökonomische und ökologische Chancen, Berlin, S. 8-9.

Arantes-Oliviera, N. (2007): A Case Study on Obstacles to the Growth of Biotechnology. In: Technological Forecasting and Social Change, Vol. 74, S. 6174.

Archibugi, D.; Evangelista, R.; Simonetti, R. (1994): On the Definition and Measurement of Product and Process Innovations. In: Shionoya, Y.; Perlman, M. (Hrsg.): Innovation in Technology, Industries and Institutions. Studies in Schumpeterian Perspectives, The University of Michigan Press, Ann Arbor, S. 7-24.

Arrow, K. J. (1962): The Economic Implications of Learning by Doing. In: Review of Economic Studies, Vol. 29, S. 155-173.

Arundel, A. (2000): Measuring the Economic Impacts of Biotechnology: From R\&D to Applications. In: De La Mothe, J.; Niosi, J. (Hrsg.): The Economic 
and Social Dynamics of Biotechnology, Kluwer Academic Publishers, Boston, S. 83-100

Arundel, A. (2002): Agro-biotechnology, Innovation and Employment. In: Science and Public Policy, Vol. 29, S. 297-306.

Arundel, A. (2003): Biotechnology Indicators and Public Policy. In: STI Working Papers, Nr. 2003/5, OECD, Paris.

Arundel, A. (2007): Bioeconomy to 2030. International Futures Project: Bioeconomy to 2030: Designing a Policy Agenda. Foliensatz für Vortrag in Athen am 6.11.2007.

Arundel, A.; Crespi, G. Patel, P. (2006): Biotechnology, Scoping Paper, Arundel, A.; Crespi, G. Patel, P. (2006): Biotechnology, Scoping Paper, Europe INNOVA, Innovation Watch

Arundel, A.; Mintzes, B. (2004): The Benefits of Biopharmaceuticals. In: Innogen Working Paper Nr. 14, Edinburgh University.

Autor, D.; Katz, L.; Krueger, A. (1998): Computing Inequality: Have Computers changed the Labour Market?, In: Quarterly Journal of Economics, Vol. 113, S. 1169-1214.

BAFA (Bundesamt für Wirtschaft und Ausfuhrkontrolle) (2008): Amtliche Mineralöldaten für die Bundesrepublik Deutschland, Eschborn.

Barben, D. (2007): Politische Ökonomie der Biotechnologie. Innovation und gesellschaftlicher Wandel im Vergleich. Reihe „Theorie und Gesellschaft".Frankfurt am Main/New York.

Barro, R. (1991): Economic Growth in a Cross-Section of Countries. In: The Quarterly Journal of Economics, Vol. 106, S. 407-444.

Basberg, B. L. (1987): Patents and the Measurement of Technological Change: A Survey of the Literature. In: Research Policy, Vol. 16, S. 131-141.

Basu, P.; Joglekar, G.; Rai, S.; Suresh, P.; Vernon, J. (2008): Analysis of Manufacturing Costs in Pharmaceutical Companies. In: Journal of Pharmaceutical Innovation, Vol. 3, S. 30-40.

Bauer, M. (1995): Resistance to New Technology. Cambridge, Mass.

BCG (2006): Medizinische Biotechnologie in Deutschland 2006 - Wirtschaftliche Bedeutung und Erfolgsfaktoren. BCG Report.

BCG (2007): Medizinische Biotechnologie in Deutschland 2007 - Wirtschaftliche Situation, Nutzen und Einsatz von Biopharmazeutika. BCG Report.

BCG (2008): Medizinische Biotechnologie in Deutschland 2008 - Wirtschaftsdaten und Innovationskraft. BCG Report.

Beckert, B.; Roloff, N.; Friedewald, M. (2006): Converging Technologies and their Impact on the Social Sciences and Humanities (CONTECS): R\&D 
Trends in Converging Technologies Deliverable D1.1. Karlsruhe: Fraunhofer ISI. http://www.contecs.fraunhofer.de/. Zugriff: 30.06.2007.

Beise, M. (2001): Lead Markets: Country-Specific Success Factors of the Global Diffusion of Innovations, ZEW Economic Studies Nr. 14, Physica-Verlag, Heidelberg.

Bernardes, A. T.; Ruiz, R. M.; Ribeiro, L. C.; Albuquerque, E. M. (2006): Modelling Economic Growth fuelled by Science and Technology. In: Discussion Paper der Universidade federal de Minas Gerais, Nr. 294.

Beucker, S.; Marscheider-Weidemann, F. (2007): Zukunftsmarkt Biokunststoffe. Fallstudie im Auftrag des Umweltbundesamtes im Rahmen des Forschungsprojektes Innovative Umweltpolitik in wichtigen Handlungsfeldern, Nr. 07/08, Berlin.

biotechnologie.de (2006): Die deutsche Biotechnologie-Branche 2006, Daten \& Fakten, www.biotechnologie.de. Zugriff: 30.09.2006.

biotechnologie.de (2007): Die deutsche Biotechnologie-Branche 2007, Daten \& Fakten, www.biotechnologie.de. Zugriff: 10.10.2007.

biotechnologie.de (2008): Die deutsche Biotechnologie-Branche 2008, Daten \& Fakten, www.biotechnologie.de. Zugriff: 02.09.2008.

Blaauw, R.; van Haveren, J.; Scott E. L.; Bos H. L. (2008): Biomass for the Dutch Chemical Industry, Opportunities for agriculture, Wageningen.

Blank, S. (2008): Small and Medium Enterprises in Agricultural Biotechnology. In: OECD International Futures Project on "The Bioeconomy to 2030: Designing a Policy Agenda", Paris.

Blattner, N. (1996): Technischer Fortschritt und Arbeitslosigkeit, in: Gahlen, B. Hesse, H.; Ramser, H. J. (Hrsg.): Arbeitslosigkeit und Möglichkeiten ihrer Überwindung, Tübingen, S. 211-228.

BMELV (Bundesministerium für Ernährung, Landwirtschaft und Verbraucherschutz) (2007): Statistisches Jahrbuch über Ernährung, Landwirtschaft und Forsten der Bundesrepublik Deutschland 2007, Wirtschaftsverlag NW, Bremerhaven.

BMU (2008): Weiterentwicklung der Strategie zur Bioenergie. April 2008, Berlin.

Bohlmann, G. (2006): Process Economic Considerations for Production of Ethanol from Biomass Feedstocks. In: Industrial Biotechnology, Vol. 2, S. 14-20.

Booth, B.; Zemmel, R. (2004): Prospects for Productivity. In: Nature Reviews Drug Discovery, Vol. 2, S. 451- 456. 
Boyer, R. (1988): Technical Change and the Theory of Régulation. In: Dosi, G.; Freeman, C.; Nelson, R.; Silverberg, G.; Soete, L. (Hrsg.): Technical Change and the Economic Theory, London, Pinter, S. 67-94.

Bräuninger, M.; Sattler, C.; Kriedel, N.; Vöpel, H.; Straubhaar, T. (2007): Gesundheitsentwicklung in Deutschland bis 2037 - Eine volkswirtschaftliche Kostensimulation. In: HWWI Policy Paper des HWWI Komptenzbereiches Wirtschaftliche Trends, Nr. 1-6, Hamburg.

Bräuninger, M.; Straubhaar, T.; Fitzner, V.; Teichmann, G. A. (2008): PolitikCheck Pharmastandort Deutschland: Potenziale erkennen - Chancen nutzen. In: HWWI Policy Report des HWWI Komptenzbereiches Wirtschaftliche Trends ,Nr. 7, Hamburg.

Bresnahan, T. F.; Trajtenberg, M. (1995): General Purpose Technologies: Engines of Growth?. In: Journal of Econometrics, Vol. 65, S. 83-108.

Bringezu, S.; Schütz, H. (2008): Auswirkungen eines verstärkten Anbaus nachwachsender Rohstoffe im globalen Maßstab. In: Technikfolgenabschätzung Theorie und Praxis, Vol. 17, S. 12-23.

Brink, J.; McKelvey, M.; Smith, K. (2004): Conceptualizing and Measuring modern Biotechnology. In: McKelvey, M.; Rickne, A.; Laage-Hellman, J. (Hrsg.): The Economic Dynamics of Modern Biotechnology, Cheltenham, S. 20-42.

Brusoni, S.; Cefis, E.; Orsenigo, L. (2006): Innovate or die? A critical Review of the Literature on Innovation and Performance. In: CESPRI Working Papers, Nr. 179.

Buchholz, S. (2006): Science to Business Center - Bio-Zentrum für »Weiße Biotechnologie« der Degussa AG. Vortrag auf der Tagung »auf Bio-raffiniert III«, 2.-3. Februar 2006, Vortragsfolien, Gelsenkirchen.

Bundesregierung (2007): Eckpunkte für ein integriertes Energie- und Klimaprogramm. Berlin.

Buttel, F. H. (1995): Biotechnology: An epoch-making Technology?. In: Fransman, M.; Junne, G.; Roobeck, A. (Hrsg.): The Biotechnology Revolution, Oxford, Blackwell, S. 25-45.

Cameron, G. (1998): Innovation and Growth: A Survey of the Empirical Evidence, Oxford.

Carlaw, K. I.; Lipsey, R. G. (2003): Productivity, Technology and Economic Growth: What is the Relationship?. In: Journal of Economic Surveys, Vol. 17, S. 457-495.

Carlsson B.; Eliasson G. (2003): Industrial Dynamics and Endogenous Growth. In: Industry and Innovation, Vol. 10, S.435-455. 
Carlsson, B.; Jacobsson, S.; Holmen, M.; Rickne, A. (2002): Innovation Systems: Analytical and Methodological Issues. In: Research Policy, Vol. 31, S. 233-245.

Carole, T.M.; Pellegrino, J.; Paster M. D. (2004): Opportunities in the Industrial Bio-based Products Industry. In: Applied Biochemistry and Biotechnology, Vol. 115, S. 871-885.

Carter, A. P. (1990): Upstream and Downstream Benefits of Innovation. In: Economic Systems Research, Vol. 2, S. 241-257.

Carter, A.P. (1970): Structural Change in the American Economy, Harvard University Press, Cambridge, Mass.

Casamassima, M.; Schade B. (2007): High Oil Prices: a comparison of the main national and international studies. Deliverable 1 of HOP! Macro-economic Impact of high Oil Price in Europe. Funded by European Commission 6. RTD Programm. Mailand.

Castaldi, C.; Dosi, G. (2008): Technical Change and Economic Growth: Some Lessons from Secular Patterns and Some Conjectures on the Current Impact of ICT Technologies. In: LEM Working Paper Series, Nr. 2008/01.

CEFIC (2004): Horizon 2015: Perspectives for the European Chemical Industry, Brüssel.

Chandler, A. D. (2005): Shaping the Industrial Century: The remarkable Story of the Evolution of the Modern Chemical and Pharmaceutical Industries, Harvard University Press, Cambridge, London.

Chataway, J.; Tait, J.; Wield, D. (2006): The Governance of Agro- and Pharmaceutical Biotechnology Innovation: Public Policy and Industrial Strategy. In: Technology Analysis and Strategic Management, Vol. 18, S. 1-17.

Chateau, B.; Biberacher, M.; Birnbaum, U.; Hamacher, T.; Lako, P.; Martinsen, D.; Patel, M.; Pospischil, W.; Quercia, N.; Smekens, K. (2005): VLEEM 2 (Very Long Term Energy Environment Model), Grenoble.

Chennells, L.; van Reenen, J. (2002); Technical Change and the Structure of Employment and Wages: A Survey of the Microeconometric Evidence. In: Greenan, N.; L'Horty, Y.; Mairess, J. (Hrsg.): Productivity, Inequality, and the Digital Economy: A Transatlantic Perspective, MIT Press, Cambridge, S.175-224.

Choumert F.; Paltsev S.; Reilly J. (2006): Improving the Refining Sector in EPPA. In: MIT Joint Program on the Science and Policy of Global Change. Technical Note No. 9, July 2006.

ColognePaper (2007): En Route to the Knowledge-Based Bio-Economy. Mai 2007, Köln. 
CONCAWE (2006): Well-to-Wheels Analysis of Future Automotive Fuels and Powertrains in the European Context. Version 2c.

Conte, A. (2006): The Evolution of the Literature on Technological Change over time: A Survey. In: Discussion Paper on Entrepreneurship, Growth and Public Policy, Max Planck Institut für Ökonomik, Jena, Nr. 107.

Cooper, C. (1994): Technology and Innovation in the International Economy, Edward Elgar, Cheltenham.

Cordes, S. (2007): Innovationspolitik im 6. Kondratieff: Hinterherlaufen oder Vorauseilen? In: Wirtschaftsdienst 87, S. 450-457.

CRA (Charles River Associates) (2004): Innovation in the Pharmaceutical Sector. A study undertaken for the European Commission, London, Brüssel.

Crank, M.; Patel, M.; Marscheider-Weidemann, F.; Schleich, J.; Hüsing, B.; Angerer, G. (2004): Techno-economic Feasibility of Large-scale Production of Bio-based Polymers in Europe (PRO-BIP). Final Report prepared for the European Commission's Institute for Prospective Technological Studies, Sevilla, Spain, Universität Utrecht, Utrecht, Fraunhofer ISI, Karlsruhe.

Cyert R. M.; Mowery, D. C. (1988): The Impact of Technological Change on Employment and Economic Growth. Mass, Ballinger, Cambridge.

Danzon, P.M.; Furukawa, M. F. (2006): Prices and Availability of Biopharmaceuticals: An International Comparisom. In: Health affairs, Vol. 25, S. 13531362.

Das, G. G. (2007): Information Age to genetic Revolution: Embodied Technology Transfer and Assimilation - A tale of two Technologies, Technological Forecasting \& Social Change, Vol. 74, 819-842

David, P. (1986): Technology diffusion, public policy and industrial competitiveness. In: Landon, R., Rosenberg, N.(Hrsg.): The Positive Sum Strategy. National Academy Press, Washington, DC., S.373-392.

David, P. A. (1990): The Dynamo and the Computer: A historical Perspective on the Productivity Paradox. In: American Economic Review, Vol. 80, 355361 .

Dechema (2004): Weiße Biotechnologie: Chancen für Deutschland. DECHEMA Gesellschaft für Chemische Technik und Biotechnologie e. V, Frankfurt/M..

Deimling, S.; Goymann, M. ; Baitz, M.; Rehl, T. (2007): Auswertung von Studien zur ökologischen Betrachtung von nachwachsenden Rohstoffen bei einer stofflichen Nutzung. Gutachten im Auftrag von der Fachagentur nachwachsende Rohstoffe e.V., Leinfelden-Echterdingen.

Deke, O. (2004): Der Preis des "Grünen Goldes" - Informationen und Informationsdefizite über den Marktwert genetischer Ressourcen. In: Kieler Arbeitspapiere, Kiel, Nr. 1233. 
Dewick, P.; Green, K.; Fleetwood, T.; Miozzo, M. (2006): Modelling creative Destruction: Technological Diffusion and Industrial Structure Change to 2050- In: Technological Forecasting and Social Change, Vol. 73, S. 10841106.

Dewick, P.; Green, K.; Miozzo, M. (2004): Technological Change, Industrial Structure and the Environment. In: Futures, Vol. 36, S. 267-294.

Dickson, M.; Gagnon, J. P. (2004): Key factors in the Rising Cost of new Drug Discovery and Development. In: Nature Reviews Drug Discovery, Vol. 3, S. 417-429.

DiMasi, J. A.; Grabowski, H. G. (2007): The Cost of Biopharmaceutical R\&D: Is Biotech Different?. In: Managerial and Decision Economics, Vol. 28, S. 469-479.

DiMasi, J.A.; Hansen, R. W.; Grabowski, H. G. (2003): The Price of Innovation: New Estimates of Drug Development Costs. In: Journal of Health Economics, Vol. 22, S. 151-185.

Döhrn, R.; Engel, D.; Stiebale, J. (2008): Außenhandel und ausländische Direktinvestitionen deutscher Unternehmen, Studien zum deutschen Innovationssystem Nr. 3-2008, Berlin.

Dolata, U. (2007): Technik und sektoraler Wandel. Technologische Eingriffstiefe, sektorale Adaptionsfähigkeit und soziotechnische Transformationsmuster. In: MPIfG Discussion Paper, Köln, Nr. 07/3.

Dominguez Lacasa, I. (2005): Exploring Technological Change in the German Pharmaceutical Industry. A history-friendly Model of Technological Change and Technology Adoption in a Science-based Industry, Karlsruhe.

Dopfer, K. (2005): Evolutionary Economics: A Theoretical Framework. In: Dopfer, K. (Hrsg.): The Evolutionary Foundations of Economics, Cambridge University Press; Cambridge, UK, S. 3-55.

Dornburg, V. (2004): Multi-functional Biomass Systems, Utrecht.

Dornburg, V.; Faaij, A.; Wicke, B. (2007): Developments in Bioenergy Production across the World - Electricity, Heat and Second Generation Biofuels. Consultancy report and database provided to the OECD Secretariat, Nr. 23.

Dosi, G. (1982): Technological Paradigms and Technological Trajectories: A suggested Interpretation of the Determinants and Directions of Technical Change. In: Research Policy, Vol. 11, S. 147-162.

Dosi, G. (1988): Source, Procedure and Microeconomic Effects of Innovation. In: Journal of Economic Literature, Vol. 26, S. 1120-1171.

Dosi, G., Orsenigo, L.; Sylos Labini, M. (2003): Technology and the Economy. In: Smelser, N.J.; Swedberg, R. (Hrsg.): Handbook of Economic Sociology, 2. Auflage, Princeton University Press, Lawrenceville, S.678-702. 
Dosi, G.; Pavitt, K.; Soete, L. (1990): The Economics of Technical Change and International Trade, Harvester Wheatsheaf, London.

Drejer, I. (1999): Technological Change and Interindustrial Linkages. Introducing Knowledge Flows in Input-Output Studies, Aalborg University.

Drumm, L. (2005): Increasing the Penetration Rate of Biotechnolgy in the Chemical Industry. In: Industrial Biotechnology, Vol. 1, S. 244-246.

Duchin, F. (1998): Structural economics. Measuring change in Technology, Lifestyles, and the Environment, Island Press, Washington,D.C., Covelo, Calif..

Duchin, F.; Lange, G. M.; Thonstad, K.; Idenburg, A.; (1994): The Future of the Environment, Ecological Economics and Technological Change. Oxford University Press, New York.

Dukes, M. N. G. (2008): OECD International Futures Project on "The Bioeconomy to 2030: Designing a Policy Agenda". Biotechnology Regulation in the Health Sector. In: Multidisciplinar Issues International Futures Programm Series der OECD, Paris.

Dunne, T.; Foster, L.; Haltiwanger, J.; Troske, K. (2000): Wage and Productivity Dispersion in U.S. Manufacturing: The Role of Computer Investment. In: NBER Working Paper, Nr. 7465.

ECCP (2001): European Climate Change Programme - Long Report. European Commission, Brüssel.

Edler, D. (1990): Ein dynamisches Input-Output-Modell zur Abschätzung der Auswirkungen ausgewählter neuer Technologien auf die Beschäftigung in der Bundesrepublik Deutschland. In: Beiträge zur Strukturforschung, Vol. 116. Berlin.

Edquist, C.; Hommen, L.; McKelvey, M. (2001): Innovation and Employment: Product Versus Process Innovation, Edward Elgar, Cheltenham.

EFPIA (European Federation of Pharmaceutical Industries and Associations) (2008): The Pharmaceutical Industry in Figures, European Federation of Pharmaceutical Industries and Associations, Brüssel.

EIA (Energy Information Administration) (2007): International Energy Outlook 2007. www.eia.doe.gov/oiaf/ieo/index.html, Zugriff: 20.02.2008.

Enzing, C.; van der Giessen, A.; van der Molen, S.; Manicad, G.; Reiß, T.; Lindner, R.; Dominguez-Lacasa, I.; European Commission (2007a): BioPolis. Inventory and Analysis of National Public Policies that Stimulate Biotechnology Research, its Exploitation and Commercialisation by Industry in Europe in the Period 2002-2005: Final Report.

Enzing, C.; van der Giessen, A.; van Zandvoort, C.; van Groenestijn, J.; Meesters, K.; Koivisto, R.; Wirtanen, G.; Miettinen-Oinonen, A.; Pere, J.; Gais- 
ser,S. (2007b): Consequences, Opportunities and Challenges of modern Biotechnology for Europe (Bio4EU) - Case Studies Report. The Impact of Industrial Biotechnology Applications.

Erber, G.; Hagemann, H.; Seiter, S.; (1998): Zukunftsperspektiven Deutschlands im internationalen Wettbewerb: Industriepolitische Implikationen der Neuen Wachstumstheorie. Heidelberg.

Ernst \& Young (2008): Deutscher Biotechnologie-Report 2008 "Auf gutem Kurs", Mannheim.

Euractiv (2008): Energie-Abkommen: EU beschließt 10\%-Ziel für ,ÖkoKraftstoffe', Downlooad: http://www.euractiv.com/de/verkehr/energie-abkommen-eu-beschliet-10-ziel-oko-kraftstoffe/article-177843, Zugriff: 08.12. 2008.

European Commission (2004): European Energy and Transports Scenarios on Key Drivers (DG TREN), Brüssel.

EWI; Prognos (2006): Auswirkungen höherer Ölpreise auf Energieangebot und -nachfrage. Ölpreisvariante der Energiewirtschaftlichen Referenzprognose 2030. Energiewirtschaftliches Institut an der Universität zu Köln, Untersuchung im Auftrag des Bundesministeriums für Wirtschaft und Technologie (BMWi), Köln, Basel.

Faber, A.; Idenburg, A.M.; Wilting, H.C. (2007): Exploring Techno-economic Scenarios in an Input-Output Model. In: Futures, Vol. 39, S. 16-37.

Fagerberg, J. (2003): Schumpeter and the Revival of Evolutionary Economics: an Appraisal of the Literature. In: Journal of Evolutionary Economics, Vol. 13 , S. 125-159.

Fagerberg, J. (2005): Innovation. A guide to the Literature. In: Fagerberg, J.; Mowery; D., Nelson; R. (Hrsg.): The Oxford Handbook of Innovation. Oxford University Press, Oxford, S.1- 25.

Fagerberg, J.; Verspagen, B. (2002): Technology-gaps, Innovation-diffusion and Transformation: an Evolutionary Interpretation. In: Research Policy, Vol. 31, S. 1291-1304.

Festel, G.; Knöll, J.; Götz, H.; Zinke, H. (2004): Der Einfluss der Biotechnologie auf Produktionsverfahren in der Chemieindustrie. In: Chemie Ingenieur Technik, Vol. 76, S. 307-312.

Feuerstein, G. (2007): Steuerungsversuche und Dynamik biotechnischer Innovationen In: Feuerstein, G. (Hrsg): Strategien biotechnischer Innovation. Analysen, Konzepte und empirische Befunde, Hamburg University Press, S. 1130. 
Fier, A.; Heneric, O. (2005): Public R\&D Policy: The Right Turns of the Wrong Screw? The Case of the German Biotechnology Industry. In: ZEW Discussion Paper Nr. 05-60, Mannheim.

Flaschel, E.; Sell, D. (2005): Charme und Chancen der Weißen Biotechnologie. In: Chemie Ingenieur Technik, Vol. 77, S. 1298-1312.

Fleissner, P.; Böhme, W.; Brautzsch, H.-U.; Höhne, H.; Siassi, J.; Stark, K. (1993): Input-Output-Analyse. Eine Einführung in Theorie und Anwendungen, Springer, New York, Wien.

FNR (Fachagentur Nachwachsende Rohstoffe e.V.) (2008): Biokraftstoffe Basisdaten Deutschland, Gülzow

FNR (Fachagentur Nachwachsende Rohstoffe)(2007): Daten und Fakten zu nachwachsenden Rohstoffen, Gülzow.

Fraunhofer ISI (2006): Working Paper Work Package 1: Identification and characterization of Environmental Technologies, EU-Projekt FORASSET, Fraunhofer ISI, Karlsruhe.

Freeman, C. (1982): The Economics of Industrial Innovation, 2. Auflage, London.

Freeman, C. (1987). Technology Policy and Economic Performance. Lessons from Japan, Pinter Publishers, London.

Freeman, C. (1994): The Economics of Technical Change: In: Cambridge Journal of Economics, Vol. 18, S. 463-514.

Freeman, C. (1995): Technological Revolutions: historical Analogies. In: Fransman, M.; Junne, G.; Roobeck, A. (Hrsg.): The Biotechnology Revolution, Oxford, Blackwell, S. 7-24.

Freeman, C. (2003): Policies for Developing New Technologies, SPRU Electronic Working Paper Series, Nr. 98.

Freeman, C.; Clark, J.; Soete, L. (1982): Unemployment and Technical Innovation., Pinter Publishers, London.

Freeman, C.; Louca, F. (2001): As Time Goes By: From the Industrial Revolution to the Information Revolution, Oxford University Press, Oxford.

Freeman, C.; Perez, C. (1988): Structural Crises of Adjustment, Business Cycles and Investment Behaviour. In: Dosi, G.; Freeman, C.; Nelson, R.; Silverberg; G.; Soete; L. (Hrsg.): Technical Change and Economic Theory, Pinter Publishers, London, S. 38-66.

Freeman, C.; Soete, L. (1987): Technical Change and Full Employment, Basil Blackwell, Oxford. 
Freeman, C.; Soete, L. (1994): Work for All or Mass Unemployment? Computerised Technical Change into the Twenty-first Century, Pinter Publishers, London.

Freeman, C.; Soete, L. (1997): The Economics of Industrial Innovation. 3. Auflage, Pinter Publishers, London.

Fröhlich, D.; Gill, C.; Krieger, H. (1992): Quantitative Beschäftigungswirkungen neuer Informationstechnologien in Anwenderbetrieben der Europäischen Gemeinschaft. In: Mitteilungen der Arbeitsmarkt- und Berufsforschung, Nr. 2, S. 192-200.

Frohn, J.; Leuchtmann, U.; Kräussl, R. (1998): Fünf makroökonometrische Modelle zur Erfassung der Wirkungen umweltpolitischer Maßnahmen - eine vergleichende Betrachtung. Band 7 der Schriftenreihe "Beiträge zu den umweltökonomischen Gesamtrechnungen", Stuttgart.

Gaisser, S.; Hoogeveen, R.; Hüsing, B. (2002): Überblick über den Stand von Wissenschaft und Technik im produktionsintegrierten Umweltschutz durch Biotechnologie (PIUS-BT), Fraunhofer ISI, Karlsruhe.

Gaisser, S.; Nusser, M.; Reiss, T. (2005): Stärkung des PharmaInnovationsstandortes Deutschland, Stuttgart.

Gambardella, A. (1995), Science and Innovation in the US Pharmaceutical Industry, Cambridge University Press, Cambridge.

Gausemeier, J.; Fink, A.; Schlake, O. (1997): Szenariotechnik. In: v. Westphalen, R. (Hrsg.): Technikfolgenabschätzung, 3. Auflage, München, S. 203221.

Gavrislescu, M.; Chisti, Y. (2005): Biotechnology - A sustainable Alternative for Chemical Industry. In: Biotechnology Advances, Vol. 23, S. 471- 499.

Gerybadze, A. (1982), Innovation, Wettbewerb und Evolution: Eine mikro- und mesoökonomische Untersuchung des Anpassungsprozesses von Herstellern und Anwendern neuer Produzentengüter, J.C.B. Mohr (Paul Siebeck), Tübingen.

Gordon, R. J. (2003): Hitech Innovation and Productivity Growth: Does Supply Create Its Own Demand? Ín: NBER Working Papers, Nr. 9437.

Greulich, M. (2001): Die Homogenität ausgewählter Wirtschaftszweige der NACE Rev. 1. In: Wirtschaft und Statistik, Nr. 08/2001, S.615-632.

Groenberg, R. (1980): Diagnose und Prognose von Veränderungen der Inputkoeffizienten in offenen Input-Output-Systemen, Augsburg.

Grossman G., M.; Helpman E. (1994): Endogenous Innovation in the Theory of Growth. In: Journal of Economic Perspectives, Vol. 8, S. 3-22.

Grossman, G.M. and Helpman, E. (1991): Innovation and Growth in the Global Economy, The MIT Press, Cambridge, MA. 
Grupp, H. (1997): Messung und Erklärung des Technischen Wandels. Berlin, Springer, Heidelberg.

Guerrieri, P.; Padoan, P.C: (2007): Modelling ICT as a General Purpose Technology, Evaluation Models and Tools for Assessment of Innovation and Sustainable Development at the EU Level, Collegium, Nr. 35.

Hagemann, H. (1985): Freisetzungs- und Kompensationseffekte neuer Technologien. In: Butler, F.; Kühl, B. (Hrsg.): Rahmen, Staat und Beschäftigung, Beiträge zur Arbeitsmarkt und Berufsforschung Nr. 88, Nürnberg, S. 291335.

Hagemann, H. (1995): Technological Unemployment. In: Arestis, P.; Marshall, M. (Hrsg.): The Political Economy of Full Employment. Conservatism, Corporatism and Institutional Change. Aldershot, Avebury, S. 36-53.

Hagemann, H.; Schreyer, M.; Seiter, S. (2001): Produktivität und Wachstum: Lernen von. den USA?. In: Wirtschaft und Gesellschaft, Vol. 27, S. 277-331.

Hagen, R. (2000): New Process to Reduce Cost Price of Polylactide. Chemical Fibres International, Vol. 50, S. 540-542.

Hamelinck, C.; Faaij A. (2006): Outlook for Advanced Biofuels. In: Energy Policy, Vol. 34, S. 3268- 3283.

Hamelinck, C. N.; Hooijdonk, G.; Faaij, A. (2005): Ethanol from Lignocellulosic Biomass: Technoeconomic Performance in Short, Middle and Longterm. In: Biomass and Bioenergy, Vol. 28, S. 384-410.

Harris, R. G. (1998): The Internet as a GPT: Factor Market Implications. In: Helpman, E. (Hrsg.): General Purpose Technologies and Economic Growth, MIT Press, Cambridge, MA, S. 145-166.

Hayashi T.; Masuda, K.; Yamamoto, M. (2008): Estimating CO2 Efficiency of Bio-ethanolProduction. Vortrag präsentiert auf der Konferenz "International Input Output Meeting on Managing the Environment" vom 9.-11.7. 2008, Sevilla.

Heller, M.; Eisenberg, R. (1998): Can Patents Deter Innovation? The Anticommons in Biomedical Research. In: Science, Vol. 280, S. 698-701.

Helpman, E. (1998): Introduction. In: Helpman, E. (Hrsg.): General Purpose Technologies and Economic Growth. MIT Press, Cambridge, S. 1-14.

Helpman, E.; Trajtenberg, M., (1998a): A Time to Sow and a Time to Reap: Growth Based on General Purpose Technologies. In: Helpman, E. (Hrsg.): General Purpose Technologies and Economic Growth. MIT Press, Cambridge, S. 55-84.

Helpman, E.; Trajtenberg, M. (1998b): Diffusion of General Purpose Technologies. In: Helpman, E. (Hrsg.): General Purpose Technologies and Economic Growth, MIT Press, Cambridge, S. 85-120. 
Henderson, R.; Orsenigo, L.; Pisano, G. P. (1999): The Pharmaceutical Industry and the Revolution in Molecular Biology. In: Mowery, D. C.; Nelson, R. R. (Hrsg.): Sources of Industrial Leadership: Studies of seven Industries. Cambridge, S. 267-309.

Heneric, O. (2006): Herausforderung Biotechnologie: Eine empirische Untersuchung von staatlicher Förderung, Humankapital und Venture Capital, Nomos Verlagsgesellschaft, Baden-Baden.

Henke, J. M. (2005): Biokraftstoffe - Eine weltwirtschaftliche Perspektive. Kieler Arbeitspapiere Nr. 1236.

Henniges, O. (2007): Die Bioethanolproduktion - Wettbewerbsfähigkeit in Deutschland unter Berücksichtigung der internationalen Konkurrenz. Josef Eul Verlag, Hohenheim.

Hepperle, G. (2004): Zukunftsorientierte Industriepolitik, Möglichkeiten und Grenzen. Hohenheimer Volkswirtschaftliche Schriften, Peter Lang, Frankfurt.

Herrera S. (2004): Industrial Biotechnology: A Chance at Redemption. In: Nature Biotechnology, Vol. 22, S. 671-675.

Hess, J. R.; Wright, C. T.; Kenney, K. L. (2007): Cellulosic Biomass Feedstocks and Logistics for Ethanol Production. In: Biofuels, Bioproduction, Biorefineries, Vol. 1, S. 181-190.

Hetmeier, H.-W.; Göbel, W.; Brugger, P. (1995): Ausgaben für biotechnologische Forschung. Metzler-Poeschel, Statistisches Bundesamt, Wiesbaden.

Hicks, J. R. (1932): The Theory of Wages. Macmillan, London.

Hicks, J. R. (1962): Value and Capital: An Inquiry into some Fundamental Principles of Economic Theory, Clarendon Press, Oxford.

Hicks, J. R. (1973): Capital and Time, Oxford.

Hine, D.; Kapeleris, J. (2006): Innovation and Entrepreneurship in Biotechnology, An International Perspective, Edward Elgar, Cheltenham.

Hirooka, M. (2006): Innovation Dynamism and Economic Growth: A nonlinear Perspective, Edward Elgar, Cheltenham.

Holub, H.-W.; Schnabl, H. (1994): Input-Output-Analyse. Oldenburg München, Wien.

Holub, H.-W.; Schnabl, H. (1994): Input-Output-Rechnung: Input-OutputAnalyse, Oldenbourg, München, Wien.

Holwegler, B. (2003): Innovation, Diffusion und Beschäftigung: Die ökonomische Theorie der Technologiediffusion und ihr Beitrag zur Erklärung technologischer Arbeitslosigkeit; Berlin. 
Hopkins, M.; Martin, P.; Nightingale, P.; Kraft, A.; Mahdi, S. (2007): The Myth of the Biotech Revolution: An assessment of Technological, Clinical and Organisational Change. In: Research Policy, Vol. 36, S. 566-589.

Hoppenheidt, K.; Mücke, W.; Peche, R.; Tronecker, D.; Roth, U.; Würdinger, E.; Hottenroth, S.; Rommel, W. (2005): Entlastungseffekte für die Umwelt durch Substitution konventioneller chemisch technischer Prozesse und Produkte durch biotechnische Verfahren. Forschungsbericht für das Umweltbundesamt, Nr. 07/05, Berlin.

Howell, D. R. (1985): The future Employment Impacts of Industrial Robots. In: Technological Forecasting and Social Change, Vol. 28, S. 297-310.

Hüsing, B.; Angerer, G.; Gaisser, S.; Marscheider-Weidemann, F. (2003): Biotechnologische Herstellung von Wertstoffen unter besonderer Berücksichtigung von Energieträgern und Biopolymeren aus Reststoffen. Forschungsbericht Nr. 64/03, Umweltbundesamt, Berlin.

Hüsing, B.; Gießler, S.; Jaeckel, G. (1998): Stand der Möglichkeiten von prozeßintegrierten biotechnischen Präventivtechniken zur Vermeidung oder zur Verminderung von Umweltbelastungen. UBA-Texte 68/98. Umweltbundesamt, Berlin.

IBTF (Industrial Biotechnology Task Force) (2004): Industrial Biotechnology: Delivering Sustainability and Competitiveness. A Draft Report. www.dti.uk, Zugriff: 15.02.2006.

Idenburg, A.M. (1993): Gearing Production Models to Ecological Economic Analysis. A Case Study, within the Input-Output Framework, of Fuels for Road Transport, Universiteit Twente, Enschede.

IEA (2008): World Energy Outlook, Paris.

IFEU (2007): Nachwachsende Rohstoffe für die chemische Industrie: Optionen und Potenziale für die Zukunft. Studie des Instituts für Energie- und Umweltforschung Heidelberg $\mathrm{GmbH}$.

IKB/Prognos (2007): Die Gesundheitsbranche: Dynamisches Wachstum im Spannungsfeld von Innovation und Intervention. In: Report Gesundheitswesen, Düsseldorf.

IPTS (2002a): Impact of Technological and Structural Change on Employment: Prospective Analysis 2020. Background Report, Sevilla.

IPTS (2002b): The Assessment of Future Environmental and Economic Impacts of Process-Integrated Biocatalysts.

IPTS (2007): Consequences, Opportunities and Challenges of Modern Biotechnology for Europe, Bio4EU Synthesis Report, http://bio4eu.jrc.es/ documents/eur22728en.pdf). Zugriff: 06.11.2007. 
Iten, R.; Peter, M.; Walz, R.; Menegale, S.; Blum, M. (2005): Auswirkungen des Umweltschutzes auf BIP. Beschäftigung und Unternehmen. In: UmweltMaterialien, Nr. 197. Bundesamt für Umwelt, Wald und Landschaft, Bern.

Johansen, L. (1972): Production Functions: An Integration of Micro and Macro, Short Run and Long run Aspects. North Holland Publishing Company, Amsterdam.

Joppi, R.; Bertele, V.; Garattini, S. (2005): Disappointing Biotech. In: British Medical Journal, Vol. 331, S. 895-897.

Jürgens, U.; Sablowski, T. (2005): Die Vielfalt sektoraler Innovationsprozesse Pharmaindustrie, Telekommunikation, Autoindustrie. In: WSI-Mitteilungen, 3/2005, S. 121-129.

Kaeb, H. (2006): Biokunststoffe \& Bioverpackungen. Informationen des Verbandes European Bioplastics für einen parlamentarischen Abend im Dezember 2006, Berlin.

Kaldor, N. (1966): Causes of the Slow Rate of Growth of the United Kingdom, Cambridge University Press, Cambridge.

Kaldor, N. (1972): The Irrelevance of Equilibrium Economics. In: Economic Journal, Vol. 82, S. 1237-1255.

Kalmbach, P. (1992): The Impact of New Technologies on Employment: The State of the Art and Perspectives for Research. In: Clauser, O. (Hrsg.): Technological Innovation, Competitiveness, and Economic Growth. Berlin, S. 169-185.

Kalmbach, P. mit "Forschungsgruppe Technologischer Wandel und Beschäftigung" (1989): Mikroelektronik und Beschäftigung: Eine Untersuchung der Auswirkungen des Einsatzes programmgesteuerter Arbeitsmittel unter Verwendung eines dynamischen Input-Output-Modells, Bremen.

Kalmbach, P.; Franke, R.; Knottenbauer, K.; Kraemer, H.; Schaefer, H. (2003): Die Bedeutung einer wettbewerbsfähigen Industrie für die Entwicklung des Dienstleistungssektors: Eine Analyse der Bestimmungsgründe der Expansion industrienaher Dienstleistungen in modernen Industriestaaten. http://www. bmwi.de/BMWi/Redaktion/PDF/C-D/die-bedeutung-einer-wettbewerbsfaehigen-industrie-fuer-die-entwicklung-des-dienstleistungssektors, property= pdf,bereich=bmwi,sprache=de,rwb=true.pdf, Zugriff: 20.02.2005.

Kalmbach, P.; Kurz, H. D. (1992): Chips und Jobs. Zu den Beschäftigungswirkungen des Einsatzes programmgesteuerter Arbeitsmittel, Metropolis, Marburg.

Kaplinsky, R. (1987): Micro-Electronics and Employment Revisited: A Review, ILO, Genf. 
Katsoulacos, Y. (1986): The Employment Effect of Technical Change. A theoretical Study of new Technology and the Labour Market, Wheatsheaf, Brighton.

Kemnitz, D. (2008): Neue Biokraftstoffe aktueller Stand und künftige Optionen. Vortrag VDLUFA Kongress Jena - 17.09.2008.

Kern, S.; Enzing, C. (2003): The Interplay between national, sectoral and technological Determinants Innovation Systems in Europe: The Case of Biotechnology. Papier eingereicht für die "International Conference on Innovation in Europe: Dynamics, Institutions and Values" at the Roskilde University, 09.05.2003.

Kinkel, S.; Friedewald, M.; Hüsing, B.; Lay, G.; Lindner, R. (2008): Arbeiten in der Zukunft: Strukturen und Trends der Industriearbeit, Edition Sigma, Berlin.

Klauder, W. (1986): Technischer Fortschritt und Beschäftigung. In: Mitteilungen der Arbeitsmarkt- und Berufsforschung, Nr. 1, S. 1-19.

Kline, S. J.; Rosenberg, N. (1986): An Overview of Innovation. In Landau, R.; Rosenberg, N. (Hrsg.): The Positive Sum Strategy: Harnessing Technology for Economic Growth, National Academy Press, Washington D.C, S. 275304.

Knottenbauer, K. (2000): Theorien des sektoralen Strukturwandels. Metropolis, Marburg.

Köhler, J. (2005): Making (Kondratiev) Waves: Simulating long run Technical Thange. In: Dewick, P.; Miozzo, M.; Green, K. (Hrsg.): Technology, Knowledge and the Firm: Implications for Strategy and Industrial Change. Edward Elgar, Cheltenham, S. 404-426.

Kranich, J. (2007): Biotechnologie und Internationalisierung, VDM Verlag Dr. Müller, Saarbrücken.

Kriegesmann B.; Kerka F.; Sieger C. (2005): Zukunftsperspektiven der Biotechnologie - Nur Umsetzungseliten schaffen Wachstum. In: Innovation: Forschung und Management, Band 24, Bochum.

Kuznets, S. (1972): Economic growth of nations, Belknap Press, Cambridge.

La Merie (2008): TOP 20 Biologics 2007/R\&D Pipeline News. A premier Information Source of Biopharmaceutical R\&D.

Lange, K. S. G. (2006): Deutsche Biotech-Unternehmen und ihre Innovationsfähigkeit im internationalen Vergleich - eine institutionentheoretische Analyse.

Lehman-Waffenschmidt, B. C. (2006): Industrieevolution und die New Economy: Eine evolutorische Analyse der Beziehung zwischen New Economy und Old Economy aus simulationsanalytischer und wirtschaftshistorischer Perspektive, Metropolis, Marburg. 
Lehr, U.; Nitsch, J.; Kratz, M.; Lutz, C.; Edler, D. (2008): Renewable Energy and Employment in Germany. In: Energy Policy, Vol. 36, S. 108-117.

Leontief, W. (1966): Input-Output Economics, Oxford University Press, New York.

Leontief, W. (1982): Werkzeuge, Maschinen, Computer, Roboter. Die Folgen für Arbeitsplätze und Einkommensverteilung. In: Spektrum der Wissenschaft, Nr. 11, S. 146-156.

Leontief, W.; Duchin, F. (1986): The Future Impact of Automation on Workers, Oxford University Press, Oxford.

Lim, D.; Choi, Y. (2006): The Economic Impact of Biotechnology and Bioindustry in Korea: Methodologies and Implications for the Bio-industry in Korea. In: Industrial Economic Review, Vol. 9, S. 25-42.

Lipsey, R. G.; Bekar, C.; Carlaw, K. (1998): What requires Explanation? In: Helpman, E. (Hrsg.): General purpose technologies and economic growth, MIT Press, Cambridge, S. 14-54.

Lipsey, R.; Carlaw, L.; Bekar, C. (2005): Economic Transformations: General Purpose Technologies and Long Term Economic Growth, Oxford University Press, Oxford.

Llerena, P.; Lorentz, A. (2004): Alternative Theories on Economic Growth and the Co-evolution of Macro-Dynamics and Technological Change: A Survey. In: LEM Discussion Paper Series, Nr. 2003/27.

Löschel, A. (2002): Technological Change in Economic Models of Environmental Policy: A Survey. In: Ecological Economics, Vol. 43, S. 105-126.

Lowe, A. (1976): The Path of Economic Growth, Cambridge University Press, Cambridge.

Lundvall, B. A. (1992): National Systems of Innovation. Towards a Theory of Innovation and Interactive Learning, London.

Lundvall, B. A. (1988). Innovation as an Interactive Process: From Userproducer Interaction to the national System of Innovation. In: Dosi, G.; Freeman, C.; Nelson, R.; Silverberg; G.; Soete; L. (Hrsg.): Technical Change and Economic Theory, Pinter Publishers, London, S. 349-369.

Luukkonen, T.; Palmberg, C. (2007): Living up to the Expectations Set by ICT? The Case of Biotechnology Commercialisation in Finland. In: Technology Analysis \& Strategic Management, Vol. 19, S. 329-349.

Lynd, L. R. (1996): Overview and Evaluation of Fuel Ethanol from Cellulosic Biomass: Technology, Economics, the Environment and Policy. In: Energy Environment, Vol. 21, S. 403-465.

Lynd, L. R.; Laser, M. S.; Bransby, D.; Dale, B. E.; Davison, B.; Hamilton, R.; Himmel, M.; Keller, M.; McMillan, J. D.; Sheehan, J.; Wyman; C. E. (2008): 
How Biotech can Transform Biofuels. In: Nature Biotechnology, Vol. 26, S. 169-172.

Malerba, F. (2002): Sectoral Systems of Innovation and Production. In: Research Policy, Vol. 32, S. 247-254.

Marschall, L. (2005): Weiße Biotechnologie: Eine zweite Chance für eine alte Technik. In: GAIA, Vol. 14, S. 314-322.

Marscheider-Weidemann, F.; Hüsing, B. (2004): Abfallvermeidung bei Produktionen für organische Spezialchemikalien durch den Einsatz hochspezifischer Katalysatoren. Forschungsbericht für das Umweltbundesamt, Nr. 21/04, Berlin.

Marx, Karl (19872): Das Kapital, 2.Auflage, Lawrence and Wishart, London.

Matzner, E. (1990): The Employment Impact of New Technology: The Case of West Germany, Aldershot, Avebury.

McKelvey, M. (1996): Evolutionary Innovations - The Business of Biotechnology. Oxford:

McKelvey, M. (2007): Biotechnology Industries. In: Hanusch, H.; Pyka, A. (Hrsg.): Companion to Neo-Schumpeterian Economics, Edward Elgar, Cheltenham.

McKelvey, M. (2008): Health Biotechnology: Emerging Business Models and Institutional Drivers. In: OECD International Futures Project on "The Bioeconomy to 2030: Designing a Policy Agenda", Paris.

McKinsey (2006): Industrial Biotechnology -- Turning Potential into Profits, Plenary at the annual World Congress on Industrial Biotechnology and Bioprocessing in Toronto 18.07.2006.

Meade, D. (1995): The Impact of the Electric Car on the U. S. Economy: 19982005. In: Economic Systems Research, Vol. 7, S. 413-438.

Meiß, K.-M.; Eisenberg, W.; Gustrau-Wissing, M. (2003): Implementationsstudie zur biotechnologischen Produktion von Biopolymeren unter Einsatz digitaler Modelle auf der Basis nachwachsender Rohstoffe und organischer Abfälle. Forschungsbericht für das Umweltbundesamt, Nr. 38/03, Berlin.

Melzig- Thiel, B. (2000): Arbeit in der Informationsgesellschaft. Frankfurt am Main.

Menrad, K.; Blind K.; Frietsch R.; Hüsing B.; Nathani C.; Reiss T.; Strobel O., Walz R., Zimmer R. (2003): Beschäftigungspotenziale in der Biotechnologie, Stuttgart.

Metcalfe, S. (1998): Evolutionary Economics and Creative Destruction, Routledge, London. 
Mettelsiefen, B.; Barens, I. (1987): Direkte und indirekte Beschäftigungswirkungen technologischer Innovationen. In: Beiträge zur Arbeitsmarkt- und Berufsforschung, Nürnberg, Nr. 112.

Meyer, R.; Grunwald, A.; Rösch, C.; Sauter; A. (2007): Chancen und Herausforderungen neuer Energiepflanzen. Basisanalyse. Arbeitsbericht für das Büro für Technikfolgen-Abschätzung beim Deutschen Bundestag, Berlin, Nr. 121.

Meyer-Krahmer, F. (1999): Innovation als Beitrag zur Lösung von Beschäftigungsproblemen? In: Mitteilungen aus der Arbeitsmarkt- und Berufsforschung, Vol. 32, S. 402-415.

Miernyk, W. (1975): The Projection of Technical Coefficients for MediumTerm Forecasting. In: Gossling, W. (Hrsg.): Medium-Term Dynamic Forecasting: The 1975 London Input-Output Conference, William Clowes \& Sons Limited, London.

Mietzsch, A. (2008): Biotechnologie. Das Jahr- und Adressbuch 2008, BIOCOM, Berlin.

Mill, J. St. (1848): Principles of Political Economy. With some of their Applications to Social Philosophy. London, reprint 1976, Fairfield.

Miller, R. E.; Blair, P.D. (1985): Input-Output Analysis: Foundations and Extensions. Prentice Hall, Englewood Cliffs, NJ.

Moser, P.; Nicholas, T. (2004): Was electricity a General Purpose Technology? In: The American Economic Review, Papers and Proceedings, Vol. 94, S. 388-394.

Mühlbauer; U. (2006): Die Machbarkeit von Milchsäure- und Polylactidanlagen: Vortrag auf der Tagung "auf Bio-raffiniert IIl«, Vortragsfolien, Gelsenkirchen, 2.-3. Februar 2006.

Mussatto, S. I.; Dragone, G.; Roberto, I. C. (2006): Brewers' spent grain: Generation, Characteristics and Potential Applications. In: Journal of Cereal Science, Vol. 43, S. 1-14.

Müssig, J.; Carus, M. (2007): Bio-Polymerwerkstoffe sowie holz- und naturfaserverstärkte Kunststoffe. In: Fachagentur Nachwachsende Rohstoffe e. V. (FNR) (Hrsg.): Marktanalyse- Nachwachsende Rohstoffe Teil II. Gutachten für das Bundesministerium für Ernährung, Landwirtschaft und Verbraucherschutz, Gülzow, S. 7-216.

MWV (2006): MWV-Prognose 2025 für die Bundesrepublik Deutschland, Hamburg.

MWV (2008): Zusammensetzung des Preises für Superbenzin 1972 bis 2007, http://www.mwv.de/cms/front_content.php?idcat=14\&idart=56, Zugriff: 10.10.2008. 
Nathani, C. (2003): Modellierung des Strukturwandels beim Übergang einer materialeffizienten Kreislaufwirtschaft. Kopplung eines Output-Modells mit einem Stoffstrommodell am Beispiel der Wertschöpfungskette, Physica Verlag, Heidelberg.

Neisser, H. (1942): Permanent Technological Unemployment. In: American Economic Review, Vol. 32, S. 50-71.

Nelson, R. (1988). Institutions upporting Technical Change in the United States. In: Dosi, G.; Freeman, C.; Nelson, R.; Silverberg; G.; Soete; L. (Hrsg.): Technical Change and Economic Theory. Pinter, London, S 312-329.

Nelson, R.; Winter, S.G. (1982): An Evolutionary Theory of Economic Change, The Belknap Press of Harvard University Press, Cambridge.

Neuwahl, F.; Löschel, A.; Mongelli, I.; Delgado, L. (2007): Employment Impacts of EU Biofuels Policy: Combining Bottom-up Technology Information and sectoral Market Simulations in an Input-Output Framework. Papier präsentiert auf der Input-Output Konferenz in Istanbul, 02-06 Juli 2007.

Neuwahl, F.; Löschel, A.; Mongelli, I.; Delgado, L. (2008): Employment Impacts of EU Biofuels Policy: Combining Bottom-up Technology Information and sectoral Market Simulations in an Input-Output Framework, Ecological Economics, S. 447-460.

Nightingale, P.; Martin, M., (2004): The Myth of the Biotech Revolution. In: Trends in Biotechnology, Vol. 22, S. 564-569.

Nordhoff, S.; Höcker, H.; Gebhardt, H. (2007): Nachwachsende Rohstoffe in der chemischen Industrie - Weg vom Öl?. In: Chemie Ingenieur Technik 79, S. 551-560.

Nusser, M. (2000): Innovative Wachstumsprozesse und zunehmende strukturelle Arbeitslosigkeit: Komplementäre Entwicklungsprozesse?. TENEA Verlag für Medien, Berlin.

Nusser, M.; Soete, B.; Wydra, S. (2007a): Wettbewerbsfähigkeit und Beschäftigungspotentiale der Biotechnologie in Deutschland. Edition der Hans Böckler Stiftung, Nr. 197, Düsseldorf.

Nusser, M.; Hüsing, B.; Wydra; S. (2007b): Potenzialanalyse der industriellen, weißen Biotechnologie. Studie im Auftrag des Bundesministeriums für Bildung und Forschung (BMBF). www.innovationsanalysen.de/de/download/ endbericht_weisse_biotechnologie_final.pdf, Zugriff: 05.07.2007:

Nusser, M.; Wydra, S.; Hartig, J.; Gaisser, S. (2007c): Forschungs- und wissensintensive Branchen: Optionen zur Stärkung ihrer internationalen Wettbewerbsfähigkeit- Innovationsreport; Arbeitsbericht Nr. 116, Berlin.

Nusser, M.; Sheridan, P.; Walz, R.; Seydel, P.; Wydra, S. (2007d): Makroökonomische Effekte des Anbaus und der Nutzung von nachwachsenden Roh- 
stoffen. Studie für das Bundesministerium für Ernährung, Landwirtschaft und Verbraucherschutz vertreten durch die Fachagentur Nachwachsende Rohstoffe e.V., Gülzow.

OECD (1982): Micro-Electronics, Robotics and Jobs, Paris.

OECD (1988): New Technologies in the 1990s. A Socio-Economic Strategy. Paris.

OECD (1989) Biotechnology: Economic and wider Impacts, Paris.

OECD (1992): Technology and the Economy. The Key Relationships, Paris.

OECD (1994), The OECD Jobs Study: Evidence and Explanations, Vol. I, Paris.

OECD (1996): Technology, Productivity and Job Creation. Vol. 2, Analytical Report, Paris.

OECD (1997): Oslo Manual: Proposed Guidelines for Collecting and Interpreting Technological Innovation Data, Paris.

OECD (1998): Biotechnology for clean Industrial Products and Processes. Towards Industrial Sustainability, Paris.

OECD (2001): The Application of Biotechnology to Industrial Sustainability, Paris.

OECD (2004): The Economic Impacts of Biotechnology - An Introduction, OECD Workshop on the Economic Impacts of Biotechnology, 26. Mai 2004, Paris.

OECD (2005a): A Framework for Biotechnology Statistics, Paris.

OECD (2005b): The OECD Health Project- Health Technology and Decision Making, Paris.

OECD (2006a): Second Workshop on Measuring the Impacts of Biotechnology, 11. Dezember 2006, Paris.

OECD (2006b): Biotechnology Statistics, OECD, Paris.

OECD (2006c): Innovation in Pharmaceutical Biotechnology. Comparing national Innovation Systems at the sectoral Level, Paris.

OECD (2008a): Compendium of Patent Statistics 2006, Paris.

OECD (2008b): Draft Analytical Report on Current Status and Trends in Research and Development for Industrial Biotechnology, Paris.

OECD (2008c): Rising Agricultural Prices: Causes, Consequences and Responses, Policy Brief, August 2008.

OECD (2008d): Economic Assessment of Biofuel Support Policies, Paris.

OECD (2008e): OECD Health Policy Studies - Pharmaceutical Pricing Policies in a Global Market, Paris. 
OECD; FAO (2007): OECD-FAO Agricultural Outlook 2007-2016, Organisation for Economic Co-operation and Development, Paris; UN Food and Agriculture Organization, Rome.

OECD; FAO (2008): OECD-FAO Agricultural Outlook 2008-2017, Organisation for Economic Co-operation and Development, Paris; UN Food and Agriculture Organization, Rome.

Oertel, D. (2007): Industrielle stoffliche Nutzung nachwachsender Rohstoffe. Sachstandsbericht zum Monitoring "Nachwachsende Rohstoffe«. Arbeitsbericht für das Büro für Technikfolgen-Abschätzung beim Deutschen Bundestag, Nr. 114, Berlin.

Orsenigo L. (1989): The Emergence of Biotechnology: Institutions and Markets in Industrial Innovation. London.

Osborne, S. (2007): Energy in 2020: Assessing the Economic Effects of Commercialization of Cellulosic Ethanol. In: Manufacturing and Services Competitiveness Report November 2007, US. Department of Commerce, International Trade Administration, Washington.

OTA (Office of Technology Assessment) (1991): Biotechnology in a Global Economy, OTA-BA 494, DC US Government Printing Office, Washington.

Otero, G. (1995): The coming Revolution of Biotechnology: a Critique of Buttel. In: Fransman, M.; Junne, G.; Roobeck, A. (Hrsg.): The Biotechnology Revolution, Blackwell, Oxford, S. 46-62.

Ott, I.; Papilloud, C.; Zülsdorf, T. (2008): What drives Innovation? Causes of and Consequences for Nanotechnologies. In: Kiel Working Papers, Nr. 1455, Kiel.

Palmberg ,C.; Nikulainen, T. (2006): Nanotechnology as a General Purpose Technology? - Insights from a Finnish viewpoint. In: Kalvet, T.; Kattel, R. (Hrsg,): Creative Destruction Management in Central and Eastern Europe: Meeting the Challenges of the Techno-Economic Paradigm shift, Talinn, S.51-72.

Pan, H. (2006): Dynamic and endogenous Change of Input-Output Structure under specific Layers of Technology. In: Structural Change and Economic Dynamics, Vol. 17, S. 200-223.

Paris, V.; Docteur, E. (2008): Pharmaceutical Pricing and Reimbursement Policies in Germany. In: OECD Health Working Papers, Nr. 39, Paris.

Pasinetti, L. (1981): Structural Change and Economic Growth, Cambridge University Press, Cambridge.

Pasinetti, L. L. (1993): Structural Economic Dynamics, Cambridge University Press Cambridge. 
Patel, M.; Crank, M.; Dornburg, V.; Hermann, B.; Roes, L.; Hüsing, B.; Overbeek, L.; Terragni, F.; Recchia, E. (2006): Medium and Long-term Opportunities and Risks of the Biotechnological Production of Bulk Chemicals from Renewable Resources. The Potential of White Biotechnology, Utrecht University, Department of Science, Technology and Society (STS)/Copernicus Institute, Utrecht.

Patel, P.; Arundel, A.; Hopkins, M. (2008): Sectoral Innovation Systems in Europe: Monitoring, Analysing Trends and Identifying Challenges in Biotechnology. Sector Report. Final Report. Europe Innova.

Patel, P.; Pavitt, K. (1995): Patterns of technological Activity: their Measurement and Interpretation. In: Stoneman, P. (Hrsg.): Handbook of the Economics of Innovation and Technical Change. Blackwell,Oxford.

Paulus, A. (2005) : Ökonomische und ökologische Effekte der Nutzung des Werkstoffs Aluminium: Ein Beitrag zur Berücksichtigung der Nutzungsphase in der modellgestützten Stoffstromanalyse, http://deposit.ddb.de/cgibin/dokserv?idn=979705290, Zugriff: 17.06.2006.

Pavitt, K. (1984): Sectoral Patterns of Technical Change. In: Research Policy, Vol. 13, S. 343-373.

Perez, C. (1983): Structural Change and the Assimilation of new Technologies in the Economic and Social Systems. In: Futures, Vol. 15, S. 357-375.

Perez, C. (2003): Technological Revolutions and Financial Capital: The dynamics of Bubbles and Golden Ages, Edward Elgar, Cheltenham.

Peter, V. (2002): Institutionen im Innovationsprozess - Eine Analyse anhand der biotechnologischen Innovationssysteme in Deutschland und Japan, PhysicaVerlag, Heidelberg.

Peters, D. (2006): Kohlenhydrate als Fermentationsrohstoff. In: Chemie Ingenieur Technik, Vol. 78, S. 229-238.

Petit, P. (1995): Employment and Technological Change. In: Stoneman, P. (Hrsg.): Handbook of the Economics of Innovation and Technological Change, North Holland, Amsterdam, S. 366-408.

Pfaffenberger W. (1995): Arbeitsplatzeffekte von Energiesystemen. In: Energiewirtschaftliche Studien, Band 6 der Vereinigung Deutscher Elektrizitätswerke, Frankfurt am Main.

Pflaum, H.,. Nusser, M.; Hartig, J.; Hüsing, B.; Kabasci, S.; Merrettig-Bruns, U.; Rettweiler, M.; Sayder, B.; Selt, J.; Schnell, U.; Wydra; S. (2008): Anwendungspotenziale der Bioverfahrenstechnik (weiße Biotechnologie) in Nordrhein-Westfalen, Karlsruhe.

Phylipsen, D.; Kerssemeeckers, M.; Blok, K.; Patel, M.; de Beer, J., Eder, P.;Wolf, O. (2002): Clean Technologies in the materials Sector-Current and 
future Environmental Performance of material Technologies. European Commission - Institute for Prospective Technological Studies (IPTS), EUR 20515 EN Seville.

Pianta, M. (2000): The Employment Impact of Product and Process Innovations. In: Vivarelli; M. ; Pianta, M. (Hrsg.): The Employment Impact of Innovation. Evidence and Policy, Routledge, London, S. 77-95.

Pianta, M. (2005): Innovation and employment. In: Fagerberg, J.; Mowery; D., Nelson; R. (Hrsg.): The Oxford Handbook of Innovation. Oxford University Press ,Oxford, S. 568-598.

Pini P. (1995): Economic Growth, Technological Change and Employment: Empirical Evidence for a Cumulative Growth Model with External Causation for nine OECD Countries, 1960-1990. In: Structural Change and Economic Dynamics, Vol. 6, S. 185-213.

Pini, P. (1996): An Integrated Cumulative Growth Model: Empirical Evidence for Nine OECD countries, 1960-1990. In: Labour, Vol. 10, S. 93-150.

Pisano, G. (2006a): Can Science Be a Business? Lessons from Biotech. In: Harvard Business Review, 84, S. 114-125.

Pisano, G.P. (2006b): Science Business: The Promise, the Reality, and the Future of Biotech. Harvard Business School Press.

PlasticsEurope(2007): The Compelling Facts About Plastics 2007: An aAnalysis of Plastics Production, Demand and Recovery for 2006 in Europe, Download: www.plasticseurope.org, Zugriff: 30.10.2007.

Posner, M. V (1961): International Trade and Technical Change. In: Oxford Economic Papers, Vol. 31, S. 323-341.

Productivity Commission (2005): Impacts of Advances in Medical Technology in Australia. In: Productivity Commission Research Report, Melbourne.

Prognos (2002): Deutschland Report 2020, Basel.

Prognos (2006): Deutschland Report 2030, Basel.

Pyka, A. (1999): Der kollektive Innovationsprozess, Duncker \& Humblot, Berlin.

Rammer, C.; Ohmstedt, J.; Binz, H.; Heneric, O. (2006): Unternehmensgründungen in der Biotechnologie in Deutschland 1991 bis 2004. In: ZEW Working Paper, Nr. 06-03, Mannheim.

Reineke, R. D.; von Armansperg, M. (2006): Farben und Lacke. In: Fachagentur nachwachsende Rohstoffe e. V. (FNR) (Hrsg.): Marktanalyse - Nachwachsende Rohstoffe Teil II. Gutachten für das Bundesministerium für Ernährung, Landwirtschaft und Verbraucherschutz, Gülzow, S. 307-332. 
Reinhardt, U. E. (2001): Perspectives on the Pharmaceutical Industry. In: Health Affairs, Vol. 20, S. 1363-1370.

Reiss, T.; Gaisser, S.; Buehrlen, B.; Enzing, C.; van der Giessen, A.; Arundel, A.; Bordoy, C.; Cozzens, S.; Catalán, P.; Gatchair, S.; Ordónez, G. (2006): Consequences, Opportunities and Challenges of modern Biotechnology for Europe (Bio4EU) - Task 1: A preparatory Study mapping modern Biotechnology Applications and Industrial Sectors, Identifying Data Needs and developing Indicators, http://bio4eu.jrc.ec.europa.eu/documents/Bio4EUTask1.pdf, Zugriff: 09.02.2007.

Reiss, T.; Gaisser, S.; Dominguez Lacasa, I.; Buehrlen, B.; Schiel, B.; Enzing, C. und 30 weiteren Autoren (2007): Consequences, opportunities and challenges of modern biotechnology for Europe (Bio4EU) - Task 2. Main report. http://bio4eu.jrc.ec.europa.eu/documents/Bio4EU-Task2Mainreport.pdf, Zugriff: 25.02.2008.

Reiss, T.; Hinze, S.; Dominguez Lacasa, I.; Mangematin, V.; Enzing, C.; van der Giessen, A.; Kern, S.; Senker, J.; Calvert, J.; Nesta, L.; Patel, P. (2003): Efficiency of Innovation Policies in High Technology Sectors in Europe (EPOHITE), Office for Official Publications of the European Communities, Luxembourg.

Reiss, T.; Koschatzky, K. (1997): Biotechnologie. Unternehmen, Innovationen, Förderinstrumente, Heidelberg.

Renneberg, R.. (2006): Biotechnologie für Einsteiger, Spektrum, Heidelberg.

Ricardo, D. (1821): On the Principles of Political Economy and Taxation. 3. Auflage. In: Sraffa, P. (Hrsg.): The Works and Correspondence if David Ricardo, Band 1, 3. Auflage Cambridge, 1973.

Richter, J. (1991): Aktualisierung und Prognose technischer Koeffizienten in gesamtwirtschaftlichen Input-Output-Modellen, Heidelberg.

Roco, M. C.; Bainbridge, W. S. (2003): Converging Technologies for Improving Human Performance: Nanotechnology, Biotechnology, Information Technology and Cognitive Science. Kluwer, Dordrecht.

Roco, M. C.; Montemagno, C. D. (2004): The Coevolution of Human Potential and Converging Technologies, Annals of the New York Academy of Sciences 1013, New York.

Rogers, E. M. (1995): Diffusion of Innovations, 4. Auflage, Free Press New York; London; Toronto.

Roland Berger (2007): Umweltpolitische Innovations- und Wachstumsmärkte aus Sicht der Unternehmen. In: Reihe „Umwelt, Innovation und Beschäftigung“ des BMU/UBA, Berlin. 
Roobeck, A. (1995): Biotechnology: A Core Technology in a new technoeconomic Paradigm. In: Fransman, M.; Junne, G.; Roobeck, A. (Hrsg.): The Biotechnology Revolution, Blackwell, Oxford, S. 62-84.

Rösch, C.; Jörissen, J.; Skarka, J.; Hartlieb, N. (2008): Flächennutzungskonflikte: Ursachen, Folgen und Lösungsansätze. Einführung in den Schwerpunkt. In: Technikfolgenabschätzung - Theorie und Praxis, Vol. 17, S. 4-11.

Rose, A. (1984): Technological Change and Input-Output Analysis: An Appraisal, Socio-Economic Planning. In: Science, Vol. 18, S. 305-318.

Rose, A. (1995): Input-output economics and computable general equilibrium models. In: Structural Change and Economic Dynamics, Vol. 6, S. 295-304.

Rose, A. (2000): A Challenge for Measuring Biotechnology Activities. In: De La Mothe J.; Niosi J. (Hrsg.): The Economic and Social Dynamics of Biotechnology, Kluwer Academic Publishers, Boston, S.71-82.

Rose, A.; McNiven, C. (2007): Biotechnology: From Measures of Activities, Linkages and Outcomes to Impact Science, Technology and Innovation Indicators in a Changing World: Responding to Policy Needs is a selection of the papers discussed at the OECD Blue Sky II Forum, Paris, S. 215-230.

Rosenberg, N. (1982): Inside the Black Box: Technology and Economics, Cambridge University Press, Cambridge.

Rosenberg, N. (1998): Chemical Engineering as a General Purpose Technology. In: Helpman, E. (Hrsg.): General Purpose Technologies and Economic Growth, MIT Press, Cambridge, S. 167-192.

Rosenberg-Yunger, Z. R. S.; Daar, A. S.; Singer, P. A.; Martin, D. K. (2008): Healthcare Sustainability and the Challenges of Innovation to Biopharmaceuticals in Canada. In: Health Policy, Vol. 87, S. 359-368.

Russell, A. (1999): Biotechnology as a Technological Paradigm in the Global Knowledge Structure. In: Technology Analysis and Strategic Management, Vol. 11, S. 235-254.

Ruttan, V. W. (1999): Biotechnology and Agriculture: A Skeptical Perspective. In: AgBioForum, Vol. 2, S. 54-60.

Ruttan, V. W. (2001): Technology, Growth and Development: An Induced Innovation Perspective, Oxford University Press, New York.

Ruttan, V. W. (2002): The Role of the Public Sector in Technology Development: Generalizations from General Purpose Technologies. In: Staff Paper Series, Department of Applied Economics College of Agricultural, Food and Environmental Sciences University of Minnesota, Nr. P01-11, St. Paul.

RWI (2005): Beschäftigungswirkungen von Forschung und Innovation, Essen.

Sagar, A. D.; Kartha. S. (2007): Bioenergy and sustainable Development? Annual Review of Environmental Resources, Vol. 32, S.131-167 
Say, J. B. (1819): Traité d'Economie Politique, 4. Auflage, Paris (1. Auflage 1803).

Schmitz, N. (2005): Innovationen bei der Bioethanolerzeugung. Schriftenreihe „Nachwachsende Rohstoffe“, Band 26, Landwirtschaftsverlag, Münster.

Schmookler, J. (1966): Invention and Economic Growth, Harvard University Press, Cambridge, Mass.

Schöpe, M.; Britschkat, G. (2006): Volkswirtschaftliche Effekte der Erzeugung von Bioethanol zum Einsatz im Kraftstoffbereich. In: Ifo Schnelldienst, Nr. 21, München, S. 27-36.

Schumpeter, J. A. (1934): Theorie der wirtschaftlichen Entwicklung: Eine Untersuchung über Unternehmensgewinn, Kapital, Kredit, Zins und den Konjunkturzyklus. Berlin.

Schwitalla, B. (1993): Messung und Erklärung industrieller Innovationsaktivitäten - mit einer empirischen Analyse für die westdeutsche Industrie, PhysicaVerlag, Heidelberg:

Searchinger, T.; Heimlich, R.; Houghton, R.A.; Dong, F.; Elobeid, A.; Fabiosa, J.; Tokgoz, S.; Hayes, D.; Yu, T. (2008): Use of U.S. Croplands for Biofuels increases Greenhouse Gases through Emissions from Land Use Change. In: Science, Vol. 319, S. 1238-1240.

Seiter, S. (1997): Der Beitrag Nicholas Kaldors zur Neuen Wachstumstheorie: Eine vergleichende Studie vor dem Hintergrund der Debatte über den Verdoorn-Zusammenhang, Lang, Frankfurt am Main.

Senker, J.; Reiss, T.; Mangematin, V.; Enzing, C. (2007): The Effects of national Policy on Biotechnology Development: The Need for a broad Policy Approach. In: International Journal of Biotechnology, Vol. 9, S. 20-38.

Silverberg, G. (1997): Evolutionary Modeling in Economics: Recent History and Immediate Prospects. Paper prepared for the workshop "Evolutionary Economics as a Scientific Research Programme," Mai 1997, Stockholm.

Silverberg, G.; Verspagen, B. (2005): Evolutionary theorizing on economic Growth. In: Dopfer, K. (Hrsg.): The Evolutionary Foundations of Economics, University Press, Cambridge, S. 506-539.

Sirilli, G. (1998): Conceptualising and Measuring technological Innovation. IDEA Report 1/1998.

Smith, K. (2001): Assessing the Economic Impact of ICT. Papier präsentiert auf der "Conference on the Future of Innovation Studies", Eindhoven Centre for Innovation Studies, 21-23 September, Eindhoven.

Soete, B. (2006): Biotechnologie im Vergleich - Wo steht Deutschland? Eine Untersuchung nationaler Innovationssysteme, Edition der Hans Böckler Stiftung 165, Düsseldorf. 
Solow, R. M. (1956): A Contribution to the Theory of Economic Growth. In: Quarterly Journal of Economics, Vol. 71, S. 65-94.

Solow, R. M. (1957), Technological Change and the Aggregate Production Function. In: Review of Economics and Statistics, Vol. 39, S. 312-320.

Spezia, V.; Vivarelli, M. (2002): Innovation and Employment: A critical Survey. In: Greenan, N.; L'Horty, Y.; Mairesse, J. (Hrsg.): Productivity, inequality, and the Digital Economy: A Transatlantic Perspective, MIT Press, Cambridge, S. 101-131.

SRU (Sachverständigenrat für Umweltfragen) (2007): Klimaschutz durch Biomasse, Sondergutachten, Erich Schmidt Verlag GmbH \& Co., Berlin.

Stahmer, C.; Bleses, P.; Meyer, B. (2000): Input-Output-Rechnung: Instrumente zur Politikberatung. Statistisches Bundesamt, Wiesbaden.

Statistics Canada (2005): Bioproducts Development Survey, The Daily, 16.05.2005, Ottawa.

Statistics Canada (2007): Biotechnology Use an Development Survey 2003, The Daily, 30.01.2007, Ottawa.

Statistisches Bundesamt (2005): Unternehmen der Biotechnologie in Deutschland. Ergebnisse der Wiederholungsbefragung 2004. Wiesbaden

Statistisches Bundesamt (2006): Produzierendes Gewerbe, Fachserie 4, Reihe 4.1.1., Wiesbaden.

Statistisches Bundesamt (2008a): Volkswirtschaftliche Gesamtrechnungen, Fachserie 18 Reihe 1.4, Inlandsproduktsberechnung - Detaillierte Jahresergebnisse 2007, Wiesbaden.

Statistisches Bundesamt (2008b): Fachserie 4 Reihe 3.1: Produktion im produzierenden Gewerbe. Wiesbaden.

Statistisches Bundesamt (2008c): Volkswirtschaftliche Gesamtrechnungen, Fachserie 18, Reihe 2, Input-Output-Tabellen 2005, Wiesbaden.

Statistisches Bundesamt (2008d): Produzierendes Gewerbe, Fachserie 4, Reihe 4.1.1. Wiesbaden.

Steininger, K.; Kettner, C.; Kufleitner, A.; Loibnegger, T.; Pack, A.; Schleicher, S.; Töglhofer, C.; Trink, T. (2008): Volkswirtschaftliche Effekte einer erweiterten Biomasse-Energie-Nutzung in der Energieregion Oststeiermark. In: Wissenschaftlicher Bericht des Wegener Zentrum für Klima und Globalen Wandel, Karl-Franzens-Universität Graz, Nr. 21-2008, Graz.

Steuart, J. (1767): An Inquiry into the Principles of Political Oeconomy. Skinner, A. S., Edinburgh, London, 1966.

Stifterverband Wissenschaft (2008): Zahlen und Fakten aus der Wissenschaftsstatistik GmbH Stifterverband, www.stifterverband.de, Zugriff: 08.08.2008. 
Stiglitz, J. E. (1987): Learning to Learn, Localized Learning and Technological Progress. In: Dasgupta, P.; Stoneman, P. (Hrsg.): Economic Policy and Technological Performance, Cambridge University Press, Cambridge, S. 125-153.

Stille, F.; Bitzer, J. (1998): Beschäftigungswirkungen von Innovationen: Analysen zu einem komplizierten Verhältnis. In: Lehner, F.; Baethge, M.; Kühl, J.; Stille, F. (Hrsg.): Beschäftigung durch Innovation. Eine Literaturstudie, Mering, München, S. 15-58.

Stoneman, P. (1983): The Economic Analysis of Technological Change, Oxford University Press, Oxford.

Suresh, P.; Basu, P. K. (2008): Improving Pharmaceutical Product Development and Manufacturing: Impact on Cost of Drug Development and Cost of Goods Sold of Pharmaceuticals. In: Journal of Pharmaceutical Innovation, Vol. 3, S. 175-187.

Swann, P. G. M.; Prevezer, M.; Stout, D. (1998): The Dynamics of Industrial Clustering. International Comparisons in Computing and Biotechnology, University Press, Oxford.

Sylos Labini, P. (1969): Oligopoly and Technical Progress, Harvard University Press, Cambridge, 1. Auflage von 1956.

Taheripour, F.; Birur, D. K.; Hertel, T. W.; Tyner, W. E. (2008): Introducing Liquid Biofuels into the GTAP Data Base. In: GTAP Research Memorandum Nr. 11, Purdue.

Tait, J. (2007): Systemic Interactions in Life Science Innovation. In: Technology Analysis and Strategic Management, Vol. 19, S. 257-277.

Tait, J.; Chataway, J.; Wield, D.; Bruce, A. (2007): Human Health Scenarios. In: OECD International Futures Project on "The Bioeconomy to 2030: Designing a Policy Agenda", Paris.

Temple, J. (1999): The New Growth Evidence. In: Journal of Economic Literature, Vol. 37, S. 112-156.

Ten Raa, T. (2006): The Economics of Input-Output analysis, Cambridge University Press Cambridge.

Tether, B. S. (2003): What is Innovation? Approaches to Distinguishing New Products and Processes from Existing Products and Processes. In: CRIC Working Paper, Nr. 12.

Thrän, D.; Weber, M.; Scheuermann, A.; Fröhlich, N.; Zeddies, J.; Henze, A.; Thoroe, C.; Schweinle, J.; Fritsche, U. R.; Jenseit, W.; Rausch, L.; Schmidt, K. (2005): Nachhaltige Biomassenutzungsstrategien im europäischen Kontext. Analysen im Spannungsfeld nationaler Vorgaben und der Konkurrenz 
zwischen festen, flüssigen und gasförmigen Bioenergieträgern. Institut für Energetik und Umwelt, Leipzig.

Tilanus, C. B. (1968): Input-Output Experiments : The Netherlands 1948-1961, Rotterdam University Press, Rotterdam.

Toro, F. (2004): Techno-Economic Assessment of Biofuel Production in the European Union, Fraunhofer ISI, Karlsruhe.

Urbanchuk, J. M. (2007): Contribution of the Ethanol Industry to the Economy of the United States. Report of the Renewable Fuels Association, http://www.ethanolrfa.org/resource/reports/,Zugriff:17.04.2008.

VCI (Verband der Chemischen Industrie) (2008): Chemiewirtschaft in Zahlen: Ausgabe 2008, Frankfurt/Main.

Vernon, R. (1966): International Investment and International Trade in the Product Cycle. In: Quarterly Journal of Economics, Vol. 80, S. 190-207.

Verspagen, B. (2001): Economic Growth and Technological Change: An Evolutionary Interpretation. In: OECD Science, Technology and Industry Working Papers 2001/1, OECD Directorate for Science, Technology and Industry, Paris.

Verspagen, B. (2002): Evolutionary Macroeconomics: A Synthesis between neo-Schumpeterian and post-Keynesian Lines of Thought. In: The Electronic Journal of Evolutionary Modeling and Economic Dynamics, Nr. 1007, http://www.e-jemed.org/1007/index.php., Zugriff:13.06.2006.

VFA (Verband forschender Arzneihersteller e.V.) (2007): Statistics 2007. Die Arzneimittelindustrie in Deutschland, Berlin.

VFA (Verband forschender Arzneihersteller e.V.) (2008): Statistics 2008. Die Arzneimittelindustrie in Deutschland, Berlin.

Villeval, M. (2002): Comments on Chapter 3. In: Greenan, N.; L'Horty, Y.; Mairesse, J. (Hrsg.): Productivity, inequality, and the Digital Economy: A Transatlantic Perspective, MIT Press, Cambridge, S. 133-137

Vivarelli , M. (2007): Innovation and Employment: A Survey, IZA Discussion Paper, Nr. 2621, Bonn.

Vivarelli, M. (1995): The Economics of Technology and Employment: Theory and Empirical Evidence, Aldershot, Avebury.

Vivarelli, M.; Pianta, M. (2000): The Employment Impact of Innovation: Evidence and Policy, Routledge, London.

VKE (2007): Kunststoffmarkt - Einsatzgebiete, Download: www.vke.de, Zugriff: 20.11.2007. 
Vogel, C. (2000): Deutschland im internationalen Technologiewettlauf. Bedeutung der Forschungs- und Technologiepolitik für die technologische Wettbewerbsfähigkeit, Duncker \& Humblot, Berlin.

Von Armansperg, M. (2006): Chemie. In: Fachagentur Nachwachsende Rohstoffe e. V. (FNR) (Hrsg.): Marktanalyse- Nachwachsende Rohstoffe Teil I. Gutachten für das Bundesministerium für Ernährung, Landwirtschaft und Verbraucherschutz, Gülzow.

Von Armansperg, M.; Patel, M. (2007): Weiße Biotechnologie. In: Fachagentur nachwachsende Rohstoffe e. V. (FNR) (Hrsg.): Marktanalyse- Nachwachsende Rohstoffe Teil II. Gutachten für das Bundesministerium für Ernährung, Landwirtschaft und Verbraucherschutz, Gülzow, S. 217-332.

Von Tunzelmann, N. (1995): Technology and Industrial Progress. The Foundations of Economic Growth. Edward Elgar, Cheltenham.

Vosskamp, R.; Schmidt-Ehmcke, J. (2006): Die Beiträge von Forschung, Entwicklung und Innovation zu Produktivität und Wachstum, Schwerpunktstudie zur »Technologischen Leistungsfähigkeit Deutschlands«. Berlin.

Wagner, S.; Graf, N.; Böchzelt, H.; Schnitzer, H. (2005): Nachwachsende Rohstoffe für die chemische Industrie. In: Berichte aus Energie- und Umweltforschung, Nr. 30/2005. Gutachten im Auftrag des BMVIT, Wien.

Walter, A.; Rosillo-Calle, F.; Dolzan, P.; Piacente, E.; Borges da Cunha, K. (2008): Perspectives on Fuel Ethanol Consumption and Trade. In: Biomass and Bioenergy, Vol. 32, S. 730-748.

Walter, H. (1969): Der technische Fortschritt in der neueren ökonomischen Theorie: Versuch einer Systematik, Duncker \& Humblot, Berlin.

Walz, R. (1999): Productivity Effects of Technology Diffusion induced by an Energy Tax. In: Energy \& Environment, Vol. 10, S. 169-180.

Walz, R. (2002): Nachhaltige Entwicklung in Deutschland. Operationalisierung, Präzisierung der Anforderungen und Politikfolgenabschätzung, Freiburg.

Walz, R. (2006): Impact of Strategies to Increase RES in Europe on Employment and Competitiveness, In: Energy \& Environment 17, S. 951-975.

Walz, R.; Dreher, C.; Marscheider-Weidemann, F.; Nathani, C.; Schirrmeister, E.; Schleich, J.;Schneider, R; Schön, M. (2001): Arbeitswelt in einer nachhaltigen Wirtschaft: Analyse der Wirkungen von Umweltschutzstrategien auf Wirtschaft und Arbeitsstrukturen. Umweltbundesamt, Texte Nr. 44/01, Berlin.

Walz, R.; Schleich, J. (2009): The Economics of Climate Change Policies Macroeconomic Effects, Structural Adjustments and Technological Change, Physica, Heidelberg, im Erscheinen. 
Warhust, A. (1995): Metal biotechnology: Trends and Implications. In: Fransman, M.; Junne, G.; Roobeck, A. (Hrsg.): The Biotechnology Revolution, Blackwell, Oxford, S. 258-273.

WBA (Wissenschaftlicher Beirat Agrarpolitik) (2007): Nutzung von Biomasse zur Energiegewinnung - Empfehlungen an die Politik. Gutachten vom November 2007, Berlin.

Welsch, J. (2006): Paradigmenwechsel beim Zusammenhang von Innovation und Beschäftigung. In: Wirtschaftsdienst, Nr. 12, S. 811-820.

Whitley, J. D.; Wilson, R. A. (1982): Quantifying the Employment Effects of Micro-electronics. In: Futures, Vol. 14, S. 486-495.

Whitley, J.D.; Wilson, R. A. (1987): Quantifying the Impact of Information Technology on Employment Using a Macroeconomic Model of the United Kingdom Economy. In: OECD, ICCP Paper, Nr. 12, Information Technology and Economic Prospects, Paris.

WI; RWI (2008): Nutzungskonkurrenzen bei Biomasse - Auswirkungen der verstärkten Nutzung von Biomasse im Energiebereich auf die stoffliche Nutzung in der Biomasse verarbeitenden Industrie und deren Wettbewerbsfähigkeit durch staatlich induzierte Förderprogramme. Ein Studie des Wuppertal Instituts für Klima, Umwelt, Energie $\mathrm{GmbH}$ (WI) und des RheinischWestfälischen Institut für Wirtschaftsforschung (RWI) für das Bundesministerium für Wirtschaft und Technologie (BMWi). Endbericht, 25. April 2008, Wuppertal, Essen.

Wicke, B. (2006): The Socio-Economic Impacts of Large-Scale Land Use Change and Export-Oriented Bio-Energy Production in Argentina, Utrecht.

Widmann, B.; Remmele, E. (2008): Biokraftstoffe - Fragen und Antworten, Technologie- und Förderzentrum im Kompetenzzentrum für Nachwachsende Rohstoffe, Weihenstephan.

Wieland, T. (2003): Paths of New Technology: Nuclear Power Reactors, Biotechnology, and West German S\&T Policy, Forschungsverbund Innovationskultur in Deutschland, Arbeitspapier 09/2003, München.

Wilting, H. C; Faber, A.; Idenburg, A. M. (2008): Investigating new Technologies in a Scenario Context: Description and Application of an Input-Output Method. In: Journal of Cleaner Production, Vol.16, S.102-112.

Wonglimpiyarat, J. (2008): Biotech Revolution: The Impact of Biotechnology on the Pharmaceutical Industry. In: International Journal of Technology, Policy and Management, Vol. 8, S. 182-193.

Youtie, J.; Iacopetta, M.; Graham, S. (2007): Assessing the nature of nanotechnology: Can we uncover an emerging General Purpose Technology?. In: Journal of Technology Transfer, Vol. 32, S. 123-130. 
Zeddies, G., (2006): Gesamtwirtschaftliche Effekte der Förderung regenerativer Energien, insbesondere der Biomasse - Eine kritische Beurteilung vor dem Hintergrund modelltheoretischer Konzeptionen, Universität Hohenheim, Institut für Landwirtschaftliche Betriebslehre, Forschungsbericht 3/2006, Stuttgart-Hohenheim. 
Sven Wydra - 978-3-631-75392-7

Downloaded from PubFactory at 01/11/2019 05:32:25AM

via free access 


\section{Anhang}

Tabelle 31: Kennzahlen zur Biotechnologie - Branche in Deutschland 20052007

\begin{tabular}{|l|c|c|c|c|c|c|c|c|}
\hline & \multicolumn{3}{|c|}{$\begin{array}{c}\text { Anzahl von Unter- } \\
\text { nehmen }\end{array}$} & \multicolumn{3}{c|}{$\begin{array}{c}\text { Anzahl von Mitar- } \\
\text { beiter }\end{array}$} & \multicolumn{2}{c|}{$\begin{array}{c}\text { Umsatz in } \\
\text { Mrd.EUR }\end{array}$} \\
\hline $\begin{array}{l}\text { Unterneh- } \\
\text { mens-typ }\end{array}$ & 2005 & 2006 & 2007 & 2005 & 2006 & 2007 & 2006 & 2007 \\
\hline $\begin{array}{l}\text { Kernbiotech- } \\
\text { no-logie- } \\
\text { unternehmen }\end{array}$ & 480 & 495 & 496 & 12973 & 14150 & 14360 & 1,76 & 2,01 \\
\hline $\begin{array}{l}\text { Diversifizierte } \\
\text { Unternehmen }\end{array}$ & 59 & 56 & 91 & 10856 & 14800 & 15210 & n.v. & n.v. \\
\hline
\end{tabular}

Quelle: Biotechnologie.de 2006, 2007, 2008 (für 2005 liegen keine Daten zu Umsätzen und F\&E-Aufwendungen vor)

Tabelle 32: Verwendete Preis- und Einkommenselastizitäten für die Berechnung der Konsumvektoren

\begin{tabular}{|l|c|c|}
\hline & $\begin{array}{c}\text { Einkommenselastizi- } \\
\text { täten }\end{array}$ & Preiselastizitäten \\
\hline Landwirtschaft & 0,414 & $-0,73$ \\
\hline Getränke und Nahrungsmitttel & 0,610 & $-0,73$ \\
\hline Textil & 0,760 & $-0,73$ \\
\hline Kraftstoffe & 0,964 & $-0,13$ \\
\hline Andere Industriegüter & 0,869 & $-0,73$ \\
\hline Dienstleistungen & 1,224 & $-0,35$ \\
\hline
\end{tabular}

Quelle: Neuwahl et al. (2007) 
AMGEN GmbH; ARTES Biotechnology GmbH; BASF SE; Baxter Deutschland GmbH; Bayer Technology Services GmbH; Biomer GmbH; Bitop AG; Boehringer Ingelheim Pharma GmbH \& Co. KG; BTU Cottbus; Cargill Deutschland $\mathrm{GmbH}$; ChemCon $\mathrm{GmbH}$; Ciba Inc.; Cognis Oleochemicals GmbH; DIREVO Biotech AG; Dow Deutschland Anlagengesellschaft mbH; DSM N.V.; Eucodis GmbH; European Chemical Industry Council (Cefic); Evonik Degussa GmbH; FKuR Kunststoff GmbH; Fraunhofer UMSICHT; GlaxoSmithKline GmbH \& Co. KG; Janssen-Cilag GmbH; Lanxess AG; LINDE AG; Lonza AG; Merck KGaA; MorphoSys AG; Novozymes A/S; Rentschler Biotechnologie $\mathrm{GmbH}$; Sächsisches Institut für Angewandte Biotechnologie e.V. ; Sandoz Industrial Products GmbH; Sanofi Pasteur MSD GmbH; Sanofi-Aventis Deutschland GmbH; Sigma-Aldrich Inc.; Universität Uutrecht; Verband Der Chemischen Industrie e.V. (VCI); Wacker Chemie AG

Tabelle 34: Zusätzlicher Flächenbedarf durch die Verwendung von Bioethanol

\begin{tabular}{|c|c|c|c|c|c|c|}
\hline & \multirow[t]{2}{*}{ Rohstoff } & $\begin{array}{l}\text { Rohstoff- } \\
\text { menge }\end{array}$ & Ertrag & $\begin{array}{l}\text { Flächen- } \\
\text { bedarf }\end{array}$ & $\begin{array}{l}\text { Anteil an } \\
\text { Weizenflä- } \\
\text { che (Stand } \\
2005)\end{array}$ & $\begin{array}{l}\text { Anteil Flä- } \\
\text { che Land- } \\
\text { wirtschaft } \\
\text { (Stand } \\
\text { 2005) }\end{array}$ \\
\hline & & (in Mio t) & (t/ha) & (in Mio. ha) & (in ha) & (in\%) \\
\hline \multirow{2}{*}{ 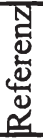 } & Weizen & 3,4 & $9,6-10,3$ & $0,33-0,36$ & $10,6-11,4 \%$ & $2,8-3,0 \%$ \\
\hline & $\begin{array}{l}\text { Zuckerrü- } \\
\text { be }\end{array}$ & 2,4 & $69,4-83,3$ & $0,03-0,04$ & $6,9-8,3 \%$ & $0,2-0,3 \%$ \\
\hline \multirow[b]{2}{*}{ 旺 } & Weizen & 4,1 & $9,6-10,3$ & $0,40-0,43$ & $12,8-13,8 \%$ & $3,4-3,6 \%$ \\
\hline & $\begin{array}{l}\text { Zuckerrü- } \\
\text { be }\end{array}$ & 2,8 & $69,4-83,3$ & $0,03-0,04$ & $8,0-9,6 \%$ & $0,3 \%$ \\
\hline
\end{tabular}

Quelle: Eigene Berechnungen; Datenbasis: BMELV (2007), Thrän et al. (2005), Nusser et al. (2007d), WI/RWI (2008) 
Tabelle 35: Zusätzlicher Flächenbedarf durch die Verwendung von Biopolymeren

\begin{tabular}{|l|l|l|l|l|l|l|}
\hline & Rohstoff & $\begin{array}{l}\text { Rohstoff- } \\
\text { menge }\end{array}$ & Ertrag & $\begin{array}{l}\text { Flächenbe- } \\
\text { darf }\end{array}$ & $\begin{array}{l}\text { Anteil an } \\
\text { Weizenflä- } \\
\text { che (Stand } \\
\text { 2005) }\end{array}$ & $\begin{array}{l}\text { Anteil Flä- } \\
\text { che Land- } \\
\text { wirtschaft } \\
\text { (Stand } \\
2005)\end{array}$ \\
\cline { 3 - 7 } & (in Mio t) & (t/ha) & (in Mio. ha) & (in ha) & (in\%) \\
\hline $\begin{array}{l}\text { Refe } \\
\text { renz }\end{array}$ & Weizen & 2,20 & $9,6-10,3$ & $0,22-0,23$ & $7,0-7,5 \%$ & $1,8-2,0 \%$ \\
\hline HD & Weizen & 3,70 & $9,6-10,3$ & $0,36-0,38$ & $11,4-12,2 \%$ & $3,0-3,2 \%$ \\
\hline
\end{tabular}

Quelle: Eigene Berechnungen; Datenbasis: BMELV (2007), Thrän et al. (2005), Nusser et al. (2007d), WI/RWI (2008) 


\section{Tabelle 36: Sektorale Produktionseffekte für Bioethanol im Referenzszena- rio (im Vergleich zum Basisszenario)}

\begin{tabular}{|c|c|c|c|c|c|c|}
\hline & Ethanol & $\begin{array}{l}\text { Etha- } \\
\text { nol } \\
\text { Invest. }\end{array}$ & \begin{tabular}{|l|} 
Substi- \\
tut \\
Benzin
\end{tabular} & $\begin{array}{l}\text { Substi- } \\
\text { tut } \\
\text { Invest. }\end{array}$ & \begin{tabular}{|l|} 
Negati- \\
ver \\
Konsum
\end{tabular} & $\begin{array}{l}\text { Ge- } \\
\text { samt }\end{array}$ \\
\hline & Mio. $€$ & Mio. $€$ & Mio. $€$ & Mio. $€$ & Mio. $€$ & Mio. $€$ \\
\hline I Erzeugnisse der Landwirtschaft und Jagd & 479,4 & 0,0 & 73,8 & 0,0 & 2,8 & 402,9 \\
\hline 2 Forstwirtschaftliche Erzeugnisse und DL & 0,9 & 0,1 & 0,2 & 0,0 & 0,1 & 0,7 \\
\hline 3 Fische und Fischereierzeugnisse & 0,0 & 0,0 & 0,0 & 0,0 & 0,0 & 0,0 \\
\hline 4 Kohle und Torf & 3,1 & 0,0 & 3,5 & 0,0 & 0,5 & $-0,8$ \\
\hline 5 Erdöl, Erdgas, DL fur Erdől-, Erdgasgewinnung & 0,4 & 0,1 & 1,0 & 0,0 & 0,3 & $-0,8$ \\
\hline 6 Uran- und Thoriumerze & 0,0 & 0,0 & 0,0 & 0,0 & 0,0 & 0,0 \\
\hline 7 Erze & 0,0 & 0,0 & 0,0 & 0,0 & 0,0 & 0,0 \\
\hline 8 Steine und Erden, sonstige Bergbauerzeugnisse & 2,2 & 0,2 & 0,2 & 0,0 & 0,2 & 2,1 \\
\hline 9 Nahrungs- und Futtermittel & 0,4 & 0,0 & 5,8 & 0,0 & 9,3 & $-14,7$ \\
\hline 10 Getränke & 0,1 & 0,0 & 0,0 & 0,0 & 2,1 & $-2,0$ \\
\hline 11 Tabakerzeugnisse & 0,0 & 0,0 & 0,0 & 0,0 & 0,5 & $-0,5$ \\
\hline 12 Textilien & 0,1 & 0,1 & 0,0 & 0,0 & 0,4 & $-0,3$ \\
\hline 13 Bekleidung & 0,1 & 0,0 & 0,0 & 0,0 & 0,8 & $-0,8$ \\
\hline 14 Leder und Lederwaren & 0,0 & 0,0 & 0,0 & 0,0 & 0,2 & $-0,2$ \\
\hline 15 Holz; Holz-, Kork-, Flechtwaren (o. M.) & 1,4 & 1,0 & 0,3 & 0,1 & 0,7 & 1,3 \\
\hline 16 Holzstoff, Zellstoff, Papier, Karton, Pappe & 0,9 & 0,2 & 0,2 & 0,0 & 0,5 & 0,4 \\
\hline 17 Papier-, Karton- und Pappewaren & 2,2 & 0,2 & 0,3 & 0,0 & 0,8 & 1,3 \\
\hline 18 Verlagserzeugnisse & 1,0 & 0,1 & 0,4 & 0,0 & 2,7 & $-2,0$ \\
\hline $\begin{array}{l}19 \text { Druckerzeugnisse, bespielte Ton-, Bild- und } \\
\text { Datenträger }\end{array}$ & 1,8 & 0,3 & 0,7 & 0,0 & 1,8 & $-0,4$ \\
\hline $\begin{array}{l}20 \text { Kokereierzeugnisse, Mineralolerzeugnisse, Spalt- } \\
\text { und Brutstoffe }\end{array}$ & 673,8 & 0,4 & 684,3 & 0,0 & 33,2 & $-43,4$ \\
\hline 21 Pharmazeutische Erzeugnisse & 1,4 & 0,0 & 0,2 & 0,0 & 0,8 & 0,4 \\
\hline $\begin{array}{l}22 \text { Chemische Erzeugnisse (o. pharmazeutische } \\
\text { Erzeugnisse) }\end{array}$ & 53,7 & 1,2 & 4,2 & 0,1 & 2,6 & 48,0 \\
\hline 23 Gummiwaren & 0,5 & 0,2 & 0,2 & 0,0 & 0,2 & 0,2 \\
\hline 24 Kunststoffwaren & 2,5 & 1,2 & 0,5 & 0,1 & 1,1 & 2,0 \\
\hline 25 Glas und Glaswaren & 1,8 & 0,2 & 0,3 & 0,0 & 0,2 & 1,4 \\
\hline 26 Keramik, bearbeitete Steine und Erden & 3,3 & 1,1 & 0,7 & 0,1 & 0,6 & 3,1 \\
\hline 27 Roheisen, Stahl, Rohre und Halbzeug daraus & 2,1 & 1,9 & 1,0 & 0,1 & 0,7 & 2,1 \\
\hline 28 NE-Metalle und Halbzeug daraus & 0,4 & 0,4 & 0,1 & 0,0 & 0,1 & 0,5 \\
\hline 29 Gießereierzeugnisse & 0,9 & 0,7 & 0,2 & 0,1 & 0,2 & 1,1 \\
\hline 30 Metallerzeugnisse & 6,3 & 5,9 & 3,1 & 0,5 & 2,2 & 6,5 \\
\hline 31 Maschinen & 28,8 & 24,3 & 3,0 & 1,9 & 1,7 & 46,5 \\
\hline $\begin{array}{l}32 \text { Buromaschinen, Datenverarbeitungsgerăte u. - } \\
\text { einrichtungen }\end{array}$ & 0,3 & 1,1 & 0,2 & 0,1 & 0,2 & 1,0 \\
\hline 33 Gerăte der Elektrizitătserzeugung, -verteilung u.a. & 5,0 & 6,8 & 2,6 & 0,5 & 1,3 & 7,3 \\
\hline $\begin{array}{l}34 \text { Nachrtechn., Rundf.- und Fernsehgerăte, elekt- } \\
\text { ron. Bauelemente }\end{array}$ & 0,3 & 3,2 & 0,1 & 0,2 & 0,5 & 2,6 \\
\hline $\begin{array}{l}35 \text { Medizin-, mess-, regelungstechn., optische Er- } \\
\text { zeugnisse; Uhren }\end{array}$ & 0,4 & 5,2 & 0,3 & 0,4 & 0,6 & 4,4 \\
\hline $36 \mathrm{Kraftwagen}$ und Kraftwagenteile & 2,8 & 0,2 & 0,8 & 0,0 & 7,8 & $-5,7$ \\
\hline $\begin{array}{l}37 \text { Sonstige Fahrzeuge (Wasser-, Schienen-, Luft- } \\
\text { fahrzeuge u.a.) }\end{array}$ & 0,2 & 0,0 & 0,1 & 0,0 & 0,5 & $-0,4$ \\
\hline 38 Mobel, Schmuck, Musikinstrumente, Sportgeratte, & 0,0 & 2,1 & 0,0 & 0,2 & 1,9 & 0,1 \\
\hline
\end{tabular}




\begin{tabular}{|c|c|c|c|c|c|c|}
\hline & Ethanol & $\begin{array}{l}\text { Etha- } \\
\text { nol } \\
\text { Invest. }\end{array}$ & \begin{tabular}{|l|} 
Substi- \\
tut \\
Benzin \\
\end{tabular} & \begin{tabular}{|l|} 
Substi- \\
tut \\
Invest.
\end{tabular} & \begin{tabular}{|l|} 
Negati- \\
ver \\
Konsum \\
\end{tabular} & $\begin{array}{l}\text { Ge- } \\
\text { samt }\end{array}$ \\
\hline \multicolumn{7}{|l|}{ Spielwaren u.ä. } \\
\hline 39 Sekundärrohstoffe & 0,4 & 0,1 & 0,1 & 0,0 & 0,2 & 0,2 \\
\hline $\begin{array}{l}40 \text { Elektrizităt, Fernwărme, DL der Elektrizităts- u. } \\
\text { Femwärmevers. }\end{array}$ & 49,6 & 0,8 & 42,4 & 0,1 & 5,8 & 2,2 \\
\hline 41 Gase, DL der Gasversorgung & 2,4 & 0,1 & 1,8 & 0,0 & 1,3 & $-0,6$ \\
\hline 42 Wasser und DL der Wasserversorgung & 7,9 & 0,1 & 4,8 & 0,0 & 1,1 & 2,1 \\
\hline $\begin{array}{l}43 \text { Vorb. Baustellenarbeiten, Hoch- } u \text {. Tiefbauarbei- } \\
\text { ten }\end{array}$ & 2,1 & 6,2 & 0,9 & 0,5 & 1,2 & 5,7 \\
\hline 44 Bauinstallations- und sonstige Bauarbeiten & 11,7 & 5,6 & 1,3 & 0,4 & 3,4 & 12,1 \\
\hline $\begin{array}{l}45 \text { Handelsleist. mit Kfz; Rep. an Kfz; Tankleistun- } \\
\text { gen }\end{array}$ & 5,8 & 0,3 & 1,5 & 0,0 & 8,0 & $-3,3$ \\
\hline 46 Handelsvermittlungs- und Großhandelsleistungen & 33,6 & 9,3 & 6,8 & 0,7 & 12,3 & 23,1 \\
\hline $\begin{array}{l}47 \text { Einzelhandelsleistungen; Reparatur an Ge- } \\
\text { brauchsgutern }\end{array}$ & 1,8 & 0,3 & 0,5 & 0,0 & 28,5 & $-26,9$ \\
\hline 48 Beherbergungs- und Gaststătten-DL & 0,2 & 0,0 & 0,1 & 0,0 & 12,5 & $-12,4$ \\
\hline 49 Eisenbahn-DL & 0,7 & 0,1 & 0,4 & 0,0 & 2,0 & $-1,5$ \\
\hline $\begin{array}{l}50 \text { Sonst. Landv.leistungen, Transportl. in Rohrfern- } \\
\text { leitungen }\end{array}$ & 11,6 & 1,1 & 8,7 & 0,1 & 5,1 & $-1,2$ \\
\hline 51 Schifffahrtsleistungen & 0,6 & 0,1 & 0,5 & 0,0 & 0,2 & 0,0 \\
\hline 52 Luftfahrtleistungen & 1,3 & 0,4 & 0,8 & 0,0 & 2,1 & $-1,2$ \\
\hline $\begin{array}{l}53 \text { DL bezuglich Hilfs- u. Nebentătigkeiten fur den } \\
\text { Verkehr }\end{array}$ & 9,0 & 2,1 & 2,9 & 0,2 & 6,6 & 1,4 \\
\hline 54 Nachrichtenubermittlungs-DL & 5,0 & 1,0 & 2,7 & 0,1 & 12,5 & $-9,3$ \\
\hline 55 DL der Kreditinstitute & 19,4 & 1,5 & 6,4 & 0,1 & 17,2 & $-2,9$ \\
\hline $\begin{array}{l}56 \text { DL der Versicherungen (ohne Sozialversiche- } \\
\text { rung) }\end{array}$ & 9,0 & 0,4 & 2,6 & 0,0 & 13,0 & $-6,2$ \\
\hline 57 DL des Kredit- und Versicherungshilfsgewerbes & 28,4 & 0,3 & 1,2 & 0,0 & 4,4 & 23,0 \\
\hline 58 DL des Grundstucks- und Wohnungswesens & 13,4 & 3,0 & 4,8 & 0,2 & 56,8 & $-45,4$ \\
\hline $\begin{array}{l}59 \text { DL der Vermietung beweglicher Sachen (o. Per- } \\
\text { sonal) }\end{array}$ & 32,1 & 1,6 & 7,0 & 0,1 & 4,4 & 22,2 \\
\hline 60 DL der Datenverarbeitung und von Datenbanken & 2,2 & 13,1 & 1,0 & 1,0 & 1,8 & 11,5 \\
\hline 6I Forschungs- und Entwicklungsleistungen & 0,3 & 0,0 & 0,0 & 0,0 & 0,1 & 0,3 \\
\hline 62 Unternehmensbezogene DL & 67,4 & 8,3 & 25,6 & 0,6 & 23,1 & 26,4 \\
\hline 63 DL der offentlichen Verwaltung, Verteidigung & 5,4 & 0,4 & 3,4 & 0,0 & 2,1 & 0,3 \\
\hline 64 DL der Sozialversicherung & 0,0 & 0,0 & 0,0 & 0,0 & 0,0 & 0,0 \\
\hline 65 Erziehungs- und Unterrichts-DL & 1,5 & 0,3 & 0,9 & 0,0 & 3,3 & $-2,3$ \\
\hline $\begin{array}{l}66 \text { DL des Gesundheits-, Veterinär- und Sozialwe- } \\
\text { sens }\end{array}$ & 4,7 & 0,0 & 0,8 & 0,0 & 11,8 & $-7,8$ \\
\hline $\begin{array}{l}67 \text { Abwasser-, Abfallbeseitigungs- u. sonst. Entsor- } \\
\text { gungsleistungen }\end{array}$ & 5,5 & 0,2 & 1,1 & 0,0 & 4,2 & 0,4 \\
\hline 68 DL von Interessenvertretungen, Kirchen u.ă. & 1,7 & 0,2 & 0,9 & 0,0 & 1,3 & $-0,3$ \\
\hline 69 Kultur-, Sport- und Unterhaltungs-DL & 2,5 & 0,3 & 0,9 & 0,0 & 7,5 & $-5,6$ \\
\hline 70 Sonstige $\mathrm{DL}$ & 2,5 & 0,3 & 0,8 & 0,0 & 6,8 & $-4,8$ \\
\hline 71 DL privater Haushalte & 0,0 & 0,0 & 0,0 & 0,0 & 1,4 & $-1,4$ \\
\hline Summe & 1618,4 & 115,9 & 921,7 & 9,0 & 341,9 & 461,7 \\
\hline
\end{tabular}

Quelle: Eigene Berechnungen

Die Gesamtsumme ergibt sich aus Spalte 1+2 abzüglich den Spalten 3-5. 


\section{Tabelle 37: Sektorale Produktionseffekte für Bioethanol im HD-Szenario (im Vergleich zum Basisszenario)}

\begin{tabular}{|c|c|c|c|c|c|c|}
\hline & Ethanol & $\begin{array}{l}\text { Ethanol } \\
\text { Invest. }\end{array}$ & \begin{tabular}{|l} 
Substi- \\
tut \\
Benzin \\
\end{tabular} & \begin{tabular}{|l|} 
Substi- \\
tut \\
Invest. \\
\end{tabular} & \begin{tabular}{|l|} 
Negati- \\
ver \\
Konsum \\
\end{tabular} & $\begin{array}{l}\text { Ge- } \\
\text { samt }\end{array}$ \\
\hline & Mio. $\epsilon$ & Mio. $€$ & Mio. $\epsilon$ & Mio. $€$ & Mio. $€$ & Mio. $€$ \\
\hline 1 Erzeugnisse der Landwirtschaft und Jagd & 918,7 & 0,0 & 176,6 & 0,0 & 2,5 & 739,6 \\
\hline 2 Forstwirtschaftliche Erzeugnisse und DL & 1,7 & 0,2 & 0,4 & 0,0 & 0,1 & 1,4 \\
\hline 3 Fische und Fischereierzeugnisse & 0,0 & 0,0 & 0,0 & 0,0 & 0,0 & 0,0 \\
\hline 4 Kohle und Torf & 8,8 & 0,1 & 9,0 & 0,0 & 0,4 & $-0,5$ \\
\hline 5 Erdol, Erdgas, DL fur Erdøl-, Erdgasgewinnung & 0,9 & 0,2 & 2,8 & 0,0 & 0,2 & $-1,9$ \\
\hline 6 Uran- und Thoriumerze & 0,0 & 0,0 & 0,0 & 0,0 & 0,0 & 0,0 \\
\hline 7 Erze & 0,0 & 0,0 & 0,0 & 0,0 & 0,0 & 0,0 \\
\hline 8 Steine und Erden, sonstige Bergbauerzeugnisse & 5,5 & 0,5 & 0,4 & 0,0 & 0,1 & 5,4 \\
\hline 9 Nahrungs- und Futtermittel & 0,7 & 0,0 & 13,9 & 0,0 & 8,2 & $-21,4$ \\
\hline 10 Getränke & 0,2 & 0,0 & 0,0 & 0,0 & 1,8 & $-1,7$ \\
\hline 11 Tabakerzeugnisse & 0,0 & 0,0 & 0,0 & 0,0 & 0,4 & $-0,4$ \\
\hline 12 Textilien & 0,3 & 0,1 & 0,1 & 0,0 & 0,4 & 0,0 \\
\hline 13 Bekleidung & 0,1 & 0,0 & 0,0 & 0,0 & 0,7 & $-0,6$ \\
\hline 14 Leder und Lederwaren & 0,0 & 0,0 & 0,0 & 0,0 & 0,2 & $-0,1$ \\
\hline 15 Holz; Holz-, Kork-, Flechtwaren (o. Møbel) & 3,1 & 2,2 & 0,6 & 0,2 & 0,6 & 3,9 \\
\hline 16 Holzstoff, Zellstoff, Papier, Karton, Pappe & 2,1 & 0,3 & 0,4 & 0,0 & 0,4 & 1,6 \\
\hline 17 Papier-, Karton- und Pappewaren & 5,0 & 0,6 & 0,7 & 0,1 & 0,7 & 4,2 \\
\hline 18 Verlagserzeugnisse & 2,2 & 0,3 & 0,8 & 0,0 & 2,4 & $-0,8$ \\
\hline $\begin{array}{l}19 \text { Druckerzeugnisse, bespielte Ton-, Bild- und } \\
\text { Datentrager }\end{array}$ & 3,9 & 0,8 & 1,4 & 0,1 & 1,6 & 1,6 \\
\hline $\begin{array}{l}20 \text { Kokereierzeugnisse, Mineralolerzeugnisse, Spalt- } \\
\text { und Brutstoffe }\end{array}$ & 1456,3 & 1,0 & 1740,9 & 0,1 & 29,6 & $-313,3$ \\
\hline 21 Pharmazeutische Erzeugnisse & 2,8 & 0,0 & 0,6 & 0,0 & 0,7 & 1,5 \\
\hline $\begin{array}{l}22 \text { Chemische Erzeugnisse (o. pharmazeutische } \\
\text { Erzeugnisse) }\end{array}$ & 142,3 & 2,7 & 9,4 & 0,2 & 2,3 & 133,1 \\
\hline 23 Gummiwaren & 1,0 & 0,4 & 0,3 & 0,0 & 0,2 & 0,8 \\
\hline 24 Kunststoffwaren & 5,8 & 2,7 & 1,1 & 0,3 & 1,0 & 6,3 \\
\hline 25 Glas und Glaswaren & 3,7 & 0,4 & 0,7 & 0,0 & 0,2 & 3,1 \\
\hline 26 Keramik, bearbeitete Steine und Erden & 6,9 & 2,5 & 1,6 & 0,2 & 0,5 & 7,1 \\
\hline 27 Roheisen, Stahl, Rohre und Halbzeug daraus & 4,9 & 4,2 & 2,2 & 0,4 & 0,7 & 5,8 \\
\hline 28 NE-Metalle und Halbzeug daraus & 1,0 & 0,8 & 0,3 & 0,1 & 0,1 & 1,3 \\
\hline 29 Gießereierzeugnisse & 1,8 & 1,5 & 0,5 & 0,1 & 0,2 & 2,5 \\
\hline 30 Metallerzeugnisse & 14,2 & 13,3 & 6,0 & 1,2 & 2,0 & 18,3 \\
\hline 31 Maschinen & 57,6 & 55,0 & 6,9 & 5,0 & 1,6 & 99,1 \\
\hline $\begin{array}{l}32 \text { Buromaschinen, Datenverarbeitungsgerăte u. - } \\
\text { einrichtungen }\end{array}$ & 0,7 & 2,5 & 0,3 & 0,2 & 0,2 & 2,5 \\
\hline 33 Gerăte der Elektrizitatserzeugung, -verteilung u.ă. & 12,9 & 15,3 & 7,4 & 1,4 & 1,2 & 18,2 \\
\hline $\begin{array}{l}34 \text { Nachrtechn., Rundf.- und Fernsehgerăte, elekt- } \\
\text { ron. Bauelemente }\end{array}$ & 0,6 & 7,2 & 0,3 & 0,7 & 0,4 & 6,4 \\
\hline $\begin{array}{l}35 \text { Medizin-, mess-, regelungstechn., optische Er- } \\
\text { zeugnisse; Uhren }\end{array}$ & 1,0 & 11,9 & 0,6 & 1,1 & 0,5 & 10,7 \\
\hline 36 Kraftwagen und Kraftwagenteile & 5,5 & 0,5 & 1,6 & 0,0 & 7,0 & $-2,7$ \\
\hline $\begin{array}{l}37 \text { Sonstige Fahrzeuge (Wasser-, Schienen-, Luft- } \\
\text { fahrzeuge u.a.) }\end{array}$ & 0,5 & 0,1 & 0,3 & 0,0 & 0,4 & $-0,1$ \\
\hline 38 Mobel, Schmuck, Musikinstrumente, Sportgerăte, & 0,1 & 4,8 & 0,0 & 0,4 & 1,7 & 2,7 \\
\hline
\end{tabular}




\begin{tabular}{|c|c|c|c|c|c|c|}
\hline & Ethanol & $\begin{array}{l}\text { Ethanol } \\
\text { Invest. }\end{array}$ & \begin{tabular}{|l|} 
Substi- \\
tut \\
Benzin
\end{tabular} & $\begin{array}{l}\text { Substi- } \\
\text { tut } \\
\text { Invest. }\end{array}$ & \begin{tabular}{|l|} 
Negati- \\
ver \\
Konsum
\end{tabular} & $\begin{array}{l}\text { Ge- } \\
\text { samt }\end{array}$ \\
\hline \multicolumn{7}{|l|}{ Spielwaren u.ă. } \\
\hline 39 Sekundărrohstoffe & 0,9 & 0,1 & $\overline{0,2}$ & 0,0 & $\overline{0,2}$ & 0,7 \\
\hline $\begin{array}{l}40 \text { Elektrizităt, Fernwărme, DL der Elektrizităts- u. } \\
\text { Fernwărmevers. }\end{array}$ & 145,1 & 1,7 & 131,1 & 0,2 & 5,2 & 10,5 \\
\hline 41 Gase, DL der Gasversorgung & 6,1 & 0,3 & 3,7 & 0,0 & 1,2 & 1,5 \\
\hline 42 Wasser und DL der Wasserversorgung & 20,2 & 0,3 & 14,3 & 0,0 & 1,0 & 5,1 \\
\hline $\begin{array}{l}43 \text { Vorb. Baustellenarbeiten, Hoch- } u \text {. Tiefbauarbei- } \\
\text { ten }\end{array}$ & 4,5 & 14,1 & 1,9 & 1,3 & 1,1 & 14,3 \\
\hline 44 Bauinstallations- und sonstige Bauarbeiten & 30,3 & 12,5 & 3,0 & 1,1 & 3,1 & 35,7 \\
\hline $\begin{array}{l}45 \text { Handelsleist. mit Kfz; Rep. an Kfz; Tankleistun- } \\
\text { gen }\end{array}$ & 11,6 & 0,7 & 3,1 & 0,1 & 7,1 & 2,1 \\
\hline 46 Handelsvermittllungs- und Großhandelsleistungen & 69,3 & 20,9 & 15,8 & 1,9 & 10,9 & 61,6 \\
\hline $\begin{array}{l}47 \text { Einzelhandelsleistungen; Reparatur an Ge- } \\
\text { brauchsgutern }\end{array}$ & 3,9 & 0,6 & 1,1 & 0,1 & 25,4 & $-22,0$ \\
\hline 48 Beherbergungs- und Gaststătten-DL & 0,4 & 0,1 & 0,2 & 0,0 & 11,1 & $-10,8$ \\
\hline 49 Eisenbahn-DL & 1,9 & 0,2 & 1,0 & 0,0 & 1,7 & $-0,6$ \\
\hline $\begin{array}{l}50 \text { Sonst. Landv.leistungen, Transportl. in Rohrfern- } \\
\text { leitungen }\end{array}$ & 25,0 & 2,4 & 13,2 & 0,2 & 4,5 & 9,4 \\
\hline 51 Schifffahrtsleistungen & 1,5 & 0,1 & 1,0 & 0,0 & 0,2 & 0,4 \\
\hline 52 Luftfahrtleistungen & 2,9 & 1,0 & 1,4 & 0,1 & 1,9 & 0,6 \\
\hline $\begin{array}{l}53 \text { DL bezuglich Hilfs- u. Nebentátigkeiten für den } \\
\text { Verkehr }\end{array}$ & 19,0 & 4,6 & 5,8 & 0,4 & 5,9 & 11,6 \\
\hline 54 Nachrichtenubermittlungs-DL & 11,2 & 2,2 & 5,0 & 0,2 & 11,2 & $-3,0$ \\
\hline 55 DL der Kreditinstitute & 40,1 & 3,4 & 12,5 & 0,3 & 15,3 & 15,4 \\
\hline $\begin{array}{l}56 \text { DL der Versicherungen (ohne Sozialversiche- } \\
\text { rung) }\end{array}$ & 19,3 & 0,9 & 5,6 & 0,1 & 11,5 & 3,0 \\
\hline 57 DL des Kredit- und Versicherungshilfsgewerbes & 63,6 & 0,6 & 2,5 & 0,1 & 3,9 & 57,7 \\
\hline $58 \mathrm{DL}$ des Grundstucks- und Wohnungswesens & 28,7 & 6,8 & 9,7 & 0,6 & 50,5 & $-25,3$ \\
\hline $\begin{array}{l}59 \text { DL der Vermietung beweglicher Sachen (o. Per- } \\
\text { sonal) }\end{array}$ & 64,4 & 3,6 & 16,5 & 0,3 & 3,9 & 47,3 \\
\hline 60 DL der Datenverarbeitung und von Datenbanken & 5,0 & 29,7 & 1,9 & 2,7 & 1,6 & 28,4 \\
\hline 61 Forschungs- und Entwicklungsleistungen & 0,8 & 0,1 & 0,1 & 0,0 & 0,1 & 0,7 \\
\hline 62 Unternehmensbezogene DL & 143,9 & 18,8 & 50,2 & 1,7 & 20,5 & 90,3 \\
\hline 63 DL der 8 ffentlichen Verwaltung, Verteidigung & 13,8 & 1,0 & 9,3 & 0,1 & 1,9 & 3,5 \\
\hline 64 DL der Sozialversicherung & 0,0 & 0,0 & 0,0 & 0,0 & 0,0 & 0,0 \\
\hline 65 Erziehungs- und Unterrichts-DL & 3,3 & 0,7 & 1,5 & 0,1 & 2,9 & $-0,5$ \\
\hline $\begin{array}{l}66 \text { DL des Gesundheits-, Veterinär- und Sozialwe- } \\
\text { sens }\end{array}$ & 9,1 & 0,0 & 1,8 & 0,0 & 10,5 & $-3,2$ \\
\hline $\begin{array}{l}67 \text { Abwasser-, Abfallbeseitigungs- u. sonst. Entsor- } \\
\text { gungsleistungen }\end{array}$ & 12,0 & 0,5 & 2,6 & 0,0 & 3,7 & 6,2 \\
\hline 68 DL von Interessenvertretungen, Kirchen u.ă. & 3,6 & 0,4 & 1,7 & 0,0 & 1,1 & 1,1 \\
\hline 69 Kultur-, Sport- und Unterhaltungs-DL & 5,3 & 0,7 & 1,9 & 0,1 & 6,6 & $-2,6$ \\
\hline 70 Sonstige $\mathrm{DL}$ & 5,3 & 0,8 & 1,6 & 0,1 & 6,0 & $-1,7$ \\
\hline 71 DL privater Haushalte & 0,0 & 0,0 & 0,0 & 0,0 & 1,3 & $-1,3$ \\
\hline Summe & 3444,9 & 261,9 & 2309,1 & 23,9 & 304,2 & 1069,6 \\
\hline
\end{tabular}

Quelle: Eigene Berechnungen

Die Gesamtsumme ergibt sich aus Spalte 1+2 abzüglich den Spalten 3-5. 


\section{Tabelle 38: Sektorale Beschäftigungseffekte für Bioethanol im Referenzsze- nario (im Vergleich zum Basisszenario)}

\begin{tabular}{|c|c|c|c|c|c|c|}
\hline & Ethanol & $\begin{array}{l}\text { Etha- } \\
\text { nol } \\
\text { lnvest. }\end{array}$ & $\begin{array}{l}\text { Substi- } \\
\text { tut } \\
\text { Benzin }\end{array}$ & $\begin{array}{l}\text { Substi- } \\
\text { tut } \\
\text { Invest. }\end{array}$ & \begin{tabular}{|l|} 
Nega- \\
tiver \\
Kon- \\
sum \\
\end{tabular} & $\begin{array}{l}\text { Ge- } \\
\text { samt }\end{array}$ \\
\hline & $\begin{array}{c}\text { Er- } \\
\text { werbs- } \\
\text { tatige } \\
\end{array}$ & \begin{tabular}{|l|} 
Er- \\
werbs- \\
tatige \\
\end{tabular} & \begin{tabular}{|l}
$\begin{array}{l}\text { Er- } \\
\text { werbs- } \\
\text { tatige }\end{array}$ \\
\end{tabular} & \begin{tabular}{|l|}
$\begin{array}{l}\text { Er- } \\
\text { werbs- } \\
\text { tatige }\end{array}$ \\
\end{tabular} & \begin{tabular}{|l} 
Er- \\
werbs- \\
tatige \\
\end{tabular} & $\begin{array}{l}\begin{array}{l}\text { Er- } \\
\text { werbs- } \\
\text { tătige }\end{array} \\
\end{array}$ \\
\hline 1 Erzeugnisse der Landwirtschaft und Jagd & 3461 & 0 & 537 & 0 & 21 & 2903 \\
\hline 2 Forstwirtschaftliche Erzeugnisse und DL & 4 & 0 & 1 & 0 & 1 & 3 \\
\hline 3 Fische und Fischereierzeugnisse & 0 & 0 & 0 & 0 & 0 & 0 \\
\hline 4 Kohle und Torf & 41 & 1 & 46 & 0 & 6 & -11 \\
\hline 5 Erdol, Erdgas, DL fur Erdol-, Erdgasgewinnung & 1 & 0 & 2 & 0 & 1 & -2 \\
\hline 6 Uran- und Thoriumerze & 0 & 0 & 0 & 0 & 0 & 0 \\
\hline 7 Erze & 0 & 0 & 0 & 0 & 0 & 0 \\
\hline 8 Steine und Erden, sonstige Bergbauerzeugnisse & 6 & 1 & 0 & 0 & 0 & 6 \\
\hline 9 Nahrungs- und Futtermittel & 3 & 0 & 50 & 0 & 79 & -126 \\
\hline 10 Getrănke & 0 & 0 & 0 & 0 & 3 & -3 \\
\hline 11 Tabakerzeugnisse & 0 & 0 & 0 & 0 & 2 & -2 \\
\hline 12 Textilien & 1 & 0 & 0 & 0 & 2 & -1 \\
\hline 13 Bekleidung & 0 & 0 & 0 & 0 & 3 & -3 \\
\hline 14 Leder und Lederwaren & 0 & 0 & 0 & 0 & 1 & -1 \\
\hline 15 Holz; Holz-, Kork-, Flechtwaren (o. Möbel) & 9 & 7 & 2 & 1 & 5 & 9 \\
\hline 16 Holzstoff, Zellstoff, Papier, Karton, Pappe & 1 & 0 & 0 & 0 & 1 & 0 \\
\hline 17 Papier-, Karton- und Pappewaren & 10 & 1 & 1 & 0 & 4 & 6 \\
\hline 18 Verlagserzeugnisse & 5 & 1 & 2 & 0 & 14 & -10 \\
\hline $\begin{array}{l}19 \text { Druckerzeugnisse, bespielte Ton-, Bild- und Daten- } \\
\text { träger }\end{array}$ & 22 & 4 & 8 & 0 & 22 & -5 \\
\hline $\begin{array}{l}20 \text { Kokereierzeugnisse, Mineralolerzeugnisse, Spalt- } \\
\text { und Brutstoffe }\end{array}$ & 314 & 0 & 49 & 0 & 14 & 251 \\
\hline 21 Pharmazeutische Erzeugnisse & 2 & 0 & 0 & 0 & 1 & 1 \\
\hline $\begin{array}{l}22 \text { Chemische Erzeugnisse (o. pharmazeutische Erzeug- } \\
\text { nisse) }\end{array}$ & 47 & 1 & 4 & 0 & 2 & 42 \\
\hline 23 Gummiwaren & 2 & 1 & 1 & 0 & 1 & 1 \\
\hline 24 Kunststoffwaren & 14 & 7 & 3 & 1 & 6 & 11 \\
\hline 25 Glas und Glaswaren & 11 & 1 & 2 & 0 & 1 & 9 \\
\hline 26 Keramik, bearbeitete Steine und Erden & 22 & 7 & 4 & 1 & 4 & 21 \\
\hline 27 Roheisen, Stahl, Rohre und Halbzeug daraus & 2 & 2 & 1 & 0 & 1 & 2 \\
\hline 28 NE-Metalle und Halbzeug daraus & 1 & 1 & 0 & 0 & 0 & 1 \\
\hline 29 Gießereierzeugnisse & 2 & 2 & 1 & 0 & 0 & 3 \\
\hline 30 Metallerzeugnisse & 47 & 44 & 23 & 3 & 16 & 48 \\
\hline 31 Maschinen & 196 & 91 & 11 & 7 & 7 & 262 \\
\hline $\begin{array}{l}32 \text { Büromaschinen, Datenverarbeitungsgerăte u. - } \\
\text { einrichtungen }\end{array}$ & 0 & 1 & 0 & 0 & 0 & 1 \\
\hline 33 Geräte der Elektrizitătserzeugung, -verteilung u.ä. & 17 & 23 & 9 & 2 & 5 & 24 \\
\hline $\begin{array}{l}34 \text { Nachrtechn., Rundf.- und Fernsehgeräte, elektron. } \\
\text { Bauelemente }\end{array}$ & 0 & 2 & 0 & 0 & 0 & 2 \\
\hline $\begin{array}{l}35 \text { Medizin-, mess-, regelungstechn., optische Erzeug- } \\
\text { nisse; Uhren }\end{array}$ & 2 & 23 & 1 & 2 & 3 & 19 \\
\hline $36 \mathrm{Kraftwagen}$ und Kraftwagenteile & 6 & 0 & 2 & 0 & 18 & -13 \\
\hline
\end{tabular}




\begin{tabular}{|c|c|c|c|c|c|c|}
\hline & Ethanol & $\begin{array}{l}\text { Etha- } \\
\text { nol } \\
\text { Invest. }\end{array}$ & $\begin{array}{l}\text { Substi- } \\
\text { tut } \\
\text { Benzin }\end{array}$ & \begin{tabular}{|l} 
Substi- \\
tut \\
Invest.
\end{tabular} & \begin{tabular}{|l|} 
Nega- \\
tiver \\
Kon- \\
sum
\end{tabular} & $\begin{array}{l}\text { Ge- } \\
\text { samt }\end{array}$ \\
\hline $\begin{array}{l}37 \text { Sonstige Fahrzeuge (Wasser-, Schienen-, Luftfahr- } \\
\text { zeuge u.a.) }\end{array}$ & 0 & 0 & 0 & 0 & 1 & 0 \\
\hline $\begin{array}{l}38 \text { Mobel, Schmuck, Musikinstrumente, Sportgerăte, } \\
\text { Spielwaren u.a.. }\end{array}$ & 0 & 16 & 0 & 1 & 14 & 1 \\
\hline 39 Sekundärrohstoffe & 2 & 0 & 0 & 0 & 1 & $\mathrm{~T}$ \\
\hline $\begin{array}{l}40 \text { Elektrizităt, Fernwărme, DL der Elektrizităts- u. } \\
\text { Fernwărmevers. }\end{array}$ & 55 & 1 & 47 & 0 & 6 & 2 \\
\hline 41 Gase, DL der Gasversorgung & 8 & 0 & $\overline{6}$ & 0 & $\overline{4}$ & -2 \\
\hline 42 Wasser und DL der Wasserversorgung & 17 & 0 & 10 & 0 & 2 & 5 \\
\hline 43 Vorb. Baustellenarbeiten, Hoch- u. Tiefbauarbeiten & 23 & 69 & 9 & 5 & 14 & 63 \\
\hline 44 Bauinstallations- und sonstige Bauarbeiten & 131 & 62 & 15 & 5 & 38 & 135 \\
\hline 45 Handelsleist. mit $\mathrm{Kfz}$; Rep. an $\mathrm{Kfz}$; Tankleistungen & 80 & 4 & 20 & 0 & 109 & -46 \\
\hline 46 Handelsvermittlungs- und Großhandelsleistungen & 211 & 58 & 43 & 5 & 77 & 144 \\
\hline $\begin{array}{l}47 \text { Einzelhandelsleistungen; Reparatur an Gebrauchsgü- } \\
\text { tern }\end{array}$ & 42 & 6 & 11 & 0 & 666 & -629 \\
\hline 48 Beherbergungs- und Gaststătten-DL & 5 & 1 & 2 & 0 & 350 & -347 \\
\hline 49 Eisenbahn-DL & 7 & 1 & 3 & 0 & 18 & -14 \\
\hline $\begin{array}{l}50 \text { Sonst. Landv.leistungen, Transportl. in Rohrfernlei- } \\
\text { tungen }\end{array}$ & 206 & 19 & 155 & 1 & 90 & -21 \\
\hline 51 Schifffahrtsleistungen & 0 & 0 & 0 & 0 & 0 & 0 \\
\hline 52 Luftfahrtleistungen & $\overline{4}$ & 1 & 2 & 0 & 6 & -4 \\
\hline $\begin{array}{l}53 \text { DL bezuglich Hilfs- u. Nebentătigkeiten fur den } \\
\text { Verkehr }\end{array}$ & 37 & 8 & 12 & 1 & 27 & 6 \\
\hline 54 Nachrichtenubermittlungs-DL & 13 & 2 & 7 & 0 & 32 & -24 \\
\hline 55 DL der Kreditinstitute & 71 & 6 & 24 & 0 & 63 & -11 \\
\hline 56 DL der Versicherungen (ohne Sozialversicherung) & 29 & 1 & 8 & 0 & 42 & -20 \\
\hline 57 DL des Kredit- und Versicherungshilfsgewerbes & 272 & 2 & 11 & 0 & 42 & 221 \\
\hline 58 DL des Grundstucks- und Wohnungswesens & 21 & 5 & 7 & 0 & 88 & -71 \\
\hline 59 DL der Vermietung beweglicher Sachen (o. Personal) & 83 & 4 & 18 & 0 & 11 & 58 \\
\hline 60 DL der Datenverarbeitung und von Datenbanken & 25 & 146 & 11 & 11 & 20 & 129 \\
\hline 61 Forschungs- und Entwicklungsleistungen & 3 & 0 & 0 & 0 & 1 & 2 \\
\hline 62 Unternehmensbezogene DL & 926 & 114 & 352 & 9 & 317 & 362 \\
\hline 63 DL der 8 ffentlichen Verwaltung, Verteidigung & 72 & 6 & 45 & 0 & 28 & 4 \\
\hline 64 DL der Sozialversicherung & 0 & 0 & 0 & 0 & 0 & 0 \\
\hline 65 Erziehungs- und Unterrichts-DL & 34 & 7 & 19 & 1 & 73 & -52 \\
\hline 66 DL des Gesundheits-, Veterinar- und Sozialwesens & 72 & 0 & 11 & 0 & 179 & -119 \\
\hline $\begin{array}{l}67 \text { Abwasser-, Abfallbeseitigungs- u. sonst. Entsor- } \\
\text { gungsleistungen }\end{array}$ & 40 & 1 & 8 & 0 & 30 & 3 \\
\hline 68 DL von Interessenvertretungen, Kirchen u.ă. & 37 & 4 & 19 & 0 & 28 & -7 \\
\hline 69 Kultur-, Sport- und Unterhaltungs-DL & 31 & 4 & 12 & 0 & 92 & -70 \\
\hline 70 Sonstige DL & 53 & 7 & 17 & 1 & 144 & -101 \\
\hline $71 \mathrm{DL}$ privater Haushalte & $\overline{0}$ & 0 & 0 & 0 & 65 & -65 \\
\hline Summe & 6858 & 777 & 1668 & 60 & 2923 & 2984 \\
\hline
\end{tabular}

Quelle: Eigene Berechnungen

Die Gesamtsumme ergibt sich aus Spalte 1+2 abzüglich den Spalten 3-5. 


\section{Tabelle 39: Sektorale Beschäftigungseffekte für Bioethanol im HD-Szenario (im Vergleich zum Basisszenario)}

\begin{tabular}{|c|c|c|c|c|c|c|}
\hline & $\begin{array}{l}\text { Etha- } \\
\text { nol }\end{array}$ & $\begin{array}{l}\text { Ethanol } \\
\text { Invest. }\end{array}$ & $\begin{array}{l}\text { Substi- } \\
\text { tut } \\
\text { Benzin }\end{array}$ & $\begin{array}{l}\text { Substi- } \\
\text { tut } \\
\text { Invest. }\end{array}$ & \begin{tabular}{|l|} 
Negati- \\
ver \\
Kon- \\
sum
\end{tabular} & $\begin{array}{l}\text { Ge- } \\
\text { samt }\end{array}$ \\
\hline & $\begin{array}{c}\text { Er- } \\
\text { werbs- } \\
\text { tắtige }\end{array}$ & $\begin{array}{c}\text { Er- } \\
\text { werbs- } \\
\text { tătige }\end{array}$ & $\begin{array}{c}\begin{array}{c}\text { Er- } \\
\text { werbs- } \\
\text { tattige }\end{array} \\
\text { tation }\end{array}$ & $\begin{array}{c}\text { Er- } \\
\text { werbs- } \\
\text { tattige }\end{array}$ & $\begin{array}{c}\text { Er- } \\
\text { werbs- } \\
\text { tatige }\end{array}$ & $\begin{array}{c}\text { Er- } \\
\text { werbs } \\
\text {-tătige }\end{array}$ \\
\hline 1 Erzeugnisse der Landwirtschaft und Jagd & 6534 & 0 & 1285 & 0 & 18 & 5231 \\
\hline 2 Forstwirtschaftliche Erzeugnisse und DL & 9 & 1 & 2 & 0 & 1 & 7 \\
\hline 3 Fische und Fischereierzeugnisse & 0 & 0 & 0 & 0 & 0 & 0 \\
\hline 4 Kohle und Torf & 118 & 1 & 120 & 0 & 6 & -7 \\
\hline 5 Erdől, Erdgas, DL für Erdচl-, Erdgasgewinnung & 2 & 0 & 6 & 0 & 1 & -4 \\
\hline 6 Uran- und Thoriumerze & $\overline{0}$ & 0 & 0 & 0 & 0 & 0 \\
\hline 7 Erze & 0 & 0 & 0 & 0 & 0 & 0 \\
\hline 8 Steine und Erden, sonstige Bergbauerzeugnisse & 16 & 2 & 1 & 0 & 0 & 16 \\
\hline 9 Nahrungs- und Futtermittel & 3 & 0 & 119 & 0 & 71 & -187 \\
\hline 10 Getrănke & 0 & 0 & 0 & 0 & 2 & -2 \\
\hline 11 Tabakerzeugnisse & 0 & 0 & 0 & 0 & 2 & -2 \\
\hline 12 Textilien & 2 & 1 & 0 & 0 & 2 & 0 \\
\hline 13 Bekleidung & 0 & 0 & 0 & 0 & 2 & -2 \\
\hline 14 Leder und Lederwaren & 0 & 0 & 0 & 0 & 1 & -1 \\
\hline 15 Holz; Holz-, Kork-, Flechtwaren (o. Mðbel) & 20 & 15 & 4 & T & 4 & 26 \\
\hline 16 Holzstoff, Zellstoff, Papier, Karton, Pappe & 2 & 0 & 0 & 0 & 0 & 2 \\
\hline 17 Papier-, Karton- und Pappewaren & 23 & 3 & $\overline{3}$ & 0 & 3 & 19 \\
\hline 18 Verlagserzeugnisse & 11 & 2 & 4 & 0 & 12 & -4 \\
\hline $\begin{array}{l}19 \text { Druckerzeugnisse, bespielte Ton-, Bild- und Daten- } \\
\text { trăger }\end{array}$ & 46 & 9 & 16 & 1 & 19 & 19 \\
\hline $\begin{array}{l}20 \text { Kokereierzeugnisse, Mineralolerzeugnisse, Spalt- } \\
\text { und Brutstoffe }\end{array}$ & 694 & 0 & 99 & 0 & 13 & 583 \\
\hline 21 Pharmazeutische Erzeugnisse & 5 & 0 & 1 & 0 & 1 & 3 \\
\hline $\begin{array}{l}22 \text { Chemische Erzeugnisse (o. pharmazeutische Er- } \\
\text { zeugnisse) }\end{array}$ & 124 & 2 & 8 & 0 & 2 & 116 \\
\hline 23 Gummiwaren & 4 & 1 & 1 & 0 & 1 & 3 \\
\hline 24 Kunststoffwaren & 33 & 15 & 6 & 1 & 5 & 35 \\
\hline 25 Glas und Glaswaren & 23 & 3 & 4 & 0 & 1 & 19 \\
\hline 26 Keramik, bearbeitete Steine und Erden & 45 & 17 & 10 & 2 & 3 & 46 \\
\hline 27 Roheisen, Stahl, Rohre und Halbzeug daraus & 6 & 5 & 3 & 0 & 1 & 7 \\
\hline 28 NE-Metalle und Halbzeug daraus & 1 & 1 & 0 & 0 & 0 & 2 \\
\hline 29 Gießereierzeugnisse & 5 & 4 & 1 & 0 & 0 & 6 \\
\hline 30 Metallerzeugnisse & 106 & 100 & 45 & 9 & 15 & 137 \\
\hline 31 Maschinen & 385 & 206 & 26 & 19 & 6 & 540 \\
\hline $\begin{array}{l}32 \text { Buromaschinen, Datenverarbeitungsgeráte u. - } \\
\text { einrichtungen }\end{array}$ & 0 & 2 & 0 & 0 & 0 & 2 \\
\hline 33 Gerăte der Elektrizitătserzeugung, -verteilung u.ä. & 43 & 52 & 25 & 5 & 4 & 61 \\
\hline $\begin{array}{l}34 \text { Nachrtechn., Rundf.- und Fernsehgerate, elektron. } \\
\text { Bauelemente }\end{array}$ & 0 & 5 & 0 & 0 & 0 & 4 \\
\hline $\begin{array}{l}35 \text { Medizin-, mess-, regelungstechn., optische Erzeug- } \\
\text { nisse; Uhren }\end{array}$ & 4 & 51 & 3 & 5 & 2 & 46 \\
\hline $36 \mathrm{Kraftwagen}$ und Kraftwagenteile & 12 & 1 & 4 & 0 & 16 & -6 \\
\hline
\end{tabular}




\begin{tabular}{|c|c|c|c|c|c|c|}
\hline & $\begin{array}{l}\text { Etha- } \\
\text { nol }\end{array}$ & $\begin{array}{l}\text { Ethanol } \\
\text { Invest. }\end{array}$ & \begin{tabular}{|l|} 
Substi- \\
tut \\
Benzin
\end{tabular} & $\begin{array}{l}\text { Substi- } \\
\text { tut } \\
\text { Invest. }\end{array}$ & \begin{tabular}{|l|} 
Negati- \\
ver \\
Kon- \\
sum \\
\end{tabular} & $\begin{array}{l}\text { Ge- } \\
\text { samt }\end{array}$ \\
\hline $\begin{array}{l}37 \text { Sonstige Fahrzeuge (Wasser-, Schienen-, Luftfahr- } \\
\text { zeuge u.a.) }\end{array}$ & 1 & 0 & 0 & 0 & 1 & 0 \\
\hline $\begin{array}{l}38 \text { Mobel, Schmuck, Musikinstrumente, Sportgerăte, } \\
\text { Spielwaren u.a. }\end{array}$ & 1 & 37 & 0 & 3 & 13 & 21 \\
\hline 39 Sekundärrohstoffe & 5 & 1 & 1 & 0 & 1 & 4 \\
\hline $\begin{array}{l}40 \text { Elektrizităt, Fernwărme, DL der Elektrizităts- u. } \\
\text { Fernwărmevers. }\end{array}$ & 161 & 2 & 145 & 0 & 6 & 12 \\
\hline 41 Gase, DL der Gasversorgung & 21 & 1 & 13 & 0 & 4 & 5 \\
\hline 42 Wasser und DL der Wasserversorgung & 43 & 1 & 31 & 0 & 2 & 11 \\
\hline 43 Vorb. Baustellenarbeiten, Hoch- u. Tiefbauarbeiten & 49 & 155 & 20 & 14 & 12 & $\overline{158}$ \\
\hline 44 Bauinstallations- und sonstige Bauarbeiten & 337 & 140 & 33 & 13 & 34 & 397 \\
\hline 45 Handelsleist. mit $\mathrm{Kfz}$; Rep. an Kfz; Tankleistungen & 159 & 9 & 42 & 1 & 97 & 28 \\
\hline 46 Handelsvermittlungs- und Großhandelsleistungen & 434 & 131 & 99 & 12 & 69 & 386 \\
\hline $\begin{array}{l}47 \text { Einzelhandelsleistungen; Reparatur an Gebrauchs- } \\
\text { gutern }\end{array}$ & 91 & 13 & 25 & 1 & 592 & -514 \\
\hline 48 Beherbergungs- und Gaststätten-DL & 11 & 2 & 4 & 0 & 312 & -303 \\
\hline 49 Eisenbahn-DL & 18 & 2 & 9 & 0 & 16 & -6 \\
\hline $\begin{array}{l}50 \text { Sonst. Landv.leistungen, Transportl. in Rohrfernlei- } \\
\text { tungen }\end{array}$ & 444 & 42 & 235 & 4 & 80 & 168 \\
\hline 51 Schifffahrtsleistungen & 0 & 0 & 0 & 0 & 0 & 0 \\
\hline 52 Luftfahrtleistungen & 9 & 3 & 4 & 0 & 6 & 2 \\
\hline $\begin{array}{l}53 \text { DL bezuglich Hilfs- u. Nebentătigkeiten fur den } \\
\text { Verkehr }\end{array}$ & 78 & 19 & 24 & 2 & 24 & 48 \\
\hline 54 Nachrichtenubermittlungs-DL & 28 & 6 & 13 & 1 & 28 & -8 \\
\hline 55 DL der Kreditinstitute & 147 & 13 & 46 & 1 & 56 & 57 \\
\hline 56 DL der Versicherungen (ohne Sozialversicherung) & 62 & 3 & 18 & 0 & 37 & 10 \\
\hline 57 DL des Kredit- und Versicherungshilfsgewerbes & 610 & 5 & 24 & 1 & 38 & 553 \\
\hline 58 DL des Grundstucks- und Wohnungswesens & 45 & 11 & 15 & 1 & 79 & -39 \\
\hline $\begin{array}{l}59 \text { DL der Vermietung beweglicher Sachen (o. Perso- } \\
\text { nal) }\end{array}$ & 168 & 9 & 43 & 1 & 10 & 123 \\
\hline 60 DL der Datenverarbeitung und von Datenbanken & 55 & 331 & 21 & 30 & 18 & 317 \\
\hline 61 Forschungs- und Entwicklungsleistungen & 7 & 1 & 1 & 0 & 0 & 6 \\
\hline 62 Unternehmensbezogene DL & 1975 & 258 & 689 & 24 & 282 & 1239 \\
\hline $63 \mathrm{DL}$ der 8 ffentlichen Verwaltung, Verteidigung & 183 & 13 & 123 & 1 & 25 & 46 \\
\hline 64 DL der Sozialversicherung & 0 & 0 & 0 & 0 & 0 & 0 \\
\hline 65 Erziehungs- und Unterrichts-DL & 75 & 16 & 35 & 1 & 65 & -11 \\
\hline 66 DL des Gesundheits-, Veterinar- und Sozialwesens & 138 & 0 & 27 & 0 & 159 & -48 \\
\hline $\begin{array}{l}67 \text { Abwasser-, Abfallbeseitigungs- u. sonst. Entsor- } \\
\text { gungsleistungen }\end{array}$ & 88 & 3 & 19 & 0. & 27 & 45 \\
\hline $68 \mathrm{DL}$ von Interessenvertretungen, Kirchen u.ă. & 79 & 8 & 37 & 1 & 25 & 24 \\
\hline 69 Kultur-, Sport- und Unterhaltungs-DL & 66 & 8 & 23 & 1 & 82 & -32 \\
\hline 70 Sonstige $\mathrm{DL}$ & 112 & 16 & 34 & 1 & 128 & -36 \\
\hline 71 DL privater Haushalte & 0 & 0 & 0 & 0 & 58 & -58 \\
\hline Summe & 13976 & 1757 & 3653 & 160 & 2601 & 9320 \\
\hline
\end{tabular}

Quelle: Eigene Berechnungen

Die Gesamtsumme ergibt sich aus Spalte 1+2 abzüglich den Spalten 3-5. 


\section{Tabelle 40: Sektorale Produktionseffekte für Biopolymere im Referenzsze- nario (im Vergleich zum Basisszenario)}

\begin{tabular}{|c|c|c|c|c|c|c|}
\hline & $\begin{array}{l}\text { Biopol } \\
y \text {-mere }\end{array}$ & $\begin{array}{l}\text { Biopol. } \\
\text { Invest. }\end{array}$ & $\begin{array}{l}\text { Substi- } \\
\text { tut } \\
\text { Polym. }\end{array}$ & $\begin{array}{c}\text { Substi- } \\
\text { tut } \\
\text { Invest. }\end{array}$ & $\begin{array}{l}\text { Positi- } \\
\text { ver } \\
\text { Kon- } \\
\text { sum }\end{array}$ & $\begin{array}{c}\text { Ge- } \\
\text { samt }\end{array}$ \\
\hline & Mio.€ & Mio. $€$ & Mio. $€$ & Mio. $€$ & Mio. $€$ & Mio. $€$ \\
\hline 1 Erzeugnisse der Landwirtschaft und Jagd & 597,5 & 0,0 & $\overline{74,3}$ & 0,0 & 0,1 & 523,3 \\
\hline 2 Forstwirtschaftliche Erzeugnisse und DL & 1,2 & 0,1 & 0,6 & 0,0 & 0,0 & $\overline{0,6}$ \\
\hline 3 Fische und Fischereierzeugnisse & 0,0 & 0,0 & 0,0 & 0,0 & 0,0 & 0,0 \\
\hline 4 Kohle und Torf & 7,9 & 0,1 & 9,7 & 0,0 & 0,0 & $-1,7$ \\
\hline 5 Erdől, Erdgas, DL fur Erdøl-, Erdgasgewinnung & 1,3 & 0,2 & 2,1 & 0,1 & 0,0 & $-0,7$ \\
\hline 6 Uran- und Thoriumerze & 0,0 & 0,0 & 0,0 & 0,0 & 0,0 & 0,0 \\
\hline 7 Erze & 0,0 & 0,0 & 0,0 & 0,0 & 0,0 & 0,0 \\
\hline 8 Steine und Erden, sonstige Bergbauerzeugnisse & 11,4 & 0,4 & 22,7 & 0,1 & 0,2 & $-10,8$ \\
\hline 9 Nahrungs- und Futtermittel & 0,4 & 0,0 & 14,0 & 0,0 & 0,4 & $-13,3$ \\
\hline 10 Getränke & 0,2 & 0,0 & 0,3 & 0,0 & 0,1 & 0,0 \\
\hline 11 Tabakerzeugnisse & 0,0 & 0,0 & 0,0 & 0,0 & 0,0 & 0,0 \\
\hline 12 Textilien & 0,2 & 0,1 & 0,1 & 0,0 & 0,0 & 0,2 \\
\hline 13 Bekleidung & 0,1 & 0,0 & 0,0 & 0,0 & 0,0 & 0,1 \\
\hline 14 Leder und Lederwaren & 0,0 & 0,0 & 0,0 & 0,0 & 0,0 & 0,0 \\
\hline 15 Holz; Holz-, Kork-, Flechtwaren (o. Mobbel) & 4,0 & 1,7 & 3,6 & 0,6 & 0,1 & 1,6 \\
\hline 16 Holzstoff, Zellstoff, Papier, Karton, Pappe & 3,1 & 0,3 & 5,2 & 0,1 & 0,1 & $-1,8$ \\
\hline 17 Papier-, Karton- und Pappewaren & 8,1 & 0,5 & 14,3 & 0,2 & 0,2 & $-5,7$ \\
\hline 18 Verlagserzeugnisse & 2,3 & 0,3 & 2,9 & 0,1 & 0,1 & $-0,4$ \\
\hline $\begin{array}{l}19 \text { Druckerzeugnisse, bespielte Ton-, Bild- und Daten- } \\
\text { träger }\end{array}$ & 4,2 & 0,7 & 5,5 & 0,2 & 0,1 & $-0,7$ \\
\hline $\begin{array}{l}20 \text { Kokereierzeugnisse, Mineralolerzeugnisse, Spalt- } \\
\text { und Brutstoffe }\end{array}$ & 23,7 & 0,8 & 54,5 & 0,3 & 0,4 & $-29,8$ \\
\hline 21 Pharmazeutische Erzeugnisse & 1,4 & 0,0 & 0,2 & 0,0 & 0,0 & 1,2 \\
\hline $\begin{array}{l}22 \text { Chemische Erzeugnisse (o. pharmazeutische Er- } \\
\text { zeugnisse) }\end{array}$ & 1750,4 & 2,3 & 2110,5 & 0,7 & 15,7 & $-342,8$ \\
\hline 23 Gummiwaren & 0,8 & 0,3 & 0,9 & 0,1 & 0,0 & 0,2 \\
\hline 24 Kunststoffwaren & 8,8 & 2,3 & 10,2 & 0,7 & 0,1 & 0,2 \\
\hline 25 Glas und Glaswaren & 3,0 & 0,3 & 2,8 & 0,1 & 0,0 & 0,5 \\
\hline 26 Keramik, bearbeitete Steine und Erden & 5,8 & 1,8 & 4,7 & 0,6 & 0,1 & 2,4 \\
\hline 27 Roheisen, Stahl, Rohre und Halbzeug daraus & 5,7 & 3,7 & 7,3 & 1,2 & 0,1 & 1,0 \\
\hline 28 NE-Metalle und Halbzeug daraus & 1,2 & 0,7 & 1,6 & 0,2 & 0,0 & 0,1 \\
\hline 29 Gießereierzeugnisse & 1,3 & 1,3 & 0,9 & 0,4 & 0,0 & 1,3 \\
\hline 30 Metallerzeugnisse & 16,8 & 11,5 & 18,2 & 3,7 & 0,2 & 6,6 \\
\hline 31 Maschinen & $\overline{40,2}$ & 49,4 & 23,5 & 15,9 & 0,3 & 50,4 \\
\hline $\begin{array}{l}32 \text { Büromaschinen, Datenverarbeitungsgerăte u. - } \\
\text { einrichtungen }\end{array}$ & 1,0 & 2,2 & 1,4 & 0,7 & 0,0 & 1,1 \\
\hline 33 Gerăte der Elektrizitátserzeugung, -verteilung u.ă. & 15,3 & 13,5 & 12,6 & 4,4 & 0,1 & 11,9 \\
\hline $\begin{array}{l}34 \text { Nachrtechn., Rundf.- und Fernsehgerăte, elektron. } \\
\text { Bauelemente }\end{array}$ & 0,6 & 6,5 & 0,8 & 2,1 & 0,0 & 4,3 \\
\hline $\begin{array}{l}35 \text { Medizin-, mess-, regelungstechn., optische Erzeug- } \\
\text { nisse; Uhren }\end{array}$ & 1,7 & 10,7 & 3,0 & 3,4 & 0,0 & 5,9 \\
\hline $36 \mathrm{Kraftwagen}$ und Kraftwagenteile & 3,5 & 0,4 & 2,4 & 0,1 & 0,2 & 1,6 \\
\hline $\begin{array}{l}37 \text { Sonstige Fahrzeuge (Wasser-, Schienen-, Luftfahr- } \\
\text { zeuge u.a.) }\end{array}$ & 0,6 & 0,1 & 0,8 & 0,0 & 0,0 & $-0,1$ \\
\hline
\end{tabular}




\begin{tabular}{|c|c|c|c|c|c|c|}
\hline & $\begin{array}{l}\text { Biopol } \\
y \text {-mere }\end{array}$ & $\begin{array}{l}\text { Biopol. } \\
\text { Invest. }\end{array}$ & $\begin{array}{l}\text { Substi- } \\
\text { tut } \\
\text { Polym. }\end{array}$ & $\begin{array}{c}\text { Substi- } \\
\text { tut } \\
\text { Invest. }\end{array}$ & $\begin{array}{c}\text { Positi- } \\
\text { ver } \\
\text { Kon- } \\
\text { sum } \\
\end{array}$ & $\begin{array}{c}\text { Ge- } \\
\text { samt }\end{array}$ \\
\hline $\begin{array}{l}38 \text { Mobel, Schmuck, Musikinstrumente, Sportgerăte, } \\
\text { Spielwaren u.ă. }\end{array}$ & 0,1 & 4,4 & 0,1 & 1,4 & 0,1 & 3,0 \\
\hline 39 Sekundärrohstoffe & 1,3 & 0,1 & 2,2 & 0,0 & 0,0 & $-0,8$ \\
\hline $\begin{array}{l}40 \text { Elektrizităt, Fernwărme, DL der Elektrizităts- u. } \\
\text { Fernwărmevers. }\end{array}$ & 130,7 & 1,5 & 159,8 & 0,5 & 0,4 & $-27,7$ \\
\hline 4I Gase, DL der Gasversorgung & 19,3 & 0,2 & 39,6 & 0,1 & 0,1 & $-20,0$ \\
\hline 42 Wasser und DL der Wasserversorgung & 18,3 & 0,2 & 35,9 & 0,1 & 0,1 & $-17,4$ \\
\hline 43 Vorb. Baustellenarbeiten, Hoch- u. Tiefbauarbeiten & 3,8 & 9,8 & 3,9 & 3,2 & 0,1 & 6,7 \\
\hline 44 Bauinstallations- und sonstige Bauarbeiten & 60,4 & 8,8 & 7,3 & 2,8 & 0,2 & 59,3 \\
\hline 45 Handelsleist. mit Kfz; Rep. an Kfz; Tankleistungen & 7,6 & 0,6 & 4,9 & 0,2 & 0,3 & 3,3 \\
\hline 46 Handelsvermittlungs- und Großhandelsleistungen & 61,2 & 18,5 & 63,6 & 6,0 & 0,9 & 11,0 \\
\hline $\begin{array}{l}47 \text { Einzelhandelsleistungen; Reparatur an Gebrauchs- } \\
\text { gutern }\end{array}$ & 4,2 & 0,5 & 4,7 & 0,2 & 0,9 & 0,7 \\
\hline 48 Beherbergungs- und Gaststatten-DL & 0,5 & 0,1 & 0,6 & 0,0 & 0,4 & 0,3 \\
\hline 49 Eisenbahn-DL & 2,7 & 0,1 & 4,0 & 0,0 & 0,1 & $-1,2$ \\
\hline $\begin{array}{l}50 \text { Sonst. Landv.leistungen, Transportl. in Rohrfern- } \\
\text { leitungen }\end{array}$ & 15,7 & 2,1 & 17,5 & 0,7 & 0,3 & $-0,1$ \\
\hline 51 Schifffahrtsleistungen & 2,0 & 0,1 & 2,9 & 0,0 & 0,0 & $-0,8$ \\
\hline 52 Luftfahrtleistungen & 3,9 & 0,9 & 5,0 & 0,3 & 0,1 & $-0,4$ \\
\hline $\begin{array}{l}53 \text { DL bezuglich Hilfs- u. Nebentătigkeiten fur den } \\
\text { Verkehr }\end{array}$ & 18,2 & 4,1 & 21,6 & 1,3 & 0,4 & $-0,2$ \\
\hline 54 Nachrichtenübermittlungs-DL & 12,6 & 1,9 & 16,2 & 0,6 & 0,5 & $-1,8$ \\
\hline 55 DL der Kreditinstitute & 32,9 & 2,9 & 22,8 & 0,9 & 0,7 & 12,7 \\
\hline 56 DL der Versicherungen (ohne Sozialversicherung) & 16,8 & 0,7 & 13,8 & 0,2 & 0,5 & 4,0 \\
\hline 57 DL des Kredit- und Versicherungshilfsgewerbes & 65,9 & 0,5 & 6,0 & 0,2 & 0,2 & 60,5 \\
\hline 58 DL des Grundstücks- und Wohnungswesens & 28,5 & 5,5 & 32,8 & 1,8 & 2,0 & 1,4 \\
\hline $\begin{array}{l}59 \text { DL der Vermietung beweglicher Sachen (o. Perso- } \\
\text { nal) }\end{array}$ & 43,6 & 3,0 & 28,0 & 1,0 & 0,3 & 18,0 \\
\hline 60 DL der Datenverarbeitung und von Datenbanken & 6,2 & 26,7 & 8,4 & 8,6 & 0,1 & 16,0 \\
\hline 61 Forschungs- und Entwicklungsleistungen & 7,3 & 0,1 & 3,3 & 0,0 & 0,0 & 4,1 \\
\hline 62 Unternehmensbezogene DL & 147,2 & 16,0 & 190,6 & 5,1 & 2,4 & $-30,2$ \\
\hline 63 DL der offentlichen Verwaltung, Verteidigung & 13,7 & 0,8 & 17,8 & 0,3 & 0,2 & $-3,4$ \\
\hline $64 \mathrm{DL}$ der Sozialversicherung & 0,0 & 0,0 & 0,0 & 0,0 & 0,0 & 0,0 \\
\hline 65 Erziehungs- und Unterrichts-DL & 4,5 & 0,6 & 5,2 & 0,2 & 0,1 & $-0,1$ \\
\hline 66 DL des Gesundheits-, Veterinär- und Sozialwesens & 5,0 & 0,0 & 1,7 & 0,0 & 0,4 & 3,7 \\
\hline $\begin{array}{l}67 \text { Abwasser-, Abfallbeseitigungs- u. sonst. Entsor- } \\
\text { gungsleistungen }\end{array}$ & 14,8 & 0,4 & 22,9 & 0,1 & 0,3 & $-7,6$ \\
\hline 68 DL von Interessenvertretungen, Kirchen u.ă. & 3,4 & 0,3 & 3,7 & 0,1 & 0,1 & 0,0 \\
\hline 69 Kultur-, Sport- und Unterhaltungs-DL & 5,5 & 0,6 & 7,3 & 0,2 & 0,3 & $-1,1$ \\
\hline 70 Sonstige DL & 5,1 & 0,7 & 6,2 & 0,2 & 0,3 & $-0,4$ \\
\hline 71 DL privater Haushalte & 0,0 & 0,0 & 0,0 & 0,0 & 0,0 & 0,0 \\
\hline Summe & 3286,5 & 224,9 & 3171,8 & 72,4 & 31,4 & 298,6 \\
\hline
\end{tabular}

\section{Quelle: Eigene Berechnungen}

Die Gesamtsumme ergibt sich aus Spalte 1+2+5 abzüglich den Spalten 3-4. 


\section{Tabelle 41: Sektorale Produktionseffekte für Biopolymere im HD-Szenario (im Vergleich zum Basisszenario)}

\begin{tabular}{|c|c|c|c|c|c|c|}
\hline & $\begin{array}{l}\text { Biopol } \\
y-\text {-mere }\end{array}$ & $\begin{array}{l}\text { Biopol. } \\
\text { Invest. }\end{array}$ & $\begin{array}{l}\text { Substi- } \\
\text { tut } \\
\text { Polym. }\end{array}$ & $\begin{array}{l}\text { Substi- } \\
\text { tut } \\
\text { Invest. }\end{array}$ & $\begin{array}{c}\text { Posi- } \\
\text { tiver } \\
\text { Kon- } \\
\text { sum } \\
\end{array}$ & $\begin{array}{l}\text { Ge- } \\
\text { samt }\end{array}$ \\
\hline & Mio. $\epsilon$ & Mio. $€$ & Mio. $\epsilon$ & Mio. $\epsilon$ & $\begin{array}{c}\text { Mio. } \\
\epsilon\end{array}$ & $\begin{array}{c}\text { Mio. } \\
\epsilon\end{array}$ \\
\hline 1 Erzeugnisse der Landwirtschaft und Jagd & 1194,9 & 0,1 & 178,9 & 0,0 & 1,6 & $\begin{array}{c}1017 \\
7\end{array}$ \\
\hline 2 Forstwirtschaftliche Erzeugnisse und DL & 2,3 & 0,3 & 1,5 & 0,1 & 0,1 & 1,1 \\
\hline 3 Fische und Fischereierzeugnisse & 0,0 & 0,0 & 0,0 & 0,0 & 0,0 & 0,0 \\
\hline 4 Kohle und Torf & 20,4 & 0,2 & 24,9 & 0,1 & 0,5 & $-4,0$ \\
\hline 5 Erdől, Erdgas, DL fur Erdচا-, Erdgasgewinnung & 2,9 & 0,3 & 5,3 & 0,1 & 0,3 & $-1,9$ \\
\hline 6 Uran- und Thoriumerze & 0,0 & 0,0 & 0,0 & 0,0 & 0,0 & 0,0 \\
\hline 7 Erze & 0,0 & 0,0 & 0,0 & 0,0 & 0,0 & 0,0 \\
\hline 8 Steine und Erden, sonstige Bergbauerzeugnisse & 23,6 & 0,8 & 55,2 & 0,3 & 3,7 & $-27,3$ \\
\hline 9 Nahrungs- und Futtermittel & 0,8 & 0,1 & 34,0 & 0,0 & 5,6 & $-27,5$ \\
\hline 10 Getrănke & 0,5 & 0,0 & 0,8 & 0,0 & 1,0 & 0,7 \\
\hline 11 Tabakerzeugnisse & 0,0 & 0,0 & 0,0 & 0,0 & 0,2 & 0,2 \\
\hline 12 Textilien & 0,5 & 0,2 & 0,3 & 0,1 & 0,2 & 0,5 \\
\hline 13 Bekleidung & 0,1 & 0,0 & 0,1 & 0,0 & 0,4 & 0,4 \\
\hline 14 Leder und Lederwaren & 0,1 & 0,1 & 0,1 & 0,0 & 0,1 & 0,2 \\
\hline 15 Holz; Holz-, Kork-, Flechtwaren (o. Möbel) & 7,9 & 3,7 & 8,7 & 1,4 & 0,8 & 2,4 \\
\hline 16 Holzstoff, Zellstoff, Papier, Karton, Pappe & 6,4 & 0,6 & 12,6 & 0,2 & 1,0 & $-4,7$ \\
\hline 17 Papier-, Karton- und Pappewaren & 16,7 & 1,0 & 34,8 & 0,4 & 2,6 & $-14,8$ \\
\hline 18 Verlagserzeugnisse & 4,7 & 0,6 & 7,1 & 0,2 & 1,6 & $-0,5$ \\
\hline $\begin{array}{l}19 \text { Druckerzeugnisse, bespielte Ton-, Bild- und Daten- } \\
\text { trăger }\end{array}$ & 8,7 & 1,4 & 13,4 & 0,5 & 1,6 & $-2,2$ \\
\hline $\begin{array}{l}20 \text { Kokereierzeugnisse, Mineralolerzeugnisse, Spalt- } \\
\text { und Brutstoffe }\end{array}$ & 48,7 & 1,8 & 173,9 & 0,7 & 6,3 & $\overline{117,8}$ \\
\hline 21 Pharmazeutische Erzeugnisse & 2,7 & 0,0 & 0,6 & 0,0 & 0,4 & 2,5 \\
\hline $\begin{array}{l}22 \text { Chemische Erzeugnisse (o. pharmazeutische Er- } \\
\text { zeugnisse) }\end{array}$ & 3690,7 & 4,9 & 5083,9 & 1,8 & 244,8 & $\begin{array}{c}- \\
1145 \\
3\end{array}$ \\
\hline 23 Gummiwaren & 1,7 & 0,7 & 2,1 & 0,3 & 0,2 & 0,3 \\
\hline 24 Kunststoffwaren & 17,6 & 5,0 & 24,9 & 1,8 & 2,1 & $-2,1$ \\
\hline 25 Glas und Glaswaren & 6,0 & 0,8 & 6,8 & 0,3 & 0,5 & 0,1 \\
\hline 26 Keramik, bearbeitete S & 11,7 & 4,0 & 11,5 & 1,5 & 0,9 & 3,6 \\
\hline 27 Roheisen, Stahl, Rohre und Halbzeug daraus & 12,5 & 8,0 & 18,4 & 2,9 & 1,0 & 0,2 \\
\hline 28 NE-Metalle und Halbzeug daraus & 2,5 & 1,5 & 4,0 & 0,6 & 0,3 & $-0,2$ \\
\hline 29 Gießereierzeugnisse & 2,6 & 2,9 & 2,2 & 1,1 & 0,2 & 2,4 \\
\hline 30 Metallerzeugnisse & 34,6 & 24,8 & 44,8 & 9,2 & 3,4 & 8,9 \\
\hline 31 Maschinen & 80,5 & 106,8 & 57,6 & 39,5 & 3,9 & 94,1 \\
\hline $\begin{array}{l}32 \text { Buromaschinen, Datenverarbeitungsgerăte u. - } \\
\text { einrichtungen }\end{array}$ & 2,0 & 4,8 & 3,5 & 1,8 & 0,3 & 1,9 \\
\hline 33 Gerăte der Elektrizitătserzeugung, -verteilung u.ä. & 33,9 & 29,2 & 32,0 & 10,8 & 1,5 & 21,8 \\
\hline $\begin{array}{l}34 \text { Nachrtechn., Rundf.- und Fernsehgerăte, elektron. } \\
\text { Bauelemente }\end{array}$ & 1,4 & 14,0 & 1,9 & 5,2 & 0,3 & 8,6 \\
\hline $\begin{array}{l}35 \text { Medizin-, mess-, regelungstechn., optische Erzeug- } \\
\text { nisse; Uhren }\end{array}$ & 3,8 & 23,0 & 7,6 & 8,5 & 0,6 & 11,3 \\
\hline
\end{tabular}




\begin{tabular}{|c|c|c|c|c|c|c|}
\hline & $\begin{array}{l}\text { Biopol } \\
y \text {-mere }\end{array}$ & $\begin{array}{l}\text { Biopol. } \\
\text { Invest. }\end{array}$ & $\begin{array}{l}\text { Substi- } \\
\text { tut } \\
\text { Polym. }\end{array}$ & $\begin{array}{l}\text { Substi- } \\
\text { tut } \\
\text { Invest. }\end{array}$ & $\begin{array}{l}\text { Posi- }- \\
\text { tiver } \\
\text { Kon- } \\
\text { sum } \\
\end{array}$ & $\begin{array}{l}\text { Ge- } \\
\text { samt }\end{array}$ \\
\hline 36 Kraftwagen und Kraftwagenteile & 7,1 & 0,9 & 5,9 & 0,3 & 3,9 & 5,6 \\
\hline $\begin{array}{l}37 \text { Sonstige Fahrzeuge (Wasser-, Schienen-, Luftfahr- } \\
\text { zeuge u.a.) }\end{array}$ & 1,4 & 0,2 & 2,0 & 0,1 & 0,3 & $-0,2$ \\
\hline $\begin{array}{l}38 \text { Mbbel, Schmuck, Musikinstrumente, Sportgerăte, } \\
\text { Spielwaren u.a.. }\end{array}$ & 0,1 & 9,4 & 0,1 & 3,5 & 0,9 & 6,8 \\
\hline 39 Sekundärrohstoffe & 2,7 & 0,3 & 5,3 & 0,1 & 0,4 & $-2,0$ \\
\hline $\begin{array}{l}40 \text { Elektrizităt, Fernwărme, DL der Elektrizităts- u. } \\
\text { Fernwărmevers. }\end{array}$ & 340,8 & 3,2 & 412,3 & 1,2 & 6,9 & $-62,5$ \\
\hline 41 Gase, DL der Gasversorgung & 49,9 & 0,5 & 106,4 & 0,2 & 1,4 & $-54,7$ \\
\hline 42 Wasser und DL der Wasserversorgung & 45,7 & 0,5 & 96,6 & 0,2 & 1,2 & $-49,4$ \\
\hline 43 Vorb. Baustellenarbeiten, Hoch- u. Tiefbauarbeiten & 8,1 & 21,3 & 9,7 & 7,9 & 1,0 & 12,7 \\
\hline 44 Bauinstallations- und sonstige Bauarbeiten & 115,1 & 19,1 & 18,3 & 7,1 & 2,3 & 111,3 \\
\hline 45 Handelsleist. mit Kfz; Rep. an Kfz; Tankleistungen & 15,2 & 1,2 & 12,2 & 0,4 & 4,2 & 8,0 \\
\hline 46 Handelsvermittlungs- und Großhandelsleistungen & 123,8 & 40,0 & 155,2 & 14,8 & 14,5 & 8,2 \\
\hline $\begin{array}{l}47 \text { Einzelhandelsleistungen; Reparatur an Gebrauchs- } \\
\text { gutern }\end{array}$ & 8,6 & 1,0 & 11,5 & 0,4 & 13,8 & 11,5 \\
\hline 48 Beherbergungs- und Gaststätten-DL & 1,0 & 0,1 & 1,5 & 0,1 & 5,9 & 5,4 \\
\hline 49 Eisenbahn-DL & 5,9 & 0,3 & 10,1 & 0,1 & 1,5 & $-2,5$ \\
\hline $\begin{array}{l}50 \text { Sonst. Landv.leistungen, Transportl. in Rohrfern- } \\
\text { leitungen }\end{array}$ & 33,1 & 4,5 & 44,1 & 1,7 & 4,5 & $-3,7$ \\
\hline 51 Schifffahrtsleistungen & 4,3 & 0,2 & 7,2 & 0,1 & 0,5 & $-2,3$ \\
\hline 52 Luftfahrtleistungen & 8,0 & 1,8 & 12,2 & 0,7 & 1,8 & $-1,2$ \\
\hline $\begin{array}{l}53 \text { DL bezuglich Hilfs- u. Nebentătigkeiten für den } \\
\text { Verkehr }\end{array}$ & 37,3 & 8,8 & 52,9 & 3,3 & 6,1 & $-4,0$ \\
\hline 54 Nachrichtenübermittlungs-DL & 26,4 & 4,2 & 40,1 & 1,5 & 7,9 & $-3,1$ \\
\hline 55 DL der Kreditinstitute & 67,5 & 6,2 & 56,2 & 2,3 & 10,6 & 25,8 \\
\hline 56 DL der Versicherungen (ohne Sozialversicherung) & 35,6 & 1,6 & 34,3 & 0,6 & 7,6 & 9,9 \\
\hline 57 DL des Kredit- und Versicherungshilfsgewerbes & 140,3 & 1,0 & 14,8 & 0,4 & 2,7 & 128,9 \\
\hline 58 DL des Grundstücks- und Wohnungswesens & 58,9 & 11,9 & 80,5 & 4,4 & 30,6 & 16,5 \\
\hline $\begin{array}{l}59 \text { DL der Vermietung beweglicher Sachen (o. Perso- } \\
\text { nal) }\end{array}$ & 88,3 & 6,5 & 69,0 & 2,4 & 5,0 & 28,4 \\
\hline $60 \mathrm{DL}$ der Datenverarbeitung und von Datenbanken & 12,9 & 57,6 & 20,6 & 21,3 & 2,0 & 30,6 \\
\hline 61 Forschungs- und Entwicklungsleistungen & 14,1 & 0,2 & 8,0 & 0,1 & 0,6 & 6,7 \\
\hline 62 Unternehmensbezogene DL & 304,1 & 34,5 & 467,5 & 12,8 & 37,0 & 104,5 \\
\hline 63 DL der offentlichen Verwaltung, Verteidigung & 32,4 & 1,7 & 45,0 & 0,6 & 2,3 & $-9,2$ \\
\hline 64 DL der Sozialversicherung & 0,0 & 0,0 & 0,0 & 0,0 & 0,0 & 0,0 \\
\hline 65 Erziehungs- und Unterrichts-DL & 9,3 & 1,4 & 12,7 & 0,5 & 2,2 & $-0,4$ \\
\hline 66 DL des Gesundheits-, Veterinär- und Sozialwesens & 9,8 & 0,0 & 4,2 & 0,0 & 5,6 & 11,2 \\
\hline $\begin{array}{l}67 \text { Abwasser-, Abfallbeseitigungs- u. sonst. Entsor- } \\
\text { gungsleistungen }\end{array}$ & 30,4 & 0,8 & 55,8 & 0,3 & 5,4 & $-19,5$ \\
\hline 68 DL von Interessenvertretungen, Kirchen u.ă. & 7,1 & 0,7 & 9,1 & 0,2 & 1,0 & $-0,5$ \\
\hline 69 Kultur-, Sport- und Unterhaltungs-DL & 11,5 & 1,2 & 17,9 & 0,5 & 4,4 & $-1,2$ \\
\hline 70 Sonstige $\mathrm{DL}$ & 10,6 & 1,4 & 15,2 & 0,5 & 4,0 & 0,2 \\
\hline $71 \mathrm{DL}$ privater Haushalte & 0,0 & 0,0 & 0,0 & 0,0 & 0,7 & 0,7 \\
\hline Summe & 6910,6 & 486,1 & 7768,1 & 179,8 & 489,1 & $-62,0$ \\
\hline
\end{tabular}

Quelle: Eigene Berechnungen

Die Gesamtsumme ergibt sich aus Spalte 1+2+5 abzüglich den Spalten 3-4. 


\section{Tabelle 42: Sektorale Beschäftigungseffekte für Biopolymere im Referenz- szenario (im Vergleich zum Basisszenario)}

\begin{tabular}{|c|c|c|c|c|c|c|}
\hline & $\begin{array}{l}\text { Biopol } \\
y \text {-mere }\end{array}$ & $\begin{array}{l}\text { Biopol. } \\
\text { Invest. }\end{array}$ & $\begin{array}{l}\text { Substi- } \\
\text { tut } \\
\text { Polym. }\end{array}$ & $\begin{array}{c}\text { Substi- } \\
\text { tut } \\
\text { Invest. }\end{array}$ & $\begin{array}{l}\text { Positi- } \\
\text { ver } \\
\text { Kon- } \\
\text { sum }\end{array}$ & $\begin{array}{l}\text { Ge- } \\
\text { samt }\end{array}$ \\
\hline & \begin{tabular}{|c|} 
Er- \\
werbs- \\
tatige
\end{tabular} & $\begin{array}{c}\text { Er- } \\
\text { werbs- } \\
\text { tătige }\end{array}$ & $\begin{array}{c}\text { Er- } \\
\text { werbs- } \\
\text { tattige }\end{array}$ & \begin{tabular}{|c|} 
Er- \\
werbs- \\
tătige
\end{tabular} & $\begin{array}{c}\text { Er- } \\
\text { werbs- } \\
\text { tătige }\end{array}$ & $\begin{array}{c}\text { Er- } \\
\text { werbs- } \\
\text { tătige }\end{array}$ \\
\hline 1 Erzeugnisse der Landwirtschaft und Jagd & 3403 & 0 & 541 & 0 & 1 & 2862 \\
\hline 2 Forstwirtschaftliche Erzeugnisse und DL & 6 & 1 & 3 & 0 & 0 & 3 \\
\hline 3 Fische und Fischereierzeugnisse & 0 & 0 & 0 & 0 & 0 & 0 \\
\hline 4 Kohle und Torf & 105 & 1 & 129 & 0 & 0 & -22 \\
\hline 5 Erdol, Erdgas, DL fur Erdð̋l-, Erdgasgewinnung & 3 & 0 & 5 & 0 & 0 & -2 \\
\hline 6 Uran- und Thoriumerze & 0 & 0 & 0 & 0 & 0 & 0 \\
\hline 7 Erze & 0 & 0 & 0 & 0 & 0 & 0 \\
\hline 8 Steine und Erden, sonstige Bergbauerzeugnisse & 34 & 1 & 67 & 0 & 1 & -32 \\
\hline 9 Nahrungs- und Futtermittel & 3 & 0 & 120 & 0 & 3 & -114 \\
\hline 10 Getränke & 0 & 0 & 0 & 0 & 0 & 0 \\
\hline 11 Tabakerzeugnisse & 0 & 0 & 0 & 0 & 0 & 0 \\
\hline 12 Textilien & 1 & 1 & 1 & 0 & 0 & 1 \\
\hline 13 Bekleidung & 0 & 0 & 0 & 0 & 0 & 0 \\
\hline 14 Leder und Lederwaren & 0 & 0 & 0 & 0 & 0 & 0 \\
\hline 15 Holz; Holz-, Kork-, Flechtwaren (o. Mobel) & 26 & 12 & 24 & 4 & 0 & 11 \\
\hline 16 Holzstoff, Zellstoff, Papier, Karton, Pappe & 4 & 0 & 6 & 0 & 0 & -2 \\
\hline 17 Papier-, Karton- und Pappewaren & 37 & 2 & 65 & 1 & 1 & -26 \\
\hline 18 Verlagserzeugnisse & 12 & 1 & 15 & 0 & 1 & -2 \\
\hline $\begin{array}{l}19 \text { Druckerzeugnisse, bespielte Ton-, Bild- und Daten- } \\
\text { träger }\end{array}$ & 50 & 8 & 64 & 2 & 1 & -8 \\
\hline $\begin{array}{l}20 \text { Kokereierzeugnisse, Mineralolerzeugnisse, Spalt- } \\
\text { und Brutstoffe }\end{array}$ & 10 & 0 & 23 & 0 & 0 & -13 \\
\hline 21 Pharmazeutische Erzeugnisse & 2 & 0 & 0 & 0 & 0 & 2 \\
\hline $\begin{array}{l}22 \text { Chemische Erzeugnisse (o. pharmazeutische Er- } \\
\text { zeugnisse) }\end{array}$ & 442 & 2 & 724 & 1 & 14 & -267 \\
\hline 23 Gummiwaren & 3 & 1 & 3 & 0 & 0 & $T$ \\
\hline 24 Kunststoffwaren & 49 & 13 & 57 & 4 & 1 & 1 \\
\hline 25 Glas und Glaswaren & 19 & 2 & 17 & 1 & 0 & 3 \\
\hline 26 Keramik, bearbeitete Steine und Erden & 38 & 12 & 31 & 4 & 0 & 16 \\
\hline 27 Roheisen, Stahl, Rohre und Halbzeug daraus & 7 & 4 & 8 & 1 & 0 & 1 \\
\hline 28 NE-Metalle und Halbzeug daraus & 2 & 1 & 3 & 0 & 0 & 0 \\
\hline 29 Gießereierzeugnisse & 3 & 4 & 2 & 1 & $\overline{0}$ & 3 \\
\hline 30 Metallerzeugnisse & 126 & 86 & 136 & 28 & 2 & 50 \\
\hline 31 Maschinen & 244 & 185 & 88 & 59 & 1 & 283 \\
\hline $\begin{array}{l}32 \text { Buromaschinen, Datenverarbeitungsgerăte u. - } \\
\text { einrichtungen }\end{array}$ & 1 & 1 & 1 & 0 & 0 & 1 \\
\hline 33 Gerăte der Elektrizitătserzeugung, -verteilung u.ă. & 51 & 45 & 43 & 15 & 0 & 40 \\
\hline $\begin{array}{l}34 \text { Nachrtechn., Rundf.- und Fernsehgerăte, elektron. } \\
\text { Bauelemente }\end{array}$ & 0 & 4 & 1 & 1 & 0 & 3 \\
\hline $\begin{array}{l}35 \text { Medizin-, mess-, regelungstechn., optische Erzeug. } \\
\text { nisse; Uhren }\end{array}$ & 7 & 46 & 13 & 15 & 0 & 26 \\
\hline 36 Kraftwagen und Kraftwagenteile & 8 & 1 & 5 & 0 & 1 & 4 \\
\hline
\end{tabular}




\begin{tabular}{|c|c|c|c|c|c|c|}
\hline & $\begin{array}{l}\text { Biopol } \\
y \text {-mere }\end{array}$ & $\begin{array}{l}\text { Biopol. } \\
\text { Invest. }\end{array}$ & $\begin{array}{c}\text { Substi- } \\
\text { tut } \\
\text { Polym. }\end{array}$ & $\begin{array}{l}\text { Substi- } \\
\text { tut } \\
\text { Invest. }\end{array}$ & $\begin{array}{l}\text { Positi- } \\
\text { ver } \\
\text { Kon- } \\
\text { sum }\end{array}$ & $\begin{array}{l}\text { Ge- } \\
\text { samt }\end{array}$ \\
\hline $\begin{array}{l}37 \text { Sonstige Fahrzeuge (Wasser-, Schienen-, Luftfahr- } \\
\text { zeuge u.a.) }\end{array}$ & 1 & 0 & 1 & 0 & 0 & 0 \\
\hline $\begin{array}{l}38 \text { Mobel, Schmuck, Musikinstrumente, Sportgerate, } \\
\text { Spielwaren u.a.. }\end{array}$ & 0 & 33 & 0 & 11 & 0 & 23 \\
\hline 39 Sekundărrohstoffe & 23 & 2 & 39 & 1 & 0 & -14 \\
\hline $\begin{array}{l}40 \text { Elektrizităt, Fernwărme, DL der Elektrizităts- u. } \\
\text { Fernwărmevers. }\end{array}$ & 145 & 2 & 177 & 1 & 0 & -31 \\
\hline 41 Gase, DL der Gasversorgung & 65 & 1 & 134 & 0 & 0 & -68 \\
\hline 42 Wasser und DL der Wasserversorgung & 39 & 0 & 77 & 0 & 0 & -37 \\
\hline 43 Vorb. Baustellenarbeiten, Hoch- u. Tiefbauarbeiten & 42 & 108 & 43 & 35 & 1 & 73 \\
\hline 44 Bauinstallations- und sonstige Bauarbeiten & 672 & 98 & 81 & 32 & 2 & 659 \\
\hline 45 Handelsleist. mit Kfz; Rep. an Kfz; Tankleistungen & 104 & 8 & 68 & 2 & 4 & 45 \\
\hline 46 Handelsvermittlungs- und Großhandelsleistungen & 383 & 116 & 398 & 37 & 6 & 69 \\
\hline $\begin{array}{l}47 \text { Einzelhandelsleistungen; Reparatur an Gebrauchs- } \\
\text { gutern }\end{array}$ & 98 & 11 & 110 & 4 & 21 & 16 \\
\hline 48 Beherbergungs- und Gaststätten-DL & 13 & 2 & 17 & $T$ & 11 & 8 \\
\hline 49 Eisenbahn-DL & 25 & 1 & 37 & 0 & 1 & -11 \\
\hline $\begin{array}{l}50 \text { Sonst. Landv.leistungen, Transportl. in Rohrfern- } \\
\text { leitungen }\end{array}$ & 279 & 37 & 310 & 12 & 5 & -1 \\
\hline 51 Schifffahrtsleistungen & 0 & 0 & 0 & 0 & 0 & 0 \\
\hline 52 Luftfahrtleistungen & 12 & 3 & 15 & 1 & 0 & -1 \\
\hline $\begin{array}{l}53 \text { DL bezuglich Hilfs- u. Nebentátigkeiten fur den } \\
\text { Verkehr }\end{array}$ & 75 & 17 & 89 & 5 & 2 & -1 \\
\hline 54 Nachrichtenübermittlungs-DL & 32 & 5 & 41 & 2 & 1 & -5 \\
\hline 55 DL der Kreditinstitute & 121 & 11 & 84 & 3 & 3 & 47 \\
\hline 56 DL der Versicherungen (ohne Sozialversicherung) & 224 & 10 & 183 & 3 & 6 & 53 \\
\hline 57 DL des Kredit- und Versicherungshilfsgewerbes & 632 & 5 & 57 & 1 & 2 & 580 \\
\hline 58 DL des Grundstücks- und Wohnungswesens & 44 & 9 & 51 & 3 & 3 & 2 \\
\hline $\begin{array}{l}59 \text { DL der Vermietung beweglicher Sachen (o. Perso- } \\
\text { nal) }\end{array}$ & 113 & 8 & 73 & 3 & 1 & 47 \\
\hline 60 DL der Datenverarbeitung und von Datenbanken & 69 & 297 & 93 & 96 & 1 & 178 \\
\hline 61 Forschungs- und Entwicklungsleistungen & 58 & 1 & 26 & 0 & 0 & 33 \\
\hline 62 Unternehmensbezogene DL & 2021 & 219 & 2617 & 71 & 33 & -415 \\
\hline 63 DL der offentlichen Verwaltung, Verteidigung & 181 & 11 & 236 & 3 & 2 & -45 \\
\hline 64 DL der Sozialversicherung & 0 & 0 & 0 & 0 & 0 & 0 \\
\hline 65 Erziehungs- und Unterrichts-DL & 101 & 14 & 116 & 5 & 3 & -2 \\
\hline 66 DL des Gesundheits-, Veterinar- und Sozialwesens & 76 & 0 & 26 & 0 & 5 & 56 \\
\hline $\begin{array}{l}67 \text { Abwasser-, Abfallbeseitigungs- u. sonst. Entsor- } \\
\text { gungsleistungen }\end{array}$ & 108 & 3 & 167 & 1 & 3 & -55 \\
\hline 68 DL von Interessenvertretungen, Kirchen u.ă. & 74 & 7 & 80 & 2 & 1 & 1 \\
\hline 69 Kultur-, Sport- und Unterhaltungs-DL & 103 & 11 & 136 & 3 & 5 & -20 \\
\hline 70 Sonstige DL & 109 & 14 & 132 & 5 & 5 & -8 \\
\hline $71 \mathrm{DL}$ privater Haushalte & 0 & 0 & 0 & 0 & 2 & 2 \\
\hline Summe & 10740 & 1498 & 7911 & 482 & 158 & 4001 \\
\hline
\end{tabular}

Quelle: Eigene Berechnungen

Die Gesamtsumme ergibt sich aus Spalte 1+2+5 abzüglich den Spalten 3-4. 


\section{Tabelle 43: Sektorale Beschäftigungseffekte für Biopolymere im HD- Szenario (im Vergleich zum Basisszenario)}

\begin{tabular}{|c|c|c|c|c|c|c|}
\hline & $\begin{array}{l}\text { Biopoly } \\
\text {-mere }\end{array}$ & $\begin{array}{l}\text { Biopol. } \\
\text { Invest. }\end{array}$ & $\begin{array}{l}\text { Substi- } \\
\text { tut } \\
\text { Polym }\end{array}$ & $\begin{array}{l}\text { Substi- } \\
\text { tut } \\
\text { Invest. }\end{array}$ & $\begin{array}{l}\text { Positi- } \\
\text { ver } \\
\text { Kon- } \\
\text { sum }\end{array}$ & $\begin{array}{l}\text { Ge- } \\
\text { samt }\end{array}$ \\
\hline & $\begin{array}{c}\text { Er- } \\
\text { werbs- } \\
\text { tătige }\end{array}$ & $\begin{array}{c}\text { Er- } \\
\text { werbs- } \\
\text { tătige }\end{array}$ & $\begin{array}{c}\begin{array}{c}\text { Er- } \\
\text { werbs- } \\
\text { tătige }\end{array} \\
\end{array}$ & $\begin{array}{c}\begin{array}{c}\text { Er- } \\
\text { werbs- } \\
\text { tattige }\end{array} \\
\end{array}$ & $\begin{array}{c}\text { Er- } \\
\text { werbs- } \\
\text { tattige }\end{array}$ & $\begin{array}{c}\text { Er- } \\
\text { werbs- } \\
\text { tattige }\end{array}$ \\
\hline 1 Erzeugnisse der Landwirtschaft und Jagd & 6553 & 0 & 1301 & 0 & 12 & 5240 \\
\hline 2 Forstwirtschaftliche Erzeugnisse und DL & 12 & 2 & 8 & 1 & 1 & 6 \\
\hline 3 Fische und Fischereierzeugnisse & 0 & 0 & 0 & 0 & 0 & $\overline{0}$ \\
\hline 4 Kohle und Torf & 271 & 3 & 332 & 1 & 6 & -53 \\
\hline 5 Erdol, Erdgas, DL fur Erdচl-, Erdgasgewinnung & 7 & 1 & 12 & 0 & 1 & -4 \\
\hline 6 Uran- und Thoriumerze & 0 & 0 & 0 & 0 & 0 & 0 \\
\hline 7 Erze & 0 & 0 & 0 & 0 & 0 & 0 \\
\hline 8 Steine und Erden, sonstige Bergbauerzeugnisse & 70 & 2 & 164 & 1 & 11 & -81 \\
\hline 9 Nahrungs- und Futtermittel & 7 & 1 & 291 & 0 & 48 & -236 \\
\hline 10 Getrănke & 1 & 0 & 1 & 0 & 1 & 1 \\
\hline 11 Tabakerzeugnisse & 0 & 0 & 0 & 0 & 1 & 1 \\
\hline 12 Textilien & 3 & 1 & 2 & 0 & 1 & 3 \\
\hline 13 Bekleidung & 0 & 0 & 0 & 0 & 1 & 1 \\
\hline 14 Leder und Lederwaren & 0 & 0 & 0 & 0 & 1 & 1 \\
\hline 15 Holz; Holz-, Kork-, Flechtwaren (o. Mobbel) & 53 & 25 & 58 & 9 & 6 & 16 \\
\hline 16 Holzstoff, Zellstoff, Papier, Karton, Pappe & 7 & 1 & 14 & 0 & 1 & -5 \\
\hline 17 Papier-, Karton- und Pappewaren & 76 & 5 & 159 & 2 & 12 & -68 \\
\hline 18 Verlagserzeugnisse & 24 & 3 & 37 & 1 & 8 & -3 \\
\hline $\begin{array}{l}19 \text { Druckerzeugnisse, bespielte Ton-, Bild- und Daten- } \\
\text { träger }\end{array}$ & 103 & 17 & 158 & 6 & 19 & -26 \\
\hline $\begin{array}{l}20 \text { Kokereierzeugnisse, Mineralolerzeugnisse, Spalt- } \\
\text { und Brutstoffe }\end{array}$ & 21 & 1 & 74 & 0 & 3 & -50 \\
\hline 21 Pharmazeutische Erzeugnisse & 5 & 0 & 1 & 0 & 1 & 4 \\
\hline $\begin{array}{l}22 \text { Chemische Erzeugnisse (o. pharmazeutische Er- } \\
\text { zeugnisse) }\end{array}$ & 951 & 4 & 1662 & 2 & 213 & -495 \\
\hline 23 Gummiwaren & 7 & 3 & 8 & 1 & 1 & 1 \\
\hline 24 Kunststoffwaren & 99 & 28 & 140 & 10 & 12 & -12 \\
\hline 25 Glas und Glaswaren & 37 & 5 & 42 & 2 & 3 & 1 \\
\hline 26 Keramik, bearbeitete Steine und Erden & 77 & 26 & 75 & 10 & 6 & 24 \\
\hline 27 Roheisen, Stahl, Rohre und Halbzeug daraus & 14 & 9 & 21 & 3 & 1 & 0 \\
\hline 28 NE-Metalle und Halbzeug daraus & 4 & 2 & 6 & 1 & 0 & 0 \\
\hline 29 Gießereierzeugnisse & 7 & 8 & 6 & 3 & 0 & 6 \\
\hline 30 Metallerzeugnisse & 259 & 186 & 335 & 69 & 26 & 67 \\
\hline 31 Maschinen & 484 & 399 & 215 & 147 & 15 & 534 \\
\hline $\begin{array}{l}32 \text { Buromaschinen, Datenverarbeitungsgerăte u. - } \\
\text { einrichtungen }\end{array}$ & 1 & 3 & 2 & 1 & 0 & 1 \\
\hline 33 Gerăte der Elektrizitătserzeugung, -verteilung u.ă. & 114 & 98 & 108 & 36 & 5 & 73 \\
\hline $\begin{array}{l}34 \text { Nachrtechn., Rundf.- und Fernsehgerăte, elektron. } \\
\text { Bauelemente }\end{array}$ & 1 & 9 & 1 & 3 & 0 & 6 \\
\hline $\begin{array}{l}35 \text { Medizin-, mess-, regelungstechn., optische Erzeug- } \\
\text { nisse; Uhren }\end{array}$ & 17 & 100 & 33 & 37 & 3 & 49 \\
\hline $36 \mathrm{Kraftwagen}$ und Kraftwagenteile & 16 & 2 & 13 & 1 & 9 & 12 \\
\hline 37 Sonstige Fahrzeuge (Wasser-, Schienen-, Luftfahr- & 2 & 0 & 3 & 0 & 0 & 0 \\
\hline
\end{tabular}




\begin{tabular}{|c|c|c|c|c|c|c|}
\hline & $\begin{array}{l}\text { Biopoly } \\
\text {-mere }\end{array}$ & $\begin{array}{l}\text { Biopol. } \\
\text { Invest. }\end{array}$ & $\begin{array}{l}\text { Substi- } \\
\text { tut } \\
\text { Polym }\end{array}$ & $\begin{array}{l}\text { Substi- } \\
\text { tut } \\
\text { Invest. }\end{array}$ & $\begin{array}{c}\text { Positi- } \\
\text { ver } \\
\text { Kon- } \\
\text { sum } \\
\end{array}$ & $\begin{array}{l}\text { Ge- } \\
\text { samt }\end{array}$ \\
\hline \multicolumn{7}{|l|}{ zeuge u.a.) } \\
\hline $\begin{array}{l}38 \text { Mobel, Schmuck, Musikinstrumente, Sportgerăte, } \\
\text { Spielwaren u.a.. }\end{array}$ & 1 & 71 & 1 & 26 & 7 & 51 \\
\hline 39 Sekundärrohstoffe & 48 & 5 & 95 & 2 & 8 & -36 \\
\hline $\begin{array}{l}40 \text { Elektrizităt, Fernwärme, DL der Elektrizităts- u. } \\
\text { Fernwärmevers. }\end{array}$ & 377 & 4 & 456 & 1 & 8 & -69 \\
\hline 41 Gase, DL der Gasversorgung & 169 & 2 & 360 & 1 & 5 & -185 \\
\hline 42 Wasser und DL der Wasserversorgung & 98 & 1 & 207 & 0 & 3 & -106 \\
\hline 43 Vorb. Baustellenarbeiten, Hoch- u. Tiefbauarbeiten & 89 & 234 & 107 & 87 & 11 & 140 \\
\hline 44 Bauinstallations- und sonstige Bauarbeiten & 1280 & 212 & 203 & 79 & 26 & 1237 \\
\hline 45 Handelsleist. mit Kfz; Rep. an Kfz; Tankleistungen & 208 & 17 & 167 & 6 & 58 & 110 \\
\hline 46 Handelsvermittlungs- und Großhandelsleistungen & 775 & 250 & 972 & 93 & 91 & 52 \\
\hline $\begin{array}{l}47 \text { Einzelhandelsleistungen; Reparatur an Gebrauchs- } \\
\text { gütern }\end{array}$ & 200 & 24 & 269 & 9 & 322 & 268 \\
\hline 48 Beherbergungs- und Gaststătten-DL & 27 & 4 & 41 & 1 & 164 & 152 \\
\hline 49 Eisenbahn-DL & 54 & 3 & 93 & 1 & 14 & -23 \\
\hline $\begin{array}{l}50 \text { Sonst. Landv.leistungen, Transportl. in Rohrfern- } \\
\text { leitungen }\end{array}$ & 588 & 80 & 784 & 30 & 80 & -66 \\
\hline 51 Schifffahrtsleistungen & 1 & 0 & 1 & 0 & 0 & 0 \\
\hline 52 Luftfahrtleistungen & 25 & 6 & 38 & 2 & 6 & -4 \\
\hline $\begin{array}{l}53 \text { DL bezüglich Hilfs- u. Nebentätigkeiten fur den } \\
\text { Verkehr }\end{array}$ & 153 & 36 & 218 & 13 & 25 & -16 \\
\hline 54 Nachrichtenubermittlungs-DL & 67 & 11 & 101 & 4 & 20 & -8 \\
\hline 55 DL der Kreditinstitute & 248 & 23 & 206 & 8 & 39 & 95 \\
\hline 56 DL der Versicherungen (ohne Sozialversicherung) & 472 & 21 & 455 & 8 & 101 & 131 \\
\hline 57 DL des Kredit- und Versicherungshilfsgewerbes & 1345 & 10 & 142 & 4 & 26 & 1236 \\
\hline 58 DL des Grundstiucks- und Wohnungswesens & 92 & 19 & 125 & 7 & 48 & 26 \\
\hline $\begin{array}{l}59 \text { DL der Vermietung beweglicher Sachen (o. Perso- } \\
\text { nal) }\end{array}$ & 230 & 17 & 180 & 6 & 13 & 74 \\
\hline 60 DL der Datenverarbeitung und von Datenbanken & 144 & 643 & 230 & 238 & 22 & 341 \\
\hline 61 Forschungs- und Entwicklungsleistungen & 112 & 1 & 64 & 1 & 4 & 53 \\
\hline 62 Unternehmensbezogene DL & 4176 & 474 & 6418 & 175 & 509 & -1435 \\
\hline 63 DL der offentlichen Verwaltung, Verteidigung & 429 & 23 & 596 & 8 & 31 & -122 \\
\hline 64 DL der Sozialversicherung & 0 & 0 & 0 & 0 & 0 & 0 \\
\hline 65 Erziehungs- und Unterrichts-DL & 208 & 30 & 284 & 11 & 50 & -8 \\
\hline 66 DL des Gesundheits-, Veterinär- und Sozialwesens & 148 & 1 & 63 & 0 & 85 & 170 \\
\hline $\begin{array}{l}67 \text { Abwasser-, Abfallbeseitigungs- u. sonst. Entsor- } \\
\text { gungsleistungen }\end{array}$ & 222 & 6 & 407 & 2 & 39 & -142 \\
\hline 68 DL von Interessenvertretungen, Kirchen u.ă. & 156 & 15 & 198 & 5 & 22 & -11 \\
\hline 69 Kultur-, Sport- und Unterhaltungs-DL & 214 & 23 & 334 & 8 & 83 & -22 \\
\hline 70 Sonstige $\mathrm{DL}$ & 224 & 31 & 323 & 11 & 84 & 4 \\
\hline $71 \mathrm{DL}$ privater Haushalte & 0 & 0 & 0 & 0 & 30 & 30 \\
\hline Summe & 21878 & 3238 & 19421 & 1197 & 2459 & 6933 \\
\hline
\end{tabular}

Quelle: Eigene Berechnungen

Die Gesamtsumme ergibt sich aus Spalte 1+2+5 abzüglich den Spalten 3-4. 


\section{Tabelle 44: Sektorale Produktionseffekte für biotechnologisch hergestellte Fein-/Spezialchemikalien im Referenzszenario (im Vergleich zum Basisszenario)}

\begin{tabular}{|c|c|c|c|c|c|c|}
\hline & $\begin{array}{l}\text { Biotech- } \\
\text { nologie }\end{array}$ & $\begin{array}{c}\text { Bio- } \\
\text { tech. } \\
\text { Invest. }\end{array}$ & $\begin{array}{c}\text { Substi- } \\
\text { tut } \\
\text { Chemie. }\end{array}$ & $\begin{array}{c}\text { Substi- } \\
\text { tut } \\
\text { Invest. }\end{array}$ & $\begin{array}{c}\text { Positi- } \\
\text { ver } \\
\text { Kon- } \\
\text { sum } \\
\end{array}$ & $\begin{array}{l}\text { Ge- } \\
\text { samt }\end{array}$ \\
\hline & Mio. $€$ & Mio. $€$ & Mio. $€$ & Mio. $€$ & Mio. $€$ & Mio. $€$ \\
\hline 1 Erzeugnisse der Landwirtschaft und Jagd & 68,8 & 0,0 & 15,0 & 0,0 & 0,8 & 54,5 \\
\hline 2 Forstwirtschaftliche Erzeugnisse und DL & 1,1 & 0,1 & 1,3 & 0,2 & 0,1 & $-0,1$ \\
\hline 3 Fische und Fischereierzeugnisse & 0,0 & 0,0 & 0,0 & 0,0 & 0,0 & 0,0 \\
\hline 4 Kohle und Torf & 4,2 & 0,0 & 5,4 & 0,1 & 0,2 & $-0,9$ \\
\hline 5 Erdol, Erdgas, DL fur Erdol-, Erdgasgewinnung & 3,0 & 0,0 & 3,8 & 0,1 & 0,2 & $-0,7$ \\
\hline 6 Uran- und Thoriumerze & 0,0 & 0,0 & 0,0 & 0,0 & 0,0 & 0,0 \\
\hline 7 Erze & 0,0 & 0,0 & 0,0 & 0,0 & 0,0 & 0,0 \\
\hline 8 Steine und Erden, sonstige Bergbauerzeugnisse & 48,5 & 0,6 & 62,0 & 0,8 & 1,8 & $-11,9$ \\
\hline 9 Nahrungs- und Futtermittel & 15,9 & 0,0 & 23,6 & 0,0 & 2,7 & $-5,0$ \\
\hline 10 Getrănke & 0,7 & 0,0 & 0,8 & 0,0 & 0,5 & 0,3 \\
\hline 11 Tabakerzeugnisse & 0,0 & 0,0 & 0,0 & 0,0 & 0,1 & 0,1 \\
\hline 12 Textilien & 0,2 & 0,0 & 0,3 & 0,1 & 0,1 & 0,1 \\
\hline 13 Bekleidung & 0,0 & 0,0 & 0,0 & 0,0 & 0,2 & 0,2 \\
\hline 14 Leder und Lederwaren & 0,1 & 0,0 & 0,1 & 0,0 & 0,1 & 0,0 \\
\hline 15 Holz; Holz-, Kork-, Flechtwaren (o. Mobbel) & 7,2 & 1,9 & 8,9 & 2,5 & 0,4 & $-1,9$ \\
\hline 16 Holzstoff, Zellstoff, Papier, Karton, Pappe & 10,9 & 0,1 & 13,6 & 0,1 & 0,5 & $-2,3$ \\
\hline 17 Papier-, Karton- und Pappewaren & 30,4 & 0,2 & 38,5 & 0,3 & 1,3 & $-6,9$ \\
\hline 18 Verlagserzeugnisse & 6,2 & 0,1 & 7,0 & 0,2 & 0,8 & 0,0 \\
\hline $\begin{array}{l}19 \text { Druckerzeugnisse, bespielte Ton-, Bild- und Daten- } \\
\text { trager }\end{array}$ & 11,2 & 0,3 & 13,1 & 0,3 & 0,8 & $-1,3$ \\
\hline $\begin{array}{l}20 \text { Kokereierzeugnisse, Mineralolerzeugnisse, Spalt- } \\
\text { und Brutstoffe }\end{array}$ & 57,0 & 0,6 & 70,6 & 0,7 & 3,0 & $-10,8$ \\
\hline 21 Pharmazeutische Erzeugnisse & 0,2 & 0,0 & 0,1 & 0,0 & 0,2 & 0,3 \\
\hline $\begin{array}{l}22 \text { Chemische Erzeugnisse (o. pharmazeutische Er- } \\
\text { zeugnisse) }\end{array}$ & 3611,4 & 1,5 & 4208,7 & 2,0 & 118,3 & $-479,5$ \\
\hline 23 Gummiwaren & 1,6 & 0,1 & 2,0 & 0,1 & 0,1 & $-0,3$ \\
\hline 24 Kunststoffwaren & 21,1 & 1,6 & 26,6 & 2,0 & 1,0 & $-4,9$ \\
\hline 25 Glas und Glaswaren & 5,5 & 0,2 & 6,8 & 0,2 & 0,2 & $-1,1$ \\
\hline 26 Keramik, bearbeitete Steine und Erden & 8,7 & 3,2 & 10,6 & 4,1 & 0,4 & $-2,4$ \\
\hline 27 Roheisen, Stahl, Rohre und Halbzeug daraus & 9,7 & 1,5 & 12,1 & 1,9 & 0,5 & $-2,3$ \\
\hline 28 NE-Metalle und Halbzeug daraus & 3,1 & 0,2 & 3,9 & 0,3 & 0,1 & $-0,8$ \\
\hline 29 Gießereierzeugnisse & 1,5 & 0,3 & 1,8 & 0,4 & 0,1 & $-0,3$ \\
\hline 30 Metallerzeugnisse & 33,5 & 5,4 & 41,9 & 7,0 & 1,6 & $-8,4$ \\
\hline 31 Maschinen & 46,7 & 10,7 & 53,9 & 14,0 & 1,9 & $-8,6$ \\
\hline $\begin{array}{l}32 \text { Buromaschinen, Datenverarbeitungsgerăte u. - } \\
\text { einrichtungen }\end{array}$ & 2,8 & 0,5 & 3,4 & 0,6 & 0,1 & $-0,6$ \\
\hline 33 Gerăte der Elektrizitătserzeugung, -verteilung u.ă. & 12,5 & 4,4 & 15,4 & 5,7 & 0,7 & $-3,5$ \\
\hline $\begin{array}{l}34 \text { Nachrtechn., Rundf.- und Fernsehgeräte, elektron. } \\
\text { Bauelemente }\end{array}$ & 1,5 & 1,3 & 1,7 & 1,7 & 0,2 & $-0,4$ \\
\hline $\begin{array}{l}35 \text { Medizin-, mess-, regelungstechn., optische Erzeug- } \\
\text { nisse; Uhren }\end{array}$ & 4,3 & 2,2 & 5,5 & 2,8 & 0,3 & $-1,5$ \\
\hline 36 Kraftwagen und Kraftwagenteile & 4,2 & 0,1 & 4,8 & 0,2 & 1,9 & 1,2 \\
\hline 37 Sonstige Fahrzeuge (Wasser-, Schienen-, Luftfahr- & 1,9 & 0,0 & 2,0 & 0,1 & 0,2 & 0,0 \\
\hline
\end{tabular}




\begin{tabular}{|c|c|c|c|c|c|c|}
\hline & $\begin{array}{l}\text { Biotech- } \\
\text { nologie }\end{array}$ & $\begin{array}{l}\text { Bio- } \\
\text { tech. } \\
\text { Invest. }\end{array}$ & $\begin{array}{c}\text { Substi- } \\
\text { tut } \\
\text { Chemie. }\end{array}$ & $\begin{array}{l}\text { Substi- } \\
\text { tut } \\
\text { Invest. }\end{array}$ & $\begin{array}{c}\text { Positi- } \\
\text { ver } \\
\text { Kon- } \\
\text { sum } \\
\end{array}$ & $\begin{array}{l}\text { Ge- } \\
\text { samt }\end{array}$ \\
\hline \multicolumn{7}{|l|}{ zeuge u.a.) } \\
\hline $\begin{array}{l}38 \text { Mobbel, Schmuck, Musikinstrumente, Sportgerăte, } \\
\text { Spielwaren u.a. }\end{array}$ & 0,1 & 0,9 & 0,1 & 1,2 & 0,4 & 0,1 \\
\hline 39 Sekundärohstoffe & 4,4 & 0,1 & 5,7 & 0,1 & 0,2 & $-1,1$ \\
\hline $\begin{array}{l}40 \text { Elektrizităt, Fernwărme, DL der Elektrizităts- u. } \\
\text { Fernwärmevers. }\end{array}$ & 59,1 & 0,7 & 75,4 & 0,9 & 3,4 & $-13,1$ \\
\hline 41 Gase, DL der Gasversorgung & 10,6 & 0,1 & 13,6 & 0,2 & 0,7 & $-2,4$ \\
\hline 42 Wasser und DL der Wasserversorgung & 9,8 & 0,1 & 12,1 & 0,1 & 0,6 & $-1,8$ \\
\hline 43 Vorb. Baustellenarbeiten, Hoch- $u$. Tiefbauarbeiten & 6,4 & 19,4 & 7,1 & 25,3 & 0,5 & $-6,2$ \\
\hline 44 Bauinstallations- und sonstige Bauarbeiten & 12,6 & 16,9 & 13,8 & 22,0 & 1,1 & $-5,2$ \\
\hline 45 Handelsleist. mit Kfz; Rep. an $\mathrm{Kfz}$; Tankleistungen & 9,8 & 0,3 & 10,3 & 0,4 & 2,0 & 1,4 \\
\hline 46 Handelsvermittlungs- und Großhandelsleistungen & 146,1 & 5,9 & 157,4 & 7,7 & 7,0 & $-6,1$ \\
\hline $\begin{array}{l}47 \text { Einzelhandelsleistungen; Reparatur an Gebrauchs- } \\
\text { gutern }\end{array}$ & 10,6 & 0,3 & 11,6 & 0,4 & 6,7 & 5,6 \\
\hline 48 Beherbergungs- und Gaststätten-DL & 1,4 & 0,0 & 1,6 & 0,0 & 2,8 & 2,7 \\
\hline 49 Eisenbahn-DL & 9,7 & 0,1 & 10,6 & 0,1 & 0,7 & $-0,2$ \\
\hline $\begin{array}{l}50 \text { Sonst. Landv.leistungen, Transportl. in Rohrfern- } \\
\text { leitungen }\end{array}$ & 38,9 & 0,8 & 42,7 & 1,1 & 2,2 & $-1,9$ \\
\hline 51 Schifffahrtsleistungen & 6,9 & 0,0 & 7,6 & 0,1 & 0,2 & $-0,5$ \\
\hline 52 Lufffahrtleistungen & 14,1 & 0,3 & 15,4 & 0,4 & 0,9 & $-0,5$ \\
\hline $\begin{array}{l}53 \text { DL bezuglich Hilfs- u. Nebentătigkeiten fur den } \\
\text { Verkehr }\end{array}$ & 50,9 & 1,4 & 55,3 & 1,8 & 3,0 & $-1,9$ \\
\hline 54 Nachrichtenubermittlungs-DL & 35,5 & 0,8 & 38,9 & 1,0 & 3,8 & 0,2 \\
\hline 55 DL der Kreditinstitute & 46,4 & 1,9 & 49,8 & 2,4 & 5,1 & 1,2 \\
\hline $56 \mathrm{DL}$ der Versicherungen (ohne Sozialversicherung) & 26,7 & 0,5 & 28,9 & 0,6 & 3,7 & 1,3 \\
\hline 57 DL des Kredit- und Versicherungshilfsgewerbes & 12,0 & 0,3 & 13,0 & 0,4 & 1,3 & 0,3 \\
\hline $58 \mathrm{DL}$ des Grundstucks- und Wohnungswesens & 72,4 & 4,7 & 79,0 & 6,2 & 14,8 & 6,7 \\
\hline $\begin{array}{l}59 \text { DL der Vermietung beweglicher Sachen (o. Perso- } \\
\text { nal) }\end{array}$ & 50,8 & 2,0 & 53,5 & 2,6 & 2,4 & $-0,9$ \\
\hline 60 DL der Datenverarbeitung und von Datenbanken & 18,8 & 5,5 & 20,7 & 7,2 & 1,0 & $-2,6$ \\
\hline 61 Forschungs- und Entwicklungsleistungen & 11,2 & 0,0 & 9,5 & 0,0 & 0,3 & 2,0 \\
\hline 62 Unternehmensbezogene DL & 437,0 & 9,1 & 474,8 & 11,9 & 17,9 & $-22,7$ \\
\hline $63 \mathrm{DL}$ der offentlichen Verwaltung, Verteidigung & 22,0 & 0,8 & 24,6 & 1,1 & 1,1 & $-1,8$ \\
\hline 64 DL der Sozialversicherung & 0,0 & 0,0 & 0,0 & 0,0 & 0,0 & 0,0 \\
\hline 65 Erziehungs- und Unterrichts-DL & 12,3 & 0,2 & 13,0 & 0,3 & 1,1 & 0,3 \\
\hline 66 DL des Gesundheits-, Veterinär- und Sozialwesens & 3,2 & 0,0 & 2,9 & 0,0 & 2,7 & 3,0 \\
\hline $\begin{array}{l}67 \text { Abwasser-, Abfallbeseitigungs- u. sonst. Entsor- } \\
\text { gungsleistungen }\end{array}$ & 46,3 & 0,2 & 60,5 & 0,3 & 2,6 & $-11,6$ \\
\hline $68 \mathrm{DL}$ von Interessenvertretungen, Kirchen u.a. & 7,2 & 0,2 & 7,9 & 0,3 & 0,5 & $-0,2$ \\
\hline 69 Kultur-, Sport- und Unterhaltungs-DL & 16,6 & 0,3 & 18,1 & 0,4 & 2,1 & 0,6 \\
\hline 70 Sonstige DL & 13,9 & 0,3 & 15,2 & 0,4 & 1,9 & 0,5 \\
\hline $71 \mathrm{DL}$ privater Haushalte & 0,0 & 0,0 & 0,0 & 0,0 & 0,3 & 0,3 \\
\hline Summe & 5259,4 & 111,5 & 6015,8 & 145,7 & 236,3 & $-554,3$ \\
\hline
\end{tabular}

Quelle: Eigene Berechnungen

Die Gesamtsumme ergibt sich aus Spalte 1+2+5 abzüglich den Spalten 3-4. 
Tabelle 45: Sektorale Produktionseffekte für biotechnologisch hergestellte Fein-/Spezialchemikalien im HD-Szenario (im Vergleich zum Basisszenario)

\begin{tabular}{|c|c|c|c|c|c|c|}
\hline & $\begin{array}{l}\text { Biotech- } \\
\text { nologie }\end{array}$ & $\begin{array}{l}\text { Bio- } \\
\text { tech. } \\
\text { Invest. }\end{array}$ & $\begin{array}{l}\text { Substi- } \\
\text { tut } \\
\text { Chemie. }\end{array}$ & $\begin{array}{c}\text { Substi- } \\
\text { tut } \\
\text { Invest. }\end{array}$ & $\begin{array}{l}\text { Positi- } \\
\text { ver } \\
\text { Kon- } \\
\text { sum }\end{array}$ & $\begin{array}{l}\text { Ge- } \\
\text { samt }\end{array}$ \\
\hline & Mio. $€$ & Mio. $\epsilon$ & Mio. $\epsilon$ & Mio. $€$ & Mio. $€$ & Mio. $\epsilon$ \\
\hline 1 Erzeugnisse der Landwirtschaft und Jagd & 113,0 & 0,0 & 29,4 & 0,0 & 2,1 & 85,7 \\
\hline 2 Forstwirtschaftliche Erzeugnisse und DL & 1,8 & 0,2 & 2,3 & 0,3 & 0,2 & $-0,4$ \\
\hline 3 Fische und Fischereierzeugnisse & 0,0 & 0,0 & 0,0 & 0,0 & 0,0 & 0,0 \\
\hline 4 Kohle und Torf & 7,0 & 0,1 & 9,6 & 0,1 & 0,6 & $-2,0$ \\
\hline 5 Erdøl, Erdgas, DL fur Erdøl-, Erdgasgewinnung & 5,0 & 0,1 & 6,8 & 0,1 & 0,4 & $-1,5$ \\
\hline 6 Uran- und Thoriumerze & 0,0 & 0,0 & 0,0 & 0,0 & 0,0 & 0,0 \\
\hline 7 Erze & 0,0 & 0,0 & 0,0 & 0,0 & 0,0 & 0,0 \\
\hline 8 Steine und Erden, sonstige Bergbauerzeugnisse & 80,3 & 1,1 & 110,7 & 1,5 & 4,8 & $-26,1$ \\
\hline 9 Nahrungs- und Futtermittel & 26,3 & 0,0 & 42,4 & 0,0 & 7,3 & $-8,9$ \\
\hline 10 Getrănke & 1,1 & 0,0 & 1,5 & 0,0 & 1,3 & 0,9 \\
\hline 11 Tabakerzeugnisse & 0,0 & 0,0 & 0,0 & 0,0 & 0,3 & 0,3 \\
\hline 12 Textilien & 0,4 & 0,1 & 0,5 & 0,1 & 0,3 & 0,2 \\
\hline 13 Bekleidung & 0,1 & 0,0 & 0,1 & 0,0 & 0,5 & 0,5 \\
\hline 14 Leder und Lederwaren & 0,1 & 0,0 & 0,1 & 0,0 & 0,1 & 0,1 \\
\hline 15 Holz; Holz-, Kork-, Flechtwaren (o. Möbel) & 12,0 & 3,2 & 16,0 & 4,5 & 1,1 & $-4,2$ \\
\hline 16 Holzstoff, Zellstoff, Papier, Karton, Pappe & 18,1 & 0,2 & 24,4 & 0,3 & 1,3 & $-5,0$ \\
\hline 17 Papier-, Karton- und Pappewaren & 50,3 & 0,3 & 68,8 & 0,5 & 3,4 & $-15,2$ \\
\hline 18 Verlagserzeugnisse & 10,7 & 0,2 & 12,5 & 0,3 & 2,1 & 0,3 \\
\hline $\begin{array}{l}19 \text { Druckerzeugnisse, bespielte Ton-, Bild- und Daten- } \\
\text { träger }\end{array}$ & 18,9 & 0,4 & 23,4 & 0,6 & 2,1 & $-2,6$ \\
\hline $\begin{array}{l}20 \text { Kokereierzeugnisse, Mineralolerzeugnisse, Spalt- } \\
\text { und Brutstoffe }\end{array}$ & 94,7 & 0,9 & 126,3 & 1,3 & 8,1 & $-23,8$ \\
\hline 21 Pharmazeutische Erzeugnisse & 0,4 & 0,0 & 0,1 & 0,0 & 0,5 & 0,7 \\
\hline $\begin{array}{l}22 \text { Chemische Erzeugnisse (o. pharmazeutische Er- } \\
\text { zeugnisse) }\end{array}$ & 6058,0 & 2,5 & 7522,5 & 3,5 & 317,1 & $\overline{1}-$ \\
\hline 23 Gummiwaren & 2,7 & 0,2 & 3,6 & 0,2 & 0,3 & $-0,7$ \\
\hline 24 Kunststoffwaren & 35,1 & 2,6 & 47,6 & 3,6 & 2,7 & $-10,8$ \\
\hline 25 Glas und Glaswaren & 9,2 & 0,3 & 12,2 & 0,4 & 0,7 & $-2,5$ \\
\hline 26 Keramik, bearbeitete Steine und Erden & 14,5 & 5,2 & 18,9 & 7,4 & 1,1 & $-5,4$ \\
\hline 27 Roheisen, Stahl, Rohre und Halbzeug daraus & 16,1 & 2,4 & 21,6 & 3,5 & 1,3 & $-5,1$ \\
\hline 28 NE-Metalle und Halbzeug daraus & 5,2 & 0,4 & 7,1 & 0,6 & 0,4 & $-1,7$ \\
\hline 29 Gießereierzeugnisse & 2,5 & 0,5 & 3,2 & 0,8 & 0,2 & $-0,7$ \\
\hline 30 Metallerzeugnisse & 55,7 & 8,9 & 75,0 & 12,5 & 4,4 & $-18,5$ \\
\hline 31 Maschinen & 77,3 & 17,7 & 96,4 & 25,0 & 5,1 & $-21,3$ \\
\hline $\begin{array}{l}32 \text { Büromaschinen, Datenverarbeitungsgeräte u. - } \\
\text { einrichtungen }\end{array}$ & 4,7 & 0,8 & 6,1 & 1,1 & 0,4 & $-1,3$ \\
\hline 33 Gerăte der Elektrizitătserzeugung, -verteilung u.ă. & 21,0 & 7,3 & 27,6 & 10,3 & 2,0 & $-7,7$ \\
\hline $\begin{array}{l}34 \text { Nachrtechn., Rundf.- und Fernsehgerate, elektron. } \\
\text { Bauelemente }\end{array}$ & 2,6 & 2,2 & 3,1 & 3,1 & 0,4 & $-0,9$ \\
\hline $\begin{array}{l}35 \text { Medizin-, mess-, regelungstechn., optische Erzeug- } \\
\text { nisse; Uhren }\end{array}$ & 7,2 & 3,6 & 9,8 & 5,0 & 0,8 & $-3,3$ \\
\hline 36 Kraftwagen und Kraftwagenteile & 7,1 & 0,2 & 8,7 & 0,3 & 5,0 & 3,4 \\
\hline 37 Sonstige Fahrzeuge (Wasser-, Schienen-, Luftfahr- & 3,2 & 0,1 & 3,7 & 0,1 & 0,4 & 0,0 \\
\hline
\end{tabular}




\begin{tabular}{|c|c|c|c|c|c|c|}
\hline & $\begin{array}{l}\text { Biotech- } \\
\text { nologie }\end{array}$ & $\begin{array}{l}\text { Bio- } \\
\text { tech. } \\
\text { Invest. }\end{array}$ & $\begin{array}{c}\text { Substi- } \\
\text { tut } \\
\text { Chemie. }\end{array}$ & $\begin{array}{l}\text { Substi- } \\
\text { tut } \\
\text { Invest. }\end{array}$ & $\begin{array}{l}\text { Positi- } \\
\text { ver } \\
\text { Kon- } \\
\text { sum }\end{array}$ & $\begin{array}{l}\text { Ge- } \\
\text { samt }\end{array}$ \\
\hline \multicolumn{7}{|l|}{ zeuge u.a.) } \\
\hline $\begin{array}{l}38 \text { Möbel, Schmuck, Musikinstrumente, Sportgerăte, } \\
\text { Spielwaren u.a. }\end{array}$ & 0,2 & 1,5 & 0,2 & 2,1 & 1,2 & 0,5 \\
\hline 39 Sekundărrohstoffe & 7,4 & 0,1 & 10,2 & 0,1 & 0,5 & $-2,3$ \\
\hline $\begin{array}{l}40 \text { Elektrizităt, Fernwărme, DL der Elektrizităts- u. } \\
\text { Fernwärmevers. }\end{array}$ & 98,1 & 1,1 & 134,8 & 1,6 & 9,0 & $-28,1$ \\
\hline 41 Gase, DL der Gasversorgung & 17,6 & 0,2 & 24,4 & 0,3 & 1,8 & $-5,1$ \\
\hline 42 Wasser und DL der Wasserversorgung & 16,3 & 0,2 & 21,7 & 0,3 & 1,6 & $-3,9$ \\
\hline 43 Vorb. Baustellenarbeiten, Hoch- u. Tiefbauarbeiten & 11,0 & 32,1 & 12,6 & 45,3 & 1,2 & $-13,6$ \\
\hline 44 Bauinstallations- und sonstige Bauarbeiten & 21,8 & 27,9 & 24,6 & 39,4 & 3,0 & $-11,3$ \\
\hline 45 Handelsleist. mit Kfz; Rep. an Kfz; Tankleistungen & 16,8 & 0,5 & 18,5 & 0,8 & 5,5 & 3,5 \\
\hline 46 Handelsvermittlungs- und Großhandelsleistungen & 253,8 & 9,8 & 281,5 & 13,8 & 18,8 & $-12,9$ \\
\hline $\begin{array}{l}47 \text { Einzelhandelsleistungen; Reparatur an Gebrauchs- } \\
\text { gutern }\end{array}$ & 18,5 & 0,6 & 20,7 & 0,8 & 17,9 & 15,4 \\
\hline 48 Beherbergungs- und Gaststatten-DL & 2,5 & 0,0 & 2,8 & 0,1 & 7,6 & 7,3 \\
\hline 49 Eisenbahn-DL & 16,9 & 0,1 & 19,0 & 0,1 & 1,9 & $-0,2$ \\
\hline $\begin{array}{l}50 \text { Sonst. Landv.leistungen, Transportl. in Rohrfern- } \\
\text { leitungen }\end{array}$ & 67,4 & 1,3 & 76,4 & 1,9 & 5,8 & $-3,7$ \\
\hline 51 Schifffahrtsleistungen & 11,9 & 0,1 & 13,6 & 0,1 & 0,7 & $-1,1$ \\
\hline 52 Luftfahrtleistungen & 24,5 & 0,5 & 27,6 & 0,7 & 2,3 & $-0,9$ \\
\hline $\begin{array}{l}53 \text { DL bezuglich Hilfs- u. Nebentătigkeiten fur den } \\
\text { Verkehr }\end{array}$ & 88,4 & 2,3 & 98,9 & 3,3 & 7,9 & $-3,5$ \\
\hline 54 Nachrichtenubermittlungs-DL & 61,6 & 1,3 & 69,5 & 1,9 & 10,3 & 1,8 \\
\hline $55 \mathrm{DL}$ der Kreditinstitute & 80,4 & 3,1 & 89,0 & 4,4 & 13,8 & 3,8 \\
\hline 56 DL der Versicherungen (ohne Sozialversicherung) & 46,2 & 0,7 & 51,7 & 1,1 & 9,8 & 4,0 \\
\hline 57 DL des Kredit- und Versicherungshilfsgewerbes & 20,9 & 0,5 & 23,2 & 0,7 & 3,6 & 1,0 \\
\hline 58 DL des Grundstucks- und Wohnungswesens & 125,6 & 7,8 & 141,2 & 11,0 & 39,6 & 20,8 \\
\hline $\begin{array}{l}59 \text { DL der Vermietung beweglicher Sachen (o. Perso- } \\
\text { nal) }\end{array}$ & 87,5 & 3,3 & 95,8 & 4,7 & 6,5 & $-3,1$ \\
\hline 60 DL der Datenverarbeitung und von Datenbanken & 32,7 & 9,1 & 36,9 & 12,8 & 2,6 & $-5,4$ \\
\hline 61 Forschungs- und Entwicklungsleistungen & 19,7 & 0,1 & 17,0 & 0,1 & 0,7 & 3,4 \\
\hline 62 Unternehmensbezogene DL & 759,9 & 15,1 & 848,9 & 21,3 & 48,0 & $-47,3$ \\
\hline $63 \mathrm{DL}$ der $\mathrm{offentlichen} \mathrm{Verwaltung,} \mathrm{Verteidigung}$ & 37,9 & 1,4 & 44,0 & 1,9 & 3,0 & $-3,7$ \\
\hline 64 DL der Sozialversicherung & 0,0 & 0,0 & 0,0 & 0,0 & 0,0 & 0,0 \\
\hline 65 Erziehungs- und Unterrichts-DL & 21,4 & 0,4 & 23,2 & 0,6 & 2,9 & 0,9 \\
\hline 66 DL des Gesundheits-, Veterinar- und Sozialwesens & 5,4 & 0,0 & 5,1 & 0,0 & 7,2 & 7,5 \\
\hline $\begin{array}{l}67 \text { Abwasser-, Abfallbeseitigungs- u. sonst. Entsor- } \\
\text { gungsleistungen }\end{array}$ & 76,8 & 0,4 & 108,1 & 0,6 & 7,0 & $-24,5$ \\
\hline 68 DL von Interessenvertretungen, Kirchen u.ă. & 12,5 & 0,3 & 14,1 & 0,5 & 1,3 & $-0,4$ \\
\hline 69 Kultur-, Sport- und Unterhaltungs-DL & 28,9 & 0,5 & 32,4 & 0,8 & 5,8 & 2,1 \\
\hline 70 Sonstige $\mathrm{DL}$ & 24,1 & 0,5 & 27,3 & 0,7 & 5,2 & 1,8 \\
\hline $71 \mathrm{DL}$ privater Haushalte & 0,0 & 0,0 & 0,0 & 0,0 & 0,9 & 0,9 \\
\hline Summe & 8877,2 & 184,4 & 10756,6 & 260,5 & 633,6 & 1321,9 \\
\hline
\end{tabular}

Quelle: Eigene Berechnungen

Die Gesamtsumme ergibt sich aus Spalte 1+2+5 abzüglich den Spalten 3-4. 
Tabelle 46: Sektorale Beschäftigungseffekte für biotechnologisch hergestellte Fein-/Spezialchemikalien im Referenzszenario (im Vergleich zum Basisszenario)

\begin{tabular}{|c|c|c|c|c|c|c|}
\hline & $\begin{array}{l}\text { Biotech- } \\
\text { nologie }\end{array}$ & $\begin{array}{l}\text { Bio- } \\
\text { tech. } \\
\text { Invest. }\end{array}$ & $\begin{array}{l}\text { Substi- } \\
\text { tut } \\
\text { Che- } \\
\text { mie. }\end{array}$ & $\begin{array}{l}\text { Substi- } \\
\text { tut } \\
\text { Invest. }\end{array}$ & $\begin{array}{l}\text { Positi- } \\
\text { ver } \\
\text { Kon- } \\
\text { sum }\end{array}$ & $\begin{array}{l}\text { Ge- } \\
\text { samt }\end{array}$ \\
\hline & $\begin{array}{c}\text { Er- } \\
\text { werbs- } \\
\text { tătige }\end{array}$ & $\begin{array}{c}\text { Er- } \\
\text { werbs- } \\
\text { tattige }\end{array}$ & $\begin{array}{c}\begin{array}{c}\text { Er- } \\
\text { werbs- } \\
\text { tattige }\end{array} \\
\end{array}$ & $\begin{array}{c}\text { Er- } \\
\text { werbs- } \\
\text { tătige }\end{array}$ & $\begin{array}{c}\text { Er- } \\
\text { werbs- } \\
\text { tătige }\end{array}$ & $\begin{array}{c}\text { Er- } \\
\text { werbs- } \\
\text { tătige }\end{array}$ \\
\hline 1 Erzeugnisse der Landwirtschaft und Jagd & 495 & 0 & 109 & 0 & 6 & 380 \\
\hline 2 Forstwirtschaftliche Erzeugnisse und DL & $\overline{6}$ & 1 & 6 & 1 & 0 & -1 \\
\hline 3 Fische und Fischereierzeugnisse & 0 & 0 & 0 & 0 & $\mathbf{0}$ & 0 \\
\hline 4 Kohle und Torf & 56 & 1 & 71 & 1 & 3 & -12 \\
\hline 5 Erdol, Erdgas, DL für Erdøl-, Erdgasgewinnung & 7 & 0 & 9 & 0 & 0 & -2 \\
\hline 6 Uran- und Thoriumerze & 0 & 0 & 0 & $\overline{0}$ & 0 & 0 \\
\hline 7 Erze & 0 & 0 & 0 & 0 & 0 & 0 \\
\hline 8 Steine und Erden, sonstige Bergbauerzeugnisse & 144 & 2 & 184 & 2 & 5 & -35 \\
\hline 9 Nahrungs- und Futtermittel & 136 & 0 & 203 & 0 & 23 & -43 \\
\hline 10 Getränke & 1 & 0 & 1 & 0 & 1 & 0 \\
\hline 11 Tabakerzeugnisse & 0 & $\overline{0}$ & 0 & 0 & 0 & 0 \\
\hline 12 Textilien & 1 & 0 & 1 & 0 & 1 & 0 \\
\hline 13 Bekleidung & 0 & 0 & 0 & 0 & 1 & 1 \\
\hline 14 Leder und Lederwaren & 0 & 0 & 0 & 0 & 0 & 0 \\
\hline 15 Holz; Holz-, Kork-, Flechtwaren (o. Möbel) & 48 & 13 & 59 & 17 & 3 & -13 \\
\hline 16 Holzstoff, Zellstoff, Papier, Karton, Pappe & 12 & 0 & 15 & 0 & 1 & -3 \\
\hline 17 Papier-, Karton- und Pappewaren & 139 & 1 & 176 & 1 & 6 & -32 \\
\hline 18 Verlagserzeugnisse & 33 & 1 & 36 & 1 & 4 & 0 \\
\hline $\begin{array}{l}19 \text { Druckerzeugnisse, bespielte Ton-, Bild- und Daten- } \\
\text { träger }\end{array}$ & 132 & 3 & 154 & 4 & 9 & -15 \\
\hline $\begin{array}{l}20 \text { Kokereierzeugnisse, Mineralolerzeugnisse, Spalt- } \\
\text { und Brutstoffe }\end{array}$ & 24 & 0 & 30 & 0 & 1 & -5 \\
\hline 21 Pharmazeutische Erzeugnisse & 0 & 0 & 0 & 0 & 0 & 1 \\
\hline $\begin{array}{l}22 \text { Chemische Erzeugnisse (o. pharmazeutische Er- } \\
\text { zeugnisse) }\end{array}$ & 3507 & 1 & 3905 & 2 & 103 & -296 \\
\hline 23 Gummiwaren & 6 & 0 & 8 & 0 & 0 & -1 \\
\hline 24 Kunststoffwaren & 119 & 9 & 149 & 11 & 6 & -28 \\
\hline 25 Glas und Glaswaren & 34 & 1 & 42 & $T$ & 2 & -7 \\
\hline 26 Keramik, bearbeitete Steine und Erden & 57 & 21 & 69 & 27 & 3 & -16 \\
\hline 27 Roheisen, Stahl, Rohre und Halbzeug daraus & 11 & 2 & 14 & 2 & 1 & -3 \\
\hline 28 NE-Metalle und Halbzeug daraus & 5 & 0 & 6 & 0 & 0 & -1 \\
\hline 29 Gießereierzeugnisse & 4 & 1 & 5 & 1 & 0 & -1 \\
\hline 30 Metallerzeugnisse & 251 & 40 & 314 & 53 & 12 & -63 \\
\hline 31 Maschinen & 191 & 40 & 201 & 52 & 7 & -16 \\
\hline $\begin{array}{l}32 \text { Buromaschinen, Datenverarbeitungsgerăte u. - } \\
\text { einrichtungen }\end{array}$ & 2 & 0 & 2 & 0 & 0 & 0 \\
\hline 33 Gerăte der Elektrizitătserzeugung, -verteilung u.ă. & 42 & 15 & 52 & 19 & 2 & -12 \\
\hline $\begin{array}{l}34 \text { Nachrtechn., Rundf.- und Fernsehgerăte, elektron. } \\
\text { Bauelemente }\end{array}$ & 1 & 1 & 1 & 1 & 0 & 0 \\
\hline $\begin{array}{l}35 \text { Medizin-, mess-, regelungstechn., optische Erzeug- } \\
\text { nisse; Uhren }\end{array}$ & 19 & 9 & 24 & 12 & 1 & -7 \\
\hline
\end{tabular}




\begin{tabular}{|c|c|c|c|c|c|c|}
\hline & $\begin{array}{l}\text { Biotech- } \\
\text { nologie }\end{array}$ & $\begin{array}{l}\text { Bio- } \\
\text { tech. } \\
\text { Invest. }\end{array}$ & $\begin{array}{c}\text { Substi- } \\
\text { tut } \\
\text { Che- } \\
\text { mie. }\end{array}$ & $\begin{array}{l}\text { Substi- } \\
\text { tut } \\
\text { Invest. }\end{array}$ & $\begin{array}{c}\text { Positi- } \\
\text { ver } \\
\text { Kon- } \\
\text { sum }\end{array}$ & $\begin{array}{l}\text { Ge- } \\
\text { samt }\end{array}$ \\
\hline $36 \mathrm{Kraftwagen}$ und Kraftwagenteile & 10 & 0 & 11 & 0 & 4 & 3 \\
\hline $\begin{array}{l}37 \text { Sonstige Fahrzeuge (Wasser-, Schienen-, Luftfahr- } \\
\text { zeuge u.a.) }\end{array}$ & 2 & 0 & 3 & 0 & 0 & 0 \\
\hline $\begin{array}{l}38 \text { Möbel, Schmuck, Musikinstrumente, Sportgerăte, } \\
\text { Spielwaren u.a.. }\end{array}$ & 1 & 7 & 1 & 9 & 3 & 1 \\
\hline 39 Sekundärrohstoffe & 24 & 0 & 31 & 0 & 1 & -6 \\
\hline $\begin{array}{l}40 \text { Elektrizităt, Fernwărme, DL der Elektrizităts- u. } \\
\text { Fernwărmevers. }\end{array}$ & 65 & 1 & 83 & 1 & 4 & -15 \\
\hline 41 Gase, DL der Gasversorgung & 36 & 0 & 46 & 1 & 2 & -8 \\
\hline 42 Wasser und DL der Wasserversorgung & 21 & 0 & 26 & 0 & 1 & -4 \\
\hline 43 Vorb. Baustellenarbeiten, Hoch- u. Tiefbauarbeiten & 71 & 214 & 78 & 279 & 5 & -68 \\
\hline 44 Bauinstallations- und sonstige Bauarbeiten & 140 & 187 & 153 & 245 & 13 & -58 \\
\hline 45 Handelsleist. mit $\mathrm{Kfz}$; Rep. an $\mathrm{Kfz}$; Tankleistungen & 134 & 4 & 142 & 6 & 28 & 19 \\
\hline 46 Handelsvermittlungs- und Großhandelsleistungen & 914 & 37 & 985 & 48 & 44 & -38 \\
\hline $\begin{array}{l}47 \text { Einzelhandelsleistungen; Reparatur an Gebrauchs- } \\
\text { gutern }\end{array}$ & 249 & 8 & 270 & 10 & 156 & 131 \\
\hline 48 Beherbergungs- und Gaststătten-DL & 40 & 1 & 44 & 1 & 79 & 75 \\
\hline 49 Eisenbahn-DL & 89 & 1 & 97 & 1 & 7 & -2 \\
\hline $\begin{array}{l}50 \text { Sonst. Landv.leistungen, Transportl. in Rohrfem- } \\
\text { leitungen }\end{array}$ & 692 & 14 & 760 & 19 & 39 & -34 \\
\hline 51 Schifffahrtsleistungen & 1 & 0 & 1 & 0 & 0 & 0 \\
\hline 52 Luftfahrtleistungen & 44 & 1 & 48 & 1 & 3 & -2 \\
\hline $\begin{array}{l}53 \text { DL bezuglich Hilfs- u. Nebentatigkeiten fur den } \\
\text { Verkehr }\end{array}$ & 209 & 6 & 227 & 7 & 12 & -8 \\
\hline 54 Nachrichtenubermittlungs-DL & 90 & 2 & 98 & 3 & 10 & 0 \\
\hline 55 DL der Kreditinstitute & 170 & 7 & 183 & 9 & 19 & 4 \\
\hline 56 DL der Versicherungen (ohne Sozialversicherung) & 85 & 1 & 92 & 2 & 12 & 4 \\
\hline 57 DL des Kredit- und Versicherungshilfsgewerbes & 115 & 3 & 125 & 4 & 13 & 3 \\
\hline $58 \mathrm{DL}$ des Grundstucks- und Wohnungswesens & 113 & 7 & 123 & 10 & 23 & 11 \\
\hline $\begin{array}{l}59 \text { DL der Vermietung beweglicher Sachen (o. Perso- } \\
\text { nal) }\end{array}$ & 132 & 5 & 139 & 7 & 6 & -2 \\
\hline 60 DL der Datenverarbeitung und von Datenbanken & 210 & 61 & 230 & 80 & 11 & -29 \\
\hline 61 Forschungs- und Entwicklungsleistungen & 89 & 0 & 75 & 0 & 2 & 16 \\
\hline 62 Unternehmensbezogene DL & 6000 & 125 & 6519 & 163 & 246 & -311 \\
\hline $63 \mathrm{DL}$ der offentlichen Verwaltung, Verteidigung & 291 & 11 & 326 & 14 & 15 & -23 \\
\hline 64 DL der Sozialversicherung & 0 & 0 & 0 & 0 & 0 & 0 \\
\hline 65 Erziehungs- und Unterrichts-DL & 274 & 6 & 290 & 7 & 24 & 7 \\
\hline 66 DL des Gesundheits-, Veterinär- und Sozialwesens & 48 & 0 & 43 & 0 & 41 & 45 \\
\hline $\begin{array}{l}67 \text { Abwasser-, Abfallbeseitigungs- u. sonst. Entsor- } \\
\text { gungsleistungen }\end{array}$ & 337 & 2 & 441 & 2 & 19 & -85 \\
\hline 68 DL von Interessenvertretungen, Kirchen u.a. & 158 & 4 & 172 & 6 & 11 & -5 \\
\hline 69 Kultur-, Sport- und Unterhaltungs-DL & 206 & 4 & 224 & 5 & 27 & 7 \\
\hline 70 Sonstige $\mathrm{DL}$ & 295 & 6 & 323 & 8 & 41 & 11 \\
\hline $71 \mathrm{DL}$ privater Haushalte & 0 & 0 & 0 & 0 & 14 & 14 \\
\hline Summe & 16837 & 889 & 18260 & 1162 & 1135 & -572 \\
\hline
\end{tabular}

Quelle: Eigene Berechnungen

Die Gesamtsumme ergibt sich aus Spalte 1+2+5 abzüglich den Spalten 3-4. 


\section{Tabelle 47: Sektorale Beschäftigungseffekte für biotechnologisch hergestell- te Fein-/Spezialchemikalien im HD-Szenario (im Vergleich zum Basisszenario)}

\begin{tabular}{|c|c|c|c|c|c|c|}
\hline & $\begin{array}{l}\text { Biotech- } \\
\text { nologie }\end{array}$ & $\begin{array}{l}\text { Bio- } \\
\text { tech. } \\
\text { Invest. }\end{array}$ & $\begin{array}{l}\text { Substi- } \\
\text { tut } \\
\text { Che- } \\
\text { mie. }\end{array}$ & $\begin{array}{l}\text { Substi- } \\
\text { tut } \\
\text { Invest. }\end{array}$ & $\begin{array}{l}\text { Positi- } \\
\text { ver } \\
\text { Kon- } \\
\text { sum }\end{array}$ & $\begin{array}{c}\text { Ge- } \\
\text { samt }\end{array}$ \\
\hline & $\begin{array}{c}\text { Er- } \\
\text { werbs- } \\
\text { tätige }\end{array}$ & $\begin{array}{c}\text { Er- } \\
\text { werbs- } \\
\text { tattige }\end{array}$ & $\begin{array}{c}\text { Er- } \\
\text { werbs- } \\
\text { tatige }\end{array}$ & $\begin{array}{c}\text { Er- } \\
\text { werbs- } \\
\text { tătige }\end{array}$ & $\begin{array}{c}\text { Er- } \\
\text { werbs- } \\
\text { tătige }\end{array}$ & $\begin{array}{c}\text { Er- } \\
\text { werbs- } \\
\text { tătige }\end{array}$ \\
\hline 1 Erzeugnisse der Landwirtschaft und Jagd & 814 & 0 & 214 & 0 & 15 & 584 \\
\hline 2 Forstwirtschaftliche Erzeugnisse und DL & 9 & 1 & 11 & 2 & 1 & -2 \\
\hline 3 Fische und Fischereierzeugnisse & 0 & 0 & 0 & 0 & 0 & 0 \\
\hline 4 Kohle und Torf & 93 & 1 & 128 & 2 & 8 & -26 \\
\hline 5 Erdol, Erdgas, DL fur Erdøl-, Erdgasgewinnung & 11 & 0 & 16 & 0 & 1 & -3 \\
\hline 6 Uran- und Thoriumerze & 0 & 0 & 0 & 0 & 0 & 0 \\
\hline 7 Erze & 0 & 0 & 0 & 0 & 0 & 0 \\
\hline 8 Steine und Erden, sonstige Bergbauerzeugnisse & 238 & 3 & 329 & 4 & 14 & -77 \\
\hline 9 Nahrungs- und Futtermittel & 226 & 0 & 364 & 0 & 62 & -76 \\
\hline 10 Getrănke & 1 & 0 & 2 & 0 & 2 & 1 \\
\hline 11 Tabakerzeugnisse & 0 & 0 & 0 & 0 & 1 & 1 \\
\hline 12 Textilien & 2 & 0 & 3 & 0 & 1 & 1 \\
\hline 13 Bekleidung & 0 & 0 & 0 & 0 & 2 & 2 \\
\hline 14 Leder und Lederwaren & 0 & 0 & 1 & 0 & 1 & 1 \\
\hline 15 Holz; Holz-, Kork-, Flechtwaren (o. Mobel) & 80 & 21 & 106 & 30 & 7 & -28 \\
\hline 16 Holzstoff, Zellstoff, Papier, Karton, Pappe & 20 & 0 & 27 & 0 & 1 & -6 \\
\hline 17 Papier-, Karton- und Pappewaren & 230 & 2 & 314 & 2 & 16 & -69 \\
\hline 18 Verlagserzeugnisse & 56 & 1 & 65 & 2 & 11 & 1 \\
\hline $\begin{array}{l}19 \text { Druckerzeugnisse, bespielte Ton-, Bild- und Daten- } \\
\text { träger }\end{array}$ & 223 & 5 & 276 & 7 & 24 & -31 \\
\hline $\begin{array}{l}20 \text { Kokereierzeugnisse, Mineralolerzeugnisse, Spalt- } \\
\text { und Brutstoffe }\end{array}$ & 40 & 0 & 54 & 1 & 3 & -10 \\
\hline 21 Pharmazeutische Erzeugnisse & 1 & 0 & 0 & 0 & 1 & 1 \\
\hline $\begin{array}{l}22 \text { Chemische Erzeugnisse (o. pharmazeutische Er- } \\
\text { zeugnisse) }\end{array}$ & 5802 & 2 & 6980 & 3 & 276 & -902 \\
\hline 23 Gummiwaren & 11 & 1 & 14 & 1 & 1 & -3 \\
\hline 24 Kunststoffwaren & 197 & 14 & 267 & 20 & 15 & -61 \\
\hline 25 Glas und Glaswaren & 57 & 2 & 76 & 2 & 4 & -15 \\
\hline 26 Keramik, bearbeitete Steine und Erden & 95 & 34 & 124 & 48 & 7 & -35 \\
\hline 27 Roheisen, Stahl, Rohre und Halbzeug daraus & 18 & 3 & 25 & 4 & 2 & -6 \\
\hline 28 NE-Metalle und Halbzeug daraus & 8 & 1 & 11 & 1 & 1 & -3 \\
\hline 29 Gießereierzeugnisse & 7 & 1 & 8 & 2 & 1 & -2 \\
\hline 30 Metallerzeugnisse & 417 & 67 & 562 & 94 & 33 & -139 \\
\hline 31 Maschinen & 316 & 66 & 360 & 93 & 19 & -52 \\
\hline $\begin{array}{l}32 \text { Büromaschinen, Datenverarbeitungsgerăte u. - } \\
\text { einrichtungen }\end{array}$ & 3 & 0 & 4 & 1 & 0 & -1 \\
\hline 33 Gerăte der Elektrizitătserzeugung, -verteilung u.ă. & 71 & 24 & 93 & 35 & 7 & -26 \\
\hline $\begin{array}{l}34 \text { Nachrtechn., Rundf.- und Fernsehgerăte, elektron. } \\
\text { Bauelemente }\end{array}$ & 2 & 1 & 2 & 2 & 0 & -1 \\
\hline $\begin{array}{l}35 \text { Medizin-, mess-, regelungstechn., optische Erzeug- } \\
\text { nisse; Uhren }\end{array}$ & 31 & 15 & 43 & 22 & 3 & -14 \\
\hline
\end{tabular}




\begin{tabular}{|c|c|c|c|c|c|c|}
\hline & $\begin{array}{c}\text { Biotech- } \\
\text { nologie }\end{array}$ & $\begin{array}{c}\text { Bio- } \\
\text { tech. } \\
\text { Invest. }\end{array}$ & $\begin{array}{c}\text { Substi- } \\
\text { tut } \\
\text { Che- } \\
\text { mie. }\end{array}$ & $\begin{array}{l}\text { Substi- } \\
\text { tut } \\
\text { Invest. }\end{array}$ & $\begin{array}{c}\text { Positi- } \\
\text { ver } \\
\text { Kon- } \\
\text { sum }\end{array}$ & $\begin{array}{l}\text { Ge- } \\
\text { samt }\end{array}$ \\
\hline 36 Kraftwagen und Kraftwagenteile & 16 & 0 & 19 & 1 & 11 & 8 \\
\hline $\begin{array}{l}37 \text { Sonstige Fahrzeuge (Wasser-, Schienen-, Luftfahr- } \\
\text { zeuge u.a.) }\end{array}$ & 4 & 0 & 5 & 0 & 1 & 0 \\
\hline $\begin{array}{l}38 \text { Möbel, Schmuck, Musikinstrumente, Sportgerăte, } \\
\text { Spielwaren u.a. }\end{array}$ & 1 & 11 & 2 & 16 & 9 & 4 \\
\hline 39 Sekundărrohstoffe & 40 & 1 & 55 & 1 & 3 & -13 \\
\hline $\begin{array}{l}40 \text { Elektrizităt, Fernwărme, DL der Elektrizităts- u. } \\
\text { Fernwärmevers. }\end{array}$ & 109 & 1 & 149 & 2 & 10 & -31 \\
\hline 41 Gase, DL der Gasversorgung & 59 & 1 & 82 & 1 & 6 & -17 \\
\hline 42 Wasser und DL der Wasserversorgung & 35 & 0 & 47 & 1 & 3 & -8 \\
\hline 43 Vorb. Baustellenarbeiten, Hoch- u. Tiefbauarbeiten & 122 & 353 & 139 & 499 & 14 & -150 \\
\hline 44 Bauinstallations- und sonstige Bauarbeiten & 242 & 310 & 274 & 438 & 34 & -126 \\
\hline 45 Handelsleist. mit $\mathrm{Kfz}$; Rep. an $\mathrm{Kfz}$; Tankleistungen & 230 & 7 & 254 & 10 & 75 & 49 \\
\hline 46 Handelsvermittlungs- und Großhandelsleistungen & 1589 & 61 & 1762 & 86 & 118 & -81 \\
\hline $\begin{array}{l}47 \text { Einzelhandelsleistungen; Reparatur an Gebrauchs- } \\
\text { gutern }\end{array}$ & 432 & 13 & 484 & 18 & 417 & 360 \\
\hline 48 Beherbergungs- und Gaststätten-DL & 70 & 1 & 79 & 2 & 212 & 203 \\
\hline 49 Eisenbahn-DL & 155 & 1 & 174 & 1 & 18 & -2 \\
\hline $\begin{array}{l}50 \text { Sonst. Landv.leistungen, Transportl. in Rohrfern- } \\
\text { leitungen }\end{array}$ & 1199 & 24 & 1359 & 34 & 104 & -66 \\
\hline 51 Schifffahrtsleistungen & 2 & 0 & 2 & 0 & 0 & 0 \\
\hline 52 Luftahrtleistungen & 76 & 1 & 86 & 2 & 7 & -3 \\
\hline $\begin{array}{l}53 \text { DL bezüglich Hilfs- u. Nebentätigkeiten fur den } \\
\text { Verkehr }\end{array}$ & 364 & 9 & 407 & 13 & 33 & -14 \\
\hline 54 Nachrichtenubermittlungs-DL & 156 & 3 & 176 & 5 & 26 & 5 \\
\hline 55 DL der Kreditinstitute & 295 & 11 & 327 & 16 & 51 & 14 \\
\hline 56 DL der Versicherungen (ohne Sozialversicherung) & 148 & 2 & 165 & 3 & 31 & 13 \\
\hline 57 DL des Kredit- und Versicherungshilfsgewerbes & 200 & 5 & 223 & 7 & 34 & 9 \\
\hline 58 DL des Grundstucks- und Wohnungswesens & 196 & 12 & 220 & 17 & 62 & 32 \\
\hline $\begin{array}{l}59 \text { DL der Vermietung beweglicher Sachen (o. Perso- } \\
\text { nal) }\end{array}$ & 228 & 9 & 249 & 12 & 17 & -8 \\
\hline 60 DL der Datenverarbeitung und von Datenbanken & 365 & 101 & 412 & 143 & 28 & -61 \\
\hline 61 Forschungs- und Entwicklungsleistungen & 156 & 0 & 135 & 1 & 6 & 27 \\
\hline 62 Unternehmensbezogene DL & 10433 & 207 & 11655 & 292 & 659 & -649 \\
\hline 63 DL der offentlichen Verwaltung, Verteidigung & 501 & 18 & 583 & 25 & 40 & -48 \\
\hline $64 \mathrm{DL}$ der Sozialversicherung & 0 & 0 & 0 & 0 & 0 & 0 \\
\hline 65 Erziehungs- und Unterrichts-DL & 478 & 9 & 518 & 13 & 64 & 20 \\
\hline 66 DL des Gesundheits-, Veterinär- und Sozialwesens & 83 & 0 & 78 & 0 & 110 & 115 \\
\hline $\begin{array}{l}67 \text { Abwasser-, Abfallbeseitigungs- u. sonst. Entsor- } \\
\text { gungsleistungen }\end{array}$ & 560 & 3 & 788 & 4 & 51 & -178 \\
\hline 68 DL von Interessenvertretungen, Kirchen u.ă. & 273 & 7 & 308 & 10 & 29 & -9 \\
\hline 69 Kultur-, Sport- und Unterhaltungs-DL & 359 & 7 & 401 & 9 & 71 & 26 \\
\hline 70 Sonstige DL & 511 & 10 & 577 & 14 & 109 & 39 \\
\hline 71 DL privater Haushalte & 0 & 0 & 0 & 0 & 39 & 39 \\
\hline Summe & 28764 & 1470 & 32668 & 2077 & 3044 & -1497 \\
\hline
\end{tabular}

Quelle: Eigene Berechnungen

Die Gesamtsumme ergibt sich aus Spalte 1+2+5 abzüglich den Spalten 3-4. 


\section{Tabelle 48: Sektorale Produktionseffekte für Biopharmazeutika im Refe- renzszenario (im Vergleich zum Basisszenario)}

\begin{tabular}{|c|c|c|c|c|c|c|}
\hline & $\begin{array}{c}\text { Bio- } \\
\text { pharma }\end{array}$ & $\begin{array}{l}\text { Biop. } \\
\text { Invest. }\end{array}$ & $\begin{array}{c}\text { Substi- } \\
\text { tut } \\
\text { Pharma. }\end{array}$ & $\begin{array}{c}\text { Substi- } \\
\text { tut } \\
\text { Invest. }\end{array}$ & $\begin{array}{l}\text { Negati- } \\
\text { ver } \\
\text { Konsum }\end{array}$ & $\begin{array}{l}\text { Ge- } \\
\text { samt }\end{array}$ \\
\hline & Mio. $\epsilon$ & Mio. $\epsilon$ & Mio. $\epsilon$ & Mio. $\epsilon$ & Mio. $\epsilon$ & Mio. $€$ \\
\hline 1 Erzeugnisse der Landwirtschaft und Jagd & 8,0 & 0,0 & 1,2 & 0,0 & 29,9 & $-23,1$ \\
\hline 2 Forstwirtschaftliche Erzeugnisse und DL & 1,0 & 0,2 & 0,2 & 0,0 & 1,3 & $-0,4$ \\
\hline 3 Fische und Fischereierzeugnisse & 0,0 & 0,0 & 0,0 & 0,0 & 0,2 & $-0,2$ \\
\hline 4 Kohle und Torf & 5,7 & 0,1 & 0,8 & 0,0 & 4,4 & 0,6 \\
\hline $\begin{array}{l}5 \text { Erdől, Erdgas, DL fur Erdől-, Erdgasgewin- } \\
\text { nung }\end{array}$ & 2,1 & 0,2 & 0,4 & 0,0 & 2,4 & $-0,6$ \\
\hline 6 Uran- und Thoriumerze & 0,0 & 0,0 & 0,0 & 0,0 & 0,0 & 0,0 \\
\hline 7 Erze & 0,0 & 0,0 & 0,0 & 0,0 & 0,0 & 0,0 \\
\hline 8 Steine und Erden, sonstige Bergbauerzeugnisse & 2,7 & 0,6 & 2,5 & 0,1 & 2,8 & $-2,1$ \\
\hline 9 Nahrungs- und Futtermittel & 36,9 & 0,0 & 5,2 & 0,0 & $\mathbf{9 8 , 8}$ & $-67,1$ \\
\hline 10 Getränke & 1,6 & 0,0 & 0,2 & 0,0 & 21,7 & $-20,3$ \\
\hline 11 Tabakerzeugnisse & 0,0 & 0,0 & 0,0 & 0,0 & 4,7 & $-4,7$ \\
\hline 12 Textilien & 21,7 & 0,1 & 2,6 & 0,0 & $\overline{5,4}$ & 13,8 \\
\hline 13 Bekleidung & 0,0 & 0,0 & 0,0 & 0,0 & 8,4 & $-8,4$ \\
\hline 14 Leder und Lederwaren & 0,0 & 0,1 & 0,0 & 0,0 & 2,3 & $-2,2$ \\
\hline 15 Holz; Holz-, Kork-, Flechtwaren (o. Möbel) & 5,8 & 2,5 & 1,1 & 0,3 & 7,7 & $-0,7$ \\
\hline 16 Holzstoff, Zellstoff, Papier, Karton, Pappe & 17,7 & 0,4 & 2,7 & 0,1 & 6,3 & 9,1 \\
\hline 17 Papier-, Karton- und Pappewaren & 85,7 & 0,6 & 11,5 & 0,1 & 13,6 & 61,1 \\
\hline 18 Verlagserzeugnisse & 7,3 & 0,3 & 1,6 & 0,0 & 28,5 & $-22,5$ \\
\hline $\begin{array}{l}19 \text { Druckerzeugnisse, bespielte Ton-, Bild- und } \\
\text { Datenträger }\end{array}$ & 24,1 & 0,8 & 3,8 & 0,1 & 20,7 & 0,4 \\
\hline $\begin{array}{l}20 \text { Kokereierzeugnisse, Mineralölerzeugnisse, } \\
\text { Spalt- und Brutstoffe }\end{array}$ & 150,8 & 1,1 & 5,6 & 0,2 & 50,7 & 95,5 \\
\hline 21 Pharmazeutische Erzeugnisse & 5370,5 & 0,0 & 980,1 & 0,0 & 450,0 & 3940,3 \\
\hline $\begin{array}{l}22 \text { Chemische Erzeugnisse (o. pharmazeutische } \\
\text { Erzeugnisse) }\end{array}$ & 90,6 & 3,1 & 157,3 & 0,4 & 97,5 & $-161,5$ \\
\hline 23 Gummiwaren & 0,9 & 0,4 & 0,2 & 0,1 & 2,2 & $-1,1$ \\
\hline 24 Kunststoffwaren & 68,3 & 3,1 & 9,1 & 0,4 & 15,2 & 46,7 \\
\hline 25 Glas und Glaswaren & 16,9 & 0,5 & 2,2 & 0,1 & 3,4 & 11,7 \\
\hline 26 Keramik, bearbeitete Steine und Erden & 5,9 & 2,9 & 1,1 & 0,4 & 6,3 & 1,0 \\
\hline 27 Roheisen, Stahl, Rohre und Halbzeug daraus & 11,7 & 4,8 & 1,8 & 0,6 & 8,2 & 5,8 \\
\hline 28 NE-Metalle und Halbzeug daraus & 3,0 & 0,9 & 0,5 & 0,1 & 1,5 & 1,8 \\
\hline 29 Gießereierzeugnisse & 1,4 & 1,7 & 0,2 & 0,2 & 2,0 & 0,6 \\
\hline 30 Metallerzeugnisse & 64,9 & 15,1 & 9,2 & 2,0 & 25,7 & 43,0 \\
\hline 31 Maschinen & 18,9 & 62,3 & 4,2 & 8,4 & 19,4 & 49,2 \\
\hline $\begin{array}{l}32 \text { Buromaschinen, Datenverarbeitungsgerăte u. } \\
\text {-einrichtungen }\end{array}$ & 6 & 2,8 & 1,2 & 0,4 & 2,7 & 7,1 \\
\hline $\begin{array}{l}33 \text { Gerăte der Elektrizitătserzeugung, -verteilung } \\
\text { u.ă. }\end{array}$ & 13,0 & 17,3 & 2,2 & 2,3 & 14,7 & 11,1 \\
\hline $\begin{array}{l}34 \text { Nachrtechn., Rundf.- und Fernsehgerăte, } \\
\text { elektron. Bauelemente }\end{array}$ & 2,5 & 8,2 & 0,5 & 1,1 & 5,2 & 3,8 \\
\hline $\begin{array}{l}35 \text { Medizin-, mess-, regelungstechn., optische } \\
\text { Erzeugnisse; Uhren }\end{array}$ & 17,2 & 13,4 & 2,2 & 1,8 & 7,0 & 19,6 \\
\hline $36 \mathrm{Kraftwagen}$ und Kraftwagenteile & 11,8 & 0,5 & 1,6 & 0,1 & 82,3 & $-71,6$ \\
\hline 37 Sonstige Fahrzeuge (Wasser-, Schienen-, & 2,6 & 0,1 & 0,6 & 0,0 & 5,5 & $-3,3$ \\
\hline
\end{tabular}




\begin{tabular}{|c|c|c|c|c|c|c|}
\hline & $\begin{array}{c}\text { Bio- } \\
\text { pharma }\end{array}$ & $\begin{array}{l}\text { Biop. } \\
\text { Invest. }\end{array}$ & $\begin{array}{l}\text { Substi- } \\
\text { tut } \\
\text { Pharma. }\end{array}$ & $\begin{array}{l}\text { Substi- } \\
\text { tut } \\
\text { Invest. }\end{array}$ & \begin{tabular}{|c|}
$\begin{array}{c}\text { Negati- } \\
\text { ver } \\
\text { Konsum }\end{array}$ \\
\end{tabular} & $\begin{array}{l}\text { Ge- } \\
\text { samt }\end{array}$ \\
\hline \multicolumn{7}{|l|}{ Luftfahrzeuge u.a.) } \\
\hline $\begin{array}{l}38 \text { Möbel, Schmuck, Musikinstrumente, Sportge- } \\
\text { răte, Spielwaren u.a. }\end{array}$ & 0,1 & 5,5 & 0,0 & 0,7 & 20,0 & $-15,1$ \\
\hline 39 Sekundärrohstoffe & 2,2 & 0,2 & 0,5 & 0,0 & 2,2 & $-0,4$ \\
\hline $\begin{array}{l}40 \text { Elektrizităt, Fernwărme, DL der Elektrizităts- } \\
\text { u. Fernwärmevers. }\end{array}$ & 78,3 & 2,0 & 12,1 & 0,3 & 64,1 & 3,8 \\
\hline 4 I Gase, DL der Gasversorgung & 12,0 & 0,3 & 1,9 & 0,0 & 14,1 & $-3,7$ \\
\hline 42 Wasser und DL der Wasserversorgung & 10,3 & 0,3 & 1,7 & 0,0 & 12,1 & $-3,3$ \\
\hline $\begin{array}{l}43 \text { Vorb. Baustellenarbeiten, Hoch- u. Tiefbau- } \\
\text { arbeiten }\end{array}$ & 19,2 & 15,9 & 2,7 & 2,1 & 13,7 & 16,5 \\
\hline 44 Bauinstallations- und sonstige Bauarbeiten & 38,7 & 14,2 & 5,6 & 1,9 & 37,9 & 7,5 \\
\hline $\begin{array}{l}45 \text { Handelsleist. mit Kfz; Rep. an Kfz; Tankleis- } \\
\text { tungen }\end{array}$ & 9,4 & 0,8 & 2,1 & 0,1 & 83,6 & $-75,6$ \\
\hline $\begin{array}{l}46 \text { Handelsvermittlungs- und Großhandelsleis- } \\
\text { tungen }\end{array}$ & 74,3 & 23,7 & 18,7 & 3,2 & 135,7 & $-59,6$ \\
\hline $\begin{array}{l}47 \text { Einzelhandelsleistungen; Reparatur an Ge- } \\
\text { brauchsgutern }\end{array}$ & 17,5 & 0,7 & 3,9 & 0,1 & 298,6 & $-284,4$ \\
\hline 48 Beherbergungs- und Gaststătten-DL & 2,3 & 0,1 & 0,5 & 0,0 & 130,5 & $-128,6$ \\
\hline 49 Eisenbahn-DL & 1,9 & 0,2 & 0,7 & 0,0 & 20,6 & $-19,2$ \\
\hline $\begin{array}{l}50 \text { Sonst. Landv. leistungen, Transportl. in Rohr- } \\
\text { fernleitungen }\end{array}$ & 20,6 & 2,7 & 4,6 & 0,4 & 49,2 & $-30,8$ \\
\hline 51 Schifffahrtsleistungen & 1,0 & 0,1 & 0,4 & 0,0 & 1,9 & $-1,2$ \\
\hline 52 Luftfahrtleistungen & 23,0 & 1,1 & 5,1 & 0,1 & 23,5 & $-4,7$ \\
\hline $\begin{array}{l}53 \text { DL bezüglich Hilfs- u. Nebentätigkeiten fur } \\
\text { den Verkehr }\end{array}$ & 36,7 & 5,3 & 8,7 & 0,7 & 71,4 & $-38,9$ \\
\hline 54 Nachrichtenübermittlungs-DL & 53,9 & 2,5 & 12,0 & 0,3 & 134,7 & $-90,7$ \\
\hline 55 DL der Kreditinstitute & 68,3 & 3,9 & 14,9 & 0,5 & 183,3 & $-126,6$ \\
\hline $\begin{array}{l}56 \text { DL der Versicherungen (ohne Sozialversiche- } \\
\text { rung) }\end{array}$ & 21,2 & 1,0 & 5,0 & 0,1 & 136,6 & $-119,5$ \\
\hline $\begin{array}{l}57 \text { DL des Kredit- und Versicherungshilfsgewer- } \\
\text { bes }\end{array}$ & 11,1 & 0,6 & 2,6 & 0,1 & 46,6 & $-37,5$ \\
\hline 58 DL des Grundstücks- und Wohnungswesens & 104,7 & 7,7 & 23,2 & 1,0 & 600,5 & $-512,3$ \\
\hline $\begin{array}{l}59 \text { DL der Vermietung beweglicher Sachen (o. } \\
\text { Personal) }\end{array}$ & 86,1 & 4,1 & 18,7 & 0,5 & 53,0 & 17,9 \\
\hline $\begin{array}{l}60 \text { DL der Datenverarbeitung und von Daten- } \\
\text { banken }\end{array}$ & 42,7 & 33,6 & 9,3 & 4,5 & 22,3 & 40,2 \\
\hline 61 Forschungs- und Entwicklungsleistungen & 42,8 & 0,1 & 8,1 & 0,0 & 4,4 & 30,5 \\
\hline 62 Unternehmensbezogene DL & 514,1 & 21,3 & 118,7 & 2,9 & 283,3 & 130,6 \\
\hline $\begin{array}{l}63 \text { DL der offentlichen Verwaltung, Verteidi- } \\
\text { gung }\end{array}$ & 26,1 & 1,1 & 5,6 & 0,2 & 24,0 & $-2,6$ \\
\hline 64 DL der Sozialversicherung & 0,0 & 0,0 & 0,0 & 0,0 & 0,0 & 0,0 \\
\hline 65 Erziehungs- und Unterrichts-DL & 17,4 & 0,8 & 3,8 & 0,1 & 35,5 & $-21,1$ \\
\hline $\begin{array}{l}66 \text { DL des Gesundheits-, Veterinar- und Sozial- } \\
\text { wesens }\end{array}$ & 0,4 & 0,0 & 0,2 & 0,0 & 122,9 & $-122,6$ \\
\hline $\begin{array}{l}67 \text { Abwasser-, Abfallbeseitigungs- u. sonst. Ent- } \\
\text { sorgungsleistungen }\end{array}$ & 15,9 & 0,5 & 5,0 & 0,1 & 45,5 & $-34,1$ \\
\hline 68 DL von Interessenvertretungen, Kirchen u.a. & 10,5 & 0,4 & 2,3 & 0,1 & 13,8 & $-5,3$ \\
\hline 69 Kultur-, Sport- und Unterhaltungs-DL & 26,3 & 0,8 & 5,9 & 0,1 & 80,0 & $-58,9$ \\
\hline 70 Sonstige DL & 19,0 & 0,9 & 4,3 & 0,1 & 72,5 & $-57,0$ \\
\hline $71 \mathrm{DL}$ privater Haushalte & 0,0 & 0,0 & 0,0 & 0,0 & 15,0 & $-15,0$ \\
\hline Summe & 7488,1 & 296,5 & 1522,5 & 39,9 & 3911,6 & 2310,5 \\
\hline
\end{tabular}




\section{Tabelle 49: Sektorale Produktionseffekte für Biopharmazeutika im HD- Szenario (im Vergleich zum Basisszenario)}

\begin{tabular}{|c|c|c|c|c|c|c|}
\hline & $\begin{array}{c}\text { Bio- } \\
\text { phar- } \\
\text { ma }\end{array}$ & $\begin{array}{c}\text { Biop. } \\
\text { Invest. }\end{array}$ & $\begin{array}{c}\text { Substi- } \\
\text { tut } \\
\text { Pharma. }\end{array}$ & $\begin{array}{c}\text { Substi- } \\
\text { tut } \\
\text { Invest. }\end{array}$ & \begin{tabular}{|c|} 
Nega- \\
tiver \\
Kon- \\
sum \\
\end{tabular} & $\begin{array}{l}\text { Ge- } \\
\text { samt }\end{array}$ \\
\hline & Mio. $€$ & Mio. $€$ & Mio. $€$ & Mio. $€$ & Mio. $€$ & Mio. $€$ \\
\hline I Erzeugnisse der Landwirtschaft und Jagd & 12,0 & 0,1 & 1,2 & 0,0 & 49,4 & $-38,5$ \\
\hline 2 Forstwirtschaftliche Erzeugnisse und DL & 1,4 & 0,3 & 0,2 & 0,0 & 2,2 & $-0,6$ \\
\hline 3 Fische und Fischereierzeugnisse & 0,0 & 0,0 & 0,0 & 0,0 & 0,3 & $-0,3$ \\
\hline 4 Kohle und Torf & 8,5 & 0,2 & 0,8 & 0,0 & 7,2 & 0,7 \\
\hline 5 Erdøl, Erdgas, DL fur Erdøl-, Erdgasgewinnung & 3,1 & 0,3 & 0,4 & 0,0 & 4,0 & $-1,0$ \\
\hline 6 Uran- und Thoriumerze & 0,0 & 0,0 & 0,0 & 0,0 & 0,0 & 0,0 \\
\hline 7 Erze & 0,0 & 0,0 & 0,0 & 0,0 & 0,0 & 0,0 \\
\hline 8 Steine und Erden, sonstige Bergbauerzeugnisse & 4,0 & 0,9 & 2,5 & 0,1 & 4,4 & $-2,1$ \\
\hline 9 Nahrungs- und Futtermittel & 55,3 & 0,1 & 5,2 & 0,0 & 163,0 & $-112,7$ \\
\hline 10 Getrănke & 2,4 & 0,0 & 0,2 & 0,0 & 35,8 & $-33,6$ \\
\hline 11 Tabakerzeugnisse & 0,0 & 0,0 & 0,0 & 0,0 & 7,8 & $-7,8$ \\
\hline 12 Textilien & 32,5 & 0,2 & 2,6 & 0,0 & 8,8 & 21,3 \\
\hline 13 Bekleidung & 0,0 & 0,0 & 0,0 & 0,0 & 14,0 & $-13,9$ \\
\hline 14 Leder und Lederwaren & 0,0 & 0,1 & 0,0 & 0,0 & 3,8 & $-3,7$ \\
\hline 15 Holz; Holz-, Kork-, Flechtwaren (o. Möbel) & 8,8 & 3,8 & 1,1 & 0,3 & 12,6 & $-1,5$ \\
\hline 16 Holzstoff, Zellstoff, Papier, Karton, Pappe & 26,6 & 0,6 & 2,7 & 0,1 & 10,2 & 14,3 \\
\hline 17 Papier-, Karton- und Pappewaren & 128,6 & 0,9 & 11,5 & 0,1 & 21,8 & 96,2 \\
\hline 18 Verlagserzeugnisse & 11,0 & 0,5 & 1,6 & 0,0 & 47,0 & $-37,1$ \\
\hline $\begin{array}{l}19 \text { Druckerzeugnisse, bespielte Ton-, Bild- und Daten- } \\
\text { träger }\end{array}$ & 36,2 & 1,3 & 3,8 & 0,1 & 33,9 & $-0,4$ \\
\hline $\begin{array}{l}20 \text { Kokereierzeugnisse, Mineralolerzeugnisse, Spalt- } \\
\text { und Brutstoffe }\end{array}$ & 226,3 & 1,7 & 5,6 & 0,2 & 83,4 & 138,8 \\
\hline 21 Pharmazeutische Erzeugnisse & 8060,6 & 0,0 & 980,1 & 0,0 & 676,8 & 6403,7 \\
\hline $\begin{array}{l}22 \text { Chemische Erzeugnisse (o. pharmazeutische Erzeug- } \\
\text { nisse) }\end{array}$ & 136,0 & 4,6 & 157,3 & 0,4 & 150,3 & $-167,4$ \\
\hline 23 Gummiwaren & 1,4 & 0,6 & 0,2 & 0,1 & 3,7 & $-1,9$ \\
\hline 24 Kunststoffwaren & 102,5 & 4,7 & 9,1 & 0,4 & 24,5 & 73,1 \\
\hline 25 Glas und Glaswaren & 25,3 & 0,7 & 2,2 & 0,1 & 5,4 & 18,3 \\
\hline 26 Keramik, bearbeitete Steine und Erden & 8,8 & 4,3 & 1,1 & 0,4 & 10,3 & 1,3 \\
\hline 27 Roheisen, Stahl, Rohre und Halbzeug daraus & 17,6 & 7,2 & 1,8 & 0,6 & 13,4 & 8,8 \\
\hline 28 NE-Metalle und Halbzeug daraus & 4,5 & 1,4 & 0,5 & 0,1 & 2,4 & 2,8 \\
\hline 29 Gießereierzeugnisse & 2,2 & 2,5 & 0,2 & 0,2 & 3,3 & 0,9 \\
\hline 30 Metallerzeugnisse & 97,3 & 22,6 & 9,2 & 2,0 & 41,8 & 67,0 \\
\hline 31 Maschinen & 28,3 & 93,5 & 4,2 & 8,4 & 31,8 & 77,5 \\
\hline $\begin{array}{l}32 \text { Buromaschinen, Datenverarbeitungsgerăte u. - } \\
\text { einrichtungen }\end{array}$ & 12,9 & 4,2 & 1,2 & 0,4 & 4,4 & 11,1 \\
\hline 33 Gerăte der Elektrizitătserzeugung, -verteilung u.a. & 19,6 & 26,0 & 2,2 & 2,3 & 24,2 & 16,9 \\
\hline $\begin{array}{l}34 \text { Nachrtechn., Rundf.- und Fernsehgerăte, elektron. } \\
\text { Bauelemente }\end{array}$ & 3,7 & 12,2 & 0,5 & 1,1 & 8,6 & 5,7 \\
\hline $\begin{array}{l}35 \text { Medizin-, mess-, regelungstechn., optische Erzeug- } \\
\text { nisse; Uhren }\end{array}$ & 25,9 & 20,1 & 2,2 & 1,8 & 11,4 & 30,5 \\
\hline 36 Kraftwagen und Kraftwagenteile & 17,8 & 0,8 & 1,6 & 0,1 & 135,9 & $-118,9$ \\
\hline $\begin{array}{l}37 \text { Sonstige Fahrzeuge (Wasser-, Schienen-, Luftfahr- } \\
\text { zeuge u.a.) }\end{array}$ & 3,9 & 0,2 & 0,6 & 0,0 & 9,0 & $-5,5$ \\
\hline
\end{tabular}




\begin{tabular}{|c|c|c|c|c|c|c|}
\hline & $\begin{array}{c}\text { Bio- } \\
\text { phar- } \\
\text { ma }\end{array}$ & $\begin{array}{l}\text { Biop. } \\
\text { Invest. }\end{array}$ & $\begin{array}{l}\text { Substi- } \\
\text { tut } \\
\text { Pharma. }\end{array}$ & $\begin{array}{l}\text { Substi- } \\
\text { tut } \\
\text { Invest. }\end{array}$ & $\begin{array}{l}\text { Nega- } \\
\text { tiver } \\
\text { Kon- } \\
\text { sum }\end{array}$ & $\begin{array}{c}\text { Ge- } \\
\text { samt }\end{array}$ \\
\hline $\begin{array}{l}38 \text { Mobel, Schmuck, Musikinstrumente, Sportgerăte, } \\
\text { Spielwaren u.a.. }\end{array}$ & 0,2 & 8,2 & 0,0 & 0,7 & 33,1 & $-25,4$ \\
\hline 39 Sekundärrohstoffe & 3,2 & 0,3 & 0,5 & 0,0 & 3,6 & $-0,7$ \\
\hline $\begin{array}{l}40 \text { Elektrizităt, Fernwărme, DL der Elektrizităts- u. } \\
\text { Fernwărmevers. }\end{array}$ & 117,5 & 3,0 & 12,1 & 0,3 & 105,1 & 3,0 \\
\hline 41 Gase, DL der Gasversorgung & 18,1 & 0,5 & 1,9 & 0,0 & 23,1 & $-6,6$ \\
\hline 42 Wasser und DL der Wasserversorgung & 15,4 & 0,4 & 1,7 & 0,0 & 19,9 & $-5,8$ \\
\hline 43 Vorb. Baustellenarbeiten, Hoch- u. Tiefbauarbeiten & 28,8 & 23,9 & 2,7 & 2,1 & 22,5 & 25,3 \\
\hline 44 Bauinstallations- und sonstige Bauarbeiten & 58,1 & 21,3 & 5,6 & 1,9 & 62,2 & 9,7 \\
\hline 45 Handelsleist. mit Kfz; Rep. an $\mathrm{Kfz}$; Tankleistungen & 14,2 & 1,1 & 2,1 & 0,1 & 138,1 & $-125,0$ \\
\hline 46 Handelsvermittlungs- und Großhandelsleistungen & 111,5 & 35,6 & 18,7 & 3,2 & 223,0 & $-97,8$ \\
\hline $\begin{array}{l}47 \text { Einzelhandelsleistungen; Reparatur an Gebrauchsgu- } \\
\text { tern }\end{array}$ & 26,3 & 1,0 & 3,9 & 0,1 & 493,2 & $-469,8$ \\
\hline 48 Beherbergungs- und Gaststătten-DL & 3,5 & 0,1 & 0,5 & 0,0 & 215,6 & $-212,5$ \\
\hline 49 Eisenbahn-DL & 2,8 & 0,3 & 0,7 & 0,0 & 34,0 & $-31,6$ \\
\hline $\begin{array}{l}50 \text { Sonst. Landv.leistungen, Transportl. in Rohrfernlei- } \\
\text { tungen }\end{array}$ & 31,0 & 4,0 & 4,6 & 0,4 & 80,9 & $-50,9$ \\
\hline 51 Schifffahrtsleistungen & 1,5 & 0,2 & 0,4 & 0,0 & 3,2 & $-1,9$ \\
\hline 52 Luftfahrtleistungen & 34,5 & 1,6 & 5,1 & 0,1 & 38,5 & $-7,7$ \\
\hline $\begin{array}{l}53 \text { DL bezüglich Hilfs- u. Nebentătigkeiten für den } \\
\text { Verkehr }\end{array}$ & 55,1 & 7,9 & 8,7 & 0,7 & 117,5 & $-63,9$ \\
\hline 54 Nachrichtenubermittlungs-DL & 80,8 & 3,8 & 12,0 & 0,3 & 221,9 & $-149,5$ \\
\hline 55 DL der Kreditinstitute & 102,5 & 5,8 & 14,9 & 0,5 & 301,9 & $-209,0$ \\
\hline 56 DL der Versicherungen (ohne Sozialversicherung) & 31,8 & 1,5 & 5,0 & 0,1 & 225,4 & $-197,2$ \\
\hline 57 DL des Kredit- und Versicherungshilfsgewerbes & 16,7 & 1,0 & 2,6 & 0,1 & 76,8 & $-61,8$ \\
\hline 58 DL des Grundstucks- und Wohnungswesens & 157,2 & 11,5 & 23,2 & 1,0 & 990,8 & $-846,3$ \\
\hline $\begin{array}{l}59 \text { DL der Vermietung beweglicher Sachen (o. Perso- } \\
\text { nal) }\end{array}$ & 129,2 & 6,1 & 18,7 & 0,5 & 86,4 & 29,7 \\
\hline $60 \mathrm{DL}$ der Datenverarbeitung und von Datenbanken & 64,1 & 50,4 & 9,3 & 4,5 & 36,2 & 64,5 \\
\hline 61 Forschungs- und Entwicklungsleistungen & 64,3 & 0,2 & 8,1 & 0,0 & 6,6 & 49,7 \\
\hline 62 Unternehmensbezogene DL & 771,7 & 32,0 & 118,7 & 2,9 & 460,1 & 222,0 \\
\hline 63 DL der offentlichen Verwaltung, Verteidigung & 39,1 & 1,7 & 5,6 & 0,2 & 39,2 & $-4,2$ \\
\hline $64 \mathrm{DL}$ der Sozialversicherung & 0,0 & 0,0 & 0,0 & 0,0 & 0,0 & 0,0 \\
\hline 65 Erziehungs- und Unterrichts-DL & 26,2 & 1,2 & 3,8 & 0,1 & 58,4 & $-34,9$ \\
\hline 66 DL des Gesundheits-, Veterinar- und Sozialwesens & 0,6 & 0,0 & 0,2 & 0,0 & 203,1 & $-202,6$ \\
\hline $\begin{array}{l}67 \text { Abwasser-, Abfallbeseitigungs- u. sonst. Entsor- } \\
\text { gungsleistungen }\end{array}$ & 23,9 & 0,8 & 5,0 & 0,1 & 74,8 & $-55,2$ \\
\hline 68 DL von Interessenvertretungen, Kirchen u.ă. & 15,7 & 0,6 & 2,3 & 0,1 & 22,7 & $-8,7$ \\
\hline 69 Kultur-, Sport- und Unterhaltungs-DL & 39,5 & 1,1 & 5,9 & 0,1 & 131,8 & $-97,1$ \\
\hline 70 Sonstige DL & 28,6 & 1,3 & 4,3 & 0,1 & 119,6 & $-94,1$ \\
\hline $71 \mathrm{DL}$ privater Haushalte & 0,0 & 0,0 & 0,0 & 0,0 & 24,7 & $-24,7$ \\
\hline Summe & $\begin{array}{l}11238 \\
9\end{array}$ & 445,1 & 1522,5 & 39,9 & 6360,4 & 3761,1 \\
\hline
\end{tabular}

Quelle: Eigene Berechnungen

Die Gesamtsumme ergibt sich aus Spalte 1+2 abzüglich den Spalten 3-5. 


\section{Tabelle 50: Sektorale Beschäftigungseffekte für Biopharmazeutika im Re- ferenzszenario (im Vergleich zum Basisszenario)}

\begin{tabular}{|c|c|c|c|c|c|c|}
\hline & $\begin{array}{l}\text { Bio- } \\
\text { pharma }\end{array}$ & $\begin{array}{l}\text { Biop. } \\
\text { Invest. }\end{array}$ & $\begin{array}{l}\text { Substi- } \\
\text { tut } \\
\text { Pharma. }\end{array}$ & $\begin{array}{c}\text { Substi- } \\
\text { tut } \\
\text { Invest. }\end{array}$ & $\begin{array}{l}\text { Nega- } \\
\text { tiver } \\
\text { Kon- } \\
\text { sum }\end{array}$ & $\begin{array}{l}\text { Ge- } \\
\text { samt }\end{array}$ \\
\hline & $\begin{array}{c}\text { Er- } \\
\text { werbs- } \\
\text { tătige }\end{array}$ & $\begin{array}{c}\text { Er- } \\
\text { werbs- } \\
\text { tatige } \\
\end{array}$ & $\begin{array}{c}\text { Er- } \\
\text { werbs- } \\
\text { tătige }\end{array}$ & \begin{tabular}{|c|} 
Er- \\
werbs- \\
tatige \\
\end{tabular} & $\begin{array}{c}\text { Er- } \\
\text { werbs- } \\
\text { tătige }\end{array}$ & $\begin{array}{c}\text { Er- } \\
\text { werbs- } \\
\text { tatige }\end{array}$ \\
\hline 1 Erzeugnisse der Landwirtschaft und Jagd & 87 & 0 & 13 & 0 & 327 & -252 \\
\hline 2 Forstwirtschaftliche Erzeugnisse und DL & 5 & 1 & 1 & $\mathbf{0}$ & 7 & -2 \\
\hline 3 Fische und Fischereierzeugnisse & 0 & 0 & 0 & 0 & 1 & -1 \\
\hline 4 Kohle und Torf & 75 & 2 & 11 & 0 & 58 & 7 \\
\hline 5 Erdől, Erdgas, DL fur Erdচا-, Erdgasgewinnung & 5 & 0 & 1 & 0 & 6 & -1 \\
\hline 6 Uran- und Thoriumerze & 0 & 0 & 0 & 0 & 0 & 0 \\
\hline 7 Erze & 0 & 0 & 0 & 0 & 0 & 0 \\
\hline 8 Steine und Erden, sonstige Bergbauerzeugnisse & 8 & 2 & 7 & 0 & 8 & -6 \\
\hline 9 Nahrungs- und Futtermittel & 316 & 0 & 44 & 0 & 848 & -576 \\
\hline 10 Getrănke & 2 & 0 & 0 & 0 & 28 & -26 \\
\hline 11 Tabakerzeugnisse & 0 & 0 & 0 & 0 & 21 & -21 \\
\hline 12 Textilien & 117 & 1 & 14 & 0 & 29 & 75 \\
\hline 13 Bekleidung & 0 & 0 & 0 & 0 & 28 & -27 \\
\hline 14 Leder und Lederwaren & 0 & 0 & 0 & 0 & 12 & -11 \\
\hline 15 Holz; Holz-, Kork-, Flechtwaren (o. Mobel) & 39 & 17 & 7 & 2 & 51 & -5 \\
\hline 16 Holzstoff, Zellstoff, Papier, Karton, Pappe & 20 & 0 & 3 & $\mathbf{0}$ & 7 & 10 \\
\hline 17 Papier-, Karton- und Pappewaren & 391 & 3 & 53 & 0 & 62 & 279 \\
\hline 18 Verlagserzeugnisse & 38 & 2 & 8 & 0 & 149 & -117 \\
\hline $\begin{array}{l}19 \text { Druckerzeugnisse, bespielte Ton-, Bild- und Daten- } \\
\text { trăger }\end{array}$ & 284 & 10 & 45 & 1 & 244 & 4 \\
\hline $\begin{array}{l}20 \text { Kokereierzeugnisse, Mineralolerzeugnisse, Spalt- } \\
\text { und Brutstoffe }\end{array}$ & 64 & 0 & 2 & 0 & 22 & 41 \\
\hline 21 Pharmazeutische Erzeugnisse & 8943 & 0 & 1627 & 0 & 740 & 6575 \\
\hline $\begin{array}{l}22 \text { Chemische Erzeugnisse (o. pharmazeutische Er- } \\
\text { zeugnisse) }\end{array}$ & 79 & 3 & 137 & 0 & 85 & -141 \\
\hline 23 Gummiwaren & 4 & 2 & 1 & 0 & 9 & -4 \\
\hline 24 Kunststoffwaren & 383 & 17 & 51 & 2 & 85 & 262 \\
\hline 25 Glas und Glaswaren & 105 & 3 & 14 & 0 & 21 & 73 \\
\hline 26 Keramik, bearbeitete Steine und Erden & 38 & 19 & 7 & 3 & 41 & 6 \\
\hline 27 Roheisen, Stahl, Rohre und Halbzeug daraus & 13 & 5 & 2 & 1 & 9 & 7 \\
\hline 28 NE-Metalle und Halbzeug daraus & 5 & 1 & 1 & 0 & 2 & 3 \\
\hline 29 Gießereierzeugnisse & 4 & 4 & 1 & 1 & 5 & 2 \\
\hline 30 Metallerzeugnisse & 486 & 113 & 69 & 15 & 192 & 322 \\
\hline 31 Maschinen & 70 & 233 & 16 & 31 & 72 & 184 \\
\hline $\begin{array}{l}32 \text { Büromaschinen, Datenverarbeitungsgerate u. - } \\
\text { einrichtungen }\end{array}$ & 5 & 2 & 1 & 0 & 2 & 4 \\
\hline 33 Gerăte der Elektrizitătserzeugung, -verteilung u.ă. & 44 & 58 & 7 & 8 & 50 & 37 \\
\hline $\begin{array}{l}34 \text { Nachrtechn., Rundf.- und Fernsehgerăte, elektron. } \\
\text { Bauelemente }\end{array}$ & 2 & 5 & 0 & 1 & 3 & 3 \\
\hline $\begin{array}{l}35 \text { Medizin-, mess-, regelungstechn., optische Erzeug- } \\
\text { nisse; Uhren }\end{array}$ & 75 & 58 & 10 & 8 & 30 & 85 \\
\hline 36 Kraftwagen und Kraftwagenteile & 27 & 1 & 4 & 0 & 184 & -160 \\
\hline
\end{tabular}




\begin{tabular}{|c|c|c|c|c|c|c|}
\hline & $\begin{array}{l}\text { Bio- } \\
\text { pharma }\end{array}$ & $\begin{array}{l}\text { Biop. } \\
\text { Invest. }\end{array}$ & $\begin{array}{c}\text { Substi- } \\
\text { tut } \\
\text { Pharma. }\end{array}$ & $\begin{array}{l}\text { Substi- } \\
\text { tut } \\
\text { Invest. }\end{array}$ & $\begin{array}{l}\text { Nega- } \\
\text { tiver } \\
\text { Kon- } \\
\text { sum }\end{array}$ & $\begin{array}{c}\text { Ge- } \\
\text { samt }\end{array}$ \\
\hline $\begin{array}{l}37 \text { Sonstige Fahrzeuge (Wasser-, Schienen-, Luftfahr- } \\
\text { zeuge u.a.) }\end{array}$ & 3 & 0 & 1 & 0 & 7 & -4 \\
\hline $\begin{array}{l}38 \text { Möbel, Schmuck, Musikinstrumente, Sportgerăte, } \\
\text { Spielwaren u.a. }\end{array}$ & 1 & 41 & 0 & 6 & 151 & -114 \\
\hline 39 Sekundärrohstoffe & 12 & 1 & 3 & 0 & 12 & -2 \\
\hline $\begin{array}{l}40 \text { Elektrizităt, Fernwărme, DL der Elektrizitäts- u. } \\
\text { Fernwărmevers. }\end{array}$ & 87 & 2 & 13 & 0 & 71 & 4 \\
\hline 41 Gase, DL der Gasversorgung & 41 & 1 & 7 & 0 & 48 & -12 \\
\hline 42 Wasser und DL der Wasserversorgung & 22 & 1 & 4 & 0 & 26 & -7 \\
\hline 43 Vorb. Baustellenarbeiten, Hoch- $u$. Tiefbauarbeiten & 211 & 176 & 30 & 24 & 151 & 182 \\
\hline 44 Bauinstallations- und sonstige Bauarbeiten & 431 & 158 & 63 & 21 & 421 & 84 \\
\hline 45 Handelsleist. mit $\mathrm{Kfz}$; Rep. an $\mathrm{Kfz}$; Tankleistungen & 129 & 10 & 29 & 1 & 1145 & -1036 \\
\hline 46 Handelsvermittlungs- und Großhandelsleistungen & 465 & 148 & 117 & 20 & 849 & -373 \\
\hline $\begin{array}{l}47 \text { Einzelhandelsleistungen; Reparatur an Gebrauchs- } \\
\text { gutern }\end{array}$ & 410 & 15 & 90 & 2 & 6974 & -6641 \\
\hline 48 Beherbergungs- und Gaststătten-DL & 65 & 2 & 15 & 0 & 3655 & -3603 \\
\hline 49 Eisenbahn-DL & 17 & 2 & 6 & 0 & 189 & -176 \\
\hline $\begin{array}{l}50 \text { Sonst. Landv.leistungen, Transportl. in Rohrfern- } \\
\text { leitungen }\end{array}$ & 367 & 48 & 82 & 6 & 874 & -547 \\
\hline 51 Schifffahrtsleistungen & 0 & 0 & 0 & 0 & 0 & 0 \\
\hline 52 Luftfahrtleistungen & 71 & 3 & 16 & 0 & 73 & -15 \\
\hline $\begin{array}{l}53 \text { DL bezuglich Hilfs- u. Nebentătigkeiten fur den } \\
\text { Verkehr }\end{array}$ & 151 & 22 & 36 & 3 & 294 & -160 \\
\hline 54 Nachrichtenubermittlungs-DL & 136 & 6 & 30 & 1 & 341 & -229 \\
\hline $55 \mathrm{DL}$ der Kreditinstitute & 251 & 14 & 55 & 2 & 673 & -464 \\
\hline 56 DL der Versicherungen (ohne Sozialversicherung) & 68 & 3 & 16 & 0 & 437 & -382 \\
\hline 57 DL des Kredit- und Versicherungshilfsgewerbes & 107 & 6 & 25 & 1 & 447 & -359 \\
\hline 58 DL des Grundstucks- und Wohnungswesens & 163 & 12 & 36 & 2 & 935 & -798 \\
\hline $\begin{array}{l}59 \text { DL der Vermietung beweglicher Sachen (o. Perso- } \\
\text { nal) }\end{array}$ & 224 & 11 & 49 & 1 & 138 & 46 \\
\hline 60 DL der Datenverarbeitung und von Datenbanken & 476 & 374 & 104 & 50 & 248 & 448 \\
\hline 61 Forschungs- und Entwicklungsleistungen & 339 & 1 & 64 & 0 & 34 & 241 \\
\hline 62 Unternehmensbezogene DL & 7059 & 293 & 1630 & 39 & 3890 & 1793 \\
\hline 63 DL der offentlichen Verwaltung, Verteidigung & 345 & 15 & 75 & 2 & 317 & -34 \\
\hline 64 DL der Sozialversicherung & 0 & 0 & 0 & 0 & 0 & 0 \\
\hline 65 Erziehungs- und Unterrichts-DL & 389 & 18 & 84 & 2 & 792 & -472 \\
\hline 66 DL des Gesundheits-, Veterinär- und Sozialwesens & 6 & 0 & 2 & 0 & 1866 & -1862 \\
\hline $\begin{array}{l}67 \text { Abwasser-, Abfallbeseitigungs- u. sonst. Entsor- } \\
\text { gungsleistungen }\end{array}$ & 116 & 4 & 37 & 1 & 331 & -249 \\
\hline 68 DL von Interessenvertretungen, Kirchen u.a. & 228 & 9 & 50 & 1 & 301 & -115 \\
\hline 69 Kultur-, Sport- und Unterhaltungs-DL & 327 & 9 & 74 & 1 & 992 & -731 \\
\hline 70 Sonstige DL & 404 & 19 & 92 & 2 & 1536 & -1208 \\
\hline $71 \mathrm{DL}$ privater Haushalte & 0 & 0 & 0 & 0 & 677 & -677 \\
\hline Summe & 24856 & 1990 & 5088 & 268 & 32336 & -10847 \\
\hline
\end{tabular}

Quelle: Eigene Berechnungen

Die Gesamtsumme ergibt sich aus Spalte 1+2 abzüglich den Spalten 3-5. 


\section{Tabelle 51: Sektorale Beschäftigungseffekte für Biopharmazeutika im HD- Szenario (im Vergleich zum Basisszenario)}

\begin{tabular}{|c|c|c|c|c|c|c|}
\hline & $\begin{array}{l}\text { Bio- } \\
\text { pharma }\end{array}$ & $\begin{array}{l}\text { Biop. } \\
\text { Invest. }\end{array}$ & \begin{tabular}{|c|} 
Substi- \\
tut \\
Phar- \\
ma. \\
\end{tabular} & $\begin{array}{l}\text { Substi- } \\
\text { tut } \\
\text { Invest. }\end{array}$ & $\begin{array}{c}\text { Nega- } \\
\text { tiver } \\
\text { Kon- } \\
\text { sum } \\
\end{array}$ & $\begin{array}{l}\text { Ge- } \\
\text { samt }\end{array}$ \\
\hline & $\begin{array}{c}\text { Er- } \\
\text { werbs- } \\
\text { tatige }\end{array}$ & $\begin{array}{c}\text { Er- } \\
\text { werbs- } \\
\text { tătige }\end{array}$ & \begin{tabular}{|c|} 
Er- \\
werbs- \\
tătige
\end{tabular} & $\begin{array}{c}\text { Er- } \\
\text { werbs- } \\
\text { tattige }\end{array}$ & $\begin{array}{c}\text { Er- } \\
\text { werbs } \\
\text {-tătige }\end{array}$ & $\begin{array}{c}\text { Er- } \\
\text { werbs- } \\
\text { tattige }\end{array}$ \\
\hline 1 Erzeugnisse der Landwirtschaft und Jagd & 131 & 1 & 13 & 0 & 539 & -420 \\
\hline 2 Forstwirtschaftliche Erzeugnisse und DL & 7 & 2 & 1 & 0 & 11 & -3 \\
\hline 3 Fische und Fischereierzeugnisse & 0 & 0 & 0 & 0 & 2 & -1 \\
\hline 4 Kohle und Torf & 113 & 3 & 11 & 0 & 96 & 9 \\
\hline 5 Erdol, Erdgas, DL für Erdচا-, Erdgasgewinnung & 7 & 1 & 1 & 0 & 9 & -2 \\
\hline 6 Uran- und Thoriumerze & 0 & 0 & 0 & 0 & 0 & 0 \\
\hline 7 Erze & 0 & 0 & 0 & 0 & 0 & 0 \\
\hline 8 Steine und Erden, sonstige Bergbauerzeugnisse & 12 & 3 & 7 & 0 & 13 & -6 \\
\hline 9 Nahrungs- und Futtermittel & 475 & 1 & 44 & 0 & 1398 & -967 \\
\hline 10 Getränke & 3 & 0 & 0 & 0 & 46 & -43 \\
\hline 11 Tabakerzeugnisse & 0 & 0 & 0 & 0 & 34 & -34 \\
\hline 12 Textilien & 176 & 1 & 14 & 0 & 48 & 115 \\
\hline 13 Bekleidung & 0 & 0 & 0 & 0 & 45 & -45 \\
\hline 14 Leder und Lederwaren & 0 & 0 & 0 & 0 & 19 & -19 \\
\hline 15 Holz; Holz-, Kork-, Flechtwaren (o. Mobel) & 58 & 25 & 7 & 2 & 84 & -10 \\
\hline 16 Holzstoff, Zellstoff, Papier, Karton, Pappe & 30 & 1 & 3 & 0 & 11 & 16 \\
\hline 17 Papier-, Karton- und Pappewaren & 587 & 4 & 53 & 0 & 99 & 439 \\
\hline 18 Verlagserzeugnisse & 57 & 3 & 8 & 0 & 245 & -194 \\
\hline $\begin{array}{l}19 \text { Druckerzeugnisse, bespielte Ton-, Bild- und Daten- } \\
\text { trager }\end{array}$ & 427 & 15 & 45 & 1 & 400 & -4 \\
\hline $\begin{array}{l}20 \text { Kokereierzeugnisse, Mineralölerzeugnisse, Spalt- } \\
\text { und Brutstoffe }\end{array}$ & 96 & 1 & 2 & 0 & 36 & 59 \\
\hline 21 Pharmazeutische Erzeugnisse & 13422 & 0 & 1627 & 0 & 1114 & 10681 \\
\hline $\begin{array}{l}22 \text { Chemische Erzeugnisse (o. pharmazeutische Er- } \\
\text { zeugnisse) }\end{array}$ & 119 & 4 & 137 & 0 & 131 & -146 \\
\hline 23 Gummiwaren & 5 & 2 & 1 & 0 & 14 & -7 \\
\hline 24 Kunststoffwaren & 574 & 26 & 51 & 2 & 138 & 410 \\
\hline 25 Glas und Glaswaren & 158 & 4 & 14 & 0 & 34 & 114 \\
\hline 26 Keramik, bearbeitete Steine und Erden & 58 & 28 & 7 & 3 & 67 & 9 \\
\hline 27 Roheisen, Stahl, Rohre und Halbzeug daraus & 20 & 8 & 2 & 1 & 15 & 10 \\
\hline 28 NE-Metalle und Halbzeug daraus & 7 & 2 & 1 & 0 & 4 & 4 \\
\hline 29 Gießereierzeugnisse & 6 & 7 & 1 & 1 & 9 & 2 \\
\hline 30 Metallerzeugnisse & 729 & 169 & 69 & 15 & 313 & 502 \\
\hline 31 Maschinen & 106 & 349 & 16 & 31 & 119 & 289 \\
\hline $\begin{array}{l}32 \text { Büromaschinen, Datenverarbeitungsgerate u. - } \\
\text { einrichtungen }\end{array}$ & 8 & 3 & 1 & 0 & 3 & 7 \\
\hline 33 Gerăte der Elektrizitătserzeugung, -verteilung u.ă. & 66 & 88 & 7 & 8 & 81 & 57 \\
\hline $\begin{array}{l}34 \text { Nachrtechn., Rundf.- und Fernsehgerăte, elektron. } \\
\text { Bauelemente }\end{array}$ & 2 & 8 & 0 & 1 & 6 & 4 \\
\hline $\begin{array}{l}35 \text { Medizin-, mess-, regelungstechn., optische Erzeug- } \\
\text { nisse; Uhren }\end{array}$ & 112 & 87 & 10 & 8 & 50 & 133 \\
\hline $36 \mathrm{Kraftwagen}$ und Kraftwagenteile & 40 & 2 & $\overline{4}$ & $\overline{0}$ & 304 & -267 \\
\hline
\end{tabular}




\begin{tabular}{|c|c|c|c|c|c|c|}
\hline & $\begin{array}{c}\text { Bio- } \\
\text { pharma }\end{array}$ & $\begin{array}{l}\text { Biop. } \\
\text { Invest. }\end{array}$ & $\begin{array}{c}\text { Substi- } \\
\text { tut } \\
\text { Phar- } \\
\text { ma. }\end{array}$ & $\begin{array}{l}\text { Substi- } \\
\text { tut } \\
\text { Invest. }\end{array}$ & $\begin{array}{c}\text { Nega- } \\
\text { tiver } \\
\text { Kon- } \\
\text { sum } \\
\end{array}$ & $\begin{array}{l}\text { Ge- } \\
\text { samt }\end{array}$ \\
\hline $\begin{array}{l}37 \text { Sonstige Fahrzeuge (Wasser-, Schienen-, Luftfahr- } \\
\text { zeuge u.a.) }\end{array}$ & 5 & 0 & 1 & 0 & 11 & -7 \\
\hline $\begin{array}{l}38 \text { Mobel, Schmuck, Musikinstrumente, Sportgerăte, } \\
\text { Spielwaren u.a. }\end{array}$ & 2 & 62 & 0 & 6 & 250 & -192 \\
\hline 39 Sekundărrohstoffe & 18 & 1 & 3 & 0 & 20 & -4 \\
\hline $\begin{array}{l}40 \text { Elektrizităt, Fernwărme, DL der Elektrizităts- u. } \\
\text { Fernwărmevers. }\end{array}$ & 130 & 3 & 13 & 0 & 116 & 3 \\
\hline 41 Gase, DL der Gasversorgung & 61 & 2 & 7 & 0 & 78 & -22 \\
\hline 42 Wasser und DL der Wasserversorgung & 33 & 1 & 4 & 0 & 43 & -13 \\
\hline 43 Vorb. Baustellenarbeiten, Hoch- u. Tiefbauarbeiten & 317 & 263 & 30 & 24 & 248 & 279 \\
\hline 44 Bauinstallations- und sonstige Bauarbeiten & 646 & 237 & 63 & 21 & 691 & 108 \\
\hline 45 Handelsleist. mit $\mathrm{Kfz}$; Rep. an $\mathrm{Kfz}$; Tankleistungen & 194 & 15 & 29 & 1 & 1891 & -1711 \\
\hline 46 Handelsvermittlungs- und Großhandelsleistungen & 698 & 223 & 117 & 20 & 1396 & -612 \\
\hline $\begin{array}{l}47 \text { Einzelhandelsleistungen; Reparatur an Gebrauchs- } \\
\text { gutern }\end{array}$ & 615 & 23 & 90 & 2 & 11518 & -10973 \\
\hline 48 Beherbergungs- und Gaststătten-DL & 98 & 4 & 15 & 0 & 6040 & -5953 \\
\hline 49 Eisenbahn-DL & 26 & 3 & 6 & 0 & 312 & -290 \\
\hline $\begin{array}{l}50 \text { Sonst. Landv.leistungen, Transportl. in Rohrfern- } \\
\text { leitungen }\end{array}$ & 551 & 72 & 82 & 6 & 1439 & -904 \\
\hline 51 Schifffahrtsleistungen & 0 & 0 & 0 & 0 & 0 & 0 \\
\hline 52 Luffahrtleistungen & 107 & 5 & 16 & 0 & 120 & -24 \\
\hline $\begin{array}{l}53 \text { DL bezulglich Hilfs- u. Nebentattigkeiten fur den } \\
\text { Verkehr }\end{array}$ & 227 & 32 & 36 & 3 & 483 & -263 \\
\hline 54 Nachrichtenubermittlungs-DL & 205 & 10 & 30 & 1 & 561 & -378 \\
\hline 55 DL der Kreditinstitute & 376 & 21 & 55 & 2 & 1108 & -767 \\
\hline 56 DL der Versicherungen (ohne Sozialversicherung) & 102 & 5 & 16 & 0 & 721 & -631 \\
\hline 57 DL des Kredit- und Versicherungshilfsgewerbes & 160 & 9 & 25 & 1 & 737 & -593 \\
\hline 58 DL des Grundstucks- und Wohnungswesens & 245 & 18 & 36 & 2 & 1543 & -1318 \\
\hline $\begin{array}{l}59 \text { DL der Vermietung beweglicher Sachen (o. Perso- } \\
\text { nal) }\end{array}$ & 336 & 16 & 49 & 1 & 225 & 77 \\
\hline 60 DL der Datenverarbeitung und von Datenbanken & 715 & 562 & 104 & 50 & 403 & 719 \\
\hline 61 Forschungs- und Entwicklungsleistungen & 509 & 1 & 64 & 0 & 53 & 394 \\
\hline 62 Unternehmensbezogene DL & 10595 & 439 & 1630 & 39 & 6317 & 3048 \\
\hline 63 DL der offentlichen Verwaltung, Verteidigung & 518 & 23 & 75 & 2 & 519 & -55 \\
\hline 64 DL der Sozialversicherung & 0 & 0 & 0 & 0 & 0 & 0 \\
\hline 65 Erziehungs- und Unterrichts-DL & 584 & 27 & 84 & 2 & 1303 & -779 \\
\hline 66 DL des Gesundheits-, Veterinarr- und Sozialwesens & 9 & 1 & 2 & 0 & 3084 & -3077 \\
\hline $\begin{array}{l}67 \text { Abwasser-, Abfallbeseitigungs- u. sonst. Entsor- } \\
\text { gungsleistungen }\end{array}$ & 174 & 6 & 37 & 1 & 545 & -402 \\
\hline 68 DL von Interessenvertretungen, Kirchen u.ă. & 342 & 14 & 50 & 1 & 495 & -190 \\
\hline 69 Kultur-, Sport- und Unterhaltungs-DL & 490 & 14 & 74 & 1 & 1634 & -1204 \\
\hline 70 Sonstige $\mathrm{DL}$ & 606 & 28 & 92 & 2 & 2533 & -1993 \\
\hline $71 \mathrm{DL}$ privater Haushalte & 0 & 0 & 0 & 0 & 1118 & -1118 \\
\hline Summe & 37306 & 2986 & 5088 & 268 & 53092 & -18156 \\
\hline
\end{tabular}

Quelle: Eigene Berechnungen

Die Gesamtsumme ergibt sich aus Spalte 1+2 abzüglich den Spalten 3-5. 


\section{HOHENHEIMER VOLKSWIRTSCHAFTLICHE SCHRIFTEN}

Band 1 Walter Deffaa: Anonymisierte Befragungen mit zufallsverschlüsselten Antworten. Die Randomized-Response-Technik (RRT). Methodische Grundlagen, Modelle und Anwendungen. 1982.

Band 2 Thomas Michael Baum: Staatsverschuldung und Stabilisierungspolitik in der Demokratie. Zur neoinstitutionalistischen Kritik der keynesianischen Fiskalpolitik. 1982.

Band 3 Klaus Schröter: Die wettbewerbspolitische Behandlung der leitungsgebundenen Energiewirtschaft. Dargestellt am Beispiel der Fernwärmewirtschaft der Bundesrepublik Deutschland. 1986.

Band 4 Hugo Mann: Theorie und Politik der Steuerreform in der Demokratie. 1987.

Band 5 Max Christoph Wewel: Intervallarithmetische Dependenzanalyse in der Ökonometrie. Ein konjekturaler Ansatz. 1987.

Band 6 Heinrich Pascher: Die U.S.-amerikanische Deregulation Policy im Luftverkehrs- und Bankenbereich. 1987.

Band 7 Harald Lob: Die Entwicklung der französischen Wettbewerbspolitik bis zur Verordnung Nr. 86-1243 vom 01. Dezember 1986. Eine exemplarische Untersuchung der Erfassung der Behinderungsstrategie auf der Grundlage des Konzepts eines wirksamen Wettbewerbs. 1988.

Band 8 Ulich Kirschner: Die Erfassung der Nachfragemacht von Handelsunternehmen. Eine Analyse der ökonomischen Beurteilungskriterien und der wettbewerbsrechtlichen Instrumente im Bereich der Verhaltenskontrolle.1988.

Band 9 Friedhelm Herb: Marktwirtschaftliche Innovationspolitik. 1988.

Band 10 Claus Schnabel: Zur ökonomischen Analyse der Gewerkschaften in der Bundesrepublik Deutschland. Theoretische und empirische Untersuchungen von Mitgliederentwicklung, Verhalten und Einfluß auf wirtschaftliche Größen. 1989.

Band 11 Jan B. Rittaler: Industrial Concentration and the Chicago School of Antitrust Analysis. A Critical Evaluation on the Basis of Effective Competition. 1989.

Band 12 Thomas Märtz: Interessengruppen und Gruppeninteressen in der Demokratie. Zur Theorie des Rent-Seeking. 1990.

Band 13 Andreas Maurer: Statistische Verfahren zur Ermittlung von oligopolistischen Strukturen. 1990.

Band 14 Peter Mendler: Zur ökonomischen und politisch-institutionellen Analyse öffentlicher Kredithilfen. 1992.

Band 15 Heinrich J. Engelke: Die Interpretation der Rundfunkfreiheit des Grundgesetzes: Eine Analyse aus ökonomischer Sicht. 1992.

Band 16 Thomas Fischer: Staat, Recht und Verfassung im Denken von Walter Eucken. Zu den staats- und rechtstheoretischen Grundlagen einer wirtschaftsordnungspolitischen Konzeption. 1993.

Band 17 Stefan Elßer: Innovationswettbewerb. Determinanten und Unternehmensverhalten. 1993.

Band 18 Reinhard Scharff: Regionalpolitik und regionale Entwicklungspotentiale. Eine kritische Analyse. 1993.

Band 19 Karin Beckmann: Probleme der Regionalpolitik im Zuge der Vollendung des Europäischen Binnenmarktes. Eine ökonomische Analyse. 1995. 
Band 20 Bernd Nolte: Engpaßfaktoren der Innovation und Innovationsinfrastruktur. Eine theoretische und empirische Analyse für ländliche Wirtschaftsräume in Baden-Württemberg. 1996.

Band 21 Klaus-Rainer Brintzinger: Die Nationalökonomie an den Universitäten Freiburg, Heidelberg und Tübingen 1918 - 1945. Eine institutionenhistorische, vergleichende Studie der wirtschaftswissenschaftlichen Fakultäten und Abteilungen südwestdeutscher Universitäten. 1996.

Band 22 Steffen Binder: Die Idee der Konsumentensouveränität in der Wettbewerbstheorie. Teleokratische vs. nomokratische Auffassung. 1996.

Band 23 Alexander Burger: Deregulierungspotentiale in der Gesetzlichen Rentenversicherung. Reformnotwendigkeiten versus Reformmöglichkeiten. 1996.

Band 24 Burkhard Scherer: Regionale Entwicklungspolitik. Konzeption einer dezentralisierten und integrierten Regionalpolitik. 1997.

Band 25 Frauke Wolf: Lorenzkurvendisparität. Neuere Entwicklungen, Erweiterungen und Anwendungen. 1997.

Band 26 Hans Pitlik: Politische Ökonomie des Föderalismus. Föderative Kompetenzverteilung im Lichte der konstitutionellen Ökonomik. 1997.

Band 27 Stephan Seiter: Der Beitrag Nicholas Kaldors zur Neuen Wachstumstheorie. Eine vergleichende Studie vor dem Hintergrund der Debatte über den Verdoorn-Zusammenhang. 1997.

Band 28 André Schmidt: Ordnungspolitische Perspektiven der europäischen Integration im Spannungsfeld von Wettbewerbs- und Industriepolitik. 1998.

Band 29 Bernd Blessin: Innovations- und Umweltmanagement in kleinen und mittleren Unternehmen. Eine theoretische und empirische Analyse. 1998.

Band 30 Oliver Letzgus: Die Ökonomie internationalen Umweltschutzes. 1999.

Band 31 Claudia Hafner: Systemwettbewerb versus Harmonisierung in Europa. Am Beispiel des Arbeitsmarktes. 1999.

Band 32 Jürgen Kulle: Ökonomie der Musikindustrie. Eine Analyse der körperlichen und unkörperlichen Musikverwertung mit Hilfe von Tonträgern und Netzen. 1998.

Band 33 Michael Ganske: Intertemporale Aspekte von Staatsverschuldung und Außenhandel. 1999.

Band 34 Margit Ströbele: Die Deregulierungswirkungen der europäischen Integration. Das Beispiel der Sondermärkte. 1999.

Band 35 Marion Benesch: Devisenmarktinterventionen in Theorie und Praxis. Eine umfassende Analyse ihrer Zielsetzungen, Wirkungsweisen und wirtschaftspolitischen Bedeutung. 1999.

Band 36 Torsten Gruber: Unterschiedliche geldpolitische Transmissionsmechanismen und Stabilitätskulturen als mögliche Ursachen geldpolitischer Spannungen in der Europäischen Währungsunion. 2000.

Band 37 Bertram Melzig-Thiel: Arbeit in der Informationsgesellschaft. Chancen und Risiken neuer Informations- und Kommunikationstechnologien für die Beschäftigung. 2000.

Band 38 Annette Fritz: Die Entsorgungswirtschaft im Spannungsfeld zwischen Abfallpolitik und Kartellrecht. Eine industrieökonomische Branchenstudie. 2001.

Band 39 Harald Strotmann: Arbeitsplatzdynamik in der baden-württembergischen Industrie. Eine Analyse mit amtlichen Betriebspaneldaten. 2002. 
Band 40 Dietrich Benner: Qualitätsungewißheit bei Gütern mit Vertrauenseigenschaften. Entwicklung und Anwendung eines entscheidungstheoretisch fundierten Analyserahmens. 2002.

Band 41 Jürgen M. Schechler: Sozialkapital und Netzwerkökonomik. 2002.

Band 42 Kay-Uwe May: Haushaltskonsolidierung durch Ausgabekürzungen. Restriktionen und Strategien. 2002.

Band 43 Peter Kühnl: Der Wechselkurs als Zwischenziel der Geldpolitik im Aufholprozess. Die monetärkeynesianische Entwicklungsstrategie der Berliner Schule vor dem Hintergrund der makroökonomischen Entwicklung ausgewählter Länder Mittel- und Osteuropas. 2003.

Band 44 Steffen Wirth: Nichtparametrische Analyse von Bildungsertragsraten. Neuere Entwicklungen und Anwendungen. 2003.

Band 45 Bernhard Holwegler: Innovation, Diffusion und Beschäftigung. Die ökonomische Theorie der Technologiediffusion und ihr Beitrag zur Erklärung technologischer Arbeitslosigkeit. 2003.

Band 46 Guntram R. M. Hepperle: Zukunftsorientierte Industriepolitik. Möglichkeiten und Grenzen. 2004.

Band 47 Udo Vullhorst: Stabilisierungspolitik bei supranationaler Geldpolitik und nationaler Fiskalpolitik. Eine spieltheoretische Betrachung. 2004.

Band 48 Matthias Rösch: Die Bedeutung von Investivlöhnen und Gewinnbeteiligungen für Einkommensverteilung und Beschäftigung. 2004.

Band 49 Michael Bubik: Erfolgskriterien für Unternehmenszusammenschlüsse. Eine theoretische und exemplarische Analyse. 2005.

Band 50 Jörg Weltin: Internationale Unternehmensbesteuerung. Allokation der Besteuerungsrechte unter veränderten Rahmenbedingungen. 2005.

Band 51 Susanne Reichart: Zum Konvergenzprozess der mittel- und osteuropäischen EU-Beitrittsländer. 2005.

Band 52 Daniel Hartmann: Geldpolitik und Beschäftigung. Die geldpolitische Strategie der Federal Reserve: Vorbild oder Auslaufmodell? 2005.

Band 53 Marc Peter Radke: Explaining Financial Crises. A Cyclical Approach. 2005.

Band 54 Katja Hölsch: Umverteilungseffekte in Europa. Eine Analyse für ausgewählte Länder. 2006.

Band 55 Ulrike Lehr: Contingent Valuation Daten und Bayes'sche Verfahren. Ein Vorschlag zur Verbesserung von Umweltbewertung und Nutzentransfer. 2006.

Band 56 Jutta Maute: Hyperinflation, Currency Board, and Bust. The Case of Argentina. 2006.

Band 57 Michael Knittel: Geldpolitik und Stabilität des Bankensystems. Das Liquiditätsproblem aus Sicht der Theoriegeschichte und der gegenwärtigen Finanzmarktentwicklung. 2007.

Band 58 Oliver Frör: Rationality Concepts in Environmental Valuation. 2007.

Band 59 Jochen Gert Arend Wiegmann: Produktivitätsentwicklung in Deutschland. 2008.

Band 60 Nicola Meier: China - The New Developmental State? An Empirical Analysis of the Automotive Industry. 2009.

Band 61 Carsten H. Wander: Logistik und Wettbewerb. Zur Rolle logistischer (Re-)Organisation in einer wettbewerbsbasierten Marktwirtschaft. 2009.

Band 62 Sven Wydra: Produktions- und Beschäftigungseffekte neuer Technologien. Am Beispiel der Biotechnologie. 2010. 
Sven Wydra - 978-3-631-75392-7

Downloaded from PubFactory at 01/11/2019 05:32:25AM

via free access 
Sven Wydra - 978-3-631-75392-7

Downloaded from PubFactory at 01/11/2019 05:32:25AM

via free access 